Supporting Information

\title{
NHC-Catalyzed Cascade Reaction between $\beta$-Methyl Enals and Dienones for Quick Construction of Complex Multicyclic \\ Lactones
}

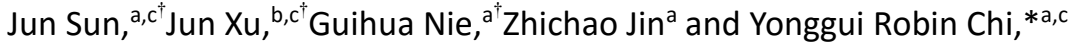

a Laboratory Breeding Base of Green Pesticide and Agricultural Bioengineering, Key Laboratory of Green Pesticide and Agricultural Bioengineering, Ministry of Education, Guizhou University, Huaxi District, Guiyang 550025, China.

${ }^{b}$ Guizhou University of Traditional Chinese Medicine, Huaxi District, Guiyang 550025, China.

${ }^{c}$ Division of Chemistry \& Biological Chemistry, School of Physical \& Mathematical Sciences, Nanyang Technological University, Singapore 637371, Singapore.
\end{abstract}

Email: robinchi@ntu.edu.sg

\section{Table of Contents}

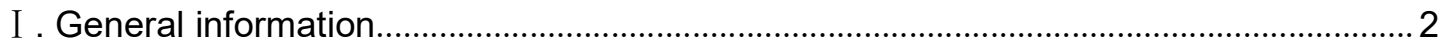

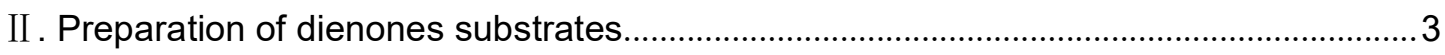

III. General procedure

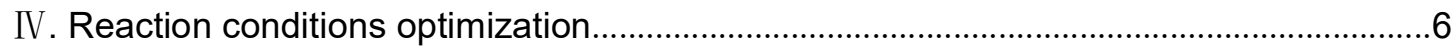

V. Stereochemistry determination via X-ray crystallographic analysis.................................

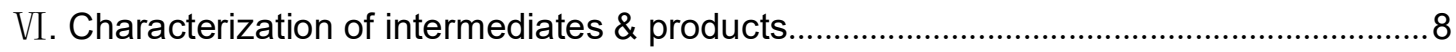

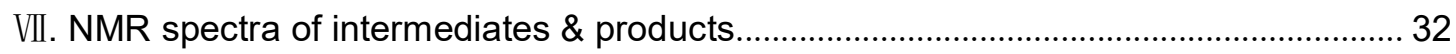

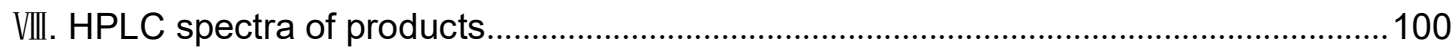




\section{General information}

Commercially available materials purchased from J\&K or Aladdin were used as received. THF was distilled over sodium. Unless otherwise specified, all reactions were carried out under an atmosphere of nitrogen in $10 \mathrm{~mL}$ dry Schlenk tube. All nuclear magnetic resonance (NMR) spectra were recorded on a Bruker $(300 \mathrm{MHz})$, Bruker $(400$ $\mathrm{MHz})$, Bruker $(500 \mathrm{MHz})$ or on a Bruker $(600 \mathrm{MHz})$ spectrometer. Chemical shifts were recorded in parts per million (ppm, $\delta$ ) relative to tetramethylsilane $(\delta 0.00)$ or chloroform $(\delta$ $=7.26$, singlet). ${ }^{1} \mathrm{H}$ NMR splitting patterns are designated as singlet (s), doublet (d), triplet $(\mathrm{t})$, quartet (q), dd (doublet of doublets); $m$ (multiplets), and etc. All first-order splitting patterns were assigned on the basis of the appearance of the multiplet. Splitting patterns that could not be easily interpreted are designated as multiplet $(\mathrm{m})$ or broad (br). The melting points (m.p.) of the title compounds were determined when left untouched on an XT-4-MP apparatus from Beijing Tech. Instrument Co. (Beijing, China). High resolution mass spectral analysis (HRMS) was performed on a quadrupole/electrostatic field orbitrap mass spectrometer. Absolute configuration of the products was determined by X-ray crystallography. HPLC analyses were measured on Waters systems with Empower3 system controller, Alliance column heater, and 2998 Diode Array Waters 2489 UV/Vis detector. Chiralcel brand chiral columns from Daicel Chemical Industries were used with models AD-H in $4.6 \times 250 \mathrm{~mm}$ size. The racemic products used to determine the er values were synthesized using racemic catalyst. Optical rotations were measured on a Insmark IP-digi Polarimeter in a $1 \mathrm{dm}$ cuvette at $26^{\circ} \mathrm{C}$. The concentration (c) is given in g/100 mL. Analytical thin-layer chromatography (TLC) was carried out on Merck 60 F254 pre-coated silica gel plate $(0.2 \mathrm{~mm}$ thickness). Visualization was performed using a UV lamp. 


\section{Preparation of dienones substrates}

1. Dienones substrates $\mathbf{2}$ were synthesized from substituted-2-hydroxy acetophenones by the combination of slightly modified literature procedure.1-3

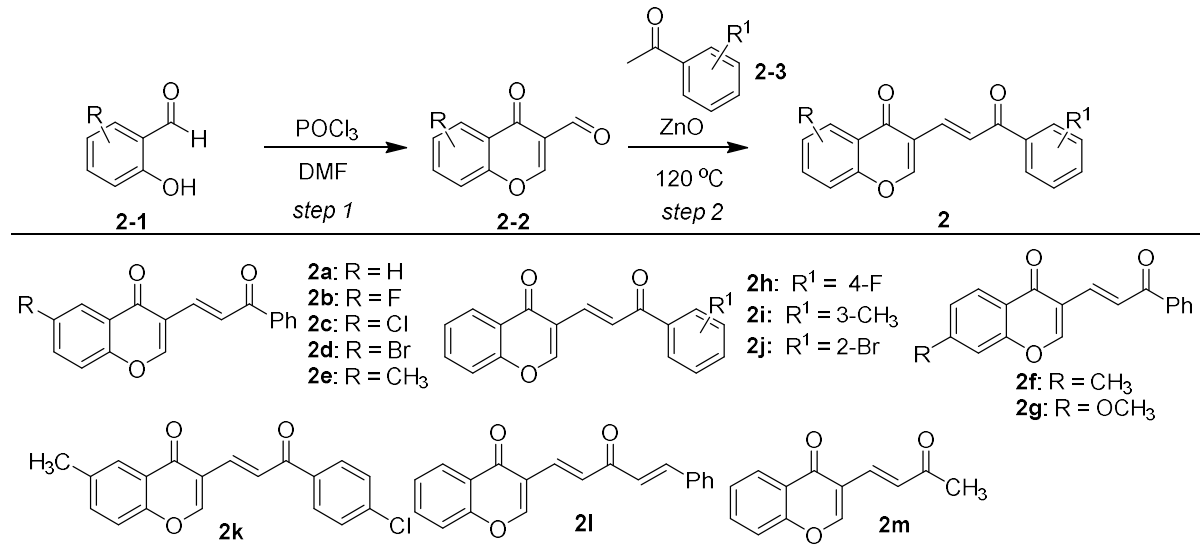

Step 1: o-Hydroxyacetophenone 2-1 $(100 \mathrm{mmol})$ was dissolved in $60 \mathrm{~mL}$ DMF. To this solution, phosphorous oxychloride $(300 \mathrm{mmol})$ was added gradually. The reaction mixture was stirred under cooling (ice-bath), left for an hour, allowed to reach room temperature, left for $24 \mathrm{~h}$ and then poured onto an ice-water mixture with stirring until complete precipitation. The obtained chromon-3-carbaldehyde was filtered off, washed with $5 \mathrm{~mL}$ cold distilled water and finally recrystallized from methanol to afford compound $\mathbf{2 - 2}$.

Step 2: A mixture of 3-formyl benzopyran-4-one 2-2 (10 mmol), acetophenone (10 $\mathrm{mmol})$, for the synthesis of chalcone with $\mathrm{ZnO}(0.1 \mathrm{mmol})$ was heated at $120^{\circ} \mathrm{C}$ (oil bath) for $30 \mathrm{~min}$ under solvent-free conditions. The reaction was monitored via TLC using hexane: ethylacetate (9:1). After the reaction completion, reaction mass was cooled $\left(15^{\circ} \mathrm{C}-20^{\circ} \mathrm{C}\right)$ and stirred with ethanol $(50 \mathrm{~mL})$ for $30 \mathrm{~min}$. Thereafter, the reaction mass was centrifuged for $10 \mathrm{~min}$ at $5000 \mathrm{rpm}$. The supernatant was collected and solvent removed under reduced pressure. The obtained products were purified by re-crystallization from $\mathrm{EtOH}$.

2. Pyranone dienones substrates 7 were synthesized from substituted-phenylacetone by literature procedure. ${ }^{3.4}$

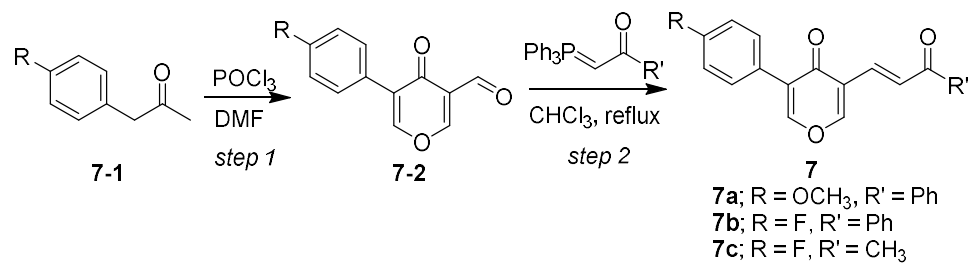

Step 1: Vilsmeier reagent was prepared by mixing ice-cold, dry DMF $(50 \mathrm{~mL})$ and $\mathrm{POCl}_{3}(2.8 \mathrm{~mL}, 30 \mathrm{mmol})$. The mixture was then stirred for $15 \mathrm{~min}$ at room temperature. Compound 7-1 (10 mmol) was dissolved in dry DMF $(5 \mathrm{~mL})$ and added over about $15 \mathrm{~min}$ 
at $0-5^{\circ} \mathrm{C}$. The reaction mixture was stirred for $72 \mathrm{~h}$ at room temperature. The mixture was then added to cold, saturated aq. $\mathrm{K}_{2} \mathrm{CO}_{3}(200 \mathrm{~mL})$ and extracted with diethyl ether $(3 \times 50$ $\mathrm{mL}$ ). The organic layer was washed with water, dried over anhydrous $\mathrm{Na}_{2} \mathrm{SO}_{4}$, and evaporated to afford the crude product, which was column chromatographed over silica gel using hexane/ ethyl acetate (9:1) as eluent to give the compound 7-2.

Step 2: Compound 7-2 $(5 \mathrm{mmol})$ was dissolved in dry $\mathrm{CHCl}_{3}(50 \mathrm{~mL})$ and Witting reagent $(5.5 \mathrm{mmol})$ was added, then the mixture was stirred for $12 \mathrm{~h}$ at reflux (oil bath) . The The reaction mixture was concentrated in vacuo and purified by flash column chromatography on silica gel with $20 \%$ EtOAc in hexanes as an eluent to afford the compound 7.

\section{General procedure.}

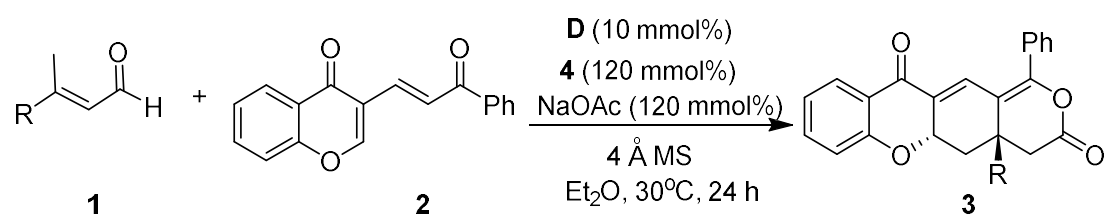

To a dry Schlenk reaction tube equipped with a magnetic stir bar was added aldehydes 1 (0.12 mmol), $2(0.1 \mathrm{mmol})$, triazolium salt $\mathbf{D}(4.1 \mathrm{mg}, 0.01 \mathrm{mmol})$, oxidant 4 (49 $\mathrm{mg}, 0.12$ $\mathrm{mmol}), \mathrm{NaOAc}(9.8 \mathrm{mg}, 0.12 \mathrm{mmol})$ and dry $4 \AA \mathrm{MS}(100 \mathrm{mg})$. The schlenk tube was then closed with septum, evacuated and refilled with $\mathrm{N}_{2}$, freshly distilled anhydrous $\mathrm{Et}_{2} \mathrm{O}(2 \mathrm{~mL})$ was added. The mixture was stirred at $30^{\circ} \mathrm{C}$ (oil bath) for $24 \mathrm{~h}$. After completion of the reaction monitored by TLC, solvent was removed under reduced pressure and the residue was purified via column chromatography on silica gel with Hexane/EtOAc (10: 1) as eluent to afford the products 3 .

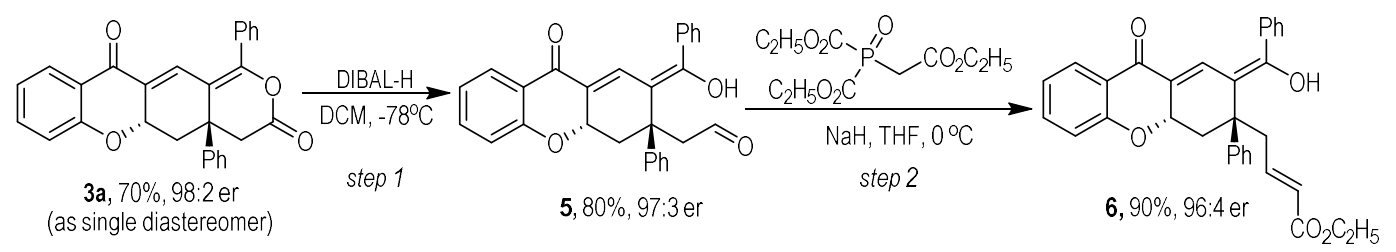

Step 1: Compound 3a $(250 \mathrm{mg}, 0.6 \mathrm{mmol})$ was dissolved in dry $\mathrm{CH}_{2} \mathrm{Cl}_{2}(5 \mathrm{~mL})$, the mixture was cold to $-78{ }^{\circ} \mathrm{C}$, then DIBAL-H $(0.72 \mathrm{mmol})$ was added dropwise at $-78{ }^{\circ} \mathrm{C}$, after that, the mixture was stirred for $2 \mathrm{~h}$. the mixture was quenched with $1 \mathrm{~N} \mathrm{HCl}$, extracted with $\mathrm{CH}_{2} \mathrm{Cl}_{2}(10 \mathrm{~mL} \times 2)$, combined the organic layer, dried over $\mathrm{Na}_{2} \mathrm{SO}_{4}$, filtrated and concentrated to give crude product, the crude product purified by column chromatography to give the compound $\mathbf{5}$ ( $200 \mathrm{mg}, 80 \%$ yield).

Step 2: To a solution of diethyl ((2-ethoxy-2-oxoethyl)phosphoryl)diformate (60 mg, 0.21 $\mathrm{mmol})$ in dry THF $(5 \mathrm{~mL}), \mathrm{NaH}(60 \% \mathrm{w}, 8.5 \mathrm{mg}, 0.21 \mathrm{mmol})$ was added at $0^{\circ} \mathrm{C}$, the mixture was stirred for $30 \mathrm{~min}$ at $0^{\circ} \mathrm{C}$, then Compound $5(60 \mathrm{mg})$ in THF $(1 \mathrm{~mL})$ was added 
dropwise, then the mixture was stirred for $6 \mathrm{~h}$ at $0^{\circ} \mathrm{C}$. the mixture was quenched with saturated $\mathrm{NH}_{4} \mathrm{Cl}$ solution, extracted with EtOAc $(5 \mathrm{~mL} \times 3)$, combined the organic layer, dried over $\mathrm{Na}_{2} \mathrm{SO}_{4}$, filtrated and concentrated to give crude product, the crude product purified by column chromatography to give the compound 6 ( $65 \mathrm{mg}, 90 \%$ yield).

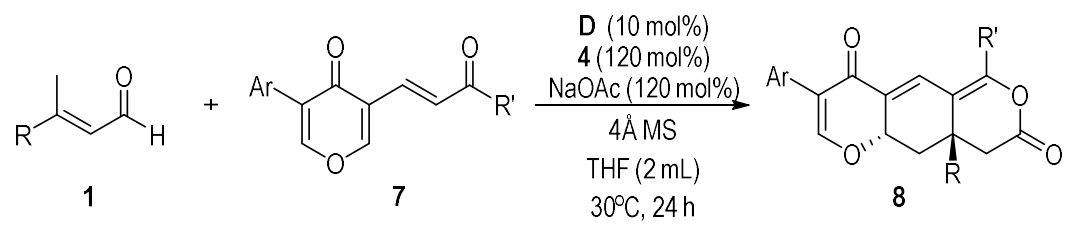

To a dry Schlenk reaction tube equipped with a magnetic stir bar was added aldehydes 1 (0.12 mmol), 7 (0.1 mmol), triazolium salt $\mathbf{D}(4.1 \mathrm{mg}, 0.01 \mathrm{mmol})$, oxidant 4 (49 $\mathrm{mg}, 0.12$ $\mathrm{mmol}), \mathrm{NaOAc}(9.8 \mathrm{mg}, 0.12 \mathrm{mmol})$ and dry $4 \AA \mathrm{MS}(100 \mathrm{mg})$. The schlenk tube was then closed with septum, evacuated and refilled with $\mathrm{N}_{2}$, freshly distilled anhydrous THF $(2 \mathrm{~mL})$ was added. The mixture was stirred at $30^{\circ} \mathrm{C}$ (oil bath) for $24 \mathrm{~h}$. The reaction was removed the solvent under reduced pressure and the residue was purified via column chromatography on silica gel with Hexane/EtOAc (10: 1) as eluent to afford the products 8.

\section{Reference:}

1. Abou-Melha, K. S. Octahedral $\mathrm{Co}(\mathrm{II})$ and $\mathrm{Ni}(\mathrm{II})$ complexes of Schiff bases, semicarbazone and thiosemicarbazone, synthesis, biological, spectral, and thermal studies. J. Coord. Chem. 61, 2053-2067 (2008).

2. Chand, S.; Sandhu, J. S. ZnO Nanoparticles: An efficient green reusable catalyst for the synthesis of 3-formyl benzopyranones chalcones by Claisen-Schmidt reaction under solvent-free condition. Indian J. Chem. B, 54, 1350-1354 (2015).

3. Dang, A.-T.; Miller, D. O.; Dawe, L. N.; Bodwell,G. J. Electron-Deficient Dienes. 5. An Inverse-Electron-Demand Diels-Alder Approach to 2-Substituted 4-Methoxyxanthones and 3,4-Dimethoxyxanthones. Org. Lett. 10, 233-236 (2008).

4. Thomas, A. D.; Josemin; Asokan, C. V. Vilsmeier-Haack reactions of carbonyl compounds: synthesis of substituted pyrones and pyridines. Tetrahedron, 60, 5069-5076 (2004). 


\section{Reaction conditions optimization}

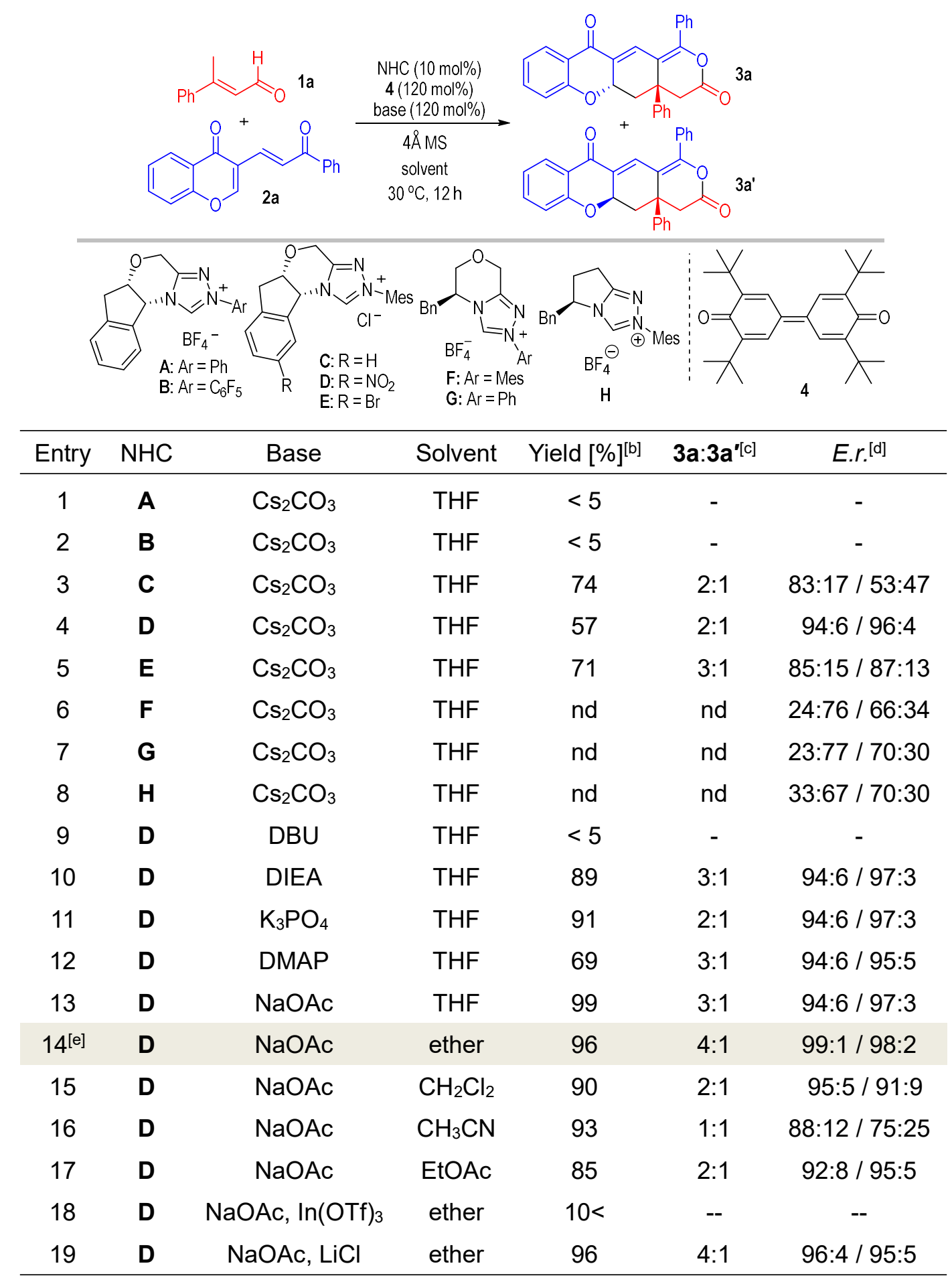

[a] Reaction conditions: $1 \mathrm{a}(0.12 \mathrm{mmol}), 2 \mathrm{a}(0.1 \mathrm{mmol}), \mathrm{NHC}(0.01 \mathrm{mmol})$, base $(0.12 \mathrm{mmol}), 4(0.12$ $\mathrm{mmol})$ and $4 \mathrm{~A} \mathrm{MS}(100 \mathrm{mg})$ in solvent $(2.0 \mathrm{~mL})$ at $30^{\circ} \mathrm{C}$. [b] Isolated yield. [c] Dr values of the products were determined by ${ }^{1} \mathrm{H}$ NMR on the crude reaction mixture. [d] Er values of $\mathbf{3 a}$ and $\mathbf{3 a}$ ' were determined via HPLC on a chiral stationary phase. [e] $24 \mathrm{~h}$. DBU = 1,8-Diazabicyclo[5.4.0]undec-7-ene, DIEA = $\mathrm{N}, \mathrm{N}$-diisopropylethylamine. DMAP $=4$-dimethylaminopyridine. 


\section{Stereochemistry determination via X-ray crystallographic analysis}

The absolute stereochemistry of $\mathbf{3 a}$ was determined by the X-ray diffraction. This crystal was deposited in the Cambridge Crystallographic Data Centre and assigned as CCDC: 1937771.

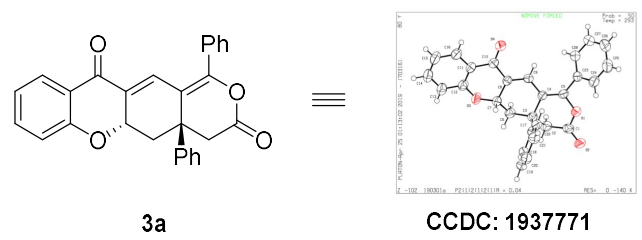

The absolute stereochemistry of $\mathbf{3 a}$ ' was determined by the X-ray diffraction. This crystal was deposited in the Cambridge Crystallographic Data Centre and assigned as CCDC: 1937772.

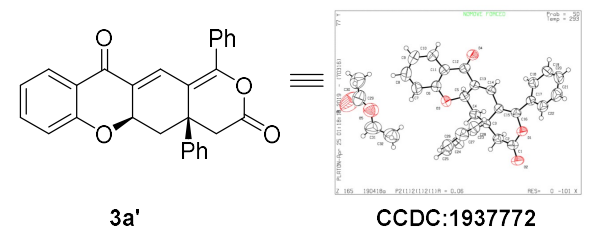

The absolute stereochemistry of $\mathbf{3} \mathbf{m}$ ' was determined by the X-ray diffraction. This crystal was deposited in the Cambridge Crystallographic Data Centre and assigned as CCDC: 1937773.

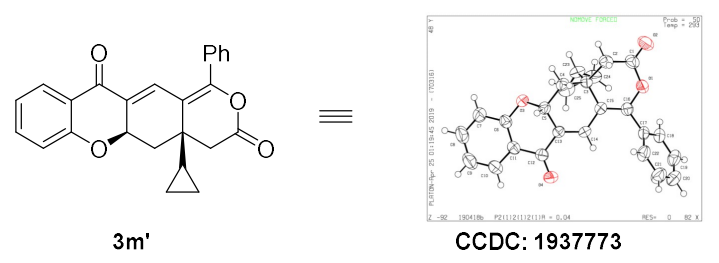

The absolute stereochemistry of $\mathbf{8} \mathbf{b}$ was determined by the X-ray diffraction. This crystal was deposited in the Cambridge Crystallographic Data Centre and assigned as CCDC: 1946481.

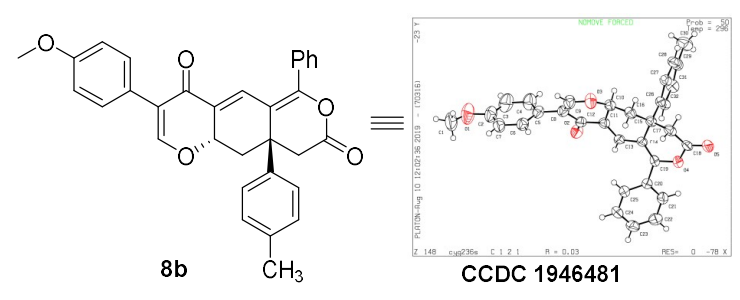




\section{Characterization of intermediates \& products}

(E)-7-methyl-3-(3-oxo-3-phenylprop-1-en-1-yl)-4H-chromen-4-one (2f)<smiles>Cc1ccc2c(=O)c(/C=C/C(=O)c3ccccc3)coc2c1</smiles>

Yellow solid, m.p $131-133^{\circ} \mathrm{C}, 200 \mathrm{mg}, 32 \%$ yield

1H NMR $\left(400 \mathrm{MHz}, \mathrm{CDCl}_{3}\right) \delta 8.68(\mathrm{~d}, \mathrm{~J}=15.3 \mathrm{~Hz}, 1 \mathrm{H}), 8.22$ $8.07(\mathrm{~m}, 4 \mathrm{H}), 7.61-7.45(\mathrm{~m}, 4 \mathrm{H}), 7.29(\mathrm{~d}, \mathrm{~J}=7.1 \mathrm{~Hz}, 2 \mathrm{H}), 2.51$ $(\mathrm{s}, 3 \mathrm{H})$.

$\underline{{ }^{13} \mathrm{C} \mathrm{NMR}}$ (101 MHz, CDCl 3 ) ס 190.7 (s), 176.3 (s), 158.8 (s), 155.6 (s), 145.6 (s), 137.9 (s), 135.5 (s), 133.0 (s), 128.7 (s), 128.6 (s), 127.5 (s), 126.0 (s), 125.6 (s), 122.1 (s), 119.5 (s), 117.9 (s), 21.8 (s).

HRMS (ESI, m/z): Mass calcd for $\mathrm{C}_{19} \mathrm{H}_{15} \mathrm{O}_{3}[\mathrm{M}+\mathrm{H}]^{+}, 291.1016$; found 291.1010.

(E)-7-methoxy-3-(3-oxo-3-phenylprop-1-en-1-yl)-4H-chromen-4-one (2g)<smiles>COc1ccc2c(=O)c(/C=C/C(=O)c3ccccc3)coc2c1</smiles>

Yellow solid, m.p $105-107^{\circ} \mathrm{C}, 500 \mathrm{mg}, 33.3 \%$ yield.

1H NMR $\left(600 \mathrm{MHz}, \mathrm{CDCl}_{3}\right) \delta 8.70(\mathrm{~d}, J=15.3 \mathrm{~Hz}, 1 \mathrm{H}), 8.22$ (dd, $J=8.9,2.4 \mathrm{~Hz}, 1 \mathrm{H}), 8.17-8.10(\mathrm{~m}, 3 \mathrm{H}), 7.64-7.57(\mathrm{~m}$, $1 \mathrm{H}), 7.51(\mathrm{dd}, J=19.0,11.5 \mathrm{~Hz}, 3 \mathrm{H}), 7.06(\mathrm{dd}, J=8.9,2.3 \mathrm{~Hz}$, $1 \mathrm{H}), 6.90(\mathrm{~d}, J=2.3 \mathrm{~Hz}, 1 \mathrm{H}), 3.94(\mathrm{~s}, 3 \mathrm{H})$.

$\underline{{ }^{13} \mathrm{C} \mathrm{NMR}}$ (151 MHz, CDCl$)_{3}$ ) 190.7 (s), 175.7 (s), 164.4 (s), 158.6 (s), 157.2 (s), 137.9 (s), 135.5 (s), 133.0 (s), 128.7 (d, J = $19.6 \mathrm{~Hz}$ ), 127.7 (s), 125.7 (s), 119.6 (s), 118.12 (s), 115.3 (s), $100.4(\mathrm{~s}), 55.9$ (s).

HRMS $(E S I, m / z)$ : Mass calcd for $\mathrm{C}_{19} \mathrm{H}_{15} \mathrm{O}_{4}[\mathrm{M}+\mathrm{H}]^{+}, 307.0965$; found 307.0960 .

(E)-3-(3-oxo-3-(m-tolyl)prop-1-en-1-yl)-4H-chromen-4-one (2i)<smiles>Cc1cccc(C(=O)/C=C/c2coc3ccccc3c2=O)c1</smiles>
Light yellow solid, m.p $135-137^{\circ} \mathrm{C}, 0.8 \mathrm{~g}, 48 \%$ yield.

${ }^{1} \mathrm{H}$ NMR $\left(400 \mathrm{MHz}, \mathrm{CDCl}_{3}\right) \delta 8.66(\mathrm{~d}, J=15.3 \mathrm{~Hz}, 1 \mathrm{H}), 8.32(\mathrm{dd}$, $J=8.0,1.6 \mathrm{~Hz}, 1 \mathrm{H}), 8.23(\mathrm{~d}, J=7.8 \mathrm{~Hz}, 1 \mathrm{H}), 7.92(\mathrm{~d}, J=4.0 \mathrm{~Hz}$, $2 \mathrm{H}), 7.76-7.68(\mathrm{~m}, 1 \mathrm{H}), 7.50(\mathrm{dt}, J=8.0,4.3 \mathrm{~Hz}, 3 \mathrm{H}), 7.43-7.37$

(m, 2H), 2.45 (s, 3H).

13. NMR (101 MHz, CDCl 3 ) ס 190.9 (s), 176.4 (s), 158.9 (s), 155.5 (s), 138.4 (s), 137.9 (s), 135.2 (s), 134.1 (s), 133.8 (s), 129.2 (s), 128.5 (s), 126.3 (s), 126.0 (s), 126.0 (s), 125.9 (s), 124.3 (s), 119.7 (s), 118.2 (s), 21.4 (s).

HRMS (ESI, m/z): Mass calcd for $\mathrm{C}_{19} \mathrm{H}_{15} \mathrm{O}_{3}[\mathrm{M}+\mathrm{H}]^{+}, 291.1016$; found 291.1015.

(E)-3-(3-(2-bromophenyl)-3-oxoprop-1-en-1-yl)-4H-chromen-4-one (2j)<smiles>O=C(/C=C/c1coc2ccccc2c1=O)c1ccccc1Br</smiles>

Light yellow solid, m.p $130-132{ }^{\circ} \mathrm{C}, 1.6 \mathrm{~g}, 65 \%$ yield

1 H NMR $\left(400 \mathrm{MHz}, \mathrm{CDCl}_{3}\right) \delta 8.27(\mathrm{dt}, J=12.1,6.1 \mathrm{~Hz}, 1 \mathrm{H}), 8.19$ $(\mathrm{s}, 1 \mathrm{H}), 8.03(\mathrm{~d}, J=15.8 \mathrm{~Hz}, 1 \mathrm{H}), 7.74-7.61(\mathrm{~m}, 2 \mathrm{H}), 7.53-7.38$ $(\mathrm{m}, 4 \mathrm{H}), 7.36-7.28(\mathrm{~m}, 2 \mathrm{H})$.

${ }^{13} \mathrm{C} \mathrm{NMR}\left(101 \mathrm{MHz}, \mathrm{CDCl}_{3}\right) \delta 194.4$ (s), 175.9 (s), 158.28 (s), 155.5 (s), 141.0 (s), 136.6 (s), 134.2 (s), 133.6 (s), 131.6 (s), 129.4 (s), 129.0 (s), 127.4 (s), 126.4 (s), 126.0 (s), 124.2 (s), 119.7 (s), 119.5 (s), 118.2 (s).

HRMS (ESI, m/z): Mass calcd for $\mathrm{C}_{18} \mathrm{H}_{12} \mathrm{O}_{3} \mathrm{Br}[\mathrm{M}+\mathrm{H}]^{+}$, 354.9964; found 354.9960. 
(E)-3-(3-(4-chlorophenyl)-3-oxoprop-1-en-1-yl)-6-methyl-4H-chromen-4-one (2k)

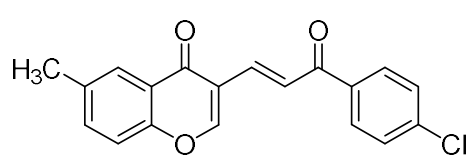

Yellow solid, m.p. $160-162{ }^{\circ} \mathrm{C}, 0.4 \mathrm{~g}, 58 \%$ yield ${ }^{1} \mathrm{H}$ NMR $\left(400 \mathrm{MHz}, \mathrm{CDCl}_{3}\right) \delta 8.66(\mathrm{~d}, \mathrm{~J}=15.3 \mathrm{~Hz}, 1 \mathrm{H})$, $8.19(\mathrm{~s}, 1 \mathrm{H}), 8.10-7.99(\mathrm{~m}, 3 \mathrm{H}), 7.56-7.44(\mathrm{~m}, 4 \mathrm{H}), 7.43-$

$7.39(\mathrm{~m}, 1 \mathrm{H}), 2.50(\mathrm{~s}, 3 \mathrm{H})$.

13. NMR (101 MHz, CDCl ${ }_{3}$ ) ס 189.4 (s), 176.4 (s), 159.2 (s), 153.8 (s), 139.4 (s), 136.3 (s), 136.2 (s), 136.1 (s), 135.4 (s), 130.2 (s), 128.9 (s), 125.6 (s), 125.0 (s), 124.0 (s), 119.3 (s), 118.0 (s), 21.1 (s).

HRMS $(\mathrm{ESI}, \mathrm{m} / \mathrm{z})$ : Mass calcd for $\mathrm{C}_{19} \mathrm{H}_{15} \mathrm{O}_{4}[\mathrm{M}+\mathrm{H}]^{+}, 325.0626$; found 325.0623 .

3-((1E,4E)-3-oxo-5-phenylpenta-1,4-dien-1-yl)-4H-chromen-4-one (2I)

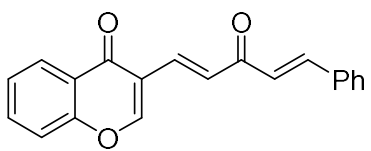

White solid, m.p. $174-176^{\circ} \mathrm{C}, 0.8 \mathrm{~g}, 48 \%$ yield.

1 ${ }^{\mathrm{H} ~ N M R}\left(400 \mathrm{MHz}, \mathrm{CDCl}_{3}\right) \delta 8.31(\mathrm{dd}, J=8.0,1.6 \mathrm{~Hz}, 1 \mathrm{H}), 8.20$ (s, 1H), $8.14(\mathrm{~d}, J=15.5 \mathrm{~Hz}, 1 \mathrm{H}), 7.80-7.69(\mathrm{~m}, 2 \mathrm{H}), 7.65-7.57$

$(\mathrm{m}, 2 \mathrm{H}), 7.49(\mathrm{dd}, J=14.0,7.0 \mathrm{~Hz}, 3 \mathrm{H}), 7.41(\mathrm{dt}, J=6.0,2.5 \mathrm{~Hz}, 3 \mathrm{H}), 7.03(\mathrm{~d}, J=16.1 \mathrm{~Hz}$, $1 \mathrm{H})$.

${ }^{13} \mathrm{C} \mathrm{NMR}\left(101 \mathrm{MHz}, \mathrm{CDCl}_{3}\right) \delta 189.2$ (s), 176.3 (s), 158.6 (s), 155.5 (s), 143.6 (s), 134.8 (s), 134.1 (s), 134.0 (s), 130.5 (s), 129.0 (s), 128.5 (s), 128.1 (s), 126.7 (s), 126.4 (s), 126.0 (s), , 124.25 (s), 119.58 (s), 118.21 (s).

HRMS (ESI, m/z): Mass calcd for $\mathrm{C}_{20} \mathrm{H}_{15} \mathrm{O}_{3}[\mathrm{M}+\mathrm{H}]^{+}, 303.1016$; found 303.1011.

(E)-3-(4-methoxyphenyl)-5-(3-oxo-3-phenylprop-1-en-1-yl)-4H-pyran-4-one (7a)

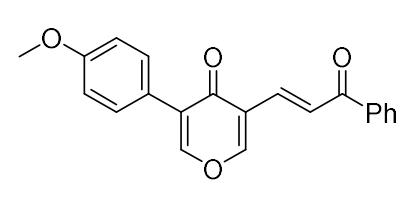

Yellow solid, m.p. $125-127^{\circ} \mathrm{C}, 500 \mathrm{mg}, 38 \%$ yield.

${ }^{1} \mathrm{H}$ NMR $\left(400 \mathrm{MHz}, \mathrm{CDCl}_{3}\right) \delta 8.93(\mathrm{~s}, 1 \mathrm{H}), 8.02-7.93(\mathrm{~m}, 2 \mathrm{H})$,

$7.83(\mathrm{~d}, J=1.0 \mathrm{~Hz}, 1 \mathrm{H}), 7.59-7.54(\mathrm{~m}, 1 \mathrm{H}), 7.47-7.41(\mathrm{~m}$,

$4 \mathrm{H}), 7.19(\mathrm{dd}, J=13.0,0.9 \mathrm{~Hz}, 1 \mathrm{H}), 7.05(\mathrm{~d}, J=13.0 \mathrm{~Hz}, 1 \mathrm{H})$,

$6.94(\mathrm{~d}, J=8.8 \mathrm{~Hz}, 2 \mathrm{H}), 3.83(\mathrm{~s}, 3 \mathrm{H})$.

${ }^{13} \mathrm{C}$ NMR (101 MHz, CDCl $)_{3}$ ) 192.2 (s), 175.9 (s), 159.9 (s), 156.6 (s), 152.0 (s), 137.5 (s), 133.3 (s), 131.6 (s), 130.0 (s), 129.2 (s), 128.8 (s), 128.7 (s), 127.5 (s), 124.3 (s), 123.2 (s), 114.0 (s), 55.3 (s).

HRMS (ESI, m/z): Mass calcd for $\mathrm{C}_{21} \mathrm{H}_{17} \mathrm{O}_{4}[\mathrm{M}+\mathrm{H}]^{+}, 333.1127$; found 333.1129.

(E)-3-(4-fluorophenyl)-5-(3-oxo-3-phenylprop-1-en-1-yl)-4H-pyran-4-one (7b)<smiles>O=C(/C=C/c1cocc(-c2ccc(F)cc2)c1=O)c1ccccc1</smiles>

Light brown solid, m.p. $210-212^{\circ} \mathrm{C}, 300 \mathrm{mg}, 21 \%$ yield.

${ }^{1} \mathrm{H}$ NMR $\left(400 \mathrm{MHz}, \mathrm{CDCl}_{3}\right) \delta 8.73(\mathrm{~d}, J=15.3 \mathrm{~Hz}, 1 \mathrm{H}), 8.09$ (dd, $J=13.0,5.8 \mathrm{~Hz}, 3 \mathrm{H}), 7.89(\mathrm{~d}, J=0.7 \mathrm{~Hz}, 1 \mathrm{H}), 7.57(\mathrm{t}, J=7.4$

$\mathrm{Hz}, 1 \mathrm{H}), 7.53-7.41(\mathrm{~m}, 5 \mathrm{H}), 7.19-7.12(\mathrm{~m}, 2 \mathrm{H})$.

${ }^{13} \mathrm{C} \mathrm{NMR}\left(101 \mathrm{MHz}, \mathrm{CDCl}_{3}\right) \delta 190.5$ (s), 175.8 (s), 163.1 (d, $\left.J=248.5 \mathrm{~Hz}\right), 158.1$ (s),

151.6 (s), 137.7 (s), 134.3 (s), 133.2 (s), 130.8 (d, J = 8.4 Hz), 129.7 (s), 128.7 (d, J = 11.4

$\mathrm{Hz}$ ), 126.9 (s), 126.5 (d, J = 3.5 Hz), 124.09 (s), 115.80 (s), 115.58 (s).

19 $\mathrm{F} \mathrm{NMR}\left(282 \mathrm{MHz}, \mathrm{CDCl}_{3}\right) \delta-112.3--112.4(\mathrm{~m})$.

HRMS (ESI, m/z): Mass calcd for $\mathrm{C}_{20} \mathrm{H}_{14} \mathrm{FO}_{3}[\mathrm{M}+\mathrm{H}]^{+}, 321.0927$; found 321.0922 . 
(E)-3-(4-fluorophenyl)-5-(3-oxobut-1-en-1-yl)-4H-pyran-4-one (7c)

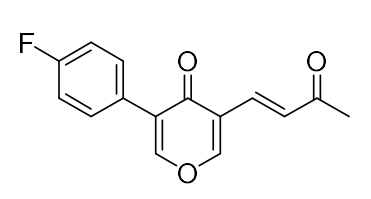

Yellow solid, m.p. $145-147^{\circ} \mathrm{C}, 400 \mathrm{mg}, 34 \%$ yield.

1H NMR $\left(300 \mathrm{MHz}, \mathrm{CDCl}_{3}\right) \delta 8.07(\mathrm{~s}, 1 \mathrm{H}), 7.89(\mathrm{~s}, 1 \mathrm{H}), 7.56-$

$7.44(\mathrm{~m}, 3 \mathrm{H}), 7.28(\mathrm{~d}, J=12.5 \mathrm{~Hz}, 1 \mathrm{H}), 7.14(\mathrm{t}, J=8.6 \mathrm{~Hz}, 2 \mathrm{H})$,

$2.35(\mathrm{~s}, 3 \mathrm{H})$.

${ }^{13} \mathrm{C} \mathrm{NMR}\left(75 \mathrm{MHz}, \mathrm{CDCl}_{3}\right) \delta 198.5$ (s), 175.4 (s), 163.1 (d, $\left.J=248.8 \mathrm{~Hz}\right), 156.6$ (s), 151.8 (s), 132.7 (s), 130.7 (d, J = 8.2 Hz), 130.6 (s), 129.4 (s), 126.5 (s), 124.1 (s), 115.6 (d, $J=$ $21.6 \mathrm{~Hz}), 28.8$ (s).

${ }^{19} \mathrm{~F}$ NMR $\left(282 \mathrm{MHz}, \mathrm{CDCl}_{3}\right) \delta-111.2--112.7(\mathrm{~m})$.

HRMS $(E S I, m / z):$ Mass calcd for $\mathrm{C}_{15} \mathrm{H}_{12} \mathrm{FO}_{3}[\mathrm{M}+\mathrm{H}]^{+}, 259.0770$; found 259.0789.

(4aS,5aS)-1,4a-diphenyl-4,4a,5,5a-tetrahydro-3H,11H-pyrano[3,4-b]xanthene-3,11-di one (3a)<smiles>O=C1CC2(c3ccccc3)CC3Oc4ccccc4C(=O)C3=CC2=C1c1ccccc1</smiles>

Yellow solid, m.p. $85-87^{\circ} \mathrm{C}, 32 \mathrm{mg}, 76 \%$ yield.

$[\alpha]_{\mathrm{D}}^{25}=89.0\left(c 1.0 \mathrm{CHCl}_{3}\right)$.

$\underline{1}{ }^{1} \mathrm{NMR}\left(600 \mathrm{MHz}, \mathrm{CDCl}_{3}\right) \delta 7.92(\mathrm{dd}, J=7.8,1.4 \mathrm{~Hz}, 1 \mathrm{H}), 7.80(\mathrm{~d}$, $J=1.9 \mathrm{~Hz}, 1 \mathrm{H}), 7.66(\mathrm{dd}, J=7.4,1.7 \mathrm{~Hz}, 2 \mathrm{H}), 7.57-7.50(\mathrm{~m}, 3 \mathrm{H})$,

$7.48-7.42(\mathrm{~m}, 1 \mathrm{H}), 7.36(\mathrm{t}, J=7.6 \mathrm{~Hz}, 2 \mathrm{H}), 7.30-7.28(\mathrm{~m}, 3 \mathrm{H}), 7.03(\mathrm{t}, J=7.5 \mathrm{~Hz}, 1 \mathrm{H})$, $6.92(\mathrm{~d}, J=8.3 \mathrm{~Hz}, 1 \mathrm{H}), 4.58(\mathrm{ddd}, J=10.6,5.0,1.8 \mathrm{~Hz}, 1 \mathrm{H}), 3.38(\mathrm{~d}, J=15.1 \mathrm{~Hz}, 1 \mathrm{H})$, $3.06(\mathrm{dd}, J=12.2,5.1 \mathrm{~Hz}, 1 \mathrm{H}), 2.98(\mathrm{~d}, J=15.1 \mathrm{~Hz}, 1 \mathrm{H}), 2.40-2.29(\mathrm{~m}, 1 \mathrm{H})$.

$\underline{{ }^{13} \mathrm{C} \mathrm{NMR}}$ (151 MHz, CDCl ${ }_{3}$ ) $\delta 180.4$ (s), 165.4 (s), 160.8 (s), 155.6 (s), 138.9 (s), 135.8 (s), 132.6 (s), 131.1 (s), 130.8 (s), 130.4 (s), 129.6 (s), 129.5 (s), 128.8 (s), 128.3 (s), 127.7 (s), 125.7 (s), 122.3 (s), 122.1 (s), 117.8 (s), 116.2 (s), 73.0 (s), 43.7 (s), 42.3 (s), 41.5 (s).

HRMS (ESI, m/z): Mass calcd for $\mathrm{C}_{28} \mathrm{H}_{21} \mathrm{O}_{4}[\mathrm{M}+\mathrm{H}]^{+}, 421.1434$; found 421.1430.

Enantiomeric ratio was measured by chiral phase HPLC (Chiralcel AD-H; IPA/Hexanes = 20/80, $0.6 \mathrm{~mL} / \mathrm{min}, 254 \mathrm{~nm}$ ), $\mathrm{Rt}_{1}$ (major) $=19.6 \mathrm{~min}, \mathrm{Rt}_{2}$ (minor) $=42.9 \mathrm{~min}$; er = 99.7:0.3).

(4aS,5aR)-1,4a-diphenyl-4,4a,5,5a-tetrahydro-3H,11 H-pyrano[3,4-b]xanthene-3,11-di one (3a')

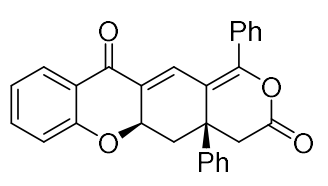

White solid, m.p. $206-208{ }^{\circ} \mathrm{C}, 8.5 \mathrm{mg}, 20 \%$ yield.

$[\alpha]_{\mathrm{D}}^{25}=115.6\left(c 1.0 \mathrm{CHCl}_{3}\right)$

$\underline{1}$ H NMR $\left(400 \mathrm{MHz}, \mathrm{CDCl}_{3}\right) \delta 7.88(\mathrm{dd}, J=7.9,1.7 \mathrm{~Hz}, 1 \mathrm{H}), 7.69-$

$7.62(\mathrm{~m}, 2 \mathrm{H}), 7.57-7.48(\mathrm{~m}, 4 \mathrm{H}), 7.36-7.29(\mathrm{~m}, 1 \mathrm{H}), 7.27-7.23$

$(\mathrm{m}, 4 \mathrm{H}), 7.21-7.13(\mathrm{~m}, 1 \mathrm{H}), 6.97-6.91(\mathrm{~m}, 1 \mathrm{H}), 6.66(\mathrm{dd}, J=8.4,0.5 \mathrm{~Hz}, 1 \mathrm{H}), 5.05-4.95$ $(\mathrm{m}, 1 \mathrm{H}), 3.24(\mathrm{dd}, J=23.5,8.1 \mathrm{~Hz}, 2 \mathrm{H}), 2.89(\mathrm{~d}, J=15.0 \mathrm{~Hz}, 1 \mathrm{H}), 2.41(\mathrm{dd}, J=15.4,6.1$ $\mathrm{Hz}, 1 \mathrm{H})$.

${ }^{13} \mathrm{C} \mathrm{NMR}$ (101 MHz, CDCl 3 ) $\delta 183.01$ (s), 165.2 (s), 161.4 (s), 155.4 (s), 139.2 (s), 135.5 (s), 132.2 (s), 130.8 (s), 130.5 (s), 129.6 (s), 129.5 (s), 128.8 (s), 128.7 (s), 127.8 (s), 127.5 (s), 126.5 (s), 122.3 (s), 121.6 (s), 117.8 (s), 115.5 (s), 72.4 (s), 44.4 (s), 40.1 (s), 39.5 (s).

HRMS (ESI, m/z): Mass calcd for $\mathrm{C}_{28} \mathrm{H}_{21} \mathrm{O}_{4}[\mathrm{M}+\mathrm{H}]^{+}, 421.1434$; found 421.1430.

Enantiomeric ratio was measured by chiral phase HPLC (Chiralcel AD-H; IPA/Hexanes = 20/80, $0.6 \mathrm{~mL} / \mathrm{min}, 254 \mathrm{~nm}$ ), $\mathrm{Rt}_{1}$ (major) $=11.3 \mathrm{~min}, \mathrm{Rt}_{2}$ (minor) $=28.6 \mathrm{~min}$; er = 98:2). 
(4aS,5aS)-4a-(4-fluorophenyl)-1-phenyl-4,4a,5,5a-tetrahydro-3H,11H-pyrano[3,4-b]x anthene-3,11-dione (3b)

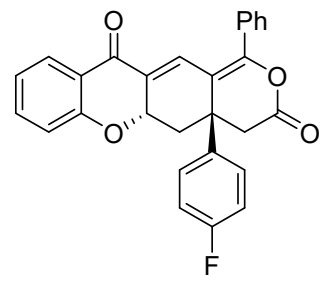

Yellow solid, m.p.176 - $178{ }^{\circ} \mathrm{C}, 30 \mathrm{mg}, 68 \%$ yield.

$[\alpha]_{D}^{29}=84.6\left(c 1.0 \mathrm{CHCl}_{3}\right)$

$\underline{{ }_{1} \mathbf{H ~ N M R}}\left(400 \mathrm{MHz}, \mathrm{CDCl}_{3}\right) \delta 7.90(\mathrm{dd}, J=7.9,1.7 \mathrm{~Hz}, 1 \mathrm{H}), 7.76(\mathrm{~d}, J=$ $2.1 \mathrm{~Hz}, 1 \mathrm{H}), 7.65-7.59(\mathrm{~m}, 2 \mathrm{H}), 7.55-7.48(\mathrm{~m}, 3 \mathrm{H}), 7.46-7.40(\mathrm{~m}, 1 \mathrm{H})$ $7.23(\mathrm{ddd}, J=8.3,5.2,2.7 \mathrm{~Hz}, 2 \mathrm{H}), 7.07-6.98(\mathrm{~m}, 3 \mathrm{H}), 6.90(\mathrm{dd}, J=8.3$, $0.7 \mathrm{~Hz}, 1 \mathrm{H}), 4.54(\mathrm{ddd}, J=10.6,5.1,2.1 \mathrm{~Hz}, 1 \mathrm{H}), 3.31(\mathrm{~d}, J=15.1 \mathrm{~Hz}$, $1 \mathrm{H}), 3.03-2.91(\mathrm{~m}, 2 \mathrm{H}), 2.32(\mathrm{dd}, J=12.3,10.7 \mathrm{~Hz}, 1 \mathrm{H})$.

$\underline{{ }^{13} \mathrm{C} \text { NMR }}\left(101 \mathrm{MHz}, \mathrm{CDCl}_{3}\right) \delta 180.3(\mathrm{~s}), 165.2(\mathrm{~s}), 162.4(\mathrm{~d}, J=248.3 \mathrm{~Hz}), 160.7(\mathrm{~s}), 155.7(\mathrm{~s})$, 135.9 (s), 134.6 (d, $J=3.1 \mathrm{~Hz}), 132.6(\mathrm{~s}), 130.9$ (d, $J=4.2 \mathrm{~Hz}), 130.2$ (s), 129.5 (s), 128.8 (s), 127.8 (s), 127.7 (s), 127.6 (s), 122.2 (d, $J=7.9$ Hz), 117.8 (s), 116.6 (s), 116.4 (s), 116.0 (s), 72.8 (s), $43.8(\mathrm{~s}), 42.4(\mathrm{~s}), 41.1(\mathrm{~s})$.

19 F NMR $\left(471 \mathrm{MHz}, \mathrm{CDCl}_{3}\right) \delta-113.5(\mathrm{~s})$.

HRMS (ESI, m/z): Mass calcd for $\mathrm{C}_{28} \mathrm{H}_{20} \mathrm{O}_{4} \mathrm{~F}[\mathrm{M}+\mathrm{H}]^{+}, 439.1340$; found 439.1342

Enantiomeric ratio was measured by chiral phase HPLC (Chiralcel AD-H; IPA/Hexanes = 30/70, $0.4 \mathrm{~mL} / \mathrm{min}, 254 \mathrm{~nm}$ ), Rt $\mathrm{Rt}_{1}$ (major) $=20.7 \mathrm{~min}, \mathrm{Rt}_{2}$ (minor) $=50.2 \mathrm{~min}$; er = 96:4).

(4aS,5aR)-4a-(4-fluorophenyl)-1-phenyl-4,4a,5,5a-tetrahydro-3H,11H-pyrano[3,4-b]x anthene-3,11-dione (3b')<smiles>O=C1C2=CC3=C(c4ccccc4)OC(=O)C3(c3ccc(F)cc3)CC2Oc2ccccc21</smiles>

White solid, m.p. $191-193^{\circ} \mathrm{C}, 12 \mathrm{mg}, 27 \%$ yield.

$[\alpha]_{\mathrm{D}}^{27}=269.9\left(c 0.33 \mathrm{CHCl}_{3}\right)$

$\underline{1}$ H NMR $\left(400 \mathrm{MHz}, \mathrm{CDCl}_{3}\right) \delta 7.88(\mathrm{dd}, J=7.9,1.7 \mathrm{~Hz}, 1 \mathrm{H}), 7.67-$ $7.62(\mathrm{~m}, 2 \mathrm{H}), 7.56-7.48(\mathrm{~m}, 4 \mathrm{H}), 7.34$ (ddd, $J=8.7,7.2,1.8 \mathrm{~Hz}$, $1 \mathrm{H}), 7.24-7.18(\mathrm{~m}, 2 \mathrm{H}), 7.00-6.89(\mathrm{~m}, 3 \mathrm{H}), 6.68(\mathrm{~d}, J=8.4 \mathrm{~Hz}$, $1 \mathrm{H}), 4.99(\mathrm{dd}, J=4.8,1.3 \mathrm{~Hz}, 1 \mathrm{H}), 3.35-3.11(\mathrm{~m}, 2 \mathrm{H}), 2.90(\mathrm{~d}, J=$ $15.0 \mathrm{~Hz}, 1 \mathrm{H}), 2.41$ (dd, $J=15.4,6.0 \mathrm{~Hz}, 1 \mathrm{H})$.

13. NMR (101 MHz, $\left.\mathrm{CDCl}_{3}\right) \delta 183.0$ (s), 165.0 (s), 161.9 (d, J = 246.8 Hz), 161.3 (s), 155.5 (s), 135.6 (s), 134.9 (d, $J=3.0 \mathrm{~Hz}$ ), 132.3 (s), 131.0 (s), 130.7 (s), 130.3 (s), 129.5 (s), 128.8 (s), 128.3 (d, $J=8.3 \mathrm{~Hz}$ ), 127.8 (s), 122.2 (s), 121.7 (s), 117.8 (s), 115.8 (s), 115.6 (s), 115.4 (s), 72.3 (s), 44.4 (s), 39.7 (s), 39.6 (d).

${ }^{19} \mathrm{~F} \mathrm{NMR}\left(471 \mathrm{MHz}, \mathrm{CDCl}_{3}\right) \delta-114.9$ (s).

HRMS (ESI, m/z): Mass calcd for $\mathrm{C}_{28} \mathrm{H}_{20} \mathrm{O}_{4} \mathrm{~F}[\mathrm{M}+\mathrm{H}]^{+}, 439.1340$; found 439.1342

Enantiomeric ratio was measured by chiral phase HPLC (Chiralcel AD-H; IPA/Hexanes = 30/70, $0.4 \mathrm{~mL} / \mathrm{min}, 254 \mathrm{~nm}$ ), Rt ${ }_{1}$ (major) $=15.7 \mathrm{~min}, \mathrm{Rt}_{2}$ (minor) $=35.8 \mathrm{~min}$; er = 99:1).

(4aS,5aS)-4a-(4-methoxyphenyl)-1-phenyl-4,4a,5,5a-tetrahydro-3H,11H-pyrano[3,4-b] xanthene-3,11-dione (3c)

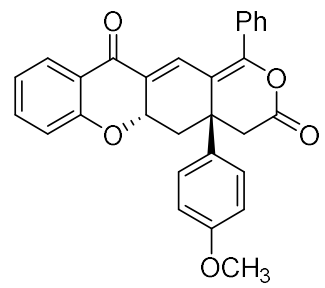

Yellow solid, m.p.106 $-108{ }^{\circ} \mathrm{C}, 30.8 \mathrm{mg}, 68 \%$ yield.

$[\alpha]_{D}^{26}=47.5\left(c 1.0 \mathrm{CHCl}_{3}\right)$

1 NMR $\left(600 \mathrm{MHz}, \mathrm{CDCl}_{3}\right) \delta 7.89(\mathrm{dd}, J=7.8,1.6 \mathrm{~Hz}, 1 \mathrm{H}), 7.76(\mathrm{~d}$, $J=2.0 \mathrm{~Hz}, 1 \mathrm{H}), 7.67-7.59(\mathrm{~m}, 2 \mathrm{H}), 7.55-7.47(\mathrm{~m}, 3 \mathrm{H}), 7.43$ (ddd, $J=8.6,7.3,1.7 \mathrm{~Hz}, 1 \mathrm{H}), 7.19-7.13(\mathrm{~m}, 2 \mathrm{H}), 7.03-6.98(\mathrm{~m}, 1 \mathrm{H})$ $6.90(\mathrm{~d}, J=8.1 \mathrm{~Hz}, 1 \mathrm{H}), 6.86-6.81(\mathrm{~m}, 2 \mathrm{H}), 4.57$ (ddd, $J=10.6$, 
5.0, $2.0 \mathrm{~Hz}, 1 \mathrm{H}$ ), 3.75 (s, 3H), 3.31 (d, J = $15.0 \mathrm{~Hz}, 1 \mathrm{H}), 2.99$ (dd, $J=12.2,5.1 \mathrm{~Hz}, 1 \mathrm{H}$ ), $2.92(\mathrm{~d}, J=15.0 \mathrm{~Hz}, 1 \mathrm{H}), 2.29$ (dd, $J=12.0,10.8 \mathrm{~Hz}, 1 \mathrm{H}$ ).

${ }^{13} \mathrm{C} \mathrm{NMR}\left(151 \mathrm{MHz}, \mathrm{CDCl}_{3}\right) \delta 180.4$ (s), 165.5 (s), 160.8 (s), 159.4 (s), 155.5 (s), 135.8 (s), 132.6 (s), 131.01 (s), 130.7 (s), 130.5 (s), 130.4 (s), 129.6 (s), 128.8 (s), 127.7 (s), 126.9 (s), 122.3 (s), 122.1 (s), 117.8 (s), 116.4 (s), 114.8 (s), 73.1 (s), 55.3 (s), 43.9 (s), 42.4 (s), 41.0 (s).

HRMS (ESI, m/z): Mass calcd for $\mathrm{C}_{29} \mathrm{H}_{23} \mathrm{O}_{5}[\mathrm{M}+\mathrm{H}]^{+}, 451.1540$; found 451.1537

Enantiomeric ratio was measured by chiral phase HPLC (Chiralcel AD-H; IPA/Hexanes = 30/70, $0.4 \mathrm{~mL} / \mathrm{min}, 254 \mathrm{~nm}$ ), Rt ${ }_{1}$ (major) $=25.4 \mathrm{~min}, \mathrm{Rt}_{2}$ (minor) $=61.8 \mathrm{~min}$; er $=94: 6$ ).

(4aS,5aR)-4a-(4-methoxyphenyl)-1-phenyl-4,4a,5,5a-tetrahydro-3H,11H-pyrano[3,4-b] xanthene-3,11-dione (3c')

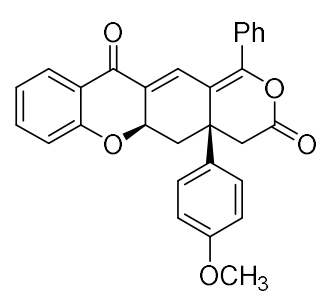

Yellow solid, m.p. $238-240{ }^{\circ} \mathrm{C}, 14.3 \mathrm{mg}, 31 \%$ yield

$[\alpha]_{\mathrm{D}}^{25}=196.6\left(c 0.33 \mathrm{CHCl}_{3}\right)$

${ }^{1} \mathrm{H}$ NMR $\left(400 \mathrm{MHz}, \mathrm{CDCl}_{3}\right) \delta 7.88(\mathrm{dd}, J=7.9,1.6 \mathrm{~Hz}, 1 \mathrm{H}), 7.64(\mathrm{dt}$, $J=4.8,1.9 \mathrm{~Hz}, 2 \mathrm{H}), 7.57-7.47(\mathrm{~m}, 4 \mathrm{H}), 7.33$ (ddd, $J=8.6,7.1,1.8$ $\mathrm{Hz}, 1 \mathrm{H}), 7.18$ - $7.11(\mathrm{~m}, 2 \mathrm{H}), 6.98-6.91(\mathrm{~m}, 1 \mathrm{H}), 6.82-6.73(\mathrm{~m}, 2 \mathrm{H})$, $6.70(\mathrm{dd}, J=8.4,0.6 \mathrm{~Hz}, 1 \mathrm{H}), 4.99(\mathrm{dd}, J=4.9,1.4 \mathrm{~Hz}, 1 \mathrm{H}), 3.72(\mathrm{~s}$, $3 \mathrm{H}), 3.19(\mathrm{dd}, J=21.6,7.7 \mathrm{~Hz}, 2 \mathrm{H}), 2.86(\mathrm{~d}, J=14.9 \mathrm{~Hz}, 1 \mathrm{H}), 2.39(\mathrm{dd}, J=15.4,6.1 \mathrm{~Hz}$, $1 \mathrm{H})$.

13. NMR (101 MHz, $\mathrm{CDCl}_{3}$ ) $\delta 181.8$ (s), 164.0 (s), 160.2 (s), 157.4 (s), 153.9 (s), 134.1 (s), 130.8 (s), 129.6 (s), 129.5 (s), 129.4 (s), 129.2 (s), 128.2 (s), 127.4 (s), 126.4 (s), 126.3 (s), 121.0 (s), 120.2 (s), 116.6 (s), 114.5 (s), 112.8 (s), 71.19 (s), 53.84 (s), 43.29 (s), 38.16 (d, $J=11.5 \mathrm{~Hz})$.

HRMS (ESI, m/z): Mass calcd for $\mathrm{C}_{29} \mathrm{H}_{23} \mathrm{O}_{5}[\mathrm{M}+\mathrm{H}]^{+}, 451.1540$; found 451.1537

Enantiomeric ratio was measured by chiral phase HPLC (Chiralcel AD-H; IPA/Hexanes = 30/70, $0.4 \mathrm{~mL} / \mathrm{min}, 254 \mathrm{~nm}$ ), Rt $\mathrm{R}_{1}$ (major) $=20.1 \mathrm{~min}, \mathrm{Rt}_{2}$ (minor) $=40.8 \mathrm{~min}$; er = 98:2).

(4aS,5aS)-1-phenyl-4a-(m-tolyl)-4,4a,5,5a-tetrahydro-3H,11H-pyrano[3,4-b]xanthene3,11-dione (3d)

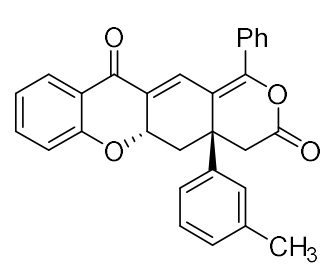

Yellow solid, m.p. $94-96{ }^{\circ} \mathrm{C}, 27.3 \mathrm{mg}, 63 \%$ yield.

$[\alpha]_{D}^{26}=70.0\left(c 1.0 \mathrm{CHCl}_{3}\right)$

1H NMR $\left(600 \mathrm{MHz}, \mathrm{CDCl}_{3}\right) \delta 7.89(\mathrm{dd}, J=7.9,1.6 \mathrm{~Hz}, 1 \mathrm{H}), 7.77(\mathrm{~d}$, $J=2.0 \mathrm{~Hz}, 1 \mathrm{H}), 7.67-7.60(\mathrm{~m}, 2 \mathrm{H}), 7.56-7.47(\mathrm{~m}, 3 \mathrm{H}), 7.45-7.37$ $(\mathrm{m}, 1 \mathrm{H}), 7.20(\mathrm{t}, J=8.0 \mathrm{~Hz}, 1 \mathrm{H}), 7.07(\mathrm{~d}, J=7.5 \mathrm{~Hz}, 1 \mathrm{H}), 7.03(\mathrm{~d}, J$ $=6.0 \mathrm{~Hz}, 2 \mathrm{H}), 7.00(\mathrm{dd}, J=11.1,3.9 \mathrm{~Hz}, 1 \mathrm{H}), 6.90(\mathrm{~d}, J=8.1 \mathrm{~Hz}$,

$1 \mathrm{H}$ ), 4.56 (ddd, $J=10.6,5.0,2.0 \mathrm{~Hz}, 1 \mathrm{H}$ ), $3.34(\mathrm{~d}, J=15.1 \mathrm{~Hz}, 1 \mathrm{H}), 3.03$ (dd, $J=12.2,5.1$ $\mathrm{Hz}, 1 \mathrm{H}), 2.93(\mathrm{~d}, J=15.1 \mathrm{~Hz}, 1 \mathrm{H}), 2.31(\mathrm{~s}, 3 \mathrm{H}), 2.31-2.27(\mathrm{~m}, 1 \mathrm{H})$.

${ }^{13} \mathrm{C} \mathrm{NMR}\left(151 \mathrm{MHz}, \mathrm{CDCl}_{3}\right) \delta 180.4$ (s), 165.5 (s), 160.8 (s), 155.5 (s), 139.1 (s), 138.8 (s), 135.8 (s), 132.6 (s), 131.1 (s), 130.7 (s), 130.5 (s), 129.6 (s), 129.3 (s), 129.1 (s), 128.8 (s), 127.7 (s), 126.2 (s), 122.8 (s), 122.3 (s), 122.1 (s), 117.8 (s), 116.3 (s), 73.04 (s), 43.79 (s), 42.34 (s), 41.46 (s), 21.76 (s).

HRMS (ESI, m/z): Mass calcd for $\mathrm{C}_{29} \mathrm{H}_{23} \mathrm{O}_{4}[\mathrm{M}+\mathrm{H}]^{+}, 435.1591$; found 435.1582

Enantiomeric ratio was measured by chiral phase HPLC (Chiralcel AD-H; IPA/Hexanes = 
30/70, $0.4 \mathrm{~mL} / \mathrm{min}, 254 \mathrm{~nm}), \mathrm{Rt}_{1}$ (major) $\left.=16.3 \mathrm{~min}, \mathrm{Rt}_{2}(\operatorname{minor})=22.3 \mathrm{~min} ; \mathrm{er}=94: 6\right)$.

(4aS,5aR)-1-phenyl-4a-(m-tolyl)-4,4a,5,5a-tetrahydro-3H,11H-pyrano[3,4-b]xanthene -3,11-dione (3d')

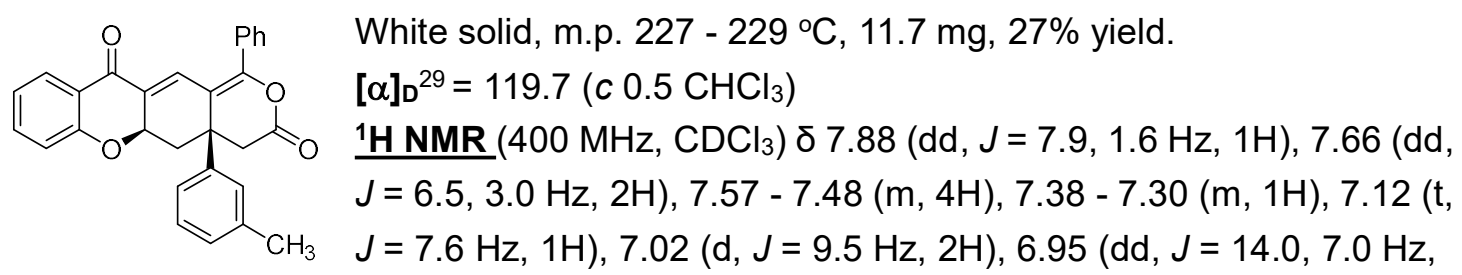

$2 \mathrm{H}), 6.68(\mathrm{~d}, J=8.3 \mathrm{~Hz}, 1 \mathrm{H}), 5.00(\mathrm{~d}, J=6.0 \mathrm{~Hz}, 1 \mathrm{H}), 3.24(\mathrm{~d}, J=15.1 \mathrm{~Hz}, 2 \mathrm{H}), 2.87(\mathrm{~d}, J$ $=15.0 \mathrm{~Hz}, 1 \mathrm{H}), 2.39(\mathrm{dd}, J=15.4,6.1 \mathrm{~Hz}, 1 \mathrm{H}), 2.27(\mathrm{~s}, 3 \mathrm{H})$.

${ }^{13} \mathrm{C} \mathrm{NMR}$ (101 MHz, $\mathrm{CDCl}_{3}$ ) $\delta 183.1$ (s), 165.4 (s), 161.5 (s), 155.3 (s), 139.1 (s), 138.2 (s), 135.5 (s), 132.1 (s), 130.9 (s), 130.8 (s), 130.6 (s), 129.5 (s), 128.7 (s), 128.6 (s), 128.4 (s), 127.8 (s), 127.0 (s), 123.5 (s), 122.3 (s), 121.5 (s), 117.9 (s), 115.7 (s), 72.5 (s), 44.6 (s), 40.0 (s), 39.4 (s), 21.8 (s).

HRMS (ESI, m/z): Mass calcd for $\mathrm{C}_{29} \mathrm{H}_{23} \mathrm{O}_{4}[\mathrm{M}+\mathrm{H}]^{+}, 435.1591$; found 435.1582 Enantiomeric ratio was measured by chiral phase HPLC (Chiralcel AD-H; IPA/Hexanes = 30/70, $0.4 \mathrm{~mL} / \mathrm{min}, 254 \mathrm{~nm}$ ), $\mathrm{Rt}_{1}$ (major) $=14.8 \mathrm{~min}, \mathrm{Rt}_{2}$ (minor) $=28.3 \mathrm{~min}$; er = 99:1).

(4aS,5aS)-4a-(3-methoxyphenyl)-1-phenyl-4,4a,5,5a-tetrahydro-3H,11H-pyrano[3,4-b] xanthene-3,11-dione (3e)

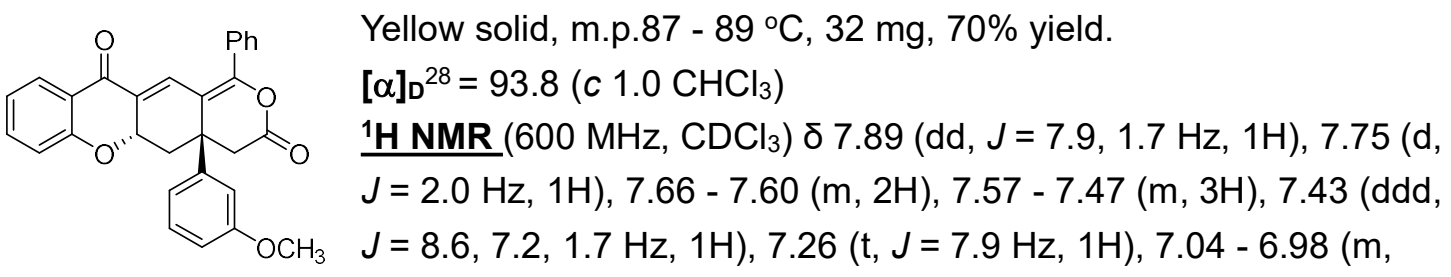
$1 \mathrm{H}), 6.90(\mathrm{~d}, J=7.9 \mathrm{~Hz}, 1 \mathrm{H}), 6.87-6.82(\mathrm{~m}, 1 \mathrm{H}), 6.78(\mathrm{dt}, J=4.3,2.1 \mathrm{~Hz}, 2 \mathrm{H}), 4.60$ (ddd, $J=10.6,5.0,2.1 \mathrm{~Hz}, 1 \mathrm{H}), 3.76(\mathrm{~s}, 3 \mathrm{H}), 3.33(\mathrm{~d}, J=15.1 \mathrm{~Hz}, 1 \mathrm{H}), 3.03(\mathrm{dd}, J=12.2,5.1$ $\mathrm{Hz}, 1 \mathrm{H}), 2.94(\mathrm{~d}, J=15.1 \mathrm{~Hz}, 1 \mathrm{H}), 2.30(\mathrm{dd}, J=12.1,10.8 \mathrm{~Hz}, 1 \mathrm{H})$.

13. NMR(151 MHz, $\mathrm{CDCl}_{3}$ ) ठ 180.3 (s), 165.4 (s), 160.8 (s), 160.2 (s), 155.6 (s), 140.6 (s), 135.8 (s), 132.6 (s), 131.1 (s), 130.8 (s), 130.6 (s), 130.3 (s), 129.6 (s), 128.8 (s), 127.7 (s), 122.3 (s), 122.1 (s), 117.9 (s), 117.8 (s), 116.2 (s), 112.7 (s), 112.6 (s), 72.99 (s), 55.25 (s), 43.73 (s), 42.23 (s), 41.53 (s).

HRMS (ESI, m/z): Mass calcd for $\mathrm{C}_{29} \mathrm{H}_{23} \mathrm{O}_{5}[\mathrm{M}+\mathrm{H}]^{+}, 451.1540$; found 451.1537 Enantiomeric ratio was measured by chiral phase HPLC (Chiralcel AD-H; IPA/Hexanes =

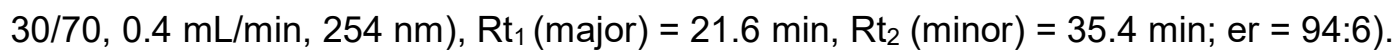

(4aS,5aR)-4a-(3-methoxyphenyl)-1-phenyl-4,4a,5,5a-tetrahydro-3H,11H-pyrano[3,4-b] xanthene-3,11-dione (3e')<smiles>COc1cccc(C2(C3C=C(c4ccccc4)OC(=O)C3)C=C3C(=O)c4ccccc4OC3C2)c1</smiles>

White solid, m.p. $174-176{ }^{\circ} \mathrm{C}, 12 \mathrm{mg}, 28 \%$ yield.

$[\alpha]_{D}{ }^{25}=317.6\left(c 0.33 \mathrm{CHCl}_{3}\right)$

${ }^{1} \mathrm{H}$ NMR $\left(400 \mathrm{MHz}, \mathrm{CDCl}_{3}\right) \delta 7.88(\mathrm{dd}, J=7.9,1.7 \mathrm{~Hz}, 1 \mathrm{H}), 7.68-$ $7.61(\mathrm{~m}, 2 \mathrm{H}), 7.58-7.48(\mathrm{~m}, 4 \mathrm{H}), 7.34$ (ddd, $J=8.6,7.2,1.7 \mathrm{~Hz}$, $1 \mathrm{H}), 7.18(\mathrm{t}, J=8.0 \mathrm{~Hz}, 1 \mathrm{H}), 6.98-6.92(\mathrm{~m}, 1 \mathrm{H}), 6.85(\mathrm{dd}, J=7.9$, 
$1.2 \mathrm{~Hz}, 1 \mathrm{H}), 6.78(\mathrm{t}, J=2.1 \mathrm{~Hz}, 1 \mathrm{H}), 6.73-6.65(\mathrm{~m}, 2 \mathrm{H}), 5.00(\mathrm{~d}, J=6.1 \mathrm{~Hz}, 1 \mathrm{H}), 3.72(\mathrm{~s}$, $3 \mathrm{H}), 3.24(\mathrm{~d}, J=15.1 \mathrm{~Hz}, 2 \mathrm{H}), 2.87(\mathrm{~d}, J=15.0 \mathrm{~Hz}, 1 \mathrm{H}), 2.40$ (dd, $J=15.4,6.1 \mathrm{~Hz}, 1 \mathrm{H})$. $\underline{{ }^{13} \mathrm{C} \mathrm{NMR}}$ (101 MHz, CDCl 3 ) $\delta 183.0$ (s), 165.2 (s), 161.4 (s), 159.7 (s), 155.4 (s), 140.9 (s), 135.5 (s), 132.1 (s), 130.9 (s), 130.8 (s), 130.5 (s), 129.8 (s), 129.5 (s), 128.7 (s), 127.8 (s), 122.3 (s), 121.6 (s), 118.8 (s), 117.9 (s), 115.5 (s), 113.2 (s), 112.2 (s), 72.5 (s), 55.2 (s), 44.5 (s), 40.1 (s), 39.3 (s).

HRMS (ESI, m/z): Mass calcd for $\mathrm{C}_{29} \mathrm{H}_{23} \mathrm{O}_{5}[\mathrm{M}+\mathrm{H}]^{+}, 451.1540$; found 451.1537 Enantiomeric ratio was measured by chiral phase HPLC (Chiralcel AD-H; IPA/Hexanes = 30/70, $0.4 \mathrm{~mL} / \mathrm{min}, 254 \mathrm{~nm}$ ), $\mathrm{Rt}_{1}$ (major) $=18.5 \mathrm{~min}, \mathrm{Rt}_{2}$ (minor) $=33.6 \mathrm{~min}$; er $=93: 7$ ).

(4aS,5aS)-4a-(2-methoxyphenyl)-1-phenyl-4,4a,5,5a-tetrahydro-3H,11H-pyrano[3,4-b] xanthene-3,11-dione (3f)

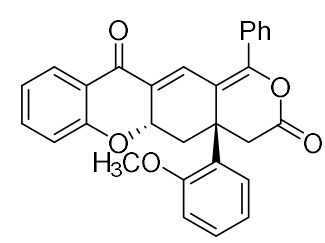

Yellow solid, m.p. $180-182{ }^{\circ} \mathrm{C}, 44 \mathrm{mg}, 97 \%$ yield.

$[\alpha]_{D}{ }^{28}=117.2\left(c 1.0 \mathrm{CHCl}_{3}\right)$

1H NMR $\left(600 \mathrm{MHz}, \mathrm{CDCl}_{3}\right) \delta 7.91(\mathrm{~d}, J=7.8 \mathrm{~Hz}, 1 \mathrm{H}), 7.78(\mathrm{~d}, J=$ $1.7 \mathrm{~Hz}, 1 \mathrm{H}), 7.69-7.62(\mathrm{~m}, 2 \mathrm{H}), 7.57-7.50(\mathrm{~m}, 3 \mathrm{H}), 7.44$ (dd, $J=$ 11.3, $4.2 \mathrm{~Hz}, 1 \mathrm{H}), 7.26(\mathrm{~d}, J=1.5 \mathrm{~Hz}, 1 \mathrm{H}), 7.02(\mathrm{t}, J=7.5 \mathrm{~Hz}, 1 \mathrm{H})$, $6.97(\mathrm{~d}, J=8.2 \mathrm{~Hz}, 1 \mathrm{H}), 6.94(\mathrm{~d}, J=8.4 \mathrm{~Hz}, 1 \mathrm{H}), 6.82(\mathrm{dt}, J=14.9,6.9 \mathrm{~Hz}, 2 \mathrm{H}), 4.56-$ $4.47(\mathrm{~m}, 1 \mathrm{H}), 4.17-4.13(\mathrm{~m}, 1 \mathrm{H}), 4.00(\mathrm{~s}, 3 \mathrm{H}), 3.75(\mathrm{dd}, J=12.0,4.9 \mathrm{~Hz}, 1 \mathrm{H}), 2.78(\mathrm{~d}, J=$ $15.1 \mathrm{~Hz}, 1 \mathrm{H}), 2.12(\mathrm{t}, J=11.4 \mathrm{~Hz}, 1 \mathrm{H})$.

${ }^{13} \mathrm{C} \mathrm{NMR}\left(151 \mathrm{MHz}, \mathrm{CDCl}_{3}\right) \delta 180.6$ (s), 166.6 (s), 160.9 (s), 157.7 (s), 155.4 (s), 135.7 (s), 132.5 (s), 131.3 (s), 130.7 (s), 130.5 (s), 130.0 (s), 129.6 (s), 129.1 (s), 128.7 (s), 127.7 (s), 124.5 (s), 122.3 (s), 122.0 (s), 121.1 (s), 117.8 (s), 117.3 (s), 112.2 (s), 73.9 (s), 55.4 (s), 41.9 (s), 39.6 (s), 38.8 (s).

HRMS (ESI, m/z): Mass calcd for $\mathrm{C}_{29} \mathrm{H}_{23} \mathrm{O}_{5}[\mathrm{M}+\mathrm{H}]^{+}, 451.1540$; found 451.1533

Enantiomeric ratio was measured by chiral phase HPLC (Chiralcel AD-H; IPA/Hexanes = 30/70, $0.4 \mathrm{~mL} / \mathrm{min}, 254 \mathrm{~nm}$ ), $\mathrm{Rt}_{1}$ (major) = $14.9 \mathrm{~min}, \mathrm{Rt}_{2}$ (minor) $=31.9 \mathrm{~min}$; er = 99:1).

(4aR,5aS)-4a-(2-chlorophenyl)-1-phenyl-4,4a,5,5a-tetrahydro-3H,11H-pyrano[3,4-b]x anthene-3,11-dione $(3 \mathrm{~g})$

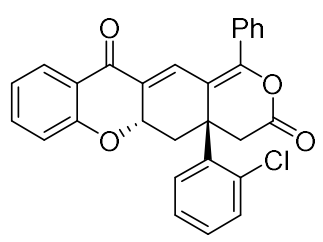

Yellow solid, m.p.92 - $94{ }^{\circ} \mathrm{C}, 43.4 \mathrm{mg}, 96 \%$ yield.

$[\alpha]_{\mathrm{D}}^{28}=91.1\left(\mathrm{c} 1.0 \mathrm{CHCl}_{3}\right)$

1 NMR $\left(600 \mathrm{MHz}, \mathrm{CDCl}_{3}\right) \delta 7.91(\mathrm{~d}, J=2.5 \mathrm{~Hz}, 1 \mathrm{H}), 7.71(\mathrm{~d}, J=$ $2.0 \mathrm{~Hz}, 1 \mathrm{H}), 7.56(\mathrm{dd}, J=7.7,1.7 \mathrm{~Hz}, 2 \mathrm{H}), 7.49-7.40(\mathrm{~m}, 4 \mathrm{H}), 7.27$ $(\mathrm{t}, J=7.6 \mathrm{~Hz}, 2 \mathrm{H}), 7.21(\mathrm{~d}, J=7.3 \mathrm{~Hz}, 1 \mathrm{H}), 7.19-7.15(\mathrm{~m}, 2 \mathrm{H}), 6.73$ (d, $J=8.8 \mathrm{~Hz}, 1 \mathrm{H}$ ), 4.46 (ddd, $J=10.6,5.0,2.0 \mathrm{~Hz}, 1 \mathrm{H}), 3.29(\mathrm{~d}, J=15.1 \mathrm{~Hz}, 1 \mathrm{H}), 2.96$ (dd, $J=12.2,5.1 \mathrm{~Hz}, 1 \mathrm{H}), 2.88(\mathrm{~d}, J=15.1 \mathrm{~Hz}, 1 \mathrm{H}), 2.23(\mathrm{dd}, J=12.1,10.8 \mathrm{~Hz}, 1 \mathrm{H}$ ).

13C NMR (151 MHz, CDCl ${ }_{3}$ ) ס 179.4 (s), 164.5 (s), 159.8 (s), 154.6 (s), 134.8 (s), 132.5 (s), 132.2 (s), 131.6 (s), 131.6 (s), 129.9 (s), 129.8 (s), 129.3 (s), 129.2 (s), 129.0 (s), 128.5 (s), 127.7 (s), 126.7 (s), 126.6 (s), 121.2 (s), 121.0 (s), 116.9 (s), 115.9 (s), 72.2 (s), 42.1 (s), 38.1 (s), 37.3 (s).

HRMS (ESI, m/z): Mass calcd for $\mathrm{C}_{28} \mathrm{H}_{20} \mathrm{O}_{4} \mathrm{Cl}[\mathrm{M}+\mathrm{H}]^{+}, 455.1045$; found 455.1046 Enantiomeric ratio was measured by chiral phase HPLC (Chiralcel AD-H; IPA/Hexanes = 30/70, $0.4 \mathrm{~mL} / \mathrm{min}, 254 \mathrm{~nm}$ ), Rt (major) $_{1} 15.48 \mathrm{~min}, \mathrm{Rt}_{2}$ (minor) $=39.2 \mathrm{~min}$; er $=99: 1$ ). 
(4aS,5aS)-4a-(naphthalen-2-yl)-1-phenyl-4,4a,5,5a-tetrahydro-3H,11H-pyrano[3,4-b]x anthene-3,11-dione (3h)

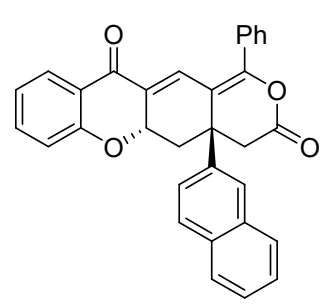

Yellow solid, m.p.153 - $155^{\circ} \mathrm{C}, 46.8 \mathrm{mg}, 98 \%$ yield.

$[\alpha]_{D}^{28}=69.4\left(c 1.0 \mathrm{CHCl}_{3}\right)$

1․ NMR $\left(600 \mathrm{MHz}, \mathrm{CDCl}_{3}\right) \delta 7.92-7.82(\mathrm{~m}, 3 \mathrm{H}), 7.78(\mathrm{dd}, J=6.0$, $3.3 \mathrm{~Hz}, 1 \mathrm{H}), 7.73(\mathrm{dd}, J=6.0,3.4 \mathrm{~Hz}, 1 \mathrm{H}), 7.68(\mathrm{dd}, J=7.1,2.1 \mathrm{~Hz}$, $2 \mathrm{H}), 7.57$ - $7.50(\mathrm{~m}, 4 \mathrm{H}), 7.50$ - $7.43(\mathrm{~m}, 3 \mathrm{H}), 7.41-7.37(\mathrm{~m}, 1 \mathrm{H})$,

$6.98(\mathrm{t}, J=7.5 \mathrm{~Hz}, 1 \mathrm{H}), 6.87(\mathrm{~d}, J=8.3 \mathrm{~Hz}, 1 \mathrm{H}), 4.53(\mathrm{ddd}, J=10.6$, 4.9, $1.9 \mathrm{~Hz}, 1 \mathrm{H}), 3.47(\mathrm{~d}, J=15.2 \mathrm{~Hz}, 1 \mathrm{H}), 3.15(\mathrm{dd}, J=12.3,5.0 \mathrm{~Hz}, 1 \mathrm{H}), 3.01(\mathrm{~d}, J=$ $15.2 \mathrm{~Hz}, 1 \mathrm{H}), 2.45-2.28(\mathrm{~m}, 1 \mathrm{H})$.

${ }^{13} \mathrm{C} \mathrm{NMR}\left(151 \mathrm{MHz}, \mathrm{CDCl}_{3}\right) \delta 180.4$ (s), 165.3 (s), 160.8 (s), 155.7 (s), 135.9 (s), 135.8 (s), 133.2 (s), 132.9 (s), 132.6 (s), 131.1 (s), 130.8 (s), 130.4 (s), 129.8 (s), 129.6 (s), 128.8 (s), 128.3 (s), 127.7 (s), 127.5 (s), 126.7 (s), 125.6 (s), 122.6 (s), 122.3 (s), 122.1 (s), 117.8 (s), 116.2 (s), 73.0 (s), 43.6 (s), 42.1 (s), 41.78 (s).

HRMS (ESI, m/z): Mass calcd for $\mathrm{C}_{32} \mathrm{H}_{23} \mathrm{O}_{4}[\mathrm{M}+\mathrm{H}]^{+}, 471.1591$; found 471.1588 Enantiomeric ratio was measured by chiral phase HPLC (Chiralcel AD-H; IPA/Hexanes = 30/70, $0.4 \mathrm{~mL} / \mathrm{min}, 254 \mathrm{~nm}$ ), Rt ${ }_{1}$ (major) $=22.3 \mathrm{~min}, \mathrm{Rt}_{2}($ minor $)=39.3 \mathrm{~min}$; er = 95:5).

(4a $R, 5 \mathrm{a} S)$-1-phenyl-4a-(thiophen-2-yl)-4,4a,5,5a-tetrahydro-3H,11H-pyrano[3,4-b]xa nthene-3,11-dione (3i)<smiles>O=C1C2=CC3=C(c4ccccc4)OC(=O)C3CC2(c2cccs2)COc2ccccc21</smiles>

Yellow solid, m.p. $203-205^{\circ} \mathrm{C}, 17 \mathrm{mg}, 40 \%$ yield.

$[\alpha]_{\mathrm{D}}^{28}=4.5\left(c \quad 0.5 \mathrm{CHCl}_{3}\right)$

1 $\mathrm{H}$ NMR $\left(400 \mathrm{MHz}, \mathrm{CDCl}_{3}\right) \delta 7.91(\mathrm{dd}, J=7.9,1.7 \mathrm{~Hz}, 1 \mathrm{H}), 7.71(\mathrm{~d}$, $J=2.1 \mathrm{~Hz}, 1 \mathrm{H}), 7.65-7.58(\mathrm{~m}, 2 \mathrm{H}), 7.56-7.48(\mathrm{~m}, 3 \mathrm{H}), 7.48-7.41$ $(\mathrm{m}, 1 \mathrm{H}), 7.23(\mathrm{dd}, J=5.1,1.2 \mathrm{~Hz}, 1 \mathrm{H}), 7.06-6.99(\mathrm{~m}, 1 \mathrm{H}), 6.93(\mathrm{dd}$, $J=8.4,0.7 \mathrm{~Hz}, 1 \mathrm{H}), 6.90(\mathrm{dd}, J=5.1,3.6 \mathrm{~Hz}, 1 \mathrm{H}), 6.85(\mathrm{dd}, J=3.6,1.2 \mathrm{~Hz}, 1 \mathrm{H}), 4.84$ (ddd, $J=10.5,5.2,2.1 \mathrm{~Hz}, 1 \mathrm{H}), 3.32(\mathrm{~d}, J=15.2 \mathrm{~Hz}, 1 \mathrm{H}), 3.05-2.92(\mathrm{~m}, 2 \mathrm{H}), 2.35$ (dd, $J$ $=12.3,10.6 \mathrm{~Hz}, 1 \mathrm{H})$.

${ }^{13} \mathrm{C} \mathrm{NMR}\left(101 \mathrm{MHz}, \mathrm{CDCl}_{3}\right) \delta 180.2$ (s), 164.9 (s), 160.8 (s), 155.6 (s), 144.1 (s), 135.8 (s), 132.1 (s), 131.0 (s), 130.9 (s), 129.7 (s), 129.5 (s), 128.8 (s), 127.8 (s), 127.5 (s), 125.8 (s), 125.4 (s), 122.3 (s), 122.1 (s), 117.8 (s), 116.2 (s), 73.1 (s), 45.1 (s), 43.2 (s), 39.6 (s).

HRMS (ESI, m/z): Mass calcd for $\mathrm{C}_{26} \mathrm{H}_{19} \mathrm{O}_{4} \mathrm{~S}[\mathrm{M}+\mathrm{H}]^{+}, 427.0999$; found 427.0994 Enantiomeric ratio was measured by chiral phase HPLC (Chiralcel AD-H; IPA/Hexanes = 30/70, $0.4 \mathrm{~mL} / \mathrm{min}, 254 \mathrm{~nm}$ ), Rt ${ }_{1}$ (major) $=23.8 \mathrm{~min}, \mathrm{Rt}_{2}($ minor $)=65.8 \mathrm{~min}$; er = 94:6).

(4aR,5aR)-1-phenyl-4a-(thiophen-2-yl)-4,4a,5,5a-tetrahydro-3H,11H-pyrano[3,4-b]xa nthene-3,11-dione (3i')

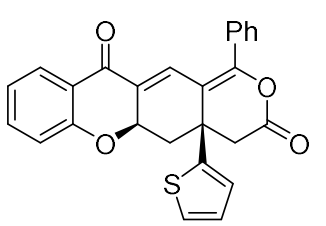

White solid, m.p. $246-248{ }^{\circ} \mathrm{C}, 18.5 \mathrm{mg}, 43 \%$ yield.

$[\alpha]_{\mathrm{D}}^{26}=171.5\left(\mathrm{c} 1.0 \mathrm{CHCl}_{3}\right)$

1H NMR $\left(400 \mathrm{MHz}, \mathrm{CDCl}_{3}\right) \delta 7.91(\mathrm{~d}, \mathrm{~J}=7.9 \mathrm{~Hz}, 1 \mathrm{H}), 7.65-7.59(\mathrm{~m}$, $2 \mathrm{H}), 7.54-7.45(\mathrm{~m}, 4 \mathrm{H}), 7.40-7.34(\mathrm{~m}, 1 \mathrm{H}), 7.16-7.11(\mathrm{~m}, 1 \mathrm{H})$, $6.98(\mathrm{t}, J=7.5 \mathrm{~Hz}, 1 \mathrm{H}), 6.89-6.75(\mathrm{~m}, 3 \mathrm{H}), 5.05(\mathrm{~d}, J=6.1 \mathrm{~Hz}, 1 \mathrm{H})$, $3.29(\mathrm{~d}, J=15.1 \mathrm{~Hz}, 1 \mathrm{H}), 3.11(\mathrm{~d}, J=15.3 \mathrm{~Hz}, 1 \mathrm{H}), 2.94(\mathrm{~d}, J=15.1 \mathrm{~Hz}, 1 \mathrm{H}), 2.47(\mathrm{dd}, J=$ $15.4,6.4 \mathrm{~Hz}, 1 \mathrm{H})$. 
13. NMR (101 MHz, $\left.\mathrm{CDCl}_{3}\right) \delta 183.0$ (s), 164.8 (s), 161.4 (s), 155.2 (s), 145.0 (s), 135.6 (s), 131.6 (s), 130.9 (s), 130.7 (s), 130.0 (s), 129.5 (s), 128.7 (s), 127.8 (s), 126.9 (s), 125.4 (s), 125.2 (s), 122.3 (s), 121.7 (s), 118.0 (s), 115.9 (s), 72.2 (s), 45.4 (s), 40.7 (s), 38.1 (s).

HRMS (ESI, m/z): Mass calcd for $\mathrm{C}_{26} \mathrm{H}_{19} \mathrm{O}_{4} \mathrm{~S}[\mathrm{M}+\mathrm{H}]^{+}, 427.0999$; found 427.0994

Enantiomeric ratio was measured by chiral phase HPLC (Chiralcel AD-H; IPA/Hexanes = 30/70, $0.4 \mathrm{~mL} / \mathrm{min}, 254 \mathrm{~nm}$ ), Rt $\mathrm{R}_{1}$ (major) $=18.3 \mathrm{~min}, \mathrm{Rt}_{2}$ (minor) $=45.6 \mathrm{~min}$; er = 99.5:0.5).

(4aR,5aS)-4a-(benzo[b]thiophen-2-yl)-1-phenyl-4,4a,5,5a-tetrahydro-3H,11H-pyrano[ 3,4-b]xanthene-3,11-dione (3j)

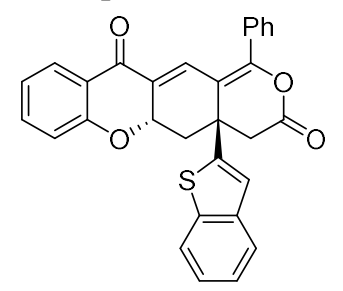

Yellow solid, m.p. $138-140{ }^{\circ} \mathrm{C}, 47.2 \mathrm{mg}, 98 \%$ yield.

$[\alpha]_{D}^{27}=86.2\left(c 1.0 \mathrm{CHCl}_{3}\right)$

1H NMR $\left(600 \mathrm{MHz}, \mathrm{CDCl}_{3}\right) \delta 7.90(\mathrm{dd}, J=7.9,1.6 \mathrm{~Hz}, 1 \mathrm{H}), 7.76(\mathrm{~d}$, $J=2.0 \mathrm{~Hz}, 1 \mathrm{H}), 7.74(\mathrm{~d}, J=7.4 \mathrm{~Hz}, 1 \mathrm{H}), 7.66(\mathrm{dd}, J=7.5,1.7 \mathrm{~Hz}$, $2 \mathrm{H}), 7.64$ - $7.61(\mathrm{~m}, 1 \mathrm{H}), 7.58-7.50(\mathrm{~m}, 3 \mathrm{H}), 7.47-7.41(\mathrm{~m}, 1 \mathrm{H})$, $7.33-7.27(\mathrm{~m}, 2 \mathrm{H}), 7.04-6.98(\mathrm{~m}, 2 \mathrm{H}), 6.92(\mathrm{~d}, J=8.3 \mathrm{~Hz}, 1 \mathrm{H})$, 4.90 (ddd, $J=10.5,5.2,2.0 \mathrm{~Hz}, 1 \mathrm{H}$ ), 3.42 (d, $J=15.3 \mathrm{~Hz}, 1 \mathrm{H}$ ), $3.16-2.96(\mathrm{~m}, 2 \mathrm{H}), 2.39$ (dd, $J=12.2,10.8 \mathrm{~Hz}, 1 \mathrm{H})$.

${ }^{13} \mathrm{C} \mathrm{NMR}\left(151 \mathrm{MHz}, \mathrm{CDCl}_{3}\right) \delta 180.3$ (s), 164.7 (s), 160.8 (s), 155.7 (s), 144.5 (s), 139.6 (s), 139.2 (s), 135.9 (s), 132.3 (s), 131.0 (s), 130.9 (s), 129.6 (s), 129.5 (s), 128.8 (s), 127.8 (s), 125.0 (s), 124.8 (s), 123.8 (s), 122.6 (s), 133.4 (s), 122.3 (s), 122.2 (s), 117.9 (s), 115.6 (s), 73.0 (s), 44.4 (s), 42.8 (s), 40.3 (s).

HRMS (ESI, m/z): Mass calcd for $\mathrm{C}_{30} \mathrm{H}_{21} \mathrm{O}_{4} \mathrm{~S}[\mathrm{M}+\mathrm{H}]^{+}, 477.1155$; found 477.1154 Enantiomeric ratio was measured by chiral phase HPLC (Chiralcel AD-H; IPA/Hexanes = 30/70, $0.4 \mathrm{~mL} / \mathrm{min}, 254 \mathrm{~nm}$ ), Rt $\mathrm{Rt}_{1}$ (major) $=22.0 \mathrm{~min}, \mathrm{Rt}_{2}$ (minor) $=36.8 \mathrm{~min}$; er = 95:5).

(4aR,5aS)-4a-(furan-2-yl)-1-phenyl-4,4a,5,5a-tetrahydro-3H,11H-pyrano[3,4-b]xanthe ne-3,11-dione (3k)<smiles>O=C1Oc2ccccc2OCC2(C3C=CO3)CC3C(=O)OC(c4ccccc4)=C3C=C12</smiles>

Yellow solid, m.p. $103-105^{\circ} \mathrm{C}, 15.7 \mathrm{mg}, 38 \%$ yield.

$[\alpha]_{D}^{28}=25.8\left(c 1.0 \mathrm{CHCl}_{3}\right)$

1 NMR $\left(400 \mathrm{MHz}, \mathrm{CDCl}_{3}\right) \delta 7.92(\mathrm{dd}, J=7.9,1.7 \mathrm{~Hz}, 1 \mathrm{H}), 7.67(\mathrm{~d}$, $J=2.1 \mathrm{~Hz}, 1 \mathrm{H}), 7.61-7.57(\mathrm{~m}, 2 \mathrm{H}), 7.52-7.43(\mathrm{~m}, 4 \mathrm{H}), 7.39(\mathrm{dd}, J$ $=1.8,0.8 \mathrm{~Hz}, 1 \mathrm{H}$ ), 7.03 (ddd, $J=8.1,7.3,1.0 \mathrm{~Hz}, 1 \mathrm{H}), 6.95$ (dd, $J=$ 8.3, $0.7 \mathrm{~Hz}, 1 \mathrm{H}), 6.24$ (dd, $J=3.3,1.9 \mathrm{~Hz}, 1 \mathrm{H}), 6.06(\mathrm{dd}, J=3.3,0.8 \mathrm{~Hz}, 1 \mathrm{H}$ ), 4.81 (ddd, J $=10.5,5.3,2.1 \mathrm{~Hz}, 1 \mathrm{H}), 3.42(\mathrm{~d}, J=15.3 \mathrm{~Hz}, 1 \mathrm{H}), 3.14(\mathrm{dd}, J=12.2,5.3 \mathrm{~Hz}, 1 \mathrm{H}), 2.86(\mathrm{~d}$, $J=15.3 \mathrm{~Hz}, 1 \mathrm{H}), 2.20$ (dd, $J=12.1,10.6 \mathrm{~Hz}, 1 \mathrm{H}$ ).

${ }^{13} \mathrm{C} \mathrm{NMR}\left(101 \mathrm{MHz}, \mathrm{CDCl}_{3}\right) \delta 180.4$ (s), 165.1 (s), 160.8 (s), 155.7 (s), 151.6 (s), 143.6 (s), 135.8 (s), 132.1 (s), 131.0 (s), 130.8 (s), 129.7 (s), 129.5 (s), 128.8 (s), 127.8 (s), 122.3 (s), 122.1 (s), 117.8 (s), 114.4 (s), 110.5 (s), 108.0 (s), 73.1 (s), 41.1 (s), 39.4 (s), 38.2 (s).

HRMS (ESI, m/z): Mass calcd for $\mathrm{C}_{26} \mathrm{H}_{19} \mathrm{O}_{5}[\mathrm{M}+\mathrm{H}]^{+}, 411.1227$; found 411.1219 Enantiomeric ratio was measured by chiral phase HPLC (Chiralcel AD-H; IPA/Hexanes = 30/70, $0.4 \mathrm{~mL} / \mathrm{min}, 254 \mathrm{~nm}$ ), $\mathrm{Rt}_{1}$ (major) $=17.6 \mathrm{~min}, \mathrm{Rt}_{2}$ (minor) $=37.8 \mathrm{~min}$; er = 90:10). 
(4aR,5aR)-4a-(furan-2-yl)-1-phenyl-4,4a,5,5a-tetrahydro-3H,11 H-pyrano[3,4-b]xanthe ne-3,11-dione (3k')

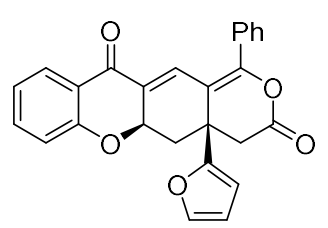

White solid, m. p.104 - $106{ }^{\circ} \mathrm{C}, 20.0 \mathrm{mg}, 45 \%$ yield.

$[\alpha]_{D}^{29}=125.4\left(c 0.5 \mathrm{CHCl}_{3}\right)$

1H NMR $\left(400 \mathrm{MHz}, \mathrm{CDCl}_{3}\right) \delta 7.92(\mathrm{dd}, J=7.9,1.7 \mathrm{~Hz}, 1 \mathrm{H}), 7.64-$

$7.58(\mathrm{~m}, 2 \mathrm{H}), 7.54$ - $7.46(\mathrm{~m}, 3 \mathrm{H}), 7.44-7.35(\mathrm{~m}, 3 \mathrm{H}), 7.05$ - $6.96(\mathrm{~m}$, $1 \mathrm{H}), 6.88-6.81(\mathrm{~m}, 1 \mathrm{H}), 6.14(\mathrm{dd}, J=3.3,1.9 \mathrm{~Hz}, 1 \mathrm{H}), 6.02(\mathrm{dd}, J=$ 3.3, $0.6 \mathrm{~Hz}, 1 \mathrm{H}), 5.05-4.99(\mathrm{~m}, 1 \mathrm{H}), 3.34(\mathrm{~d}, J=15.3 \mathrm{~Hz}, 1 \mathrm{H}), 3.17(\mathrm{~d}, J=15.3 \mathrm{~Hz}, 1 \mathrm{H})$, $2.80(\mathrm{~d}, J=15.3 \mathrm{~Hz}, 1 \mathrm{H}), 2.30(\mathrm{dd}, J=15.3,6.4 \mathrm{~Hz}, 1 \mathrm{H})$.

13. NMR (101 MHz, $\mathrm{CDCl}_{3}$ ) ס 183.1 (s), 165.0 (s), 161.4 (s), 155.4 (s), 152.5 (s), 142.8 (s), 135.6 (s), 131.3 (s), 130.9 (s), 130.8 (s), 130.0 (s), 129.5 (s), 128.7 (s), 127.9 (s), 122.4 (s), 121.7 (s), 118.0 (s), 113.7 (s), 110.3 (s), 107.7 (s), 71.9 (s), $41.3(\mathrm{~s}), 37.2$ (s), 36.5(s).

HRMS (ESI, m/z): Mass calcd for $\mathrm{C}_{26} \mathrm{H}_{19} \mathrm{O}_{5}[\mathrm{M}+\mathrm{H}]^{+}, 411.1227$; found 411.1219 Enantiomeric ratio was measured by chiral phase HPLC (Chiralcel AD-H; IPA/Hexanes = 30/70, $0.4 \mathrm{~mL} / \mathrm{min}, 254 \mathrm{~nm}$ ), Rt ${ }_{1}$ (major) $=15.5 \mathrm{~min}, \mathrm{Rt}_{2}($ minor $)=39.2 \mathrm{~min}$; er = 98:2).

(4aS,5aR)-4a-isopropyl-1-phenyl-4,4a,5,5a-tetrahydro-3H,11H-pyrano[3,4-b]xanthen e-3,11-dione (3l')<smiles>CC(C)C12CC(=O)OC(c3ccccc3)=C1C=C1C(=O)c3ccccc3OC1C2</smiles>

Yellow solid, m.p. $150-152{ }^{\circ} \mathrm{C}, 33.3 \mathrm{mg}, 86 \%$ yield.

$[\alpha]_{\mathrm{D}}^{27}=213.9\left(\right.$ c $\left.1.0 \mathrm{CHCl}_{3}\right)$

$\underline{1}$ H NMR $\left(400 \mathrm{MHz}, \mathrm{CDCl}_{3}\right) \delta 7.98(\mathrm{dd}, J=7.9,1.6 \mathrm{~Hz}, 1 \mathrm{H}), 7.56-$ $7.52(\mathrm{~m}, 2 \mathrm{H}), 7.51-7.44(\mathrm{~m}, 4 \mathrm{H}), 7.34(\mathrm{~d}, J=1.6 \mathrm{~Hz}, 1 \mathrm{H}), 7.04(\mathrm{~d}, J$ $=1.0 \mathrm{~Hz}, 1 \mathrm{H}), 6.98(\mathrm{dd}, J=8.4,0.7 \mathrm{~Hz}, 1 \mathrm{H}), 5.10-4.97(\mathrm{~m}, 1 \mathrm{H}), 3.04(\mathrm{~d}, J=16.3 \mathrm{~Hz}, 1 \mathrm{H})$, $2.81(\mathrm{dd}, J=15.5,0.9 \mathrm{~Hz}, 1 \mathrm{H}), 2.34(\mathrm{~d}, J=16.4 \mathrm{~Hz}, 1 \mathrm{H}), 2.26(\mathrm{dt}, J=13.8,6.9 \mathrm{~Hz}, 1 \mathrm{H})$, $1.93(\mathrm{dd}, J=15.5,6.4 \mathrm{~Hz}, 1 \mathrm{H}), 1.10(\mathrm{~d}, J=6.8 \mathrm{~Hz}, 3 \mathrm{H}), 0.91(\mathrm{~d}, J=7.1 \mathrm{~Hz}, 3 \mathrm{H})$.

${ }^{13} \mathrm{C} \mathrm{NMR}\left(101 \mathrm{MHz}, \mathrm{CDCl}_{3}\right) \delta 182.9$ (s), 167.2 (s), 161.2 (s), 154.0 (s), 135.6 (s), 131.2 (s), 131.0 (s), 130.5 (s), 130.3 (s), 129.5 (s), 128.5 (s), 128.0 (s), 122.7 (s), 121.9 (s), 118.1 (s), 116.1 (s), 72.9 (s), 39.3 (s), 37.1 (s), 35.7 (s), 31.0 (s), 18.2 (s), 16.1 (s).

HRMS (ESI, m/z): Mass calcd for $\mathrm{C}_{25} \mathrm{H}_{23} \mathrm{O}_{4}[\mathrm{M}+\mathrm{H}]^{+}, 387.1591$; found 387.1586 Enantiomeric ratio was measured by chiral phase HPLC (Chiralcel AD-H; IPA/Hexanes = 30/70, $0.4 \mathrm{~mL} / \mathrm{min}, 254 \mathrm{~nm}$ ), Rt (major) $12.3 \mathrm{~min}, \mathrm{Rt}_{2}$ (minor) $=18.5 \mathrm{~min}$; er = 92:8).

(4aS,5aS)-4a-cyclopropyl-1-phenyl-4,4a,5,5a-tetrahydro-3H,11H-pyrano[3,4-b]xanth ene-3,11-dione $(3 \mathrm{~m})$<smiles></smiles>

White solid, m.p. $68-70{ }^{\circ} \mathrm{C}, 10.2 \mathrm{mg}, 26 \%$ yield.

$[\alpha]_{D}{ }^{29}=54.9\left(c 0.2 \mathrm{CHCl}_{3}\right)$

$\underline{{ }^{1} \mathrm{H} \mathrm{NMR}}\left(400 \mathrm{MHz}, \mathrm{CDCl}_{3}\right) \delta 7.95(\mathrm{dd}, J=7.8,1.7 \mathrm{~Hz}, 1 \mathrm{H}), 7.56(\mathrm{~d}$, $J=2.0 \mathrm{~Hz}, 1 \mathrm{H}), 7.54-7.40(\mathrm{~m}, 6 \mathrm{H}), 7.06(\mathrm{t}, J=7.5 \mathrm{~Hz}, 1 \mathrm{H}), 6.98(\mathrm{~d}$, $J=8.2 \mathrm{~Hz}, 1 \mathrm{H}$ ), 5.48 (ddd, $J=10.4,5.4,2.1 \mathrm{~Hz}, 1 \mathrm{H}), 2.99$ (d, $J=15.3 \mathrm{~Hz}, 1 \mathrm{H}), 2.77-2.62$ $(\mathrm{m}, 2 \mathrm{H}), 2.14(\mathrm{dd}, J=12.0,10.6 \mathrm{~Hz}, 1 \mathrm{H}), 0.87-0.83(\mathrm{~m}, 1 \mathrm{H}), 0.53-0.43(\mathrm{~m}, 1 \mathrm{H}), 0.42-$ $0.34(\mathrm{~m}, 1 \mathrm{H}), 0.24$ (td, $J=10.4,5.4 \mathrm{~Hz}, 1 \mathrm{H}), 0.08$ (td, $J=10.5,5.5 \mathrm{~Hz}, 1 \mathrm{H})$.

${ }^{13} \mathrm{C} \mathrm{NMR}\left(101 \mathrm{MHz}, \mathrm{CDCl}_{3}\right) \delta 179.1$ (s), 165.2 (s), 159.6 (s), 154.6 (s), 134.5 (s), 130. 9 (s), 129.9 (s), 129.8 (s), 129.3 (s), 128.2 (s), 127.4 (s), 126.5 (s), 121.1 (s), 120.8 (s), 116.6 (s), 110.9 (s), 72.0 (s), 41.5 (s), 41.3 (s), 35.9 (s), 16.8 (s), 0.00 (s), -1.4 (s). 
HRMS (ESI, m/z): Mass calcd for $\mathrm{C}_{25} \mathrm{H}_{23} \mathrm{O}_{4}[\mathrm{M}+\mathrm{H}]^{+}, 387.1591$; found 387.1586

Enantiomeric ratio was measured by chiral phase HPLC (Chiralcel AD-H; IPA/Hexanes = 30/70, $0.4 \mathrm{~mL} / \mathrm{min}, 254 \mathrm{~nm}$ ), $\mathrm{Rt}_{1}$ (major) = $15.4 \mathrm{~min}, \mathrm{Rt}_{2}$ (minor) $=26.3 \mathrm{~min}$; er = 80:20).

(4aS,5aR)-4a-cyclopropyl-1-phenyl-4,4a,5,5a-tetrahydro-3H,11H-pyrano[3,4-b]xanth ene-3,11-dione (3m')<smiles>O=C1CC2(CC2)CC2Oc3ccccc3C(=O)C2=C1c1ccccc1</smiles>

Yellow solid, m.p. $69-71^{\circ} \mathrm{C}, 23.5 \mathrm{mg}, 61 \%$ yield.

$[\alpha]_{D}{ }^{29}=193.5\left(c 0.5 \mathrm{CHCl}_{3}\right)$

1H NMR $\left(400 \mathrm{MHz}, \mathrm{CDCl}_{3}\right) \delta 7.98(\mathrm{dd}, J=7.9,1.7 \mathrm{~Hz}, 1 \mathrm{H}), 7.55-$ $7.43(\mathrm{~m}, 6 \mathrm{H}), 7.31(\mathrm{~d}, J=1.4 \mathrm{~Hz}, 1 \mathrm{H}), 7.09-7.02(\mathrm{~m}, 1 \mathrm{H}), 7.00$ (dd, $J=8.4,0.6 \mathrm{~Hz}, 1 \mathrm{H}), 5.05(\mathrm{dd}, J=5.2,1.2 \mathrm{~Hz}, 1 \mathrm{H}), 2.92(\mathrm{~d}, J=15.4 \mathrm{~Hz}, 1 \mathrm{H}), 2.73-2.51$ $(\mathrm{m}, 2 \mathrm{H}), 2.22(\mathrm{dd}, J=15.2,6.4 \mathrm{~Hz}, 1 \mathrm{H}), 0.97(\mathrm{tt}, J=8.3,5.7 \mathrm{~Hz}, 1 \mathrm{H}), 0.55-0.45(\mathrm{~m}, 1 \mathrm{H})$, 0.36 (tt, $J=9.1,5.5 \mathrm{~Hz}, 1 \mathrm{H}$ ), 0.21 (td, $J=10.6,5.7 \mathrm{~Hz}, 1 \mathrm{H}), 0.07$ (dq, $J=9.8,5.7 \mathrm{~Hz}, 1 \mathrm{H}$ ). ${ }^{13} \mathrm{C} \mathrm{NMR}$ (101 MHz, $\mathrm{CDCl}_{3}$ ) ठ 181.6 (s), 164.9 (s), 159.9 (s), 153.6 (s), 134.0 (s), 130.0 (s), 129.2 (s), 129.0 (s), 128.9 (s), 127.8 (s), 126.9 (s), 126.4 (s), 120.9 (s), 120.1 (s), 116.3 (s), 111.1 (s), 70.8 (s), 39.9 (s), 38.4 (s), 33.8 (s), 16.9 (s), 1.8 (s).

HRMS (ESI, m/z): Mass calcd for $\mathrm{C}_{25} \mathrm{H}_{21} \mathrm{O}_{4}[\mathrm{M}+\mathrm{H}]^{+}, 385.1434$; found 385.1432

Enantiomeric ratio was measured by chiral phase HPLC (Chiralcel AD-H; IPA/Hexanes = 30/70, $0.4 \mathrm{~mL} / \mathrm{min}, 254 \mathrm{~nm}$ ), Rt ${ }_{1}$ (major) $=13.5 \mathrm{~min}, \mathrm{Rt}_{2}$ (minor) $=30.7 \mathrm{~min}$; er = 91:9).

(4aS,5aS)-9-fluoro-1,4a-diphenyl-4,4a,5,5a-tetrahydro-3H,11H-pyrano[3,4-b]xanthen e-3,11-dione (3n)

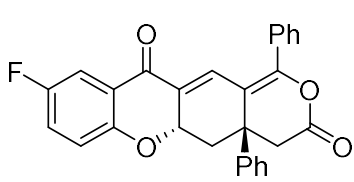

Yellow solid, m.p.199 - $201{ }^{\circ} \mathrm{C}, 27.6 \mathrm{mg}, 63 \%$ yield. $[\alpha]_{D^{26}}=74.1\left(c 1.0 \mathrm{CHCl}_{3}\right)$

1․ NMR $\left(600 \mathrm{MHz}, \mathrm{CDCl}_{3}\right) \delta 7.81(\mathrm{~d}, J=2.0 \mathrm{~Hz}, 1 \mathrm{H}), 7.66(\mathrm{dd}, J$ = 7.6, $1.7 \mathrm{~Hz}, 2 \mathrm{H}), 7.60-7.50(\mathrm{~m}, 4 \mathrm{H}), 7.36(\mathrm{t}, J=7.6 \mathrm{~Hz}, 2 \mathrm{H})$, $7.31(\mathrm{~d}, J=7.3 \mathrm{~Hz}, 1 \mathrm{H}), 7.29-7.26(\mathrm{~m}, 2 \mathrm{H}), 7.17$ (ddd, $J=9.0,7.8,3.2 \mathrm{~Hz}, 1 \mathrm{H}), 6.91$ (dd, $J=9.1,4.2 \mathrm{~Hz}, 1 \mathrm{H}$ ), 4.55 (ddd, $J=10.6,5.0,2.0 \mathrm{~Hz}, 1 \mathrm{H}), 3.38(\mathrm{~d}, J=15.1 \mathrm{~Hz}, 1 \mathrm{H}), 3.05$ (dd, $J=12.3,5.1 \mathrm{~Hz}, 1 \mathrm{H}), 2.97(\mathrm{~d}, J=15.1 \mathrm{~Hz}, 1 \mathrm{H}), 2.33$ (dd, $J=12.1,10.8 \mathrm{~Hz}, 1 \mathrm{H}$ ). ${ }^{13} \mathrm{C} \mathrm{NMR}\left(151 \mathrm{MHz}, \mathrm{CDCl}_{3}\right) \delta 179.7$ (s), 165.2 (s), 158.4 (s), 157.6 (d, $J=242.0 \mathrm{~Hz}$ ), 157.0 (s), 156.9 (d, J = 22.9 Hz), 156.0 (s), 138.8 (s), 131.8 (s), 131.1 (s), 130.9 (d, J = $14.8 \mathrm{~Hz}$ ), 129.6 (d, J = 5.7 Hz), 128.8 (s), 128.4 (s), 125.7 (s), 123.2 (d, J = $24.7 \mathrm{~Hz}$ ), 122.9 (d, $J=7.0 \mathrm{~Hz}$ ), 119.5 (d, $J=7.3 \mathrm{~Hz}), 116.1$ (s), 112.7 (d, $J=23.4 \mathrm{~Hz}$ ), 73.2 (s), 43.7 (s), $42.2(\mathrm{~s}), 41.5(\mathrm{~s})$.

19 F NMR $\left(471 \mathrm{MHz}, \mathrm{CDCl}_{3}\right) \delta-120.2(\mathrm{~s})$.

HRMS (ESI, m/z): Mass calcd for $\mathrm{C}_{28} \mathrm{H}_{20} \mathrm{O}_{4} \mathrm{~F}[\mathrm{M}+\mathrm{H}]^{+}$, 439.1340; found 439.1341.

Enantiomeric ratio was measured by chiral phase HPLC (Chiralcel AD-H; IPA/Hexanes = 30/70, $0.4 \mathrm{~mL} / \mathrm{min}, 254 \mathrm{~nm}$ ), $\mathrm{Rt}_{1}$ (major) = $17.3 \mathrm{~min}, \mathrm{Rt}_{2}$ (minor) = $36.4 \mathrm{~min}$; er = 96:4). 
(4aS,5a R)-9-fluoro-1,4a-diphenyl-4,4a,5,5a-tetrahydro-3H,11H-pyrano[3,4-b]xanthen e-3,11-dione (3n')<smiles>O=C1C[C@H]2COc3ccc(F)cc3C(=O)C2C=C1c1ccccc1</smiles>

White solid, m.p. $225-227^{\circ} \mathrm{C}, 9.3 \mathrm{mg}, 21 \%$ yield.

$[\alpha]_{\mathrm{D}}{ }^{25}=226.9\left(\mathrm{c} 0.25 \mathrm{CHCl}_{3}\right)$

1․ NMR $\left(400 \mathrm{MHz}, \mathrm{CDCl}_{3}\right) \delta 7.62-7.54(\mathrm{~m}, 2 \mathrm{H}), 7.52-7.41(\mathrm{~m}$, $5 \mathrm{H}), 7.19-7.14(\mathrm{~m}, 4 \mathrm{H}), 7.13-7.07(\mathrm{~m}, 1 \mathrm{H}), 6.97$ (ddd, $J=9.1$,

7.7, $3.2 \mathrm{~Hz}, 1 \mathrm{H}$ ), 6.57 (dd, $J=9.1,4.2 \mathrm{~Hz}, 1 \mathrm{H}), 4.90(\mathrm{~d}, J=6.1 \mathrm{~Hz}, 1 \mathrm{H}), 3.18$ (dd, $J=20.4$, $15.2 \mathrm{~Hz}, 2 \mathrm{H}), 2.82(\mathrm{~d}, J=15.0 \mathrm{~Hz}, 1 \mathrm{H}), 2.34(\mathrm{dd}, J=15.4,6.1 \mathrm{~Hz}, 1 \mathrm{H})$.

${ }^{13} \mathrm{C} \mathrm{NMR}$ (101 MHz, $\mathrm{CDCl}_{3}$ ) ठ 182.2 (s), 165.1 (s), 157.6 (s), 157.3 (d, $J=241.9 \mathrm{~Hz}$ ).

155.8 (s), 139.0 (s), 131.4 (d, J = 15.1 Hz), 131.0 (s), 130.8 (s), 129.6 (s), 128.8 (s), 128.7 (s), 127.6 (s), 126.46 (s), 123.16 (s), 122.9 (s), 119.4 (d, J = $7.3 \mathrm{~Hz}$ ), 115.4 (s), 112.7 (s), 112.5 (s), 72.67 (s), 44.39 (s), 40.11 (s), 39.52 (s).

${ }^{19} \mathrm{~F}$ NMR $\left(471 \mathrm{MHz}, \mathrm{CDCl}_{3}\right) \delta-121.1$ (s).

HRMS (ESI, m/z): Mass calcd for $\mathrm{C}_{28} \mathrm{H}_{20} \mathrm{O}_{4} \mathrm{~F}[\mathrm{M}+\mathrm{H}]^{+}$, 439.1340; found 439.1341.

Enantiomeric ratio was measured by chiral phase HPLC (Chiralcel AD-H; IPA/Hexanes = 30/70, $0.4 \mathrm{~mL} / \mathrm{min}, 254 \mathrm{~nm}$ ), Rt ${ }_{1}$ (major) = $16.9 \mathrm{~min}, \mathrm{Rt}_{2}$ (minor) = $29.4 \mathrm{~min}$; er = 99:1).

(4aS,5aS)-9-chloro-1,4a-diphenyl-4,4a,5,5a-tetrahydro-3H,11H-pyrano[3,4-b]xanthen e-3,11-dione (3o)<smiles>O=C1Oc2ccc(Cl)cc2OC2CC3(c4ccccc4)C(=O)OC(c4ccccc4)=C3C=C12</smiles>

Yellow solid, m.p. $126-128{ }^{\circ} \mathrm{C}, 34.0 \mathrm{mg}, 75 \%$ yield.

$[\alpha]_{D}^{30}=63.5\left(c 1.0 \mathrm{CHCl}_{3}\right)$

1H NMR $\left(600 \mathrm{MHz}, \mathrm{CDCl}_{3}\right) \delta 7.86(\mathrm{~d}, J=2.6 \mathrm{~Hz}, 1 \mathrm{H}), 7.81(\mathrm{~d}, J=$ $2.0 \mathrm{~Hz}, 1 \mathrm{H}), 7.69-7.62(\mathrm{~m}, 2 \mathrm{H}), 7.59-7.52(\mathrm{~m}, 3 \mathrm{H}), 7.40-7.34$

$(\mathrm{m}, 3 \mathrm{H}), 7.32-7.26(\mathrm{~m}, 3 \mathrm{H}), 6.88(\mathrm{~d}, J=8.8 \mathrm{~Hz}, 1 \mathrm{H}), 4.56(\mathrm{ddd}, J=10.6,5.0,2.0 \mathrm{~Hz}, 1 \mathrm{H})$, $3.38(\mathrm{~d}, J=15.1 \mathrm{~Hz}, 1 \mathrm{H}), 3.05(\mathrm{dd}, J=12.2,5.1 \mathrm{~Hz}, 1 \mathrm{H}), 2.97(\mathrm{~d}, J=15.1 \mathrm{~Hz}, 1 \mathrm{H}), 2.33$ (dd, $J=12.1,10.8 \mathrm{~Hz}, 1 \mathrm{H}$ ).

$\underline{{ }^{13} \mathrm{C} \mathrm{NMR}}$ (151 MHz, CDCl$)_{3}$ ) 179.3 (s), 165.2 (s), 159.2 (s), 156.1 (s), 138.7 (s), 135.5 (s), 131.6 (s), 131.3 (s), 131.0 (s), 130.9 (s), 129.6 (s), 129.5 (s), 128.8 (s), 128.4 (s), 127.5 (s), 127.0 (s), 125.7 (s), 123.1 (s), 119.5 (s), 116.0 (s), 73.23 (s), 43.68 (s), 42.18 (s), 41.50 (s).

HRMS (ESI, m/z): Mass calcd for $\mathrm{C}_{28} \mathrm{H}_{20} \mathrm{O}_{4} \mathrm{Cl}[\mathrm{M}+\mathrm{H}]^{+}, 455.1045$; found 455.1040 Enantiomeric ratio was measured by chiral phase HPLC (Chiralcel AD-H; IPA/Hexanes = 30/70, $0.4 \mathrm{~mL} / \mathrm{min}, 254 \mathrm{~nm}$ ), $\mathrm{Rt}_{1}$ (major) = $18.5 \mathrm{~min}, \mathrm{Rt}_{2}$ (minor) = $37.4 \mathrm{~min}$; er = 95:5).

(4aS,5aR)-9-chloro-1,4a-diphenyl-4,4a,5,5a-tetrahydro-3H,11H-pyrano[3,4-b]xanthen e-3,11-dione (3o')

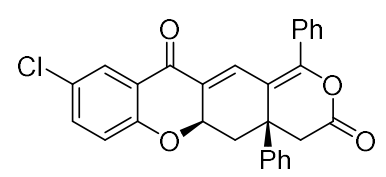

White solid, m.p. $121-123^{\circ} \mathrm{C}, 10.0 \mathrm{mg}, 22 \%$ yield.

$[\alpha]_{\mathrm{D}}^{29}=213.7\left(c \quad 0.1 \mathrm{CHCl}_{3}\right)$

$\underline{1}$ H NMR $\left(400 \mathrm{MHz}, \mathrm{CDCl}_{3}\right) \delta 7.81(\mathrm{~d}, \mathrm{~J}=2.7 \mathrm{~Hz}, 1 \mathrm{H}), 7.67-$

$7.62(\mathrm{~m}, 2 \mathrm{H}), 7.57-7.48(\mathrm{~m}, 4 \mathrm{H}), 7.21$ (ddd, $J=14.0,8.8,6.6$

$\mathrm{Hz}, 6 \mathrm{H}), 6.61(\mathrm{~d}, J=8.9 \mathrm{~Hz}, 1 \mathrm{H}), 4.97(\mathrm{~d}, J=6.0 \mathrm{~Hz}, 1 \mathrm{H}), 3.25(\mathrm{dd}, J=20.6,15.2 \mathrm{~Hz}, 2 \mathrm{H})$, $2.89(\mathrm{~d}, J=15.0 \mathrm{~Hz}, 1 \mathrm{H}), 2.41(\mathrm{dd}, J=15.4,6.0 \mathrm{~Hz}, 1 \mathrm{H})$. 
${ }^{13} \mathrm{C} \mathrm{NMR}$ (101 MHz, CDCl 3 ) ठ 181.9 (s), 165.0 (s), 159.8 (s), 155.9 (s), 139.0 (s), 135.3 (s), 131.4 (s), 131.2 (s), 131.0 (s), 130.7 (s), 129.6 (s), 128.8 (s), 128.7 (s), 127.6 (s), 127.1 (s), 127.0 (s), 126.4 (s), 123.1 (s), 119.5 (s), 115.3 (s), 72.7 (s), 44.3 (s), 40.1 (s), 39.5 (s). HRMS (ESI, m/z): Mass calcd for $\mathrm{C}_{28} \mathrm{H}_{20} \mathrm{O}_{4} \mathrm{Cl}[\mathrm{M}+\mathrm{H}]^{+}, 455.1045$; found 455.1040 Enantiomeric ratio was measured by chiral phase HPLC (Chiralcel AD-H; IPA/Hexanes = 30/70, $0.4 \mathrm{~mL} / \mathrm{min}, 254 \mathrm{~nm}), \mathrm{Rt}_{1}$ (major) $=16.9 \mathrm{~min}, \mathrm{Rt}_{2}($ minor $)=37.9 \mathrm{~min}$; er = 99:1).

(4aS,5aS)-9-bromo-1,4a-diphenyl-4,4a,5,5a-tetrahydro-3H,11H-pyrano[3,4-b]xanthen e-3,11-dione (3p)

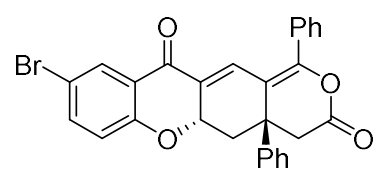

Yellow solid, m.p. $168-170{ }^{\circ} \mathrm{C}, 28.9 \mathrm{mg}, 63 \%$ yield. $[\alpha]_{D}^{28}=48.9\left(c 1.0 \mathrm{CHCl}_{3}\right)$.

1H NMR $\left(600 \mathrm{MHz}, \mathrm{CDCl}_{3}\right) \delta 7.91(\mathrm{~d}, J=2.5 \mathrm{~Hz}, 1 \mathrm{H}), 7.71(\mathrm{~d}, J$ $=2.0 \mathrm{~Hz}, 1 \mathrm{H}), 7.56(\mathrm{dd}, J=7.7,1.7 \mathrm{~Hz}, 2 \mathrm{H}), 7.49-7.40(\mathrm{~m}, 4 \mathrm{H})$, $7.27(\mathrm{t}, J=7.6 \mathrm{~Hz}, 2 \mathrm{H}), 7.21(\mathrm{~d}, J=7.3 \mathrm{~Hz}, 1 \mathrm{H}), 7.19-7.15(\mathrm{~m}, 2 \mathrm{H}), 6.73(\mathrm{~d}, J=8.8 \mathrm{~Hz}$, $1 \mathrm{H}$ ), 4.46 (ddd, $J=10.6,5.0,2.0 \mathrm{~Hz}, 1 \mathrm{H}$ ), $3.29(\mathrm{~d}, J=15.1 \mathrm{~Hz}, 1 \mathrm{H}), 2.96$ (dd, $J=12.2,5.1$ $\mathrm{Hz}, 1 \mathrm{H}), 2.88(\mathrm{~d}, J=15.1 \mathrm{~Hz}, 1 \mathrm{H}), 2.23(\mathrm{dd}, J=12.1,10.8 \mathrm{~Hz}, 1 \mathrm{H})$.

${ }^{13} \mathrm{C} \mathrm{NMR}$ (151 MHz, CDCl 3 ) $\delta 178.1$ (s), 164.2 (s), 158.6 (s), 155.1 (s), 137.7 (s), 137.3 (s), 130.5 (s), 130.2 (s), 129.9 (s), 129.8 (s), 129.1 (s), 128.5 (s), 128.5 (s), 127.8 (s), 127.4 (s), 124.6 (s), 122.5 (s), 118.8 (s), 114.9 (s), 113.6 (s), 72.2 (s), 42.6 (s), 41.1 (s), 40.0 (s).

HRMS (ESI, m/z): Mass calcd for $\mathrm{C}_{28} \mathrm{H}_{20} \mathrm{O}_{4} \mathrm{Br}[\mathrm{M}+\mathrm{H}]^{+}, 499.0540$; found 499.0540 Enantiomeric ratio was measured by chiral phase HPLC (Chiralcel AD-H; IPA/Hexanes = 30/70, $0.4 \mathrm{~mL} / \mathrm{min}, 254 \mathrm{~nm}$ ), $\mathrm{Rt}_{1}$ (major) $=20.6 \mathrm{~min}, \mathrm{Rt}_{2}$ (minor) $=41.4 \mathrm{~min}$; er = 99.3:0.7).

(4aS,5aR)-9-bromo-1,4a-diphenyl-4,4a,5,5a-tetrahydro-3H,11H-pyrano[3,4-b]xanthe ne-3,11-dione (3p')

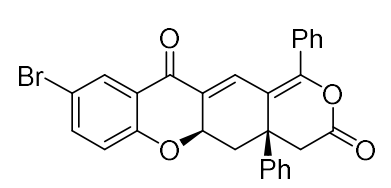

White solid, m.p. $167-169{ }^{\circ} \mathrm{C}, 16.9 \mathrm{mg}, 29 \%$ yield.

$[\alpha]_{\mathrm{D}}^{25}=287.6\left(c 1.0 \mathrm{CHCl}_{3}\right)$

${ }^{1} \mathrm{H}$ NMR $\left(400 \mathrm{MHz}, \mathrm{CDCl}_{3}\right) \delta 7.88(\mathrm{~d}, J=2.5 \mathrm{~Hz}, 1 \mathrm{H}), 7.62-$ $7.53(\mathrm{~m}, 2 \mathrm{H}), 7.50-7.40(\mathrm{~m}, 4 \mathrm{H}), 7.29(\mathrm{dd}, J=8.8,2.5 \mathrm{~Hz}, 1 \mathrm{H})$, $7.18-7.07(\mathrm{~m}, 5 \mathrm{H}), 6.47(\mathrm{~d}, J=8.8 \mathrm{~Hz}, 1 \mathrm{H}), 4.89(\mathrm{~d}, J=6.0 \mathrm{~Hz}, 1 \mathrm{H}), 3.17(\mathrm{dd}, J=20.6$, $15.2 \mathrm{~Hz}, 2 \mathrm{H}$ ), $2.82(\mathrm{~d}, J=15.0 \mathrm{~Hz}, 1 \mathrm{H}), 2.33(\mathrm{dd}, J=15.4,6.0 \mathrm{~Hz}, 1 \mathrm{H})$.

${ }^{13} \mathrm{C} \mathrm{NMR}\left(101 \mathrm{MHz}, \mathrm{CDCl}_{3}\right) \delta 180.8$ (s), 164.0 (s), 159.2 (s), 154.9 (s), 137.9 (s), 137.0 (s), 130.4 (s), 130.1 (s), 130.0 (s), 129.65 (s), 129.05 (s), 128.51 (s), 127.8 (s), 127.7 (s), 126.6 (s), 125.4 (s), 122.5 (s), 118.8 (s), 114.3 (s), 113.2 (s), 71.6 (s), 43.3 (s), 39.0 (s), 38.4 (s).

HRMS (ESI, m/z): Mass calcd for $\mathrm{C}_{28} \mathrm{H}_{20} \mathrm{O}_{4} \mathrm{Br}$ [M+H] ${ }^{+}$, 499.0540; found 499.0540 Enantiomeric ratio was measured by chiral phase HPLC (Chiralcel AD-H; IPA/Hexanes = 30/70, $0.4 \mathrm{~mL} / \mathrm{min}, 254 \mathrm{~nm}$ ), Rt ${ }_{1}$ (major) $=18.0 \mathrm{~min}, \mathrm{Rt}_{2}$ (minor) $=51.3 \mathrm{~min}$; er $=98: 2$ ).

(4aS,5aS)-9-methyl-1,4a-diphenyl-4,4a,5,5a-tetrahydro-3H,11H-pyrano[3,4-b]xanthe ne-3,11-dione (3q)<smiles>Cc1ccc2c(c1)C(=O)C1=CC3=C(c4ccccc4)OC(=O)CC3(c3ccccc3)CC1O2</smiles>

Yellow solid, m.p. $119-121^{\circ} \mathrm{C}, 43.1 \mathrm{mg}, 98 \%$ yield.

$[\alpha]_{\mathrm{D}}^{27}=80.0\left(c 1.0 \mathrm{CHCl}_{3}\right)$

$\underline{{ }^{1} \mathrm{H} \text { NMR }}\left(600 \mathrm{MHz}, \mathrm{CDCl}_{3}\right) \delta 7.79(\mathrm{~d}, J=2.0 \mathrm{~Hz}, 1 \mathrm{H}), 7.70(\mathrm{~d}, J$ 
$=1.8 \mathrm{~Hz}, 1 \mathrm{H}), 7.68-7.63(\mathrm{~m}, 2 \mathrm{H}), 7.57-7.50(\mathrm{~m}, 3 \mathrm{H}), 7.35(\mathrm{t}, J=7.5 \mathrm{~Hz}, 2 \mathrm{H}), 7.30-7.25$ (m, 4H), $6.82(\mathrm{~d}, J=8.4 \mathrm{~Hz}, 1 \mathrm{H}), 4.53$ (ddd, $J=10.6,5.0,2.1 \mathrm{~Hz}, 1 \mathrm{H}), 3.37(\mathrm{~d}, J=15.1 \mathrm{~Hz}$, $1 \mathrm{H}), 3.03(\mathrm{dd}, J=12.2,5.1 \mathrm{~Hz}, 1 \mathrm{H}), 2.97(\mathrm{~d}, J=15.1 \mathrm{~Hz}, 1 \mathrm{H}), 2.36-2.31(\mathrm{~m}, 1 \mathrm{H}), 2.30(\mathrm{~s}$, $3 \mathrm{H})$.

${ }^{13} \mathrm{C}$ NMR (151 MHz, CDCl 3 ) ठ 180.5 (s), 165.4 (s), 158.8 (s), 155.4 (s), 138.9 (s), 136.9 (s), 132.9 (s), 131.6 (s), 131.1 (s), 130.7 (s), 130.2 (s), 129.6 (s), 129.5 (s), 128.8 (s), 128.3 (s), 127.3 (s), 125.7 (s), 122.0 (s), 117.6 (s), 116.2 (s), 72.9 (s), 43.7 (s), 42.3 (s), 41.5 (s), 20.5 (s).

HRMS (ESI, m/z): Mass calcd for $\mathrm{C}_{29} \mathrm{H}_{23} \mathrm{O}_{4}[\mathrm{M}+\mathrm{H}]^{+}, 435.1591$; found 435.1595 Enantiomeric ratio was measured by chiral phase HPLC (Chiralcel AD-H; IPA/Hexanes = 30/70, $0.4 \mathrm{~mL} / \mathrm{min}, 254 \mathrm{~nm}$ ), $\mathrm{Rt}_{1}$ (major) = $18.3 \mathrm{~min}, \mathrm{Rt}_{2}$ (minor) $=49.9 \mathrm{~min}$; er = 98:2).

(4aS,5aS)-8-methyl-1,4a-diphenyl-4,4a,5,5a-tetrahydro-3H,11H-pyrano[3,4-b]xanthe ne-3,11-dione (3r)<smiles></smiles>

Yellow solid, m.p. $160-162{ }^{\circ} \mathrm{C}, 34.3 \mathrm{mg}, 79 \%$ yield.

$[\alpha]_{D}^{28}=40.0\left(c 0.5 \mathrm{CHCl}_{3}\right)$

$\underline{{ }^{1} \mathrm{H} \text { NMR }}\left(400 \mathrm{MHz}, \mathrm{CDCl}_{3}\right) \delta 7.76(\mathrm{dd}, J=11.1,5.0 \mathrm{~Hz}, 2 \mathrm{H})$, 7.68 - $7.59(\mathrm{~m}, 2 \mathrm{H}), 7.56-7.45(\mathrm{~m}, 3 \mathrm{H}), 7.39-7.30(\mathrm{~m}, 2 \mathrm{H})$, $7.29-7.23(\mathrm{~m}, 3 \mathrm{H}), 6.82(\mathrm{dd}, J=8.0,0.8 \mathrm{~Hz}, 1 \mathrm{H}), 6.70(\mathrm{~s}, 1 \mathrm{H}), 4.52$ (ddd, $J=10.6,5.0$, $2.0 \mathrm{~Hz}, 1 \mathrm{H}$ ), $3.34(\mathrm{~d}, J=15.1 \mathrm{~Hz}, 1 \mathrm{H}), 3.01(\mathrm{dd}, J=12.2,5.1 \mathrm{~Hz}, 1 \mathrm{H}), 2.94(\mathrm{~d}, J=15.1 \mathrm{~Hz}$, $1 \mathrm{H}), 2.34-2.26(\mathrm{~m}, 4 \mathrm{H})$.

${ }^{13} \mathrm{C}$ NMR (101 MHz, $\mathrm{CDCl}_{3}$ ) ठ 180.1 (s), 165.4 (s), 160.8 (s), 155.3 (s), 147.4 (s), 138.9 (s), 132.8 (s), 131.1 (s), 130.7 (s), 129.9 (s), 129.6 (s), 129.4 (s), 128.74 (s), 128.3 (s), 127.6 (s), 125.7 (s), 123.4 (s), 120.0 (s), 117.8 (s), 116.2 (s), 72.9 (s), 43.7 (s), 42.4 (s), 41.5 (s), 22.0 (s).

HRMS (ESI, m/z): Mass calcd for $\mathrm{C}_{29} \mathrm{H}_{23} \mathrm{O}_{4}[\mathrm{M}+\mathrm{H}]^{+}, 435.1591$; found 435.1593.

Enantiomeric ratio was measured by chiral phase HPLC (Chiralcel AD-H; IPA/Hexanes = 30/70, $0.4 \mathrm{~mL} / \mathrm{min}, 254 \mathrm{~nm}$ ), Rt ${ }_{1}$ (major) $=51.1 \mathrm{~min}, \mathrm{Rt}_{2}$ (minor) $=81.3 \mathrm{~min}$; er $=99: 1$ ).

(4aS,5aR)-8-methyl-1,4a-diphenyl-4,4a,5,5a-tetrahydro-3H,11H-pyrano[3,4-b]xanthe ne-3,11-dione (3r')<smiles>Cc1ccc2c(c1)OC1C[C@H](c3ccccc3)CC(=O)OC1=CC2=O</smiles>

White solid, m.p. $238-240{ }^{\circ} \mathrm{C}, 6.8 \mathrm{mg}, 15 \%$ yield.

$[\alpha]_{D}^{28}=154.4\left(c 0.25 \mathrm{CHCl}_{3}\right)$.

1H NMR $\left(400 \mathrm{MHz}, \mathrm{CDCl}_{3}\right) \delta 7.76(\mathrm{~d}, \mathrm{~J}=8.0 \mathrm{~Hz}, 1 \mathrm{H}), 7.72-$ $7.61(\mathrm{~m}, 2 \mathrm{H}), 7.59-7.46(\mathrm{~m}, 4 \mathrm{H}), 7.26-7.22(\mathrm{~m}, 4 \mathrm{H}), 7.21$ $7.14(\mathrm{~m}, 1 \mathrm{H}), 6.75(\mathrm{~d}, J=8.1 \mathrm{~Hz}, 1 \mathrm{H}), 6.47(\mathrm{~s}, 1 \mathrm{H}), 4.96(\mathrm{~d}, J=6.1 \mathrm{~Hz}, 1 \mathrm{H}), 3.24(\mathrm{dd}, J=$ 20.1, $15.2 \mathrm{~Hz}, 2 \mathrm{H}$ ), 2.89 (d, J = 15.0 Hz, 1H), 2.40 (dd, $J=15.3,6.1 \mathrm{~Hz}, 1 \mathrm{H}), 2.24(\mathrm{~s}, 3 \mathrm{H}$ ). $\underline{{ }^{13} \mathrm{C} \mathrm{NMR}}$ (101 MHz, CDCl 3 ) ठ 182.9 (s), 165.4 (s), 161.5 (s), 155.2 (s), 147.1 (s), 139.2 (s), 132.4 (s), 130.9 (s), 130.8 (s), 130.1 (s), 129.5 (s), 128.8 (s), 128.7 (s), 127.6 (s), 127.5 (s), 126.5 (s), 123.0 (s), 120.0 (s), 117.8 (s), 115.6 (s), 72.4 (s), 44.5 (s), 40.1 (s), 39.6 (s), 21.8 (s).

HRMS (ESI, m/z): Mass calcd for $\mathrm{C}_{29} \mathrm{H}_{23} \mathrm{O}_{4}[\mathrm{M}+\mathrm{H}]^{+}, 435.1591$; found 435.1593.

Enantiomeric ratio was measured by chiral phase HPLC (Chiralcel AD-H; IPA/Hexanes = 30/70, $0.4 \mathrm{~mL} / \mathrm{min}, 254 \mathrm{~nm}$ ), Rt $\mathrm{R}_{1}$ (major) $=12.8 \mathrm{~min}, \mathrm{Rt}_{2}$ (minor) $=30.0 \mathrm{~min}$; er $=83: 17$ ). 
(4aS,5aS)-8-methoxy-1,4a-diphenyl-4,4a,5,5a-tetrahydro-3H,11H-pyrano[3,4-b]xanth ene-3,11-dione (3s)

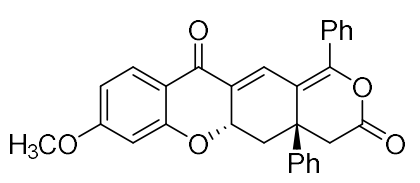

Yellow solid, m.p. $120-122{ }^{\circ} \mathrm{C}, 24.8 \mathrm{mg}, 55 \%$ yield. $[\alpha]_{\mathrm{D}}{ }^{30}=93.1\left(c 1.0 \mathrm{CHCl}_{3}\right)$.

1․ NMR $\left(600 \mathrm{MHz}, \mathrm{CDCl}_{3}\right) \delta 7.86(\mathrm{~d}, J=8.8 \mathrm{~Hz}, 1 \mathrm{H}), 7.76(\mathrm{~d}$, $J=2.0 \mathrm{~Hz}, 1 \mathrm{H}), 7.68-7.63(\mathrm{~m}, 2 \mathrm{H}), 7.57-7.49(\mathrm{~m}, 3 \mathrm{H}), 7.35(\mathrm{t}$, $J=7.5 \mathrm{~Hz}, 2 \mathrm{H}$ ), 7.29 (td, $J=7.7,2.0 \mathrm{~Hz}, 3 \mathrm{H}), 6.59(\mathrm{dd}, J=8.8,2.4 \mathrm{~Hz}, 1 \mathrm{H}), 6.37(\mathrm{~d}, J=$ $2.4 \mathrm{~Hz}, 1 \mathrm{H}$ ), 4.56 (ddd, $J=10.6,5.0,2.0 \mathrm{~Hz}, 1 \mathrm{H}), 3.83(\mathrm{~s}, 3 \mathrm{H}), 3.37(\mathrm{~d}, J=15.1 \mathrm{~Hz}, 1 \mathrm{H}$ ), $3.03(\mathrm{dd}, J=12.2,5.1 \mathrm{~Hz}, 1 \mathrm{H}$ ), $2.97(\mathrm{~d}, J=15.1 \mathrm{~Hz}, 1 \mathrm{H}$ ), 2.33 (dd, $J=12.1,10.8 \mathrm{~Hz}, 1 \mathrm{H}$ ). ${ }^{13} \mathrm{C} \mathrm{NMR}\left(151 \mathrm{MHz}, \mathrm{CDCl}_{3}\right) \delta 179.2$ (s), 166.0 (s), 165.5 (s), 162.7 (s), 155.1 (s), 138.9 (s), 132.6 (s), 131.1 (s), 130.7 (s), 129.6 (s), 129.5 (s), 129.5 (s), 129.4 (s), 128.7 (s), 128.3 (s), 125.7 (s), 116.1 (s), 116.0 (s), 110.48 (s), 100.85 (s), 73.27 (s), 55.67 (s), 43.74 (s), 42.35 (s), 41.54 (s).

HRMS (ESI, m/z): Mass calcd for $\mathrm{C}_{28} \mathrm{H}_{20} \mathrm{O}_{4} \mathrm{Cl}[\mathrm{M}+\mathrm{H}]^{+}$, 451.1540; found 451.1530. Enantiomeric ratio was measured by chiral phase HPLC (Chiralcel AD-H; IPA/Hexanes = 30/70, $0.4 \mathrm{~mL} / \mathrm{min}, 254 \mathrm{~nm}$ ), Rt ${ }_{1}$ (major) $=67.5 \mathrm{~min}, \mathrm{Rt}_{2}$ (minor) $=73.4 \mathrm{~min}$; er = 98:2).

(4aS,5aR)-8-methoxy-1,4a-diphenyl-4,4a,5,5a-tetrahydro-3H,11H-pyrano[3,4-b]xanth ene-3,11-dione (3s')<smiles>COc1ccc2c(c1)OC1CC3(c4ccccc4)CC(=O)OC3C=CC1=C2c1ccccc1</smiles>
White solid, m.p. $102-104{ }^{\circ} \mathrm{C}, 15 \mathrm{mg}, 33 \%$ yield. $[\alpha]_{\mathrm{D}}^{28}=292.1\left(\right.$ c $\left.0.1 \mathrm{CHCl}_{3}\right)$.

1 NMR $\left(400 \mathrm{MHz}, \mathrm{CDCl}_{3}\right) \delta 7.74(\mathrm{~d}, J=8.8 \mathrm{~Hz}, 1 \mathrm{H}), 7.58(\mathrm{dd}$, $\mathrm{J}=6.5,3.2 \mathrm{~Hz}, 2 \mathrm{H}), 7.48-7.40(\mathrm{~m}, 4 \mathrm{H}), 7.19-7.08(\mathrm{~m}, 5 \mathrm{H})$, $6.44(\mathrm{dd}, \mathrm{J}=8.9,2.4 \mathrm{~Hz}, 1 \mathrm{H}), 6.02(\mathrm{~d}, \mathrm{~J}=2.4 \mathrm{~Hz}, 1 \mathrm{H}), 4.92(\mathrm{~d}, J=6.2 \mathrm{~Hz}, 1 \mathrm{H}), 3.66(\mathrm{~d}, J$ $=8.2 \mathrm{~Hz}, 3 \mathrm{H}), 3.17(\mathrm{dd}, J=29.7,15.2 \mathrm{~Hz}, 2 \mathrm{H}), 2.91-2.75(\mathrm{~m}, 1 \mathrm{H}), 2.34(\mathrm{dd}, J=15.3,6.1$ $\mathrm{Hz}, 1 \mathrm{H})$.

${ }^{13} \mathrm{C}$ NMR $\left(101 \mathrm{MHz}, \mathrm{CDCl}_{3}\right) \delta 182.0$ (s), 165.7 (s), 165.4 (s), 163.4 (s), 155.1 (s), 139.2 (s), 132.3 (s), 130.9 (s), 130.8 (s), 129.9 (s), 129.5 (s), 129.4 (s), 128.8 (s), 128.7 (s), 127.5 (s), 126.5 (s), 116.1 (s), 115.6 (s), 110.2 (s), 100.7 (s), 72.7 (s), 55.6 (s), 44.4 (s), 40.1 (s), 39.6 (s).

HRMS (ESI, m/z): Mass calcd for $\mathrm{C}_{28} \mathrm{H}_{20} \mathrm{O}_{4} \mathrm{Cl}[\mathrm{M}+\mathrm{H}]^{+}$, 451.1540; found 451.1529.

Enantiomeric ratio was measured by chiral phase HPLC (Chiralcel AD-H; IPA/Hexanes = 30/70, $0.4 \mathrm{~mL} / \mathrm{min}, 254 \mathrm{~nm}$ ), $\mathrm{Rt}_{1}$ (major) $=26.5 \mathrm{~min}, \mathrm{Rt}_{2}$ (minor) $=50.8 \mathrm{~min}$; er = 98:2).

(4aS,5aS)-1-(2-bromophenyl)-4a-phenyl-4,4a,5,5a-tetrahydro-3H,11H-pyrano[3,4-b]x anthene-3,11-dione (3t)

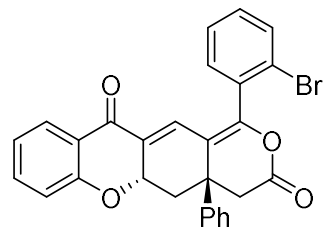

Yellow solid, m.p. $98-100{ }^{\circ} \mathrm{C}, 28.9 \mathrm{mg}, 58 \%$ yield.

$[\alpha]_{D}{ }^{30}=45.3\left(c 1.0 \mathrm{CHCl}_{3}\right)$.

1 ${ }^{\mathrm{H}}$ NMR $\left(400 \mathrm{MHz}, \mathrm{CDCl}_{3}\right) \delta 7.86(\mathrm{dd}, J=7.9,1.7 \mathrm{~Hz}, 1 \mathrm{H}), 7.71(\mathrm{~d}$, $J=7.6 \mathrm{~Hz}, 1 \mathrm{H}), 7.57(\mathrm{~s}, 1 \mathrm{H}), 7.53-7.45(\mathrm{~m}, 1 \mathrm{H}), 7.45-7.33(\mathrm{~m}$, $5 \mathrm{H}), 7.29$ (t, $J=5.6 \mathrm{~Hz}, 3 \mathrm{H}$ ), 6.99 (td, $J=7.7,1.0 \mathrm{~Hz}, 1 \mathrm{H}), 6.88$ (dd, $J=8.4,0.7 \mathrm{~Hz}, 1 \mathrm{H}), 4.58(\mathrm{~d}, J=6.5 \mathrm{~Hz}, 1 \mathrm{H}), 3.43(\mathrm{~d}, J=15.1 \mathrm{~Hz}, 1 \mathrm{H}), 3.00(\mathrm{t}, J=15.3 \mathrm{~Hz}$, $2 \mathrm{H}), 2.30(\mathrm{t}, J=11.4 \mathrm{~Hz}, 1 \mathrm{H})$. 
${ }^{13} \mathrm{C} \mathrm{NMR}$ (101 MHz, CDCl 3 ) ס 180.1(s), 165.3 (s), 160.7 (s), 138.9 (s), 135.9 (s), 133.4 (s), 133.0 (s), 132.2 (s), 131.9 (s), 131.8 (s), 129.5 (s), 129.3 (s), 128.4 (s), 127.8 (s), 127.7 (s), 126.1 (s), 125.6 (s), 123.8 (s), 122.2 (s), 122.1 (s), 118.4 (s), 117.8 (s), 73.0 (s), 43.5 (s), $42.8(\mathrm{~s}), 41.0(\mathrm{~s})$.

HRMS (ESI, m/z): Mass calcd for $\mathrm{C}_{28} \mathrm{H}_{20} \mathrm{O}_{4} \mathrm{Br}[\mathrm{M}+\mathrm{H}]^{+}$, 499.0540; found 499.0531. Enantiomeric ratio was measured by chiral phase HPLC (Chiralcel AD-H; IPA/Hexanes = 30/70, $0.4 \mathrm{~mL} / \mathrm{min}, 254 \mathrm{~nm}$ ), Rt $\mathrm{R}_{1}$ (major) $=20.3 \mathrm{~min}, \mathrm{Rt}_{2}$ (minor) $=50.3 \mathrm{~min}$; er = 97:3).

(4aS,5aR)-1-(2-bromophenyl)-4a-phenyl-4,4a,5,5a-tetrahydro-3H,11H-pyrano[3,4-b]x anthene-3,11-dione (3t')

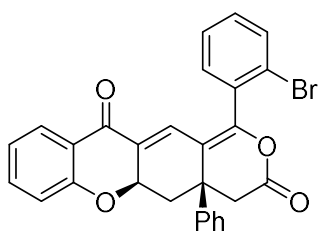

White solid, m.p. $68-70^{\circ} \mathrm{C}, 14 \mathrm{mg}, 28 \%$ yield.

$[\alpha]_{D^{27}}=163.2\left(c 0.5 \mathrm{CHCl}_{3}\right)$.

1․ NMR $\left(400 \mathrm{MHz}, \mathrm{CDCl}_{3}\right) \delta 7.87(\mathrm{dd}, J=7.9,1.6 \mathrm{~Hz}, 1 \mathrm{H}), 7.72(\mathrm{~d}$, $J=7.9 \mathrm{~Hz}, 1 \mathrm{H}), 7.57-7.27(\mathrm{~m}, 8 \mathrm{H}), 7.21(\mathrm{t}, J=6.9 \mathrm{~Hz}, 1 \mathrm{H}), 7.09(\mathrm{~s}$, $1 \mathrm{H}), 6.98-6.90(\mathrm{~m}, 1 \mathrm{H}), 6.64(\mathrm{~d}, J=8.4 \mathrm{~Hz}, 1 \mathrm{H}), 4.94(\mathrm{~d}, J=5.8 \mathrm{~Hz}$, $1 \mathrm{H}), 3.36(\mathrm{~d}, J=15.2 \mathrm{~Hz}, 1 \mathrm{H}), 3.14(\mathrm{~d}, J=14.8 \mathrm{~Hz}, 1 \mathrm{H}), 2.98(\mathrm{~d}, J=14.7 \mathrm{~Hz}, 1 \mathrm{H}), 2.39$ (dd, $J=15.2,5.7 \mathrm{~Hz}, 1 \mathrm{H}$ ).

${ }^{13} \mathrm{C} \mathrm{NMR}$ (101 MHz, $\mathrm{CDCl}_{3}$ ) $\delta 183.3$ (s), 171.2 (s), 165.1 (s), 161.5 (s), 139.6 (s), 135.6 (s), 133.6 (s), 133.5 (s), 132.3 (s), 132.2 (s), 131.9 (s), 131.3 (s), 131.1 (s), 129.1 (s), 128.8 (s), 127.8 (s), 127.6 (s), 126.6 (s), 126.3 (s), 122.2 (s), 121.6 (s), 117.9 (s), 72.3 (s), 60.4 (s), 44.3 (s), 39.7 (s).

HRMS (ESI, m/z): Mass calcd for $\mathrm{C}_{28} \mathrm{H}_{20} \mathrm{O}_{4} \mathrm{Br}[\mathrm{M}+\mathrm{H}]^{+}$, 499.0540; found 499.0531 . Enantiomeric ratio was measured by chiral phase HPLC (Chiralcel AD-H; IPA/Hexanes = 30/70, $0.4 \mathrm{~mL} / \mathrm{min}, 254 \mathrm{~nm}$ ), Rt ${ }_{1}$ (major) $=20.0 \mathrm{~min}, \mathrm{Rt}_{2}($ minor $)=32.5 \mathrm{~min}$; er = 93:7).

(4aS,5aS)-4a-phenyl-1-(m-tolyl)-4,4a,5,5a-tetrahydro-3H,11H-pyrano[3,4-b]xanthene3,11-dione (3u)

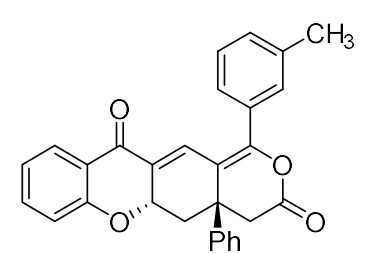

Yellow solid, m.p. $78-80^{\circ} \mathrm{C}, 25.9 \mathrm{mg}, 60 \%$ yield.

$[\alpha]_{\mathrm{D}}^{29}=97.8\left(c 1.0 \mathrm{CHCl}_{3}\right)$.

1ㅂ NMR $\left(600 \mathrm{MHz}, \mathrm{CDCl}_{3}\right) \delta 7.92(\mathrm{dd}, J=7.9,1.7 \mathrm{~Hz}, 1 \mathrm{H}), 7.80(\mathrm{~d}$, $J=2.0 \mathrm{~Hz}, 1 \mathrm{H}), 7.50-7.39(\mathrm{~m}, 4 \mathrm{H}), 7.36(\mathrm{dd}, J=9.1,6.0 \mathrm{~Hz}, 3 \mathrm{H})$,

$7.32-7.26(\mathrm{~m}, 3 \mathrm{H}), 7.03(\mathrm{t}, J=7.5 \mathrm{~Hz}, 1 \mathrm{H}), 6.92(\mathrm{~d}, J=8.3 \mathrm{~Hz}$,

1H), 4.57 (ddd, $J=10.6,5.0,2.0 \mathrm{~Hz}, 1 \mathrm{H}$ ), 3.37 (d, $J=15.1 \mathrm{~Hz}, 1 \mathrm{H}), 3.06$ (dd, $J=12.2,5.1$ $\mathrm{Hz}, 1 \mathrm{H}), 2.97(\mathrm{~d}, J=15.1 \mathrm{~Hz}, 1 \mathrm{H}), 2.46(\mathrm{~s}, 3 \mathrm{H}), 2.33(\mathrm{dd}, J=12.0,10.9 \mathrm{~Hz}, 1 \mathrm{H})$.

${ }^{13} \mathrm{C} \mathrm{NMR}\left(151 \mathrm{MHz}, \mathrm{CDCl}_{3}\right) \delta 180.4$ (s), 165.5 (s), 160.8 (s), 155.8 (s), 138.9 (s), 138.6 (s), 135.8 (s), 132.4 (s), 131.6 (s), 131.0 (s), 130.6 (s), 129.9 (s), 129.5 (s), 128.6 (s), 128.3 (s), 127.7 (s), 126.9 (s), 125.7 (s), 122.3 (s), 122.1 (s), 117.8 (s), 116.0 (s), 73.0 (s), 43.7 (s), 42.3 (s), 41.5 (s), 21.5 (s).

HRMS (ESI, m/z): Mass calcd for $\mathrm{C}_{29} \mathrm{H}_{23} \mathrm{O}_{4}[\mathrm{M}+\mathrm{H}]^{+}, 435.1591$; found 435.1586.

Enantiomeric ratio was measured by chiral phase HPLC (Chiralcel AD-H; IPA/Hexanes = 30/70, $0.4 \mathrm{~mL} / \mathrm{min}, 254 \mathrm{~nm}$ ), $\mathrm{Rt}_{1}$ (major) $=16.0 \mathrm{~min}, \mathrm{Rt}_{2}$ (minor) $=33.9 \mathrm{~min}$; er = 96:4). 
(4aS,5aR)-4a-phenyl-1-(m-tolyl)-4,4a,5,5a-tetrahydro-3H,11H-pyrano[3,4-b]xanthene -3,11-dione (3u')

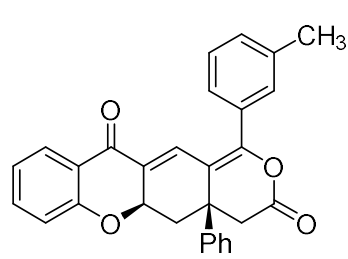

White solid, m.p. $82-84^{\circ} \mathrm{C}, 10.2 \mathrm{mg}, 23 \%$ yield.

$[\alpha]_{\mathrm{D}}^{27}=127.2\left(c 0.2 \mathrm{CHCl}_{3}\right)$.

${ }^{1} \mathrm{H}$ NMR $\left(400 \mathrm{MHz}, \mathrm{CDCl}_{3}\right) \delta 7.87(\mathrm{dd}, J=7.9,1.7 \mathrm{~Hz}, 1 \mathrm{H}), 7.53$

$7.47(\mathrm{~m}, 2 \mathrm{H}), 7.41(\mathrm{dd}, J=6.5,2.2 \mathrm{~Hz}, 2 \mathrm{H}), 7.36-7.27(\mathrm{~m}, 4 \mathrm{H})$,

$7.24(\mathrm{~s}, 2 \mathrm{H}), 6.93(\mathrm{dd}, J=11.1,3.9 \mathrm{~Hz}, 1 \mathrm{H}), 6.66(\mathrm{~d}, J=8.3 \mathrm{~Hz}$, $1 \mathrm{H}), 4.99(\mathrm{~d}, J=6.1 \mathrm{~Hz}, 1 \mathrm{H}), 3.23(\mathrm{~d}, J=14.2 \mathrm{~Hz}, 2 \mathrm{H}), 2.88(\mathrm{~d}, J=15.0 \mathrm{~Hz}, 1 \mathrm{H}), 2.45(\mathrm{~s}$, $3 \mathrm{H}), 2.40$ (dd, $J=15.4,6.1 \mathrm{~Hz}, 1 \mathrm{H})$.

13. NMR (101 MHz, CDCl ${ }_{3}$ ) $\delta 183.1$ (s), 165.4 (s), 161.4 (s), 155.6 (s), 139.2 (s), 138.6 (s), 135.4 (s), 131.9 (s), 131.7 (s), 130.7 (s), 129.8 (s), 129.4 (s), 128.8 (s), 128.5 (s), 127.8 (s), 127.5 (s), 127.0 (s), 126.5 (s), 122.3 (s), 121.5 (s), 117.8 (s), 115.4 (s), 72.5 (s), 44.5 (s), 40.1 (s), 39.5 (s), 21.4 (s).

HRMS (ESI, m/z): Mass calcd for $\mathrm{C}_{29} \mathrm{H}_{23} \mathrm{O}_{4}[\mathrm{M}+\mathrm{H}]^{+}, 435.1591$; found 435.1586.

Enantiomeric ratio was measured by chiral phase HPLC (Chiralcel AD-H; IPA/Hexanes = 30/70, $0.4 \mathrm{~mL} / \mathrm{min}, 254 \mathrm{~nm}$ ), Rt ${ }_{1}$ (major) = $13.7 \mathrm{~min}, \mathrm{Rt}_{2}$ (minor) $=25.0 \mathrm{~min}$; er = 99:1).

(4aS,5aS)-1-(4-fluorophenyl)-4a-phenyl-4,4a,5,5a-tetrahydro-3H,11H-pyrano[3,4-b]x anthene-3,11-dione (3v)

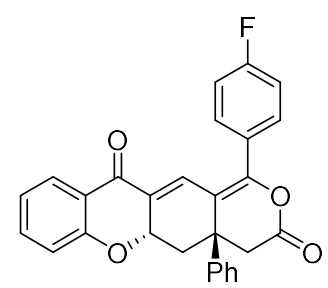

Yellow solid, m.p. $166-168{ }^{\circ} \mathrm{C}, 34.3 \mathrm{mg}, 78 \%$ yield.

$[\alpha]_{D}^{30}=105.7\left(c 1.0 \mathrm{CHCl}_{3}\right)$.

1 NMR $\left(600 \mathrm{MHz}, \mathrm{CDCl}_{3}\right) \delta 7.92(\mathrm{dd}, J=7.9,1.6 \mathrm{~Hz}, 1 \mathrm{H}), 7.74(\mathrm{~d}$, $J=2.0 \mathrm{~Hz}, 1 \mathrm{H}), 7.70-7.62(\mathrm{~m}, 2 \mathrm{H}), 7.51-7.42(\mathrm{~m}, 1 \mathrm{H}), 7.36(\mathrm{t}, J=$ $7.6 \mathrm{~Hz}, 2 \mathrm{H}), 7.32-7.21(\mathrm{~m}, 5 \mathrm{H}), 7.03(\mathrm{t}, J=7.5 \mathrm{~Hz}, 1 \mathrm{H}), 6.92(\mathrm{~d}, J=$ $8.3 \mathrm{~Hz}, 1 \mathrm{H}$ ), 4.57 (ddd, $J=10.6,5.0,2.0 \mathrm{~Hz}, 1 \mathrm{H}), 3.38(\mathrm{~d}, J=15.1$

$\mathrm{Hz}, 1 \mathrm{H}$ ), $3.06(\mathrm{dd}, J=12.2,5.1 \mathrm{~Hz}, 1 \mathrm{H}), 2.97(\mathrm{~d}, J=15.1 \mathrm{~Hz}, 1 \mathrm{H}), 2.33(\mathrm{dd}, J=12.1,10.8$ $\mathrm{Hz}, 1 \mathrm{H})$.

13. NMR (151 MHz, CDCl ${ }_{3}$ ) 180.3 (s), 165.2 (s), 164.0 (d, J = $\left.252.9 \mathrm{~Hz}\right), 160.8$ (s), 154.4 (s), 138.8 (s), 135.9 (s), 132.8 (s), 131.7 (d, J = 8.6 Hz), 129.9 (s), 129.5 (s), 128.4 (s), 127.8 (s), 127.2 (d, J = 3.5 Hz), 125.7 (s), 122.3 (s), 122.2 (s), 117.8 (s), 116.1 (s), 116.16 (d, $J=16.3 \mathrm{~Hz}$ ), 72.93 (s), 43.68 (s), 42.26 (s), 41.57 (s).

${ }^{19} \mathrm{~F} \mathrm{NMR}\left(471 \mathrm{MHz}, \mathrm{CDCl}_{3}\right) \delta-108.2(\mathrm{~s})$.

HRMS (ESI, m/z): Mass calcd for $\mathrm{C}_{28} \mathrm{H}_{20} \mathrm{O}_{4} \mathrm{~F}[\mathrm{M}+\mathrm{H}]^{+}, 439.1340$; found 439.1342 .

Enantiomeric ratio was measured by chiral phase HPLC (Chiralcel AD-H; IPA/Hexanes = 30/70, $0.4 \mathrm{~mL} / \mathrm{min}, 254 \mathrm{~nm}$ ), Rt ${ }_{1}$ (major) $=32.2 \mathrm{~min}, \mathrm{Rt}_{2}$ (minor) $=69.5 \mathrm{~min}$; er $=96: 4$ ).

(4aS,5aS)-1-(4-chlorophenyl)-9-methyl-4a-phenyl-4,4a,5,5a-tetrahydro-3H,11H-pyran o[3,4-b]xanthene-3,11-dione (3w)

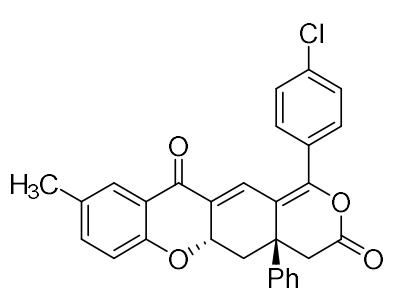

Yellow solid, m.p. $131-133^{\circ} \mathrm{C}, 45.0 \mathrm{mg}, 96 \%$ yield.

$[\alpha]_{D}^{30}=73.0\left(c 1.0 \mathrm{CHCl}_{3}\right)$.

1H NMR $\left(400 \mathrm{MHz}, \mathrm{CDCl}_{3}\right) \delta 7.68(\mathrm{dd}, J=5.1,2.0 \mathrm{~Hz}, 2 \mathrm{H})$, $7.62-7.54(\mathrm{~m}, 2 \mathrm{H}), 7.53-7.46(\mathrm{~m}, 2 \mathrm{H}), 7.36-7.30(\mathrm{~m}, 2 \mathrm{H})$, $7.27(\mathrm{dd}, J=5.5,1.7 \mathrm{~Hz}, 1 \mathrm{H}), 7.23(\mathrm{ddd}, J=5.0,3.6,2.0 \mathrm{~Hz}$, $3 \mathrm{H}), 6.80(\mathrm{~d}, J=8.4 \mathrm{~Hz}, 1 \mathrm{H}), 4.50(\mathrm{ddd}, J=10.6,5.0,2.1 \mathrm{~Hz}$, 
1H), $3.35(\mathrm{~d}, J=15.1 \mathrm{~Hz}, 1 \mathrm{H}), 3.01(\mathrm{dd}, J=12.2,5.1 \mathrm{~Hz}, 1 \mathrm{H}), 2.93(\mathrm{~d}, J=15.1 \mathrm{~Hz}, 1 \mathrm{H})$, $2.36-2.22(\mathrm{~m}, 4 \mathrm{H})$.

${ }^{13} \mathrm{C} \mathrm{NMR}\left(101 \mathrm{MHz}, \mathrm{CDCl}_{3}\right) \delta 180.1$ (s), 165.2 (s), 158.8 (s), 154.0 (s), 138.7 (s), 137.0 (s), 136.9 (s), 133.4 (s), 131.7 (s), 130.8 (s), 129.5 (s), 129.5 (s), 129.4 (s), 129.1 (s), 128.4 (s), 127.3 (s), 125.6 (s), 121.9 (s), 117.6 (s), 116.7 (s), 72.82 (s), 43.66 (s), 42.29 (s), 41.62 (s), 20.47 (s).

HRMS (ESI, m/z): Mass calcd for $\mathrm{C}_{29} \mathrm{H}_{22} \mathrm{O}_{4} \mathrm{Cl}[\mathrm{M}+\mathrm{H}]^{+}, 469.1201$; found 469.1191.

Enantiomeric ratio was measured by chiral phase HPLC (Chiralcel AD-H; IPA/Hexanes = 30/70, $0.4 \mathrm{~mL} / \mathrm{min}, 254 \mathrm{~nm}$ ), Rt ${ }_{1}$ (major) $=28.7 \mathrm{~min}, \mathrm{Rt}_{2}$ (minor) $=73.9 \mathrm{~min}$; er = 98:2).

(4aS,5aS)-4a-phenyl-1-((E)-styryl)-4,4a,5,5a-tetrahydro-3H,11H-pyrano[3,4-b]xanthe ne-3,11-dione (3x)

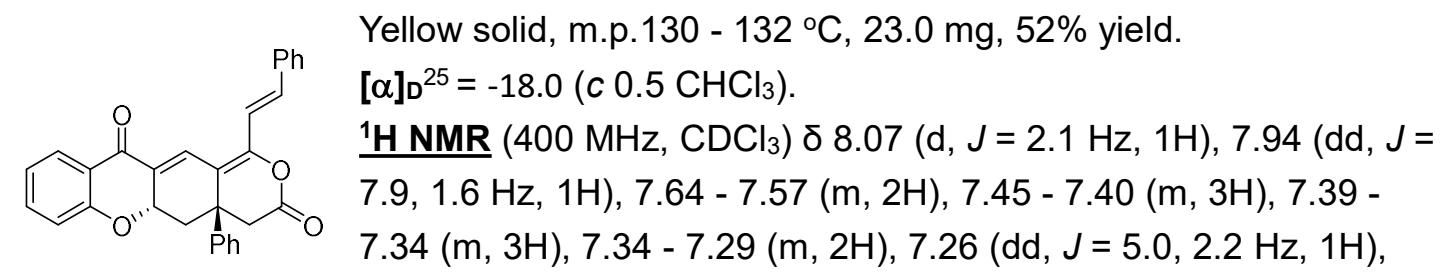

7.22 (dd, $J=6.3,4.8 \mathrm{~Hz}, 2 \mathrm{H}), 7.05-6.99(\mathrm{~m}, 1 \mathrm{H}), 6.89$ (dd, $J=8.3,0.6 \mathrm{~Hz}, 1 \mathrm{H}$ ), 4.57 (ddd, $J=10.7,4.9,2.0 \mathrm{~Hz}, 1 \mathrm{H}), 3.36(\mathrm{~d}, J=15.2 \mathrm{~Hz}, 1 \mathrm{H}), 2.93(\mathrm{dd}, J=12.1,5.0 \mathrm{~Hz}, 1 \mathrm{H}), 2.86$ (d, $J=15.2 \mathrm{~Hz}, 1 \mathrm{H}), 2.22(\mathrm{dd}, J=12.0,10.9 \mathrm{~Hz}, 1 \mathrm{H})$.

${ }^{13} \mathrm{C} \mathrm{NMR}\left(101 \mathrm{MHz}, \mathrm{CDCl}_{3}\right) \delta 180.1$ (s), 165.2 (s), 160.8 (s), 152.2 (s), 139.0 (s), 135.8 (s), 135.6 (s), 135.6 (s), 132.1 (s), 129.6 (s), 129.4 (s), 129.0 (s), 128.3 (s), 127.8 (s), 127.8 (s), 127.7 (s), 125.7 (s), 122.4 (s), 122.1 (s), 117.8 (s), 116.9 (s), 114.5 (s), 73.0 (s), 43.6 (s), 42.7 (s), 41.4 (s).

HRMS (ESI, m/z): Mass calcd for $\mathrm{C}_{30} \mathrm{H}_{22} \mathrm{O}_{4}[\mathrm{M}+\mathrm{Na}]^{+}$, 469.1410; found 469.1392.

Enantiomeric ratio was measured by chiral phase HPLC (Chiralcel AD-H; IPA/Hexanes = 30/70, $0.4 \mathrm{~mL} / \mathrm{min}, 254 \mathrm{~nm}$ ), $\mathrm{Rt}_{1}$ (minor) = $38.1 \mathrm{~min}, \mathrm{Rt}_{2}$ (major) = $66.1 \mathrm{~min}$; er = 90:10).

(4aS,5aR)-4a-phenyl-1-((E)-styryl)-4,4a,5,5a-tetrahydro-3H,11H-pyrano[3,4-b]xanthe ne-3,11-dione (3x')

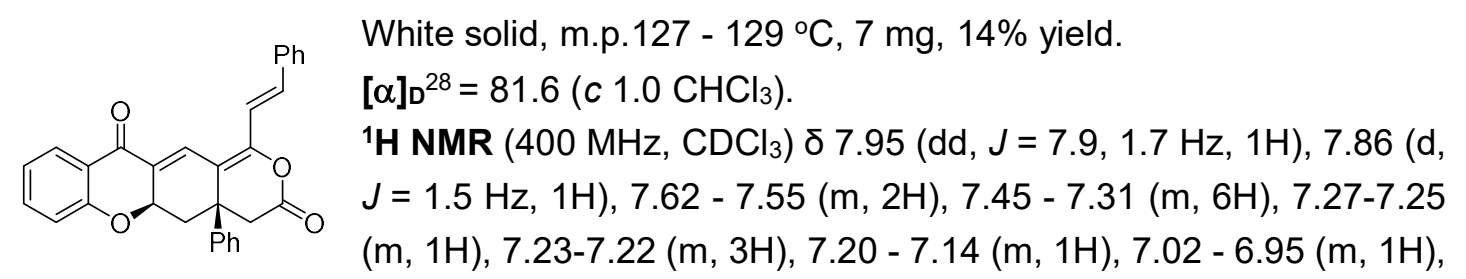

$6.67(\mathrm{dd}, J=8.4,0.6 \mathrm{~Hz}, 1 \mathrm{H}), 5.02-4.90(\mathrm{~m}, 1 \mathrm{H}), 3.29(\mathrm{~d}, J=15.2 \mathrm{~Hz}, 1 \mathrm{H}), 3.13(\mathrm{dd}, J=$ 15.3, $1.3 \mathrm{~Hz}, 1 \mathrm{H}$ ), 2.85 (d, $J=15.2 \mathrm{~Hz}, 1 \mathrm{H}), 2.33$ (dd, $J=15.3,6.0 \mathrm{~Hz}, 1 \mathrm{H}$ ).

${ }^{13} \mathrm{C} \mathrm{NMR}\left(101 \mathrm{MHz}, \mathrm{CDCl}_{3}\right) \delta 183.2$ (s), 165.0 (s), 161.5 (s), 152.1 (s), 139.6 (s), 135.6 (s), 135.5 (s), 135.4 (s), 131.6 (s), 129.5 (s), 129.0 (s), 128.8 (s), 127.9 (s), 127.7 (s), 127.6 (s), 127.5 (s), 126.3 (s), 122.4 (s), 121.6 (s), 117.9 (s), 116.2 (s), 114.4 (s), 72.2 (s), 44.4 (s), 40.0 (s), 39.7 (s).

HRMS (ESI, m/z): Mass calcd for $\mathrm{C}_{30} \mathrm{H}_{22} \mathrm{O}_{4}[\mathrm{M}+\mathrm{Na}]^{+}$, 469.1410; found 469.1392.

Enantiomeric ratio was measured by chiral phase HPLC (Chiralcel AD-H; IPA/Hexanes = 30/70, $0.4 \mathrm{~mL} / \mathrm{min}, 254 \mathrm{~nm}$ ), Rt ${ }_{1}$ (minor) $=36.7 \mathrm{~min}, \mathrm{Rt}_{2}$ (major) $=42.7 \mathrm{~min}$; er = 99:1). 
(4aS,5aS)-1-methyl-4a-phenyl-4,4a,5,5a-tetrahydro-3H,11H-pyrano[3,4-b]xanthene-3, 11-dione (3y)<smiles>CC1=C2C=C3C(=O)c4ccccc4O[C@@H]3C[C@]2(c2ccccc2)CC(=O)O1</smiles>

White solid, m.p. $98-100{ }^{\circ} \mathrm{C}, 14.8 \mathrm{mg}, 41 \%$ yield.

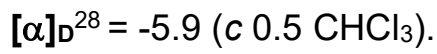

1H NMR $\left(400 \mathrm{MHz}, \mathrm{CDCl}_{3}\right) \delta 7.92(\mathrm{dd}, J=7.9,1.7 \mathrm{~Hz}, 1 \mathrm{H}), 7.74(\mathrm{~d}, J$ $=2.1 \mathrm{~Hz}, 1 \mathrm{H}), 7.42(\mathrm{ddd}, J=8.4,7.2,1.8 \mathrm{~Hz}, 1 \mathrm{H}), 7.35-7.28(\mathrm{~m}$,

$2 \mathrm{H}), 7.26-7.22(\mathrm{~m}, 1 \mathrm{H}), 7.21-7.14(\mathrm{~m}, 2 \mathrm{H}), 7.06-6.97(\mathrm{~m}, 1 \mathrm{H}), 6.88(\mathrm{dd}, J=8.3,0.7 \mathrm{~Hz}$, $1 \mathrm{H}), 4.56-4.45(\mathrm{~m}, 1 \mathrm{H}), 3.29(\mathrm{~d}, J=15.1 \mathrm{~Hz}, 1 \mathrm{H}), 2.89(\mathrm{dd}, J=12.1,5.0 \mathrm{~Hz}, 1 \mathrm{H}), 2.81(\mathrm{t}$, $J=9.1 \mathrm{~Hz}, 1 \mathrm{H}), 2.37(\mathrm{~d}, J=0.8 \mathrm{~Hz}, 3 \mathrm{H}), 2.14(\mathrm{dd}, J=12.0,10.8 \mathrm{~Hz}, 1 \mathrm{H})$.

${ }^{13} \mathrm{C} \mathrm{NMR}\left(101 \mathrm{MHz}, \mathrm{CDCl}_{3}\right) \delta 180.3$ (s), 165.5 (s), 160.58 (s), 155.1 (s), 139.2 (s), 135.8 (s), 131.5 (s), 129.3 (s), 129.2 (s), 128.18 (s), 127.73 (s), 125.65 (s), 122.3 (s), 122.1 (s), 117.8 (s), 114.8 (s), 73.0 (s), 43.7 (s), 42.5 (s), 40.7 (s), 16.2 (s).

HRMS (ESI, m/z): Mass calcd for $\mathrm{C}_{23} \mathrm{H}_{19} \mathrm{O}_{4}[\mathrm{M}+\mathrm{H}]^{+}, 359.1277$; found 359.1182

Enantiomeric ratio was measured by chiral phase HPLC (Chiralcel AD-H; IPA/Hexanes = 30/70, $0.4 \mathrm{~mL} / \mathrm{min}, 254 \mathrm{~nm}$ ), $\mathrm{Rt}_{1}$ (major) $=30.9 \mathrm{~min}, \mathrm{Rt}_{2}$ (minor) $=43.5 \mathrm{~min}$; $\mathrm{er}=97: 3$ ).

(4aS,5aR)-1-methyl-4a-phenyl-4,4a,5,5a-tetrahydro-3H,11H-pyrano[3,4-b]xanthene-3, 11-dione (3y')

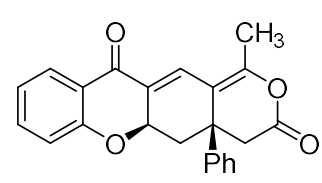

White solid, m.p. $88-90^{\circ} \mathrm{C}, 14.0 \mathrm{mg}, 39 \%$ yield.

$[\alpha]_{D}^{28}=-206.2\left(c 0.5 \mathrm{CHCl}_{3}\right)$.

$\underline{1}$ H NMR $\left(400 \mathrm{MHz}, \mathrm{CDCl}_{3}\right) \delta 7.92(\mathrm{dd}, J=7.9,1.6 \mathrm{~Hz}, 1 \mathrm{H}), 7.53(\mathrm{~d}, J$ $=1.0 \mathrm{~Hz}, 1 \mathrm{H}), 7.37-7.29(\mathrm{~m}, 1 \mathrm{H}), 7.19$ (ddd, $J=23.0,11.0,4.3 \mathrm{~Hz}$,

$5 \mathrm{H}), 7.03-6.91(\mathrm{~m}, 1 \mathrm{H}), 6.65(\mathrm{~d}, J=8.3 \mathrm{~Hz}, 1 \mathrm{H}), 4.91(\mathrm{~d}, J=5.8 \mathrm{~Hz}, 1 \mathrm{H}), 3.22(\mathrm{~d}, J=15.2$ $\mathrm{Hz}, 1 \mathrm{H}), 3.09(\mathrm{~d}, J=15.3 \mathrm{~Hz}, 1 \mathrm{H}), 2.78(\mathrm{~d}, J=15.2 \mathrm{~Hz}, 1 \mathrm{H}), 2.33(\mathrm{~s}, 3 \mathrm{H}), 2.24$ (dd, $J=$ $15.3,6.0 \mathrm{~Hz}, 1 \mathrm{H})$.

${ }^{13} \mathrm{C} \mathrm{NMR}\left(101 \mathrm{MHz}, \mathrm{CDCl}_{3}\right) \delta 183.4$ (s), 165.4 (s), 161.5 (s), 154.8 (s), 139.6 (s), 135.5 (s), 131.0 (s), 129.0 (s), 128.7 (s), 127.8 (s), 127.4 (s), 126.2 (s), 122.3 (s), 121.6 (s), 117.9 (s), 113.9 (s), 72.4 (s), 44.6 (s), 39.8 (s), 39.4 (s), 16.0 (s).

HRMS (ESI, m/z): Mass calcd for $\mathrm{C}_{23} \mathrm{H}_{19} \mathrm{O}_{4}[\mathrm{M}+\mathrm{H}]^{+}, 359.1277$; found 359.1182.

Enantiomeric ratio was measured by chiral phase HPLC (Chiralcel AD-H; IPA/Hexanes = 30/70, $0.4 \mathrm{~mL} / \mathrm{min}, 254 \mathrm{~nm}$ ), Rt $\mathrm{Rt}_{1}$ (major) $=21.2 \mathrm{~min}, \mathrm{Rt}_{2}$ (minor) $=41.1 \mathrm{~min}$; er = 98:2).

2-((3S,4aS,Z)-2-(hydroxy(phenyl)methylene)-9-oxo-3-phenyl-2,4,4a,9-tetrahydro-3Hxanthen-3-yl)acetaldehyde (5)<smiles>O=CC1(c2ccccc2)CC2Oc3ccccc3C(=O)C2=CC1=C(O)c1ccccc1</smiles>

Yellow solid, m.p. $110-112{ }^{\circ} \mathrm{C}, 200 \mathrm{mg}, 80 \%$ yield.

$[\alpha]_{D}{ }^{28}=-123.9\left(c 1.0 \mathrm{CHCl}_{3}\right)$.

$\underline{{ }^{1} \mathrm{H} \text { NMR }}\left(300 \mathrm{MHz}, \mathrm{CDCl}_{3}\right) \delta 9.73(\mathrm{~d}, J=1.2 \mathrm{~Hz}, 1 \mathrm{H}), 7.91-7.77(\mathrm{~m}$, $2 \mathrm{H}$ ), 7.57 (ddd, $J=6.3,3.7,1.4 \mathrm{~Hz}, 1 \mathrm{H}), 7.53-7.44(\mathrm{~m}, 2 \mathrm{H}), 7.36-$

$7.28(\mathrm{~m}, 2 \mathrm{H}), 7.21(\mathrm{dd}, J=10.1,4.4 \mathrm{~Hz}, 3 \mathrm{H}), 7.18-7.08(\mathrm{~m}, 2 \mathrm{H}), 7.01(\mathrm{dd}, J=7.5,1.5 \mathrm{~Hz}$, $1 \mathrm{H}), 6.86(\mathrm{td}, J=7.5,1.0 \mathrm{~Hz}, 1 \mathrm{H}), 6.76(\mathrm{~d}, J=8.1 \mathrm{~Hz}, 1 \mathrm{H}), 6.58(\mathrm{~d}, J=1.6 \mathrm{~Hz}, 1 \mathrm{H}), 4.49$ (ddd, $J=11.9,5.0,2.1 \mathrm{~Hz}, 1 \mathrm{H}), 3.80(\mathrm{~d}, J=18.0 \mathrm{~Hz}, 1 \mathrm{H}), 3.20(\mathrm{dd}, J=18.1,1.7 \mathrm{~Hz}, 1 \mathrm{H}$ ), $2.79(\mathrm{t}, J=12.0 \mathrm{~Hz}, 1 \mathrm{H}), 2.55(\mathrm{dd}, J=11.9,4.9 \mathrm{~Hz}, 1 \mathrm{H})$.

${ }^{13} \mathrm{C} \mathrm{NMR}$ (75 MHz, CDCl ${ }_{3}$ ) 200.4 (s), 196.5 (s), 155.3 (s), 144.6 (s), 141.0 (s), 140.8 (s), 138.4 (s), 132.0 (s), 130.6 (s), 130.3 (s), 130.0 (s), 128.6 (s), 128.4 (s), 128.1 (s), 127.8 (s), 
126.8 (s), 126.1 (s), 123.5 (s), 122.0 (s), 116.1 (s), 71.1 (s), 52.4 (s), 44.7 (s), 42.7 (s).

HRMS (ESI, m/z): Mass calcd for $\mathrm{C}_{28} \mathrm{H}_{23} \mathrm{O}_{4}[\mathrm{M}+\mathrm{H}]^{+}, 423.1596$; found 435.1587.

Enantiomeric ratio was measured by chiral phase HPLC (Chiralcel AD-H; IPA/Hexanes = 30/70, $0.4 \mathrm{~mL} / \mathrm{min}, 254 \mathrm{~nm}$ ), Rt ${ }_{1}$ (major) $=31.0 \mathrm{~min}, \mathrm{Rt}_{2}$ (minor) $=45.2 \mathrm{~min}$; er $=97: 3$ ).

Ethyl (E)-4-((3R,4aS,Z)-2-(hydroxy(phenyl)methylene)-9-oxo-3-phenyl-2,4,4a,9tetrahydro-3H-xanthen-3-yl)but-2-enoate (6)<smiles>CCOC(=O)/C=C/C[C@@H]1/C(=C(/O)c2ccccc2)C=C2C(=O)c3ccccc3C[C@@H]21</smiles>

Yellow solid, m.p. $83-85^{\circ} \mathrm{C}, 63 \mathrm{mg}, 90 \%$ yield.

$[\alpha]_{D}^{22}=76.4\left(c 1.0 \mathrm{CHCl}_{3}\right)$.

$\underline{1}$ H NMR $\left(300 \mathrm{MHz}, \mathrm{CDCl}_{3}\right) \delta 7.77-7.67(\mathrm{~m}, 2 \mathrm{H}), 7.60-7.50$ $(\mathrm{m}, 1 \mathrm{H}), 7.45(\mathrm{t}, J=7.4 \mathrm{~Hz}, 2 \mathrm{H}), 7.31(\mathrm{dd}, J=9.8,5.0 \mathrm{~Hz}, 2 \mathrm{H})$, 7.26 - $7.18(\mathrm{~m}, 3 \mathrm{H}), 7.15-7.05(\mathrm{~m}, 2 \mathrm{H}), 7.04-6.94(\mathrm{~m}, 2 \mathrm{H}), 6.86(\mathrm{td}, J=7.5,0.9 \mathrm{~Hz}, 1 \mathrm{H})$, $6.77(\mathrm{~d}, J=8.1 \mathrm{~Hz}, 1 \mathrm{H}), 6.56(\mathrm{~s}, 1 \mathrm{H}), 5.77(\mathrm{~d}, J=15.4 \mathrm{~Hz}, 1 \mathrm{H}), 4.58$ (ddd, $J=11.9,4.9$, $2.0 \mathrm{~Hz}, 1 \mathrm{H}$ ), 4.08 (q, $J=7.1 \mathrm{~Hz}, 2 \mathrm{H}$ ), 3.41 (dd, $J=14.0,8.8 \mathrm{~Hz}, 1 \mathrm{H}$ ), 2.96 (dd, $J=14.0$, $6.6 \mathrm{~Hz}, 1 \mathrm{H}$ ), $2.67(\mathrm{t}, J=12.0 \mathrm{~Hz}, 1 \mathrm{H}), 2.48(\mathrm{dd}, J=12.1,5.0 \mathrm{~Hz}, 1 \mathrm{H}), 1.18(\mathrm{t}, J=7.1 \mathrm{~Hz}$, $3 \mathrm{H})$.

${ }^{13} \mathrm{C}$ NMR (101 MHz, $\mathrm{CDCl}_{3}$ ) ठ 195.8 (s), 165.9 (s), 155.3 (s), 145.8 (s), 143.9 (s), 141.6 (s), 140.8 (s), 138.4 (s), 131.9 (s), 130.6 (s), 130.2 (s), 129.8 (s), 128.4 (s), 128.2 (s), 127.9 (s), 127.8 (s), 126.6 (s), 125.9 (s), 125.9 (s), 123.4 (s), 122.0 (s), 116.0 (s), 71.1 (s), 60.2 (s), 47.5 (s), 42.4 (s), 40.8 (s), 14.2 (s).

HRMS (ESI, m/z): Mass calcd for $\mathrm{C}_{32} \mathrm{H}_{29} \mathrm{O}_{5}[\mathrm{M}+\mathrm{H}]^{+}, 493.2015$; found 493.2020.

Enantiomeric ratio was measured by chiral phase HPLC (Chiralcel AD-H; IPA/Hexanes = 30/70, $0.4 \mathrm{~mL} / \mathrm{min}, 254 \mathrm{~nm}$ ), $\mathrm{Rt}_{1}$ (major) $=29.1 \mathrm{~min}, \mathrm{Rt}_{2}$ (minor) $=38.5 \mathrm{~min}$; er =96:4).

(9aS,10aS)-3-(4-methoxyphenyl)-6,9a-diphenyl-9,9a,10,10a-tetrahydro-4H,8H-pyran o[3,4-g]chromene-4,8-dione (8a)

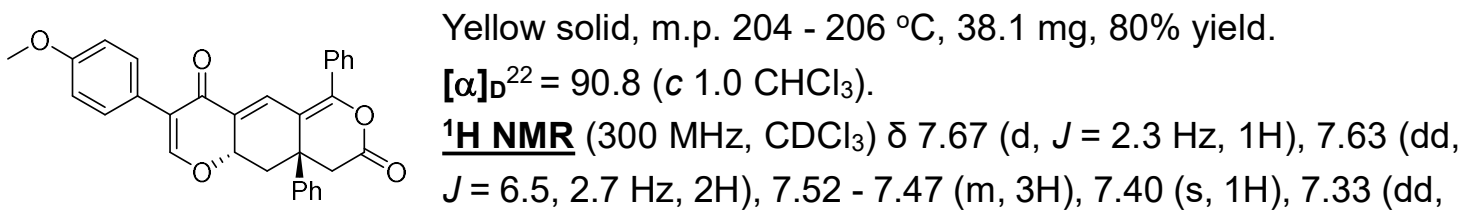

$J=12.4,7.2 \mathrm{~Hz}, 3 \mathrm{H}), 7.28(\mathrm{~d}, J=1.8 \mathrm{~Hz}, 2 \mathrm{H}), 7.26(\mathrm{~d}, J=1.8 \mathrm{~Hz}, 2 \mathrm{H}), 6.87(\mathrm{~d}, J=8.6 \mathrm{~Hz}$, 2H), 4.63 (ddd, $J=10.6,4.8,2.2 \mathrm{~Hz}, 1 \mathrm{H}), 3.79(\mathrm{~s}, 3 \mathrm{H}), 3.34(\mathrm{~d}, J=15.0 \mathrm{~Hz}, 1 \mathrm{H}), 3.06-$ $2.85(\mathrm{~m}, 2 \mathrm{H}), 2.36-2.22(\mathrm{~m}, 1 \mathrm{H})$.

${ }^{13} \mathrm{C} \mathrm{NMR}\left(75 \mathrm{MHz}, \mathrm{CDCl}_{3}\right) \delta 179.2$ (s), 165.5 (s), 159.8(s), 159.1 (s), 154.8 (s), 138.8 (s), 132.0 (s), 131.2 (s), 130.6 (s), 129.9 (s), 129.6 (s), 129.5 (s), 128.9 (s), 128.7 (s), 128.4 (s), 125.8 (s), 125.0 (s), 120.5 (s), 115.8 (s), 113.8 (s), 74.7 (s), 55.3 (s), 43.8 (s), 42.1 (s), $41.6(\mathrm{~s})$.

HRMS (ESI, m/z): Mass calcd for $\mathrm{C}_{31} \mathrm{H}_{25} \mathrm{O}_{5}[\mathrm{M}+\mathrm{H}]^{+}, 477.1702$; found 477.1711.

Enantiomeric ratio was measured by chiral phase HPLC (Chiralcel AD-H; IPA/Hexanes = 30/70, $0.4 \mathrm{~mL} / \mathrm{min}, 254 \mathrm{~nm}$ ), $\mathrm{Rt}_{1}$ (major) $=57.2 \mathrm{~min}, \mathrm{Rt}_{2}$ (minor) $=67.6 \mathrm{~min}$; $\mathrm{er}=99: 1$ ). 
(9aS,10aS)-3-(4-methoxyphenyl)-6-phenyl-9a-(p-tolyl)-9,9a,10,10a-tetrahydro-4H,8Hpyrano[3,4-g]chromene-4,8-dione (8b)

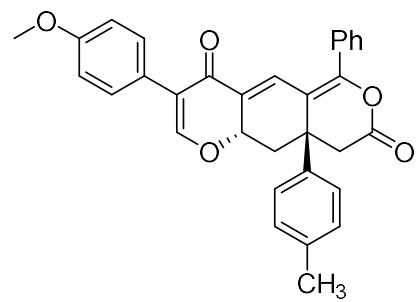

Yellow solid, m.p. $205-207^{\circ} \mathrm{C}, 36.8 \mathrm{mg}, 75 \%$ yield.

$[\alpha]_{D}^{22}=82.3\left(c 1.0 \mathrm{CHCl}_{3}\right)$.

$\underline{{ }^{1} \mathrm{H} \mathrm{NMR}}\left(500 \mathrm{MHz}, \mathrm{CDCl}_{3}\right) \delta 7.68(\mathrm{~s}, 1 \mathrm{H}), 7.63(\mathrm{~s}, 2 \mathrm{H}), 7.51$ (s, 3H), $7.42(\mathrm{~s}, 1 \mathrm{H}), 7.28(\mathrm{~d}, J=7.2 \mathrm{~Hz}, 2 \mathrm{H}), 7.17(\mathrm{~s}, 4 \mathrm{H})$, $6.89(\mathrm{~d}, J=8.0 \mathrm{~Hz}, 2 \mathrm{H}), 4.67(\mathrm{~d}, J=6.2 \mathrm{~Hz}, 1 \mathrm{H}), 3.81(\mathrm{~s}$, $3 \mathrm{H}), 3.33(\mathrm{~d}, J=14.8 \mathrm{~Hz}, 1 \mathrm{H}), 2.99(\mathrm{dd}, J=11.5,4.1 \mathrm{~Hz}$,

1H), $2.92(\mathrm{~d}, J=14.9 \mathrm{~Hz}, 1 \mathrm{H}), 2.34(\mathrm{~s}, 3 \mathrm{H}), 2.29(\mathrm{t}, J=11.4 \mathrm{~Hz}, 1 \mathrm{H})$.

13. NMR (75 MHz, CDCl ${ }_{3}$ ) 179.2 (s), 165.6 (s), 159.8 (s), 159.1 (s), 154.6 (s), 138.2 (s), 135.7 (s), 132.0 (s), 131.2 (s), 130.6 (s), 130.2 (s), 129.9 (s), 129.5 (s), 128.9 (s), 128.7 (s), 125.6 (s), 125.0 (s), 120.5 (s), 116.0 (s), 113.8 (s), 74.8 (s), 55.3 (s), 43.8 (s), 42.1 (s), $41.3(\mathrm{~s}), 21.0(\mathrm{~s})$.

HRMS (ESI, m/z): Mass calcd for $\mathrm{C}_{32} \mathrm{H}_{27} \mathrm{O}_{5}[\mathrm{M}+\mathrm{H}]^{+}, 491.1858$; found 491.1851.

Enantiomeric ratio was measured by chiral phase HPLC (Chiralcel AD-H; IPA/Hexanes = 30/70, $0.4 \mathrm{~mL} / \mathrm{min}, 254 \mathrm{~nm}$ ), Rt ${ }_{1}$ (major) $=48.4 \mathrm{~min}, \mathrm{Rt}_{2}$ (minor) $=53.3 \mathrm{~min}$; er = 99:1).

(9aS,10aS)-9a-(4-fluorophenyl)-3-(4-methoxyphenyl)-6-phenyl-9,9a,10,10a-tetrahydr 0-4H,8H-pyrano[3,4-g]chromene-4,8-dione (8c)

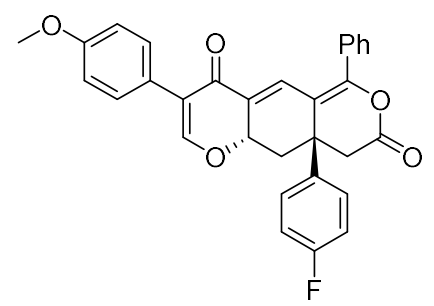

Yellow solid, m.p. $141-143^{\circ} \mathrm{C}, 34.5 \mathrm{mg}, 70 \%$ yield.

$[\alpha]_{D}^{22}=62.4\left(c 1.0 \mathrm{CHCl}_{3}\right)$.

1H NMR $\left(300 \mathrm{MHz}, \mathrm{CDCl}_{3}\right) \delta 7.65(\mathrm{~d}, J=2.3 \mathrm{~Hz}, 1 \mathrm{H}), 7.61(\mathrm{dd}$, $J=6.6,2.9 \mathrm{~Hz}, 2 \mathrm{H}), 7.49(\mathrm{dd}, J=5.0,1.7 \mathrm{~Hz}, 3 \mathrm{H}), 7.41(\mathrm{~s}$, $1 \mathrm{H}), 7.28(\mathrm{~s}, 1 \mathrm{H}), 7.26-7.21(\mathrm{~m}, 3 \mathrm{H}), 7.05(\mathrm{t}, J=8.5 \mathrm{~Hz}, 2 \mathrm{H})$,

$6.87(\mathrm{~d}, J=8.7 \mathrm{~Hz}, 2 \mathrm{H}), 4.62$ (ddd, $J=10.6,4.9,2.2 \mathrm{~Hz}, 1 \mathrm{H})$,

$3.79(\mathrm{~s}, 3 \mathrm{H}), 3.29(\mathrm{~d}, J=15.0 \mathrm{~Hz}, 1 \mathrm{H}), 3.00-2.86(\mathrm{~m}, 2 \mathrm{H}), 2.38-2.23(\mathrm{~m}, 1 \mathrm{H})$.

${ }^{13} \mathrm{C} \mathrm{NMR}\left(75 \mathrm{MHz}, \mathrm{CDCl}_{3}\right) \delta 179.1$ (s), 165.2 (s), 162.5 (d, J = 248.6 Hz), 159.7 (s), 159.2 (s), 154.8 (s), 134.5 (s), 132.0 (s), 131.0 (s), 130.7 (s), 129.9 (s), 129.5 (s), 128.7 (d, J = $3.0 \mathrm{~Hz}$ ), 127.7 (d, J = 8.3 Hz), 124.9 (s), 120.6 (s), 116.7 (s), 116.4 (s), 115.7 (s), 113.8 (s), 74.5 (s), 55.3 (s), 43.8 (s), 42.1 (s), 41.2 (s).

${ }^{19} \mathrm{~F} \mathrm{NMR}\left(282 \mathrm{MHz}, \mathrm{CDCl}_{3}\right) \delta-113.31--113.64(\mathrm{~m})$.

HRMS (ESI, m/z): Mass calcd for $\mathrm{C}_{31} \mathrm{H}_{24} \mathrm{FO}_{5}[\mathrm{M}+\mathrm{H}]^{+}, 495.1608$; found 495.1638.

Enantiomeric ratio was measured by chiral phase HPLC (Chiralcel AD-H; IPA/Hexanes = 30/70, $0.4 \mathrm{~mL} / \mathrm{min}, 254 \mathrm{~nm}$ ), Rt ${ }_{1}$ (minor) $=70.2 \mathrm{~min}, \mathrm{Rt}_{2}$ (major) $=90.2 \mathrm{~min}$; er = 99:1).

(9aR,10aS)-9a-(2-chlorophenyl)-3-(4-methoxyphenyl)-6-phenyl-9,9a,10,10a-tetrahyd ro-4H,8H-pyrano[3,4-g]chromene-4,8-dione (8d)

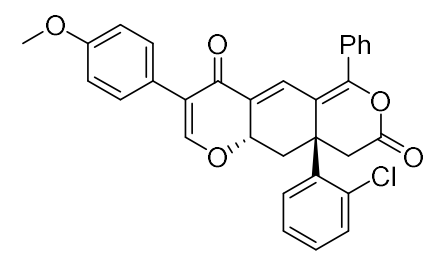

Yellow solid, m.p. $160-162{ }^{\circ} \mathrm{C}, 21.8 \mathrm{mg}, 42 \%$ yield.

$[\alpha]_{\mathrm{D}}^{22}=74.5\left(c 1.0 \mathrm{CHCl}_{3}\right)$.

1 $\mathrm{H}$ NMR $\left(300 \mathrm{MHz}, \mathrm{CDCl}_{3}\right) \delta 7.67-7.58(\mathrm{~m}, 3 \mathrm{H}), 7.53-7.44$ $(\mathrm{m}, 4 \mathrm{H}), 7.42(\mathrm{~s}, 1 \mathrm{H}), 7.26(\mathrm{t}, J=4.3 \mathrm{~Hz}, 3 \mathrm{H}), 7.16(\mathrm{t}, J=7.6$ $\mathrm{Hz}, 1 \mathrm{H}), 7.00(\mathrm{~d}, J=7.9 \mathrm{~Hz}, 1 \mathrm{H}), 6.87(\mathrm{~d}, J=8.7 \mathrm{~Hz}, 2 \mathrm{H}), 4.65$ - $4.52(\mathrm{~m}, 1 \mathrm{H}), 4.34(\mathrm{~d}, J=15.3 \mathrm{~Hz}, 1 \mathrm{H}), 3.99(\mathrm{dd}, J=12.5,4.8 \mathrm{~Hz}, 1 \mathrm{H}), 3.79(\mathrm{~s}, 3 \mathrm{H}), 2.81$ $(\mathrm{d}, J=15.3 \mathrm{~Hz}, 1 \mathrm{H}), 2.16(\mathrm{t}, J=11.8 \mathrm{~Hz}, 1 \mathrm{H})$. 
${ }^{13} \mathrm{C} \mathrm{NMR}$ (75 MHz, CDCl ${ }_{3}$ ) $\delta 179.2$ (s), 165.6 (s), 159.9 (s), 159.2 (s), 154.9 (s), 133.5 (s), 33.3 (s), 132.6 (s), 132.1 (s), 131.1 (s), 130.8 (s), 130.6 (s), 130.2 (s), 129.9 (s), 129.5 (s), 128.8 (s), 127.9 (s), 124.9 (s), 120.5 (s), 116.6 (s), 113.8 (s), 75.0 (s), 55.3 (s), 43.2 (s), 39.3 (s), $38.2(\mathrm{~s})$.

HRMS (ESI, m/z): Mass calcd for $\mathrm{C}_{31} \mathrm{H}_{24} \mathrm{ClO}_{5}[\mathrm{M}+\mathrm{H}]^{+}, 511.1312$; found 511.1308.

Enantiomeric ratio was measured by chiral phase HPLC (Chiralcel AD-H; IPA/Hexanes = 30/70, $0.4 \mathrm{~mL} / \mathrm{min}, 254 \mathrm{~nm}$ ), $\mathrm{Rt}_{1}$ (major) $=49.0 \mathrm{~min}, \mathrm{Rt}_{2}$ (minor) $=80.9 \mathrm{~min}$; er = 99.5:0.5).

(9aS,10aS)-3-(4-methoxyphenyl)-9a-(naphthalen-2-yl)-6-phenyl-9,9a,10,10a-tetrahyd ro-4H,8H-pyrano[3,4-g]chromene-4,8-dione (8e)

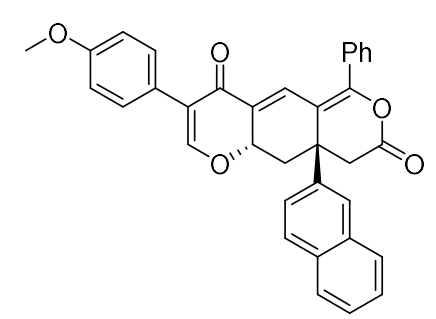

Yellow solid, m.p. $159-161^{\circ} \mathrm{C}, 32.1 \mathrm{mg}, 61 \%$ yield.

$[\alpha]_{D}^{22}=118.9\left(c 1.0 \mathrm{CHCl}_{3}\right)$.

1․ NMR $\left(300 \mathrm{MHz}, \mathrm{CDCl}_{3}\right) \delta 7.90(\mathrm{~d}, J=8.7 \mathrm{~Hz}, 1 \mathrm{H}), 7.85-$ $7.78(\mathrm{~m}, 1 \mathrm{H}), 7.75(\mathrm{t}, J=5.6 \mathrm{~Hz}, 2 \mathrm{H}), 7.66(\mathrm{dd}, J=6.5,2.9$ $\mathrm{Hz}, 2 \mathrm{H}), 7.55$ (s, 1H), $7.54-7.43(\mathrm{~m}, 6 \mathrm{H}), 7.37(\mathrm{~s}, 1 \mathrm{H}), 7.23$ $(\mathrm{d}, J=8.8 \mathrm{~Hz}, 2 \mathrm{H}), 6.84(\mathrm{~d}, J=8.7 \mathrm{~Hz}, 2 \mathrm{H}), 4.67-4.56(\mathrm{~m}$, $1 \mathrm{H}), 3.77(\mathrm{~s}, 3 \mathrm{H}), 3.46(\mathrm{~d}, J=15.1 \mathrm{~Hz}, 1 \mathrm{H}), 3.11(\mathrm{dd}, J=12.2$,

$5.0 \mathrm{~Hz}, 1 \mathrm{H}), 2.98(\mathrm{~d}, J=15.1 \mathrm{~Hz}, 1 \mathrm{H}), 2.35(\mathrm{t}, J=11.5 \mathrm{~Hz}, 1 \mathrm{H})$.

$\underline{{ }^{13} \mathrm{C} \mathrm{NMR}}$ (75 MHz, CDCl 3 ) $\delta 179.5$ (s), 165.7 (s), 160.0 (s), 159.5 (s), 155.2 (s), 136.1 (s), 133.6 (s), 133.3 (s), 132.4 (s), 131.6 (s), 131.0 (s), 130.2 (s), 129.9 (s), 129.3 (s), 129.1 (s), 128.7 (s), 127.9 (s), 127.1 (s), 126.1 (s), 125.3 (s), 122.9 (s), 121.0 (s), 116.2 (s), 114.1 (s), 75.1 (s), 55.6 (s), 44.0 (s), 42.3 (s), 42.2 (s).

HRMS (ESI, m/z): Mass calcd for $\mathrm{C}_{35} \mathrm{H}_{27} \mathrm{O}_{5}[\mathrm{M}+\mathrm{H}]^{+}, 527.1858$; found 527.1853.

Enantiomeric ratio was measured by chiral phase HPLC (Chiralcel AD-H; IPA/Hexanes = 30/70, $0.3 \mathrm{~mL} / \mathrm{min}, 254 \mathrm{~nm}$ ), Rt ${ }_{1}$ (minor) $=97.7 \mathrm{~min}, \mathrm{Rt}_{2}$ (major) $=112.9 \mathrm{~min}$; er = 97:3).

(9aR,10aS)-3-(4-methoxyphenyl)-6-methyl-9a-(thiophen-3-yl)-9,9a,10,10a-tetrahydro -4H,8H-pyrano[3,4-g]chromene-4,8-dione (8f)

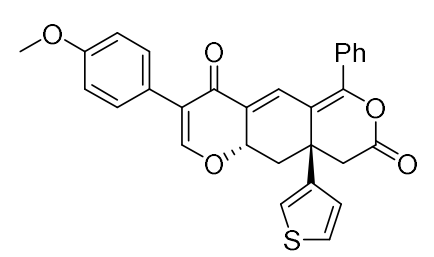

Yellow solid, m.p. $137-139{ }^{\circ} \mathrm{C}, 35.5 \mathrm{mg}, 84 \%$ yield.

$[\alpha]_{\mathrm{D}}^{22}=59.8\left(c 1.0 \mathrm{CHCl}_{3}\right)$.

1․ NMR $\left(300 \mathrm{MHz}, \mathrm{CDCl}_{3}\right) \delta 7.59(\mathrm{dd}, \mathrm{J}=8.4,4.4 \mathrm{~Hz}, 3 \mathrm{H})$,

$7.48(\mathrm{~s}, 3 \mathrm{H}), 7.41(\mathrm{~s}, 1 \mathrm{H}), 7.39-7.34(\mathrm{~m}, 1 \mathrm{H}), 7.27(\mathrm{~d}, J=8.7$

$\mathrm{Hz}, 2 \mathrm{H}), 7.04(\mathrm{~d}, J=5.0 \mathrm{~Hz}, 1 \mathrm{H}), 6.98(\mathrm{~s}, 1 \mathrm{H}), 6.87(\mathrm{~d}, J=8.5$

$\mathrm{Hz}, 2 \mathrm{H}), 4.75(\mathrm{~d}, J=7.6 \mathrm{~Hz}, 1 \mathrm{H}), 3.78(\mathrm{~s}, 3 \mathrm{H}), 3.26(\mathrm{~d}, J=15.1 \mathrm{~Hz}, 1 \mathrm{H}), 3.02-2.80(\mathrm{~m}$, $2 \mathrm{H}), 2.24(\mathrm{t}, J=11.4 \mathrm{~Hz}, 1 \mathrm{H})$.

13. NMR (75 MHz, $\mathrm{CDCl}_{3}$ ) $\delta 179.2$ (s), 165.4 (s), 159.8 (s), 159.1 (s), 154.4 (s), 140.4 (s), 131.7 (s), 131.1 (s), 130.6 (s), 129.9 (s), 129.5 (s), 128.7 (s), 128.5 (s), 128.3 (s), 125.0 (s), 124.5 (s), 122.8 (s), 120.5 (s), 116.3 (s), 113.8 (s), 74.8 (s), 55.3 (s), 43.5 (s), 41.7 (s), 39.3 (s).

HRMS (ESI, m/z): Mass calcd for $\mathrm{C}_{29} \mathrm{H}_{23} \mathrm{O}_{5} \mathrm{~S}[\mathrm{M}+\mathrm{H}]^{+}$, 483.1266; found 483.1249. Enantiomeric ratio was measured by chiral phase HPLC (Chiralcel AD-H; IPA/hexanes = 30/70, 0.4 mL/min, $254 \mathrm{~nm}$ ), Rt ${ }_{1}$ (major) $=70.2 \mathrm{~min}, \mathrm{Rt}_{2}($ minor $)=82.6 \mathrm{~min}$; er = 99:1). 
(9aS,10aS)-3-(4-fluorophenyl)-6,9a-diphenyl-9,9a,10,10a-tetrahydro-4H,8H-pyrano[3, 4-g]chromene-4,8-dione (8g)

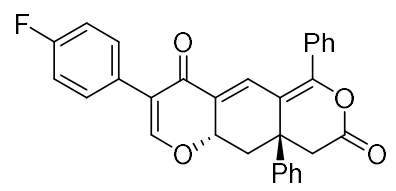

Yellow solid, m.p. $197-199{ }^{\circ} \mathrm{C}, 39 \mathrm{mg}, 84 \%$ yield.

$[\alpha]_{\mathrm{D}}^{22}=118.7\left(\right.$ ( $\left.1.0 \mathrm{CHCl}_{3}\right)$.

1H NMR $\left(300 \mathrm{MHz}, \mathrm{CDCl}_{3}\right) \delta 7.74(\mathrm{~d}, J=2.4 \mathrm{~Hz}, 1 \mathrm{H}), 7.69(\mathrm{dd}$, $J=6.7,3.0 \mathrm{~Hz}, 2 \mathrm{H}), 7.55(\mathrm{dd}, J=4.9,1.7 \mathrm{~Hz}, 3 \mathrm{H}), 7.46(\mathrm{~d}, J=$

$3.3 \mathrm{~Hz}, 1 \mathrm{H}$ ), $7.45-7.30(\mathrm{~m}, 7 \mathrm{H}), 7.07$ (t, $J=8.7 \mathrm{~Hz}, 2 \mathrm{H}), 4.71$ (ddd, $J=10.6,4.9,2.3 \mathrm{~Hz}$, $1 \mathrm{H}), 3.41(\mathrm{~d}, J=15.0 \mathrm{~Hz}, 1 \mathrm{H}), 3.06(\mathrm{dd}, J=12.1,5.1 \mathrm{~Hz}, 1 \mathrm{H}), 3.00(\mathrm{t}, J=11.8 \mathrm{~Hz}, 1 \mathrm{H})$, $2.43-2.30(\mathrm{~m}, 1 \mathrm{H})$.

${ }^{13} \mathrm{C} \mathrm{NMR}\left(75 \mathrm{MHz}, \mathrm{CDCl}_{3}\right) \delta 178.9$ (s), 165.4 (s), 162.3 (d, J = 246.5 Hz), 160.2 (s), 155.0 (s), 138.8 (s), 131.6 (s), 131.1 (s), 130.7 (s), 130.4 (d, $J=8.1 \mathrm{~Hz}$ ), 129.6 (d, J = 5.6 Hz), 129.3 (s), 128.8 (s), 128.6 (d, J = 3.3 Hz), 128.5 (s), 125.8 (s), 120.0 (s), 115.7 (s), 115.3 (s), 115.1 (s), 74.9 (s), 43.7 (s), 42.0 (s), 41.6 (s).

19 $\mathrm{F} \mathrm{NMR}\left(282 \mathrm{MHz}, \mathrm{CDCl}_{3}\right) \delta-114.7$ (s).

HRMS (ESI, m/z): Mass calcd for $\mathrm{C}_{30} \mathrm{H}_{22} \mathrm{FO}_{4}[\mathrm{M}+\mathrm{H}]^{+}, 465.1502$; found 465.1519.

Enantiomeric ratio was measured by chiral phase HPLC (Chiralcel AD-H; IPA/hexanes = 30/70, $0.4 \mathrm{~mL} / \mathrm{min}, 254 \mathrm{~nm}$ ), Rt $\mathrm{R}_{1}$ (major) $=19.8 \mathrm{~min}, \mathrm{Rt}_{2}$ (minor) $=43.6 \mathrm{~min}$; er = 96:4).

(9aS,10aS)-3-(4-fluorophenyl)-6-methyl-9a-phenyl-9,9a,10,10a-tetrahydro-4H,8H-pyr ano[3,4-g]chromene-4,8-dione (8h)

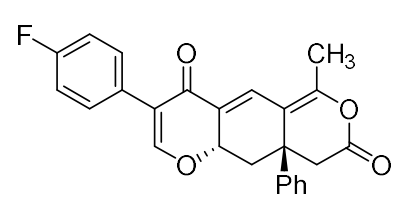

Yellow solid, m.p. $138-140{ }^{\circ} \mathrm{C}, 32.5 \mathrm{mg}, 80 \%$ yield.

$[\alpha]_{D}{ }^{22}=65.8\left(c 1.0 \mathrm{CHCl}_{3}\right)$.

1 $\mathrm{H} \mathrm{NMR}\left(300 \mathrm{MHz}, \mathrm{CDCl}_{3}\right) \delta 7.67(\mathrm{~d}, J=2.4 \mathrm{~Hz}, 1 \mathrm{H}), 7.46-$

$7.33(\mathrm{~m}, 6 \mathrm{H}), 7.28-7.19(\mathrm{~m}, 2 \mathrm{H}), 7.09(\mathrm{dd}, J=12.1,5.4 \mathrm{~Hz}$,

2H), $4.66(\mathrm{~d}, J=8.1 \mathrm{~Hz}, 1 \mathrm{H}), 3.34(\mathrm{~d}, J=15.1 \mathrm{~Hz}, 1 \mathrm{H}), 2.92(\mathrm{dd}, J=12.0,5.0 \mathrm{~Hz}, 1 \mathrm{H})$, $2.84(\mathrm{~d}, J=15.1 \mathrm{~Hz}, 1 \mathrm{H}), 2.39(\mathrm{~s}, 3 \mathrm{H}), 2.23-2.11(\mathrm{~m}, 1 \mathrm{H})$.

${ }^{13} \mathrm{C} \mathrm{NMR}\left(75 \mathrm{MHz}, \mathrm{CDCl}_{3}\right) \delta 178.8$ (s), 165.5 (s), 162.3 (d, J = 246.5 Hz), 160.2 (s), 154.4 (s), 139.1 (s), 130.5 (s), 130.4 (d, $J=8.1 \mathrm{~Hz}$ ), 129.4 (s), 128.7 (d, J=3.3 Hz), 128.2 (d, J = $21.7 \mathrm{~Hz}$ ), 125.7 (s), 119.8 (s), 115.4 (s), 115.1 (s), 114.4 (s), 74.9 (s), 43.8 (s), 42.2 (s), 40.7 (s), 16.1 (s).

${ }^{19}$ F NMR $\left(282 \mathrm{MHz}, \mathrm{CDCl}_{3}\right) \delta-114.7$ (s).

HRMS (ESI, m/z): Mass calcd for $\mathrm{C}_{25} \mathrm{H}_{20} \mathrm{FO}_{4}[\mathrm{M}+\mathrm{H}]^{+}$, 403.1346; found 403.1348.

Enantiomeric ratio was measured by chiral phase HPLC (Chiralcel AD-H; IPA/hexanes = 30/70, $0.5 \mathrm{~mL} / \mathrm{min}, 254 \mathrm{~nm}$ ), Rt ${ }_{1}$ (minor) $=68.5 \mathrm{~min}, \mathrm{Rt}_{2}$ (major) $=128.1 \mathrm{~min}$; er = 99:1).

(9aS,10aS)-3-(4-fluorophenyl)-9a-isopropyl-6-methyl-9,9a,10,10a-tetrahydro-4H,8Hpyrano[3,4-g]chromene-4,8-dione (8i)

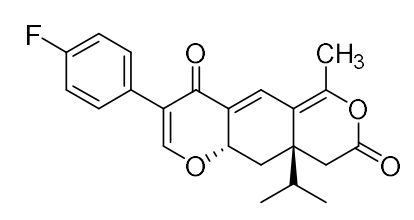

Yellow solid, m.p. $153-155^{\circ} \mathrm{C}, 15.3 \mathrm{mg}, 41 \%$ yield.

$[\alpha]_{D}^{22}=164.6\left(c 1.0 \mathrm{CHCl}_{3}\right)$.

1 NR $\left(400 \mathrm{MHz}, \mathrm{CDCl}_{3}\right) \delta 7.46(\mathrm{~s}, 1 \mathrm{H}), 7.43-7.33(\mathrm{~m}, 2 \mathrm{H})$, $7.30(\mathrm{~d}, J=1.6 \mathrm{~Hz}, 1 \mathrm{H}), 7.06(\mathrm{t}, J=8.7 \mathrm{~Hz}, 2 \mathrm{H}), 5.03(\mathrm{~d}, J=$ $6.0 \mathrm{~Hz}, 1 \mathrm{H}), 2.93(\mathrm{~d}, J=16.5 \mathrm{~Hz}, 1 \mathrm{H}), 2.66(\mathrm{~d}, J=15.7 \mathrm{~Hz}$,

$1 \mathrm{H}), 2.22(\mathrm{~d}, J=16.5 \mathrm{~Hz}, 1 \mathrm{H}), 2.19(\mathrm{~d}, J=7.4 \mathrm{~Hz}, 3 \mathrm{H}), 2.06(\mathrm{dd}, J=13.8,6.9 \mathrm{~Hz}, 1 \mathrm{H}$ ), $1.75(\mathrm{dd}, J=15.6,6.3 \mathrm{~Hz}, 1 \mathrm{H}), 1.00(\mathrm{~d}, J=6.8 \mathrm{~Hz}, 3 \mathrm{H}), 0.85(\mathrm{~d}, J=7.0 \mathrm{~Hz}, 3 \mathrm{H})$. 
${ }^{13} \mathrm{C} \mathrm{NMR}\left(75 \mathrm{MHz}, \mathrm{CDCl}_{3}\right.$ ) ठ 182.2 (s), 1671 (s), 162.4 (d, J = 246.5 Hz), 160.6 (s), 153.1 (s), 130.5 (d, J = 7.9 Hz), 128.9 (s), 128.6 (s), 121.0 (s), 115.4 (s), 115.1 (s), 113.7 (s), 74.6 (s), 38.7 (s), 36.4 (s), 36.1 (s), 31.2 (s), 18.3 (s), 16.3 (s), 16.0 (s).

${ }^{19} \mathrm{~F} \mathrm{NMR}\left(377 \mathrm{MHz}, \mathrm{CDCl}_{3}\right) \delta-114.7$ (dd, $\left.J=9.4,4.0 \mathrm{~Hz}\right)$.

HRMS (ESI, m/z): Mass calcd for $\mathrm{C}_{22} \mathrm{H}_{22} \mathrm{FO}_{4}[\mathrm{M}+\mathrm{H}]^{+}, 369.1502$; found 369.1510.

Enantiomeric ratio was measured by chiral phase HPLC (Chiralcel AD-H; IPA/hexanes = 30/70, $0.4 \mathrm{~mL} / \mathrm{min}, 254 \mathrm{~nm}$ ), $\mathrm{Rt}_{1}$ (major) $=22.3 \mathrm{~min}, \mathrm{Rt}_{2}$ (minor) $=35.5 \mathrm{~min}$; er = 82:18). 


\section{NMR spectra of intermediates \& products}

2f ${ }^{1} \mathbf{H}$ NMR $\left(400 \mathrm{MHz}, \mathrm{CDCl}_{3}\right)$

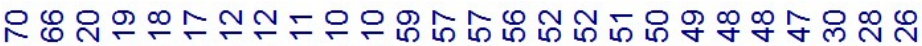

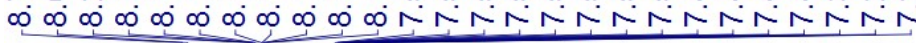

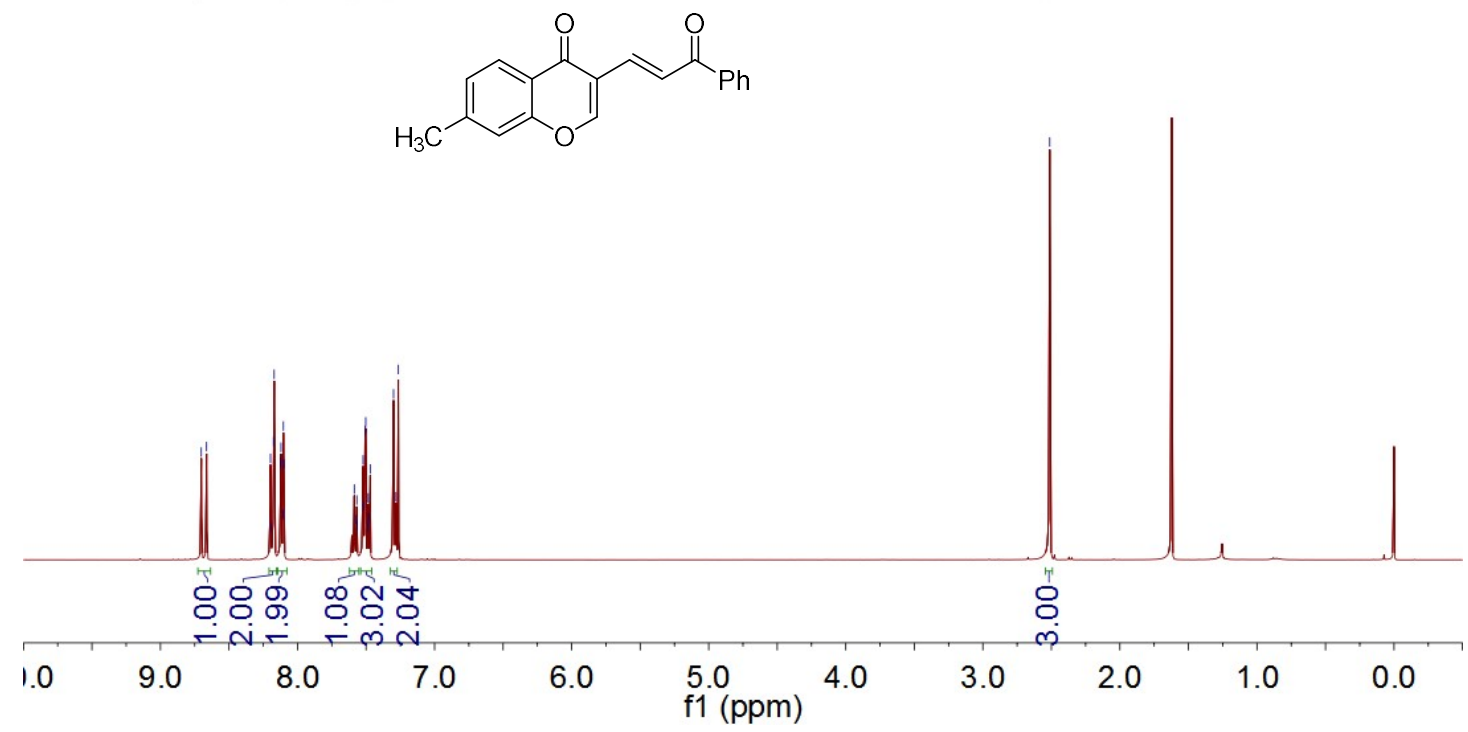

2f ${ }^{13} \mathrm{C}$ NMR $\left(101 \mathrm{MHz}, \mathrm{CDCl}_{3}\right)$

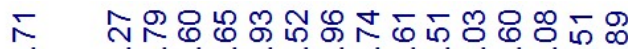

穴

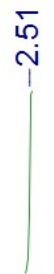<smiles>Cc1ccc2c(=O)c(/C=C/C(=O)c3ccccc3)coc2c1</smiles>

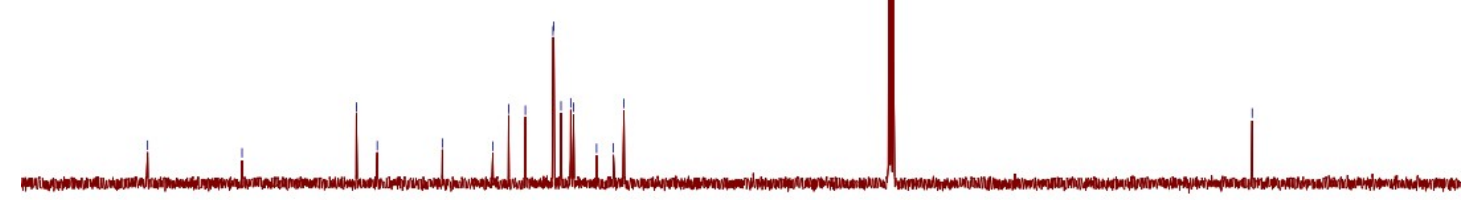


2g ${ }^{1} \mathrm{H}$ NMR $\left(600 \mathrm{MHz}, \mathrm{CDCl}_{3}\right)$

1.

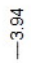<smiles>COc1ccc2c(=O)c(/C=C/C(=O)c3ccccc3)coc2c1</smiles>

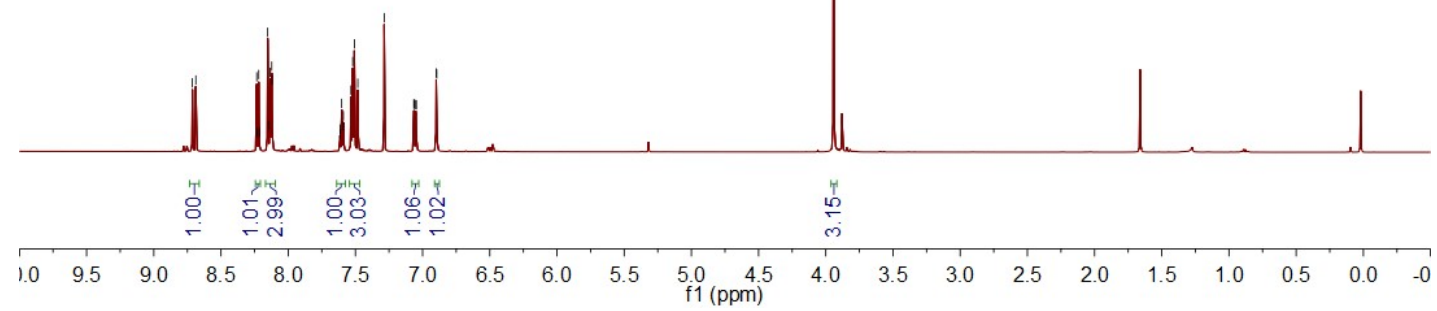

2g ${ }^{13} \mathrm{C}$ NMR $\left(151 \mathrm{MHz}, \mathrm{CDCl}_{3}\right)$

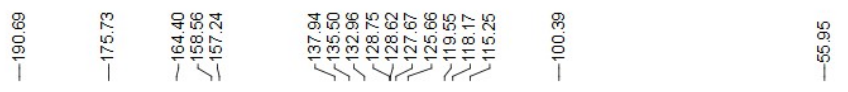<smiles>COc1ccc2c(=O)c(/C=C/C(=O)c3ccccc3)coc2c1</smiles>

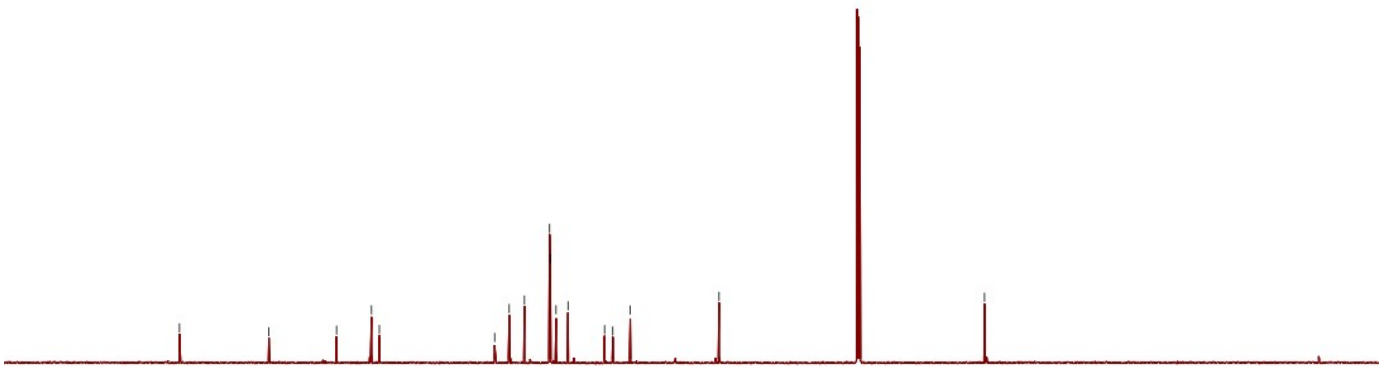

\begin{tabular}{llllllllllllllllllllllllllll}
\hline 0 & 210 & 200 & 190 & 180 & 170 & 160 & 150 & 140 & 130 & 120 & $\begin{array}{c}110 \\
\mathrm{f} 1(\mathrm{ppm})\end{array}$ & 90 & 80 & 70 & 60 & 50 & 40 & 30 & 20 & 10 & 0 & -1
\end{tabular} 
2i ${ }^{1} \mathrm{HNMR}\left(400 \mathrm{MHz}, \mathrm{CDCl}_{3}\right)$

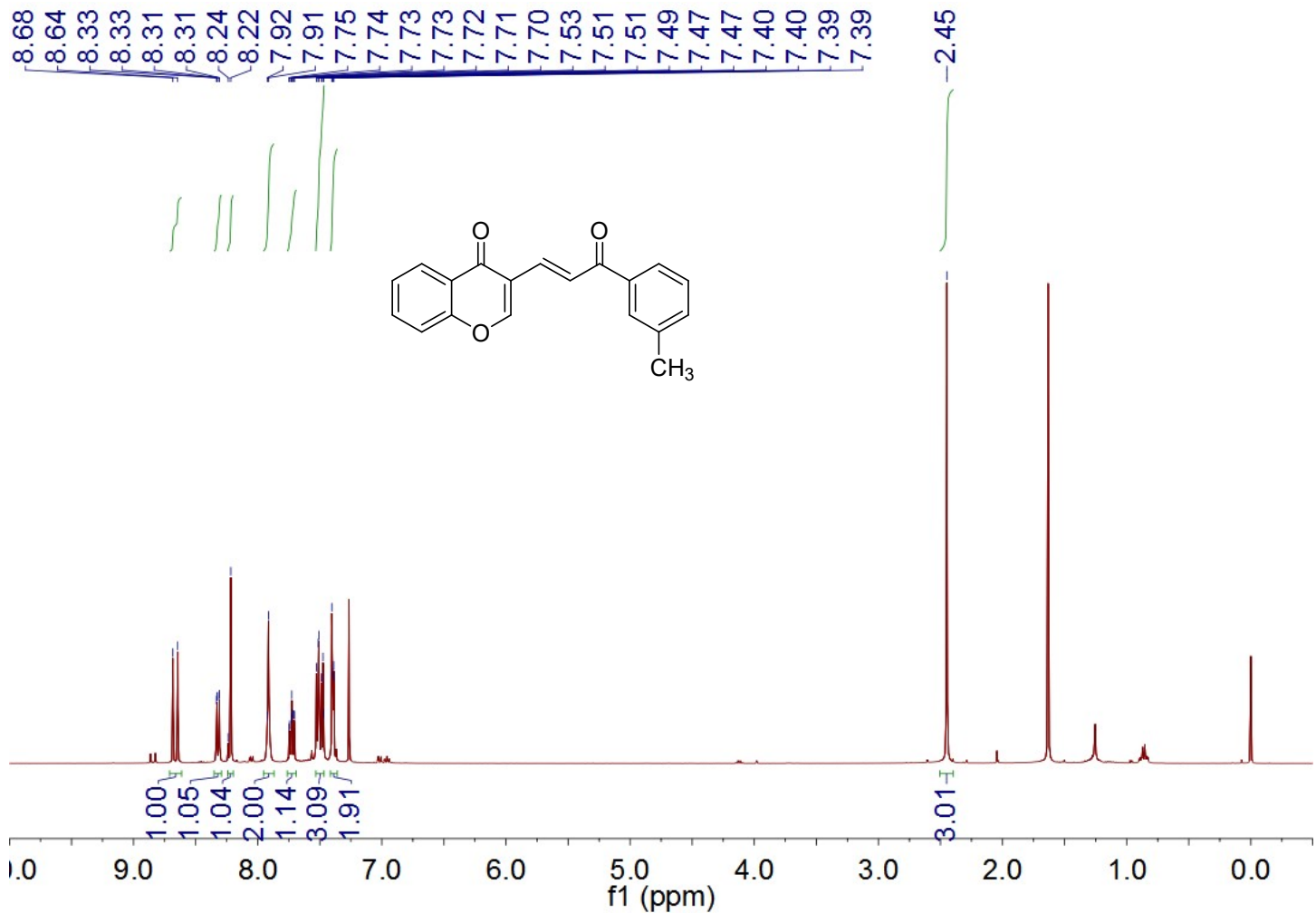

2i ${ }^{13} \mathbf{C}$ NMR $\left(101 \mathrm{MHz}, \mathrm{CDCl}_{3}\right)$

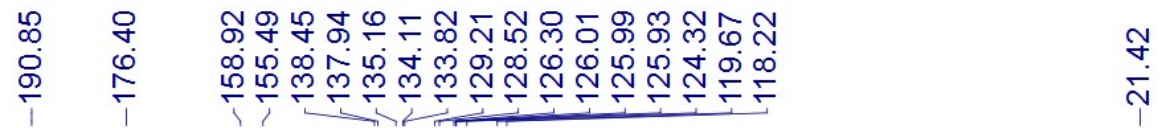<smiles>Cc1cccc(C(=O)/C=C/c2coc3ccccc3c2=O)c1</smiles>

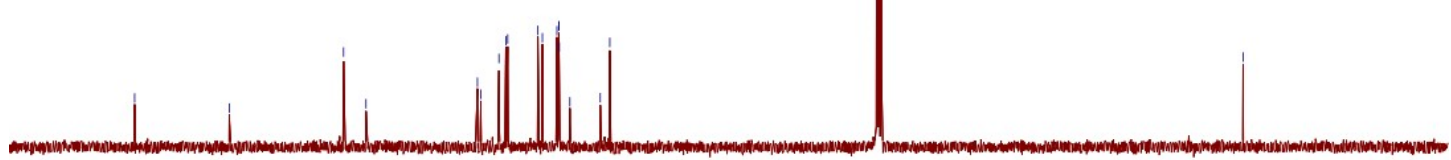

200

180

160

140

120

100

60

20 
2j ${ }^{1} \mathrm{H}$ NMR (400 MHz, $\left.\mathrm{CDCl}_{3}\right)$

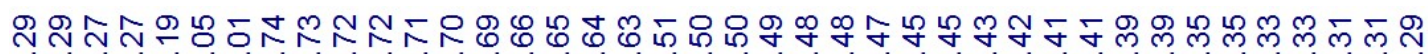
$\infty \infty \infty \infty \infty \infty \infty N N$ N<smiles>O=C(/C=C/c1coc2ccccc2c1=O)c1ccccc1Br</smiles>

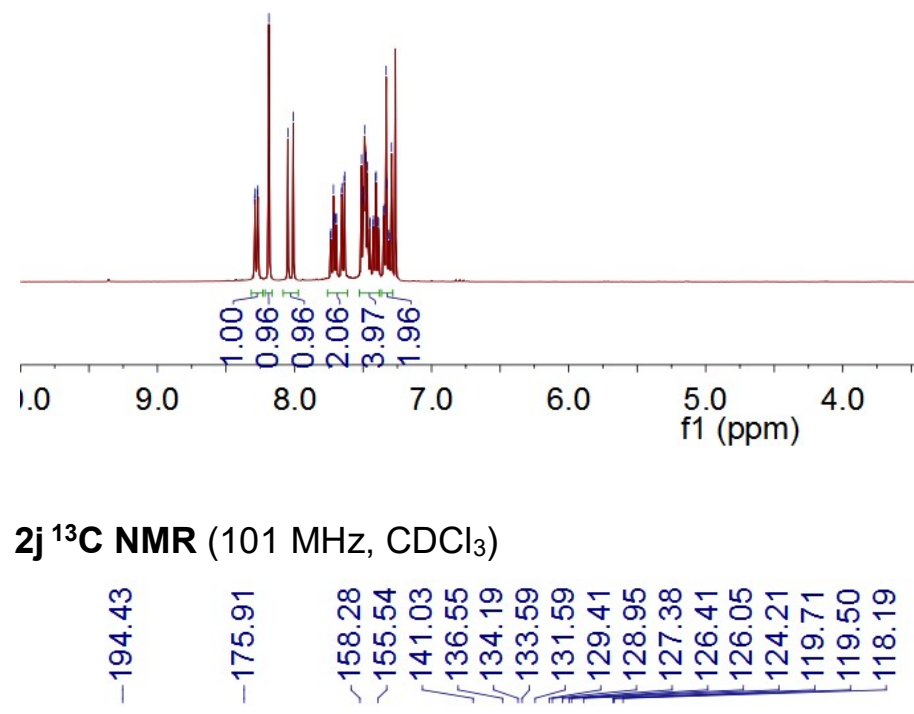<smiles>O=C(/C=C/c1coc2ccccc2c1=O)c1ccccc1Br</smiles>

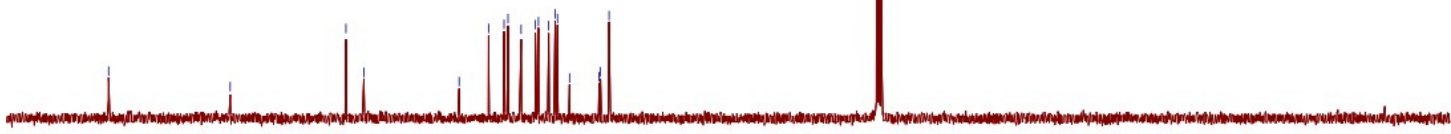

$\left.200 \quad 180 \quad 160 \quad 140 \quad 120 \begin{array}{c}100 \\ \mathrm{f} 1(\mathrm{ppm})\end{array}\right)$


2k ${ }^{1} \mathbf{H}$ NMR $\left(400 \mathrm{MHz}, \mathrm{CDCl}_{3}\right)$

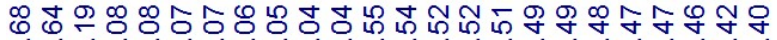

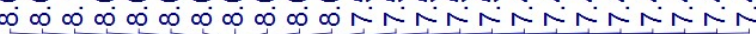

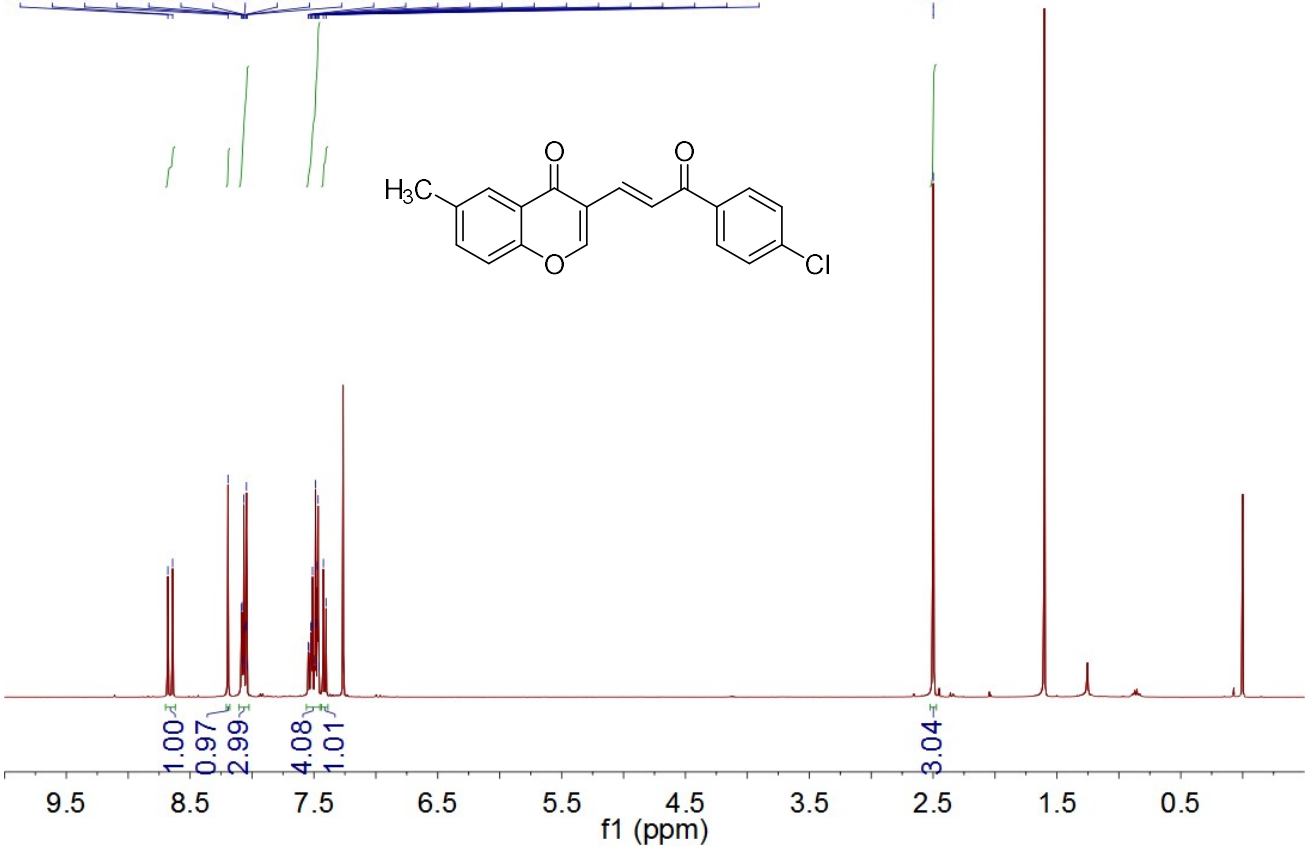

우

2k ${ }^{13} \mathbf{C}$ NMR $\left(101 \mathrm{MHz}, \mathrm{CDCl}_{3}\right)$

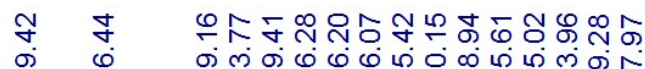

穴

$\stackrel{\infty}{\stackrel{\infty}{\leftarrow}}$<smiles>Cc1ccc2occ(/C=C/C(=O)c3ccc(Cl)cc3)c(=O)c2c1</smiles>

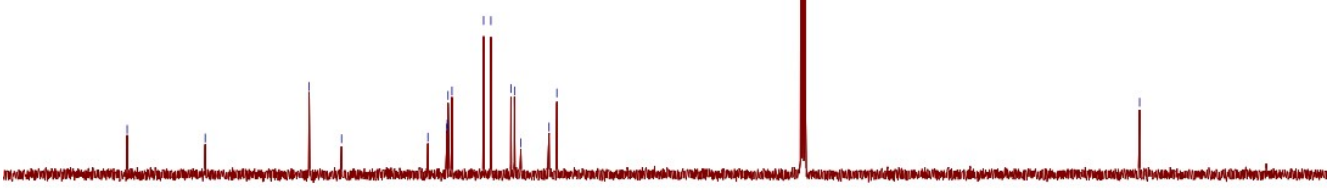

200

180

160

140

120

f1 100

80

60

20

0 
2I ${ }^{1} \mathbf{H}$ NMR $\left(400 \mathrm{MHz}, \mathrm{CDCl}_{3}\right)$

m

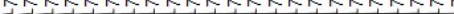<smiles>O=C(/C=C/c1coc2ccccc2c1=O)c1ccccc1</smiles>

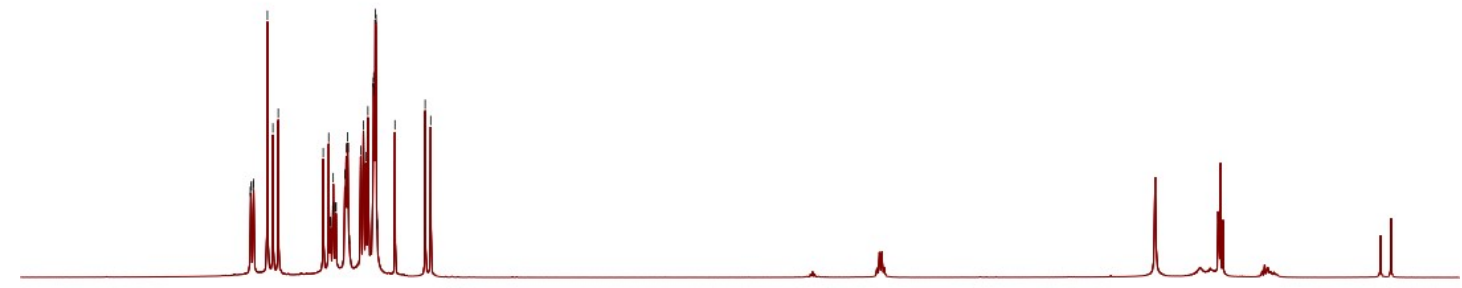

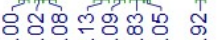

$\begin{array}{llllllllllllllllllllllllll}1.0 & 9.5 & 9.0 & 8.5 & 8.0 & 7.5 & 7.0 & 6.5 & 6.0 & 5.5 & \begin{array}{c}5.0 \\ \mathrm{f} 1(\mathrm{ppm})\end{array} & 4.5 & 4.0 & 3.5 & 3.0 & 2.5 & 2.0 & 1.5 & 1.0 & 0.5 & 0.0 & -0\end{array}$

2I ${ }^{13} \mathrm{C}$ NMR (101 MHz, $\left.\mathrm{CDCl}_{3}\right)$

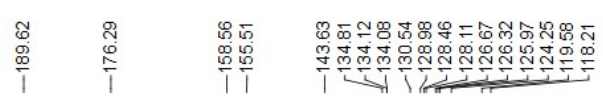<smiles>O=C(/C=C/c1ccccc1)/C=C/c1coc2ccccc2c1=O</smiles>

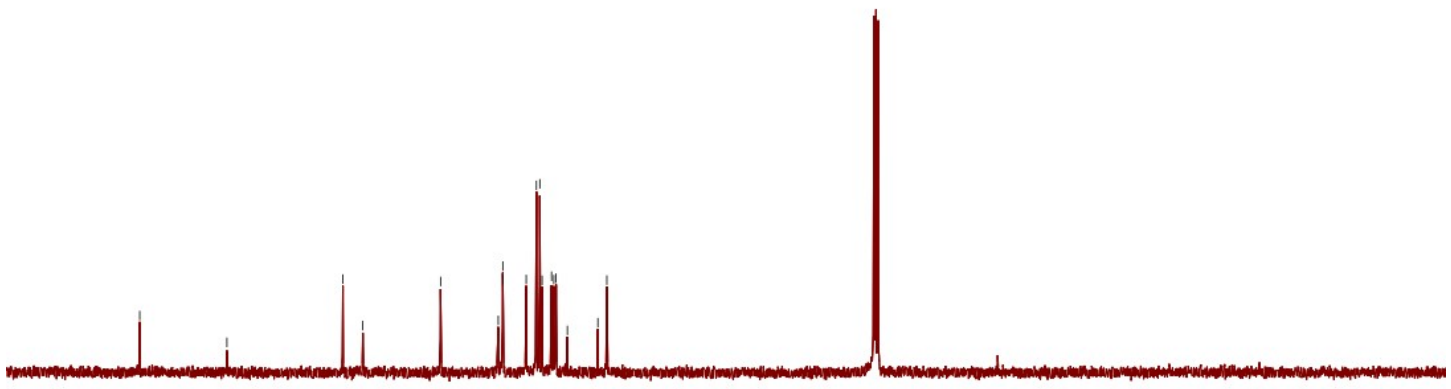

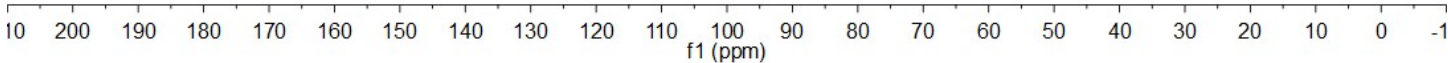


7a ${ }^{1} \mathrm{H}$ NMR $\left(400 \mathrm{MHz}, \mathrm{CDCl}_{3}\right)$

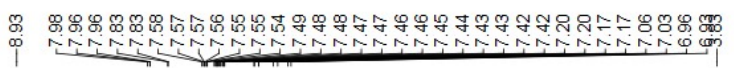<smiles>COc1ccc(-c2cocc(/C=C/C(=O)c3ccccc3)c2=O)cc1</smiles>

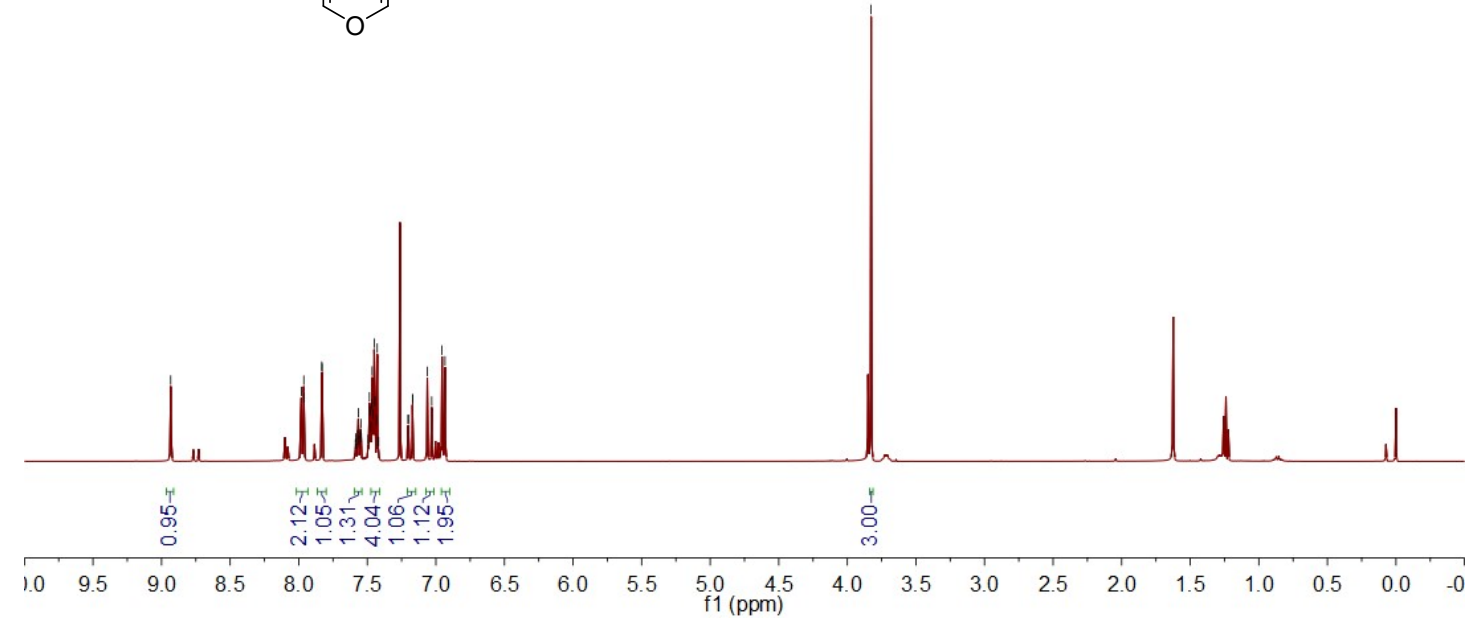

7a ${ }^{13} \mathrm{C}$ NMR $\left(101 \mathrm{MHz}, \mathrm{CDCl}_{3}\right)$

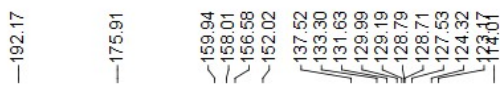<smiles>COc1ccc(-c2cocc(/C=C/C(=O)c3ccccc3)c2=O)cc1</smiles>

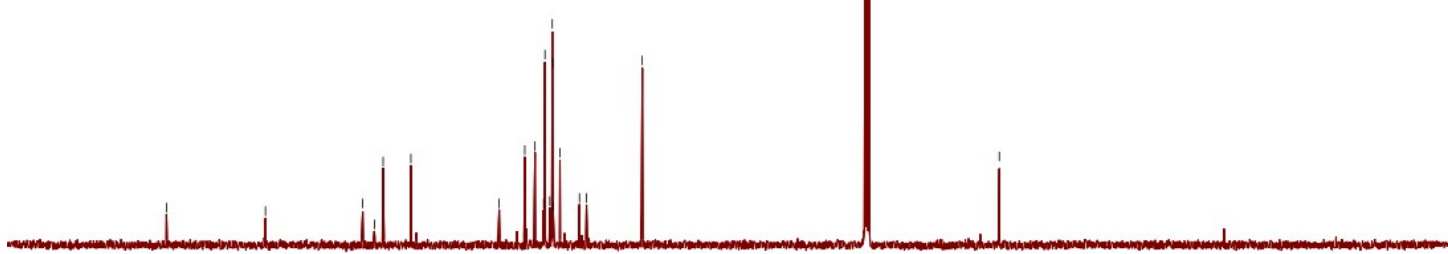

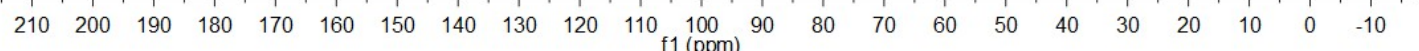


7b ${ }^{1} \mathrm{H}$ NMR $\left(400 \mathrm{MHz}, \mathrm{CDCl}_{3}\right)$

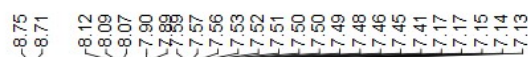<smiles>O=C(/C=C/c1cocc(-c2ccc(F)cc2)c1=O)c1ccccc1</smiles>

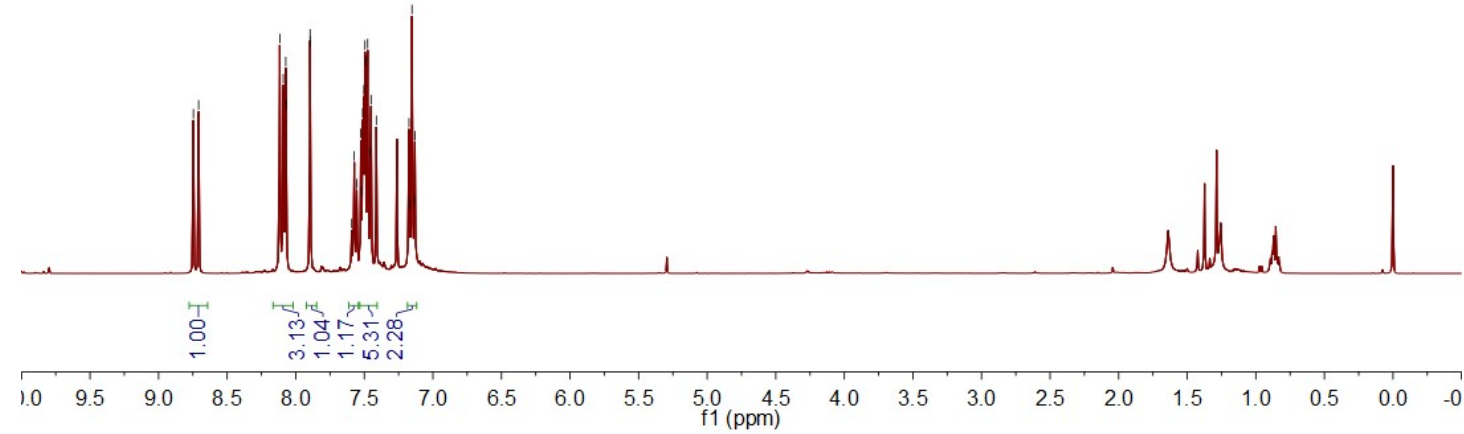

7b ${ }^{13} \mathrm{C}$ NMR $\left(101 \mathrm{MHz}, \mathrm{CDCl}_{3}\right)$

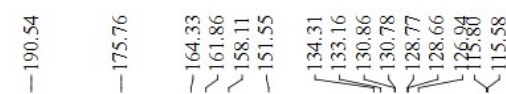

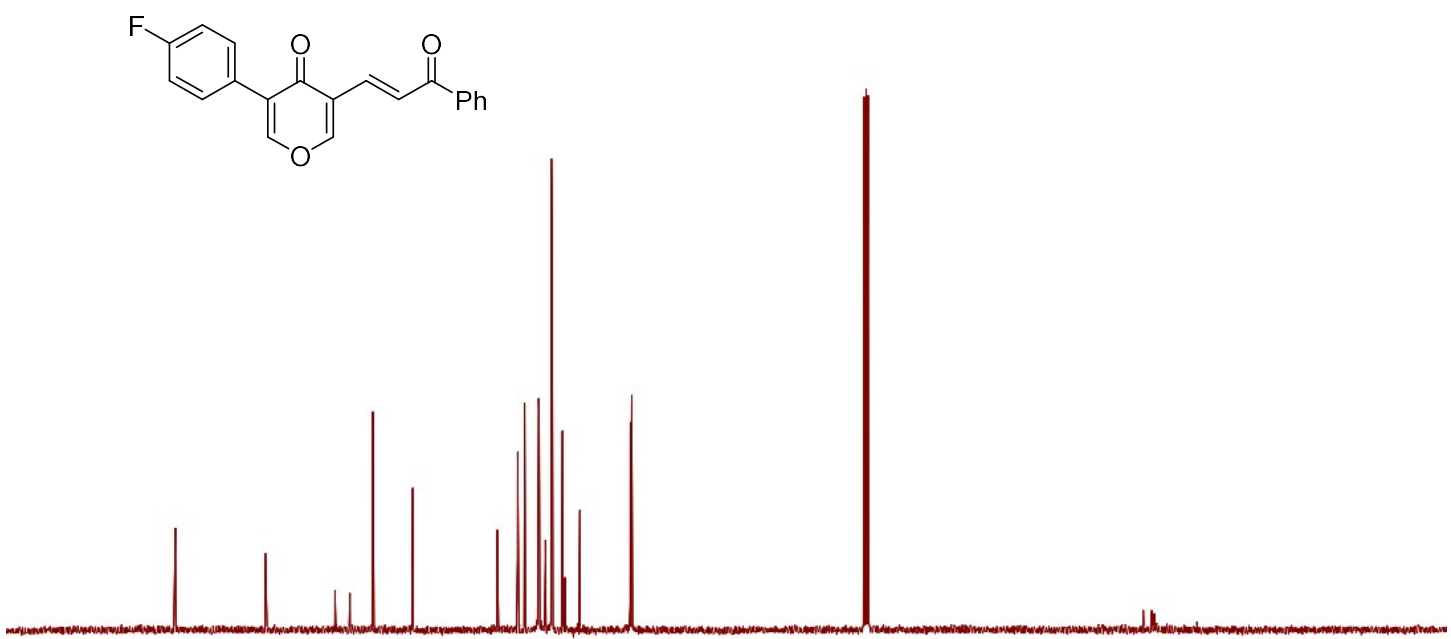

$\begin{array}{llllllllllllllllllllllllll}210 & 200 & 190 & 180 & 170 & 160 & 150 & 140 & 130 & 120 & 110 & 100 & 10 & 10 & 10 & 10 & 50 & 40 & 30 & 20 & 10 & 0 & -10\end{array}$ 
7b ${ }^{19} \mathrm{~F}$ NMR $\left(282 \mathrm{MHz}, \mathrm{CDCl}_{3}\right)$

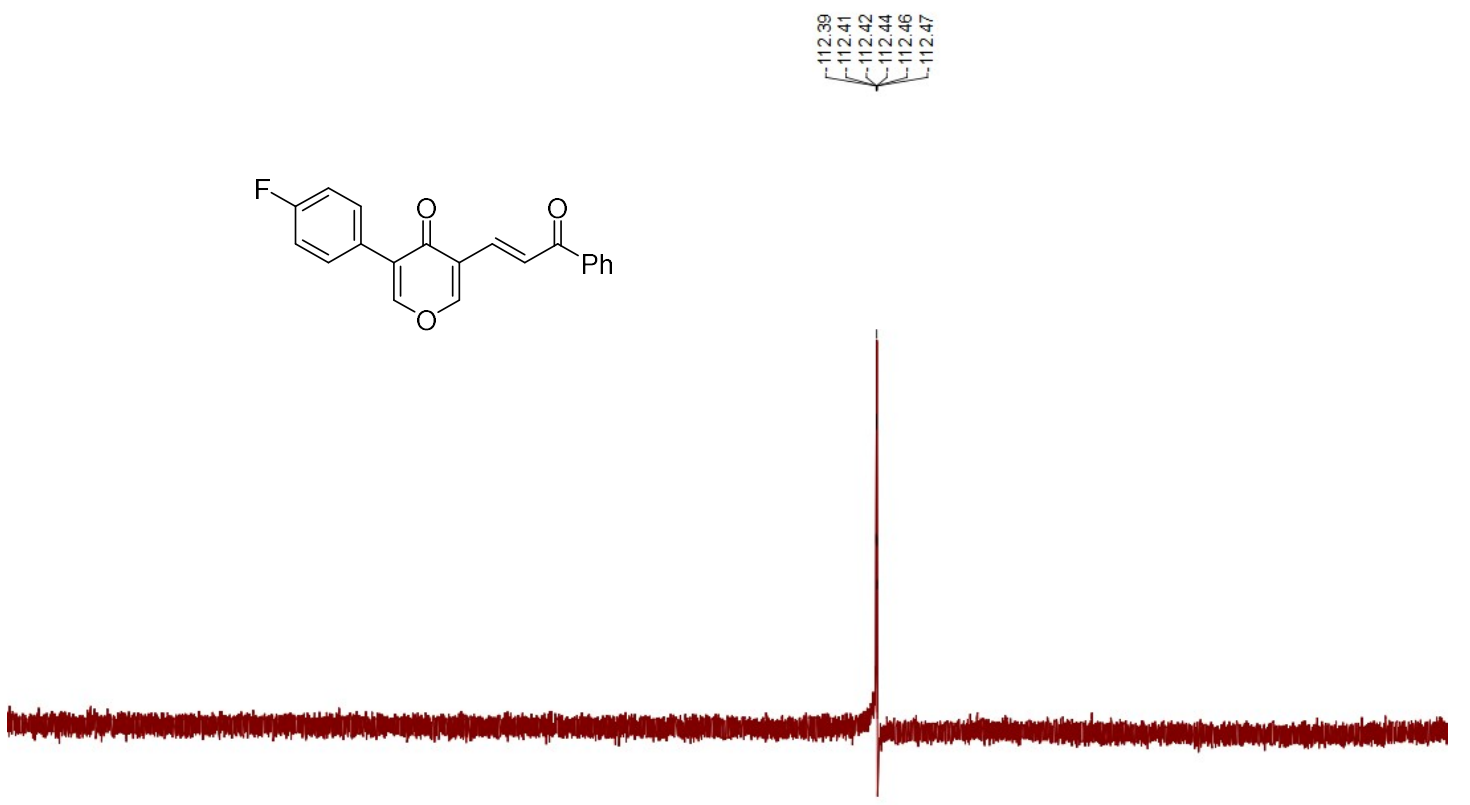

\begin{tabular}{lllllllllllllllll}
\hline 0 & -45 & -50 & -55 & -60 & -65 & -70 & -75 & -80 & -85 & -90 & -95 & & \\
$\mathrm{f} 1(\mathrm{ppm})$ & -105 & -115 & -125 & -135 & -145 & -155
\end{tabular} 
Tc ${ }^{1} \mathrm{H}$ NMR (300 MHz, $\left.\mathrm{CDCl}_{3}\right)$

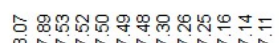

$\stackrel{\mathscr{8}}{i}$
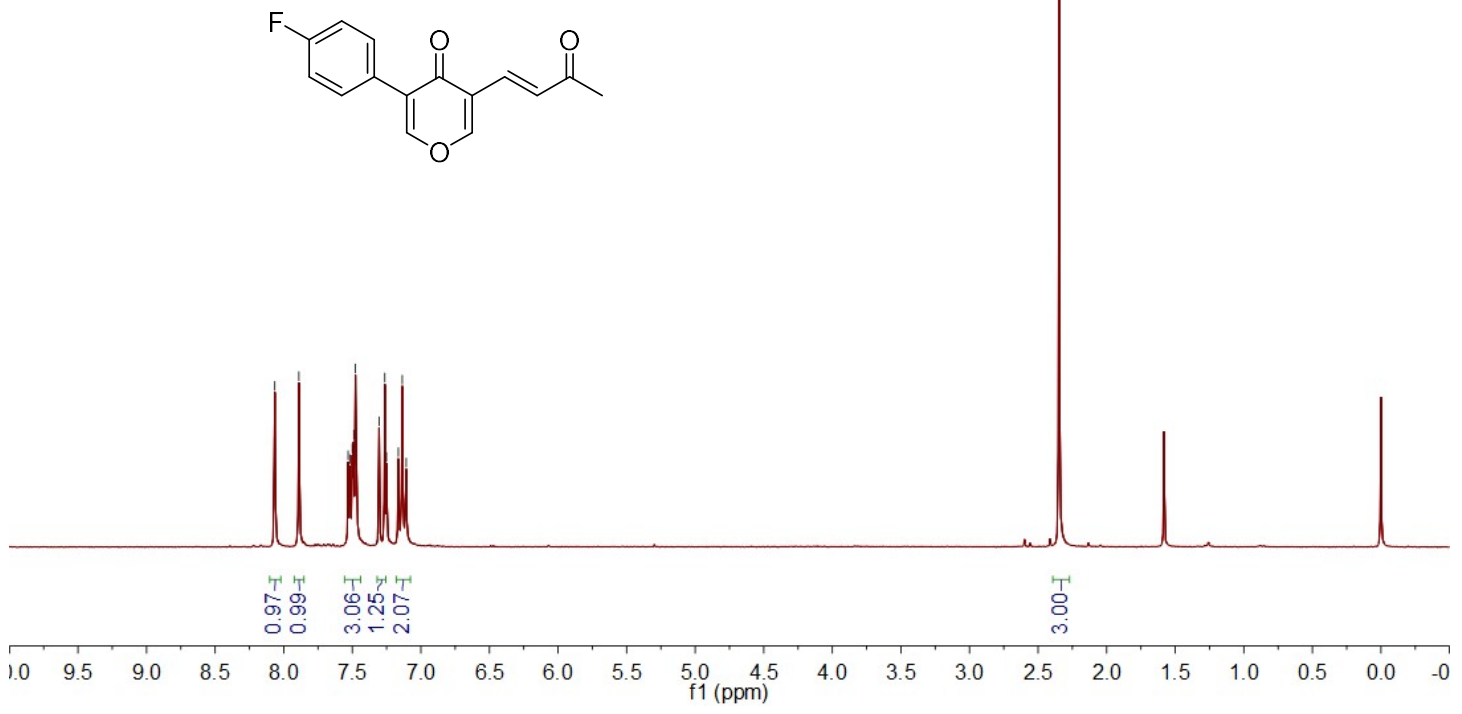

Tc ${ }^{13} \mathrm{C}$ NMR $\left(75 \mathrm{MHz}, \mathrm{CDCl}_{3}\right)$

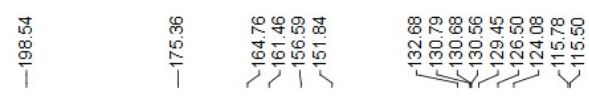

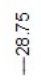
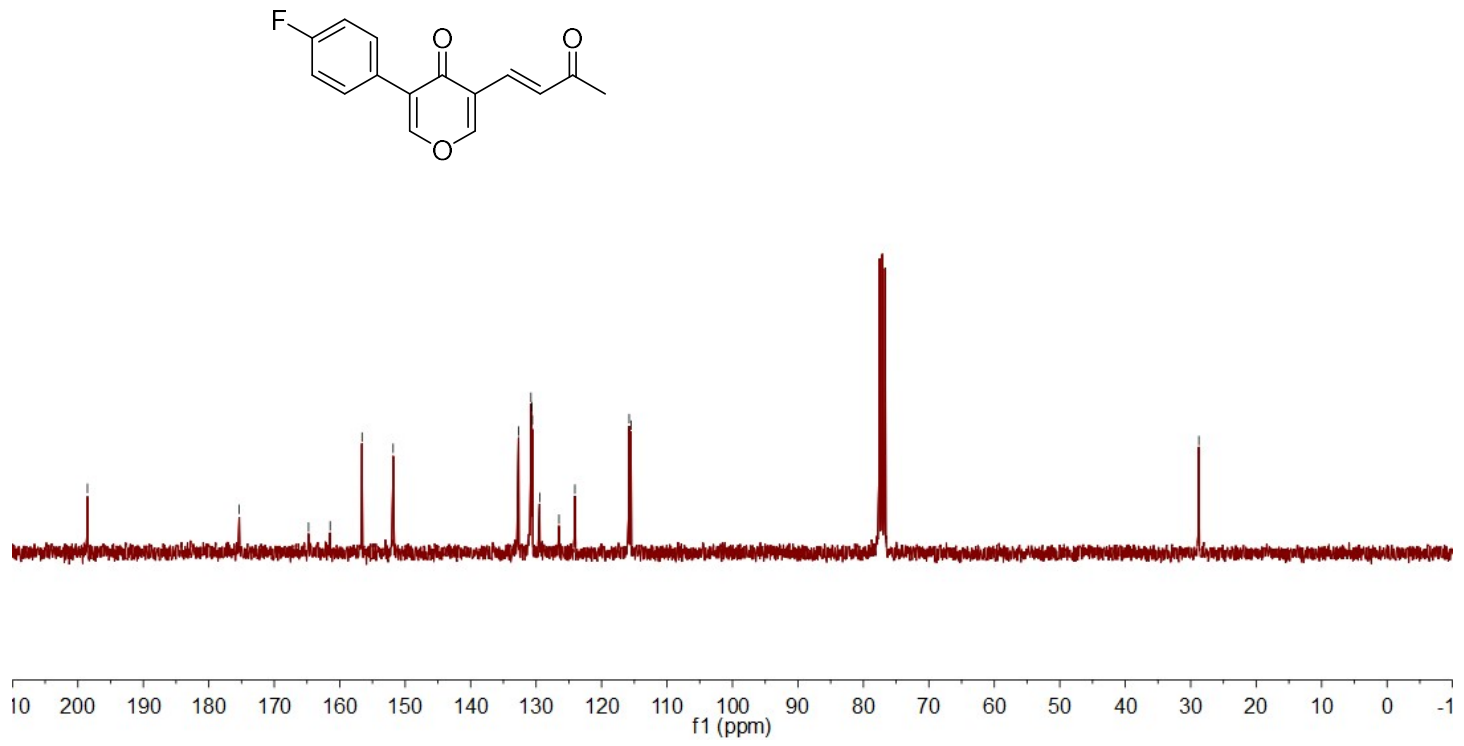

41 
7c ${ }^{19} \mathrm{~F}$ NMR $\left(282 \mathrm{MHz}, \mathrm{CDCl}_{3}\right)$

象
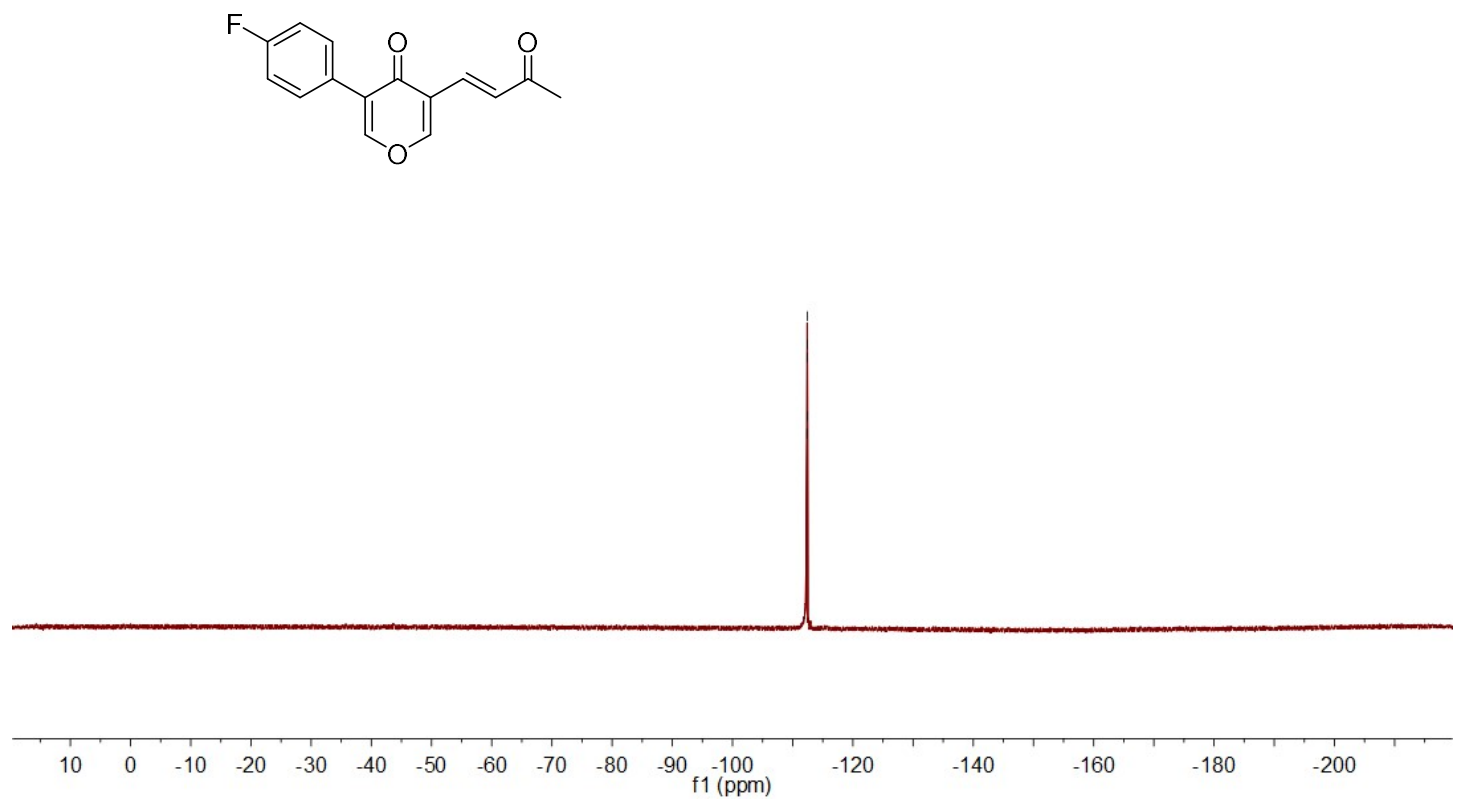

42 
3a ${ }^{1} \mathbf{H}$ NMR $\left(600 \mathrm{MHz}, \mathrm{CDCl}_{3}\right)$

及

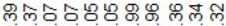<smiles>O=C1C[C@]2(c3ccccc3)C[C@H]3Oc4ccccc4C(=O)C3=CC2=C(c2ccccc2)O1</smiles>

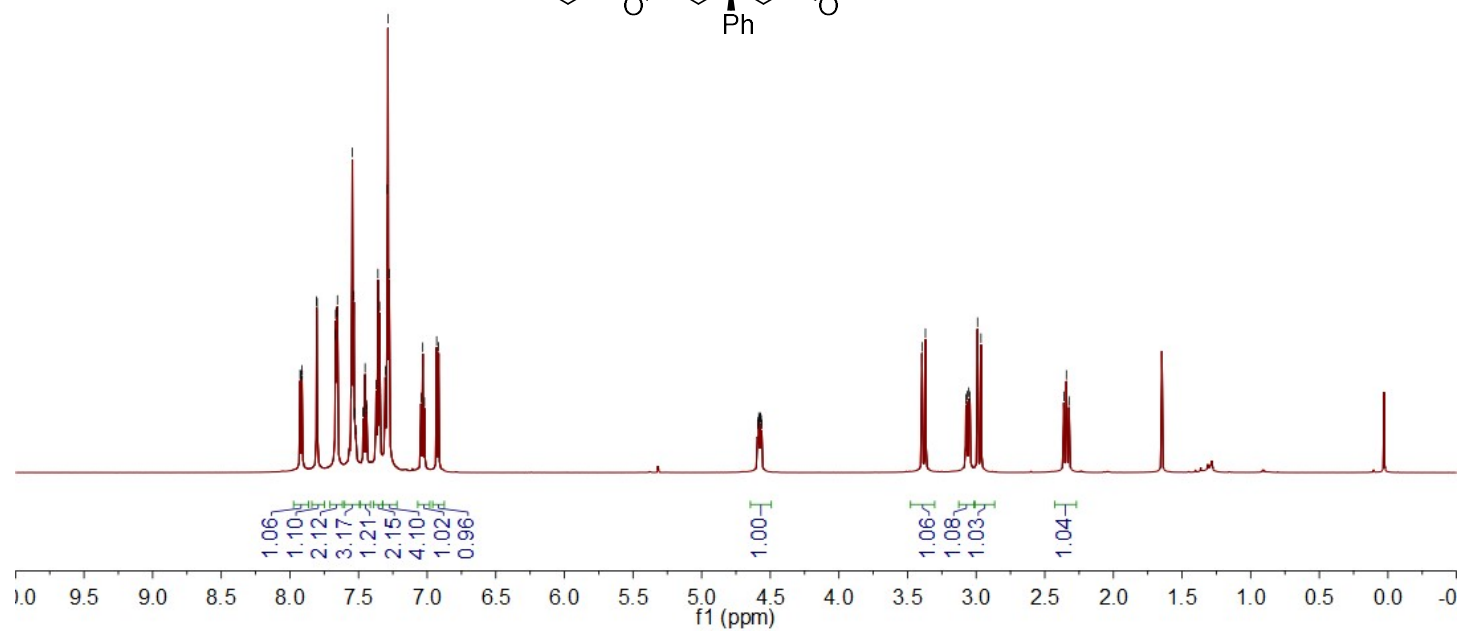

3a ${ }^{13} \mathrm{C}$ NMR $\left(151 \mathrm{MHz}, \mathrm{CDCl}_{3}\right)$

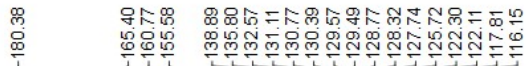

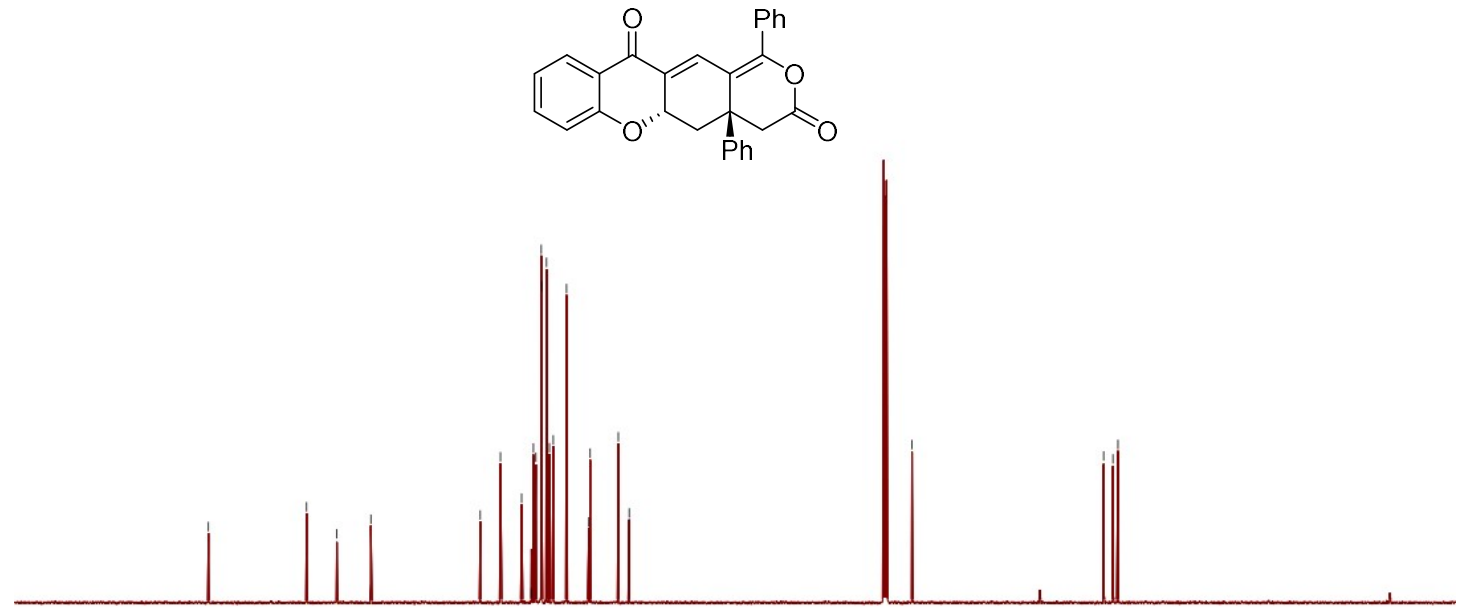

$\begin{array}{lllllllllllllllllllllllllll}10 & 200 & 190 & 180 & 170 & 160 & 150 & 140 & 130 & 120 & 110 & 100 & 90 & 80 & 70 & 60 & 50 & 40 & 30 & 20 & 10 & 0 & -1\end{array}$ 
3a' ${ }^{1} \mathbf{H}$ NMR $\left(400 \mathrm{MHz}, \mathrm{CDCl}_{3}\right)$

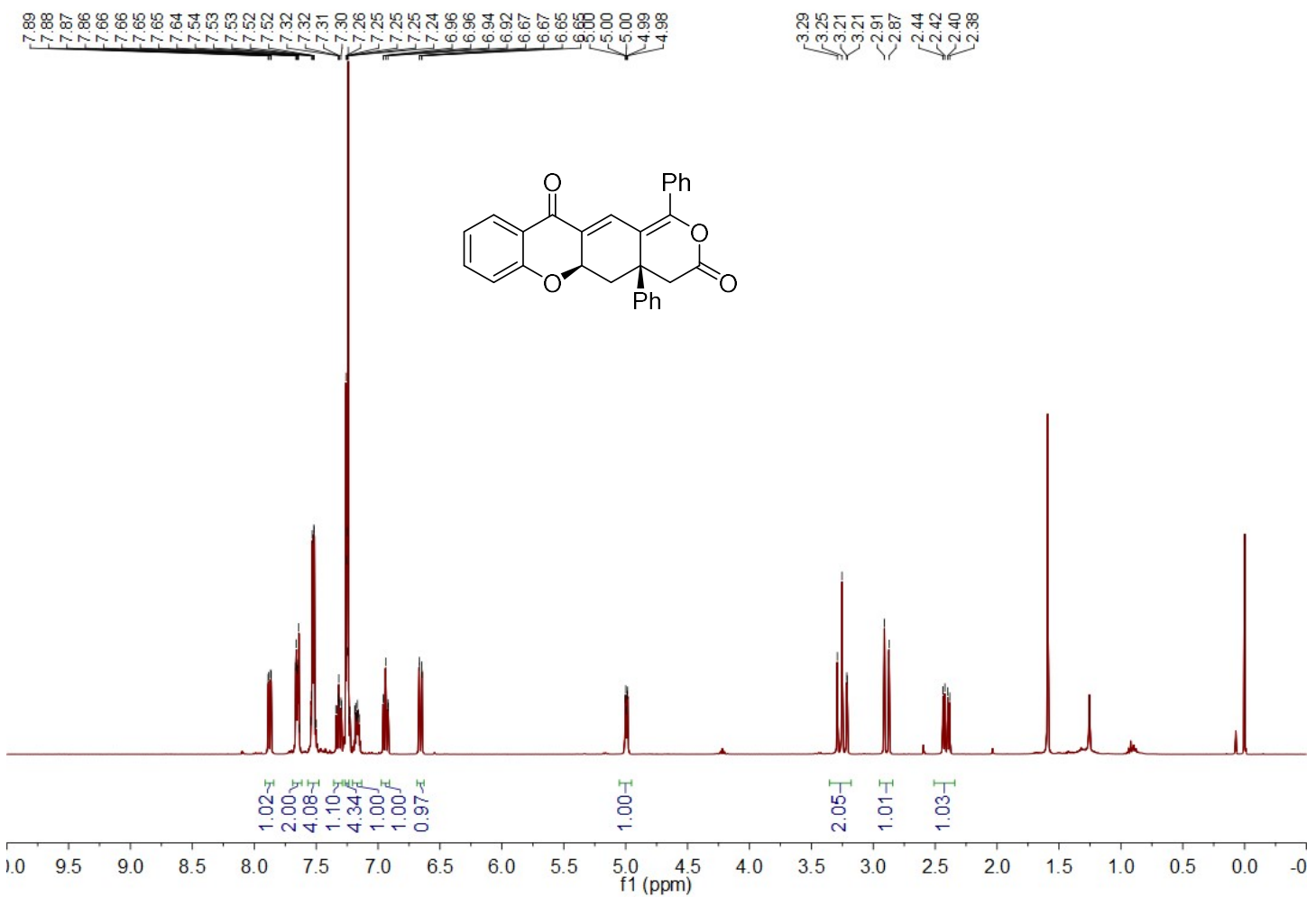

3a' ${ }^{13} \mathrm{C}$ NMR (101 MHz, $\left.\mathrm{CDCl}_{3}\right)$
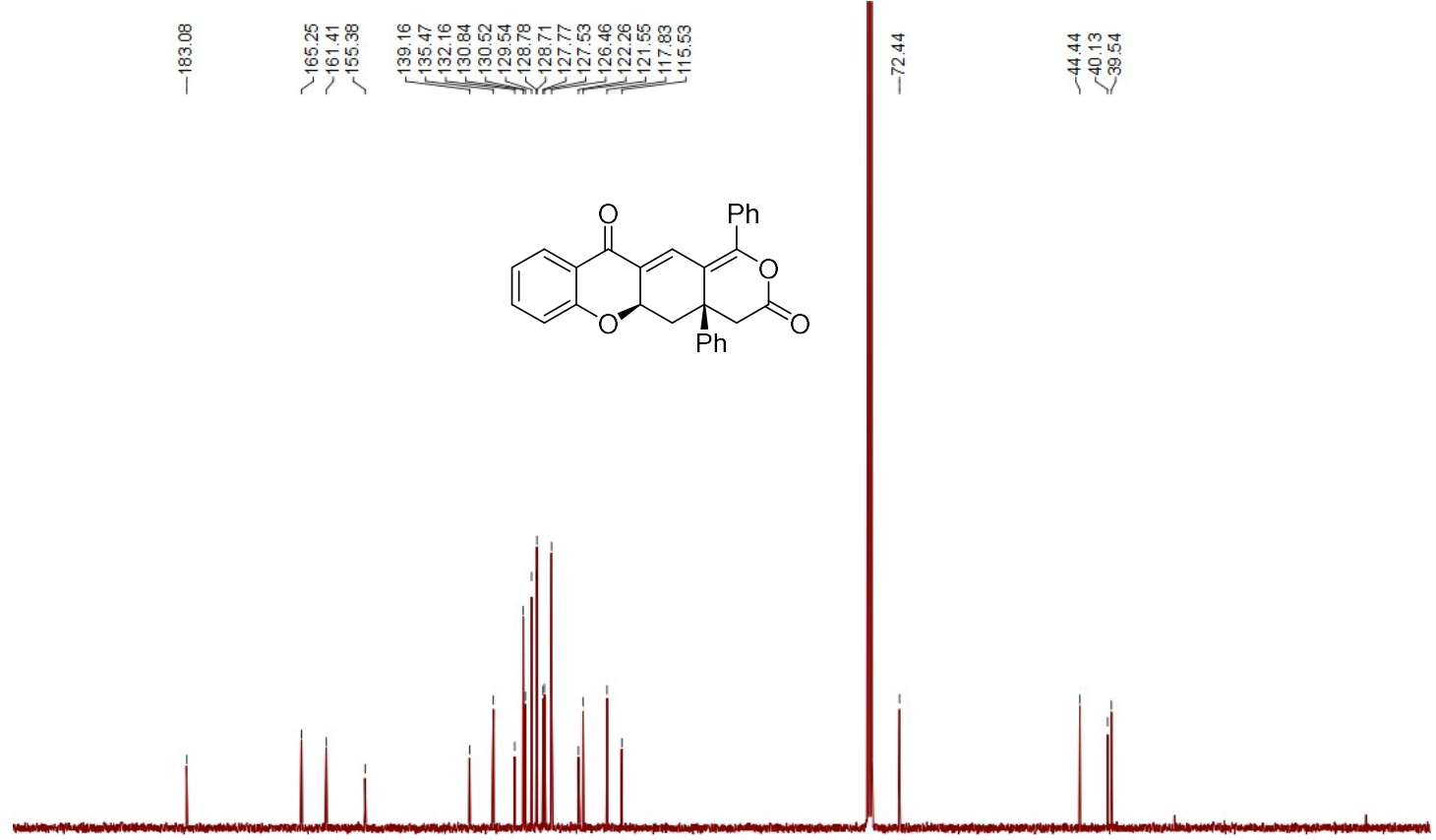

$\begin{array}{lllllllllllllllllllllllllll}10 & 200 & 190 & 180 & 170 & 160 & 150 & 140 & 130 & 120 & 110 & 100 & 90 & 80 & 70 & 60 & 50 & 40 & 30 & 20 & 10 & 0 & -1\end{array}$ 
<smiles>O=C1C[C@]2(c3ccc(F)cc3)C[C@H]3Oc4ccccc4C(=O)C3=CC2=C(c2ccccc2)O1</smiles>

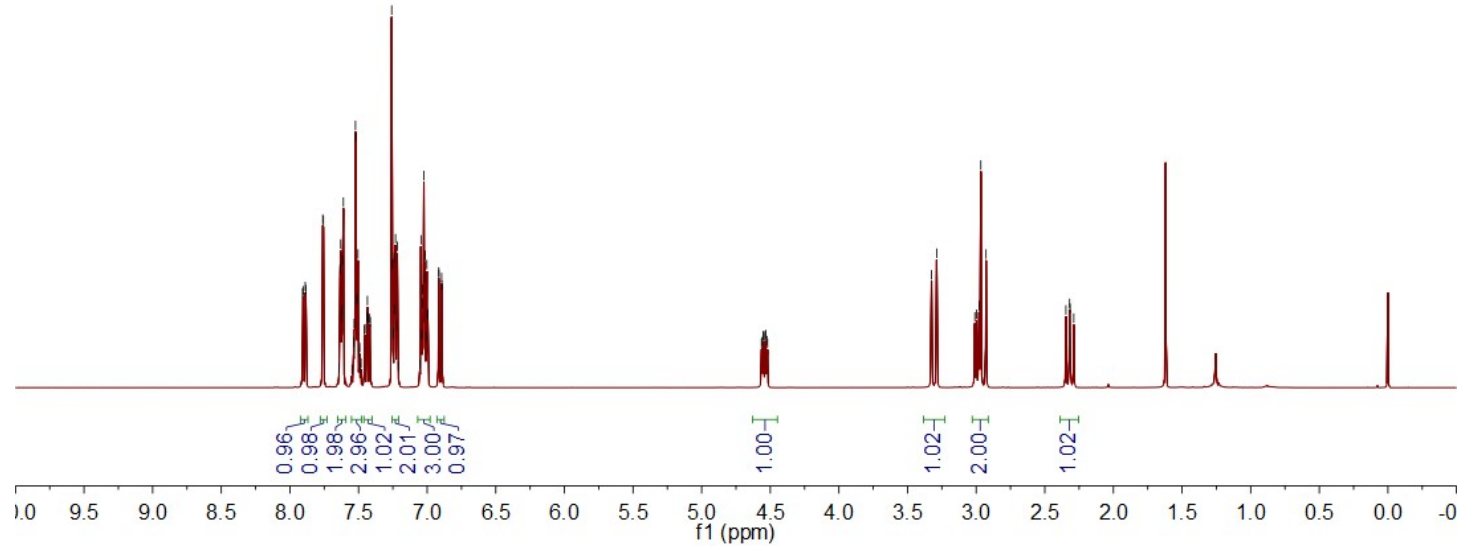

\section{3b ${ }^{13} \mathrm{C}$ NMR (101 MHz, $\mathrm{CDCl}_{3}$ )}

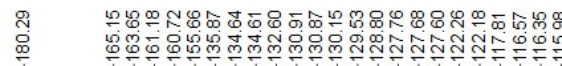<smiles>O=C1CC2(c3ccc(F)cc3)C[C@H]3Oc4ccccc4C(=O)C3=CC2=C(c2ccccc2)O1</smiles>

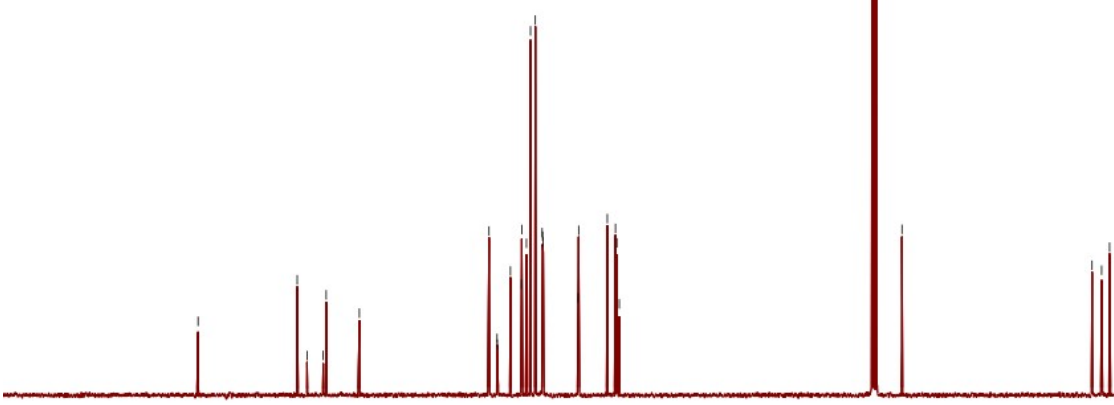

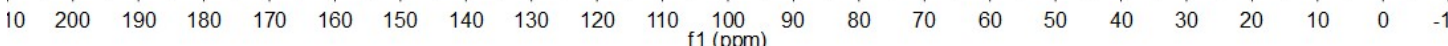


3b ${ }^{19}$ F NMR $\left(471 \mathrm{MHz}, \mathrm{CDCl}_{3}\right)$

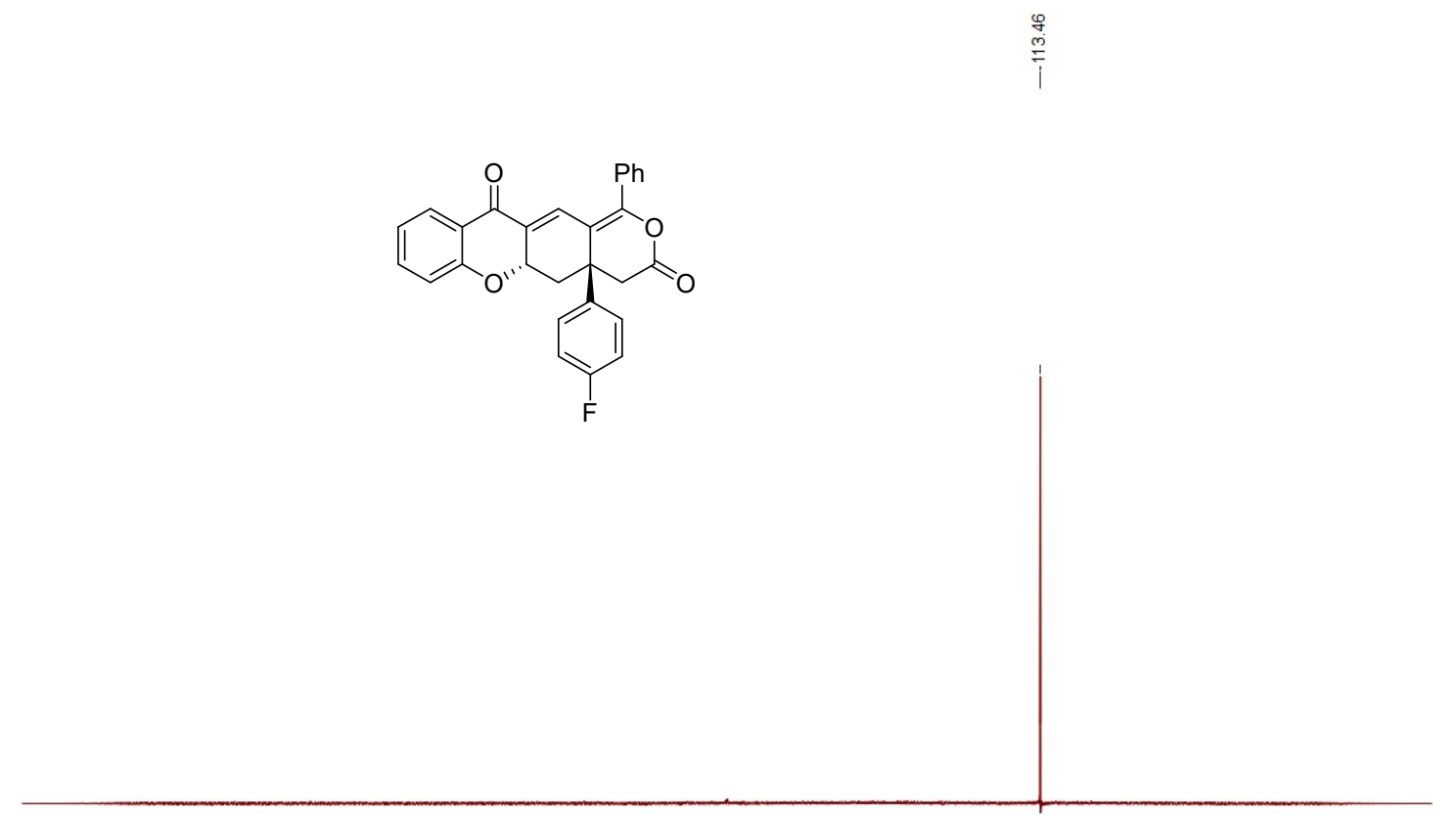

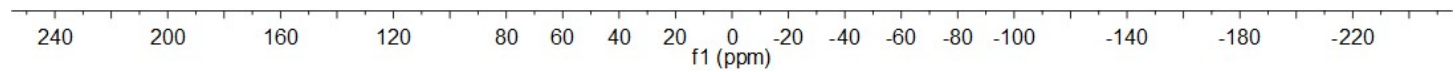

3b' ${ }^{1} \mathbf{H}$ NMR $\left(400 \mathrm{MHz}, \mathrm{CDCl}_{3}\right)$

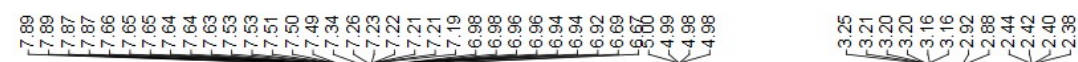

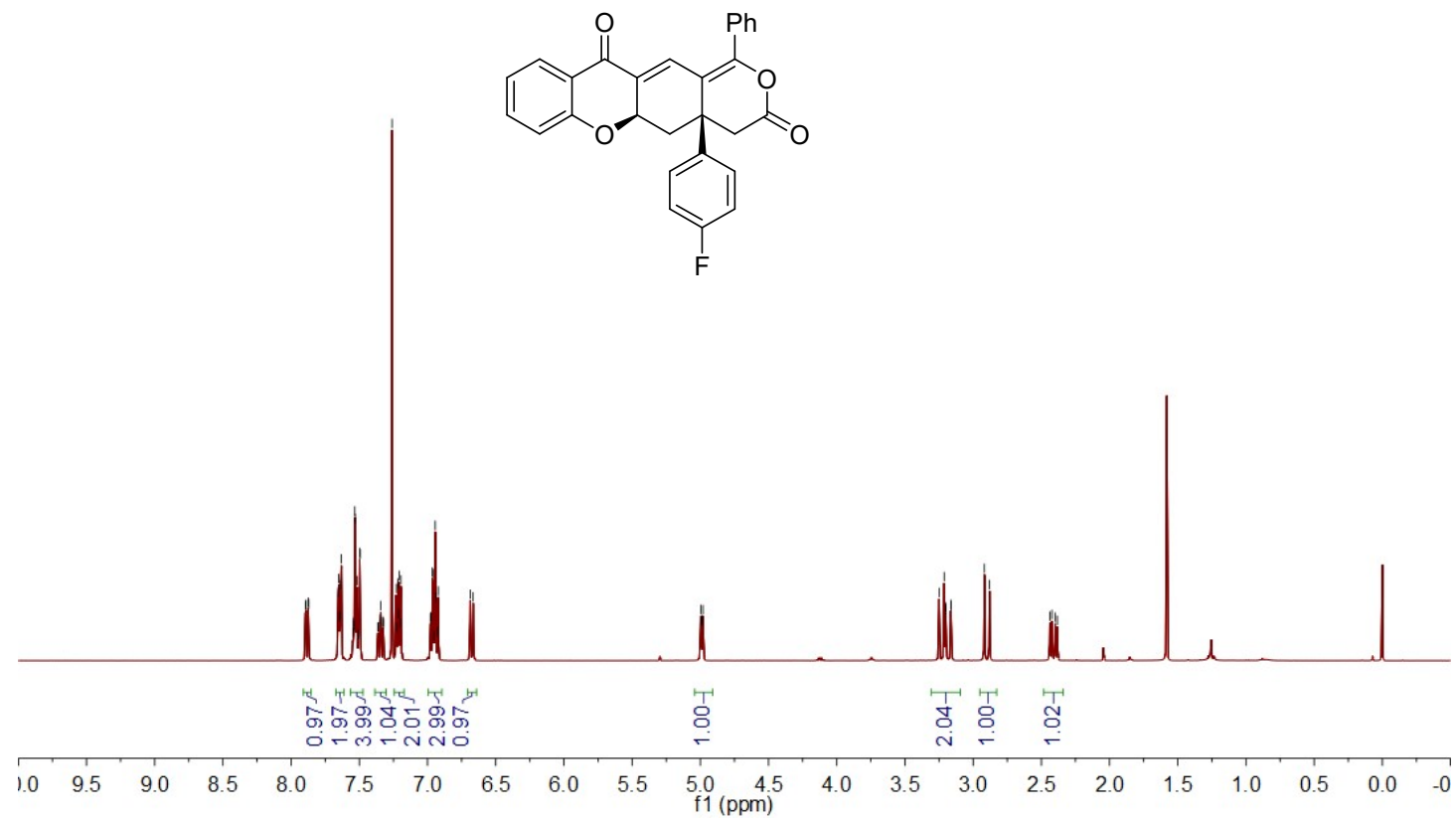


3b' ${ }^{13} \mathbf{C}$ NMR $\left(101 \mathrm{MHz}, \mathrm{CDCl}_{3}\right)$

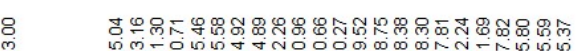

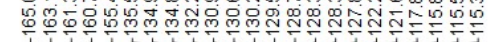

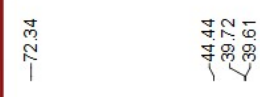

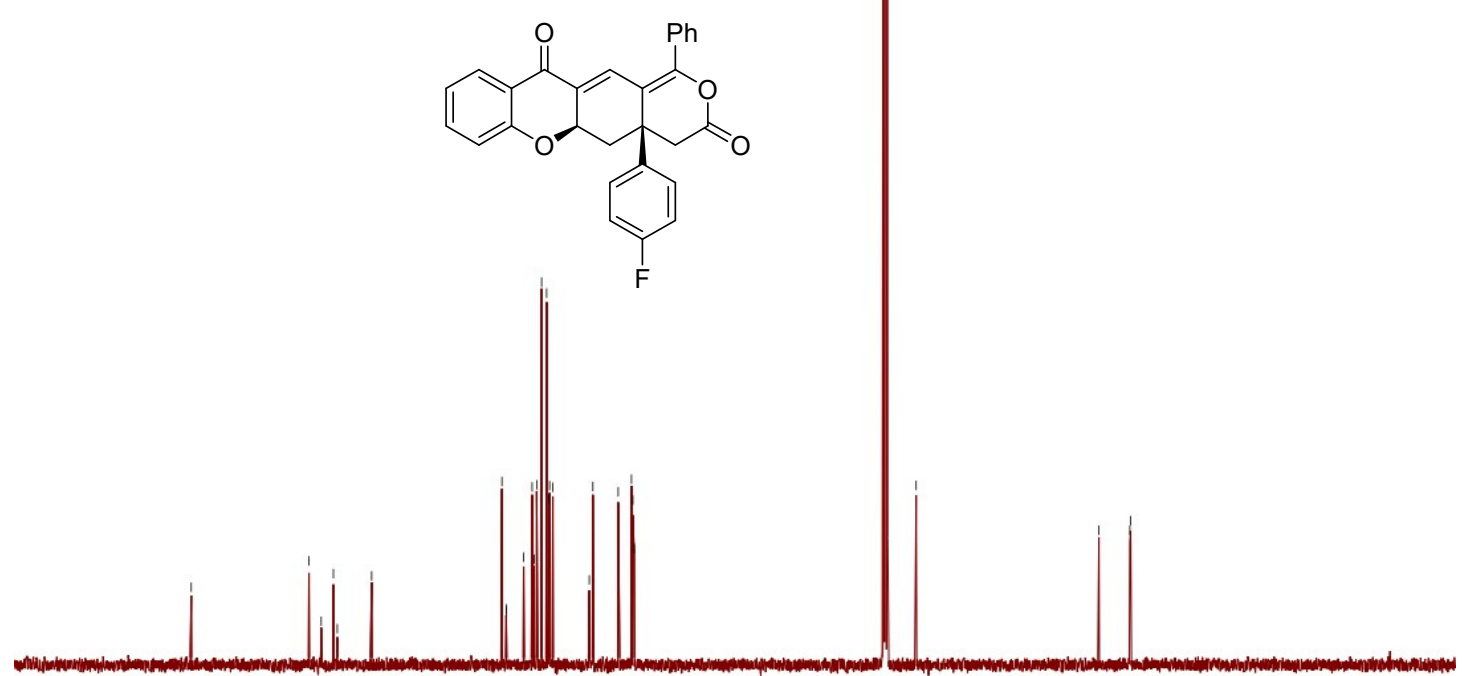

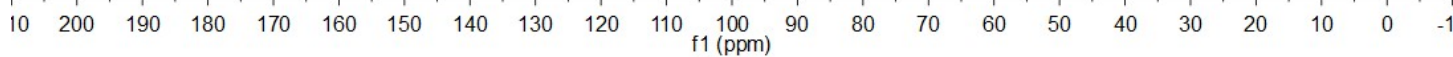

3b' ${ }^{19} \mathrm{~F}$ NMR $\left(471 \mathrm{MHz}, \mathrm{CDCl}_{3}\right)$<smiles>O=C1C2=CC3C(=O)OC(c4ccccc4)C3(c3ccc(F)cc3)CC2Oc2ccccc21</smiles>

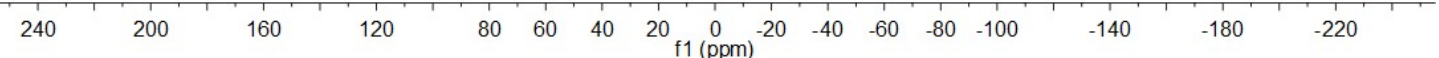


3c ${ }^{1} \mathbf{H}$ NMR $\left(600 \mathrm{MHz}, \mathrm{CDCl}_{3}\right)$

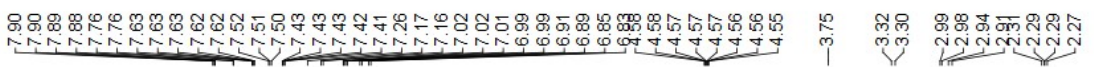<smiles>COc1ccc(C23CC(=O)OC(c4ccccc4)=CC2=CC3=O)cc1</smiles>

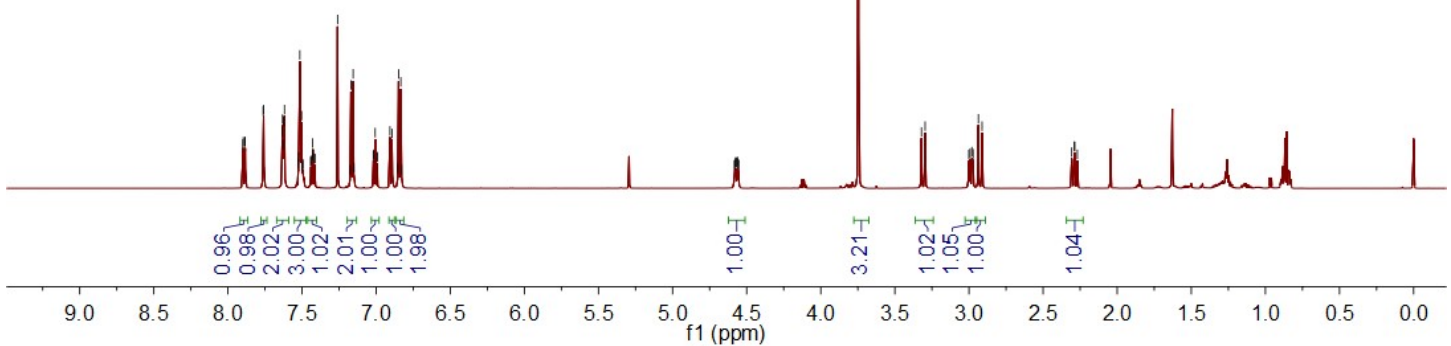

$3 \mathbf{c}^{13} \mathrm{C}$ NMR $\left(151 \mathrm{MHz}, \mathrm{CDCl}_{3}\right)$

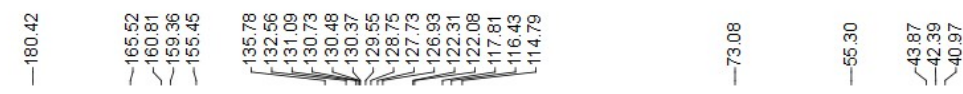

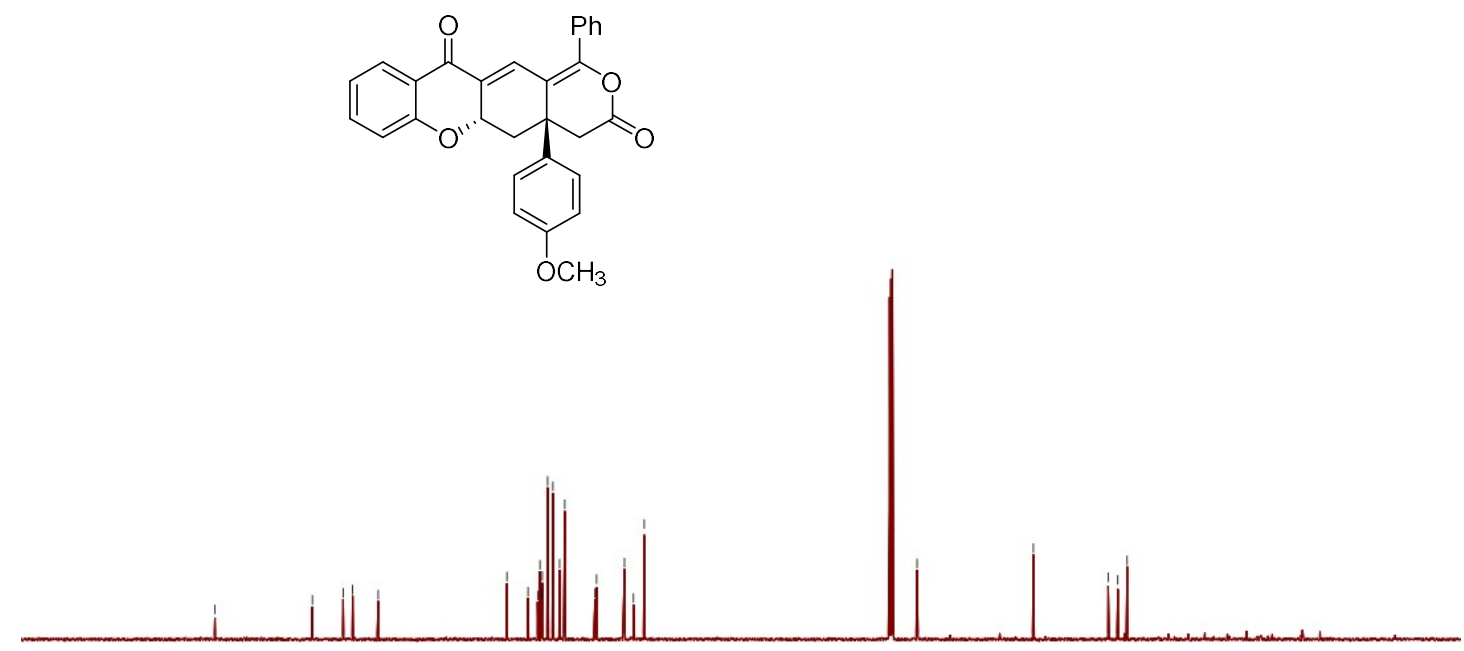

$\begin{array}{llllllllllllllllllllllllllll}10 & 200 & 190 & 180 & 170 & 160 & 150 & 140 & 130 & 120 & 110 & 100 & 90 & 80 & 70 & 60 & 50 & 40 & 30 & 20 & 10 & 0 & -1\end{array}$ 
3c' ${ }^{1} \mathrm{H}$ NMR $\left(400 \mathrm{MHz}, \mathrm{CDCl}_{3}\right)$

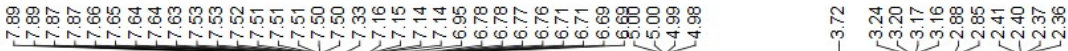<smiles>COc1ccc(C23CC(=O)OC(c4ccccc4)C2C=C3Oc2ccccc2C=O)cc1</smiles>

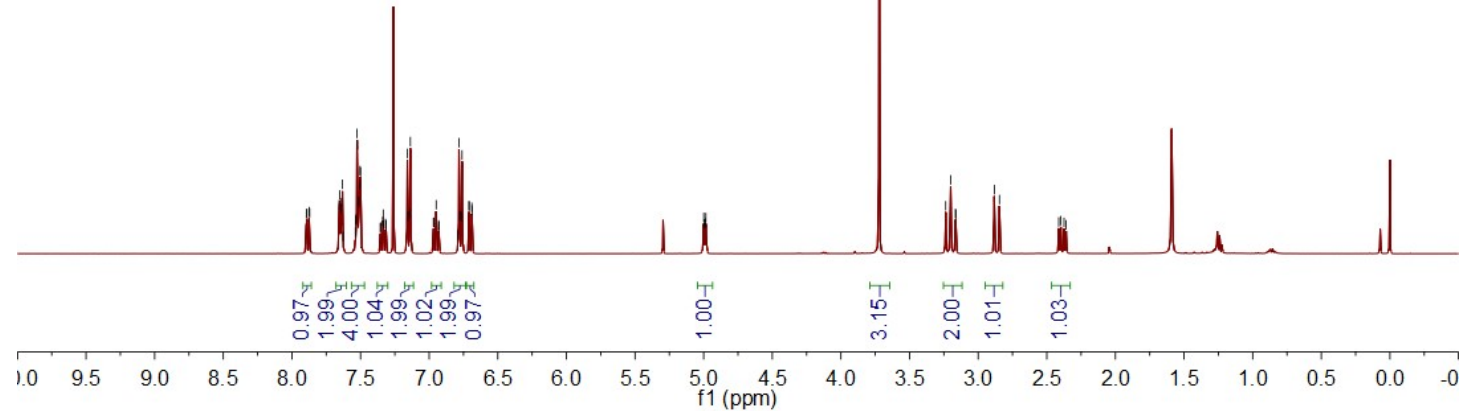

3c' ${ }^{13} \mathrm{C}$ NMR $\left(101 \mathrm{MHz}, \mathrm{CDCl}_{3}\right)$

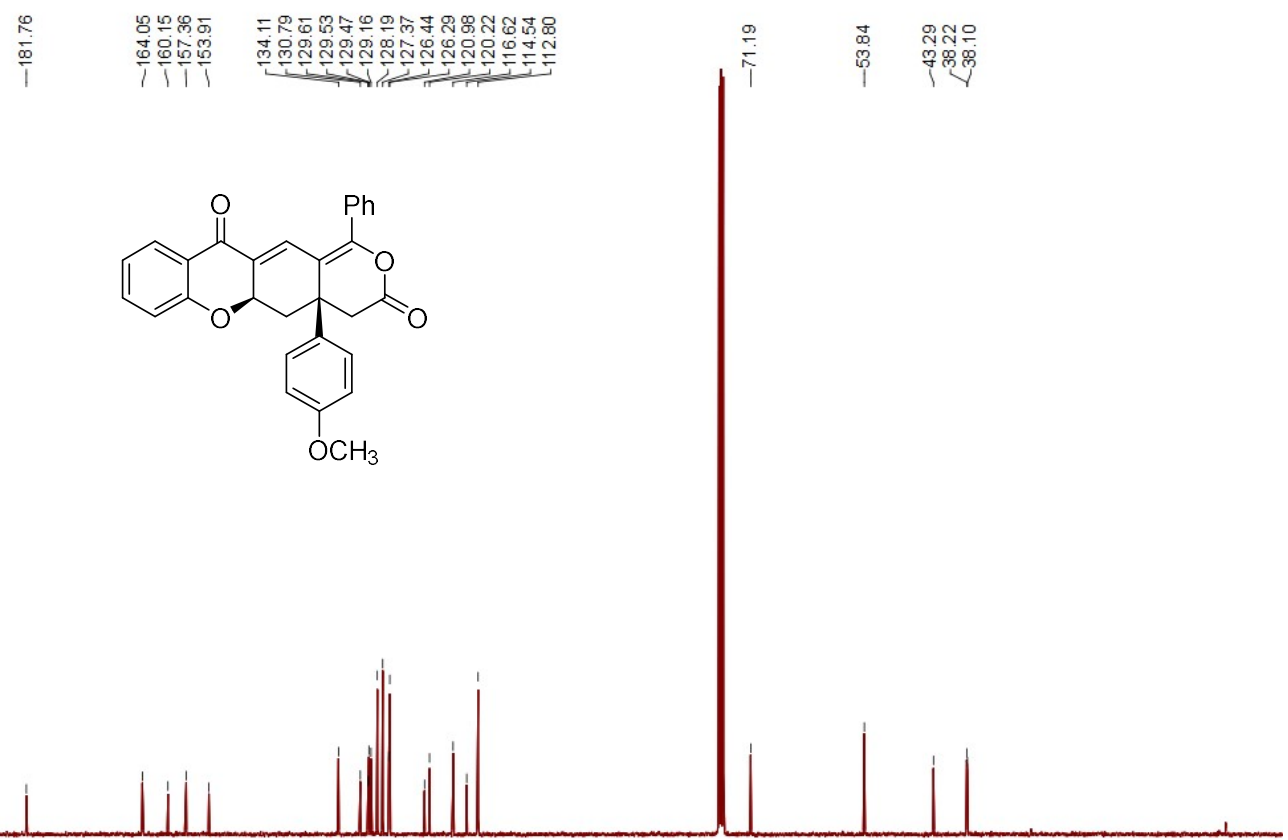

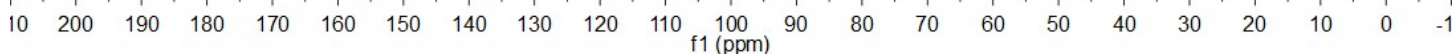


3d ${ }^{1} \mathbf{H}$ NMR $\left(600 \mathrm{MHz}, \mathrm{CDCl}_{3}\right)$

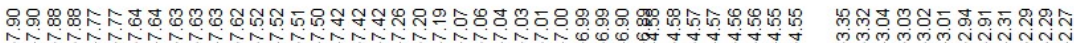<smiles>Cc1cccc(C23CC(=O)OC(c4ccccc4)=C(C=C4C(=O)c5ccccc5OC4C2)C3)c1</smiles>

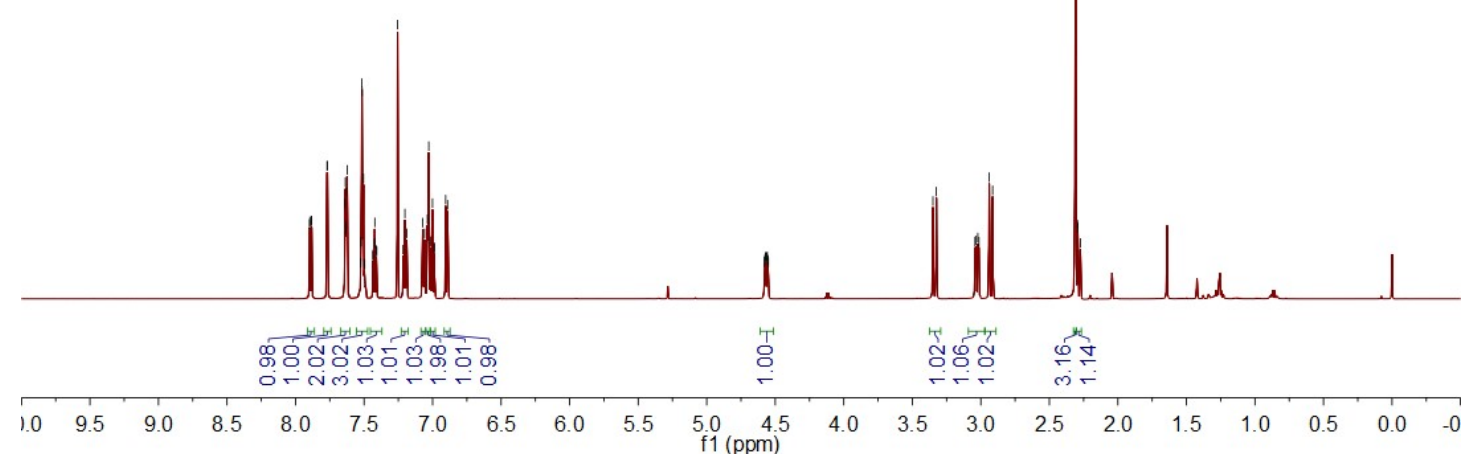

3d ${ }^{13} \mathrm{C}$ NMR (151 MHz, $\left.\mathrm{CDCl}_{3}\right)$

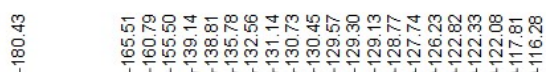

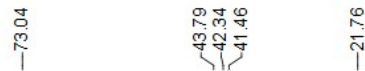<smiles>Cc1cccc(C23CC(=O)OC(c4ccccc4)=C(C=C4C(=O)c5ccccc5OC4C2)C3)c1</smiles>

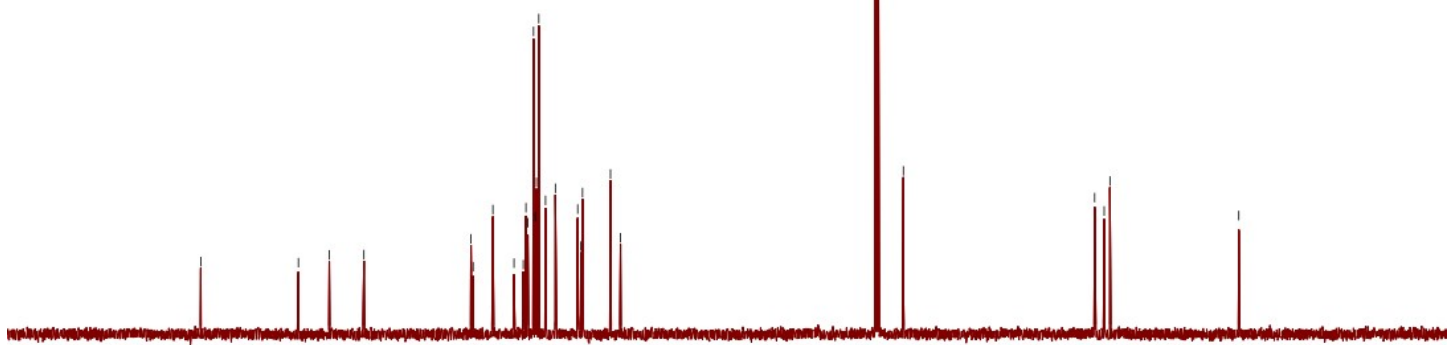

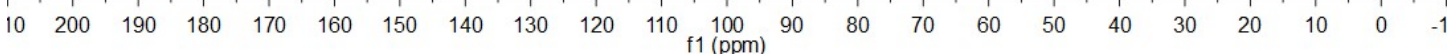


3d' ${ }^{1} \mathbf{H}$ NMR (400 MHz, $\left.\mathrm{CDCl}_{3}\right)$

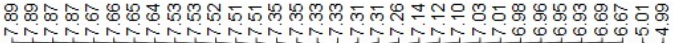

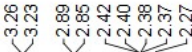<smiles></smiles>

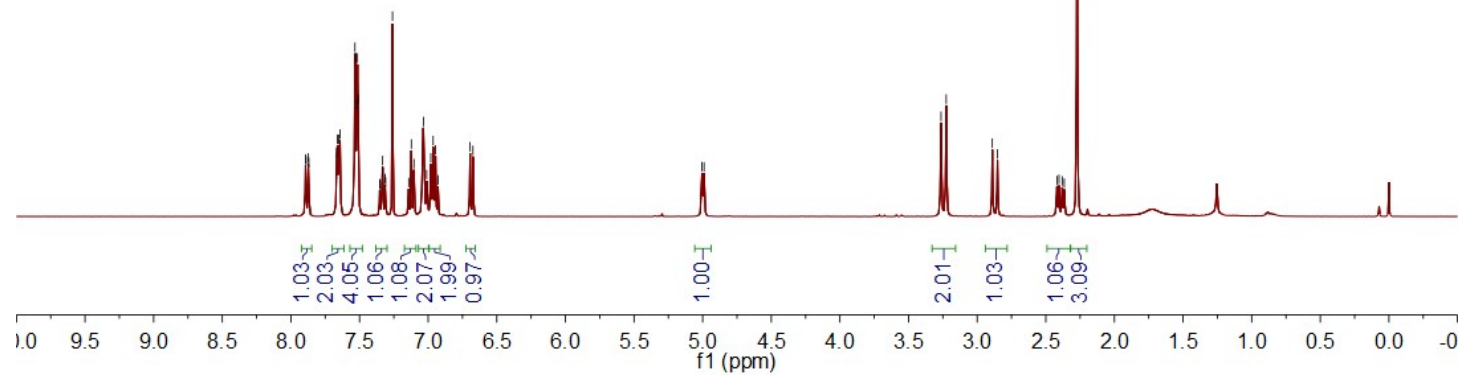

3d' ${ }^{13}$ C NMR (101 MHz, $\left.\mathrm{CDCl}_{3}\right)$
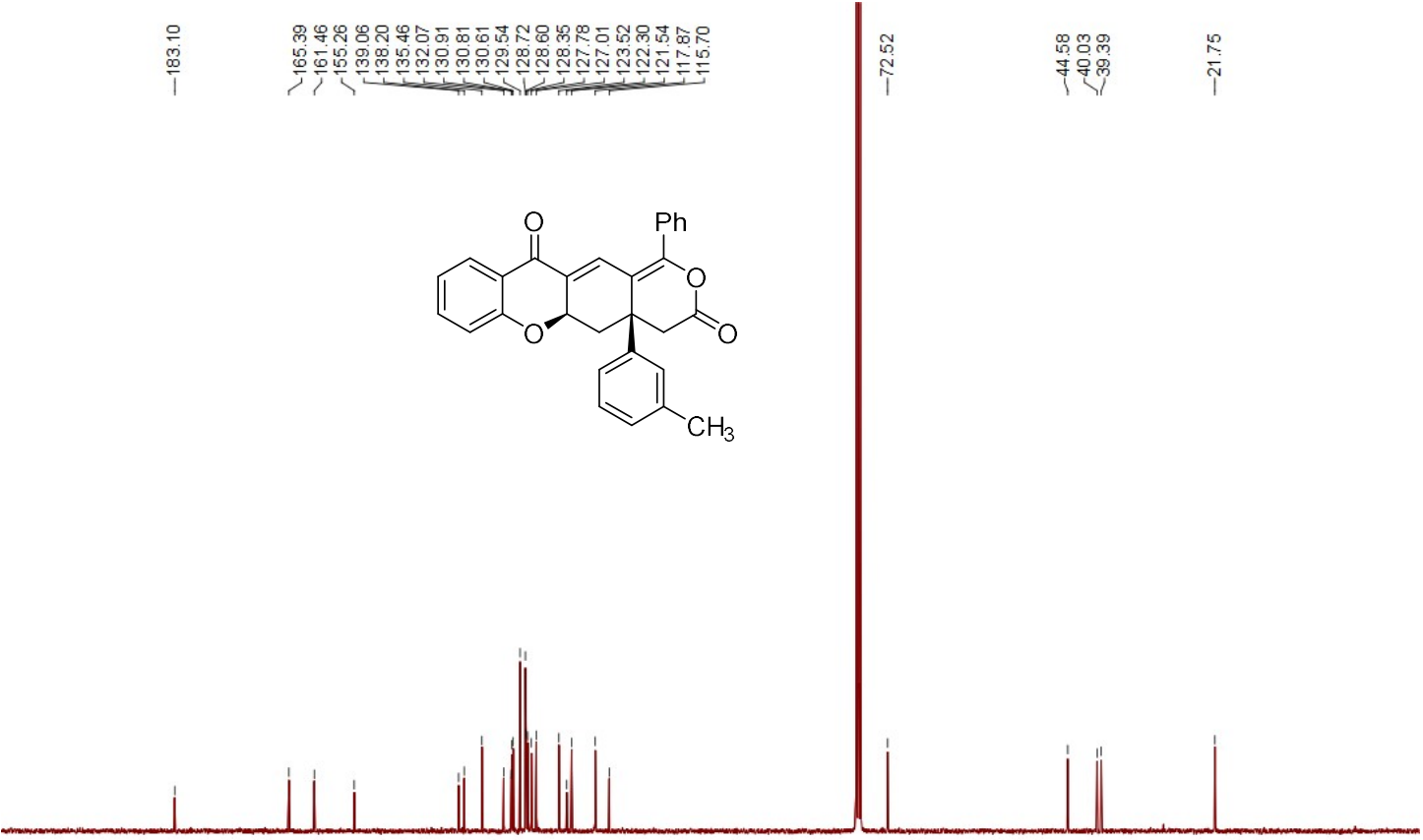

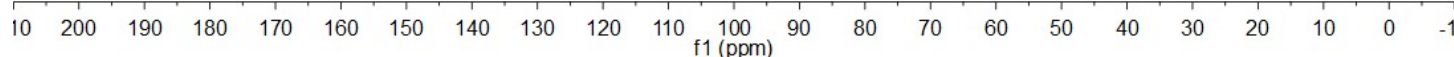


$3 \mathbf{e}^{1} \mathbf{H}$ NMR $\left(600 \mathrm{MHz}, \mathrm{CDCl}_{3}\right)$

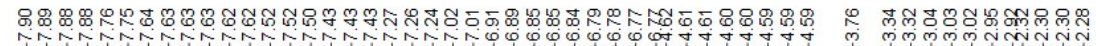<smiles>COc1cccc([C@]23CC(=O)OC(c4ccccc4)=C2C=C2C(=O)c4ccccc4O[C@H]2C3)c1</smiles>

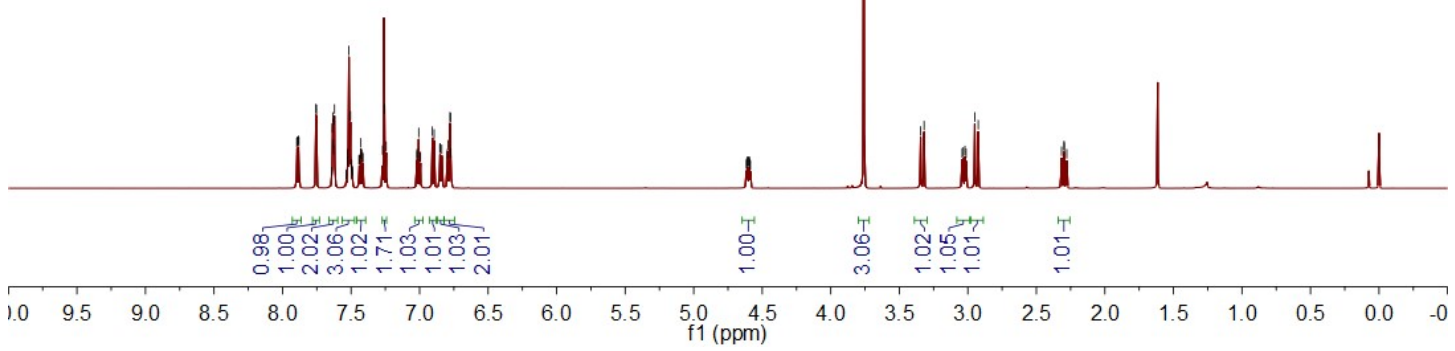

$3 \mathbf{e}^{13} \mathrm{C}$ NMR $\left(151 \mathrm{MHz}, \mathrm{CDCl}_{3}\right)$

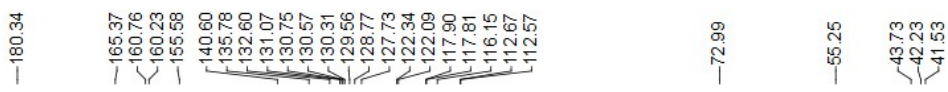<smiles>[CH-]c1ccccc1C(=O)C1=CC2=C(c3ccccc3)OC(=O)CC12c1cccc(OC)c1</smiles>

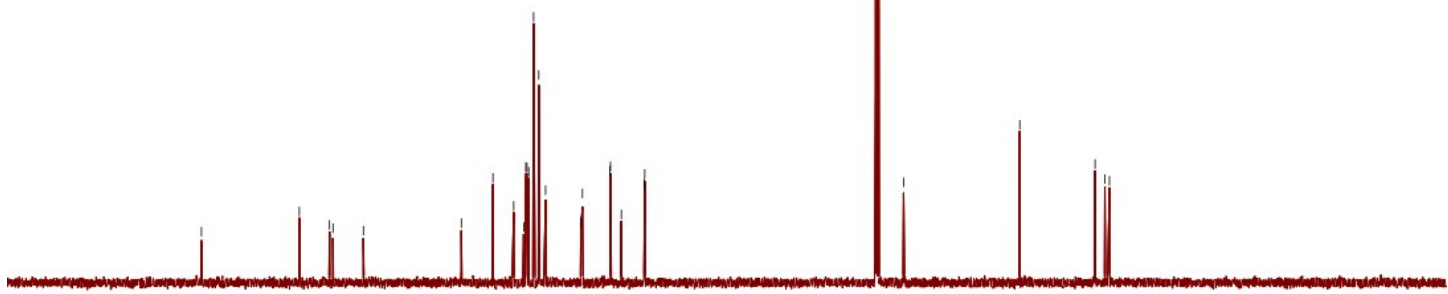

$\begin{array}{lllllllllllllllllllllll}10 & 200 & 190 & 180 & 170 & 160 & 150 & 140 & 130 & 120 & 110 & 100 & 90 & 80 & 70 & 60 & 50 & 40 & 30 & 20 & 10 & 0 & -1\end{array}$ 
3e' ${ }^{1} \mathbf{H}$ NMR $\left(400 \mathrm{MHz}, \mathrm{CDCl}_{3}\right)$

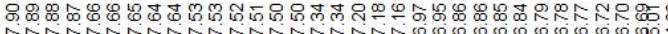

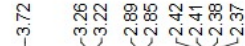<smiles>COc1cccc(C23CC(=O)OC(c4ccccc4)=C2C=C3C(=O)c2ccccc2OC)c1</smiles>

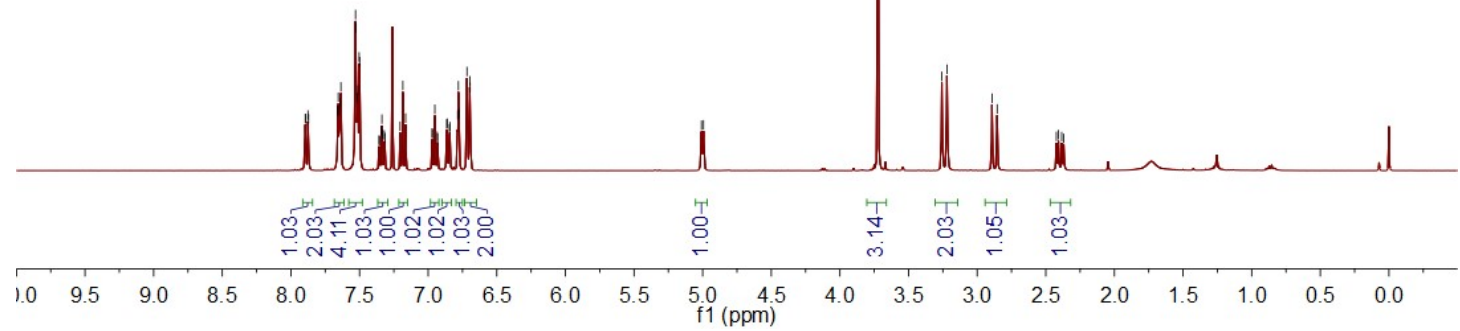

3e' ${ }^{13} \mathrm{C}$ NMR $\left(101 \mathrm{MHz}, \mathrm{CDCl}_{3}\right)$
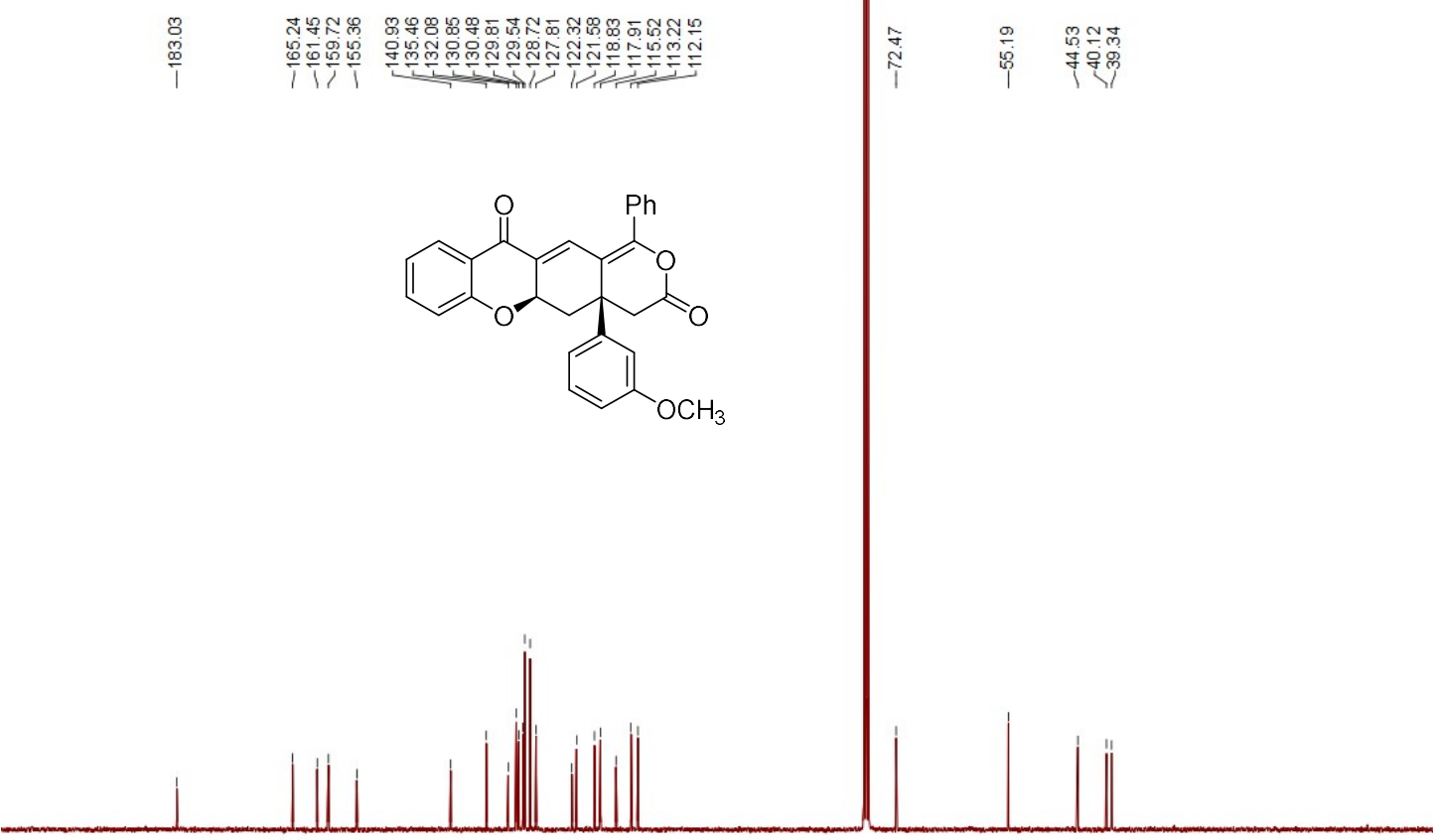

$\begin{array}{lllllllllllllllllllllllllllll}10 & 200 & 190 & 180 & 170 & 160 & 150 & 140 & 130 & 120 & 110 & 100 & 90 & 80 & 70 & 60 & 50 & 40 & 30 & 20 & 10 & 0 & -1\end{array}$ 
$3 \mathrm{f}^{1} \mathrm{H}$ NMR $\left(600 \mathrm{MHz}, \mathrm{CDCl}_{3}\right)$

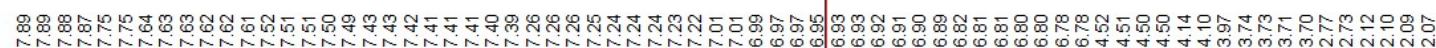<smiles>COc1ccccc1C12CC(=O)OC(c3ccccc3)=C1C=C1C(=O)c3ccccc3OC12</smiles>

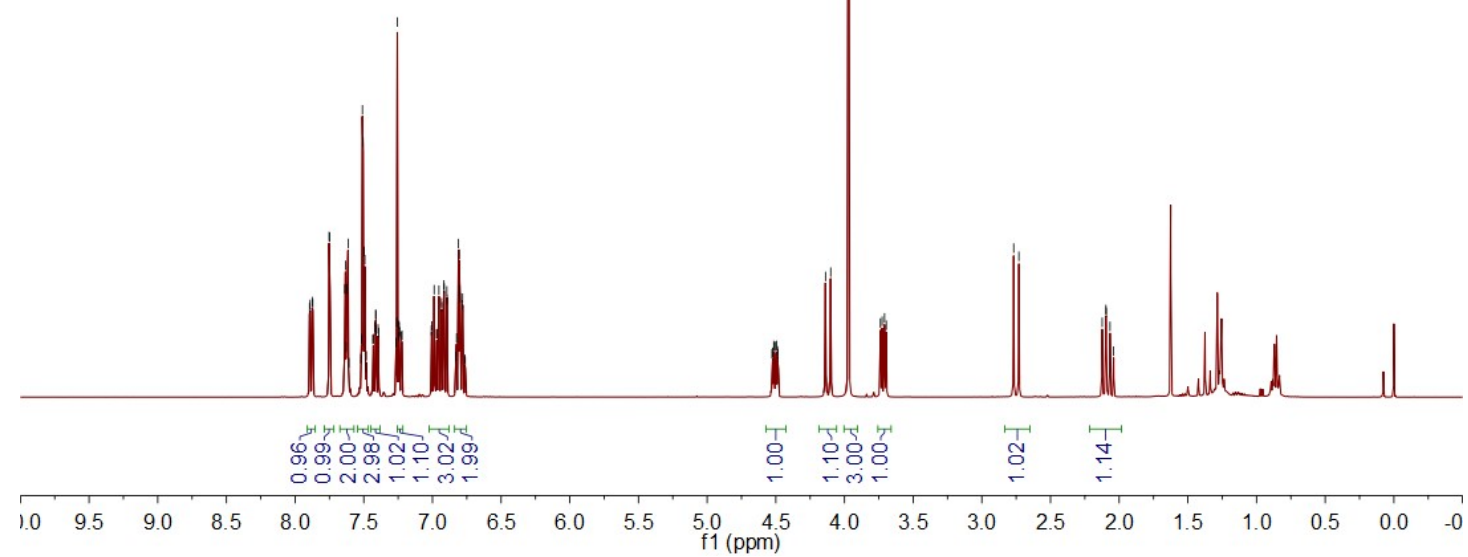

3f ${ }^{13} \mathrm{C}$ NMR $\left(151 \mathrm{MHz}, \mathrm{CDCl}_{3}\right)$

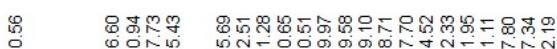

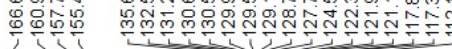

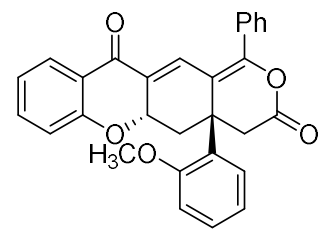

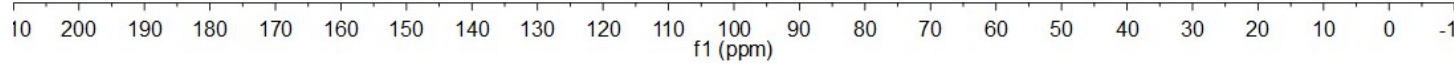


$3 \mathbf{g}^{1} \mathbf{H}$ NMR $\left(600 \mathrm{MHz}, \mathrm{CDCl}_{3}\right)$

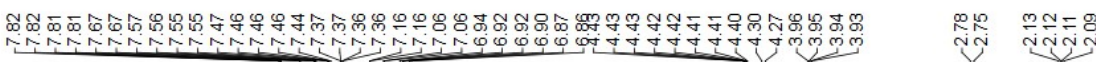

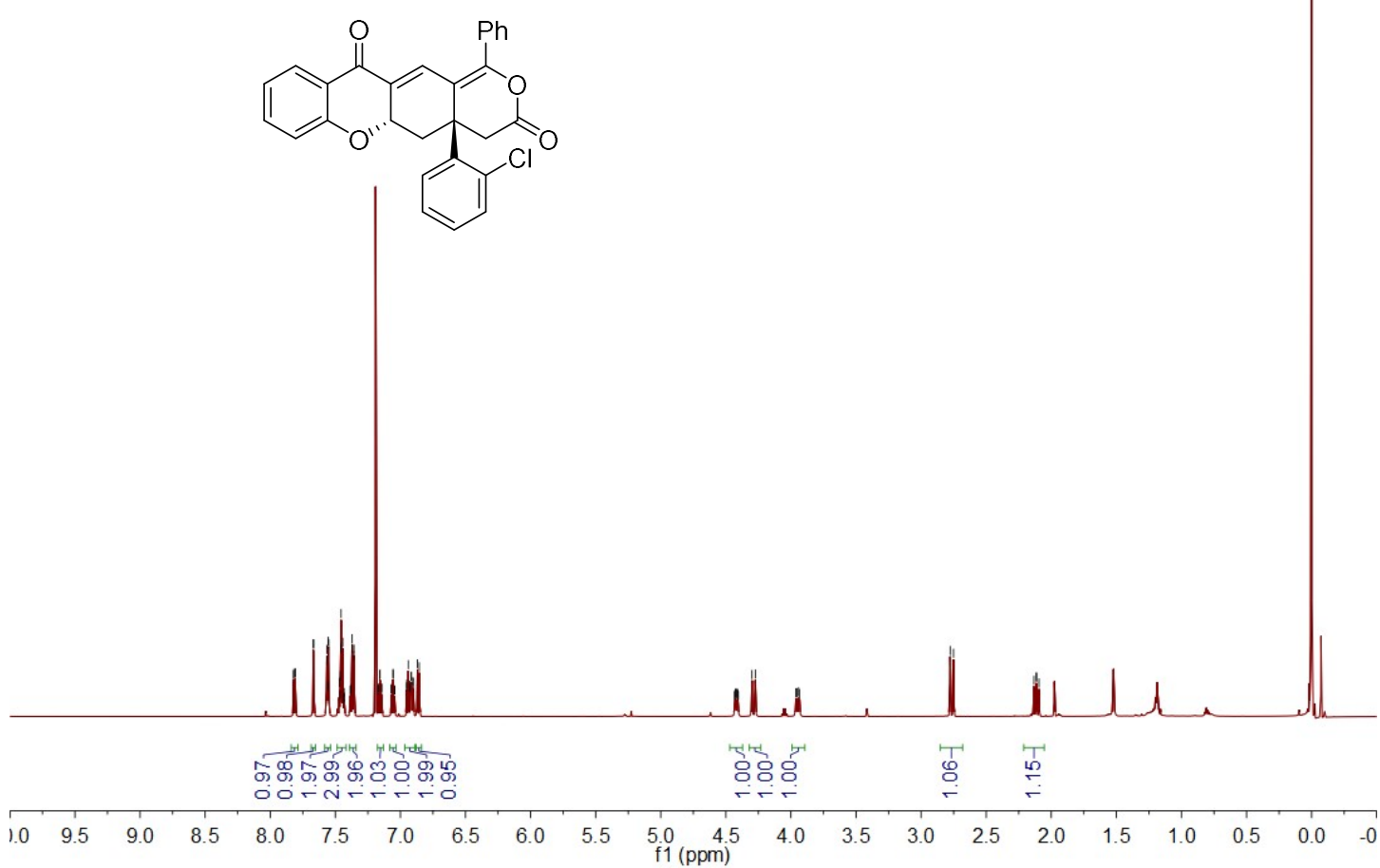

3g ${ }^{13} \mathrm{C}$ NMR $\left(151 \mathrm{MHz}, \mathrm{CDCl}_{3}\right)$

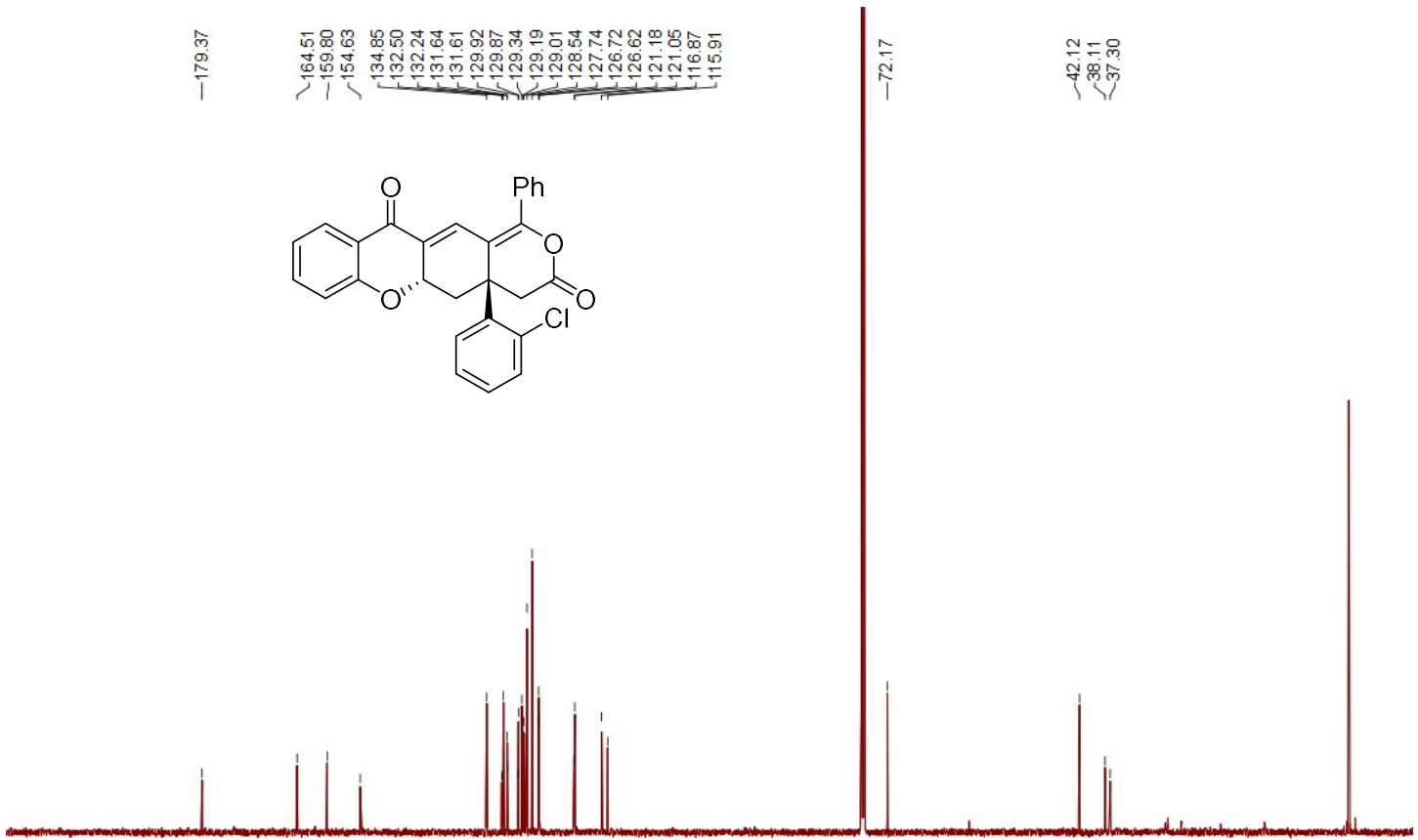

$\begin{array}{llllllllllllllllllllllllllll}10 & 200 & 190 & 180 & 170 & 160 & 150 & 140 & 130 & 120 & 110 & 100 & 90 & 80 & 70 & 60 & 50 & 40 & 30 & 20 & 10 & 0 & -1\end{array}$ 
3h ${ }^{1} \mathbf{H}$ NMR $\left(600 \mathrm{MHz}, \mathrm{CDCl}_{3}\right)$

ळ<smiles>O=C1CC2(c3ccc4ccccc4c3)CC3Oc4ccccc4C3=CC2=C1c1ccccc1</smiles>

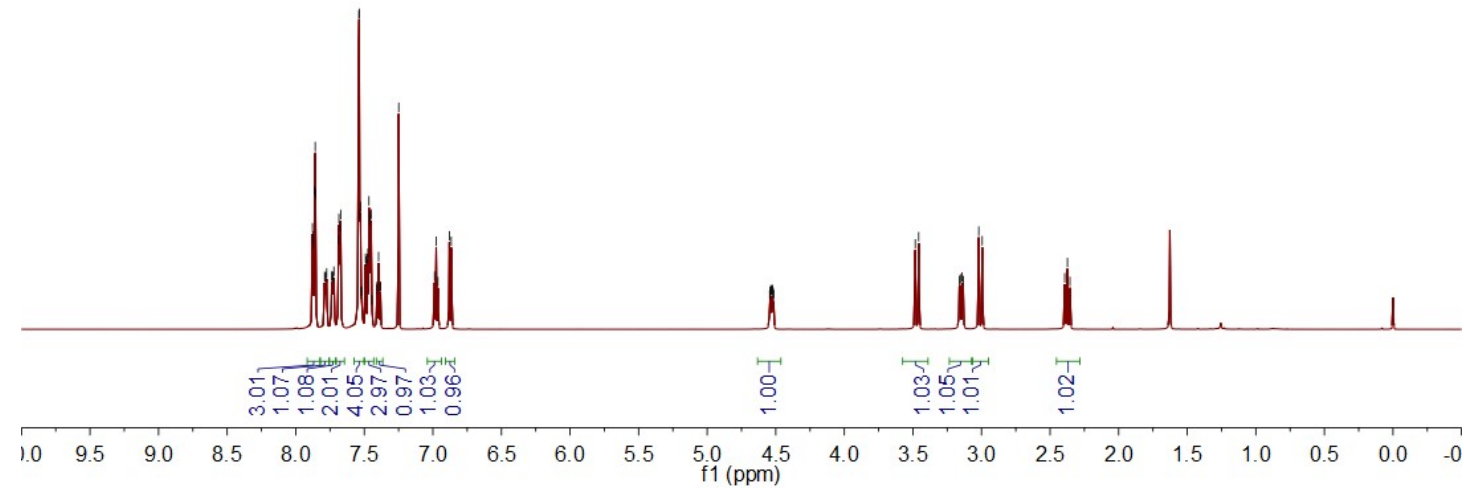

3h ${ }^{13} \mathbf{C}$ NMR (151 MHz, $\left.\mathrm{CDCl}_{3}\right)$

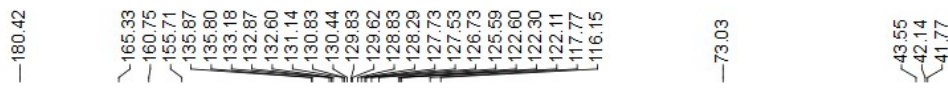

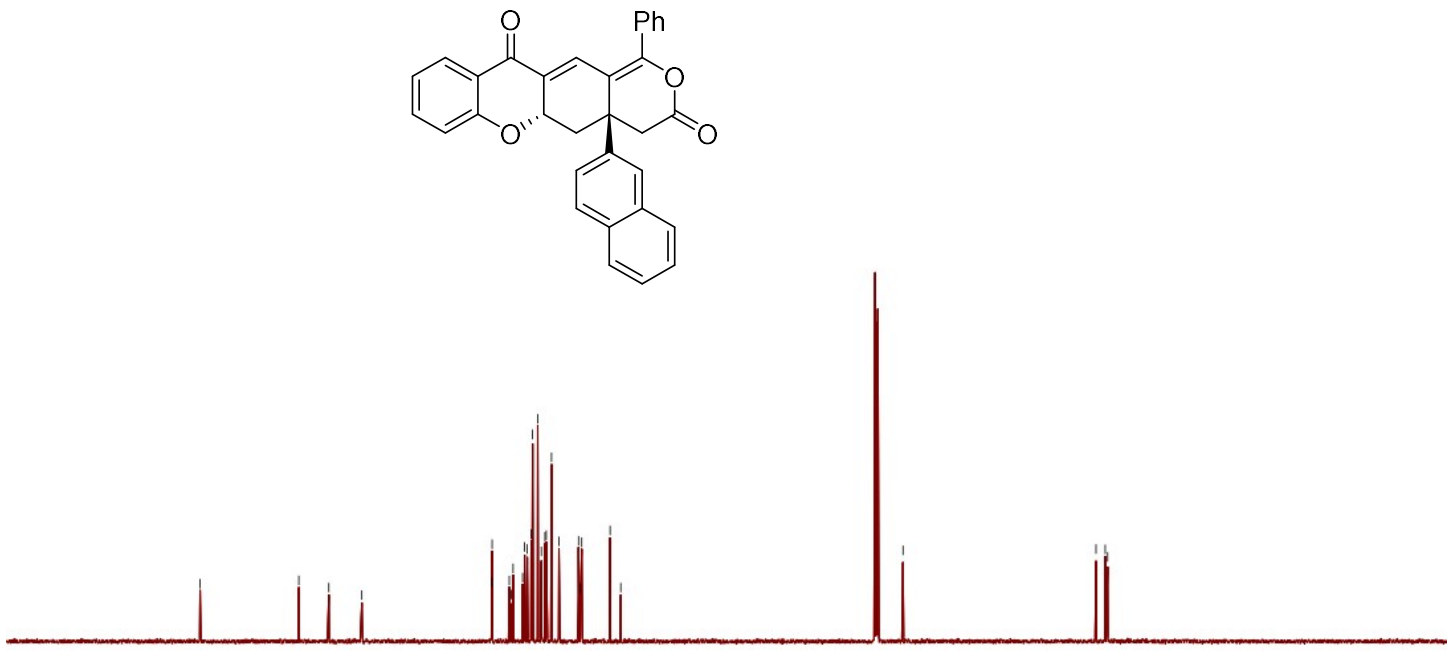

$\begin{array}{lllllllllllllllllllllllllll}10 & 200 & 190 & 180 & 170 & 160 & 150 & 140 & 130 & 120 & 110 & 100 & 90 & 80 & 70 & 60 & 50 & 40 & 30 & 20 & 10 & 0 & -1\end{array}$ 
$3 \mathbf{i}^{1} \mathbf{H}$ NMR $\left(400 \mathrm{MHz}, \mathrm{CDCl}_{3}\right)$

बढ.

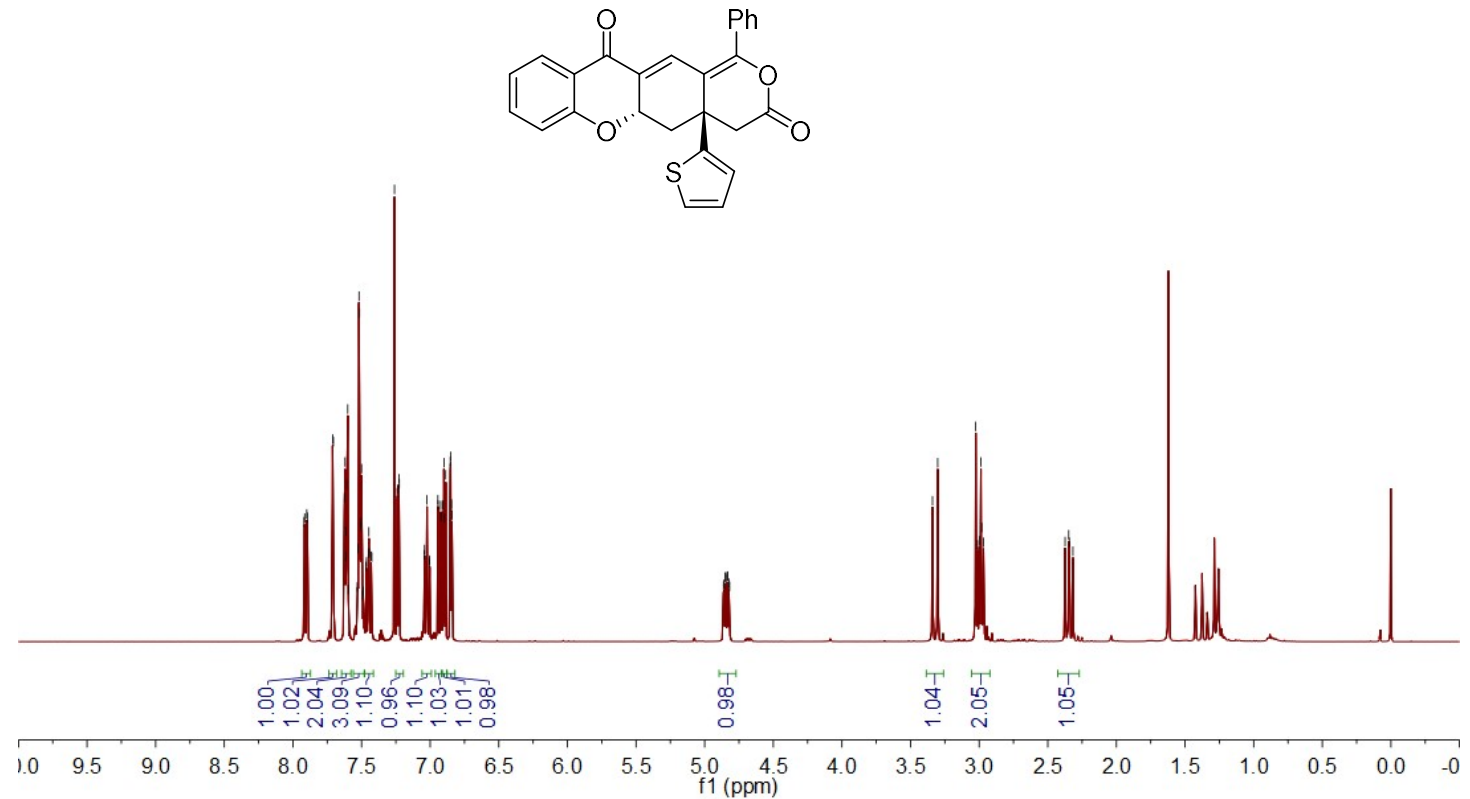

3i ${ }^{13} \mathbf{C}$ NMR $\left(101 \mathrm{MHz}, \mathrm{CDCl}_{3}\right)$

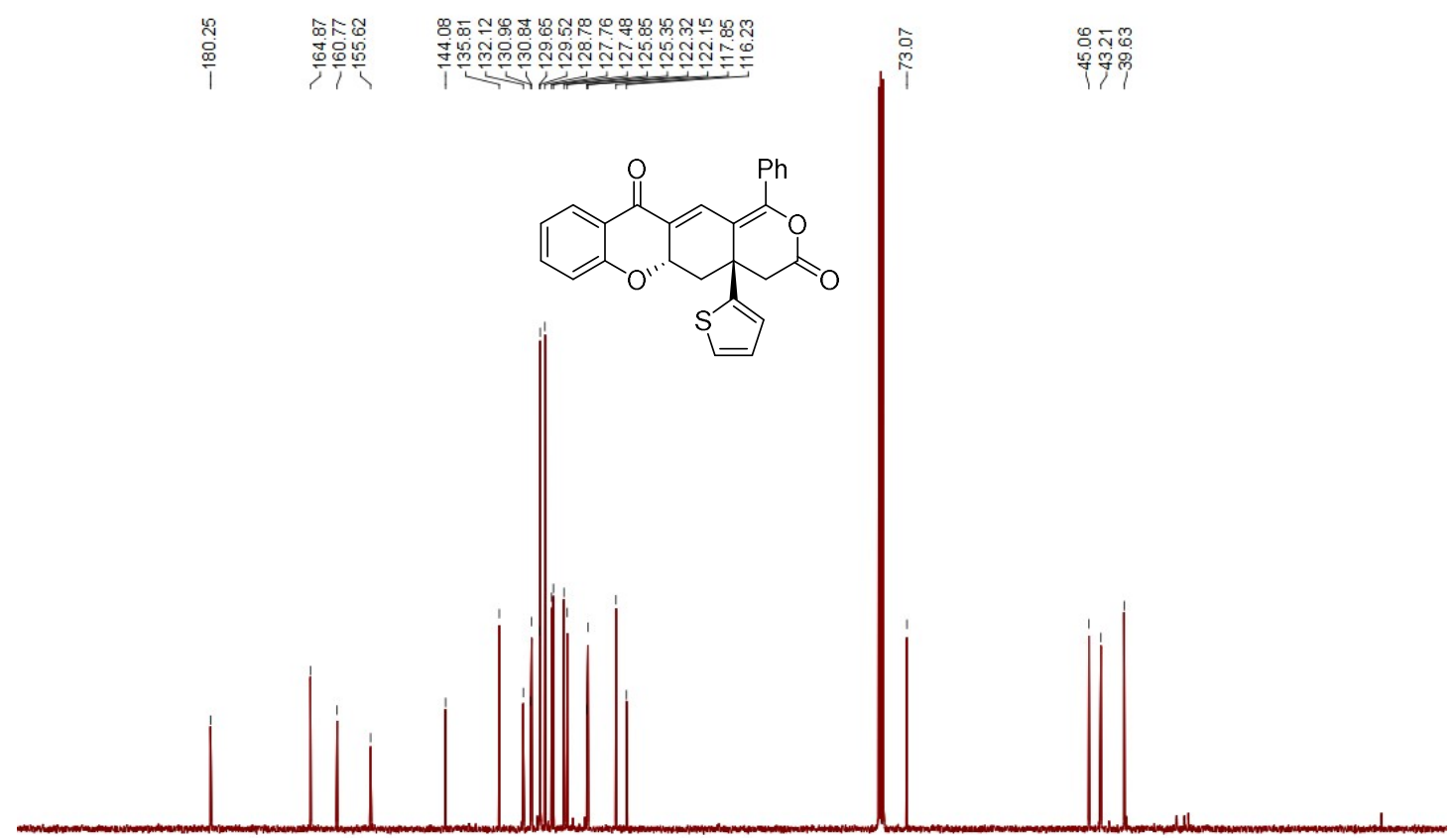

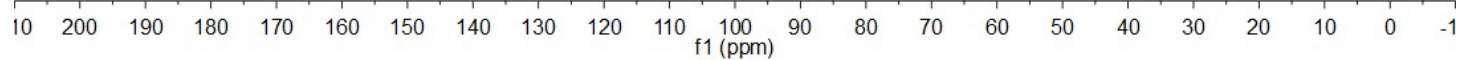


3i' ${ }^{1} \mathbf{H}$ NMR $\left(400 \mathrm{MHz}, \mathrm{CDCl}_{3}\right)$

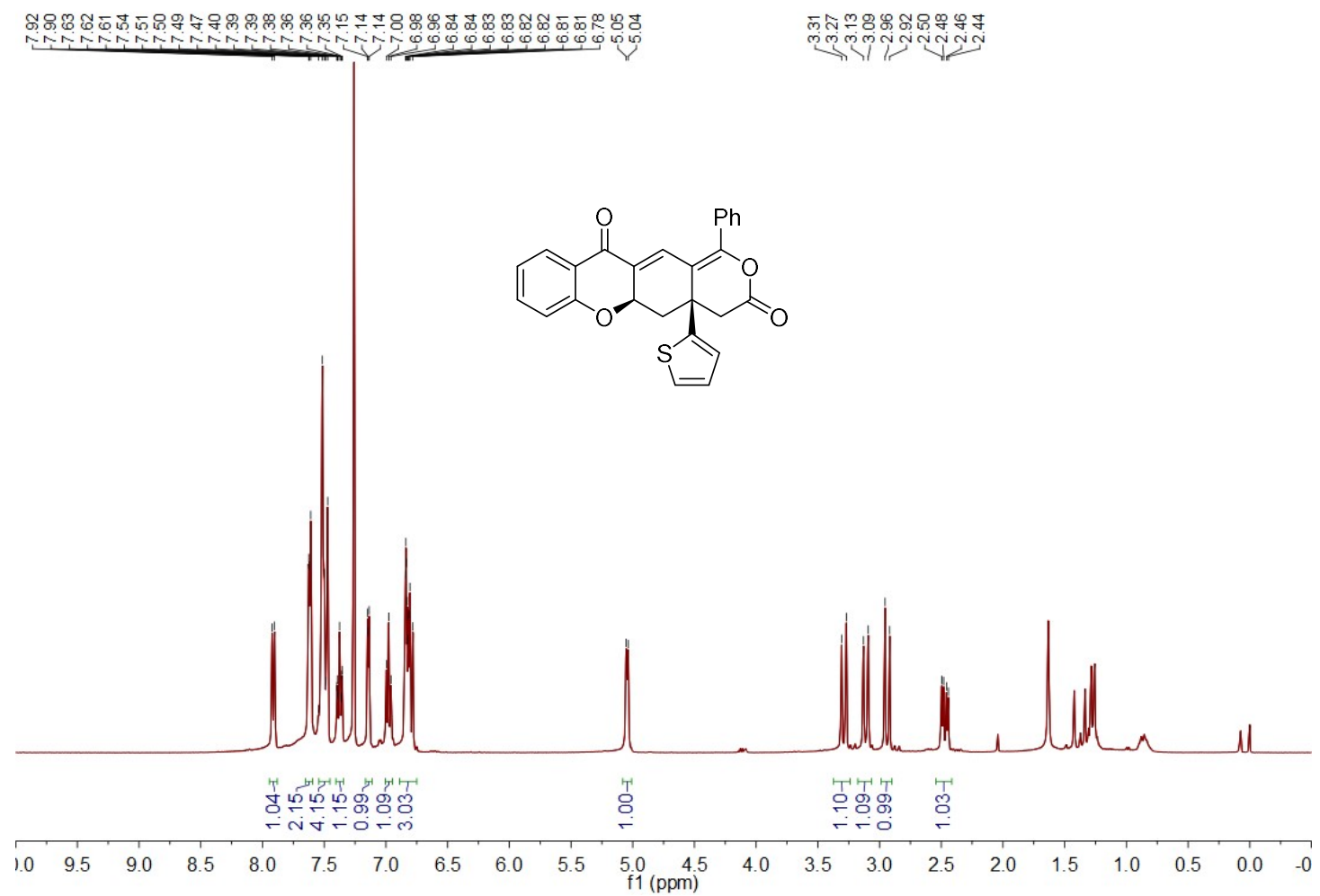

3i' ${ }^{13} \mathrm{C}$ NMR (101 MHz, $\left.\mathrm{CDCl}_{3}\right)$

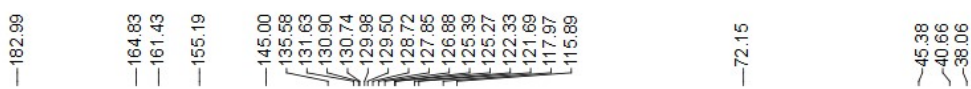
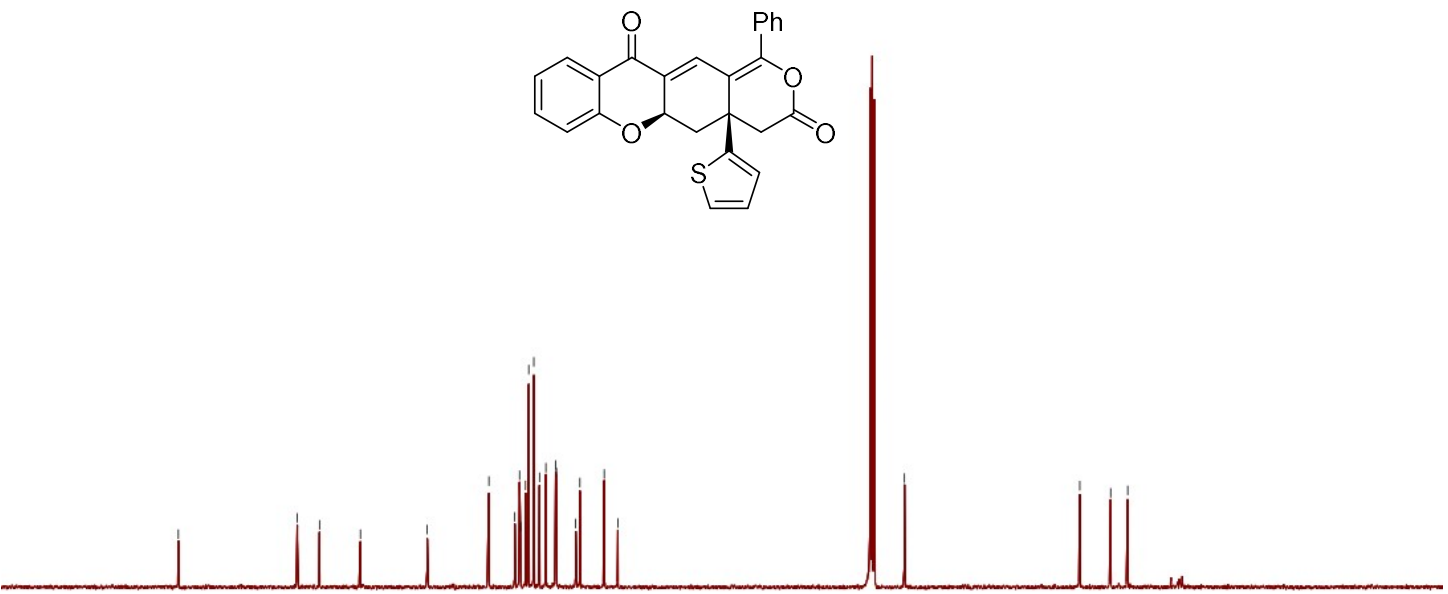

\begin{tabular}{|llllllllllllllllllllllll}
\hline 0 & 200 & 190 & 180 & 170 & 160 & 150 & 140 & 130 & 120 & 110 & 100 & 90 & 80 & 70 & 60 & 50 & 40 & 30 & 20 & 10 & 0 & -1
\end{tabular} 
3j ${ }^{1} \mathbf{H}$ NMR $\left(600 \mathrm{MHz}, \mathrm{CDCl}_{3}\right)$

8.

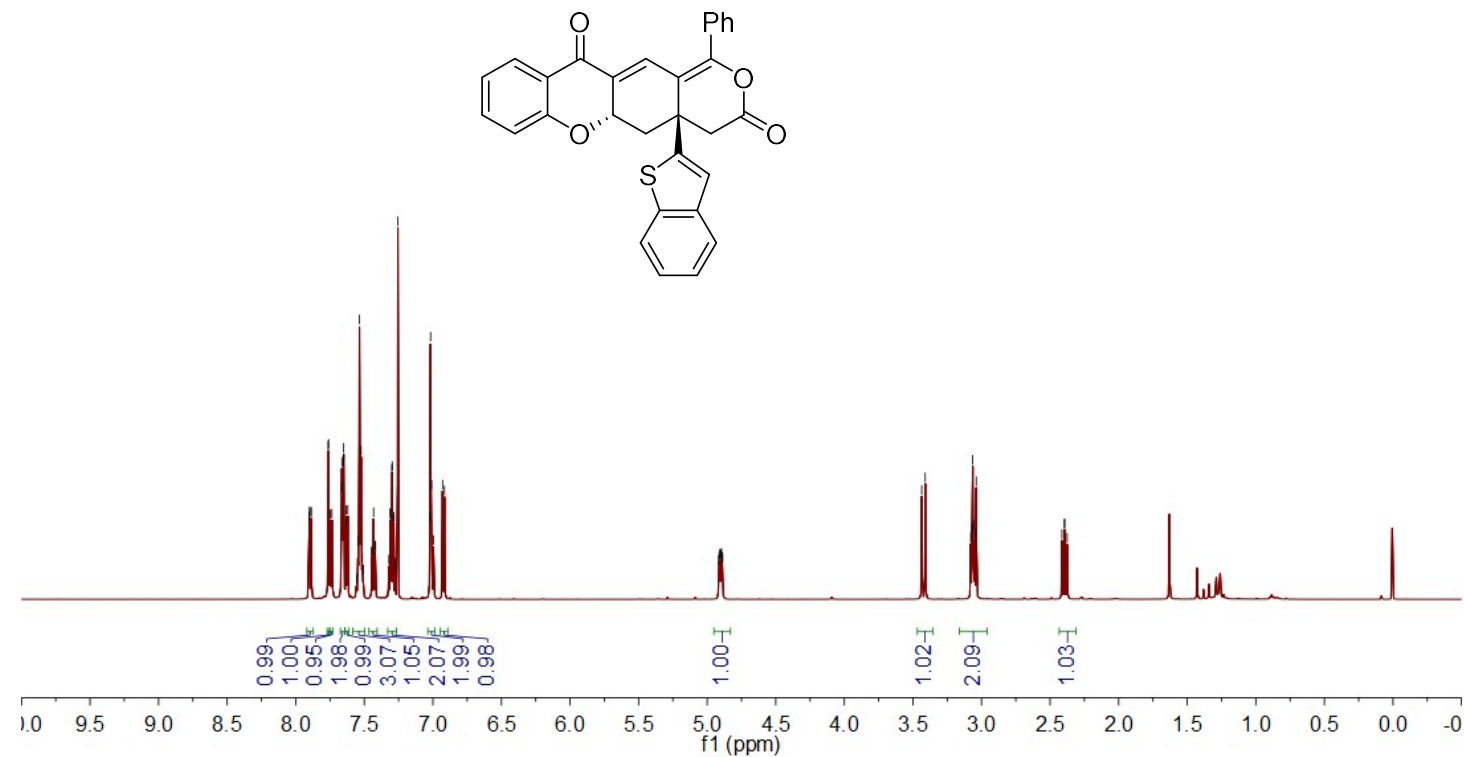

3j ${ }^{13} \mathrm{C}$ NMR (151 MHz, $\left.\mathrm{CDCl}_{3}\right)$

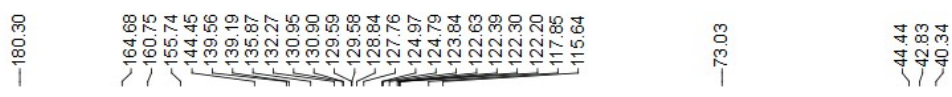

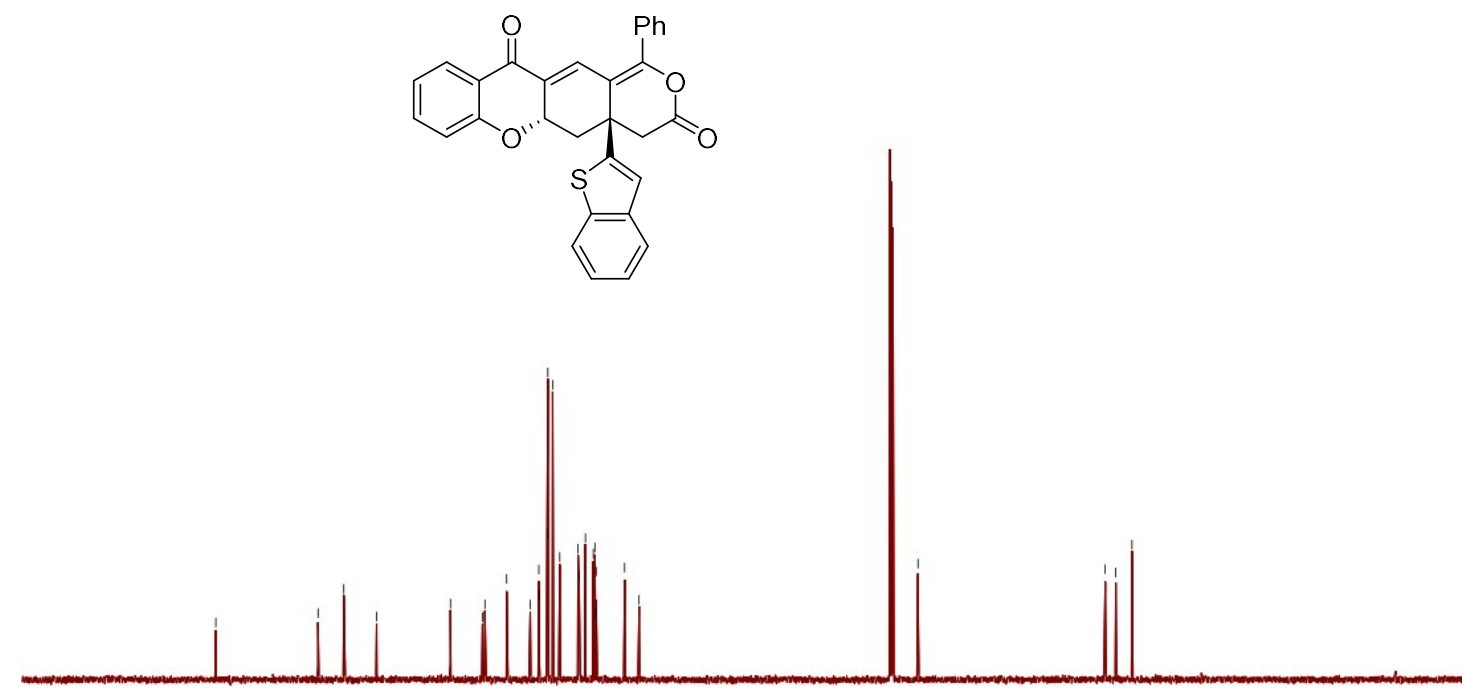

$\begin{array}{lllllllllllllllllllllllllll}10 & 200 & 190 & 180 & 170 & 160 & 150 & 140 & 130 & 120 & 110 & 100 & 90 & 80 & 70 & 60 & 50 & 40 & 30 & 20 & 10 & 0 & -1\end{array}$ 
3k ${ }^{1} \mathbf{H}$ NMR $\left(400 \mathrm{MHz}, \mathrm{CDCl}_{3}\right)$

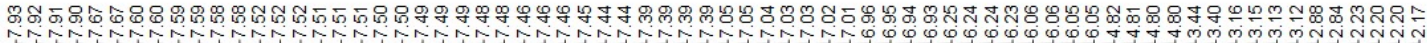

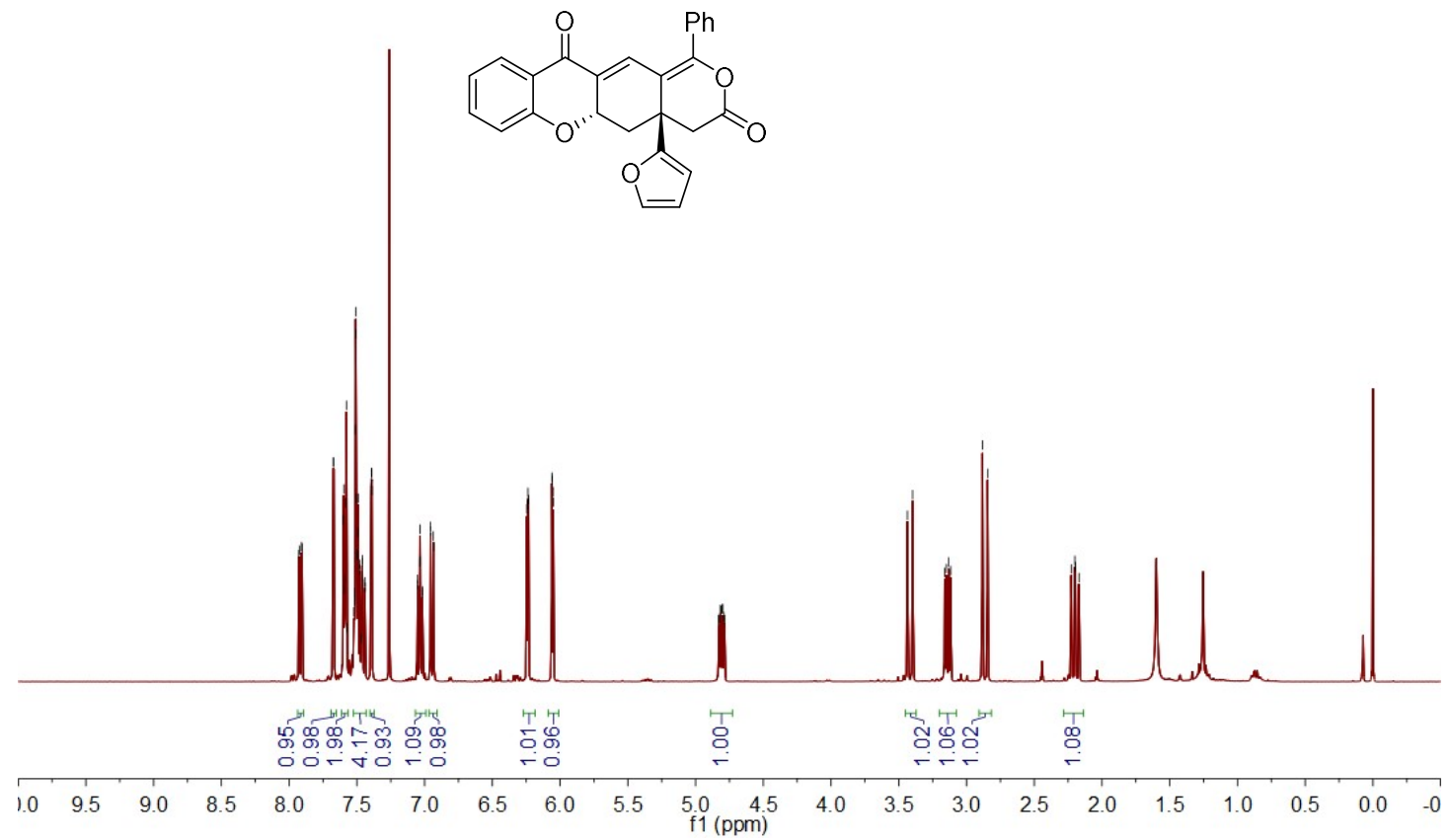

3k ${ }^{13} \mathbf{C}$ NMR $\left(101 \mathrm{MHz}, \mathrm{CDCl}_{3}\right)$
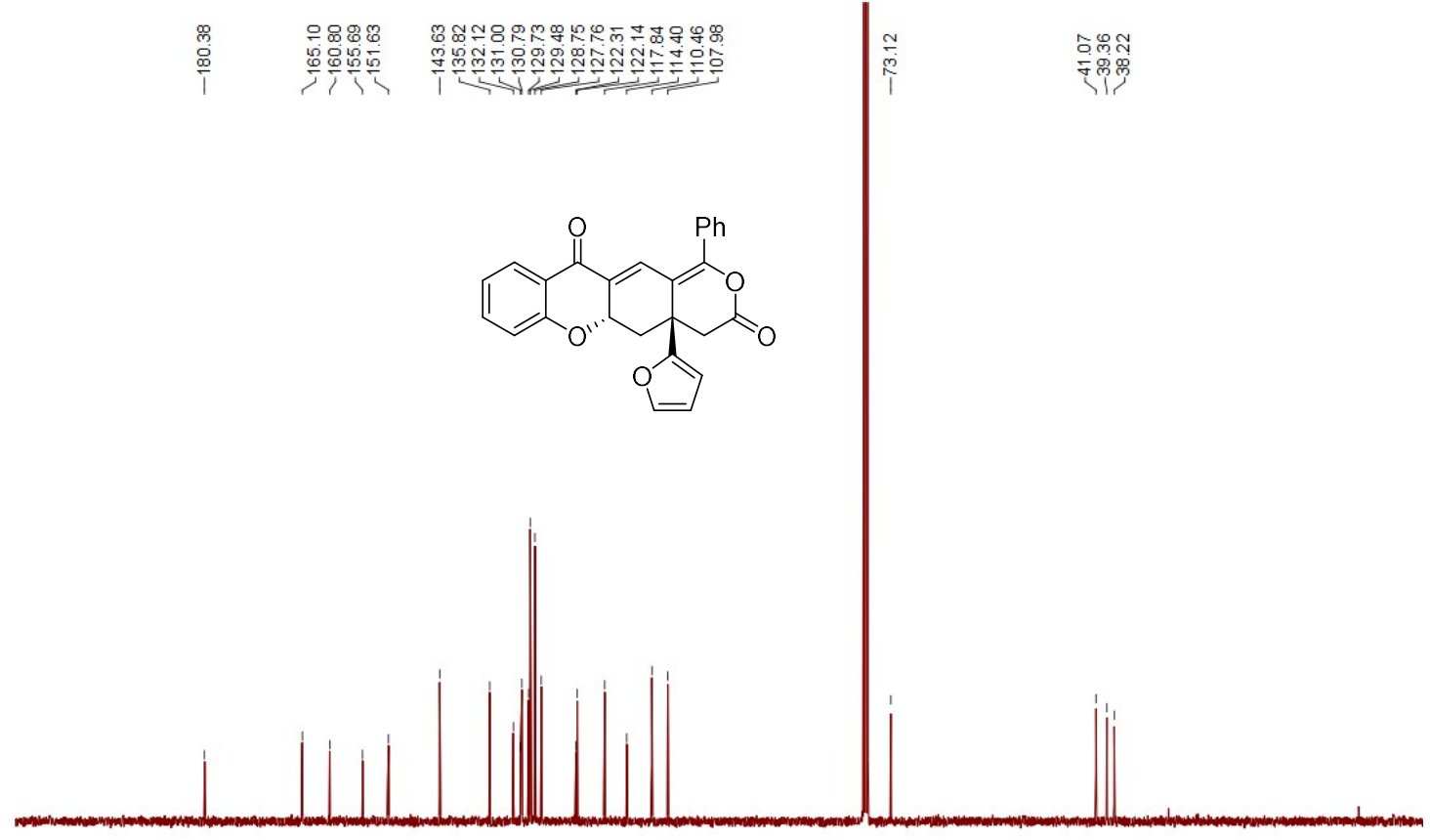

$\begin{array}{lllllllllllllllllllllllllllll}10 & 200 & 190 & 180 & 170 & 160 & 150 & 140 & 130 & 120 & 110 & 100 & 90 & 80 & 70 & 60 & 50 & 40 & 30 & 20 & 10 & 0 & -1\end{array}$ 
3k' ${ }^{1}$ H NMR $\left(400 \mathrm{MHz}, \mathrm{CDCl}_{3}\right)$

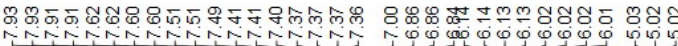

ஜூలై

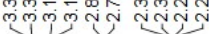<smiles>O=C1C[C@@]2(c3ccco3)C[C@H]3Oc4ccccc4C(=O)C3=CC2=C(c2ccccc2)O1</smiles>

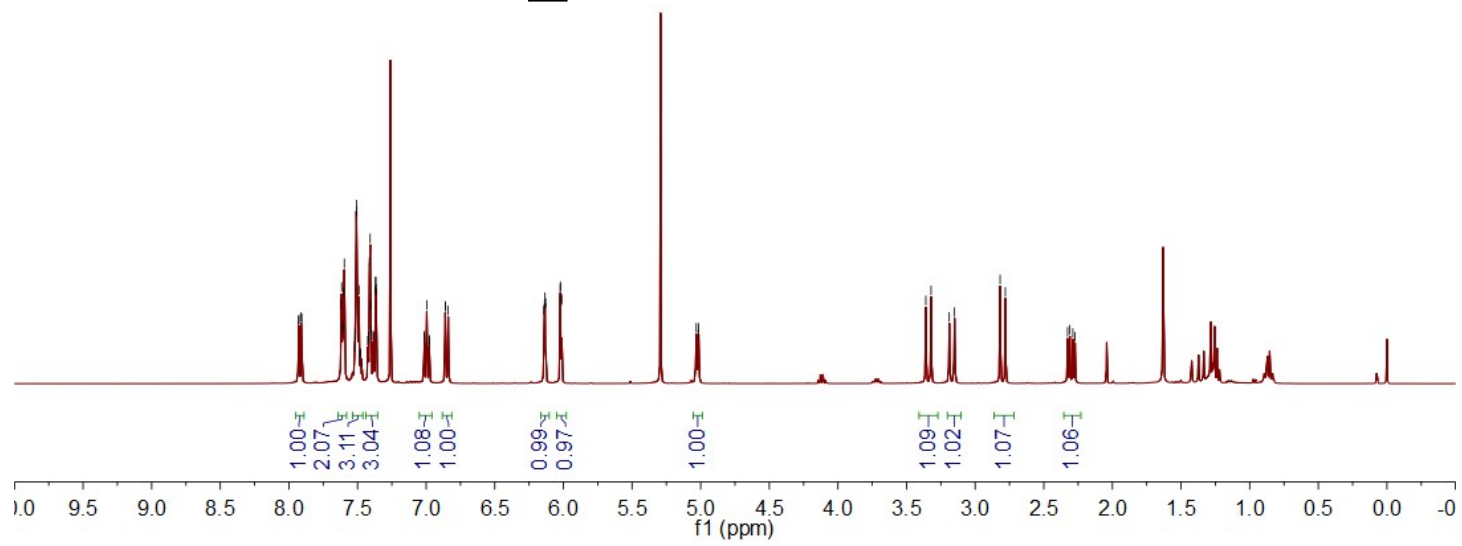

3k' ${ }^{13} \mathrm{C}$ NMR $\left(101 \mathrm{MHz}, \mathrm{CDCl}_{3}\right)$

\begin{tabular}{|c|c|c|}
\hline 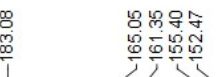 & 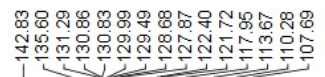 & $\stackrel{\text { T. }}{=}$ \\
\hline
\end{tabular}<smiles>O=C1C[C@]2(c3ccco3)C[C@H]3Oc4ccccc4C(=O)C3=CC2=C(c2ccccc2)O1</smiles>

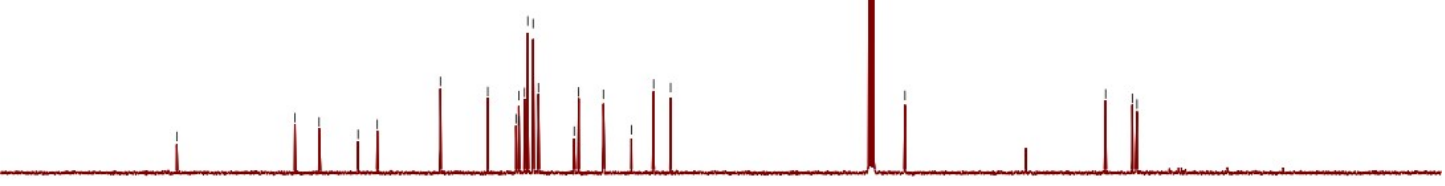

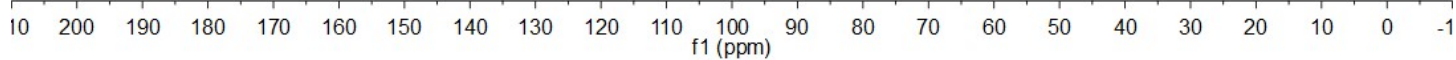


3l' ${ }^{1} \mathbf{H}$ NMR $\left(400 \mathrm{MHz}, \mathrm{CDCl}_{3}\right)$

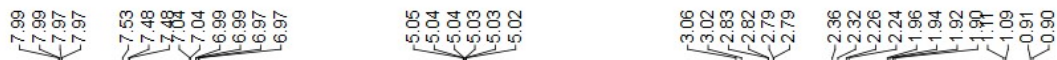<smiles>CC(C)C12CC(=O)OC(c3ccccc3)=C1C=C1C(=O)c3ccccc3OC1C2</smiles>

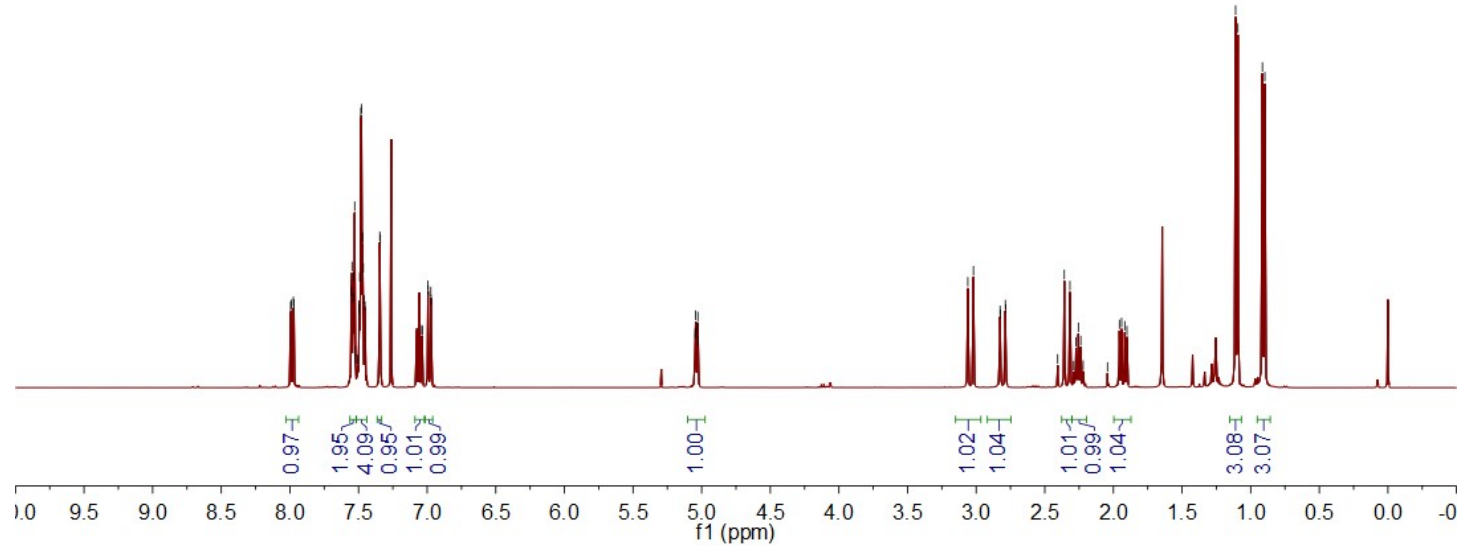

3' ${ }^{13} \mathrm{C}$ NMR (101 MHz, $\left.\mathrm{CDCl}_{3}\right)$

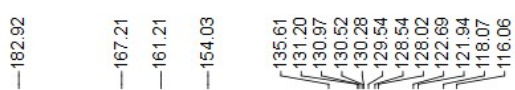<smiles>CC(C)C12CC(=O)OC(c3ccccc3)=C1C=C1C(=O)c3ccccc3O[C@H]1C2</smiles>

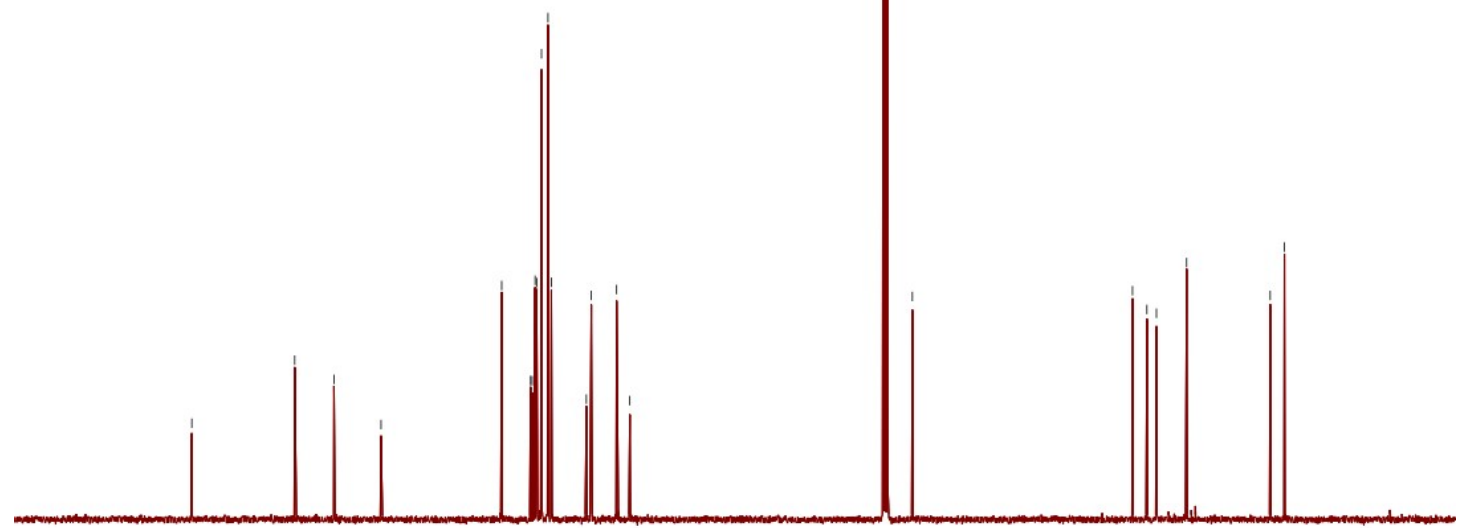

$\begin{array}{lllllllllllllllllllllllll}10 & 200 & 190 & 180 & 170 & 160 & 150 & 140 & 130 & 120 & 110 & 100 & 90 & 80 & 70 & 60 & 50 & 40 & 30 & 20 & 10 & 0 & -1\end{array}$ 
$3 \mathrm{~m}^{1} \mathrm{H}$ NMR $\left(400 \mathrm{MHz}, \mathrm{CDCl}_{3}\right)$

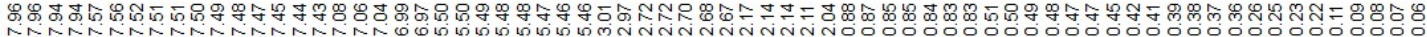

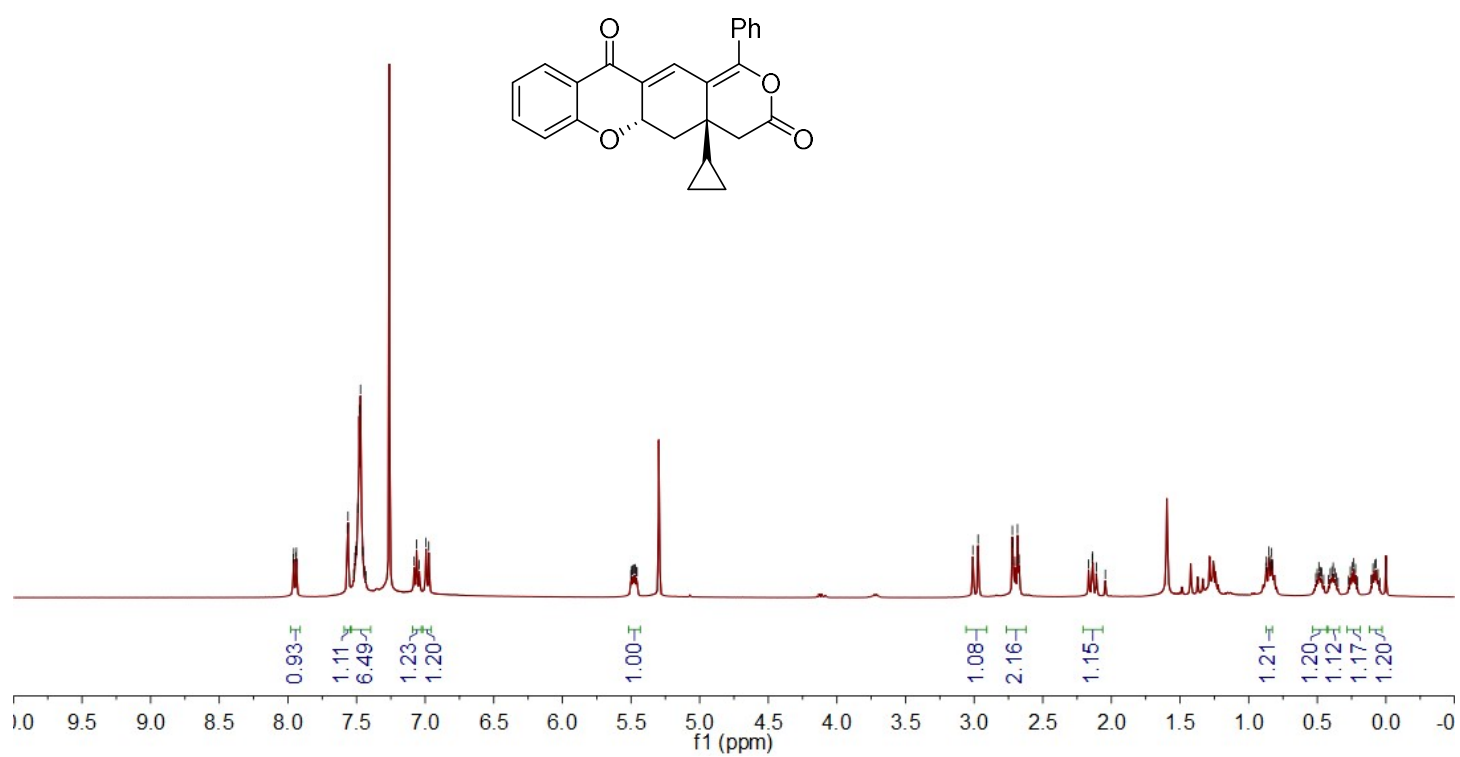

3m ${ }^{13} \mathrm{C}$ NMR $\left(101 \mathrm{MHz}, \mathrm{CDCl}_{3}\right)$<smiles>C=C1CC2(CC2)CC(=Cc2coc3ccccc3c2=O)C=C1c1ccccc1</smiles>

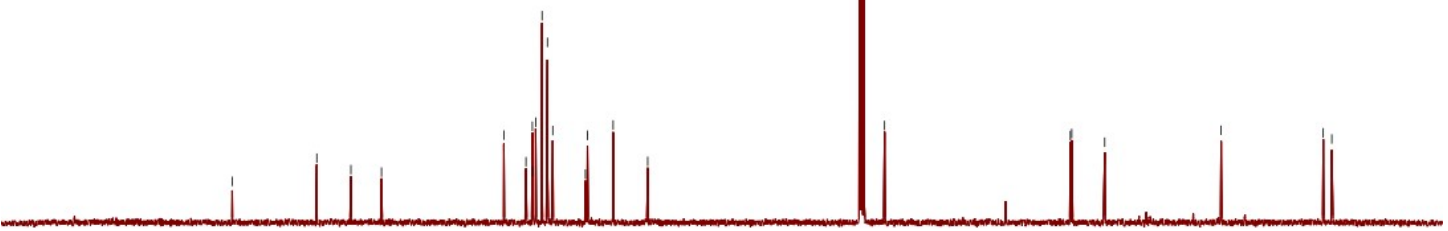

$\begin{array}{lllllllllllllllllllllll}210 & 200 & 190 & 180 & 170 & 160 & 150 & 140 & 130 & 120 & 110 & \begin{array}{c}100 \\ \mathrm{f} 1(\mathrm{ppm})\end{array} & 90 & 80 & 70 & 60 & 50 & 40 & 30 & 20 & 10 & 0 & -10\end{array}$ 
3m' ${ }^{1} \mathrm{H}$ NMR (400 MHz, $\left.\mathrm{CDCl}_{3}\right)$

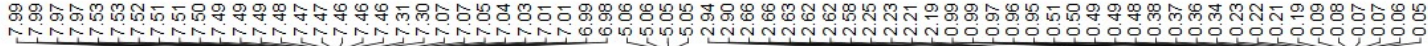<smiles>O=C1CC2(C3CC3)C[C@H]3Oc4ccccc4C(=O)C3=CC2=C1c1ccccc1</smiles>

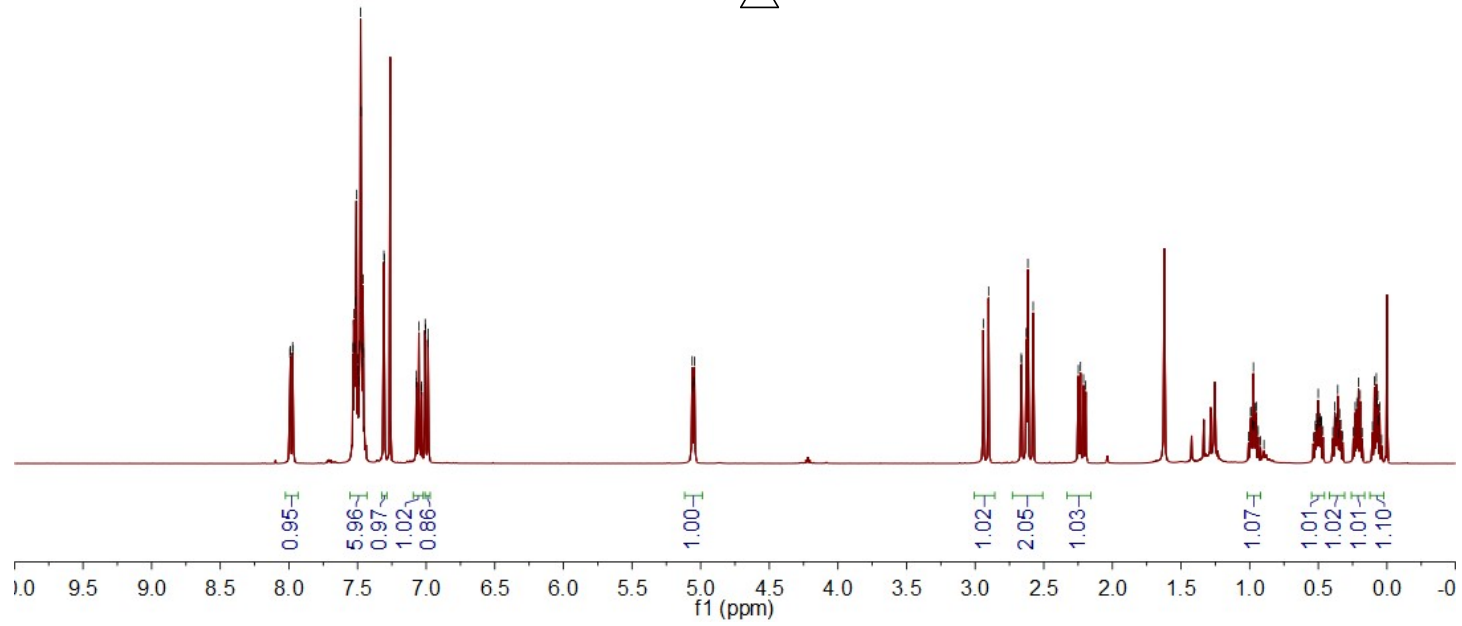

3m' ${ }^{13}$ C NMR (101 MHz, $\left.\mathrm{CDCl}_{3}\right)$

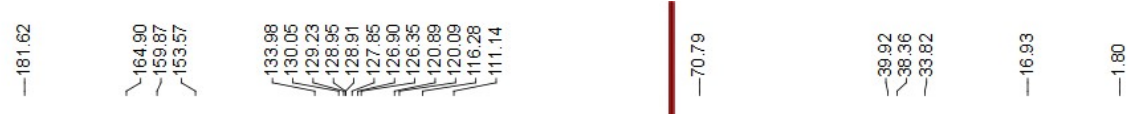<smiles>O=C1CC2(C3CC3)C[C@H]3Oc4ccccc4C(=O)C3=CC2=C1c1ccccc1</smiles>

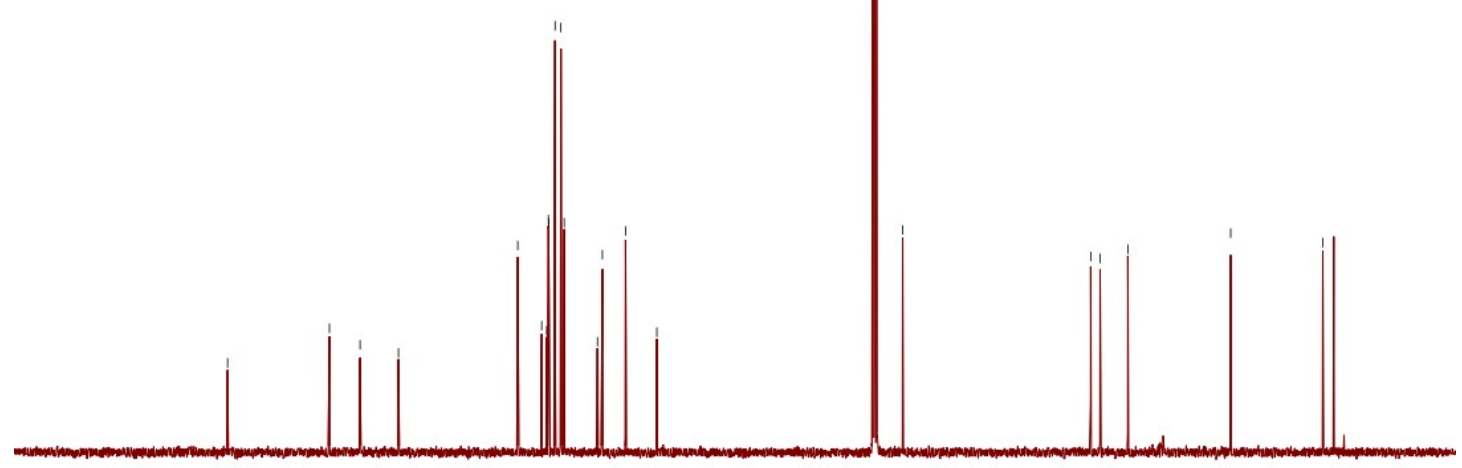

$\begin{array}{lllllllllllllllllllllll}210 & 200 & 190 & 180 & 170 & 160 & 150 & 140 & 130 & 120 & 110 & 100 & 90 & 80 & 70 & 60 & 50 & 40 & 30 & 20 & 10 & 0 & -10\end{array}$ 
3n ${ }^{1} \mathbf{H}$ NMR $\left(600 \mathrm{MHz}, \mathrm{CDCl}_{3}\right)$

б.<smiles>O=C1CC2(c3ccccc3)C[C@H]3Oc4ccc(F)cc4C(=O)C3=CC2=C(c2ccccc2)O1</smiles>

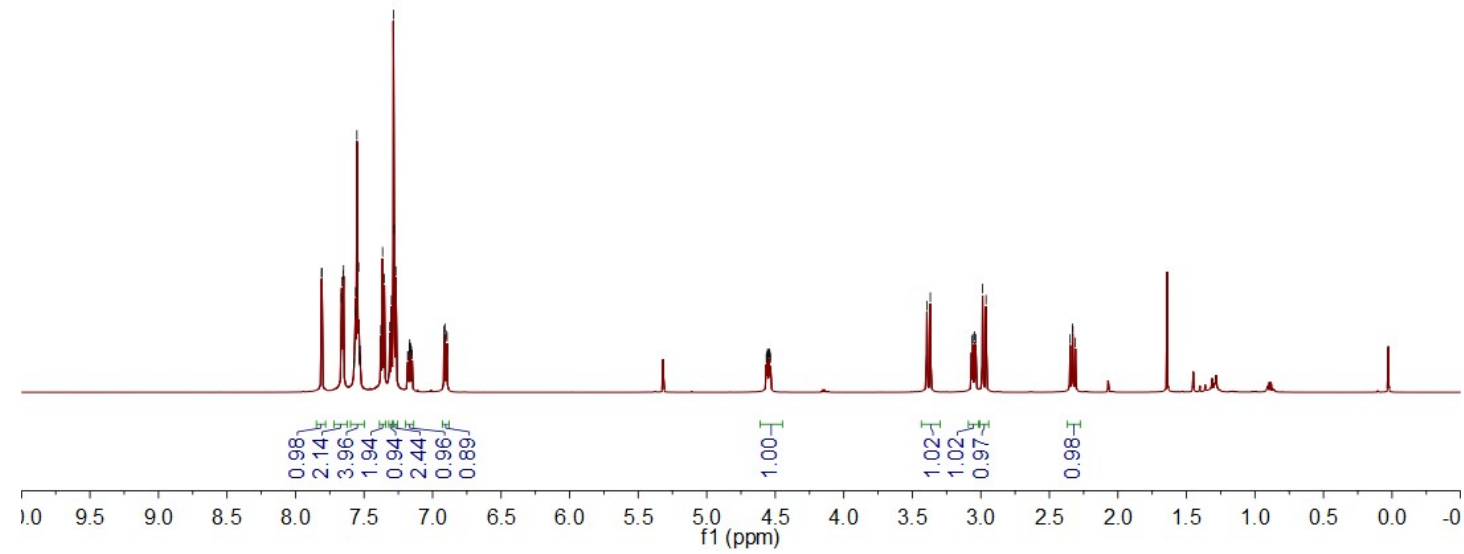

3n ${ }^{13} \mathrm{C}$ NMR $\left(151 \mathrm{MHz}, \mathrm{CDCl}_{3}\right)$

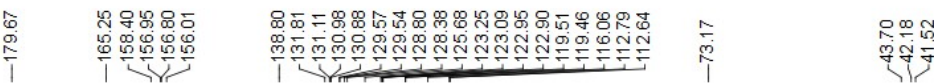<smiles>O=C1CC2(c3ccccc3)C[C@H]3Oc4ccc(F)cc4C(=O)C3=CC2=C(c2ccccc2)O1</smiles>

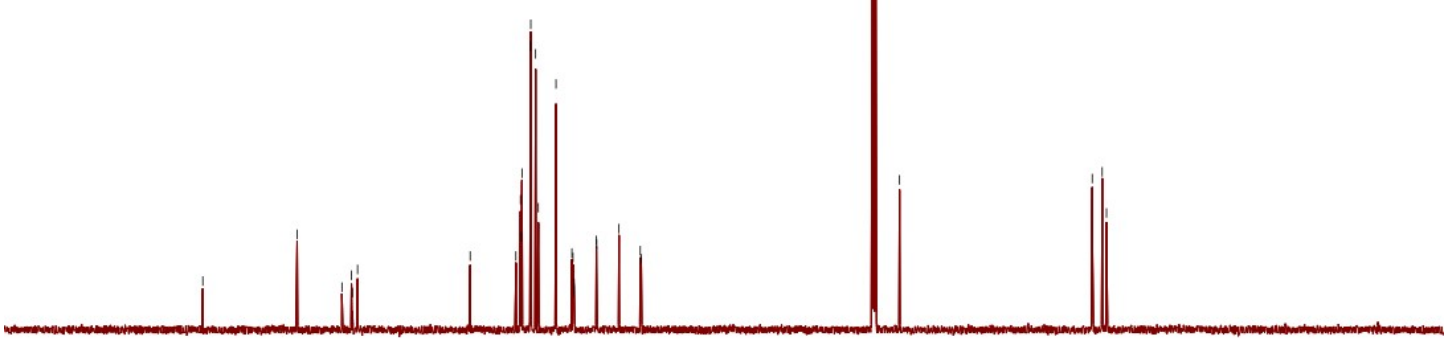

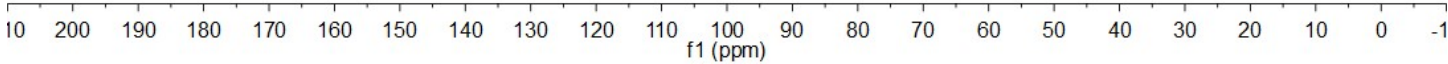


3n ${ }^{19} \mathrm{~F}$ NMR $\left(471 \mathrm{MHz}, \mathrm{CDCl}_{3}\right)$
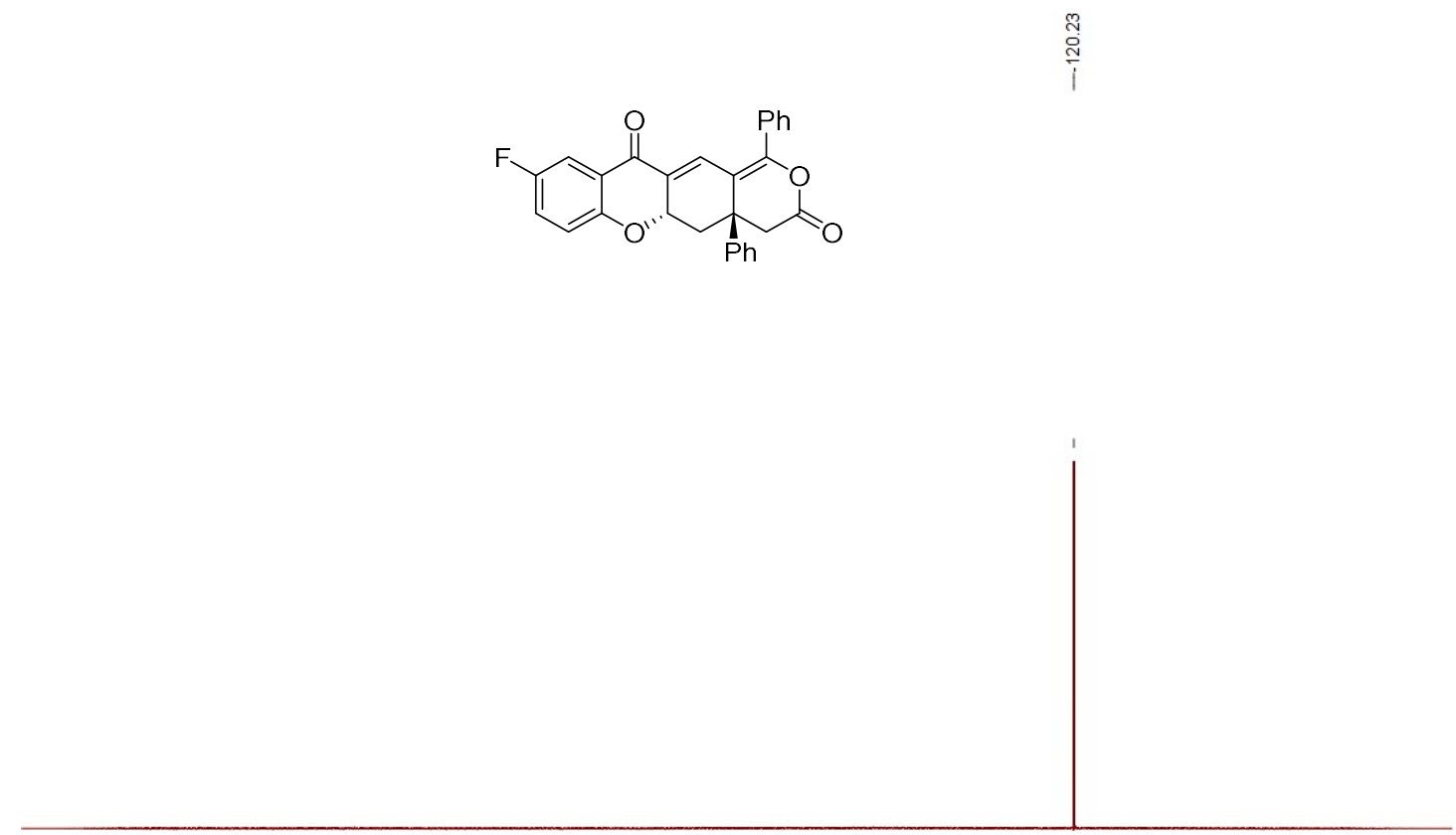

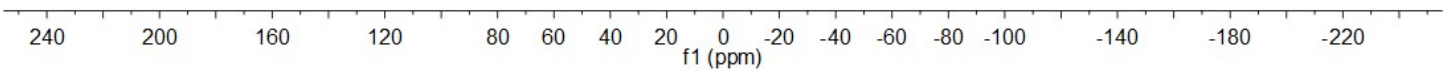

3n' ${ }^{1} \mathrm{H}$ NMR $\left(400 \mathrm{MHz}, \mathrm{CDCl}_{3}\right)$

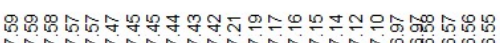

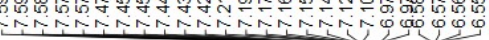<smiles></smiles>

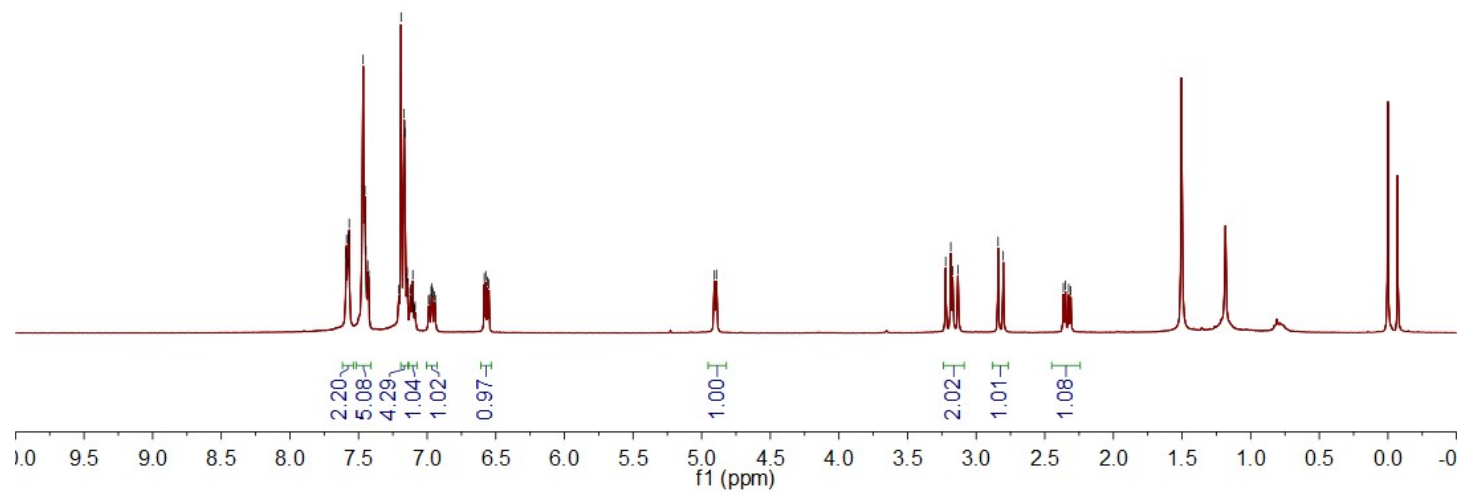


3n' ${ }^{13} \mathrm{C}$ NMR $\left(101 \mathrm{MHz}, \mathrm{CDCl}_{3}\right)$

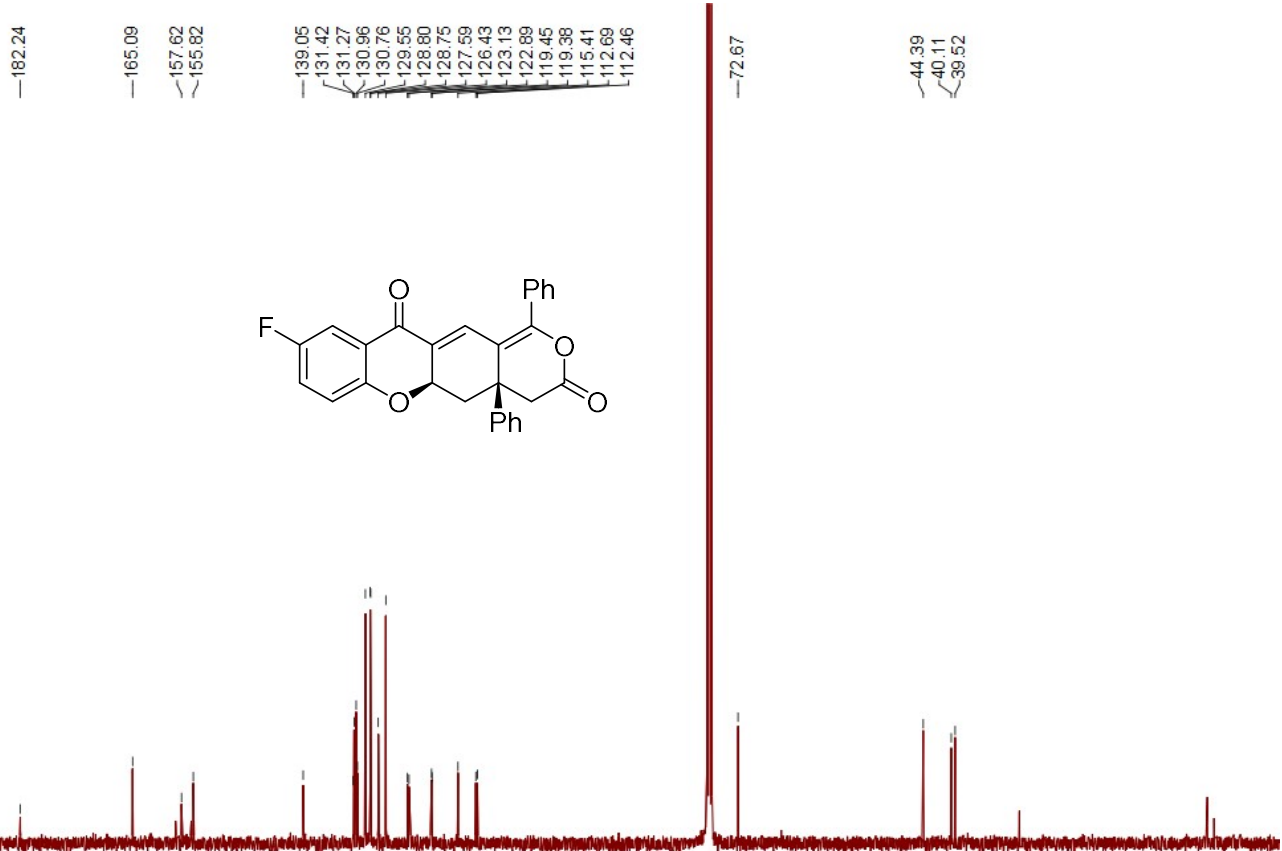

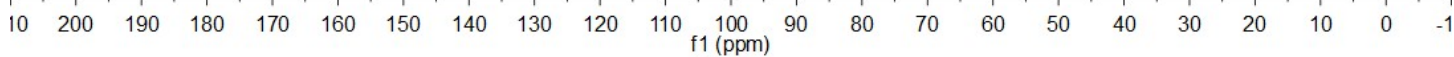

3n' ${ }^{19} \mathrm{~F}$ NMR $\left(471 \mathrm{MHz}, \mathrm{CDCl}_{3}\right)$<smiles>O=C1OC(=O)C2CC3c4ccc(F)cc4OC34C(=O)OC(c3ccccc3)=C4C=C12</smiles>

$\begin{array}{llllllllllllllllll}240 & 200 & 160 & 120 & 80 & 60 & 40 & 20 & 0 & 0 & -20 & -40 & -60 & -80 & -100 & -140 & -180 & -220\end{array}$ 
30 ${ }^{1} \mathrm{H}$ NMR $\left(600 \mathrm{MHz}, \mathrm{CDCl}_{3}\right)$

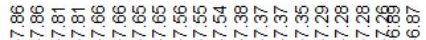

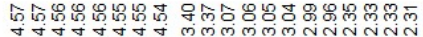

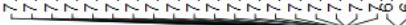<smiles>O=C1C[C@H](c2ccccc2)C2COc3ccc(Cl)cc3C(=O)C2=C1c1ccccc1</smiles>

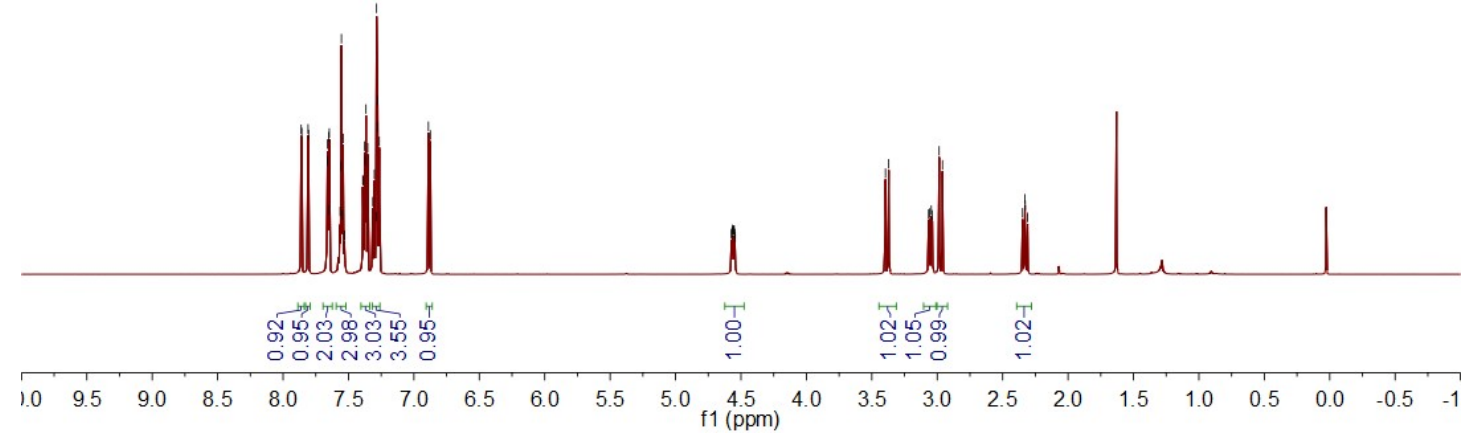

3o ${ }^{13} \mathrm{C}$ NMR $\left(151 \mathrm{MHz}, \mathrm{CDCl}_{3}\right)$

- $\quad \overline{4}=\frac{1}{2} \quad$ क

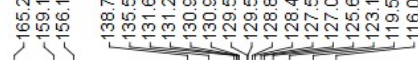<smiles>O=C1CC2(c3ccccc3)C[C@H]3Oc4ccc(Cl)cc4C(=O)C3=CC2=C(c2ccccc2)O1</smiles>

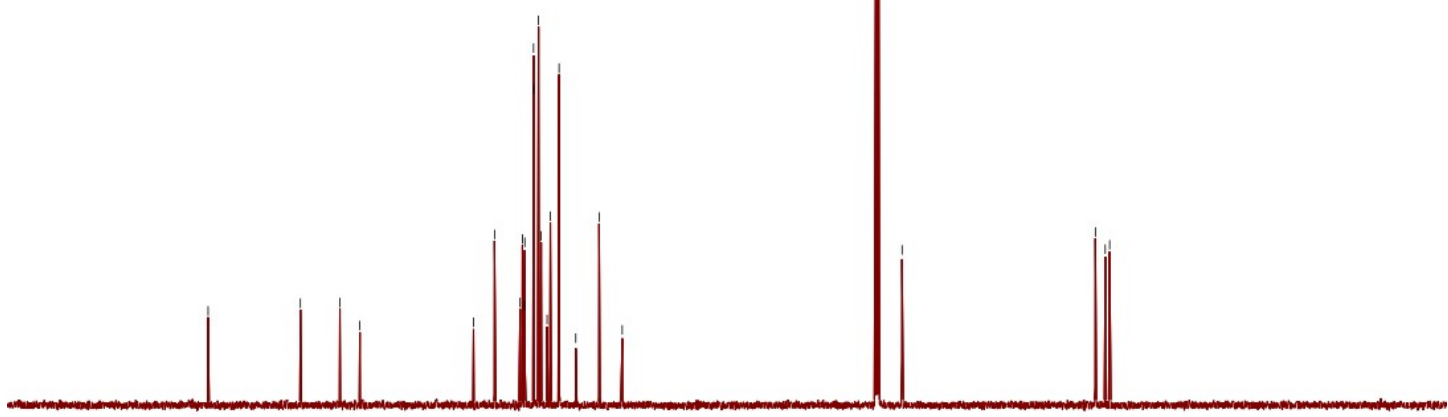

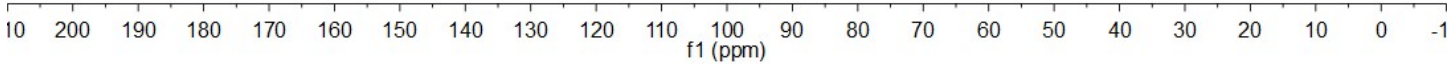


3o' ${ }^{1} \mathrm{H}$ NMR $\left(400 \mathrm{MHz}, \mathrm{CDCl}_{3}\right)$

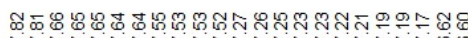

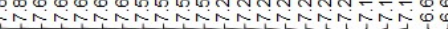

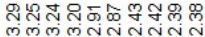

momenannina

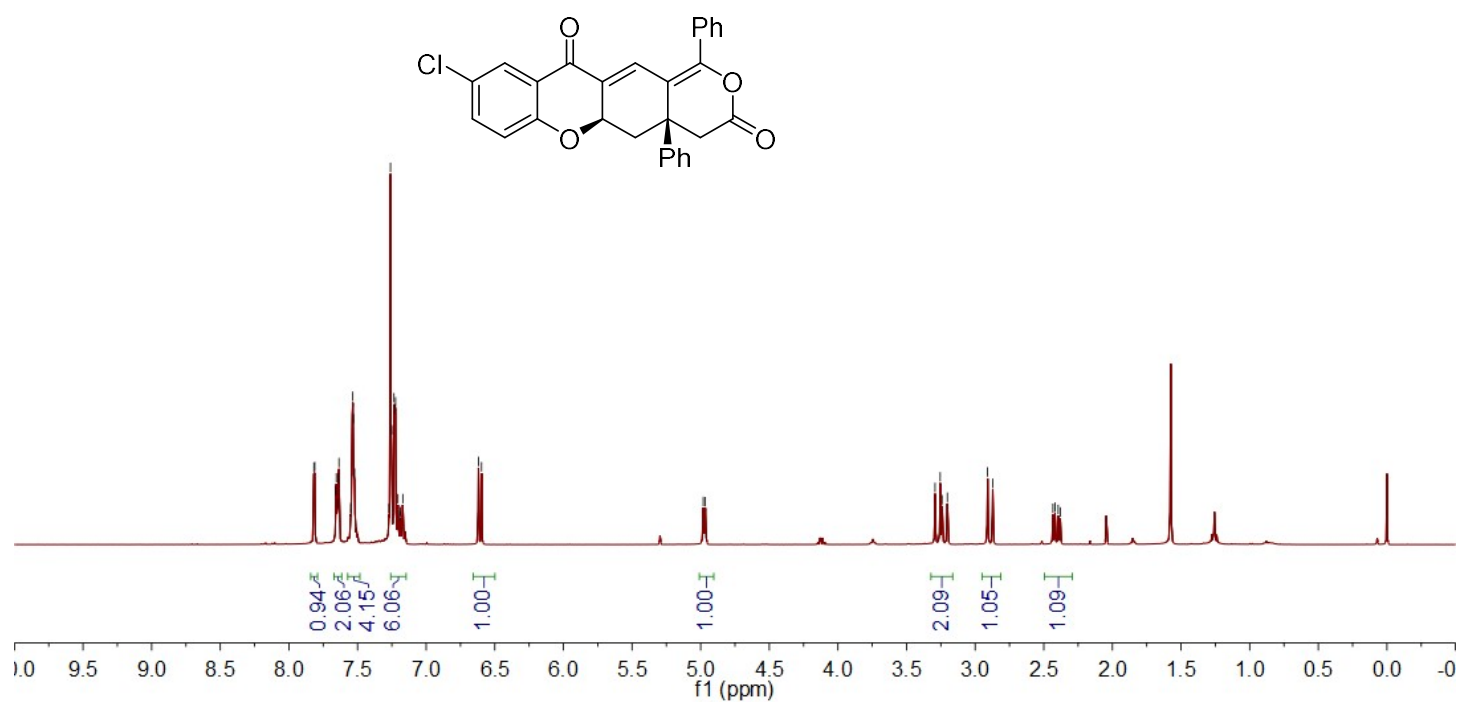

3o'13 C NMR (101 MHz, $\left.\mathrm{CDCl}_{3}\right)$

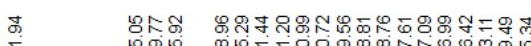

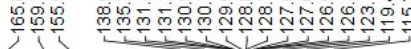

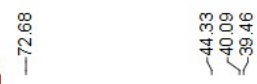<smiles>O=C1OC(Oc2ccc(Cl)cc2)=CC2CC3C(=O)OC(c4ccccc4)=C3C=C12</smiles>

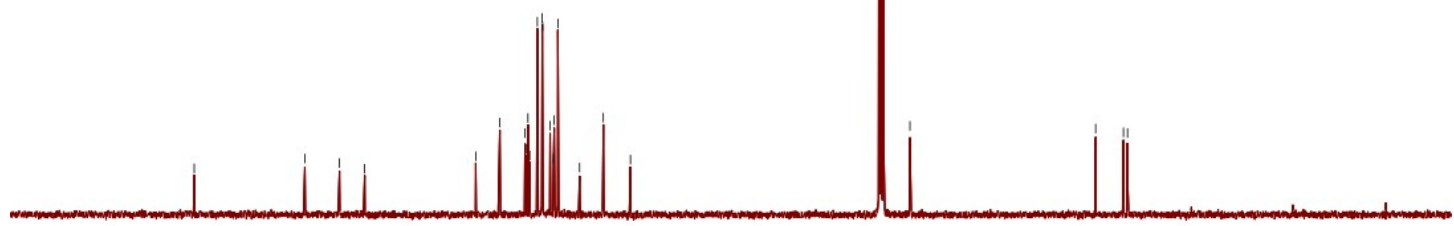

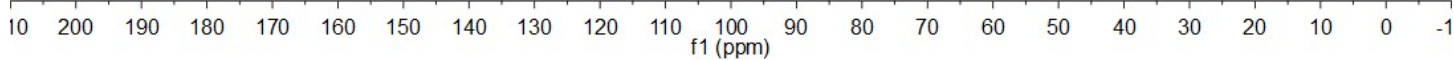


3p ${ }^{1} \mathrm{H}$ NMR $\left(600 \mathrm{MHz}, \mathrm{CDCl}_{3}\right)$

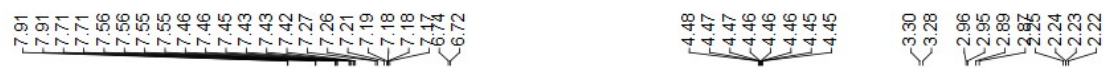

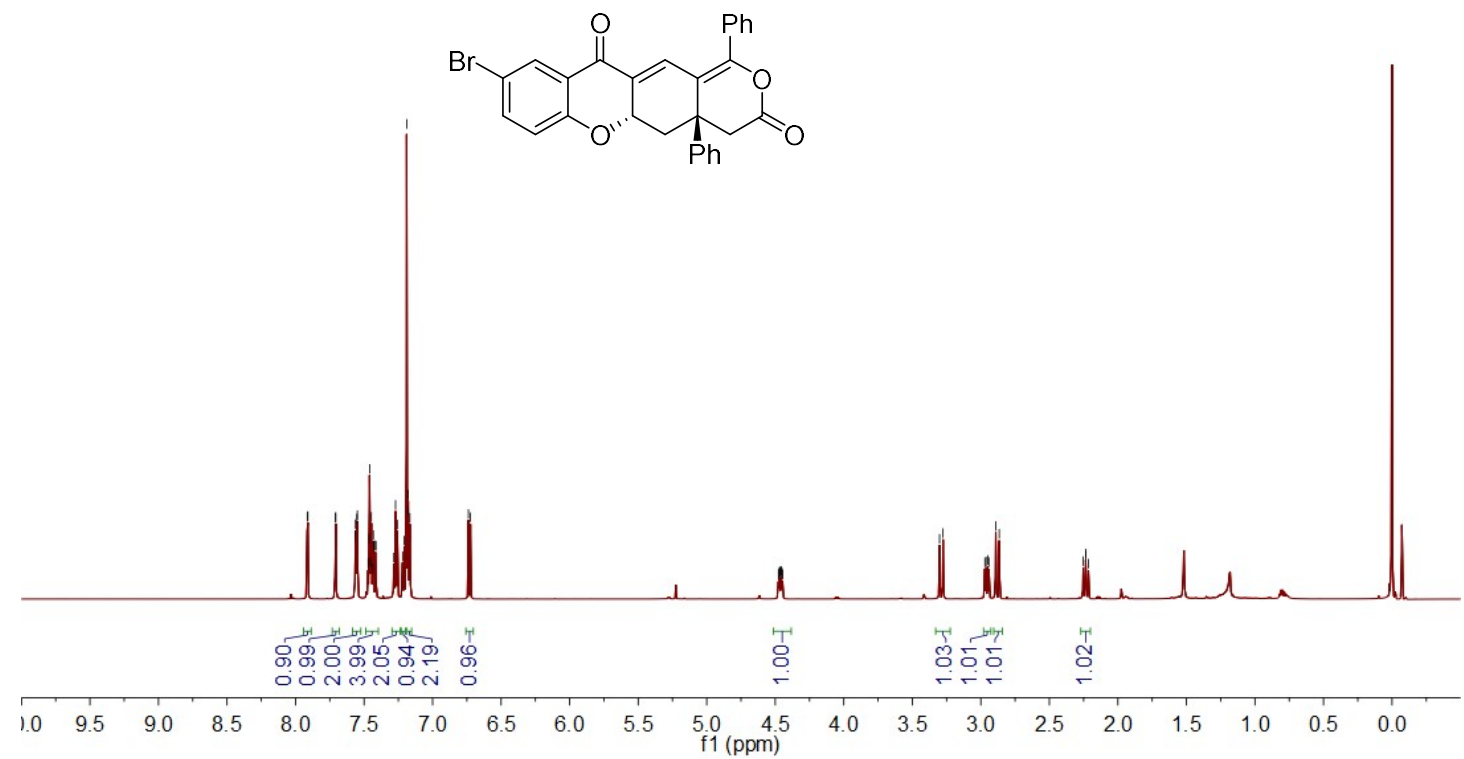

3P ${ }^{13} \mathrm{C}$ NMR $\left(151 \mathrm{MHz}, \mathrm{CDCl}_{3}\right)$

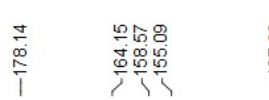
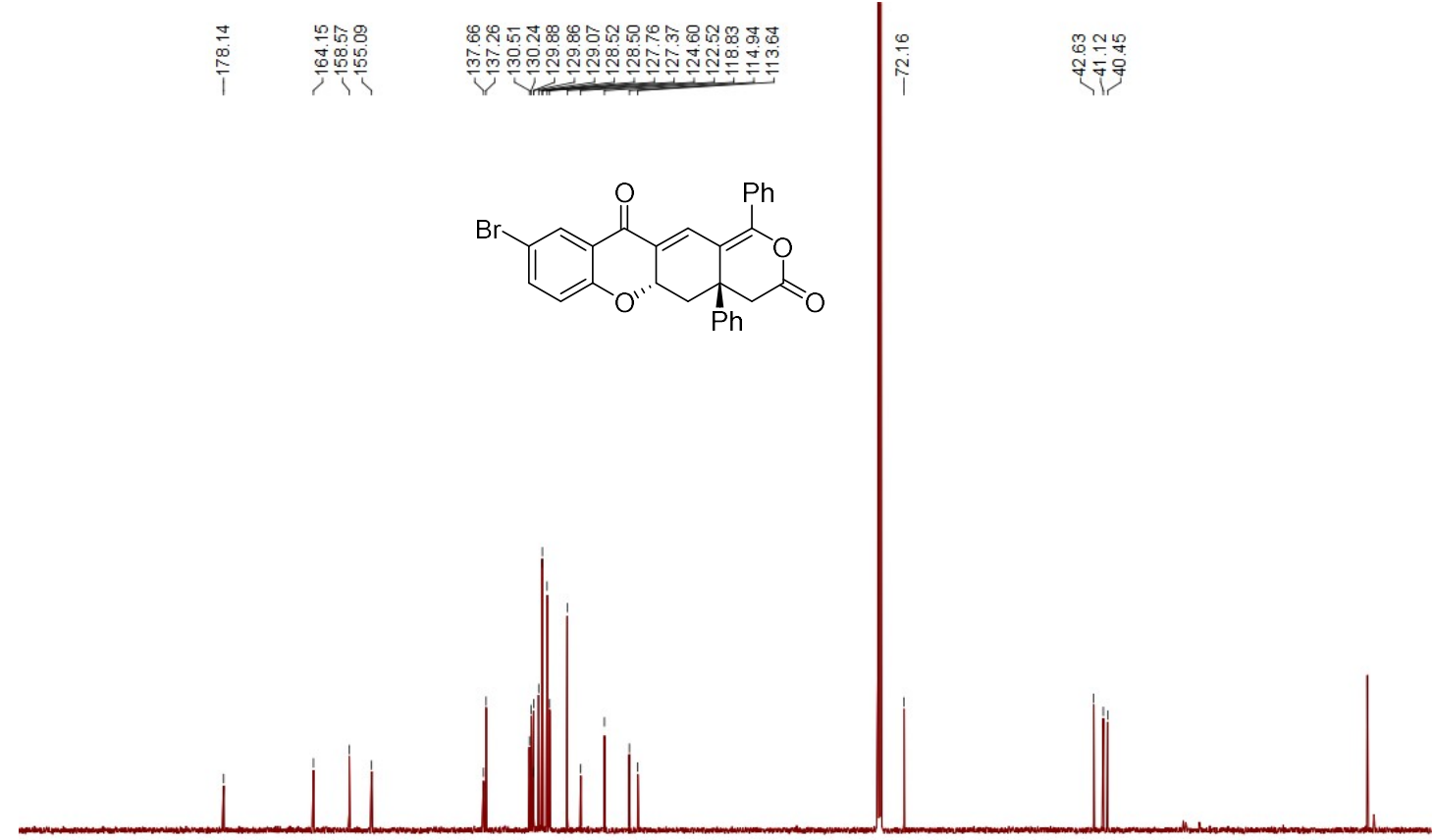

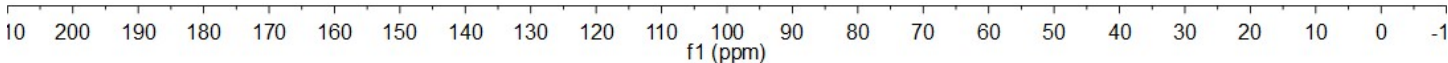


3p' ${ }^{1} \mathrm{H}$ NMR $\left(400 \mathrm{MHz}, \mathrm{CDCl}_{3}\right)$

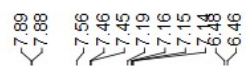
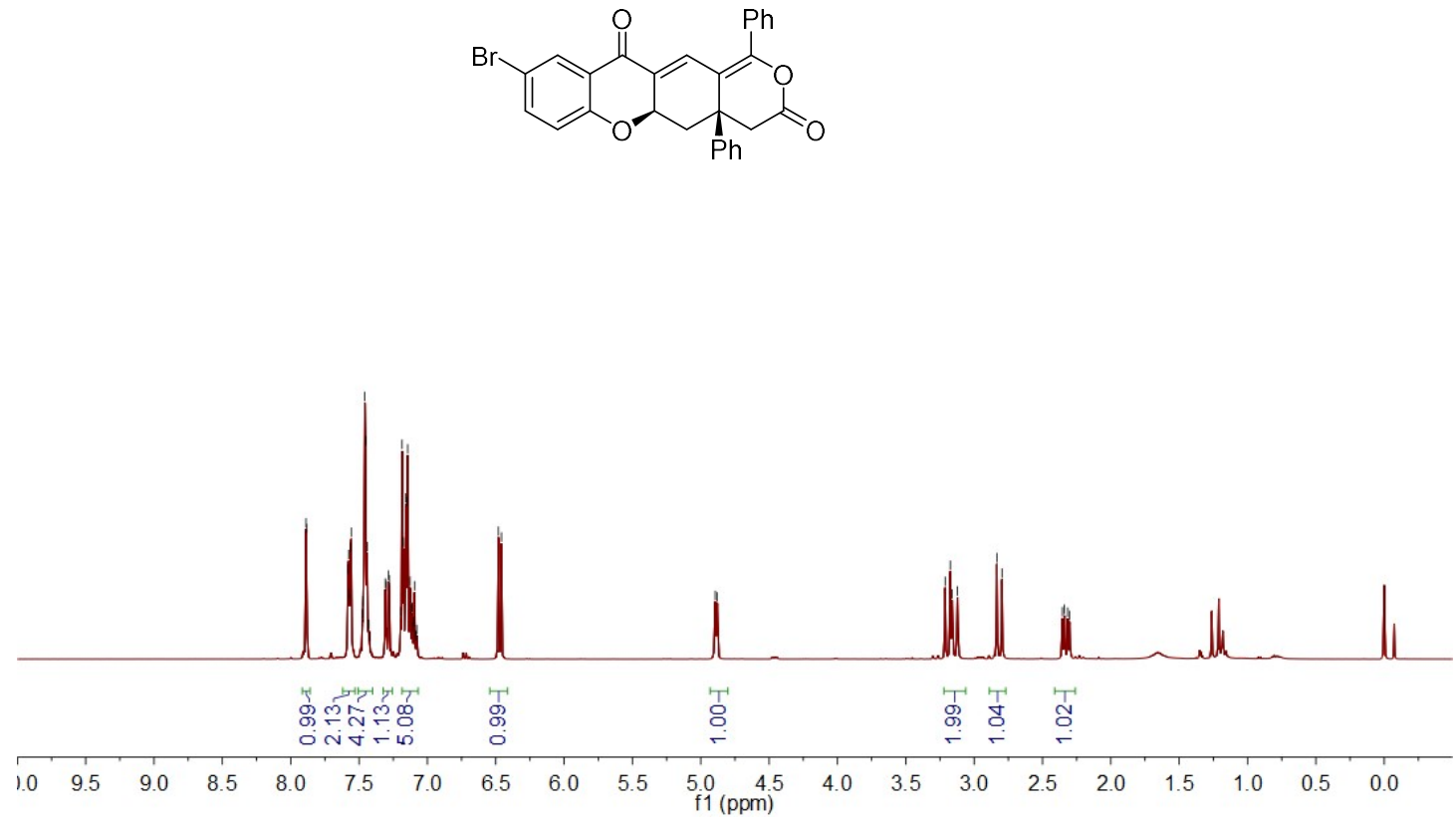

3P' ${ }^{13} \mathrm{C}$ NMR $\left(101 \mathrm{MHz}, \mathrm{CDCl}_{3}\right)$

․

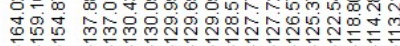<smiles></smiles>

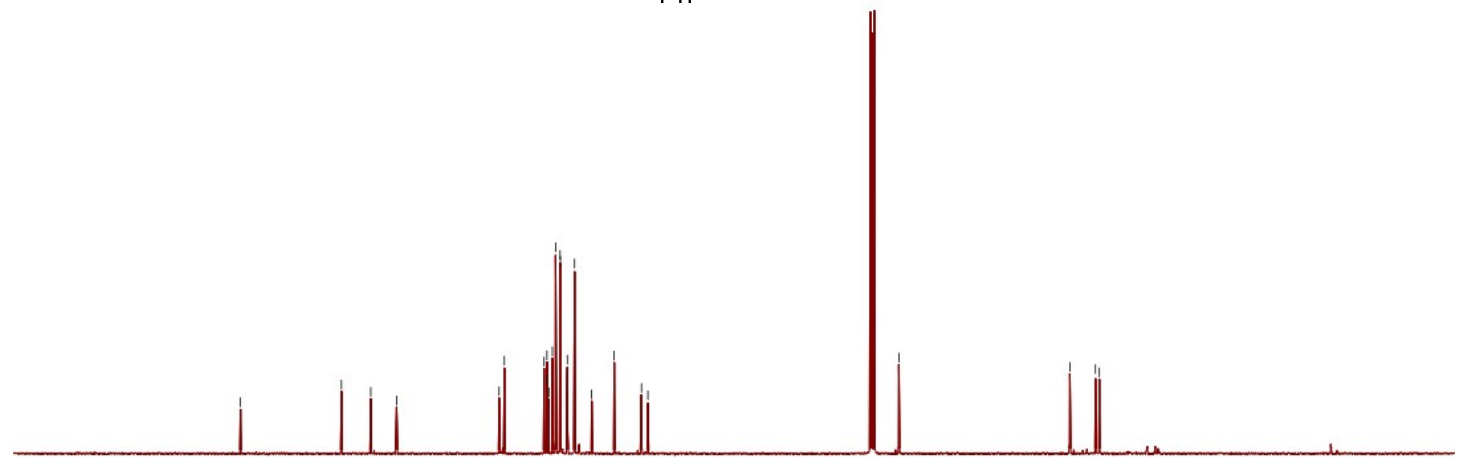

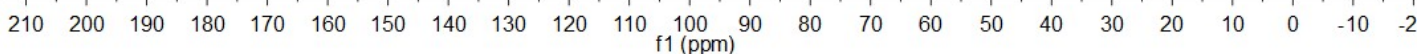


$3 \mathbf{q}^{1} \mathbf{H}$ NMR $\left(600 \mathrm{MHz}, \mathrm{CDCl}_{3}\right)$

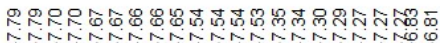

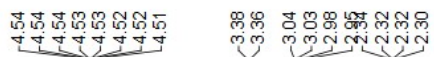<smiles>Cc1ccc2c(c1)C(=O)C1=CC3=C(c4ccccc4)OC(=O)CC3(c3ccccc3)C[C@@H]1O2</smiles>

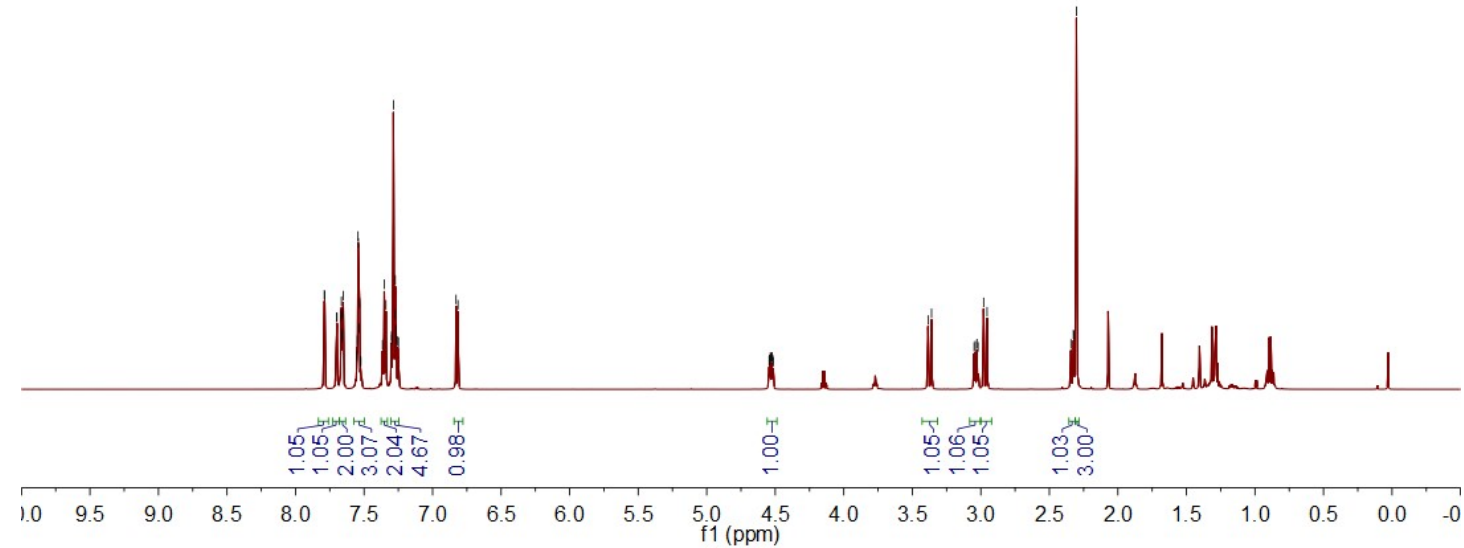

3q ${ }^{13} \mathrm{C}$ NMR $\left(151 \mathrm{MHz}, \mathrm{CDCl}_{3}\right)$

$\begin{array}{lll}1 & 1\end{array}$<smiles>Cc1ccc2c(c1)C(=O)C1=CC3=C(c4ccccc4)OC(=O)C[C@]3(c3ccccc3)C[C@H]1O2</smiles>

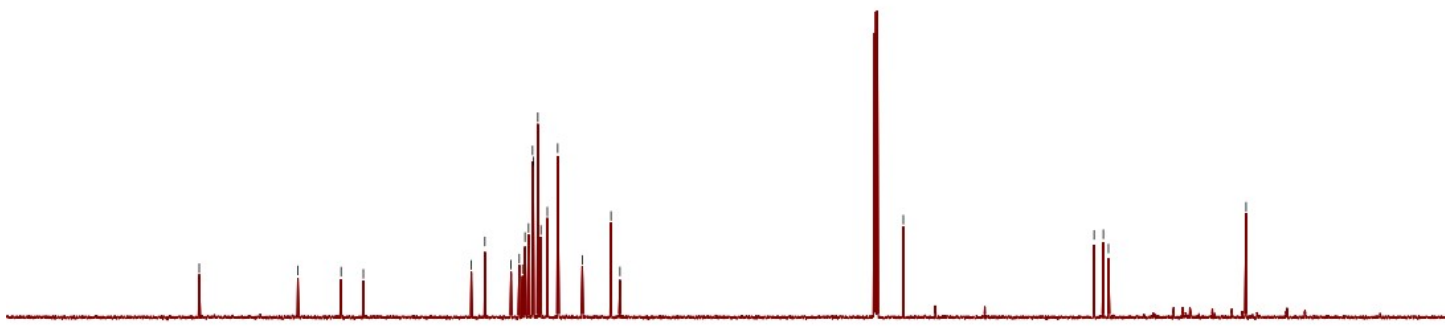

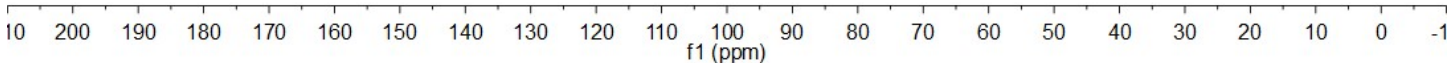


$3 \mathbf{r}^{1} \mathbf{H}$ NMR $\left(400 \mathrm{MHz}, \mathrm{CDCl}_{3}\right)$

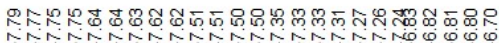

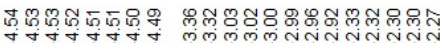

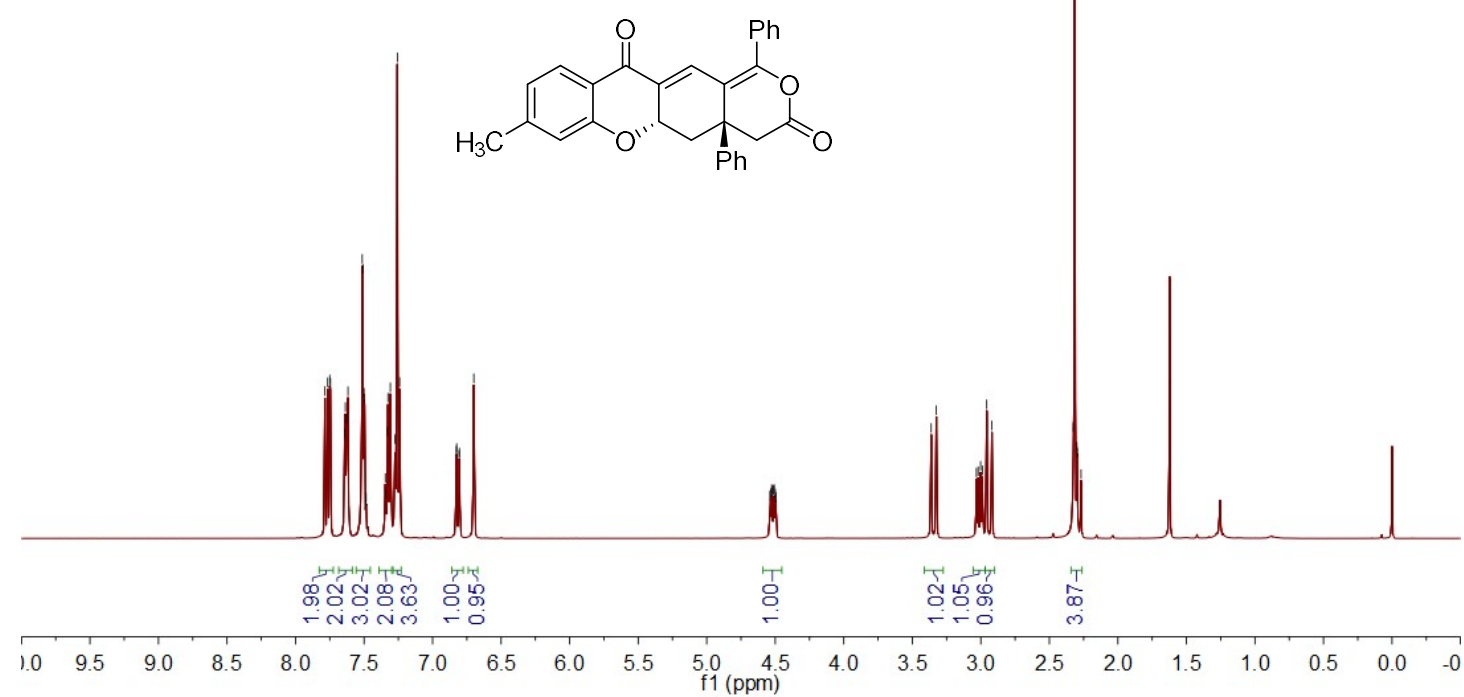

$3 \mathbf{r}^{13} \mathrm{C}$ NMR $\left(101 \mathrm{MHz}, \mathrm{CDCl}_{3}\right)$

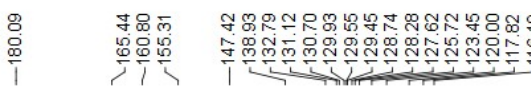

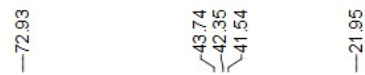<smiles>Cc1ccc2c(c1)OC1C[C@H]3CC(=O)OC(c4ccccc4)=C3C=C1C2=O</smiles>

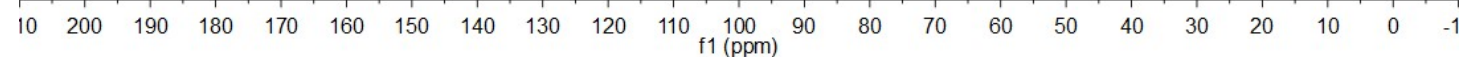


3s ${ }^{1} \mathbf{H}$ NMR $\left(600 \mathrm{MHz}, \mathrm{CDCl}_{3}\right)$<smiles>COc1ccc2c(c1)O[C]C1CC(C=C=O)(c3ccccc3)CC(=O)OC1=C2c1ccccc1</smiles>

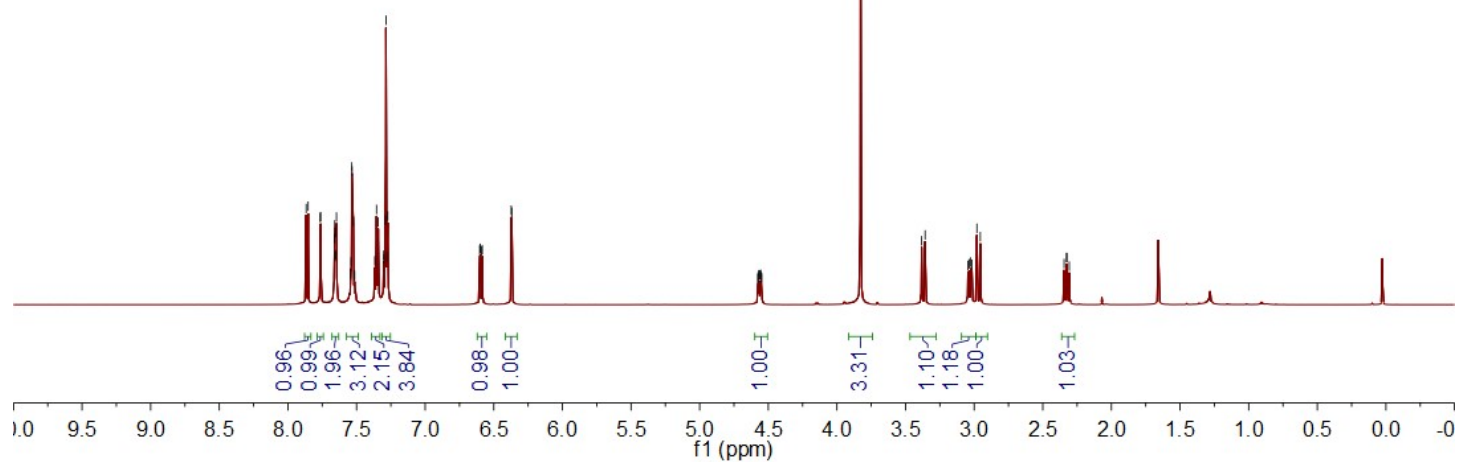

3s ${ }^{13} \mathrm{C}$ NMR $\left(151 \mathrm{MHz}, \mathrm{CDCl}_{3}\right)$

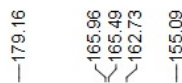

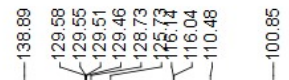

过<smiles>COc1ccc2c(c1)O[CH+]C1CC3(c4ccccc4)CC(=O)OC(c4ccccc4)=C3C=C1C2=O</smiles>

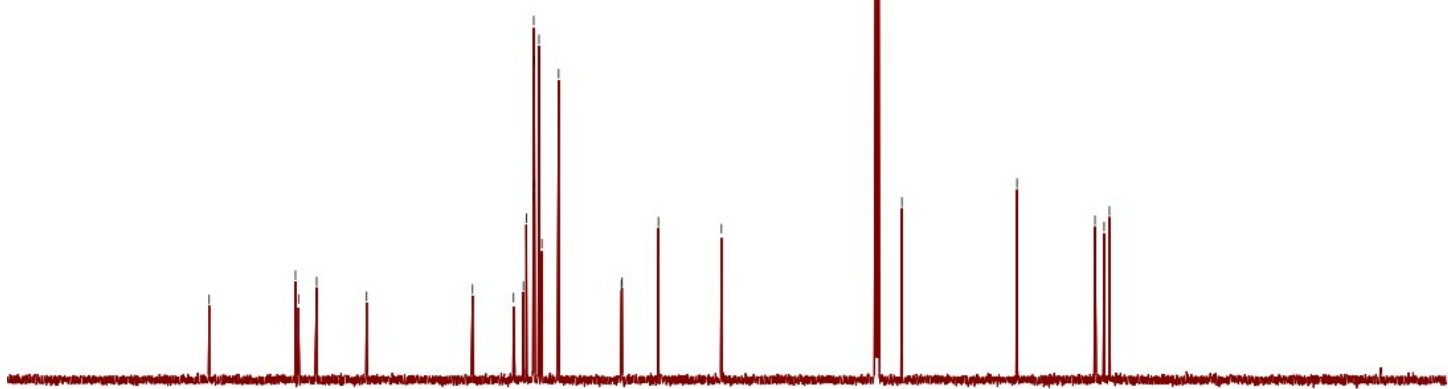

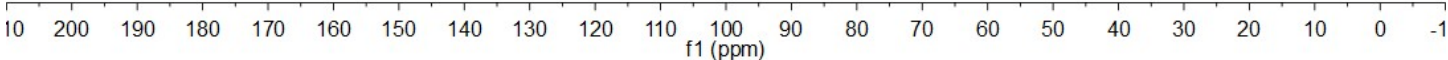


3s' ${ }^{1} \mathrm{H}$ NMR $\left(400 \mathrm{MHz}, \mathrm{CDCl}_{3}\right)$

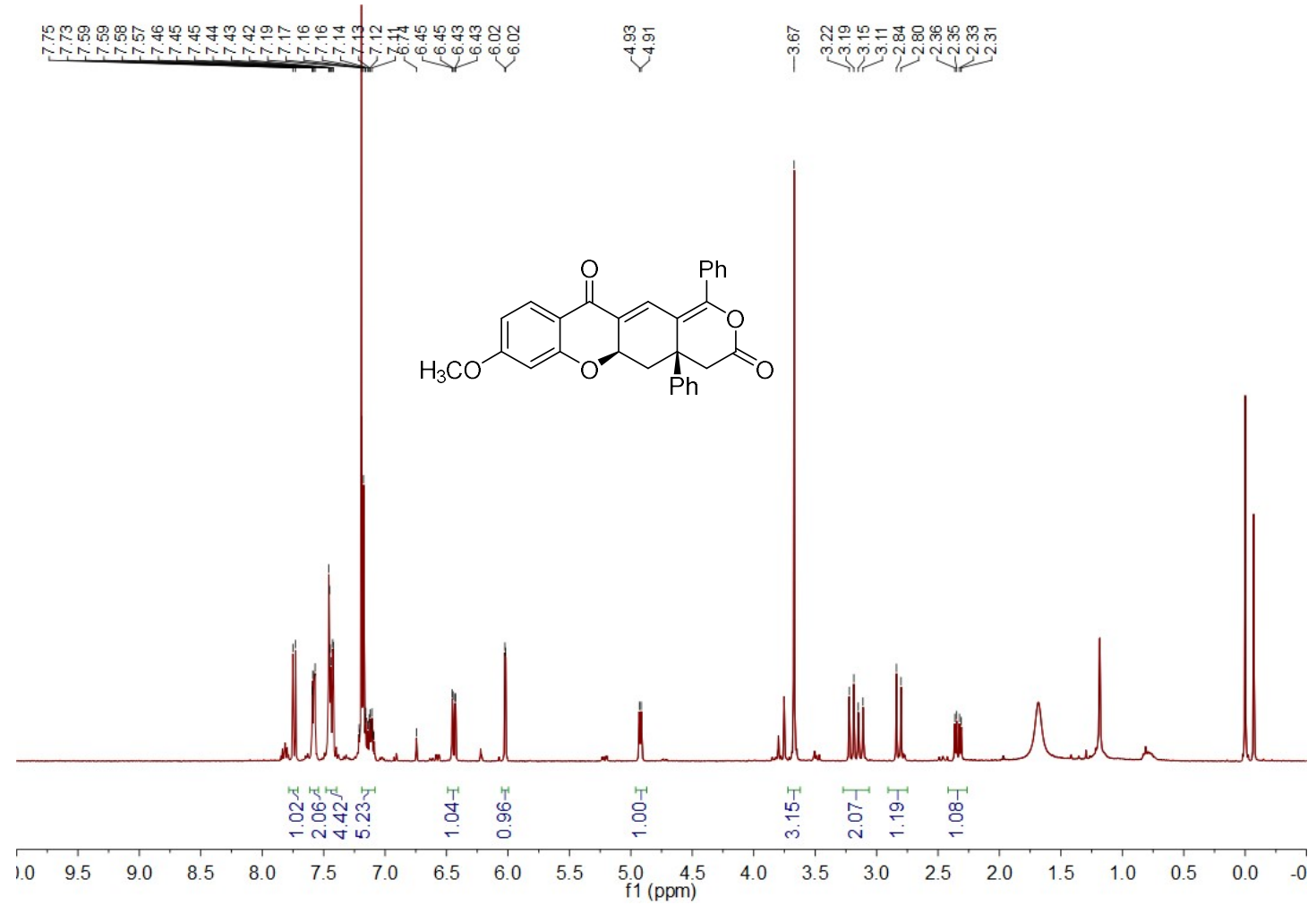

3s' ${ }^{13} \mathrm{C}$ NMR $\left(101 \mathrm{MHz}, \mathrm{CDCl}_{3}\right)$
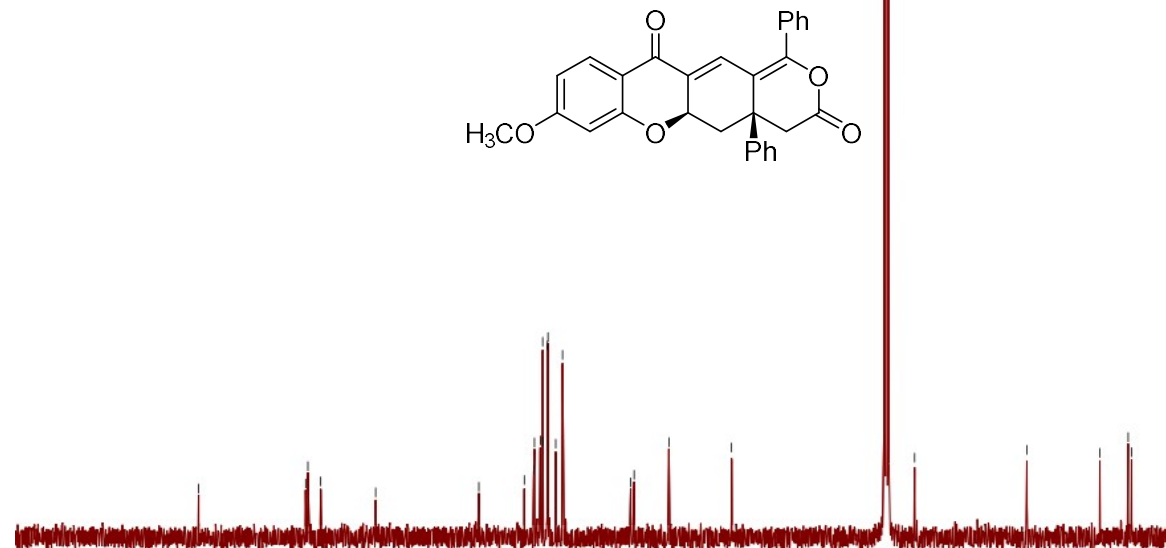

$\begin{array}{lllllllllllllllllllllll}10 & 200 & 190 & 180 & 170 & 160 & 150 & 140 & 130 & 120 & 110 & 100 & 90 & 80 & 70 & 60 & 50 & 40 & 30 & 20 & 10 & 0 & -1\end{array}$ 
3t ${ }^{1} \mathbf{H}$ NMR (400 MHz, $\left.\mathrm{CDCl}_{3}\right)$

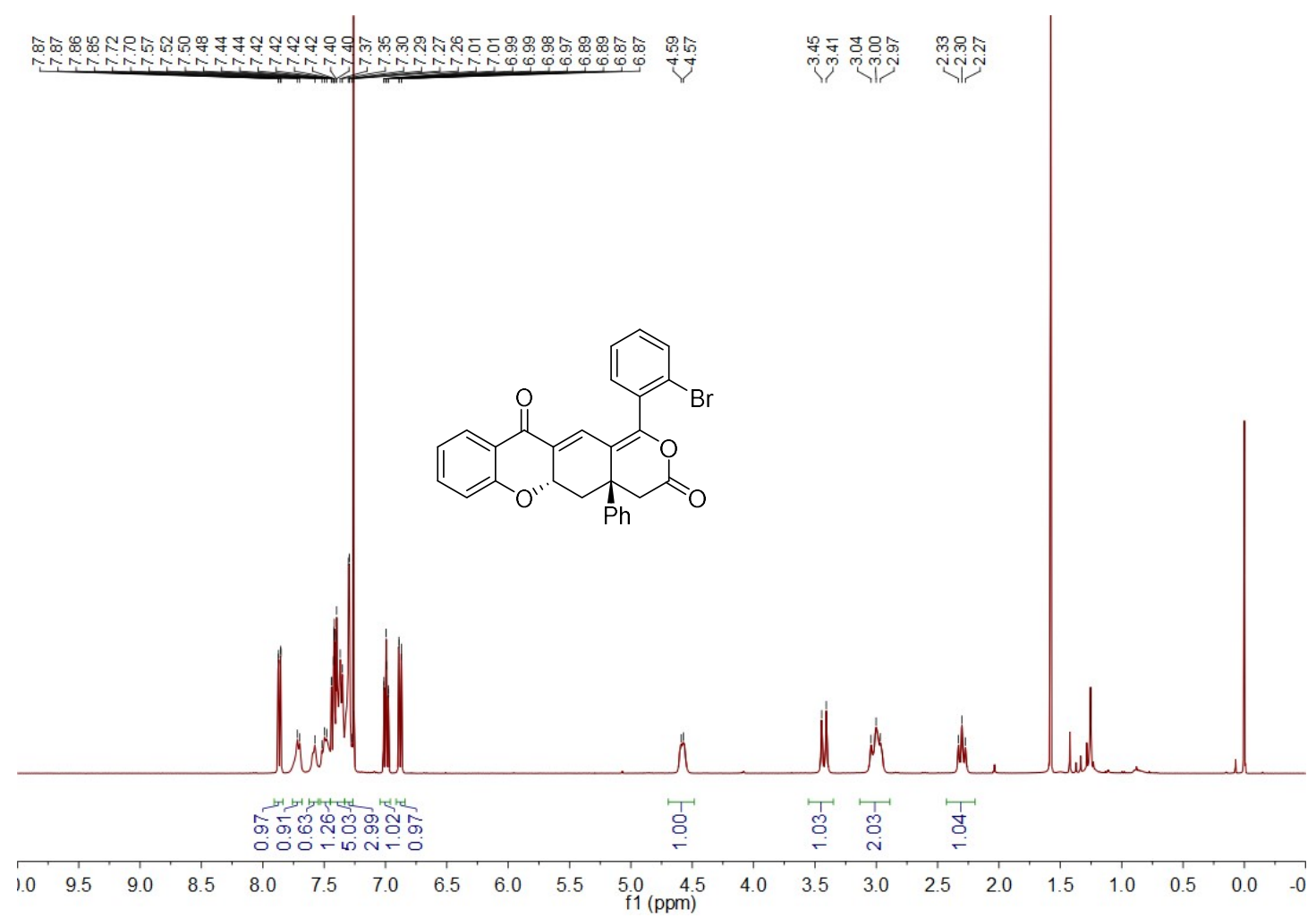

3t ${ }^{13} \mathrm{C}$ NMR $\left(101 \mathrm{MHz}, \mathrm{CDCl}_{3}\right)$
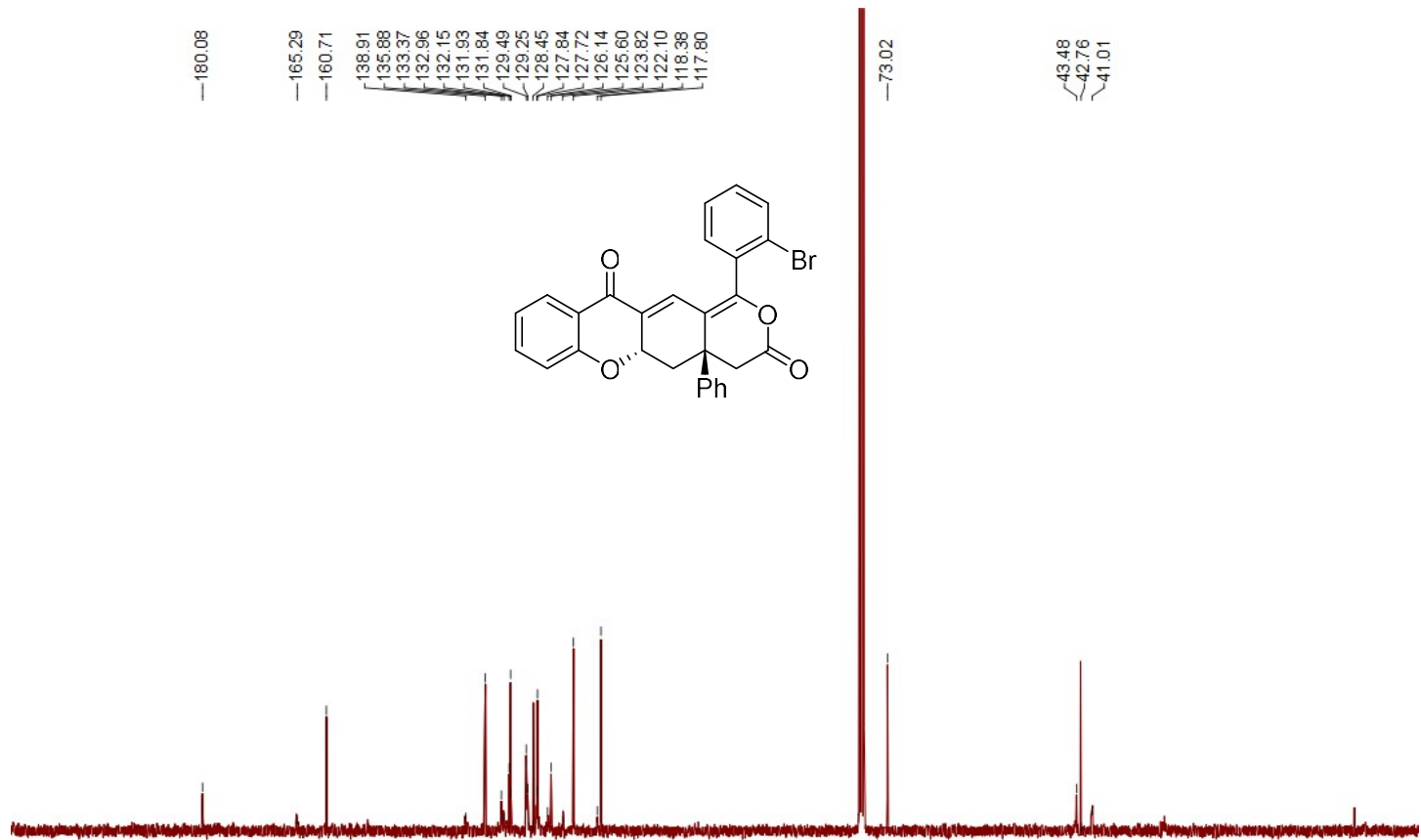

\begin{tabular}{lllllllllllllllllllllll}
\hline 10 & 200 & 190 & 180 & 170 & 160 & 150 & 140 & 130 & 120 & 110 & 100 & 90 & 80 & 70 & 60 & 50 & 40 & 30 & 20 & 10 & 0 & -1
\end{tabular} 
3t' ${ }^{1} \mathbf{H}$ NMR $\left(400 \mathrm{MHz}, \mathrm{CDCl}_{3}\right)$

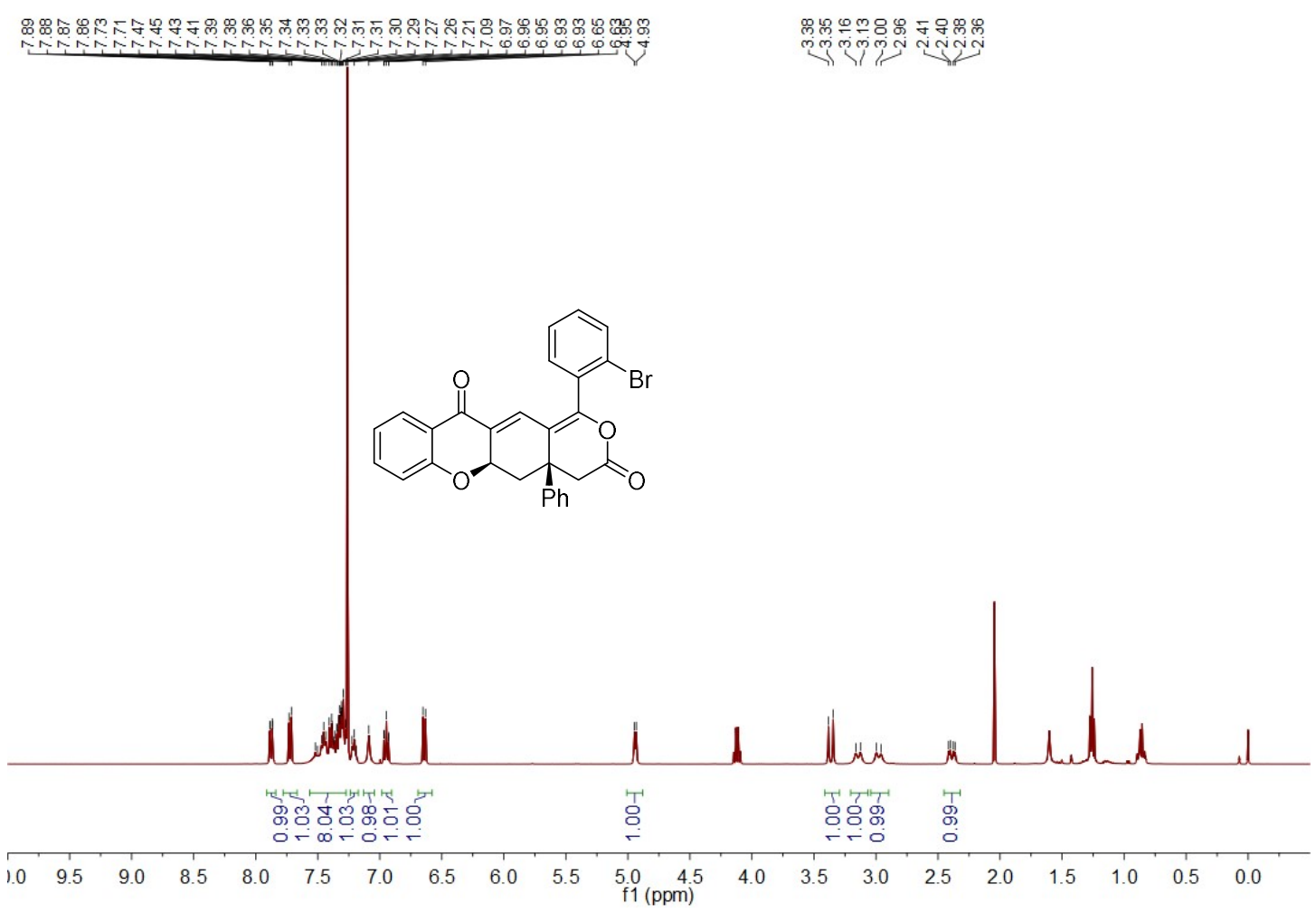

3t' ${ }^{13} \mathrm{C}$ NMR $\left(101 \mathrm{MHz}, \mathrm{CDCl}_{3}\right)$
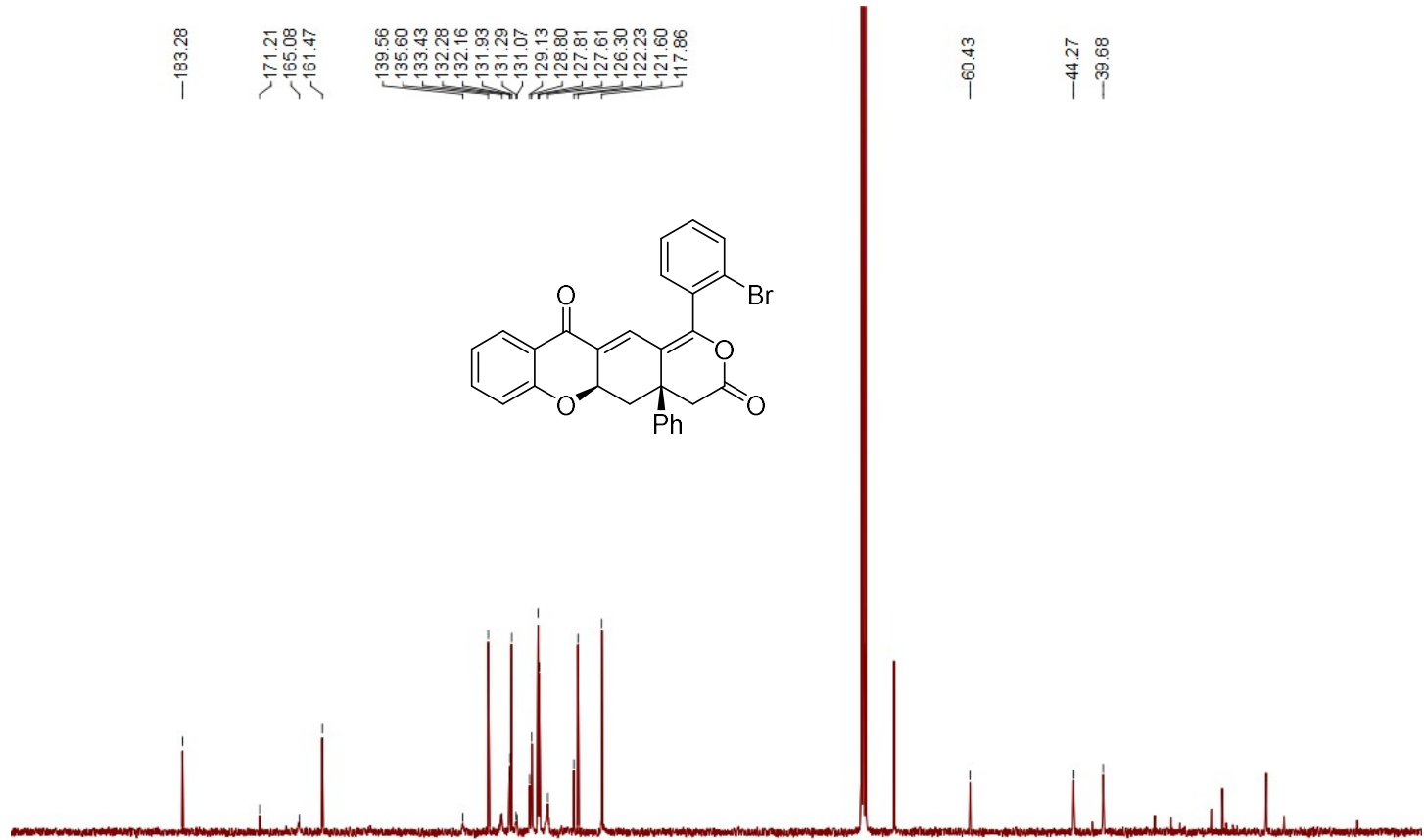

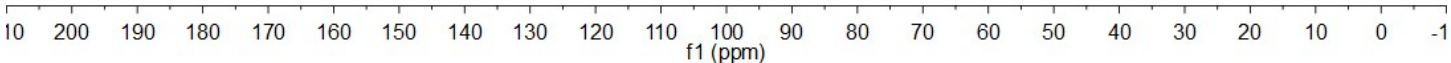


3u ${ }^{1} \mathbf{H}$ NMR $\left(600 \mathrm{MHz}, \mathrm{CDCl}_{3}\right)$

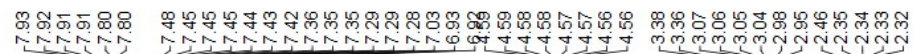
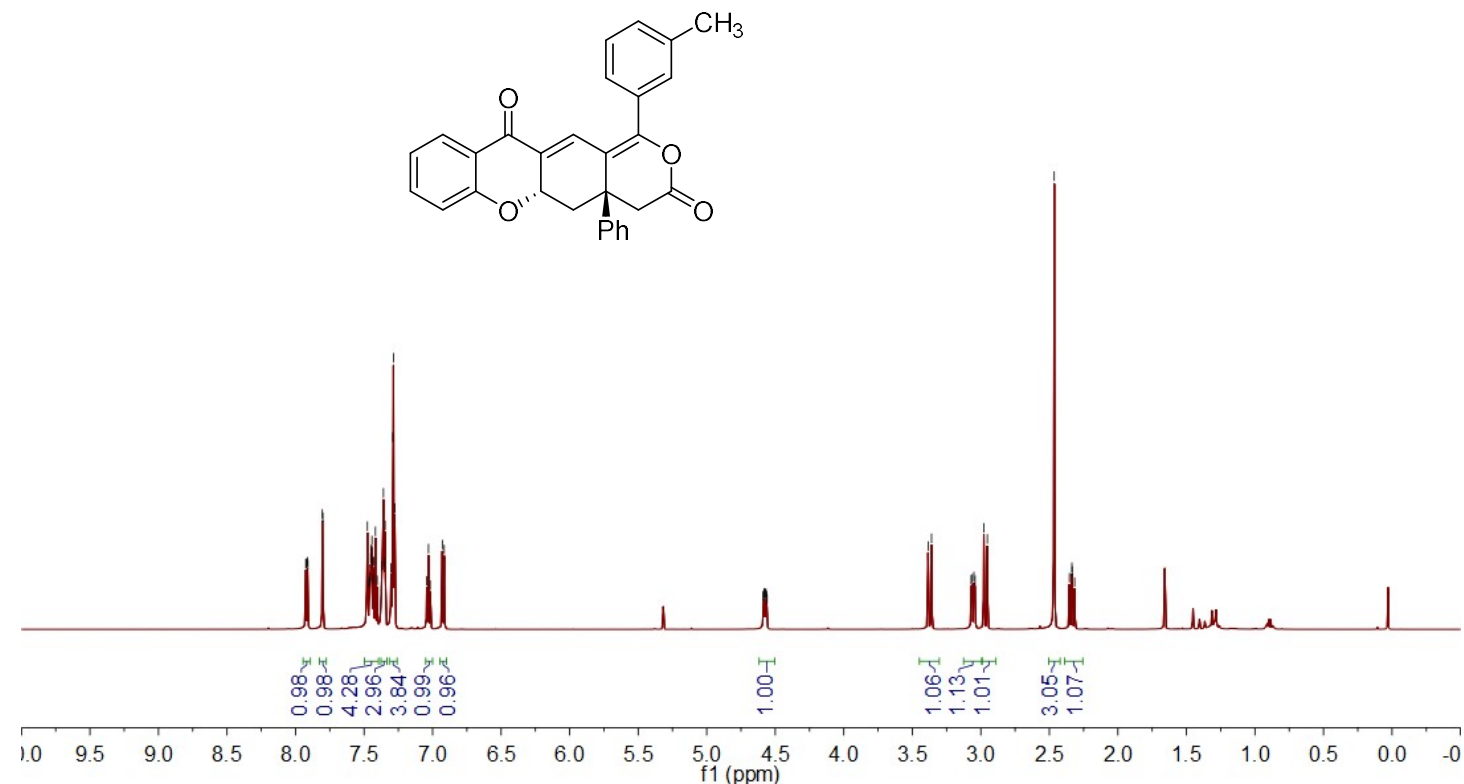

3u ${ }^{13} \mathrm{C}$ NMR $\left(151 \mathrm{MHz}, \mathrm{CDCl}_{3}\right)$

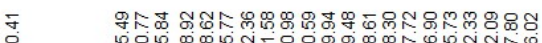

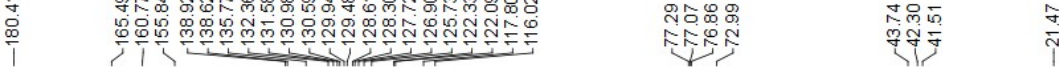
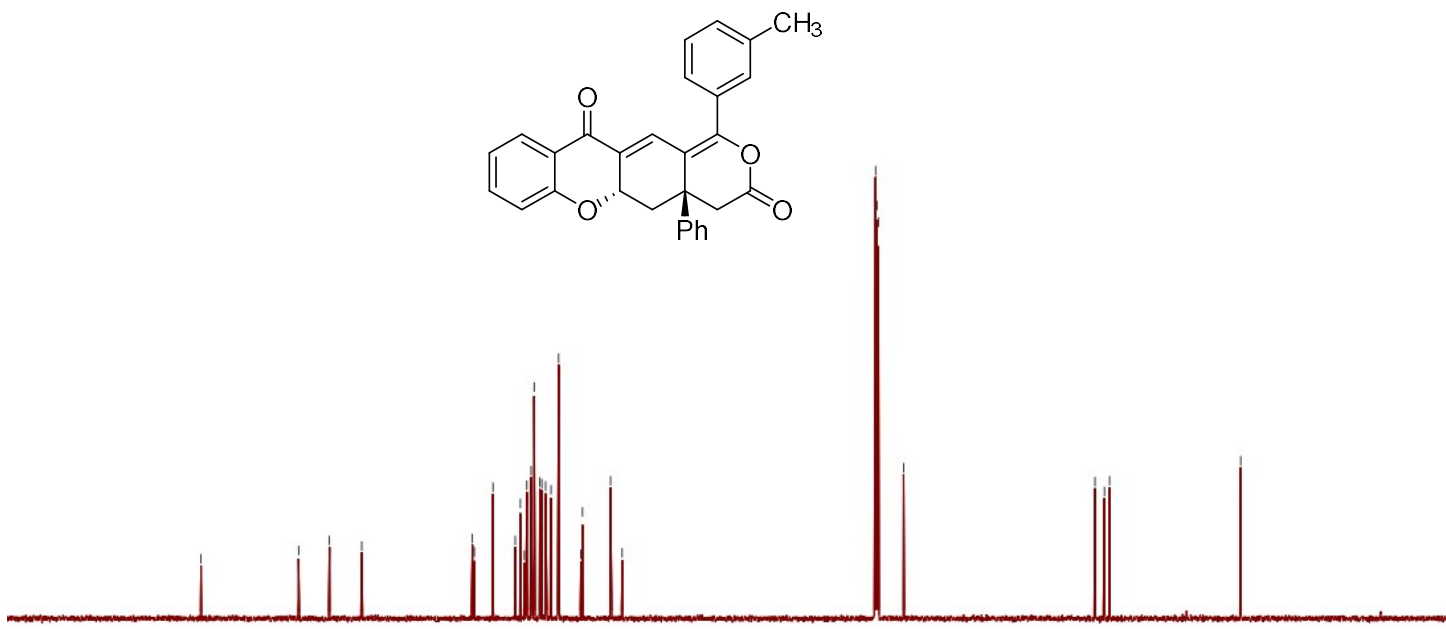

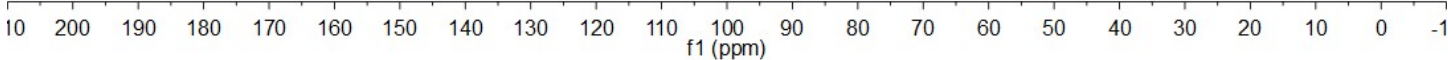


3u' ${ }^{1} \mathbf{H}$ NMR (400 MHz, $\left.\mathrm{CDCl}_{3}\right)$

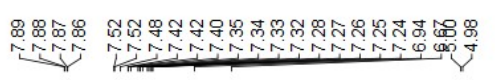

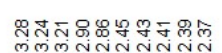
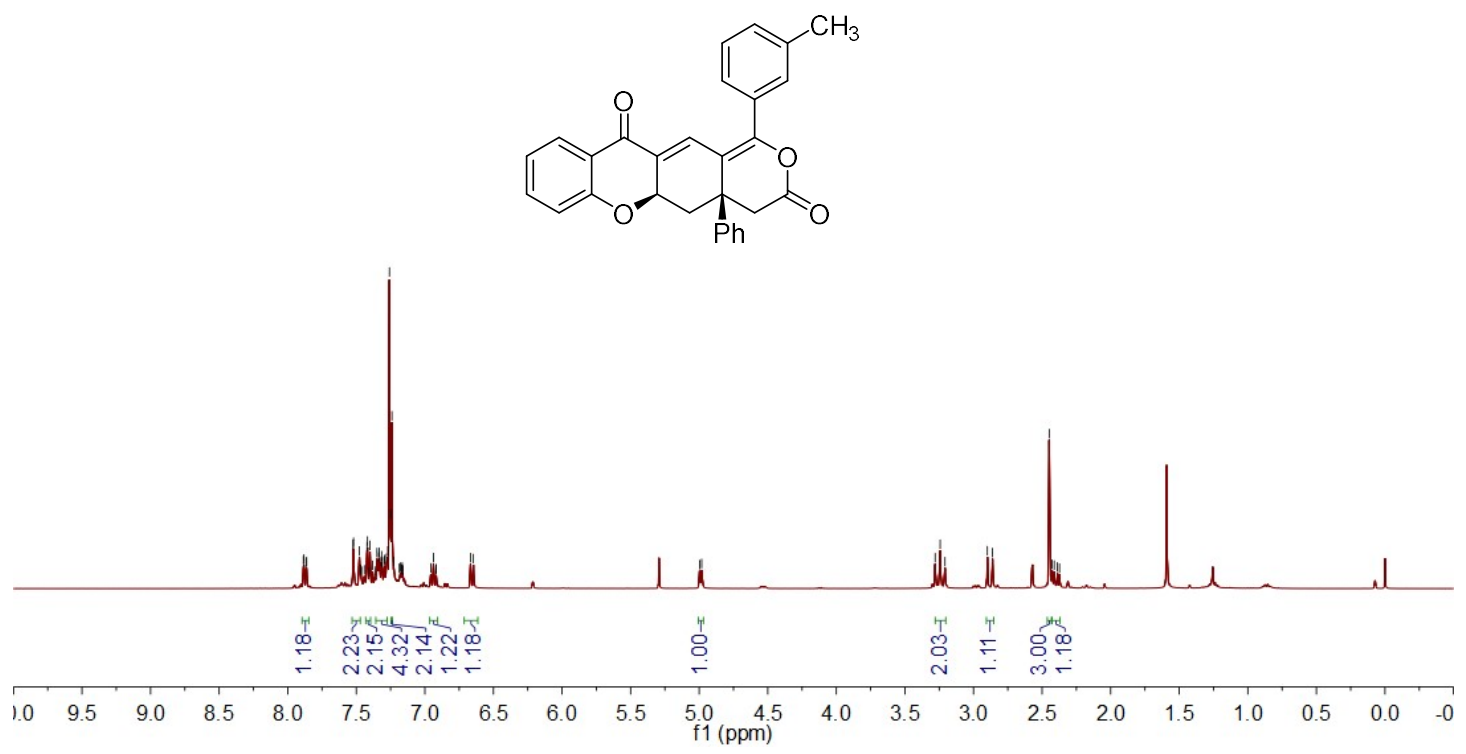

3u' ${ }^{13} \mathrm{C}$ NMR (101 MHz, $\left.\mathrm{CDCl}_{3}\right)$
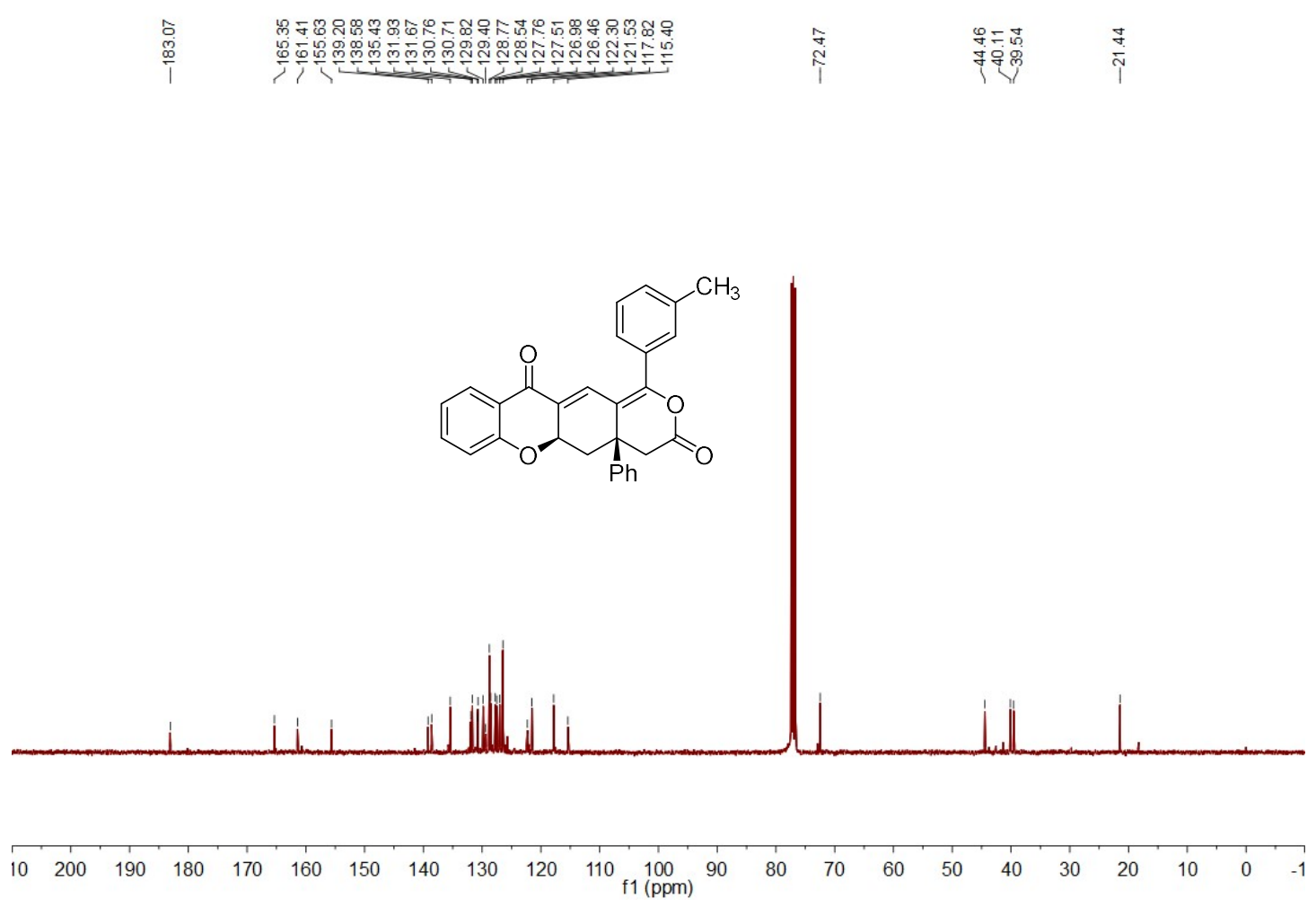

79 
3v ${ }^{1} \mathrm{H}$ NMR $\left(600 \mathrm{MHz}, \mathrm{CDCl}_{3}\right)$

及

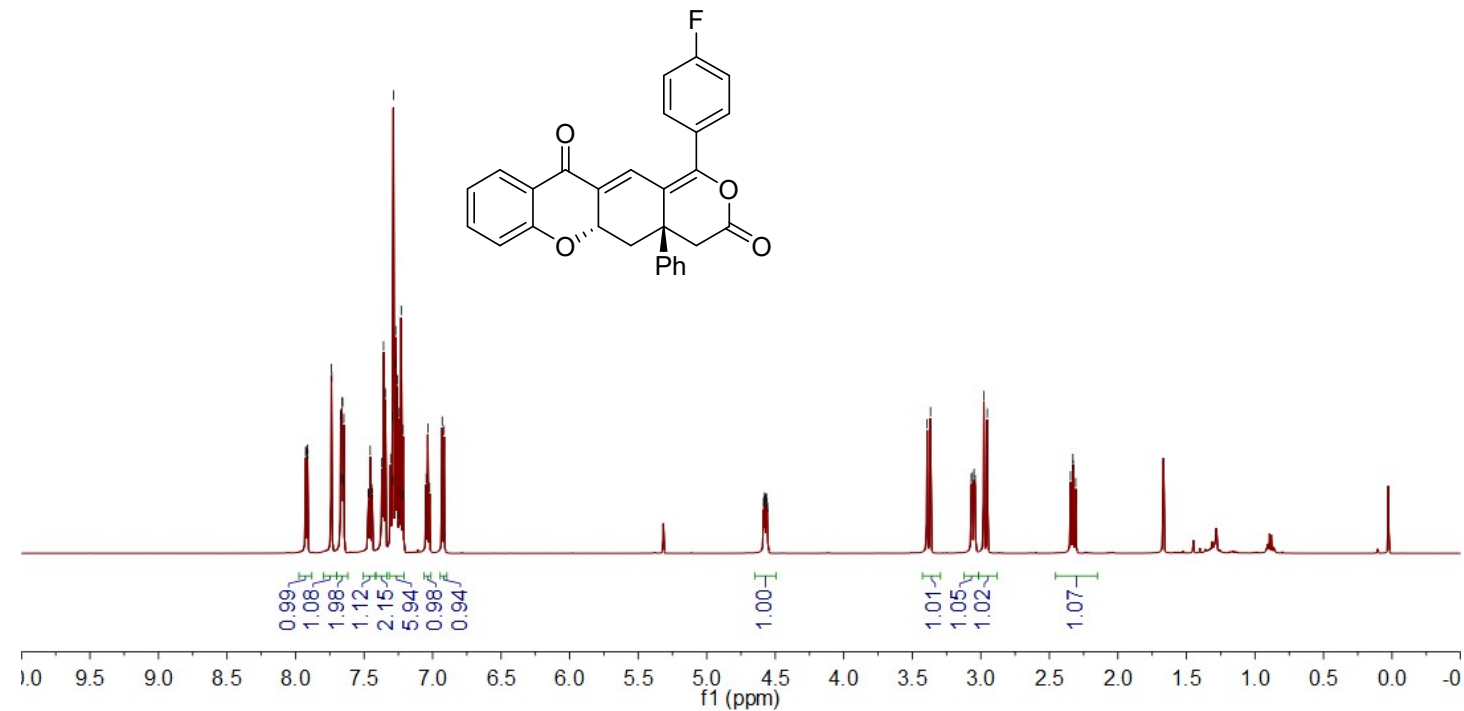

3v ${ }^{13} \mathrm{C}$ NMR $\left(151 \mathrm{MHz}, \mathrm{CDCl}_{3}\right)$

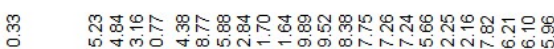

o
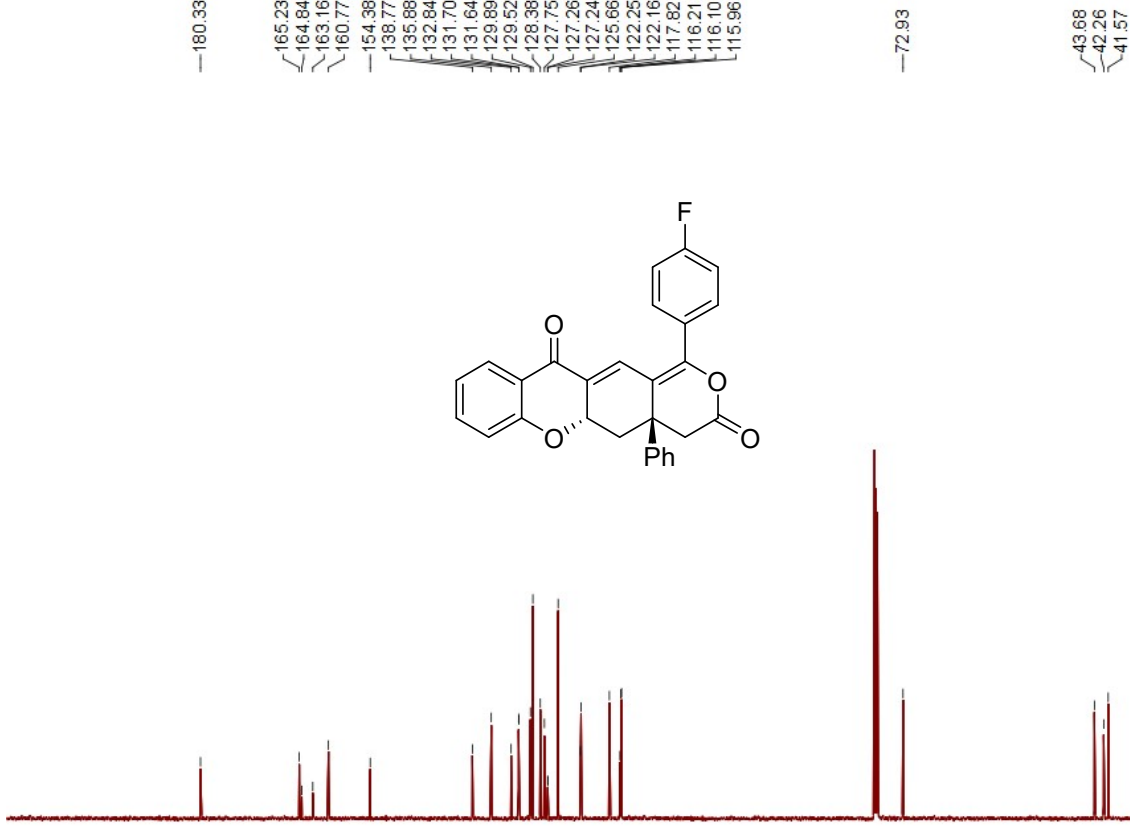

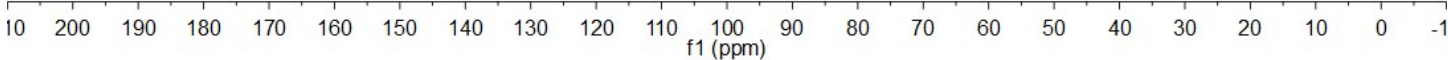


$3 v^{19}$ F NMR $\left(471 \mathrm{MHz}, \mathrm{CDCl}_{3}\right)$

$\stackrel{\infty}{\infty}$<smiles>O=C1CC2(c3ccccc3)CC3Oc4ccccc4C(=O)C3=CC2=C(c2ccc(F)cc2)O1</smiles> 
3w ${ }^{1} \mathrm{H}$ NMR $\left(400 \mathrm{MHz}, \mathrm{CDCl}_{3}\right)$

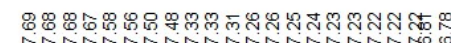

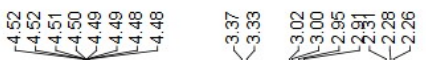<smiles>Cc1ccc2c(c1)C(=O)C1=CC3=C(c4ccc(Cl)cc4)OC(=O)C3C1c1ccccc1O2</smiles>

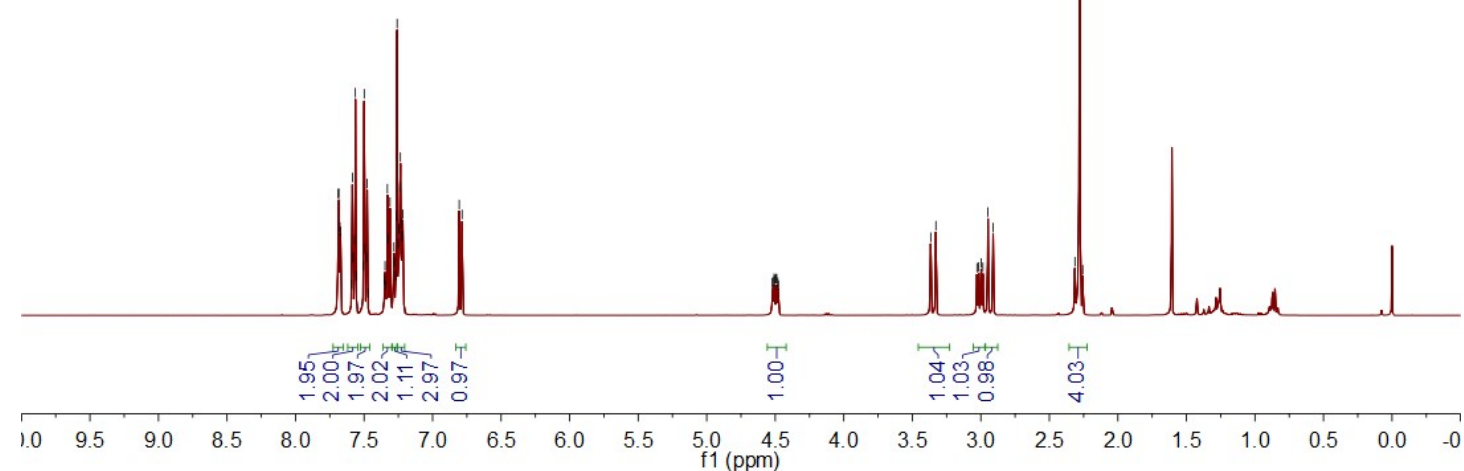

3w ${ }^{13} \mathrm{C}$ NMR $\left(101 \mathrm{MHz}, \mathrm{CDCl}_{3}\right)$

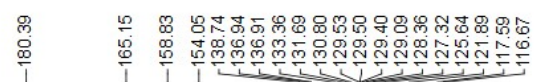

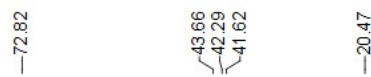<smiles>Cc1ccc2c(c1)C(=O)C1=CC3=C(c4ccc(Cl)cc4)OC(=O)CC3(c3ccccc3)CC1O2</smiles>

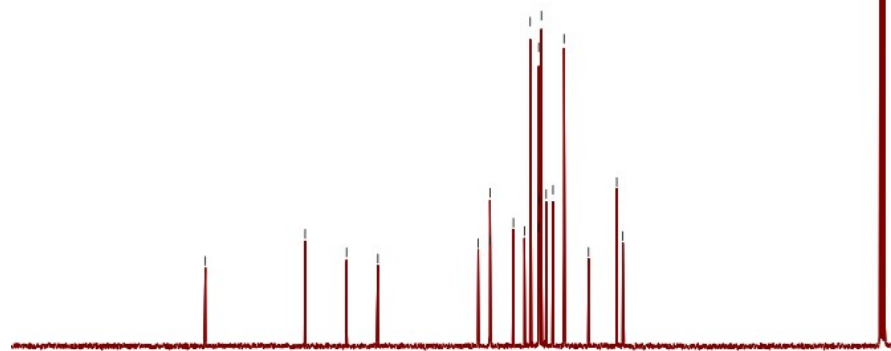

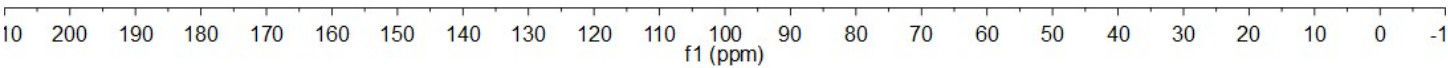


3x ${ }^{1} \mathrm{H}$ NMR $\left(400 \mathrm{MHz}, \mathrm{CDCl}_{3}\right)$

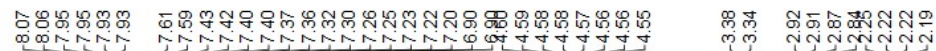<smiles>O=C1C[C@]2(c3ccccc3)C[C@H]3Oc4ccccc4C(=O)C3=CC2=C(/C=C/c2ccccc2)O1</smiles>

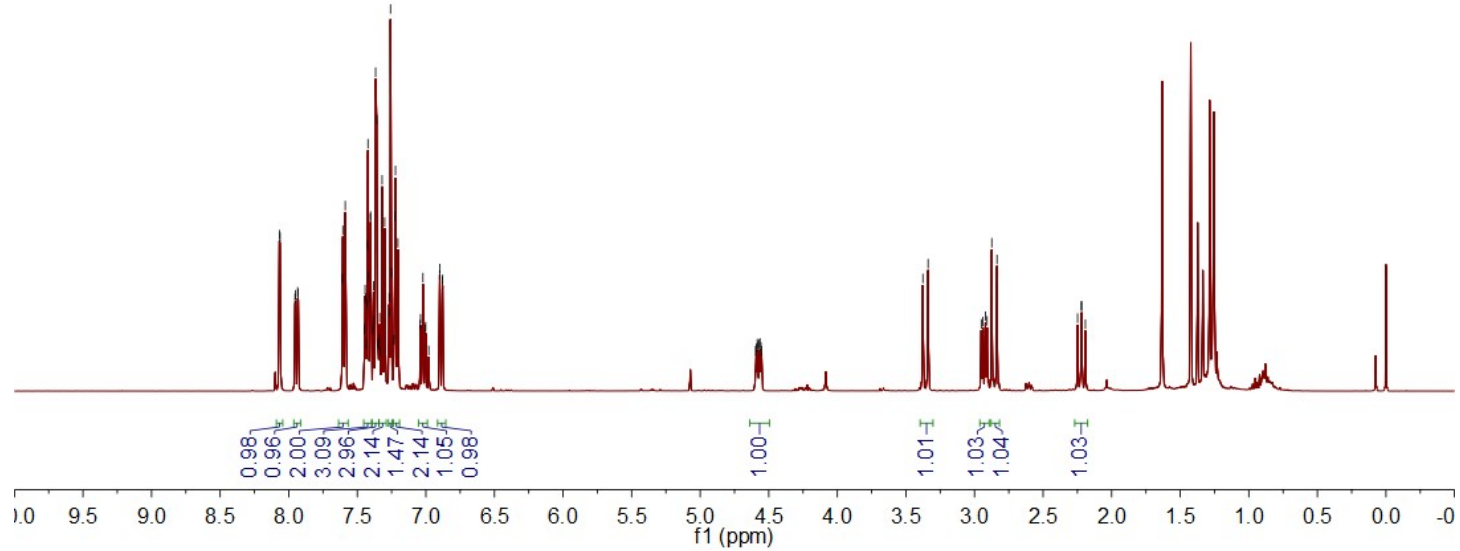

3x ${ }^{13} \mathrm{C}$ NMR $\left(101 \mathrm{MHz}, \mathrm{CDCl}_{3}\right)$

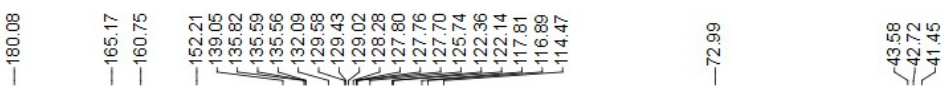<smiles>O=C1C[C@@H]2CC3Oc4ccccc4C(=O)C3=CC2=C(C=Cc2ccccc2)O1</smiles>

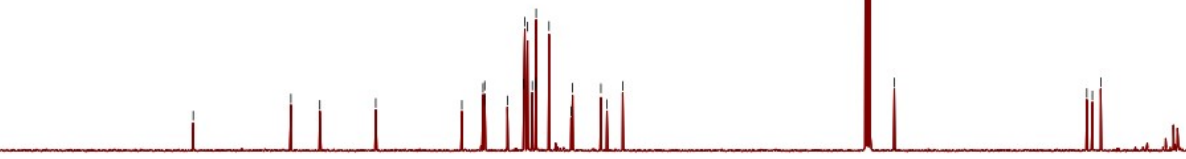

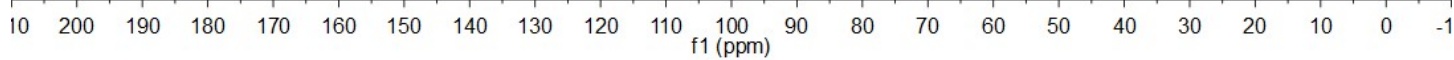


3x' ${ }^{1} \mathrm{H}$ NMR $\left(400 \mathrm{MHz}, \mathrm{CDCl}_{3}\right)$

\&.

miñ

作<smiles>O=C1C2=CC3=C(C=Cc4ccccc4)OC(=O)[C@@H]3CC2Oc2ccccc21</smiles>

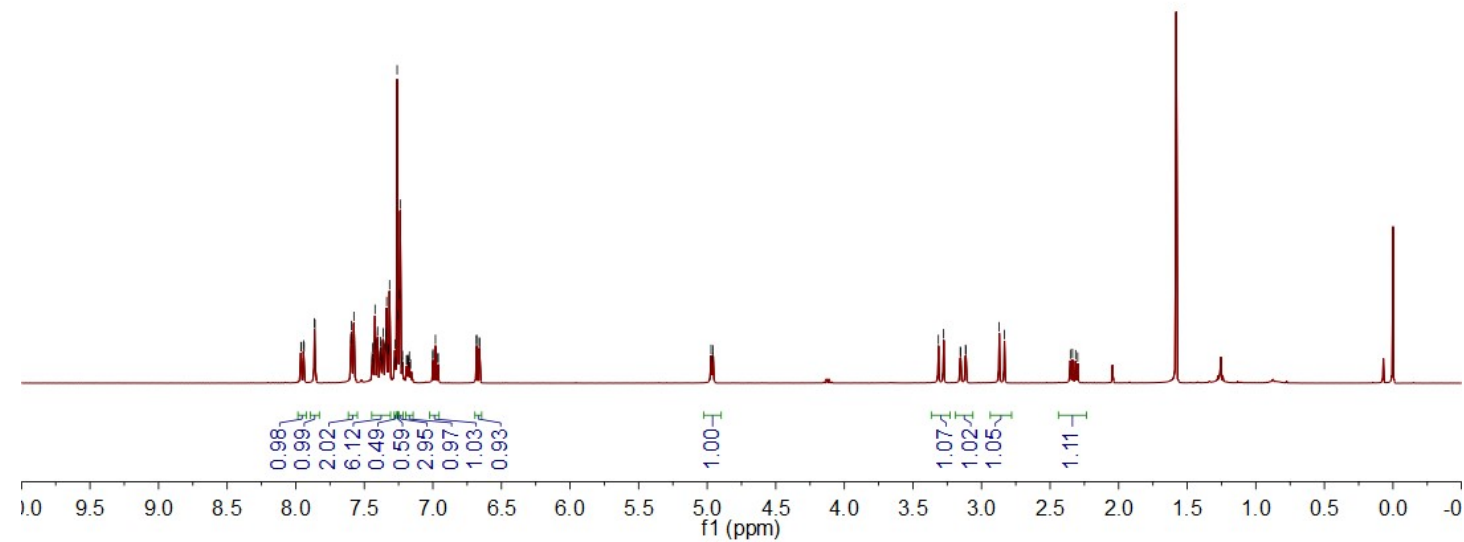

3x' ${ }^{13} \mathrm{C}$ NMR $\left(101 \mathrm{MHz}, \mathrm{CDCl}_{3}\right)$
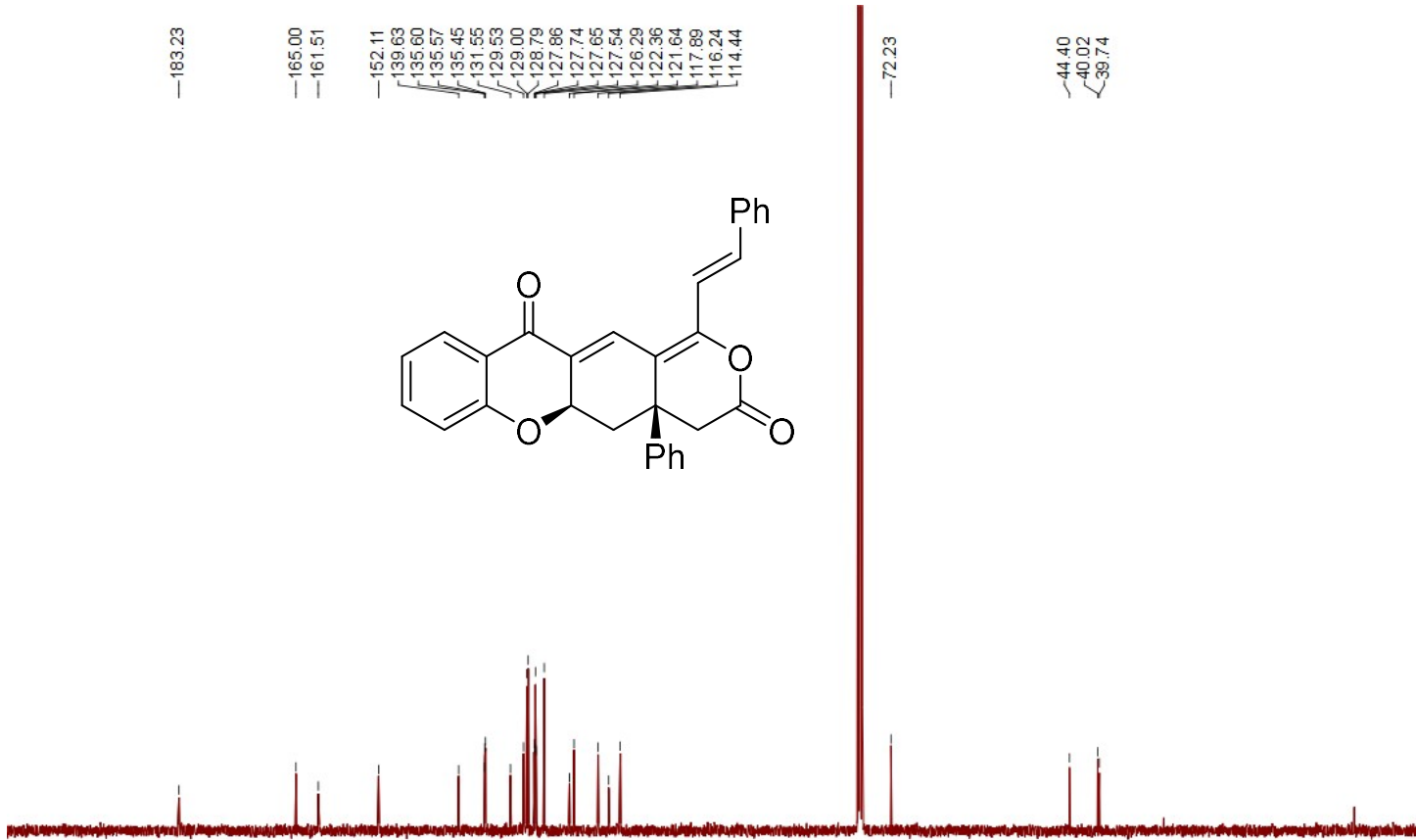

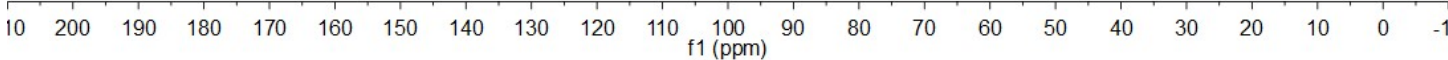


3y ${ }^{1} \mathrm{H}$ NMR $\left(400 \mathrm{MHz}, \mathrm{CDCl}_{3}\right)$

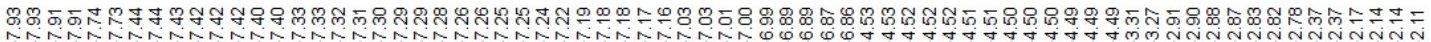<smiles>CC1=C2C=C3C(=O)c4ccccc4OC3C[C@]2(c2ccccc2)CC(=O)O1</smiles>

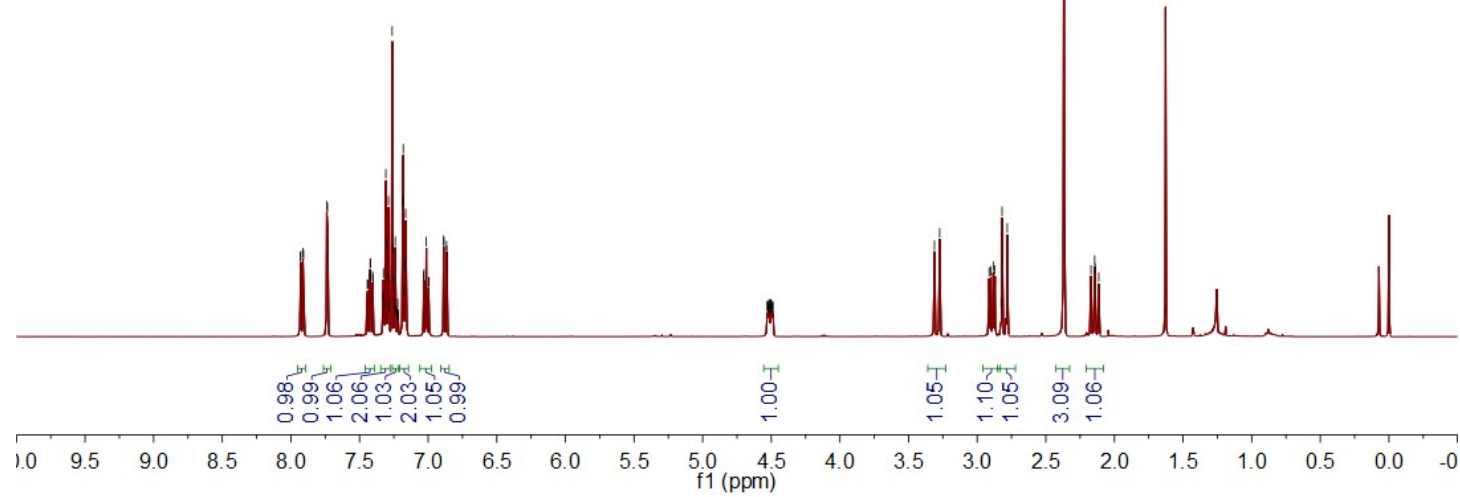

3y ${ }^{13} \mathrm{C}$ NMR $\left(101 \mathrm{MHz}, \mathrm{CDCl}_{3}\right)$

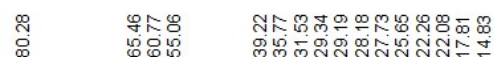

乐品

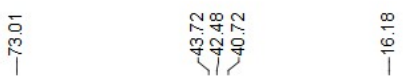
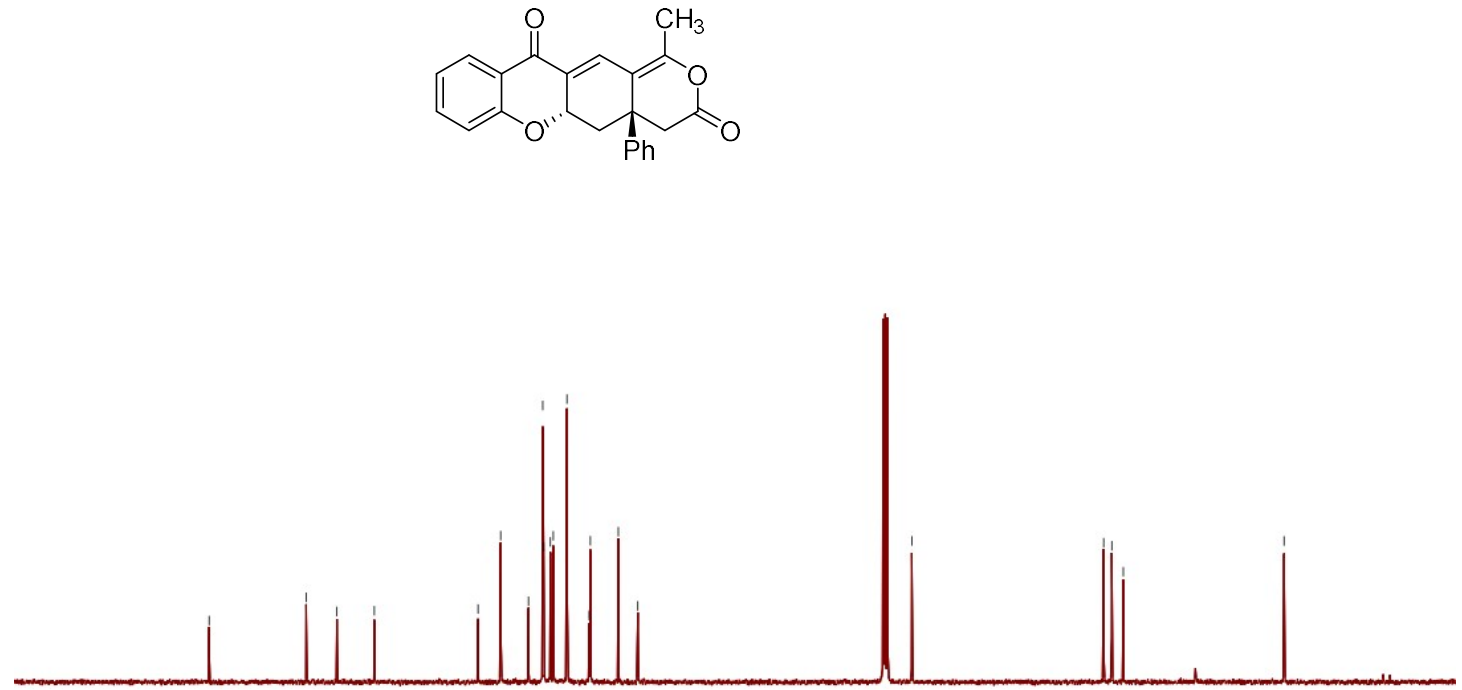

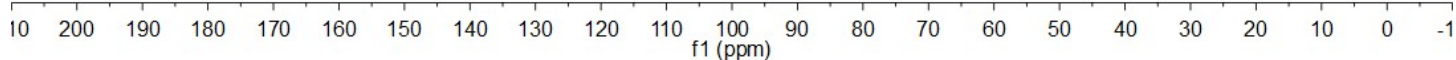


3y' ${ }^{1} \mathbf{H}$ NMR $\left(400 \mathrm{MHz}, \mathrm{CDCl}_{3}\right)$

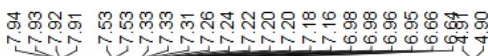

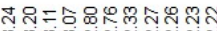

mimmininining<smiles>CC1=C2C=C3C(=O)c4ccccc4OC3CC2(c2ccccc2)CC(=O)O1</smiles>

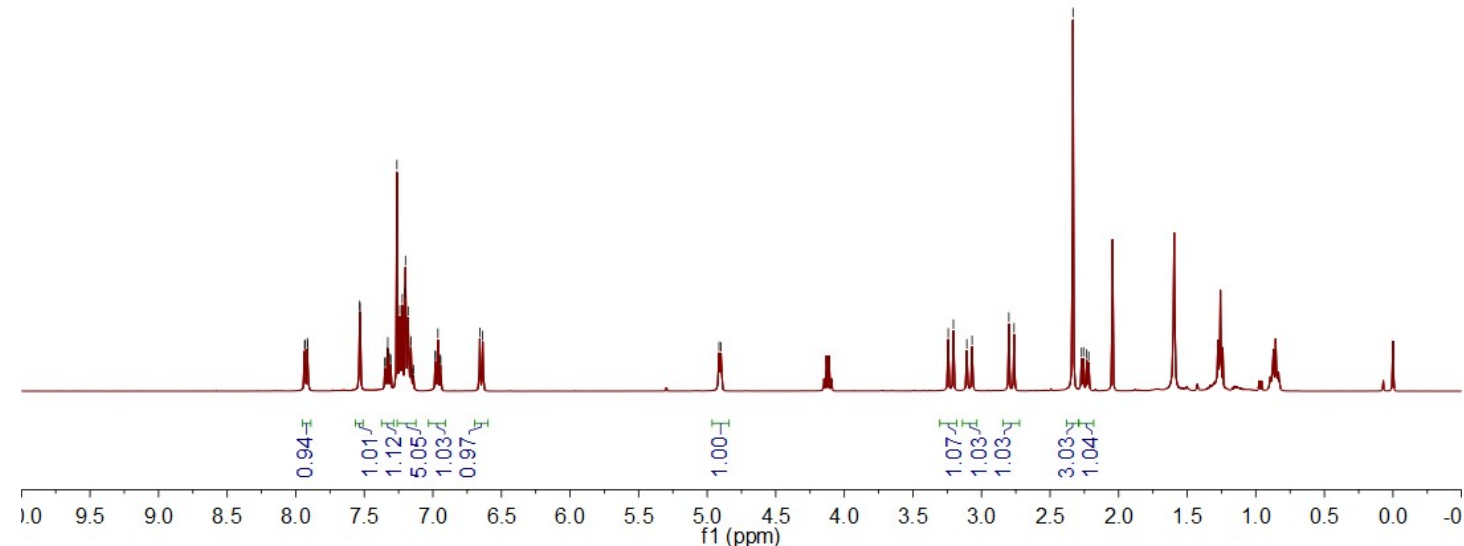

3y' ${ }^{13} \mathrm{C}$ NMR (101 MHz, $\left.\mathrm{CDCl}_{3}\right)$
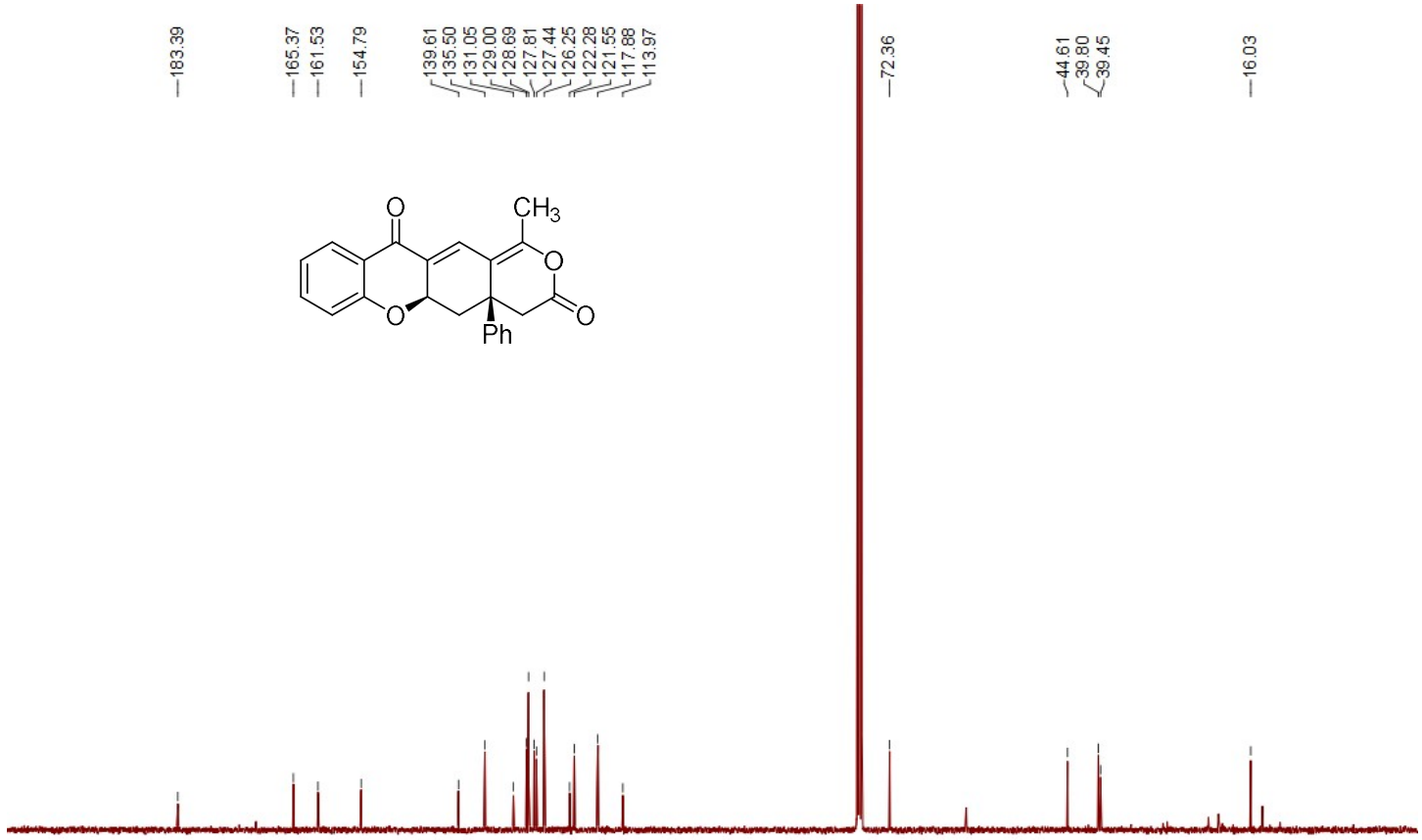

$\begin{array}{llllllllllllllllllllllllll}10 & 200 & 190 & 180 & 170 & 160 & 150 & 140 & 130 & 120 & 110 & 100 & 90 & 80 & 70 & 60 & 50 & 40 & 30 & 20 & 10 & 0 & -1\end{array}$ 
$5^{1} \mathrm{H}$ NMR $\left(300 \mathrm{MHz}, \mathrm{CDCl}_{3}\right)$

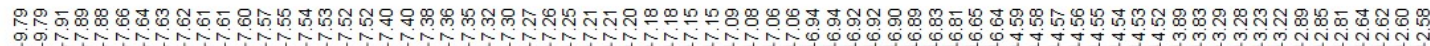

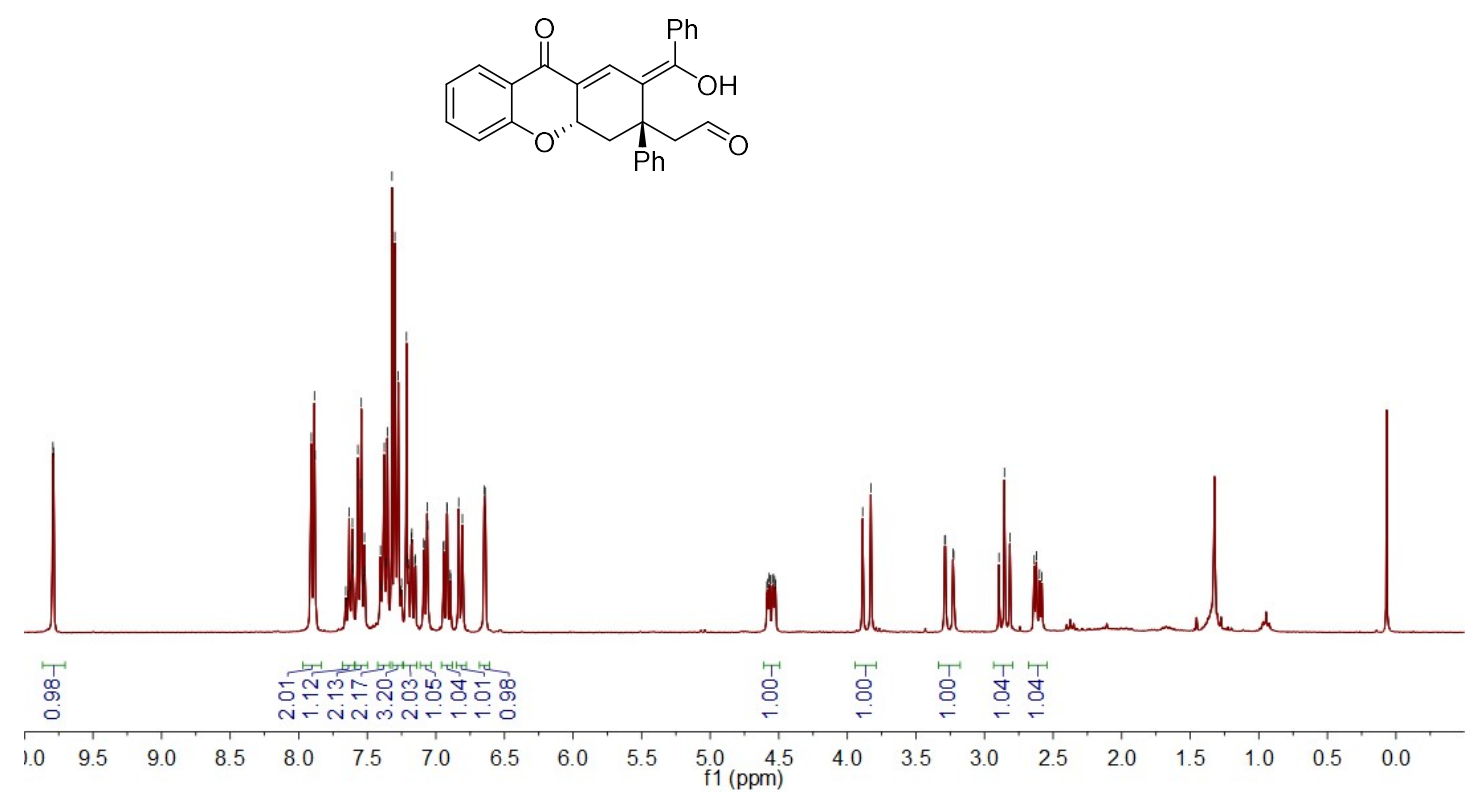

$5{ }^{13} \mathrm{C}$ NMR $\left(75 \mathrm{MHz}, \mathrm{CDCl}_{3}\right)$

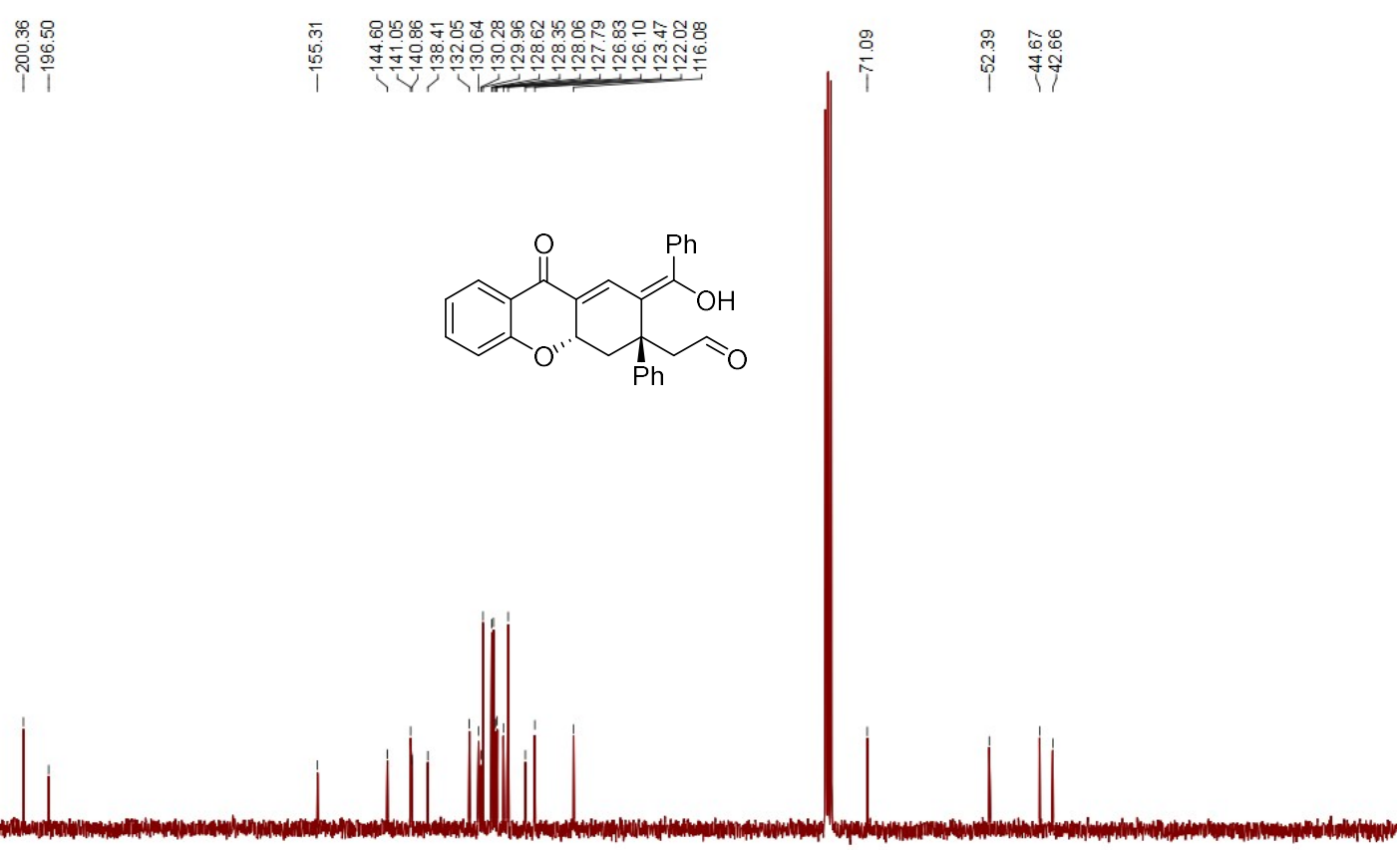

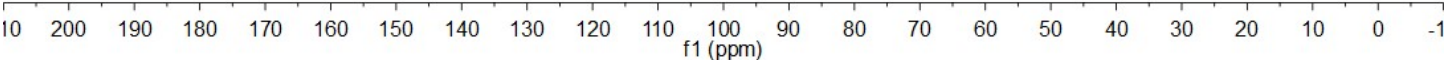


<smiles>CCOC(=O)/C=C/CC1(c2ccccc2)C[C@@H]2Oc3ccccc3C(=O)C2=C/C1=C(\O)c1ccccc1</smiles>

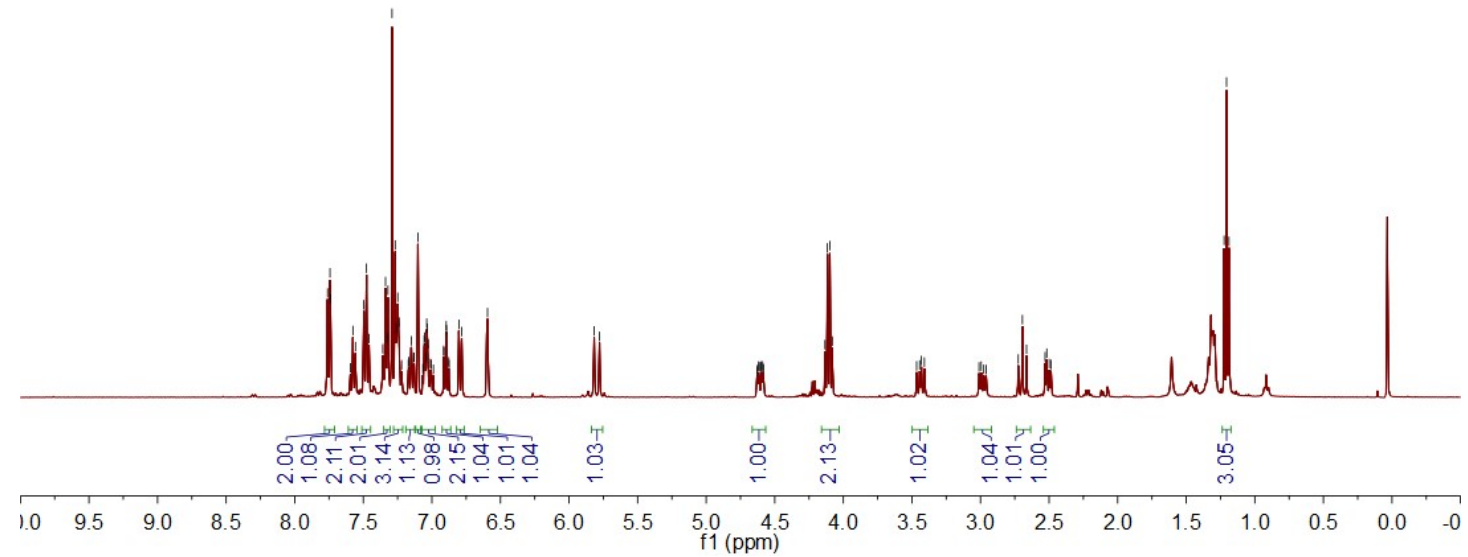

$6{ }^{13} \mathrm{C}$ NMR $\left(101 \mathrm{MHz}, \mathrm{CDCl}_{3}\right)$

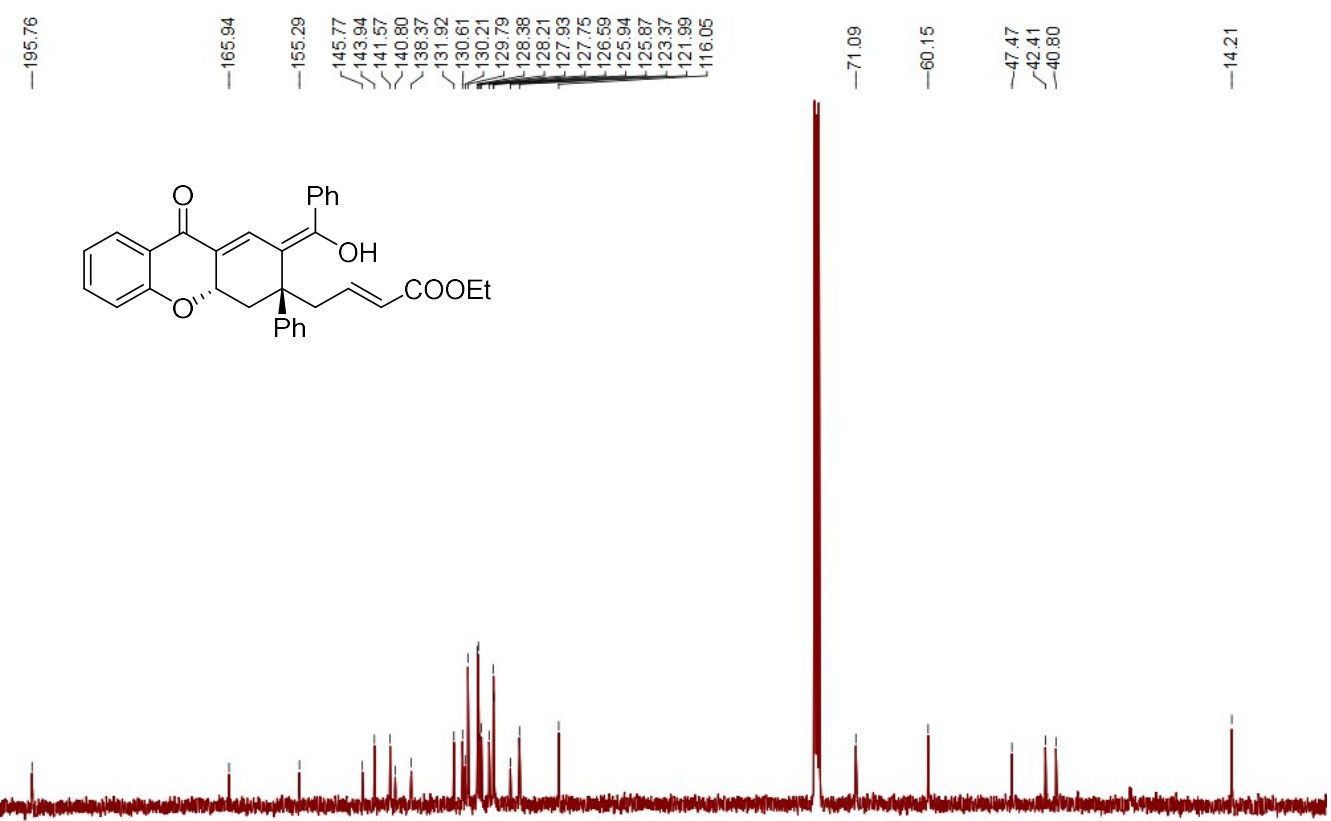

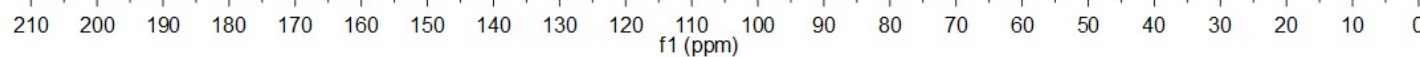


8a ${ }^{1} \mathrm{H}$ NMR $\left(400 \mathrm{MHz}, \mathrm{CDCl}_{3}\right)$

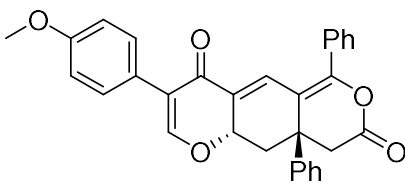

$1-8$

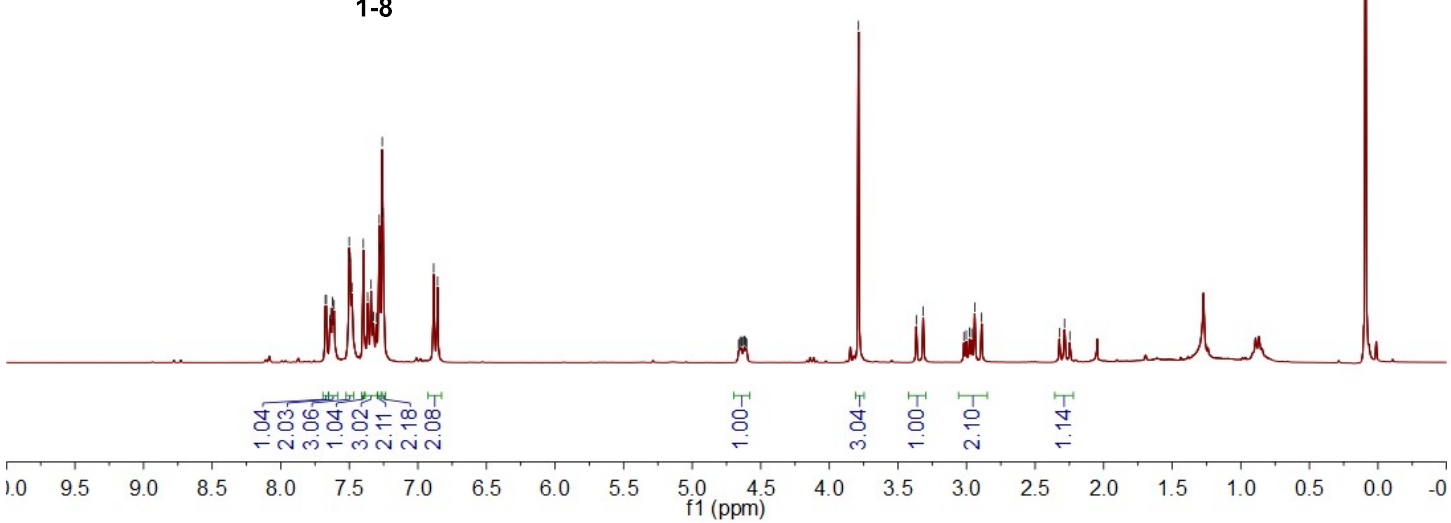

8a ${ }^{13} \mathrm{C}$ NMR $\left(75 \mathrm{MHz}, \mathrm{CDCl}_{3}\right)$

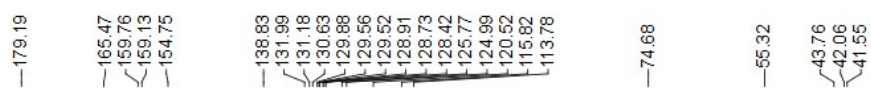<smiles>COc1ccc(C2=CO[CH+]C3CC4(c5ccccc5)CC(=O)OC(c5ccccc5)=C4C=C2C3=O)cc1</smiles>

$1-8$

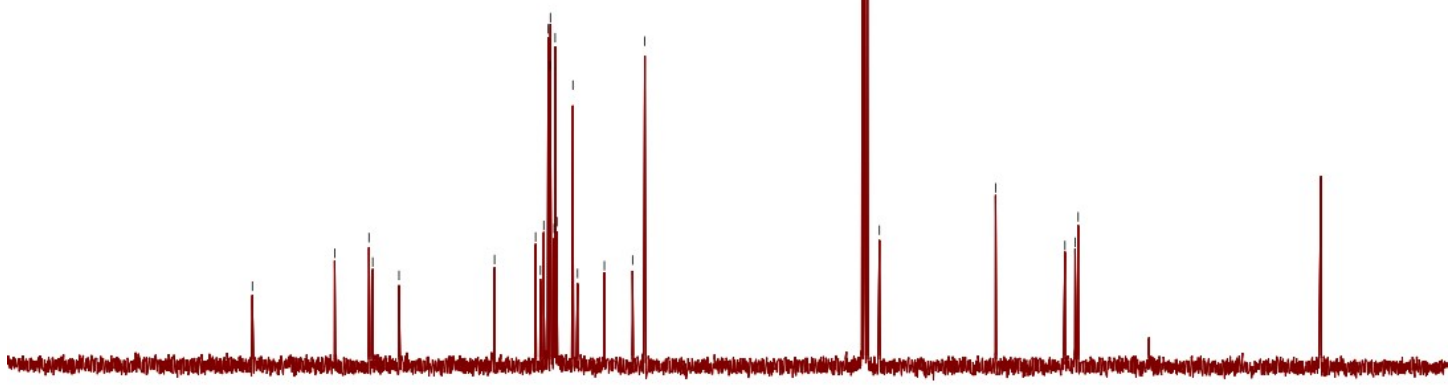

$\begin{array}{llllllllllllllllllllllllll}20 & 210 & 200 & 190 & 180 & 170 & 160 & 150 & 140 & 130 & 120 & 110 & 100 & 90 & 80 & 70 & 60 & 50 & 40 & 30 & 20 & 10 & 0 & -10 & -2\end{array}$ 
8b ${ }^{1} \mathbf{H}$ NMR $\left(500 \mathrm{MHz}, \mathrm{CDCl}_{3}\right)$

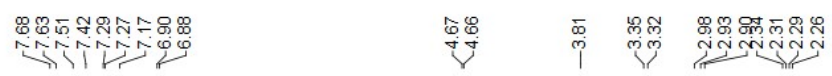

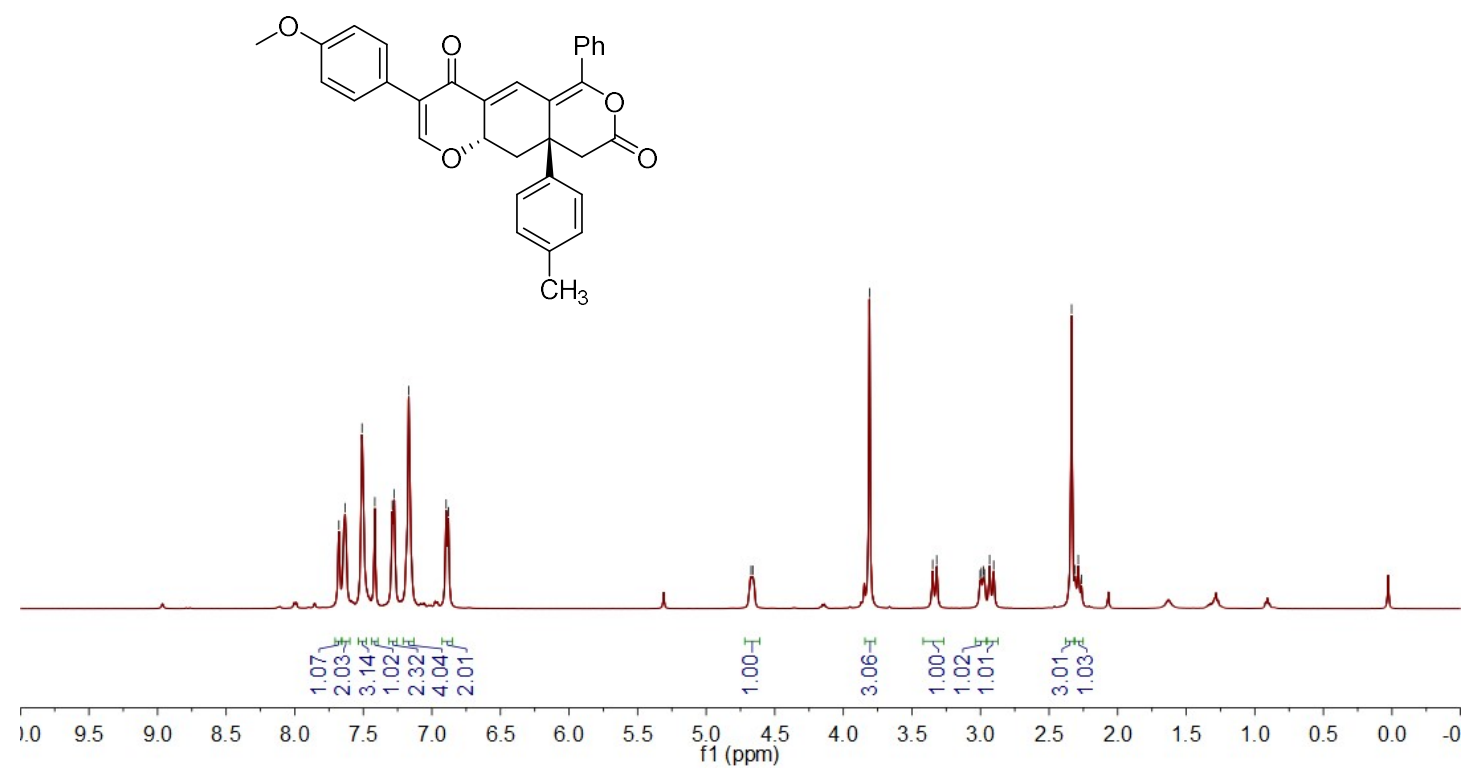

8b ${ }^{13} \mathrm{C}$ NMR $\left(75 \mathrm{MHz}, \mathrm{CDCl}_{3}\right)$

స.

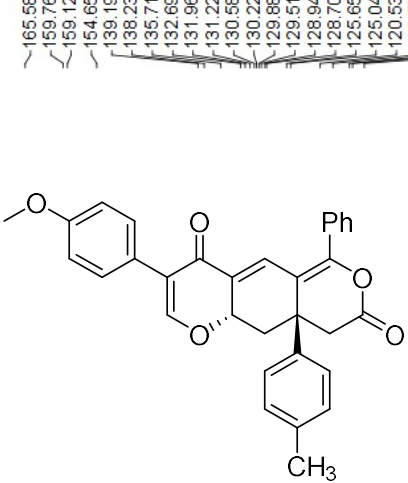

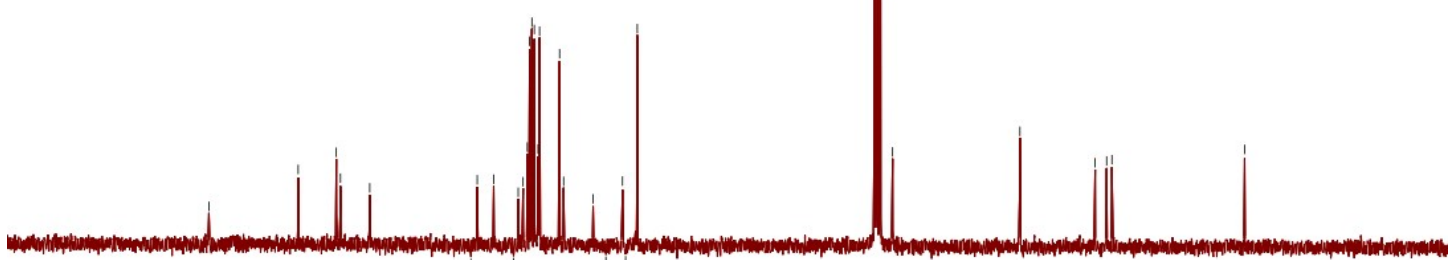

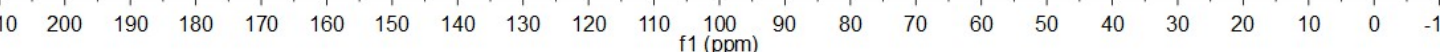


8c ${ }^{1} \mathrm{H}$ NMR $\left(400 \mathrm{MHz}, \mathrm{CDCl}_{3}\right)$

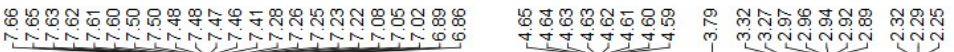

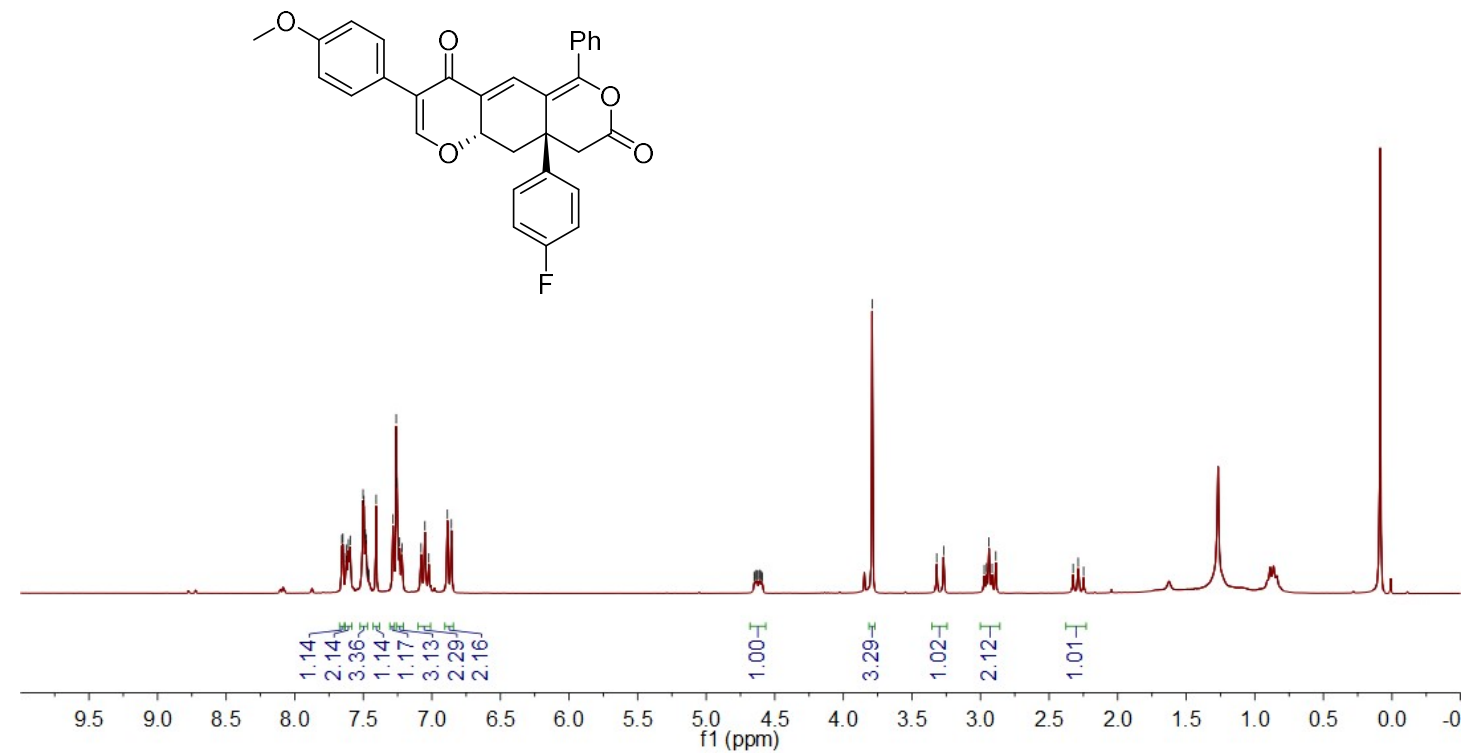

8c ${ }^{13} \mathrm{C}$ NMR $\left(75 \mathrm{MHz}, \mathrm{CDCl}_{3}\right)$

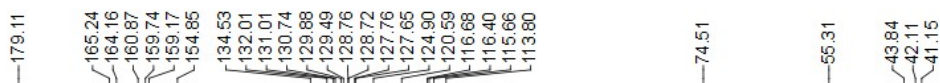<smiles>COc1ccc(C2=CO[C@H]3C[C@]4(c5ccc(F)cc5)CC(=O)OC(c5ccccc5)=C4C=C3C2=O)cc1</smiles>

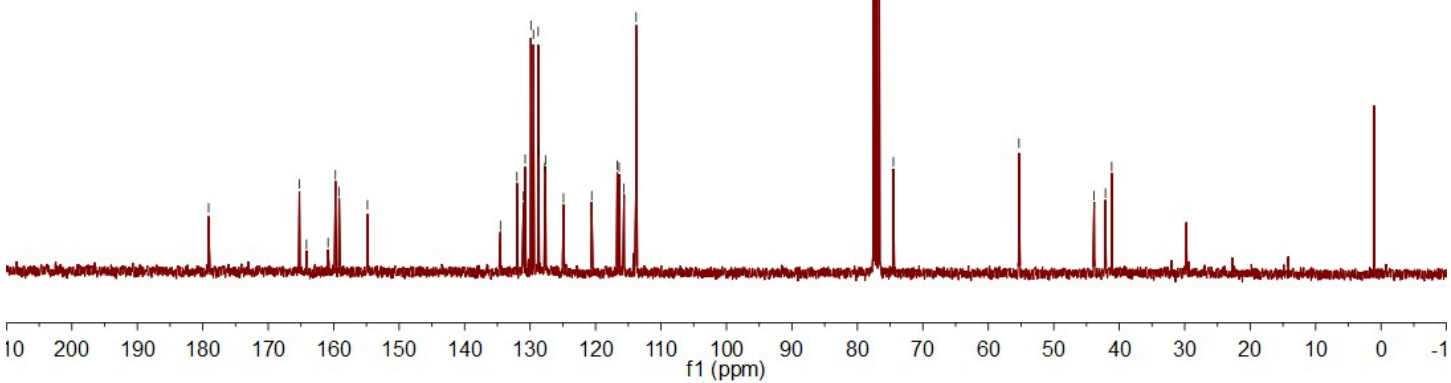


8c ${ }^{19}$ FNMR $\left(282 \mathrm{MHz}, \mathrm{CDCl}_{3}\right)$

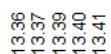

=둔<smiles>COc1ccc(C2=CO[C@H]3CC4(c5ccc(F)cc5)CC(=O)OC(c5ccccc5)=C4C=C3C2=O)cc1</smiles>

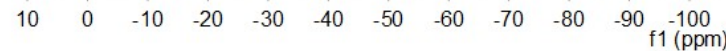

8d ${ }^{1} \mathbf{H}$ NMR (300 MHz, $\left.\mathrm{CDCl}_{3}\right)$

ชำ NTi心o

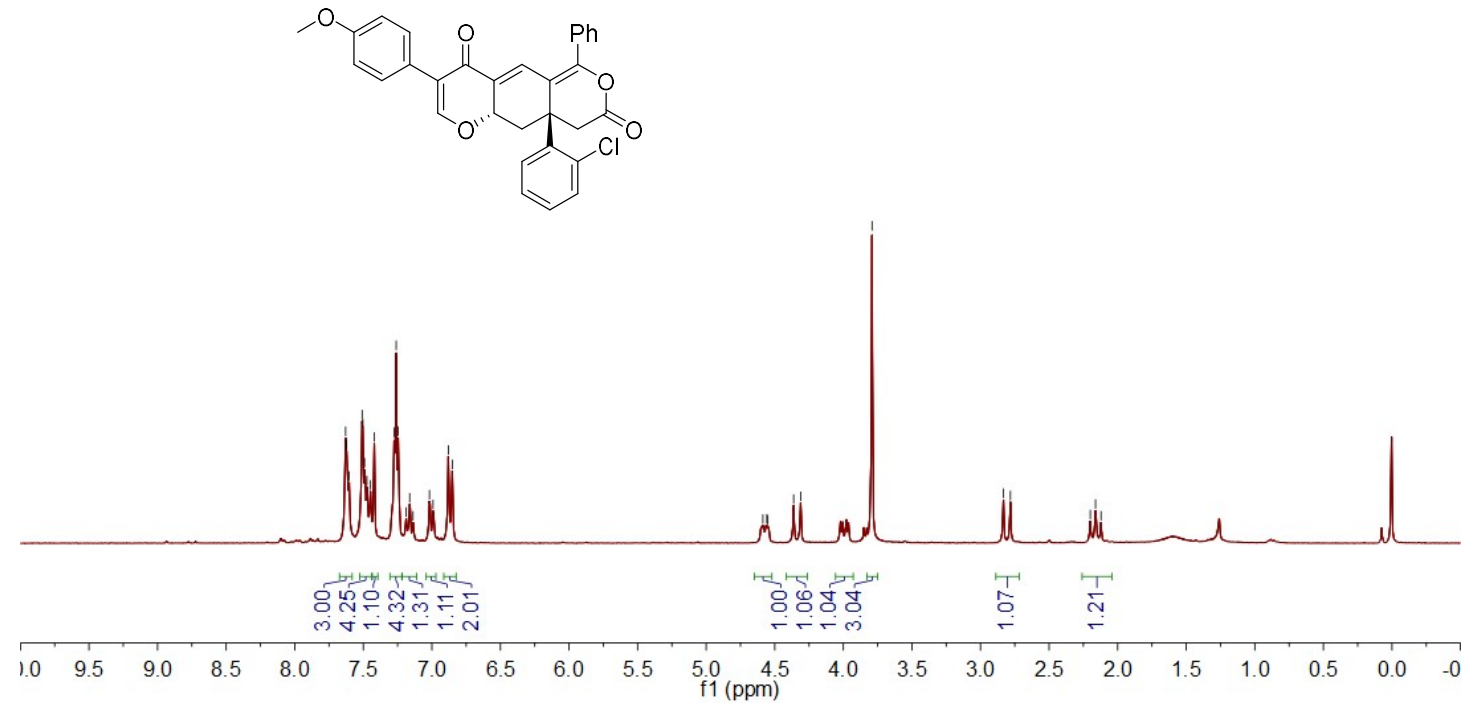


8d ${ }^{13} \mathrm{C}$ NMR $\left(75 \mathrm{MHz}, \mathrm{CDCl}_{3}\right)$

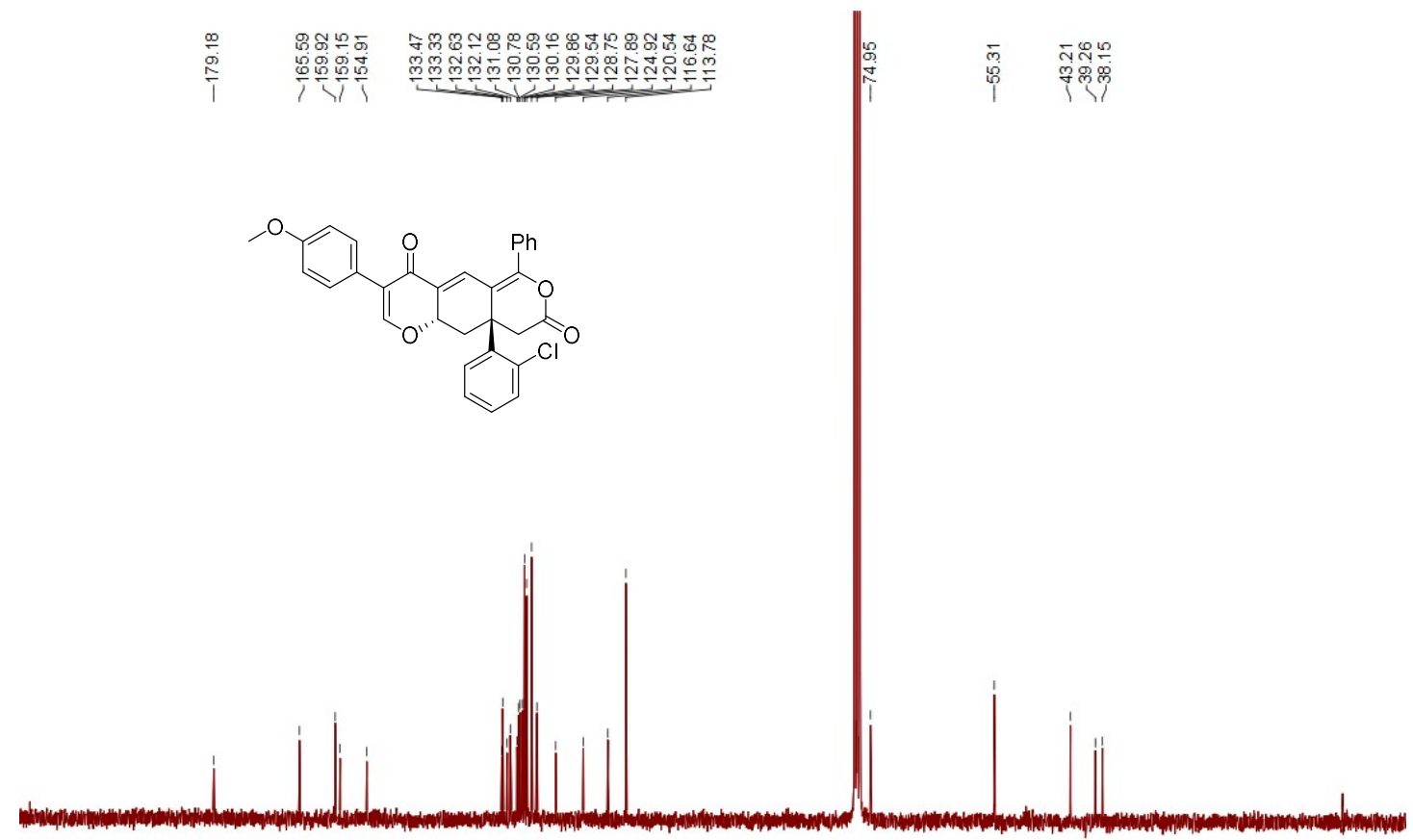

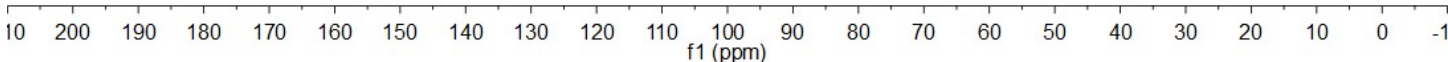

8e ${ }^{1} \mathbf{H}$ NMR $\left(300 \mathrm{MHz}, \mathrm{CDCl}_{3}\right)$

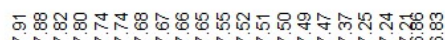

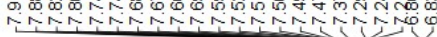

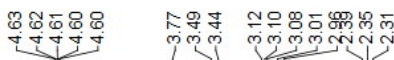
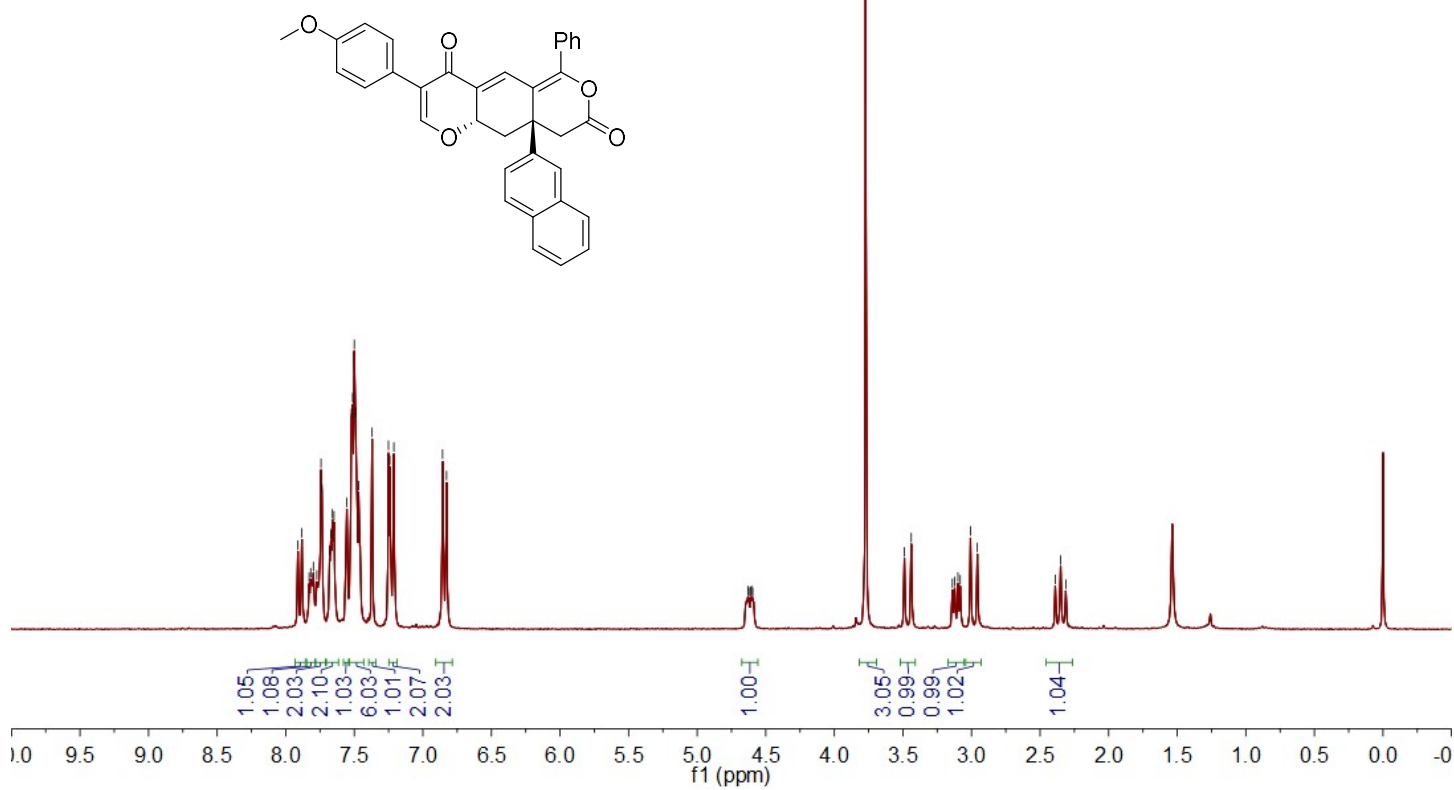
8e ${ }^{13} \mathrm{C}$ NMR $\left(75 \mathrm{MHz}, \mathrm{CDCl}_{3}\right)$

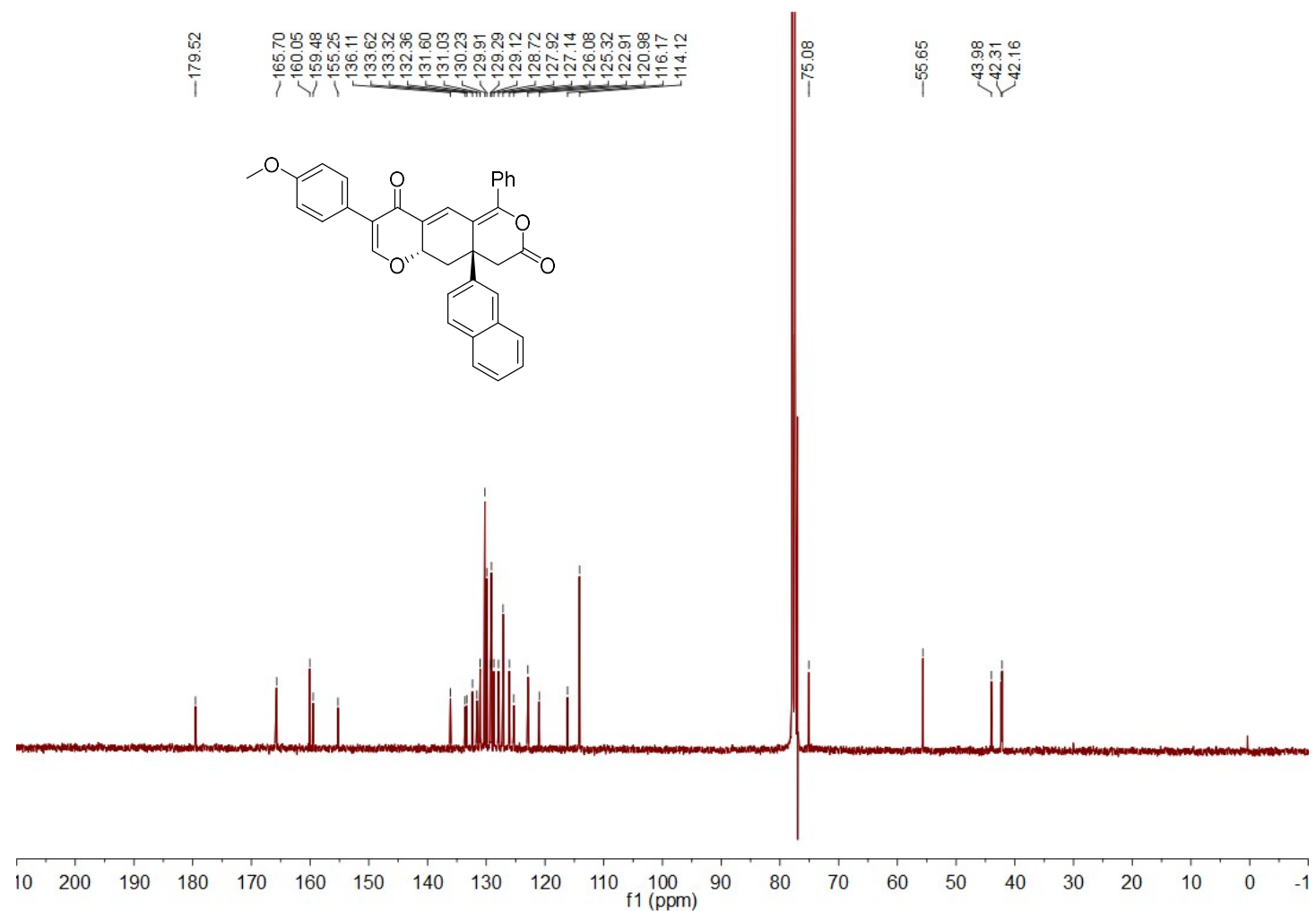

$8 \mathbf{f}^{1} \mathbf{H}$ NMR (300 MHz, $\left.\mathrm{CDCl}_{3}\right)$

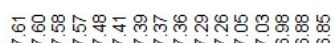
rinntiving

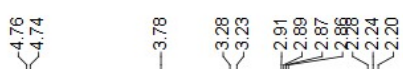

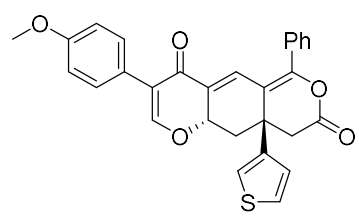

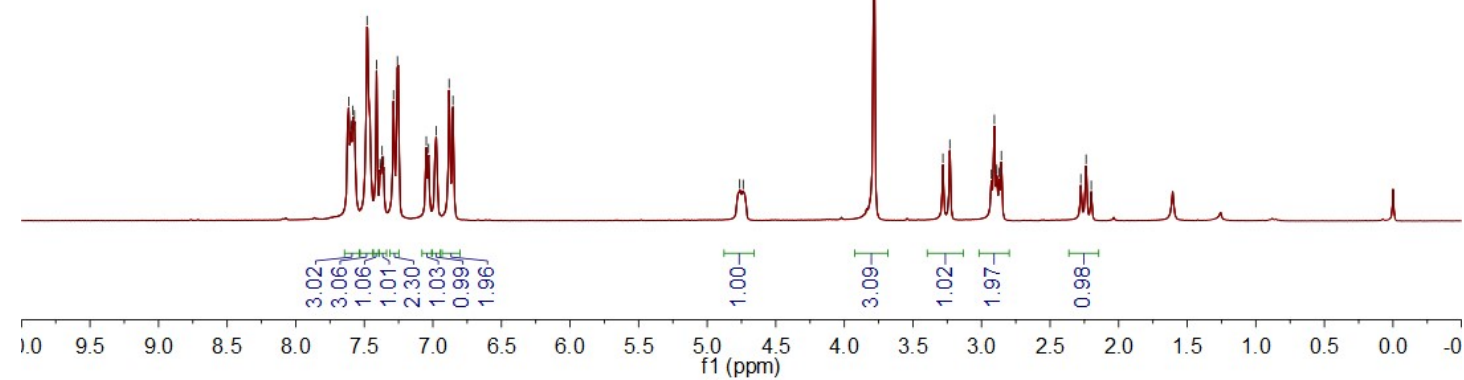


$8 \mathrm{f}^{13} \mathrm{C}$ NMR $\left(75 \mathrm{MHz}, \mathrm{CDCl}_{3}\right)$

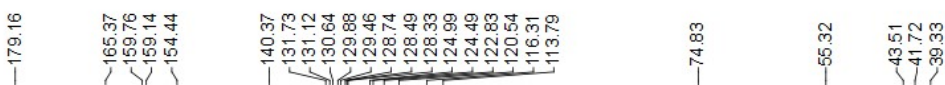

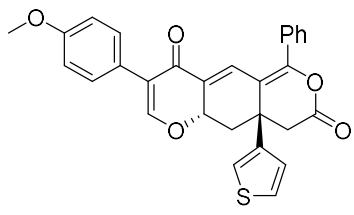

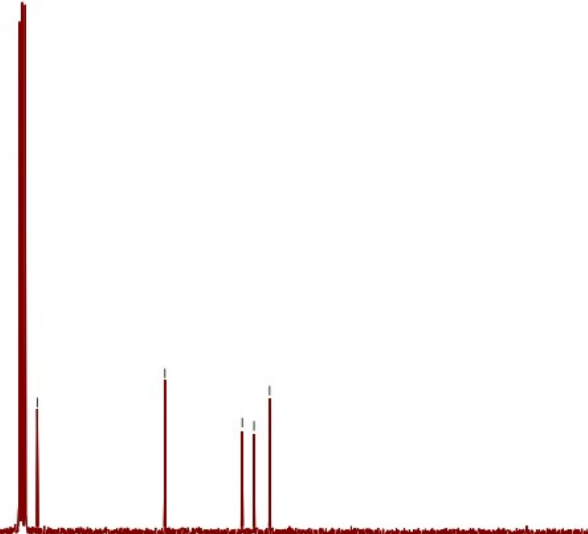

$\begin{array}{lllllllllllllllllllllll}10 & 200 & 190 & 180 & 170 & 160 & 150 & 140 & 130 & 120 & 110 & \begin{array}{c}100 \\ \mathrm{f} 1(\mathrm{ppm})\end{array} & 90 & 80 & 70 & 60 & 50 & 40 & 30 & 20 & 10 & 0 & -1\end{array}$

8g ${ }^{1} \mathrm{H}$ NMR (300 MHz, $\left.\mathrm{CDCl}_{3}\right)$

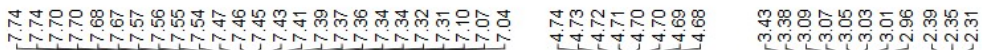<smiles>O=C1CC2(c3ccccc3)CC3OC=C(c4ccc(F)cc4)C(=O)C3=CC2=C1c1ccccc1</smiles>

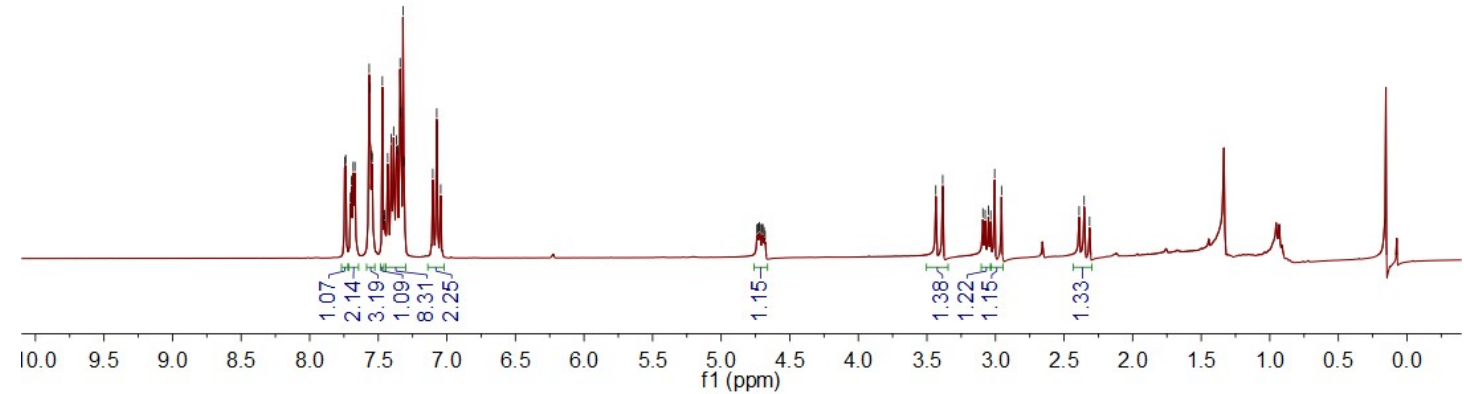


$8{ }^{13} \mathrm{C}$ NMR $\left(75 \mathrm{MHz}, \mathrm{CDCl}_{3}\right)$

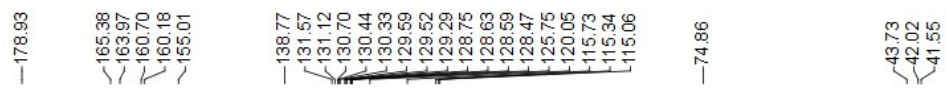<smiles>O=C1CC2(c3ccccc3)C[C@H]3OC=C(c4ccc(F)cc4)C(=O)C3=CC2=C1c1ccccc1</smiles>

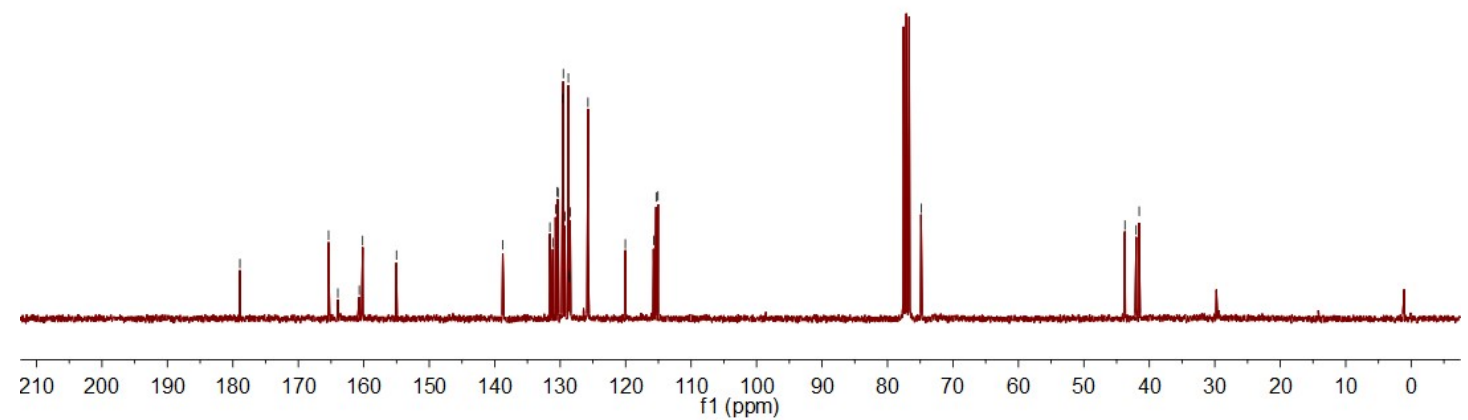

8g ${ }^{19}$ F NMR $\left(282 \mathrm{MHz}, \mathrm{CDCl}_{3}\right)$

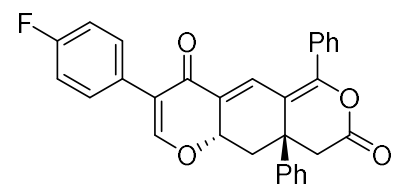

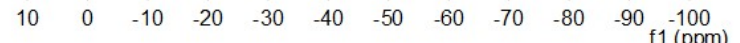


8h ${ }^{1} \mathbf{H}$ NMR $\left(300 \mathrm{MHz}, \mathrm{CDCl}_{3}\right)$

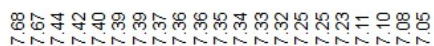

:

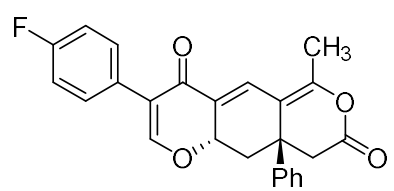

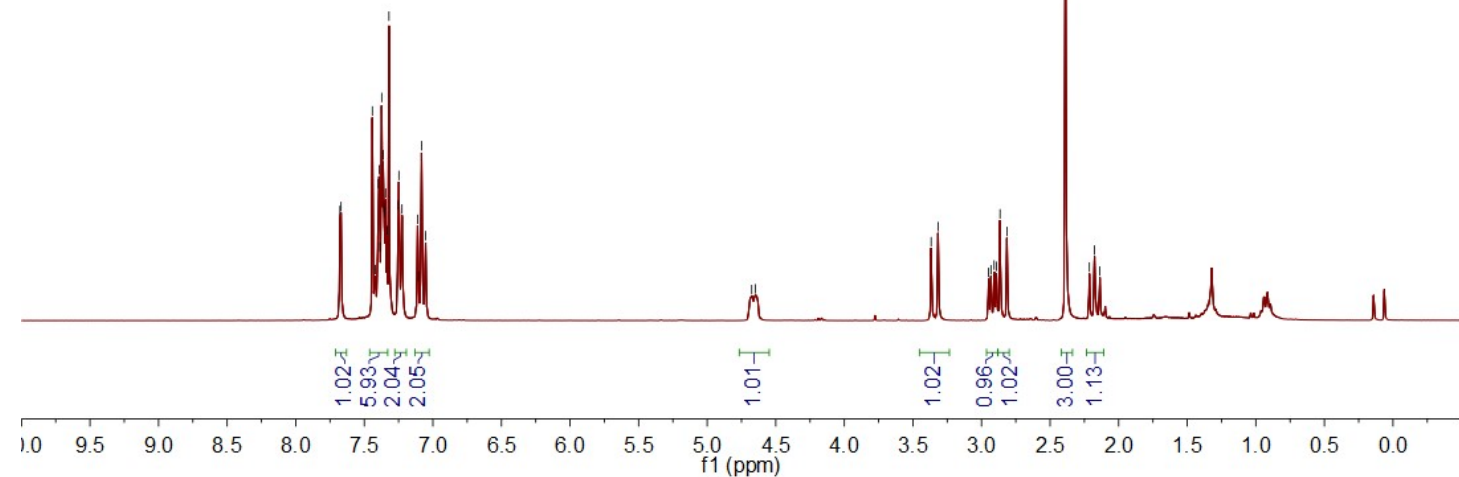

8h ${ }^{13} \mathrm{C}$ NMR (75 MHz, $\left.\mathrm{CDCl}_{3}\right)$

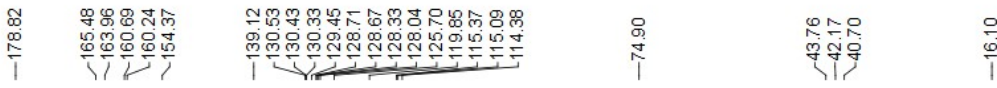

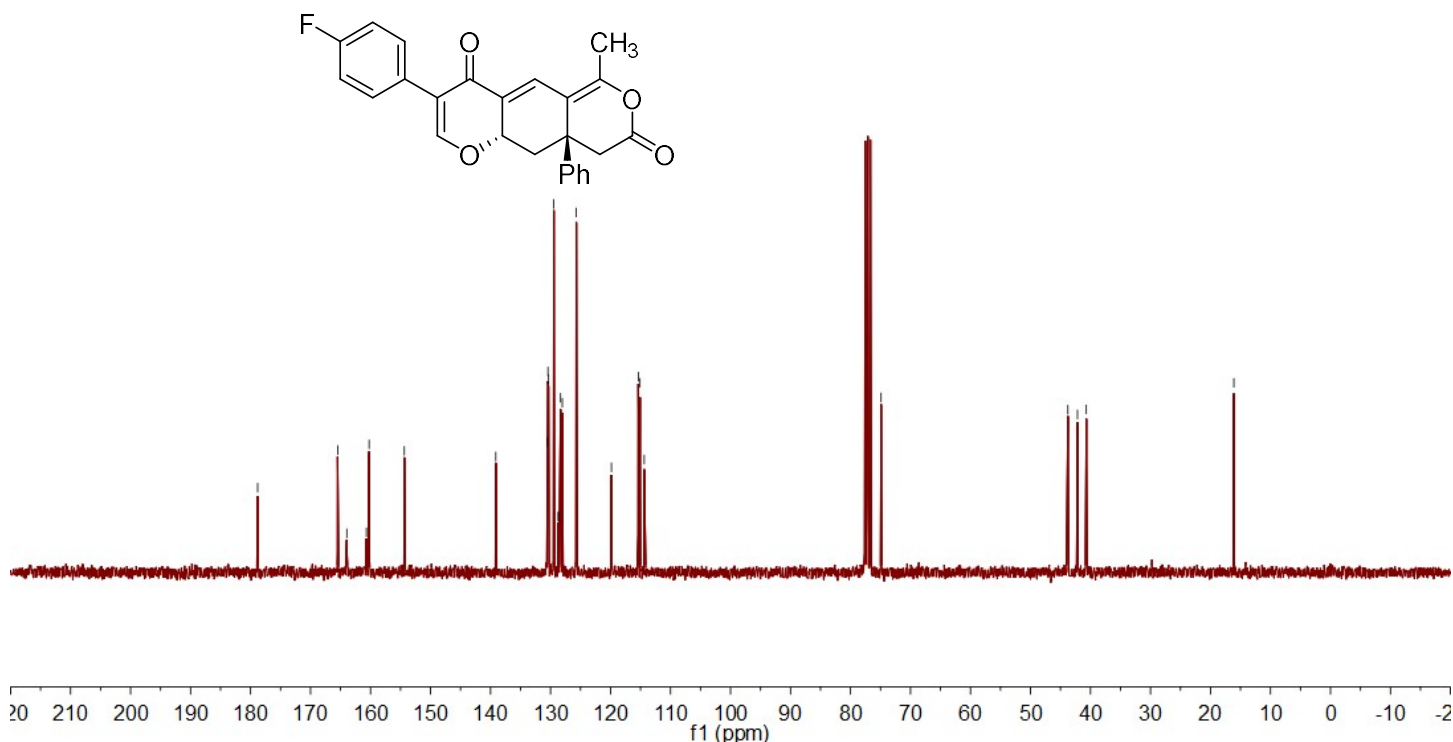


$8 \mathbf{h}{ }^{19} \mathrm{~F}$ NMR $\left(282 \mathrm{MHz}, \mathrm{CDCl}_{3}\right)$

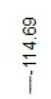<smiles>CC1=CC23CC4COC=C(c5ccc(F)cc5)C4=CC2(CC(=O)O1)C3</smiles>

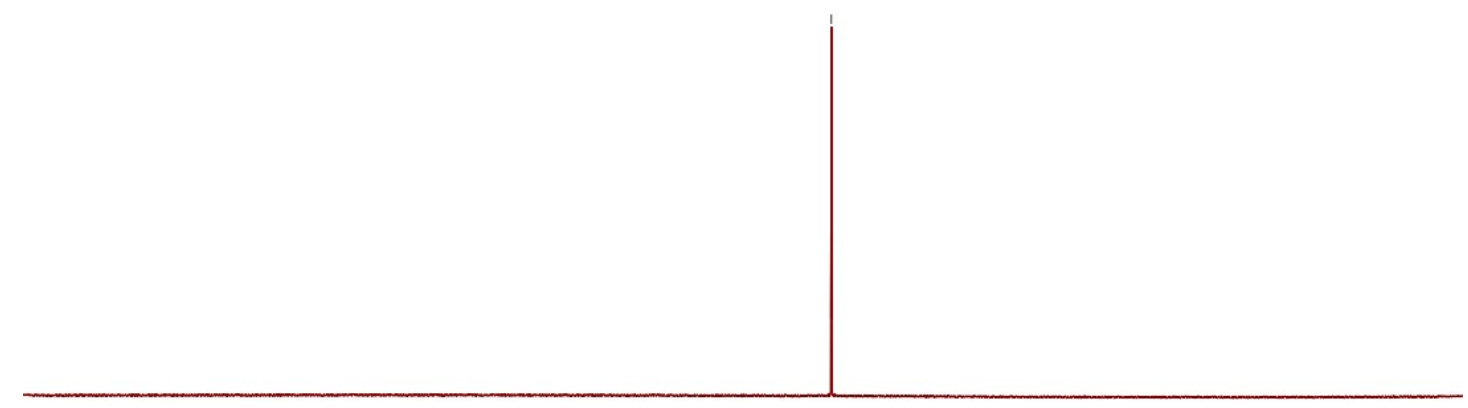

$\begin{array}{lllllllllll}10 & 0 & -10 & -20 & -30 & -40 & -50 & -60 & -70 & -80 & -90 \\ \mathrm{f} 1(\mathrm{ppm})\end{array}$

8i ${ }^{1} \mathbf{H}$ NMR (400 MHz, $\left.\mathrm{CDCl}_{3}\right)$

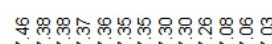
incisinatis

タ๐

Nini nniñ<smiles>CC1=C2C=C3C(=O)C(c4ccc(F)cc4)=CO[C@H]3CC2(C(C)C)CC(=O)O1</smiles>

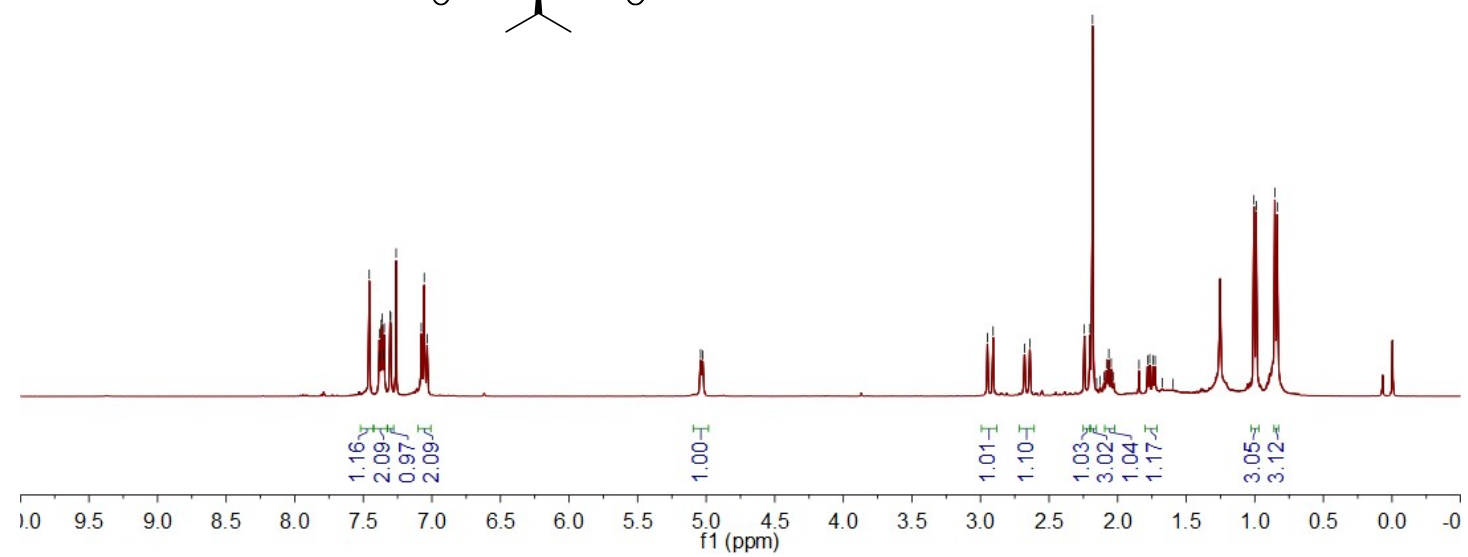


$\mathbf{8 i}^{13} \mathrm{C}$ NMR $\left(75 \mathrm{MHz}, \mathrm{CDCl}_{3}\right)$

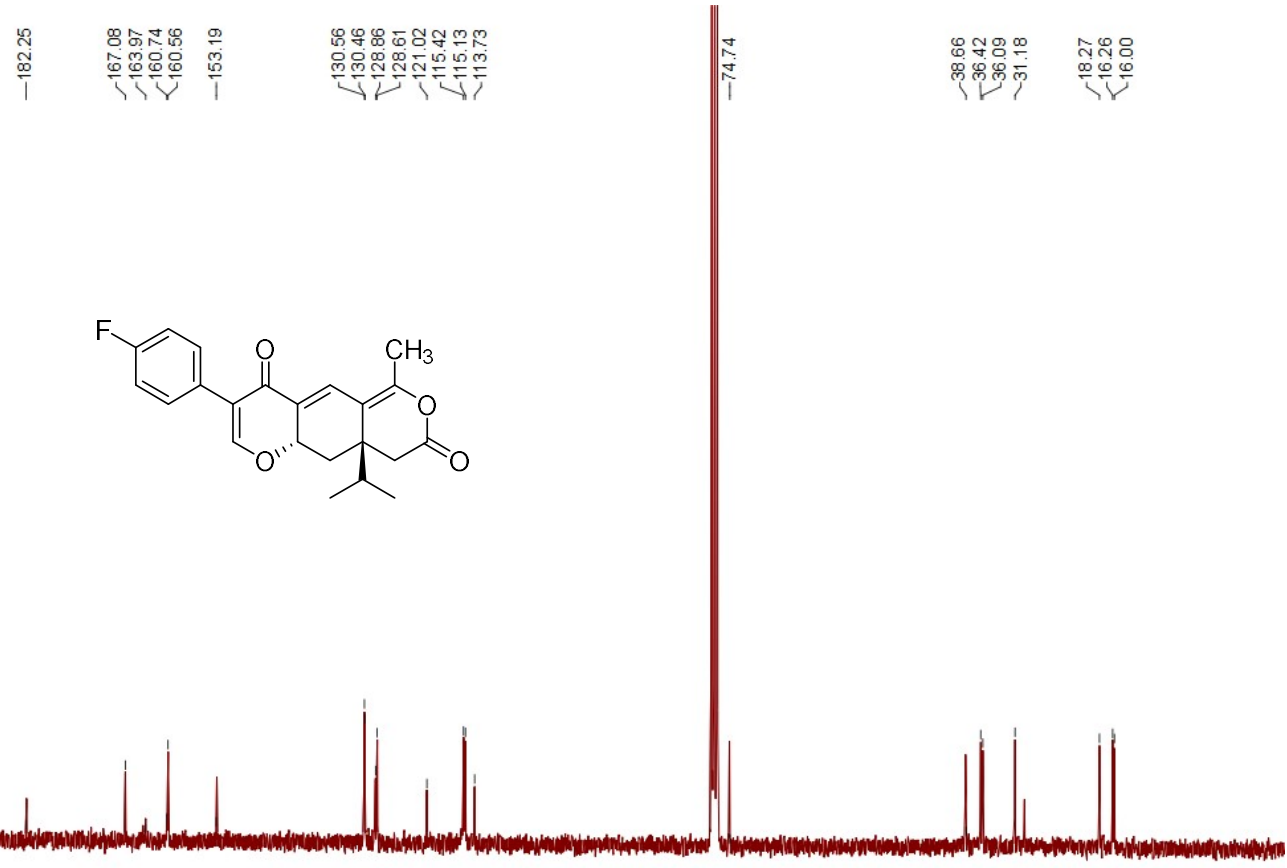

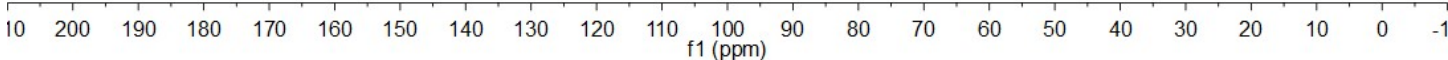

$8 \mathbf{i}^{19} \mathrm{~F}$ NMR $\left(377 \mathrm{MHz}, \mathrm{CDCl}_{3}\right)$

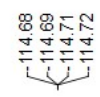<smiles>CC1=CC23CC(C(C)C)OC=C(c4ccc(F)cc4)C2C(=O)CC3CC1=O</smiles>

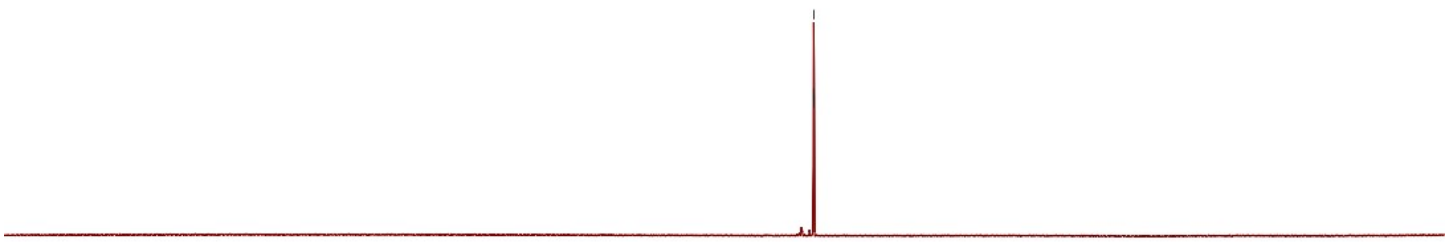

$\begin{array}{llllllllllll}10 & 0 & -10 & -20 & -30 & -40 & -50 & -60 & -70 & -80 & -90 & -100 \\ & & & \end{array}$ $\begin{array}{llllll}-120 & -140 & -160 & -180 & -200\end{array}$ 


\section{HPLC spectra of products}

\section{Racemic 3a}

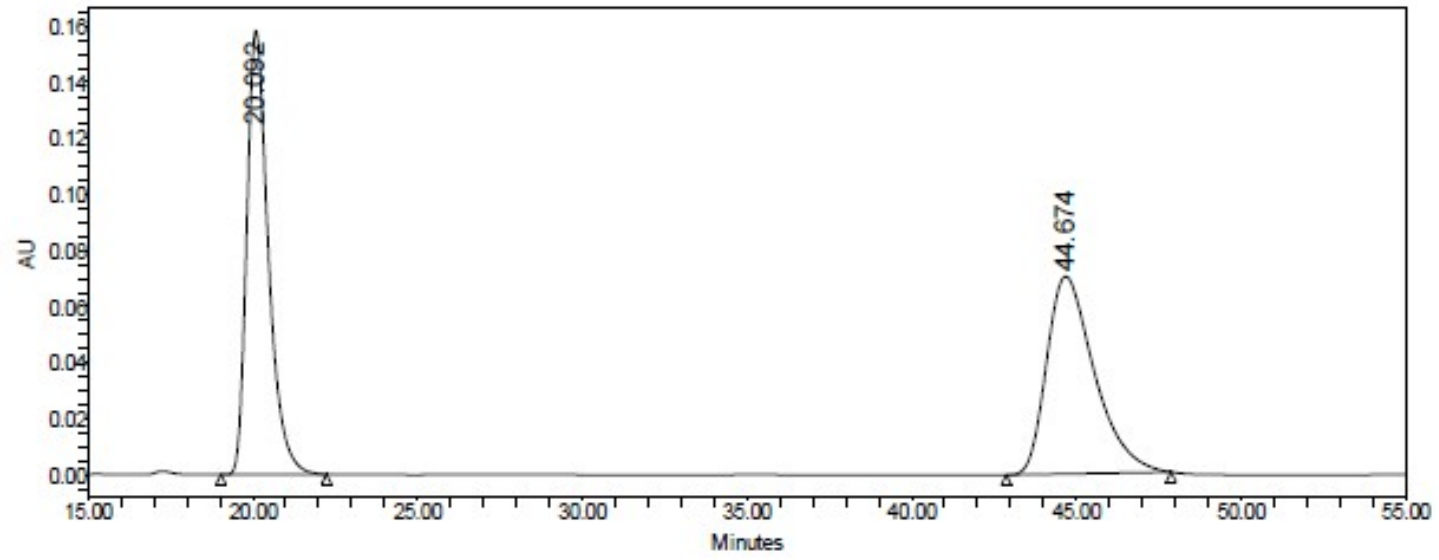

\begin{tabular}{|c|c|l|c|c|c|c|}
\hline & RT (min) & Int Type & $\begin{array}{c}\text { Width } \\
(\mathrm{sec})\end{array}$ & Area & Height & \% Area \\
\hline 1 & 20.092 & $\mathrm{bb}$ & 192.000 & 7197409 & 158387 & 50.36 \\
\hline 2 & 44.674 & $\mathrm{bb}$ & 300.500 & 7095690 & 70444 & 49.64 \\
\hline Sum & & & & 14293099.0 & 228831.8 & 100.0 \\
\hline
\end{tabular}

\section{Enantioenriched 3a}

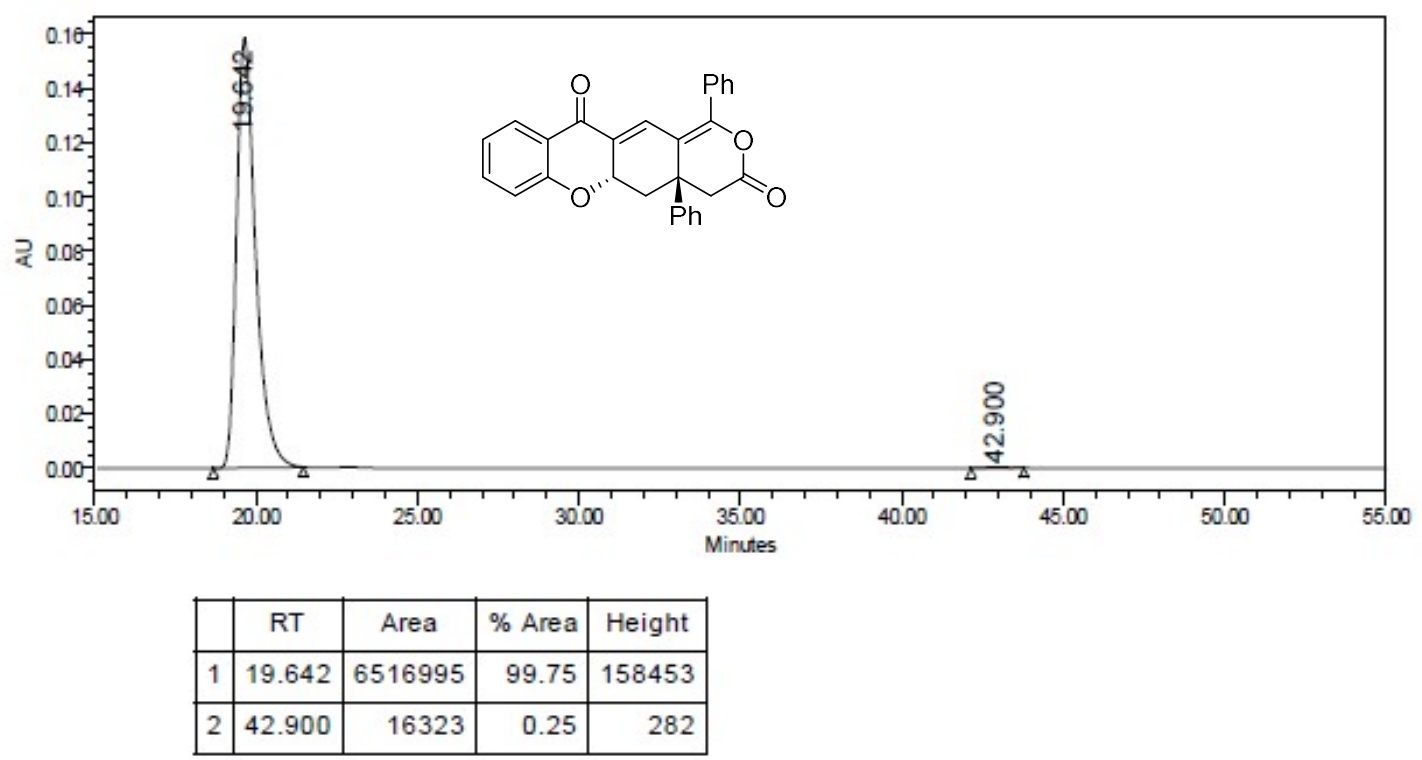




\section{Racemic 3a'}

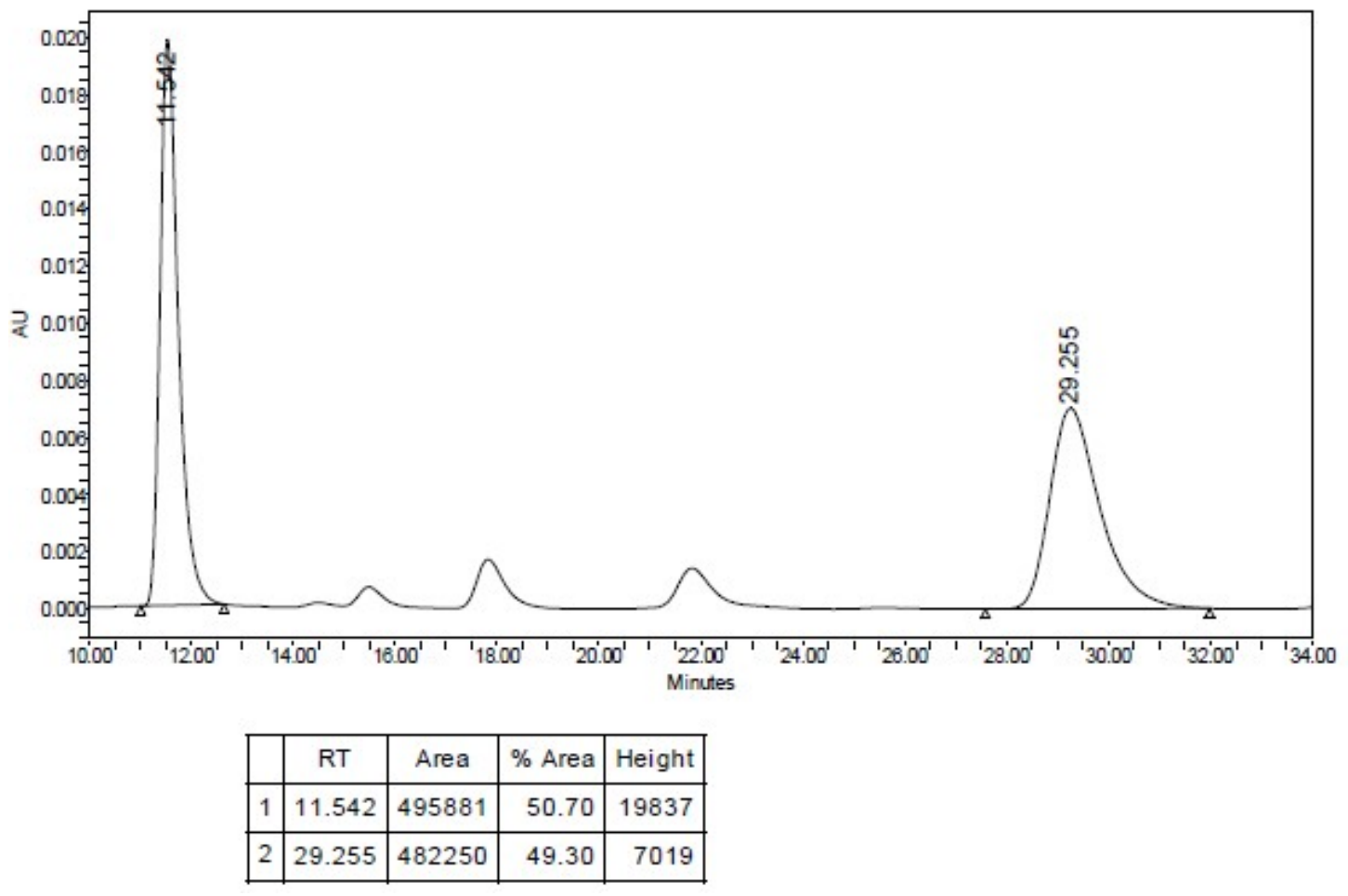

\section{Enantioenriched 3a'}

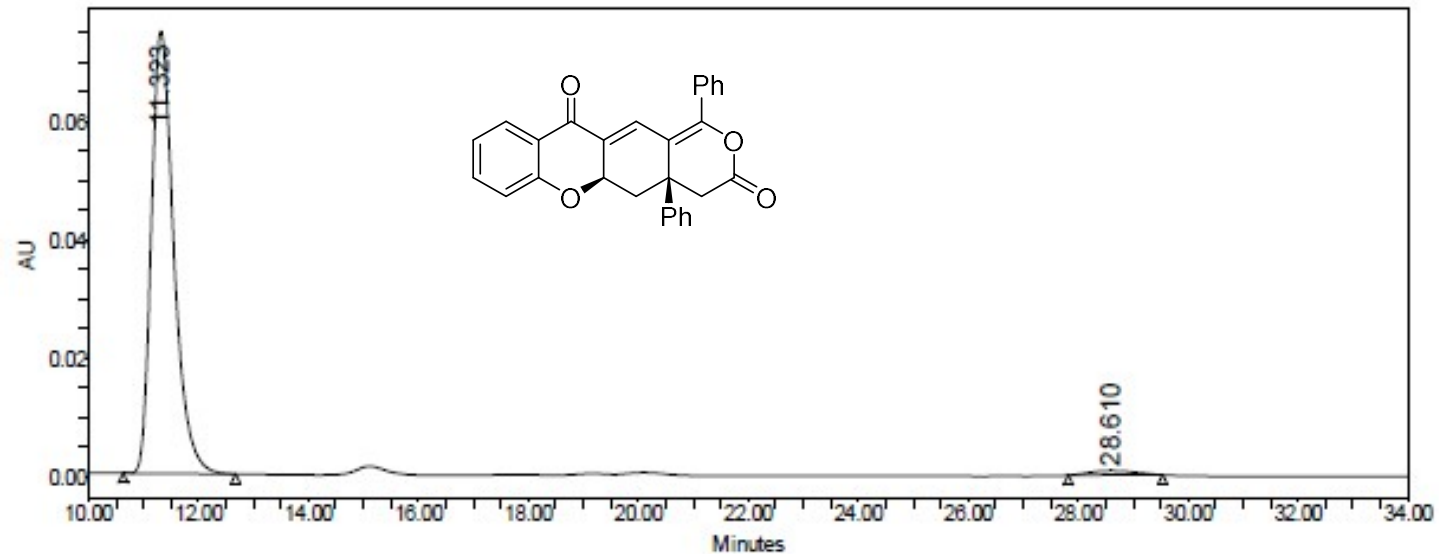

\begin{tabular}{|c|c|l|c|c|l|l|}
\hline & RT (min) & Int Type & $\begin{array}{c}\text { Width } \\
(\mathrm{sec})\end{array}$ & Area & Height & \% Area \\
\hline 1 & 11.323 & bb & 123.000 & 2118037 & 74817 & 97.99 \\
\hline 2 & 28.610 & bb & 103.000 & 43344 & 765 & 2.01 \\
\hline Sum & & & & 2161381.3 & 75581.6 & 100.0 \\
\hline
\end{tabular}




\section{Racemic 3b}

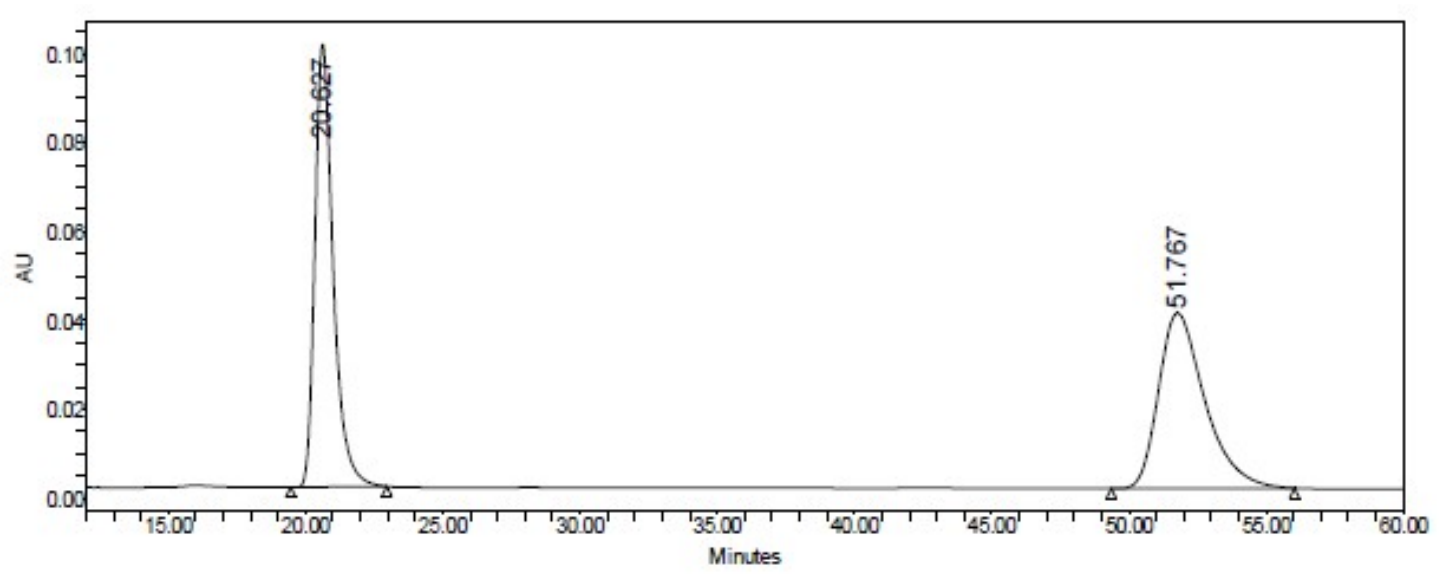

\begin{tabular}{|c|l|l|c|c|c|c|}
\hline & RT (min) & Int Type & $\begin{array}{c}\text { Width } \\
(\mathrm{sec})\end{array}$ & Area & Height & $\%$ Area \\
\hline 1 & 20.627 & $\mathrm{bb}$ & 210.000 & 4629157 & 99682 & 49.98 \\
\hline 2 & 51.767 & $\mathrm{bb}$ & 401.000 & 4633036 & 39522 & 50.02 \\
\hline Sum & & & & 9262193.2 & 139204.3 & 100.0 \\
\hline
\end{tabular}

\section{Enantioenriched 3b}

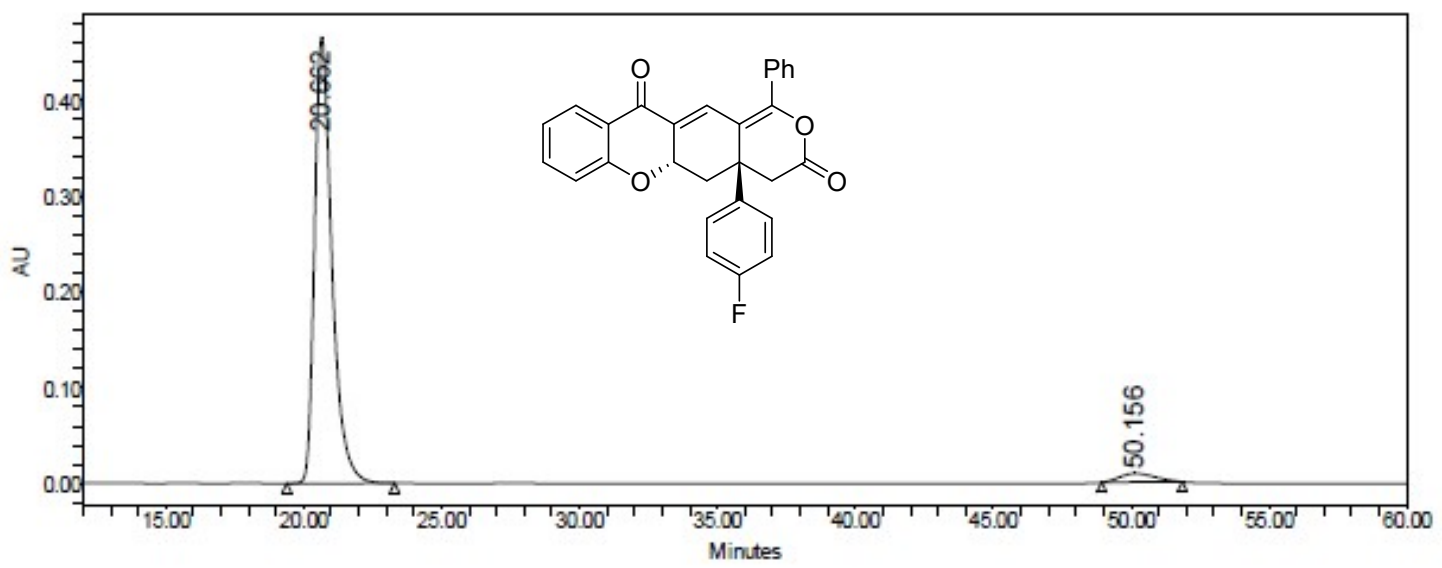

\begin{tabular}{|c|c|l|c|c|l|l|}
\hline & RT(min) & Int Type & $\begin{array}{c}\text { Width } \\
(\mathrm{sec})\end{array}$ & Area & Height & \% Area \\
\hline 1 & 20.662 & bb & 234.000 & 20431503 & 466087 & 96.20 \\
\hline 2 & 50.156 & bb & 175.000 & 806536 & 9084 & 3.80 \\
\hline Sum & & & & 21238039.5 & 475171.1 & 100.0 \\
\hline
\end{tabular}




\section{Racemic 3b'}

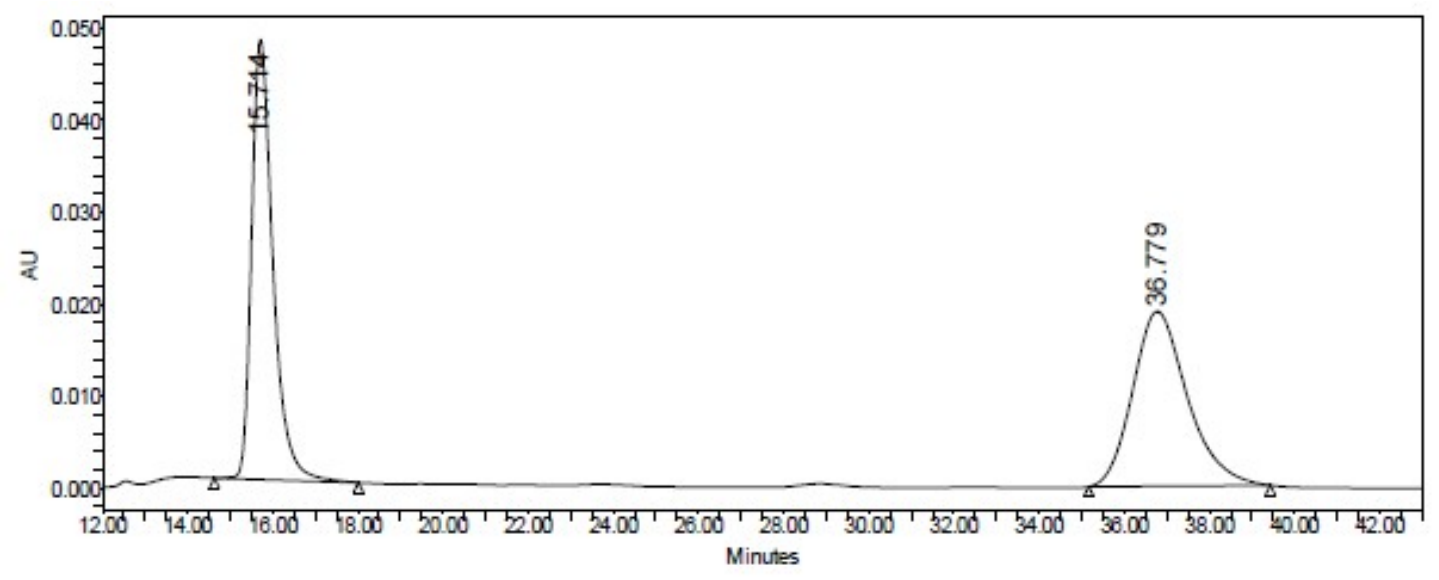

\begin{tabular}{|c|c|l|c|c|c|c|}
\hline & RT(min) & Int Type & $\begin{array}{c}\text { Width } \\
(\mathrm{sec})\end{array}$ & Area & Height & $\%$ Area \\
\hline 1 & 15.714 & bb & 203.000 & 1664344 & 47777 & 49.55 \\
\hline 2 & 36.779 & bb & 256.000 & 1694460 & 18990 & 50.45 \\
\hline Sum & & & & 3358803.2 & 66767.4 & 100.0 \\
\hline
\end{tabular}

\section{Enantioenriched 3b'}

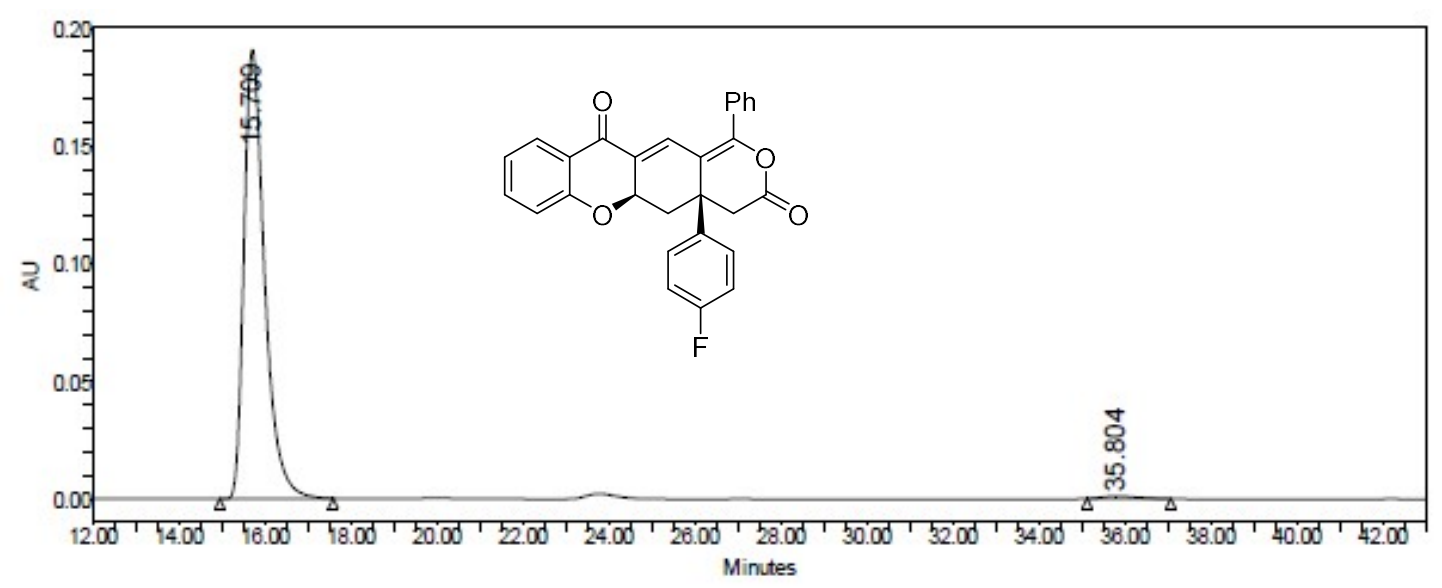

\begin{tabular}{|c|c|c|c|c|c|l|}
\hline & RT (min) & Int Type & $\begin{array}{c}\text { Width } \\
(\mathrm{sec})\end{array}$ & Area & Height & \% Area \\
\hline 1 & 15.709 & bb & 158.000 & 6348328 & 190665 & 99.06 \\
\hline 2 & 35.804 & bb & 117.000 & 60015 & 1000 & 0.94 \\
\hline Sum & & & & 6408342.9 & 191664.7 & 100.0 \\
\hline
\end{tabular}




\section{Racemic 3c}

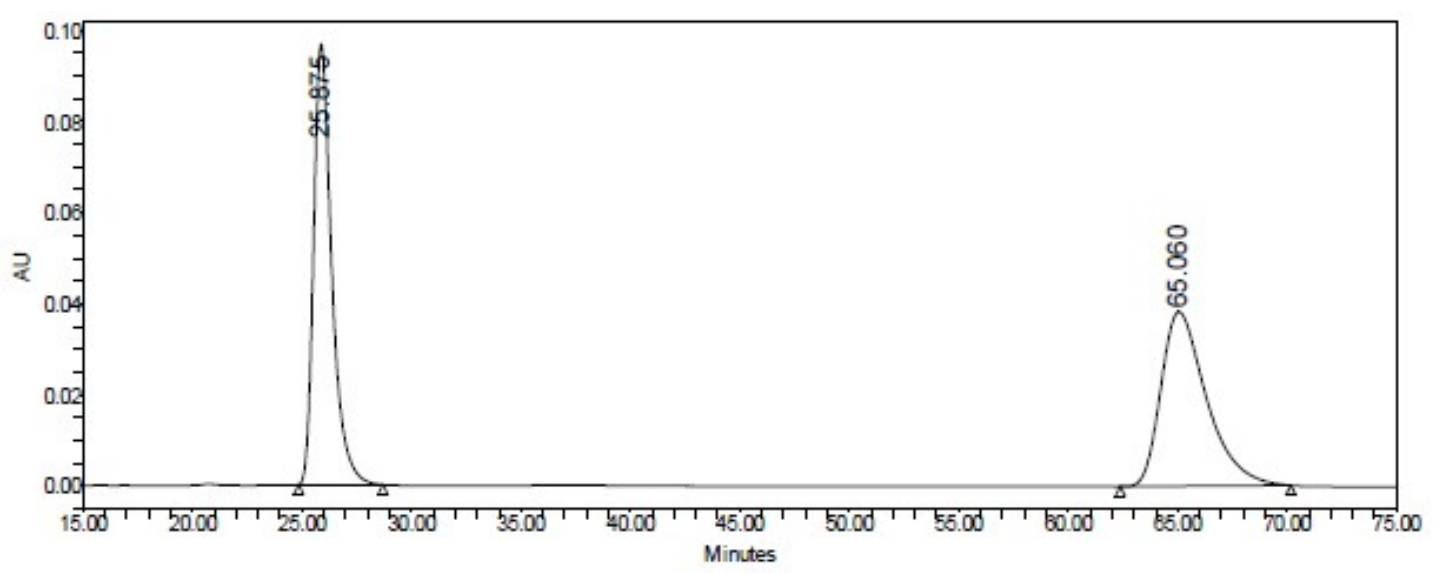

\begin{tabular}{|c|c|c|c|c|c|c|}
\hline & RT (min) & Int Type & $\begin{array}{c}\text { Width } \\
(\mathrm{sec})\end{array}$ & Area & Height & $\%$ Area \\
\hline 1 & 25.875 & bb & 231.000 & 5559192 & 96795 & 50.04 \\
\hline 2 & 65.060 & bb & 467.000 & 5550388 & 38341 & 49.96 \\
\hline Sum & & & & 11109579.3 & 135135.2 & 100.0 \\
\hline
\end{tabular}

\section{Enantioenriched 3c}

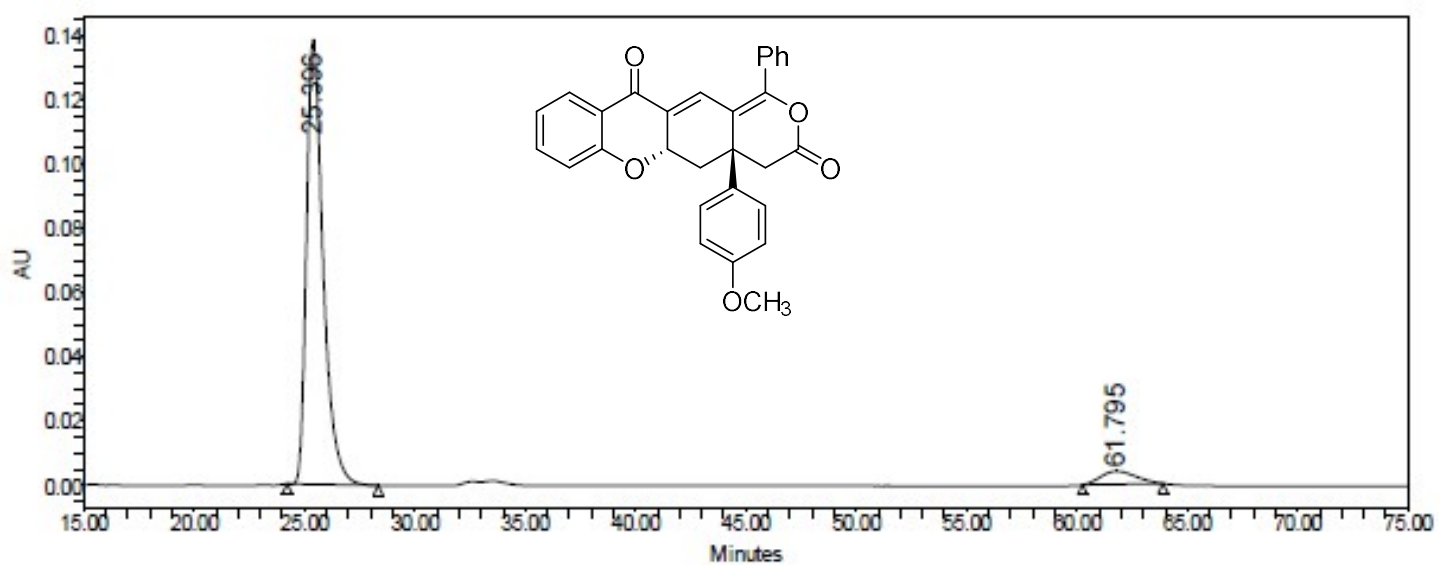

\begin{tabular}{|c|l|l|c|c|l|l|}
\hline & RT (min) & Int Type & $\begin{array}{c}\text { Width } \\
(\mathrm{sec})\end{array}$ & Area & Height & \% Area \\
\hline 1 & 25.396 & bb & 247.000 & 7219420 & 138276 & 94.08 \\
\hline 2 & 61.795 & bb & 219.000 & 454137 & 4098 & 5.92 \\
\hline Sum & & & & 7673556.4 & 142374.4 & 100.0 \\
\hline
\end{tabular}




\section{Racemic 3c'}

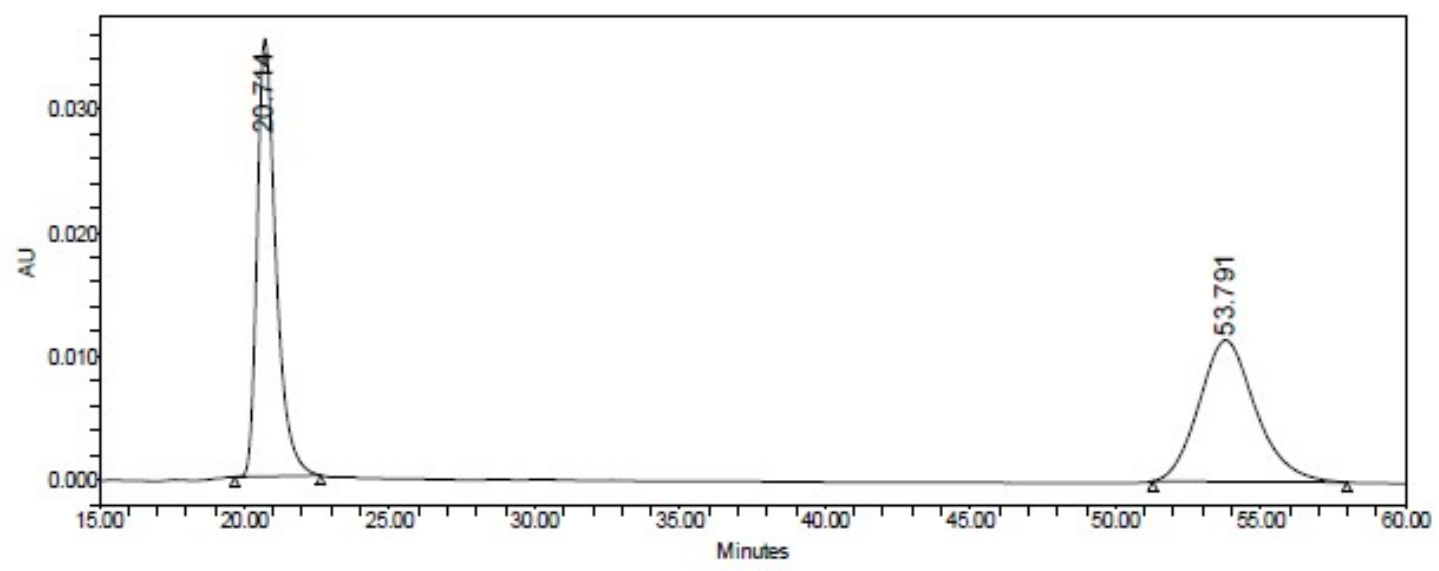

\begin{tabular}{|c|c|l|c|c|c|c|}
\hline & RT (min) & Int Type & $\begin{array}{c}\text { Width } \\
(\mathrm{sec})\end{array}$ & Area & Height & $\%$ Area \\
\hline 1 & 20.714 & $\mathrm{bb}$ & 178.000 & 1591374 & 35365 & 50.59 \\
\hline 2 & 53.791 & $\mathrm{bb}$ & 401.000 & 1554221 & 11434 & 49.41 \\
\hline Sum & & & & 3145595.3 & 46799.2 & 100.0 \\
\hline
\end{tabular}

\section{Enantioenriched 3c'}

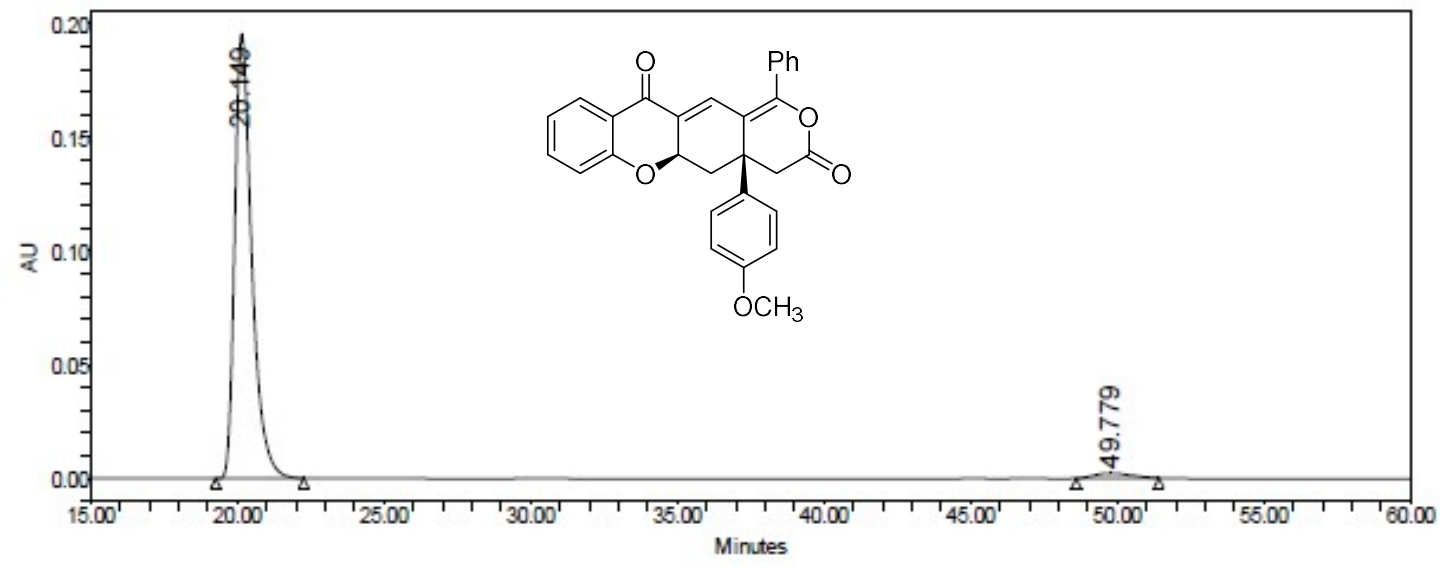

\begin{tabular}{|c|c|l|c|c|c|l|}
\hline & RT (min) & Int Type & $\begin{array}{c}\text { Width } \\
(\mathrm{sec})\end{array}$ & Area & Height & \% Area \\
\hline 1 & 20.149 & bb & 180.000 & 7918301 & 195717 & 97.65 \\
\hline 2 & 49.779 & bb & 169.000 & 190785 & 2242 & 2.35 \\
\hline Sum & & & & 8109085.8 & 197958.3 & 100.0 \\
\hline
\end{tabular}




\section{Racemic 3d}

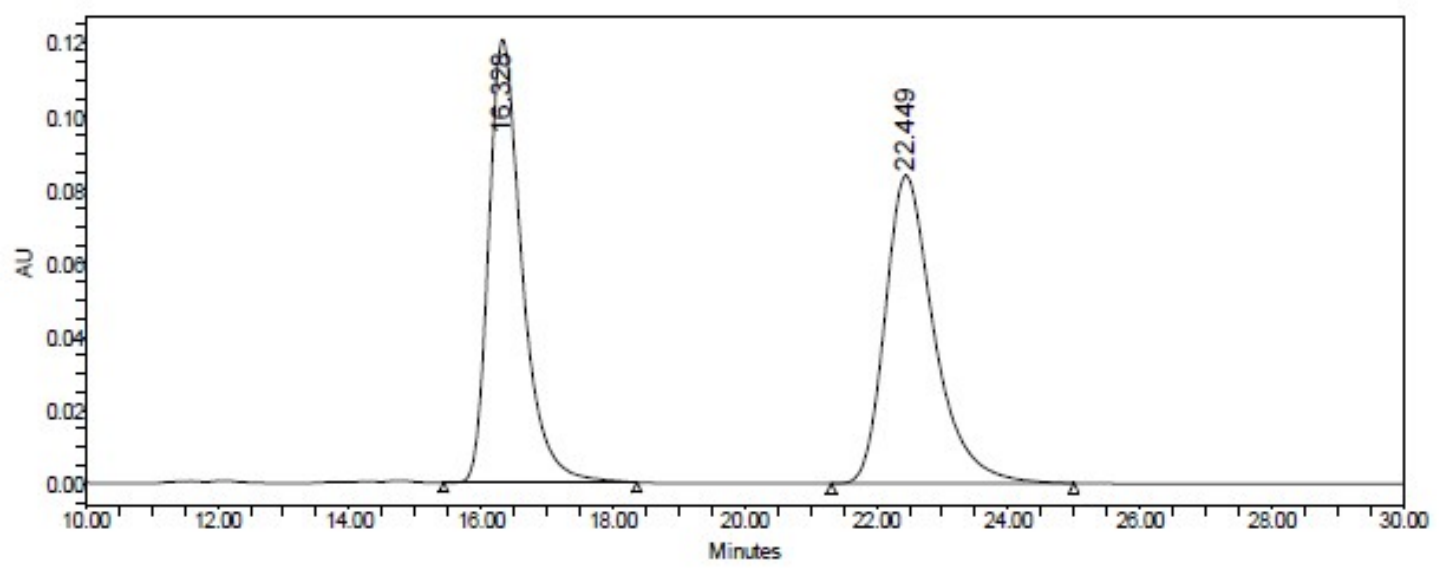

\begin{tabular}{|c|l|l|c|c|l|l|}
\hline & RT (min) & Int Type & $\begin{array}{c}\text { Width } \\
(\mathrm{sec})\end{array}$ & Area & Height & \% Area \\
\hline 1 & 16.328 & bb & 176.000 & 4236801 & 120658 & 49.47 \\
\hline 2 & 22.449 & bb & 221.000 & 4327219 & 83833 & 50.53 \\
\hline Sum & & & & 8564019.8 & 204491.2 & 100.0 \\
\hline
\end{tabular}

\section{Enantioenriched 3d}

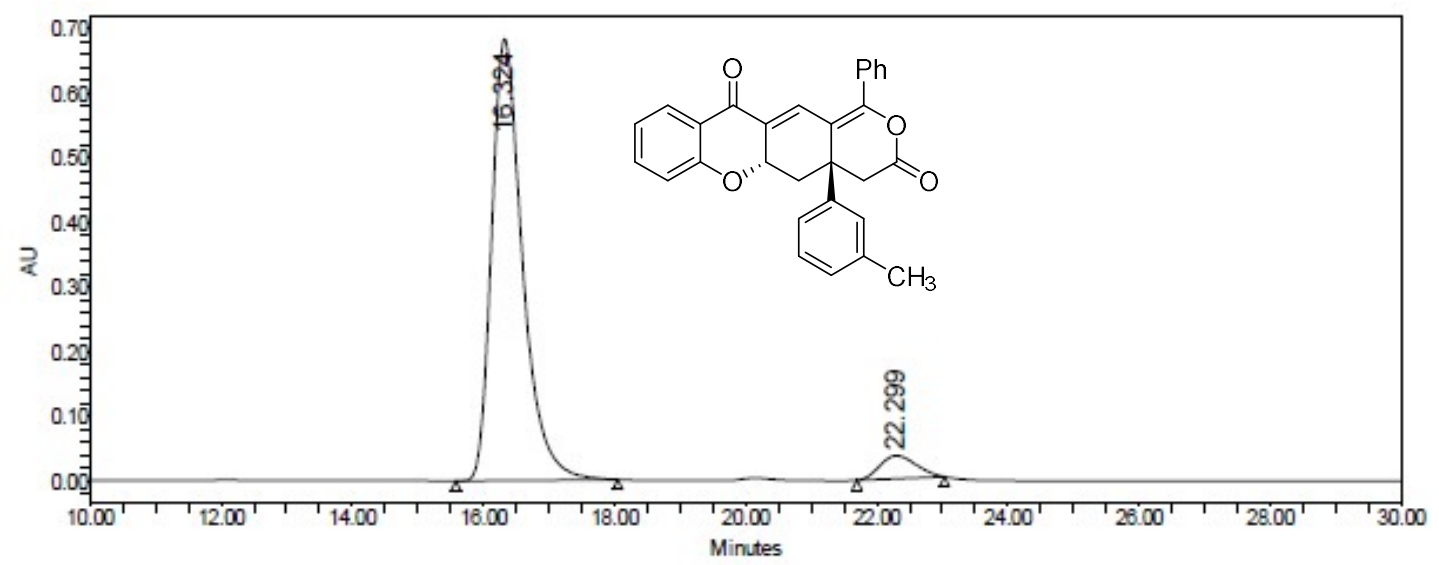

\begin{tabular}{|c|l|l|c|c|l|l|}
\hline & $R T(\min )$ & Int Type & $\begin{array}{c}\text { Width } \\
(\mathrm{sec})\end{array}$ & Area & Height & \% Area \\
\hline 1 & 16.324 & bb & 148.000 & 22335554 & 683466 & 94.21 \\
\hline 2 & 22.299 & bb & 80.000 & 1373601 & 35673 & 5.79 \\
\hline Sum & & & & 23709154.8 & 719139.0 & 100.0 \\
\hline
\end{tabular}




\section{Racemic 3d'}

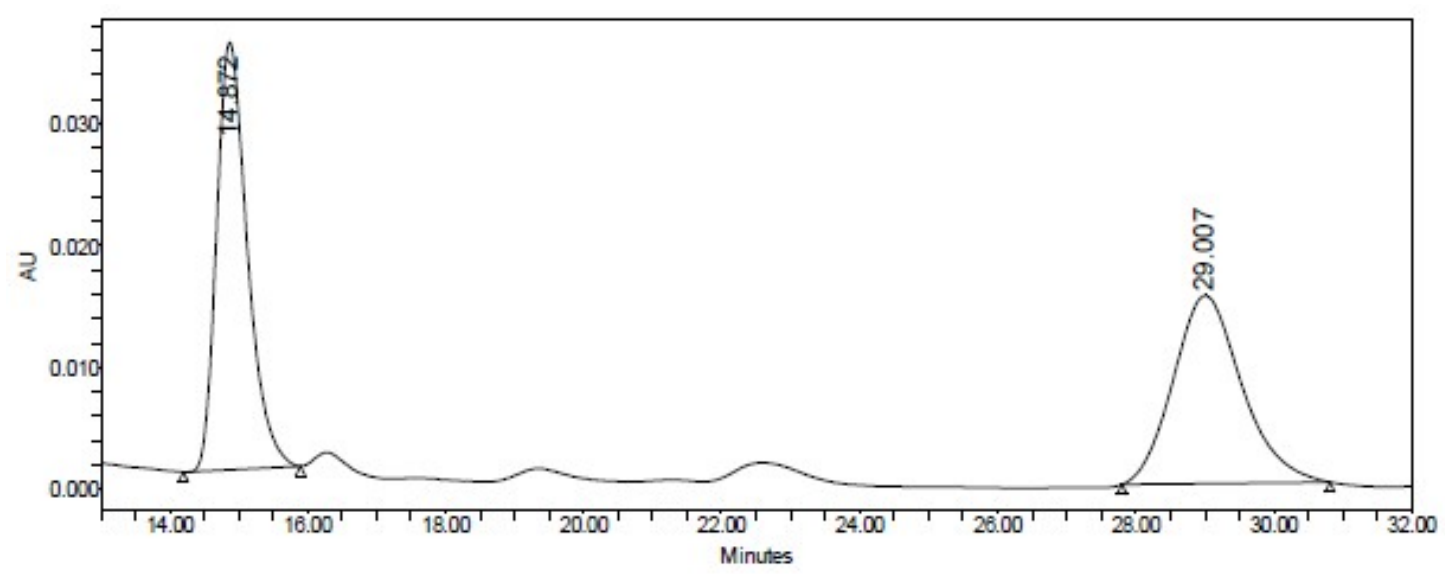

\begin{tabular}{|c|c|c|c|c|c|c|}
\hline & RT (min) & Int Type & $\begin{array}{c}\text { Width } \\
(\text { sec })\end{array}$ & Area & Height & $\%$ Area \\
\hline 1 & 14.872 & bb & 102.000 & 1086205 & 35079 & 50.45 \\
\hline 2 & 29.007 & bb & 181.000 & 1066734 & 15422 & 49.55 \\
\hline Sum & & & & 2152939.5 & 50501.7 & 100.0 \\
\hline
\end{tabular}

\section{Enantioenriched 3d'}

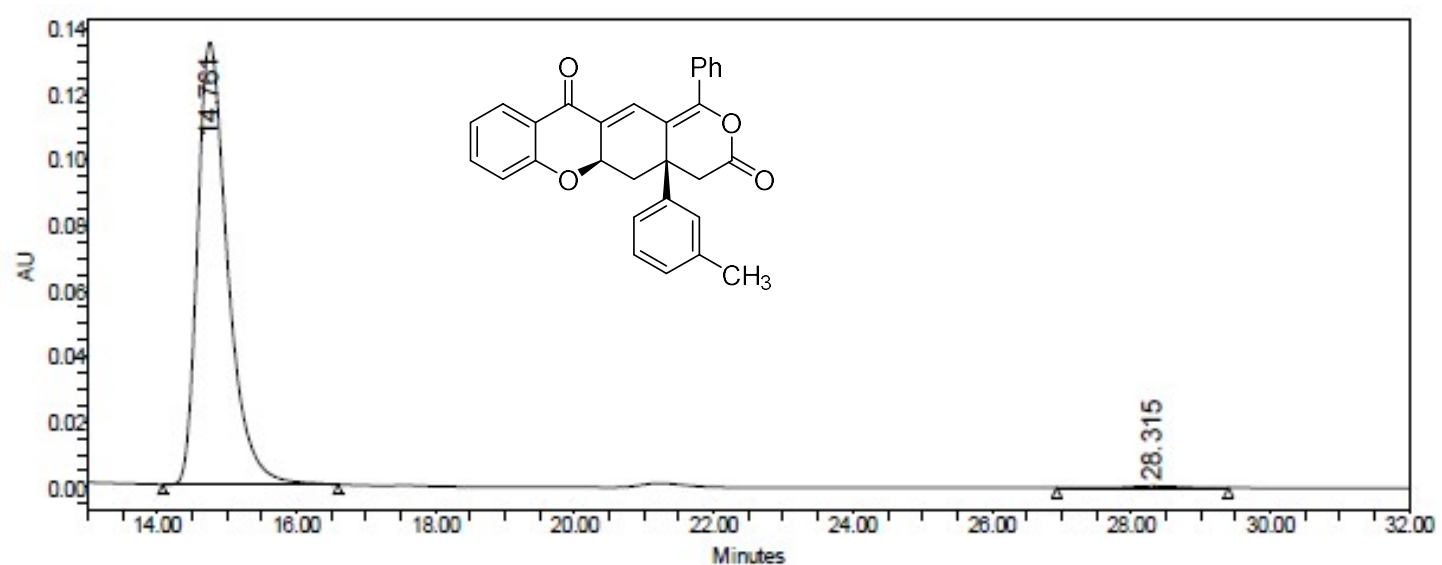

\begin{tabular}{|c|l|l|c|c|c|l|}
\hline & RT (min) & Int Type & $\begin{array}{c}\text { Width } \\
(\mathrm{sec})\end{array}$ & Area & Height & \% Area \\
\hline 1 & 14.761 & bb & 151.000 & 4077855 & 135140 & 99.28 \\
\hline 2 & 28.315 & bb & 148.000 & 29764 & 553 & 0.72 \\
\hline Sum & & & & 4107618.9 & 135692.3 & 100.0 \\
\hline
\end{tabular}




\section{Racemic 3e}

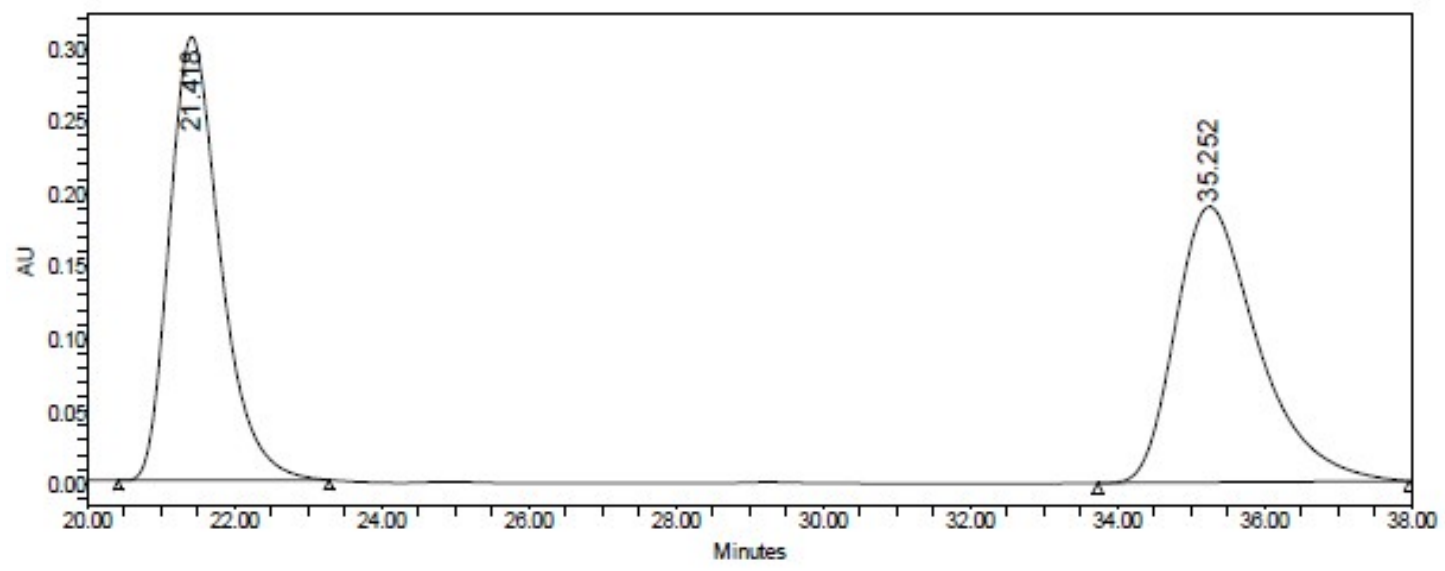

\begin{tabular}{|c|c|l|c|c|c|c|}
\hline & RT (min) & Int Type & $\begin{array}{c}\text { Width } \\
(\mathrm{sec})\end{array}$ & Area & Height & \% Area \\
\hline 1 & 21.418 & bb & 172.000 & 14666241 & 305499 & 49.68 \\
\hline 2 & 35.252 & bb & 254.000 & 14852400 & 189976 & 50.32 \\
\hline Sum & & & & 29518640.9 & 495475.1 & 100.0 \\
\hline
\end{tabular}

\section{Racemic 3e}

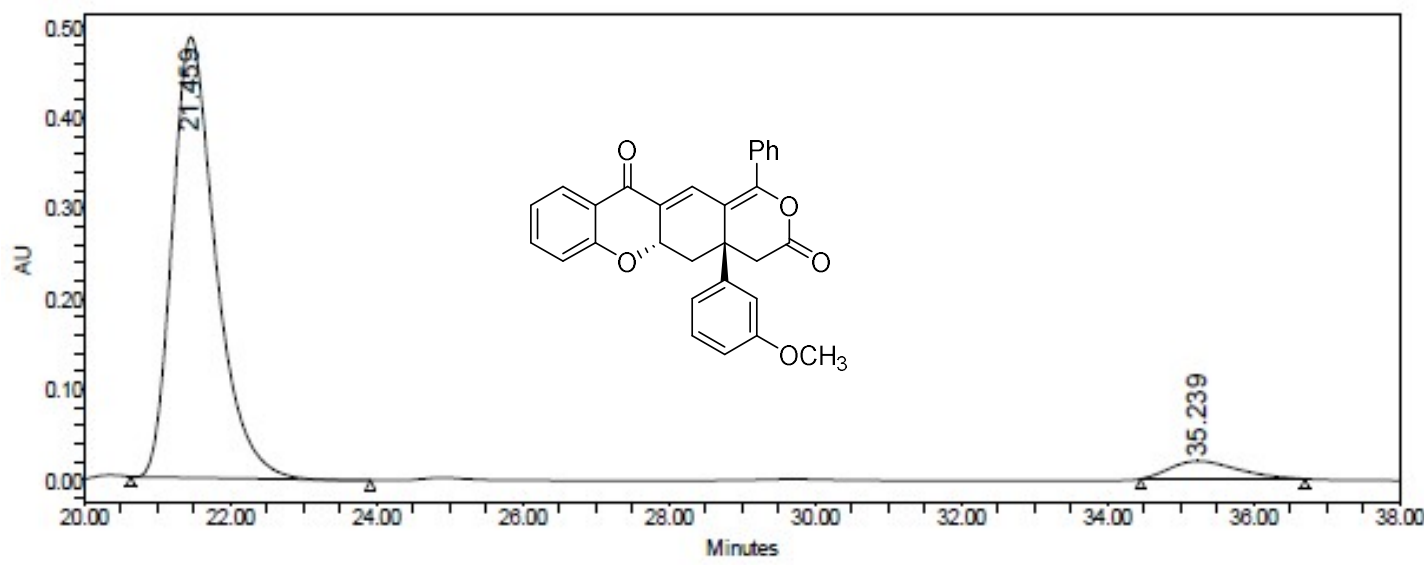

\begin{tabular}{|c|l|l|c|c|c|l|}
\hline & RT (min) & Int Type & $\begin{array}{c}\text { Width } \\
(\mathrm{sec})\end{array}$ & Area & Height & \% Area \\
\hline 1 & 21.459 & $\mathrm{bb}$ & 197.000 & 20088673 & 486651 & 94.13 \\
\hline 2 & 35.239 & $\mathrm{bb}$ & 135.000 & 1252362 & 19619 & 5.87 \\
\hline Sum & & & & 21341035.3 & 506270.5 & 100.0 \\
\hline
\end{tabular}




\section{Racemic 3e'}

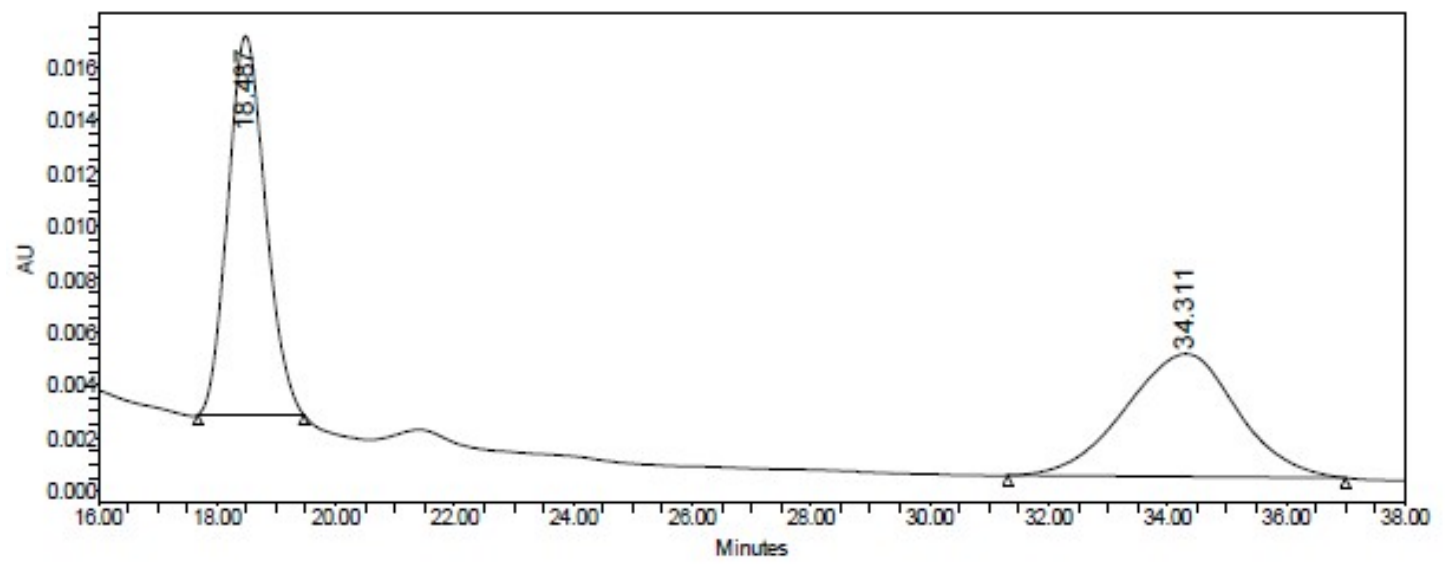

\begin{tabular}{|c|c|l|c|c|c|c|}
\hline & RT (min) & Int Type & $\begin{array}{c}\text { Width } \\
(\mathrm{sec})\end{array}$ & Area & Height & $\%$ Area \\
\hline 1 & 18.487 & bb & 107.000 & 628787 & 14280 & 50.82 \\
\hline 2 & 34.311 & bb & 341.000 & 608437 & 4623 & 49.18 \\
\hline Sum & & & & 1237224.0 & 18903.0 & 100.0 \\
\hline
\end{tabular}

\section{Enantioenriched 3e'}

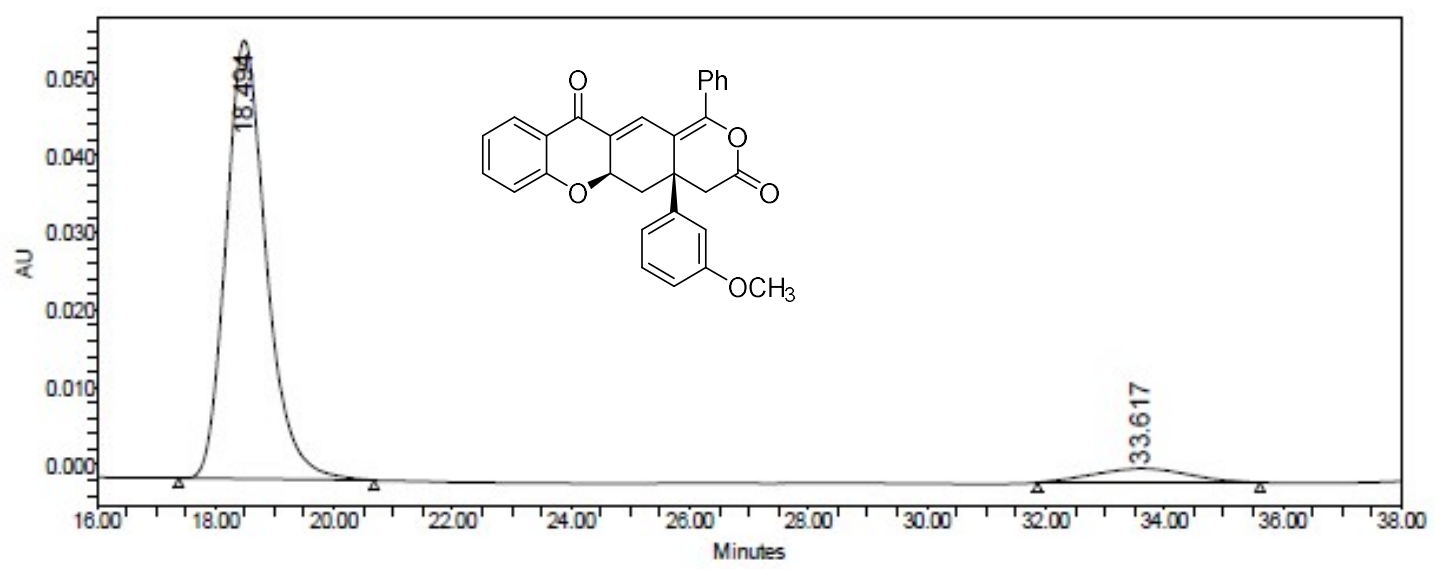

\begin{tabular}{|c|l|l|c|c|l|l|}
\hline & RT(min) & Int Type & $\begin{array}{c}\text { Width } \\
(\mathrm{sec})\end{array}$ & Area & Height & $\%$ Area \\
\hline 1 & 18.494 & $\mathrm{bb}$ & 198.000 & 2672562 & 56730 & 93.09 \\
\hline 2 & 33.617 & $\mathrm{bb}$ & 225.000 & 198326 & 1801 & 6.91 \\
\hline Sum & & & & 2870888.4 & 58531.0 & 100.0 \\
\hline
\end{tabular}




\section{Racemic 3f}

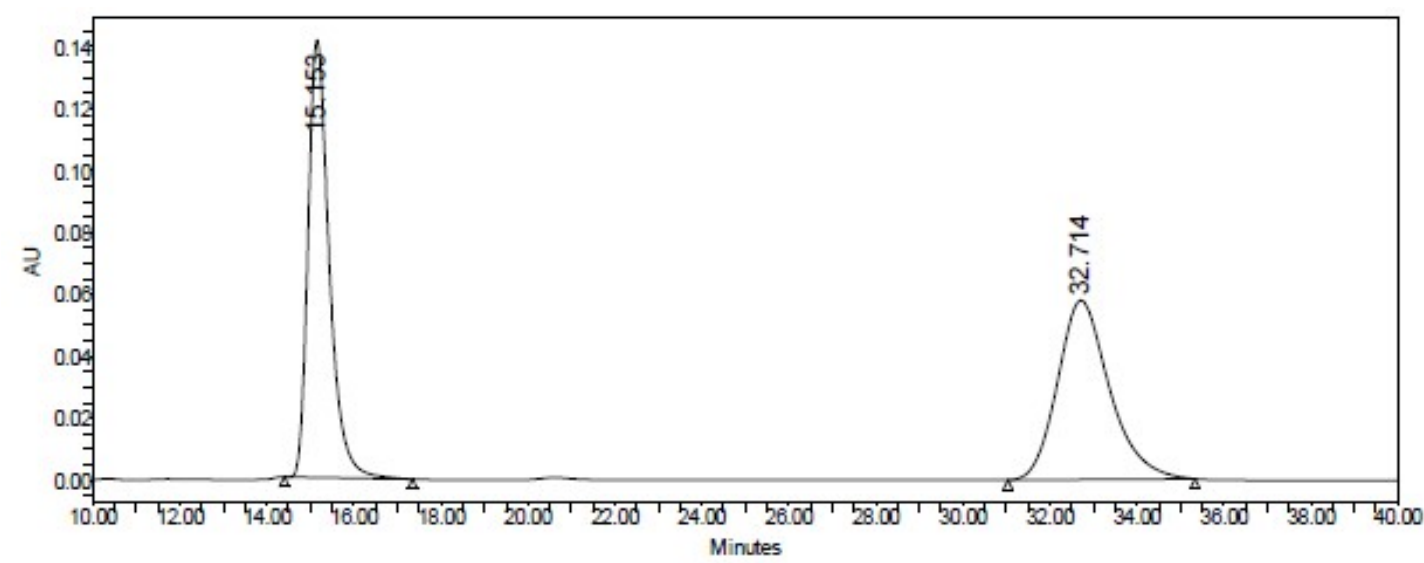

\begin{tabular}{|c|l|l|c|c|l|l|}
\hline & RT (min) & Int Type & $\begin{array}{c}\text { Width } \\
(\mathrm{sec})\end{array}$ & Area & Height & \% Area \\
\hline 1 & 15.153 & bb & 177.000 & 4745986 & 141155 & 49.71 \\
\hline 2 & 32.714 & bb & 258.000 & 4801938 & 57882 & 50.29 \\
\hline Sum & & & & 9547924.0 & 199037.5 & 100.0 \\
\hline
\end{tabular}

\section{Enantioenriched $3 f$}

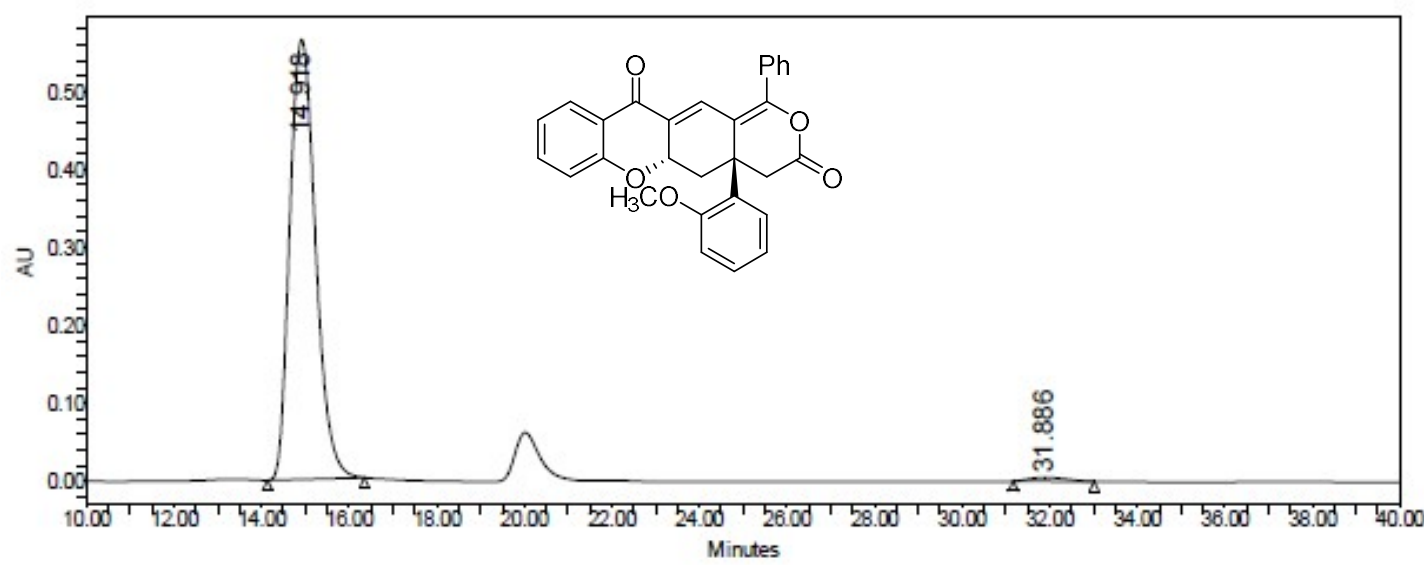

\begin{tabular}{|c|c|l|c|c|l|l|}
\hline & RT (min) & Int Type & $\begin{array}{c}\text { Width } \\
(\mathrm{sec})\end{array}$ & Area & Height & $\%$ Area \\
\hline 1 & 14.918 & bb & 133.000 & 23055696 & 564300 & 98.73 \\
\hline 2 & 31.886 & bb & 111.000 & 296842 & 5159 & 1.27 \\
\hline Sum & & & & 23352537.8 & 569458.7 & 100.0 \\
\hline
\end{tabular}




\section{Racemic 3g}

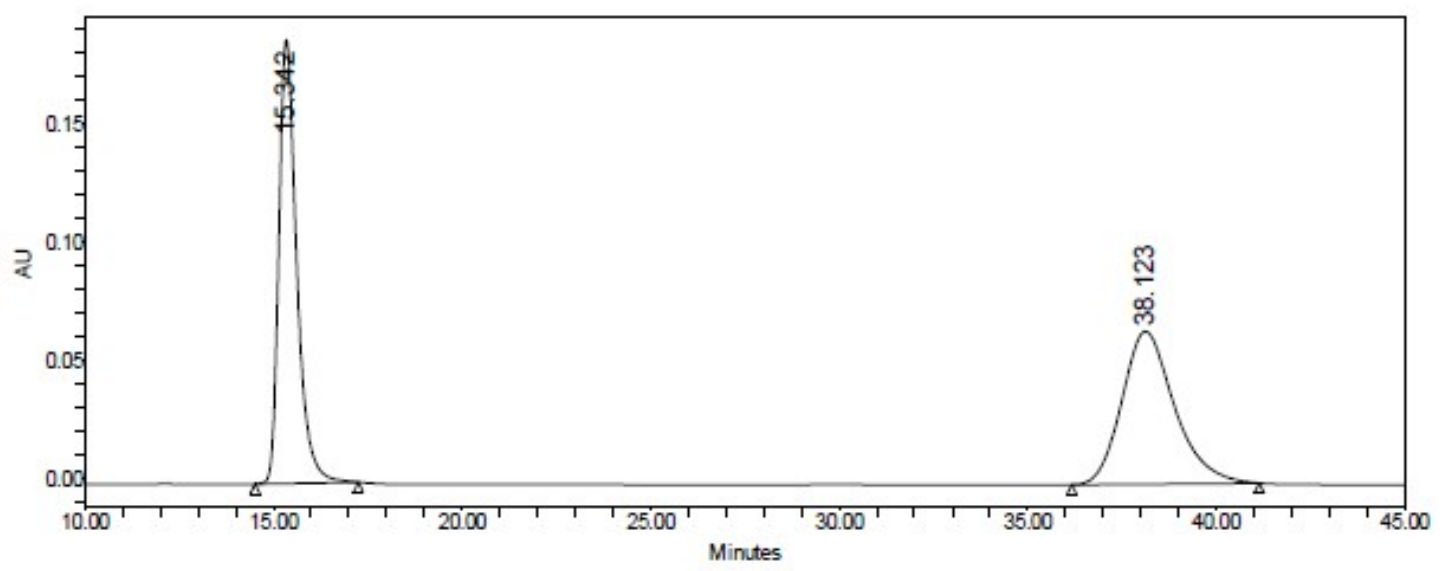

\begin{tabular}{|c|l|l|c|c|c|c|}
\hline & RT (min) & Int Type & $\begin{array}{c}\text { Width } \\
(\mathrm{sec})\end{array}$ & Area & Height & \% Area \\
\hline 1 & 15.342 & bb & 164.000 & 6159906 & 187096 & 49.72 \\
\hline 2 & 38.123 & bb & 298.000 & 6228572 & 64371 & 50.28 \\
\hline Sum & & & & 12388477.9 & 251467.5 & 100.0 \\
\hline
\end{tabular}

\section{Enantioenriched 3g}

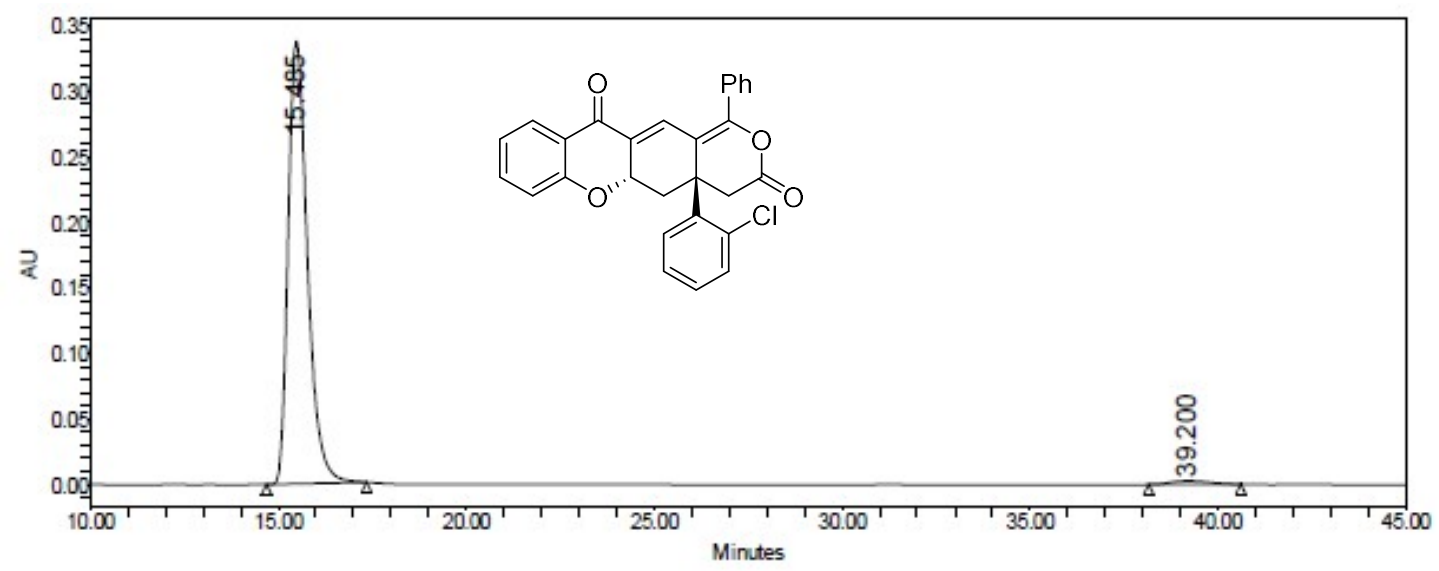

\begin{tabular}{|c|c|c|c|c|c|l|}
\hline & $R T(m i n)$ & Int Type & $\begin{array}{c}\text { Width } \\
(\mathrm{sec})\end{array}$ & Area & Height & \% Area \\
\hline 1 & 15.485 & $\mathrm{bb}$ & 160.000 & 12014113 & 337341 & 98.38 \\
\hline 2 & 39.200 & $\mathrm{bb}$ & 147.000 & 197520 & 2784 & 1.62 \\
\hline Sum & & & & 12211632.7 & 340125.2 & 100.0 \\
\hline
\end{tabular}


Racemic 3h

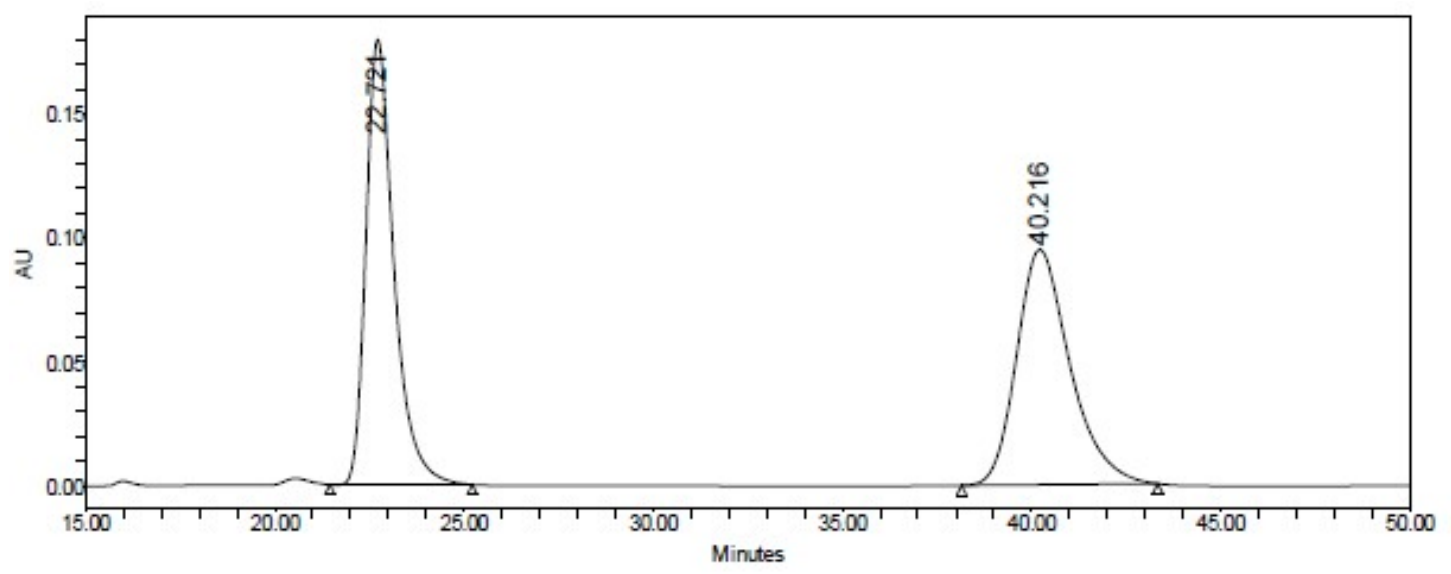

\begin{tabular}{|c|l|l|c|c|l|l|}
\hline & RT (min) & Int Type & $\begin{array}{c}\text { Width } \\
(\mathrm{sec})\end{array}$ & Area & Height & $\%$ Area \\
\hline 1 & 22.721 & bb & 226.000 & 9220750 & 179416 & 49.81 \\
\hline 2 & 40.216 & bb & 311.000 & 9289496 & 94810 & 50.19 \\
\hline Sum & & & & 18510246.5 & 274225.4 & 100.0 \\
\hline
\end{tabular}

\section{Enantioenriched 3h}

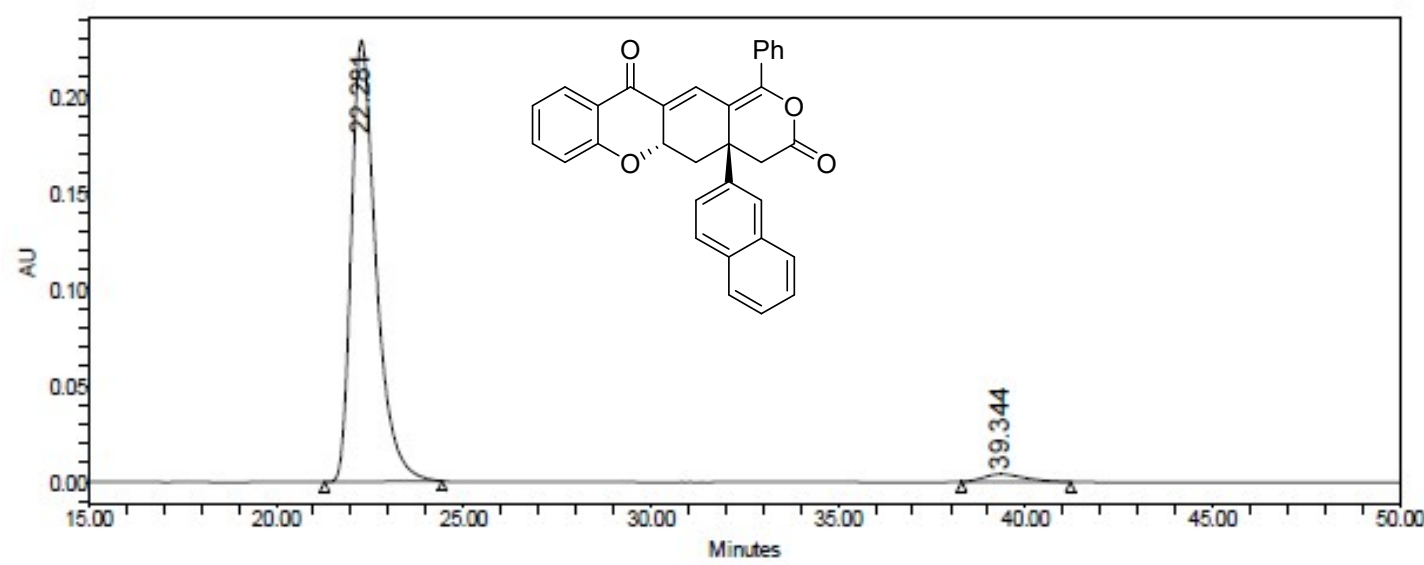

\begin{tabular}{|c|l|l|c|c|c|l|}
\hline & RT (min) & Int Type & $\begin{array}{c}\text { Width } \\
(\mathrm{sec})\end{array}$ & Area & Height & $\%$ Area \\
\hline 1 & 22.281 & bb & 188.000 & 10497256 & 228717 & 97.22 \\
\hline 2 & 39.344 & bb & 174.000 & 299703 & 3941 & 2.78 \\
\hline Sum & & & & 10796958.3 & 232658.0 & 100.0 \\
\hline
\end{tabular}




\section{Racemic 3h'}

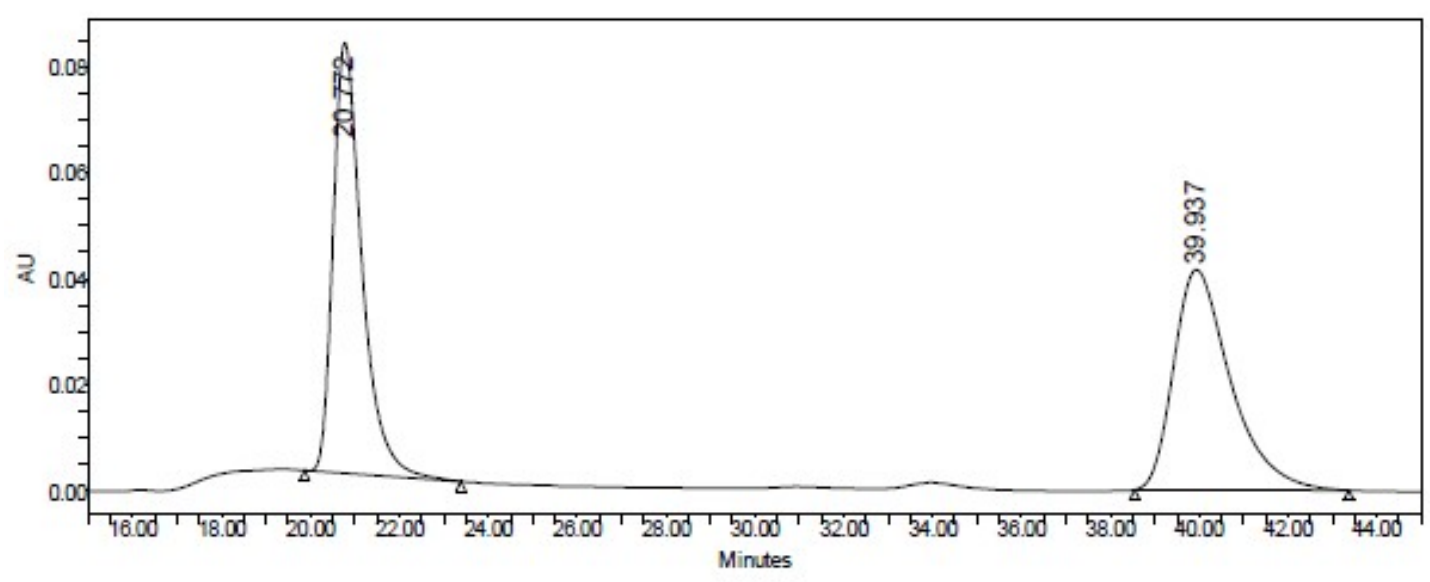

\begin{tabular}{|c|c|l|c|c|c|c|}
\hline & RT (min) & Int Type & $\begin{array}{c}\text { Width } \\
(\mathrm{sec})\end{array}$ & Area & Height & \% Area \\
\hline 1 & 20.772 & bb & 211.000 & 3668816 & 81358 & 49.79 \\
\hline 2 & 39.937 & bb & 289.000 & 3699190 & 41618 & 50.21 \\
\hline Sum & & & & 7368005.5 & 122975.4 & 100.0 \\
\hline
\end{tabular}

\section{Enantioenriched 3h'}

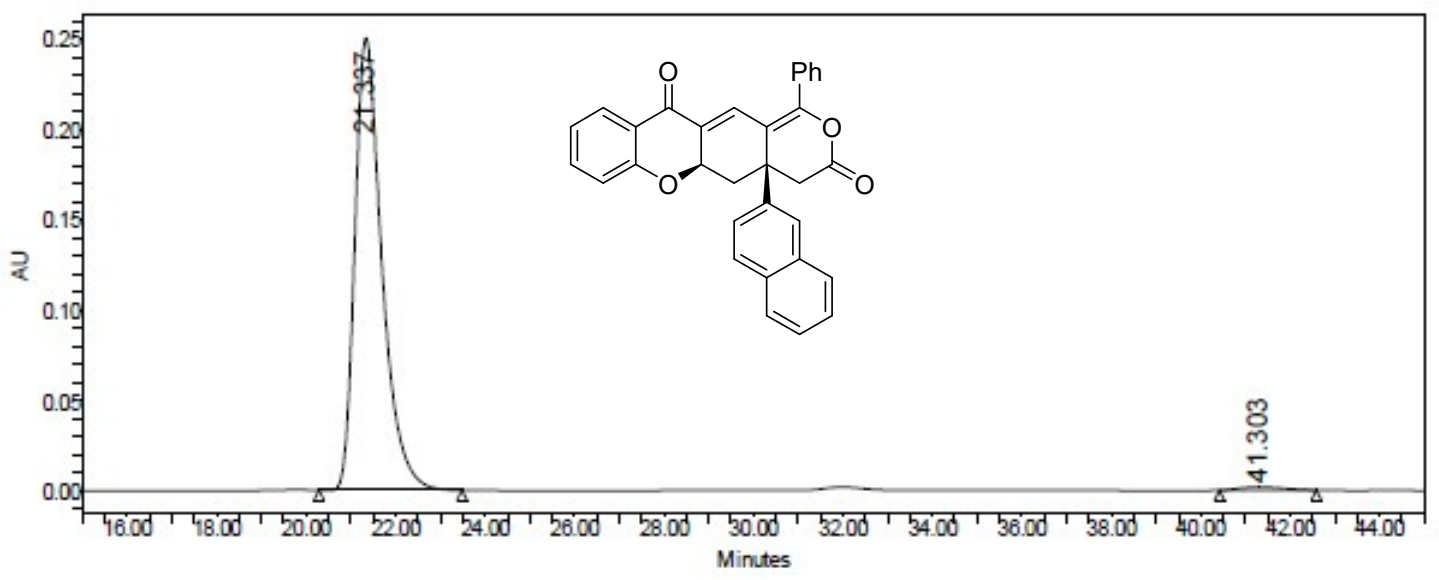

\begin{tabular}{|c|l|l|c|c|l|l|}
\hline & RT (min) & Int Type & $\begin{array}{c}\text { Width } \\
(\mathrm{sec})\end{array}$ & Area & Height & $\%$ Area \\
\hline 1 & 21.337 & bb & 192.000 & 10501001 & 250370 & 98.93 \\
\hline 2 & 41.303 & bb & 130.000 & 113872 & 1654 & 1.07 \\
\hline Sum & & & & 10614873.1 & 252023.5 & 100.0 \\
\hline
\end{tabular}




\section{Racemic 3i}

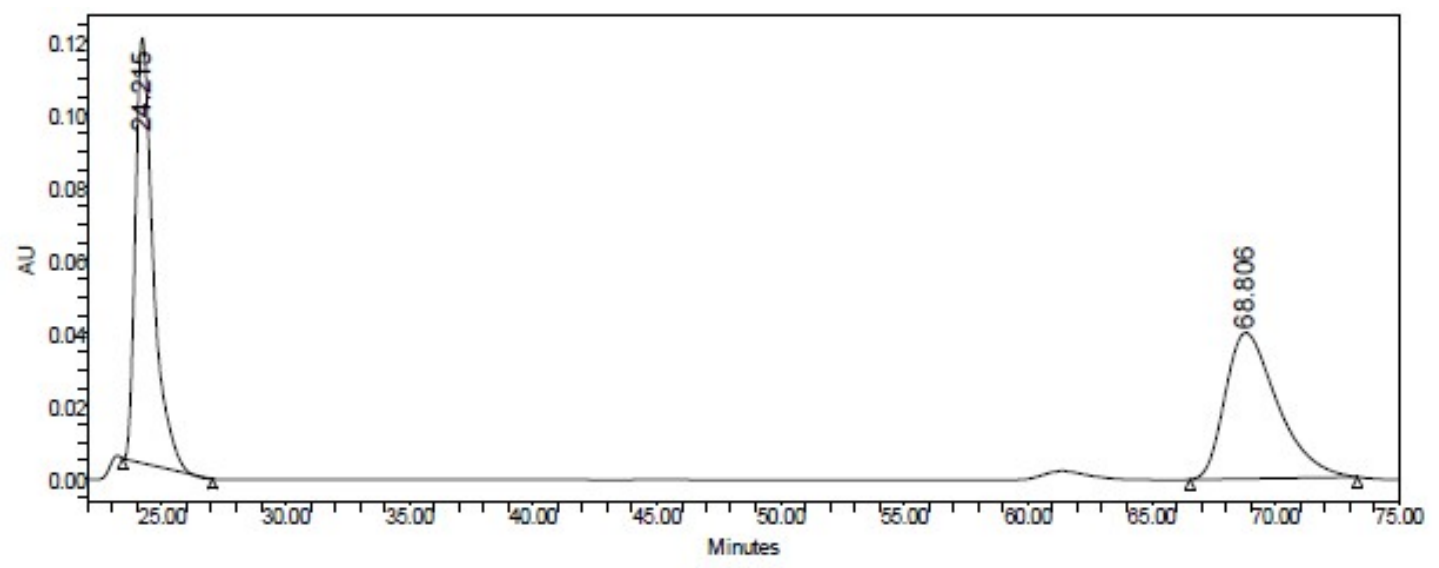

\begin{tabular}{|c|l|l|c|c|l|l|}
\hline & RT (min) & Int Type & $\begin{array}{c}\text { Width } \\
(\mathrm{sec})\end{array}$ & Area & Height & $\%$ Area \\
\hline 1 & 24.215 & bb & 218.000 & 5884964 & 116833 & 49.92 \\
\hline 2 & 68.806 & bb & 406.000 & 5903456 & 40089 & 50.08 \\
\hline Sum & & & & 11788419.6 & 156921.9 & 100.0 \\
\hline
\end{tabular}

\section{Enantioenriched 3i}

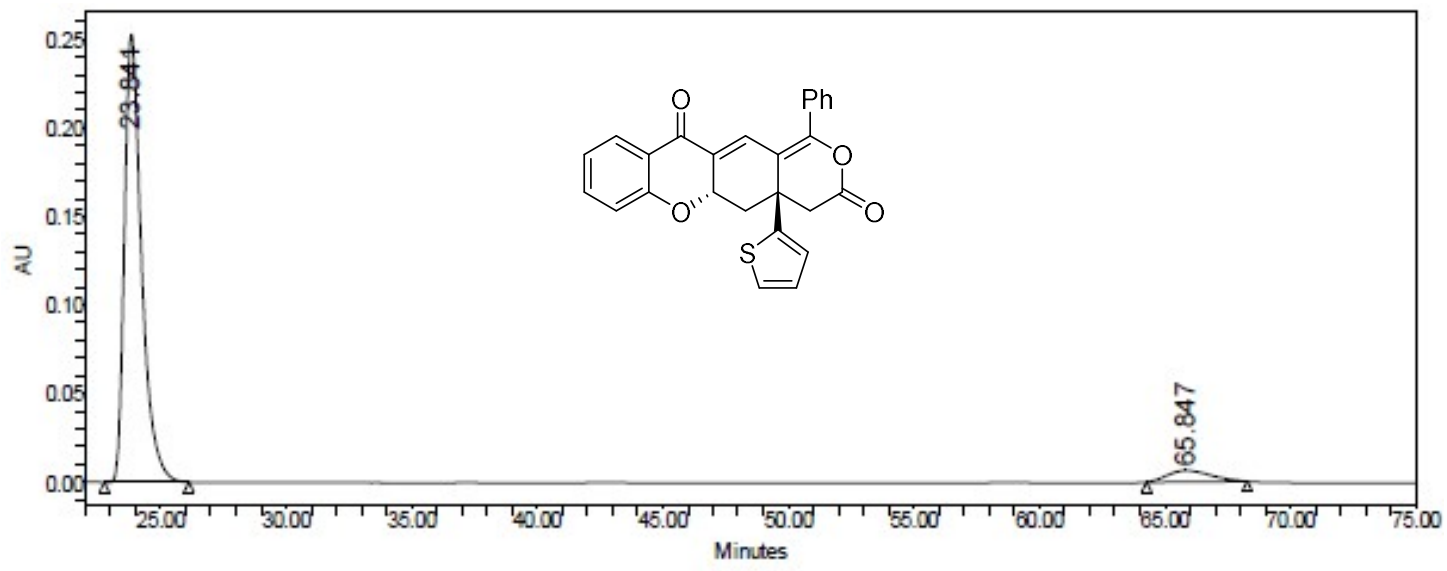

\begin{tabular}{|c|l|l|c|c|c|l|}
\hline & RT (min) & Int Type & $\begin{array}{c}\text { Width } \\
(\mathrm{sec})\end{array}$ & Area & Height & \% Area \\
\hline 1 & 23.841 & bb & 202.000 & 11983109 & 252868 & 94.00 \\
\hline 2 & 65.847 & bb & 240.000 & 764414 & 6449 & 6.00 \\
\hline Sum & & & & 12747523.0 & 259317.2 & 100.0 \\
\hline
\end{tabular}




\section{Enantioenriched 3i'}

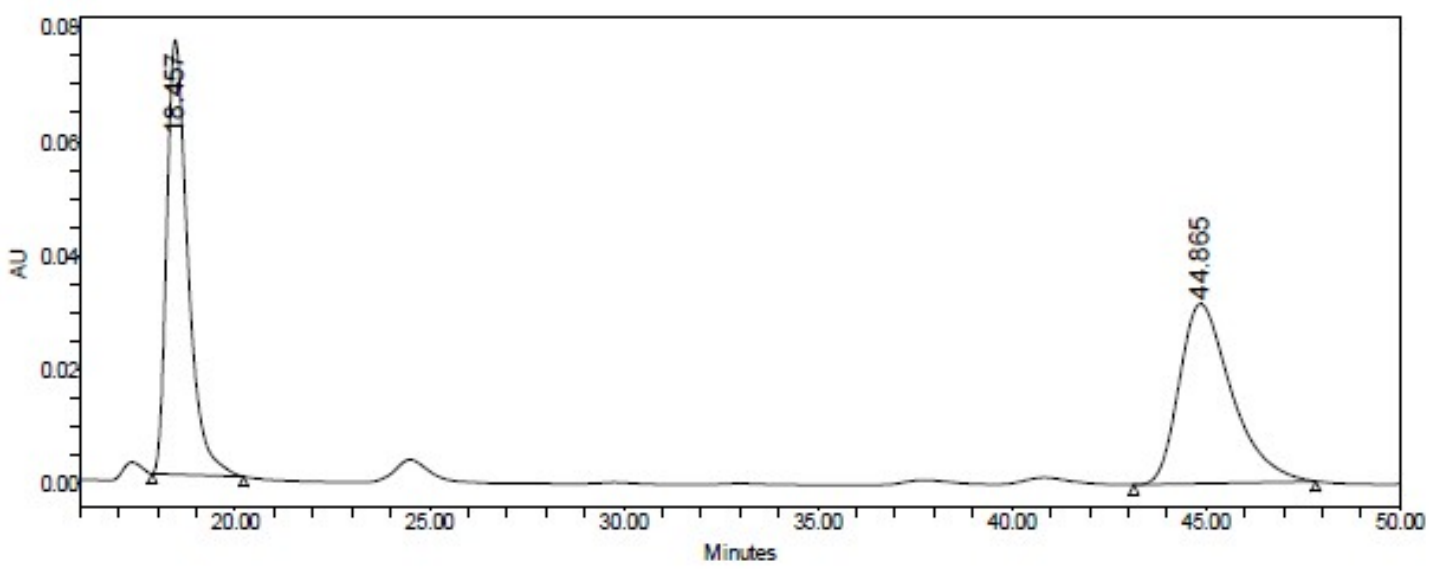

\begin{tabular}{|c|l|l|c|c|c|c|}
\hline & RT (min) & Int Type & $\begin{array}{c}\text { Width } \\
(\mathrm{sec})\end{array}$ & Area & Height & \% Area \\
\hline 1 & 18.457 & bb & 141.000 & 2873907 & 76107 & 49.36 \\
\hline 2 & 44.865 & bb & 281.000 & 2948793 & 31467 & 50.64 \\
\hline Sum & & & & 5822700.4 & 107574.6 & 100.0 \\
\hline
\end{tabular}

\section{Racemic 3i'}

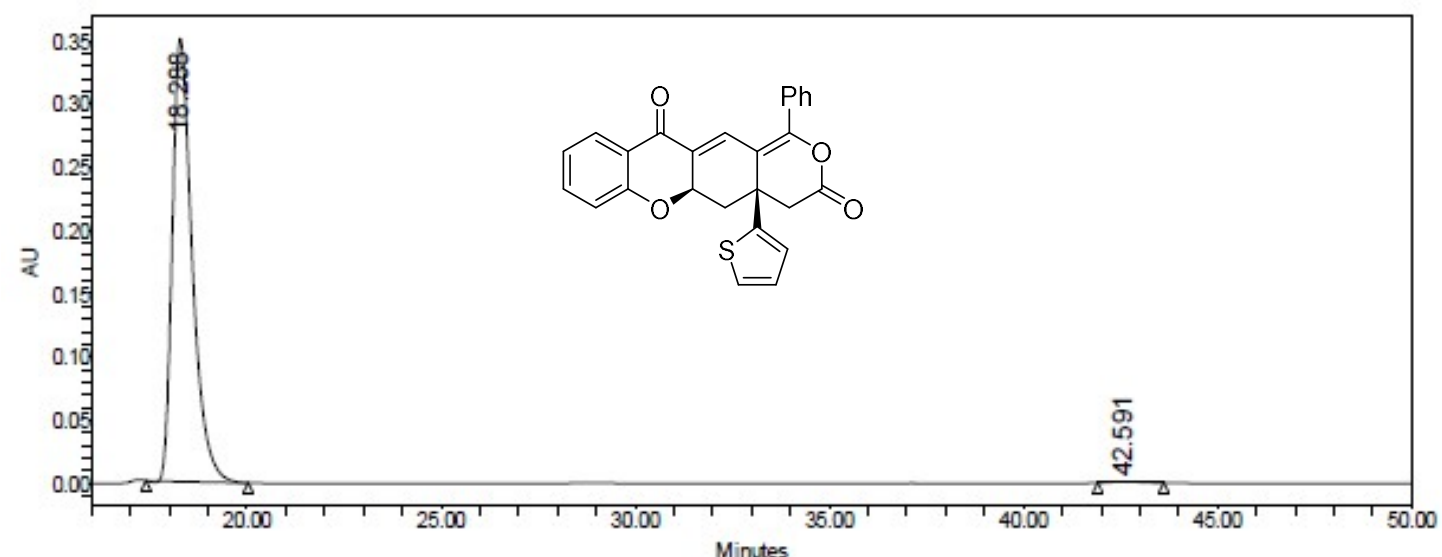

\begin{tabular}{|c|c|l|c|c|c|c|}
\hline & RT (min) & Int Type & $\begin{array}{c}\text { Width } \\
(\mathrm{sec})\end{array}$ & Area & Height & \% Area \\
\hline 1 & 18.288 & $\mathrm{bb}$ & 158.000 & 12470657 & 350238 & 99.52 \\
\hline 2 & 42.591 & $\mathrm{bb}$ & 102.000 & 60684 & 1048 & 0.48 \\
\hline Sum & & & & 12531340.8 & 351286.1 & 100.0 \\
\hline
\end{tabular}




\section{Racemic 3j}

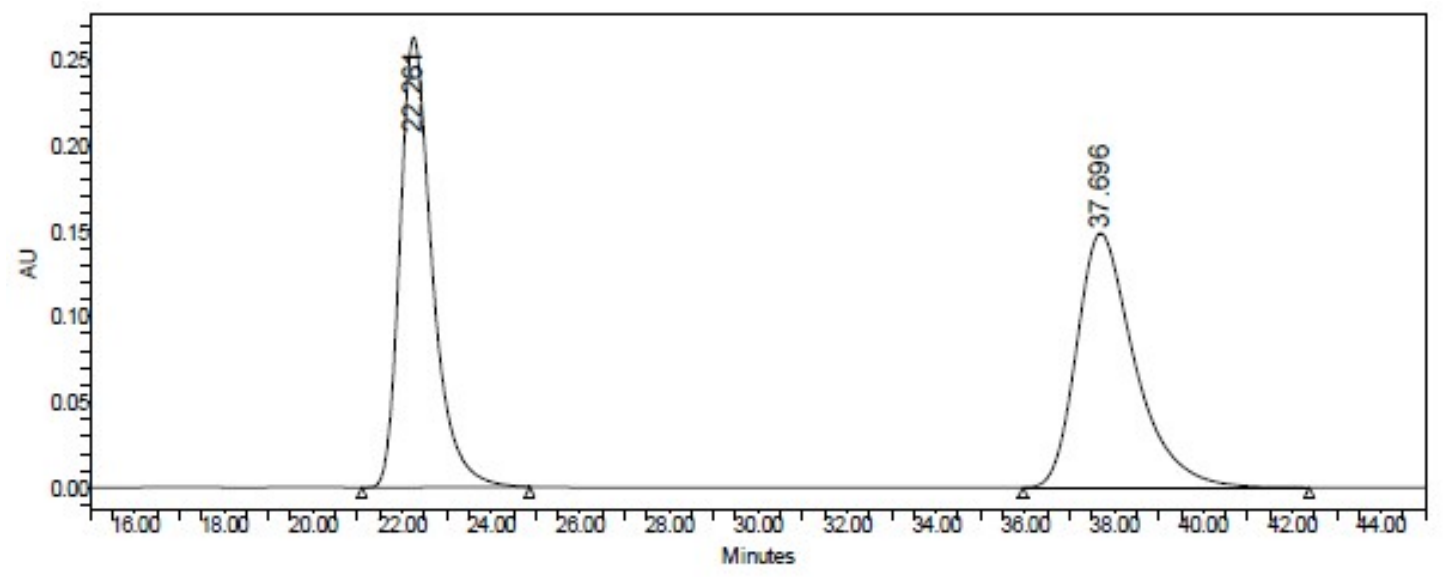

\begin{tabular}{|c|c|c|c|c|c|c|}
\hline & RT (min) & Int Type & $\begin{array}{c}\text { Width } \\
(\mathrm{sec})\end{array}$ & Area & Height & \% Area \\
\hline 1 & 22.261 & bb & 226.000 & 13226138 & 262567 & 49.54 \\
\hline 2 & 37.696 & bb & 386.000 & 13470758 & 148353 & 50.46 \\
\hline Sum & & & & 26696895.4 & 410920.3 & 100.0 \\
\hline
\end{tabular}

\section{Enantioenriched 3j}

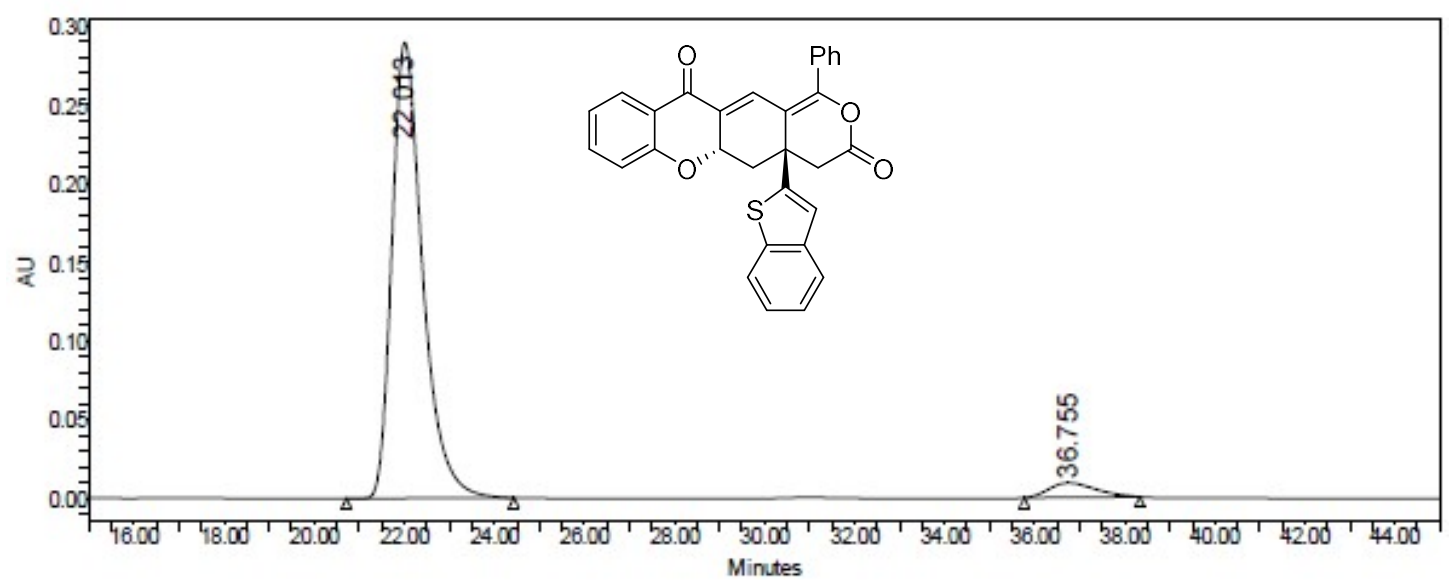

\begin{tabular}{|c|c|l|c|c|l|l|}
\hline & RT (min) & Int Type & $\begin{array}{c}\text { Width } \\
(\mathrm{sec})\end{array}$ & Area & Height & \% Area \\
\hline 1 & 22.013 & bb & 223.000 & 13716422 & 289670 & 95.31 \\
\hline 2 & 36.755 & bb & 154.000 & 674344 & 9348 & 4.69 \\
\hline Sum & & & & 14390766.6 & 299018.4 & 100.0 \\
\hline
\end{tabular}


Racemic 3j'

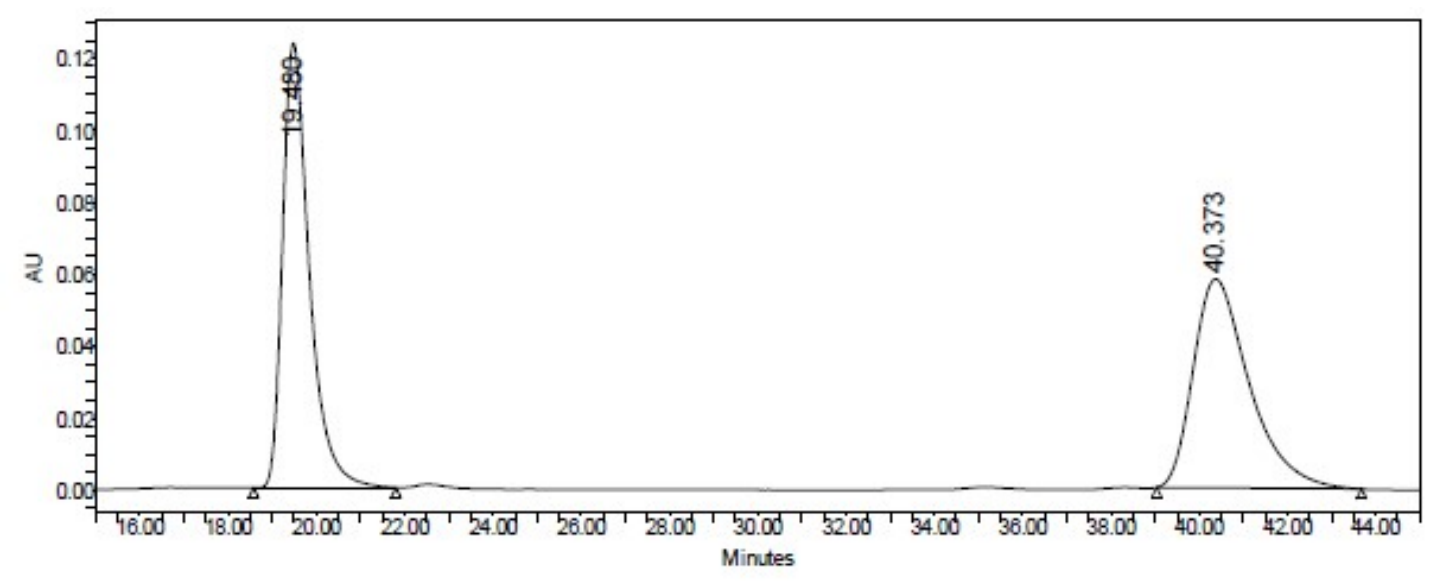

\begin{tabular}{|c|c|c|c|c|c|c|}
\hline & RT (min) & Int Type & $\begin{array}{c}\text { Width } \\
(\mathrm{sec})\end{array}$ & Area & Height & $\%$ Area \\
\hline 1 & 19.480 & bb & 193.000 & 5213221 & 123687 & 50.40 \\
\hline 2 & 40.373 & bb & 279.000 & 5129787 & 58127 & 49.60 \\
\hline Sum & & & & 10343008.4 & 181813.3 & 100.0 \\
\hline
\end{tabular}

\section{Enantioenriched 3j'}

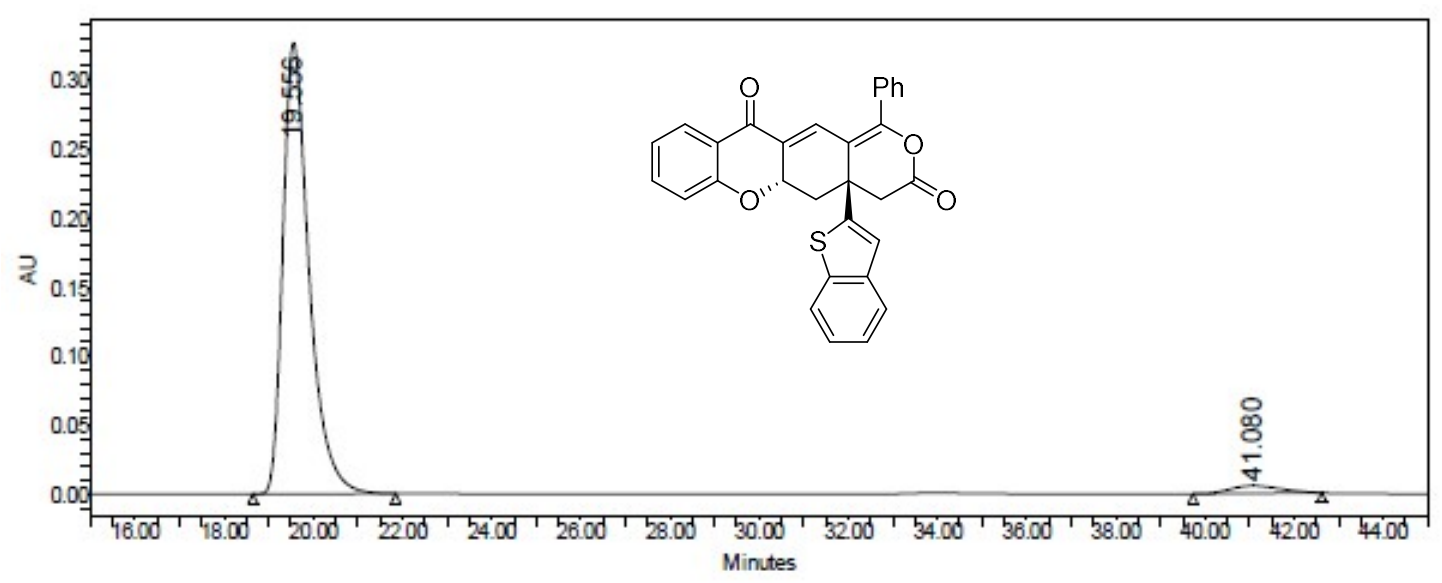

\begin{tabular}{|c|c|c|c|c|c|l|}
\hline & RT (min) & Int Type & $\begin{array}{c}\text { Width } \\
(\mathrm{sec})\end{array}$ & Area & Height & $\%$ Area \\
\hline 1 & 19.556 & bb & 192.000 & 13302844 & 326256 & 96.86 \\
\hline 2 & 41.080 & bb & 173.000 & 431328 & 5481 & 3.14 \\
\hline Sum & & & & 13734172.1 & 331736.3 & 100.0 \\
\hline
\end{tabular}


Racemic 3k

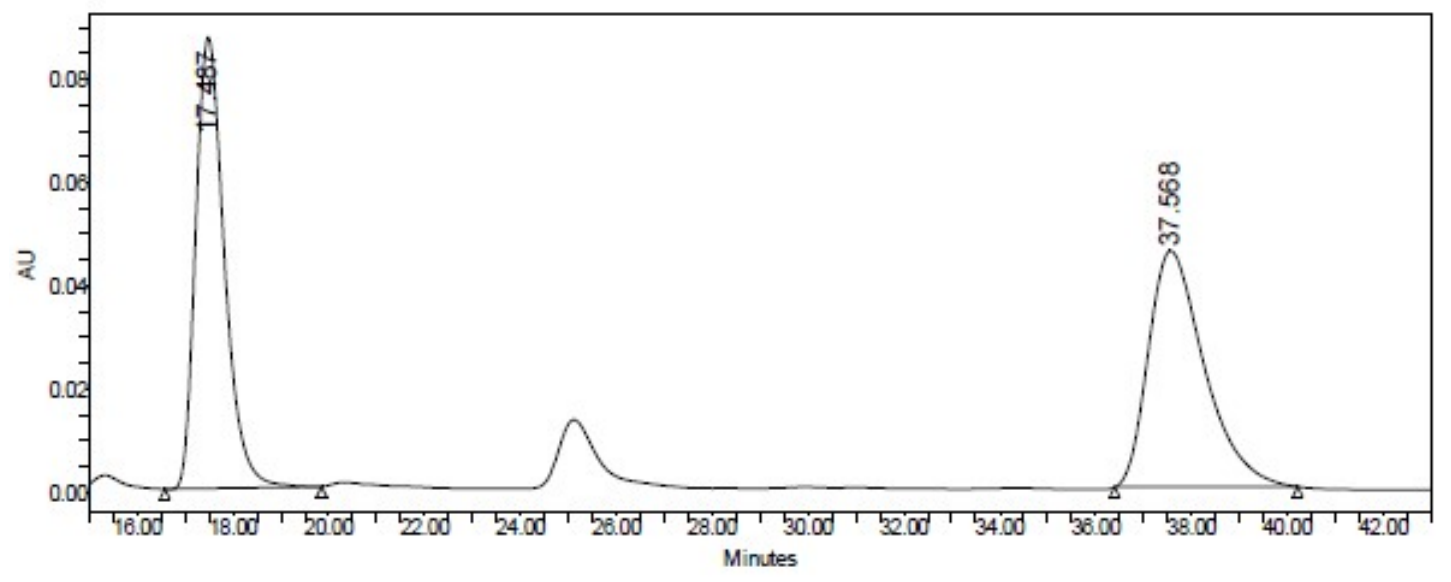

\begin{tabular}{|c|c|l|c|c|c|c|}
\hline & RT (min) & Int Type & $\begin{array}{c}\text { Width } \\
(\mathrm{sec})\end{array}$ & Area & Height & $\%$ Area \\
\hline 1 & 17.487 & bb & 196.000 & 3643597 & 87452 & 49.55 \\
\hline 2 & 37.568 & bb & 229.000 & 3710266 & 45786 & 50.45 \\
\hline Sum & & & & 7353863.2 & 133238.4 & 100.0 \\
\hline
\end{tabular}

\section{Enantioenriched 3k}

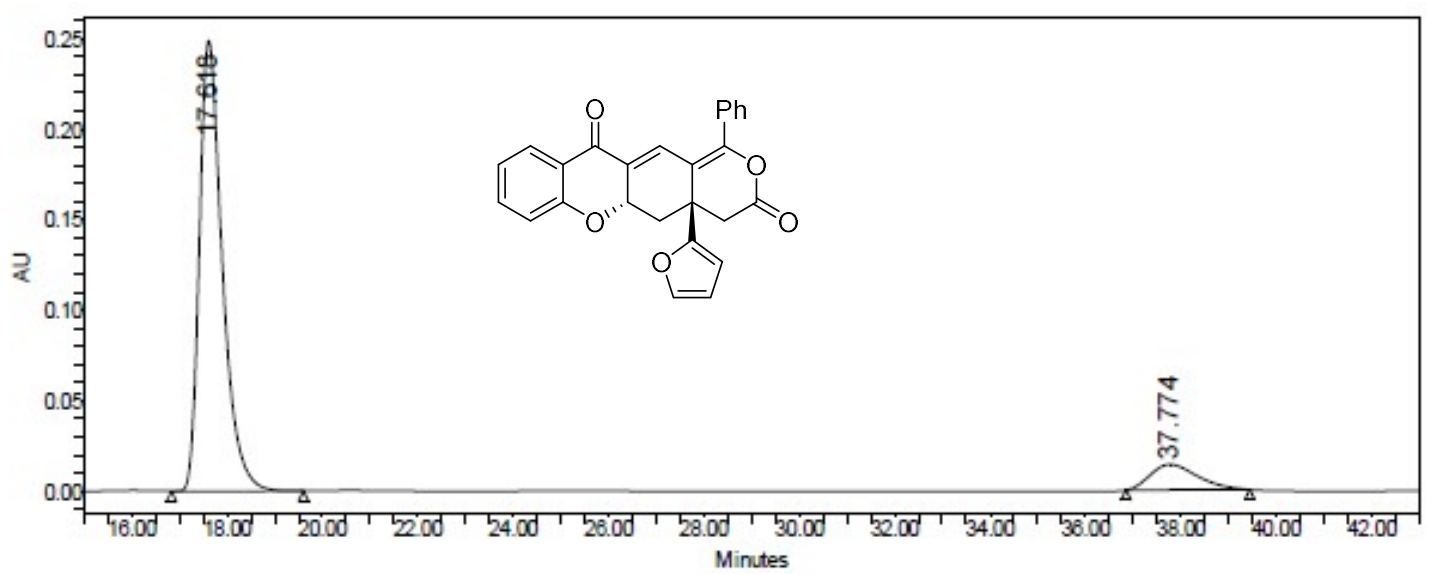

\begin{tabular}{|c|c|c|c|c|c|c|}
\hline & RT (min) & Int Type & $\begin{array}{c}\text { Width } \\
(\mathrm{sec})\end{array}$ & Area & Height & $\%$ Area \\
\hline 1 & 17.618 & bb & 166.000 & 8331031 & 248951 & 89.53 \\
\hline 2 & 37.774 & bb & 156.000 & 973810 & 14070 & 10.47 \\
\hline Sum & & & & 9304840.2 & 263021.0 & 100.0 \\
\hline
\end{tabular}


Racemic 3k'

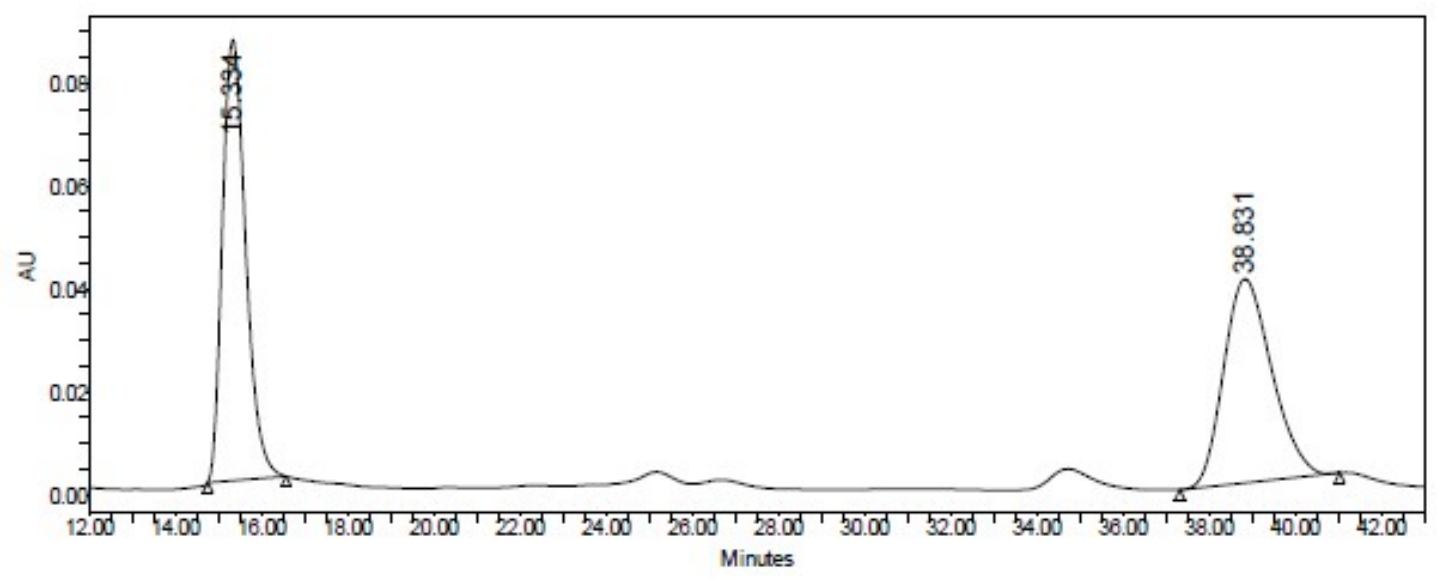

\begin{tabular}{|c|c|l|c|c|c|c|}
\hline & RT (min) & Int Type & $\begin{array}{c}\text { Width } \\
(\text { sec })\end{array}$ & Area & Height & $\%$ Area \\
\hline 1 & 15.334 & bb & 109.000 & 3214552 & 85794 & 51.14 \\
\hline 2 & 38.831 & bb & 223.000 & 3071006 & 39683 & 48.86 \\
\hline Sum & & & & 6285558.1 & 125477.4 & 100.0 \\
\hline
\end{tabular}

\section{Enantioenriched 3k'}

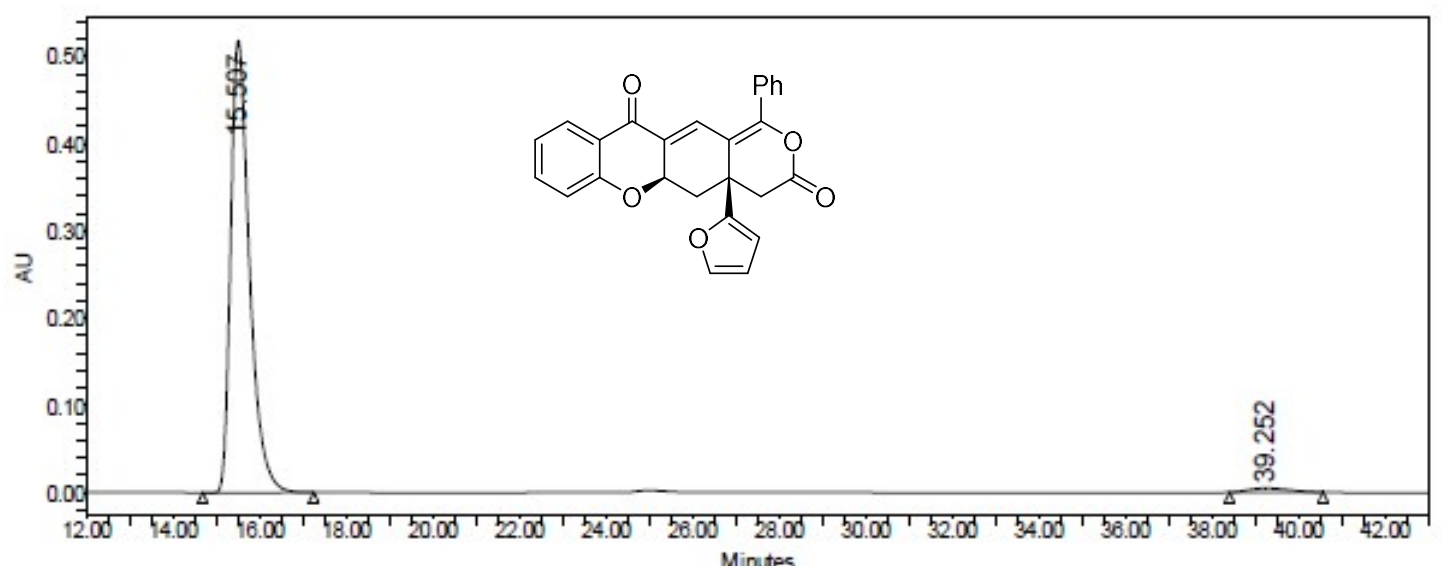

\begin{tabular}{|c|c|l|c|c|c|l|}
\hline & RT (min) & Int Type & $\begin{array}{c}\text { Width } \\
(\mathrm{sec})\end{array}$ & Area & Height & $\%$ Area \\
\hline 1 & 15.507 & bb & 154.000 & 15593621 & 518716 & 98.02 \\
\hline 2 & 39.252 & bb & 130.000 & 314259 & 4877 & 1.98 \\
\hline Sum & & & & 15907880.6 & 523593.6 & 100.0 \\
\hline
\end{tabular}


Racemic 3I

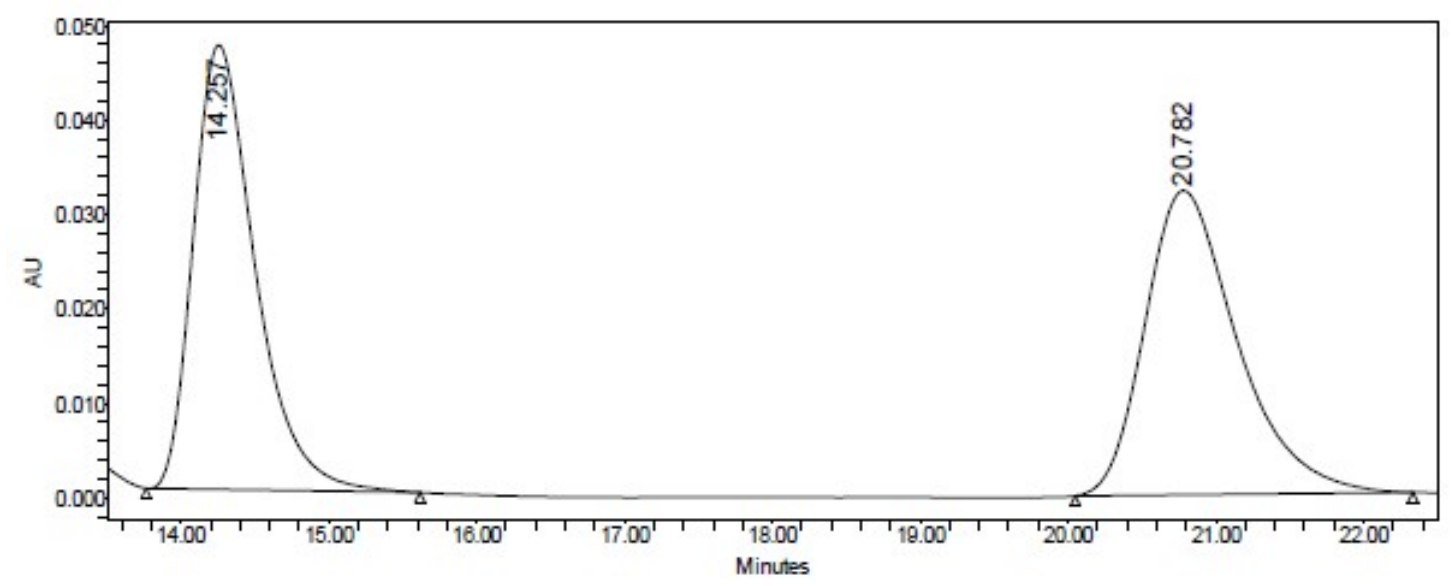

\begin{tabular}{|c|c|l|c|c|c|c|}
\hline & RT (min) & Int Type & $\begin{array}{c}\text { Width } \\
(\mathrm{sec})\end{array}$ & Area & Height & $\%$ Area \\
\hline 1 & 14.257 & bb & 111.000 & 1367293 & 47092 & 49.52 \\
\hline 2 & 20.782 & bb & 137.000 & 1393827 & 32209 & 50.48 \\
\hline Sum & & & & 2761120.0 & 79300.8 & 100.0 \\
\hline
\end{tabular}

\section{Enantioenriched 3I}

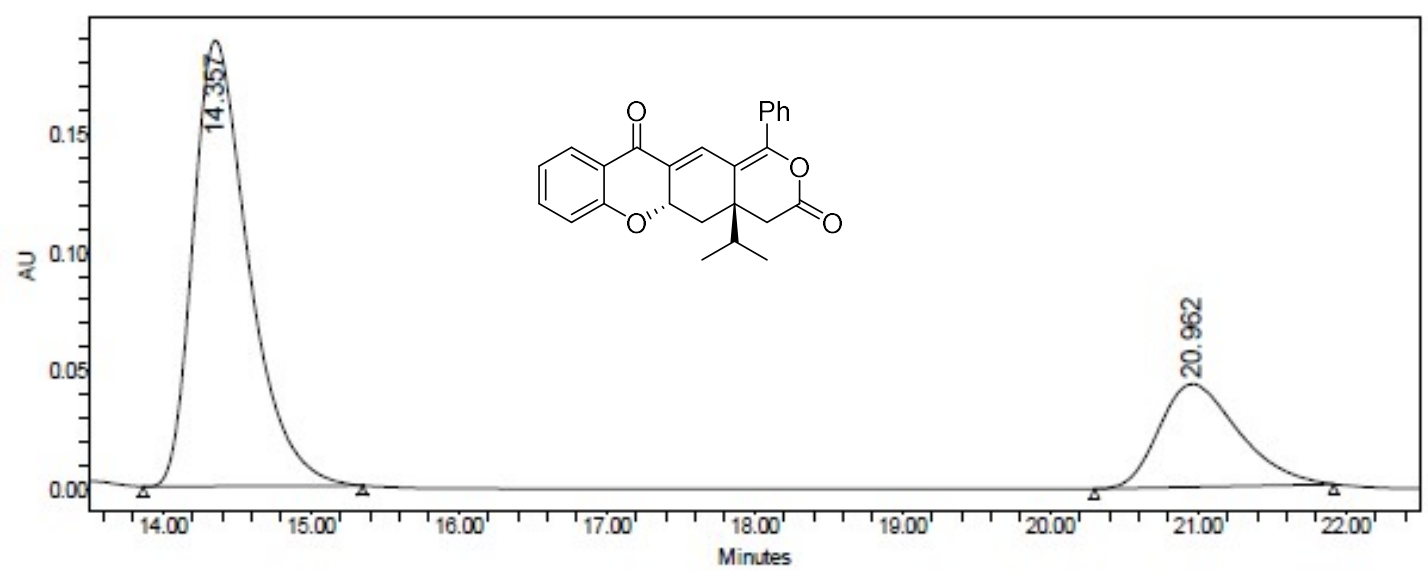

\begin{tabular}{|c|l|l|c|c|c|c|}
\hline & RT (min) & Int Type & $\begin{array}{c}\text { Width } \\
(\text { sec })\end{array}$ & Area & Height & \% Area \\
\hline 1 & 14.357 & bb & 89.000 & 4909703 & 189005 & 75.08 \\
\hline 2 & 20.962 & bb & 97.000 & 1630024 & 43534 & 24.92 \\
\hline Sum & & & & 6539726.1 & 232538.5 & 100.0 \\
\hline
\end{tabular}


Racemic 3l'

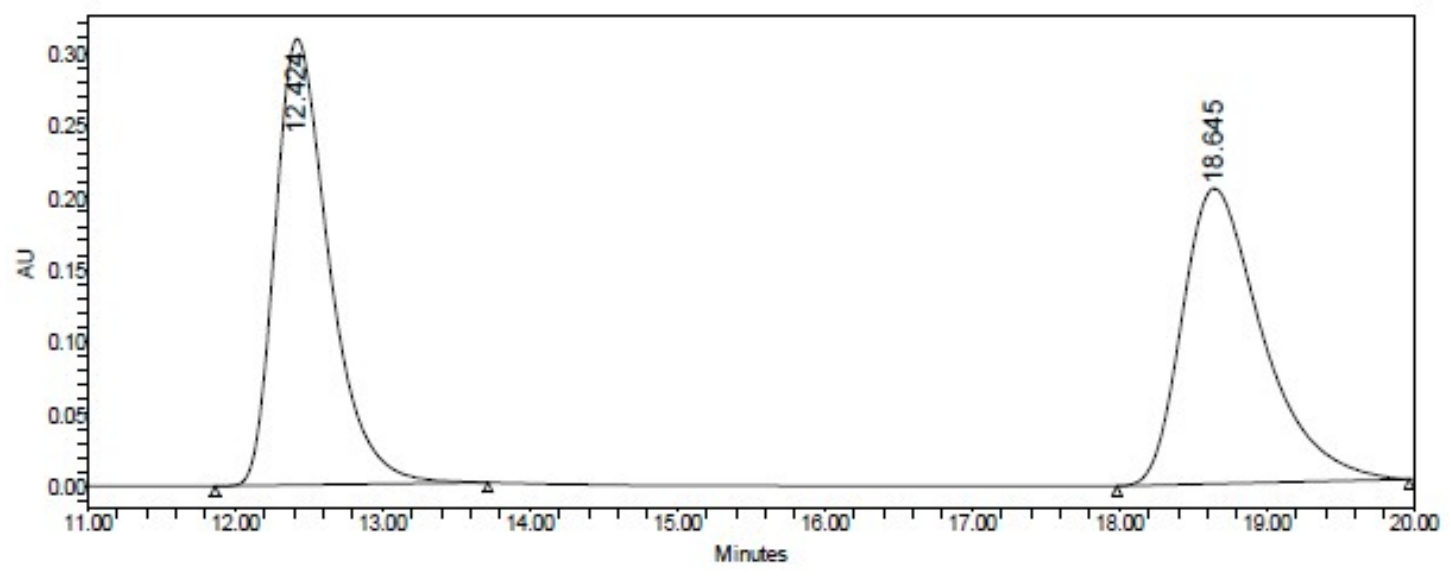

\begin{tabular}{|c|l|l|c|c|c|c|}
\hline & RT (min) & Int Type & $\begin{array}{c}\text { Width } \\
(\mathrm{sec})\end{array}$ & Area & Height & $\%$ Area \\
\hline 1 & 12.424 & bb & 111.000 & 7886974 & 309107 & 50.50 \\
\hline 2 & 18.645 & bb & 119.000 & 7729886 & 204471 & 49.50 \\
\hline Sum & & & & 15616859.9 & 513577.8 & 100.0 \\
\hline
\end{tabular}

Enantioenriched 3l'

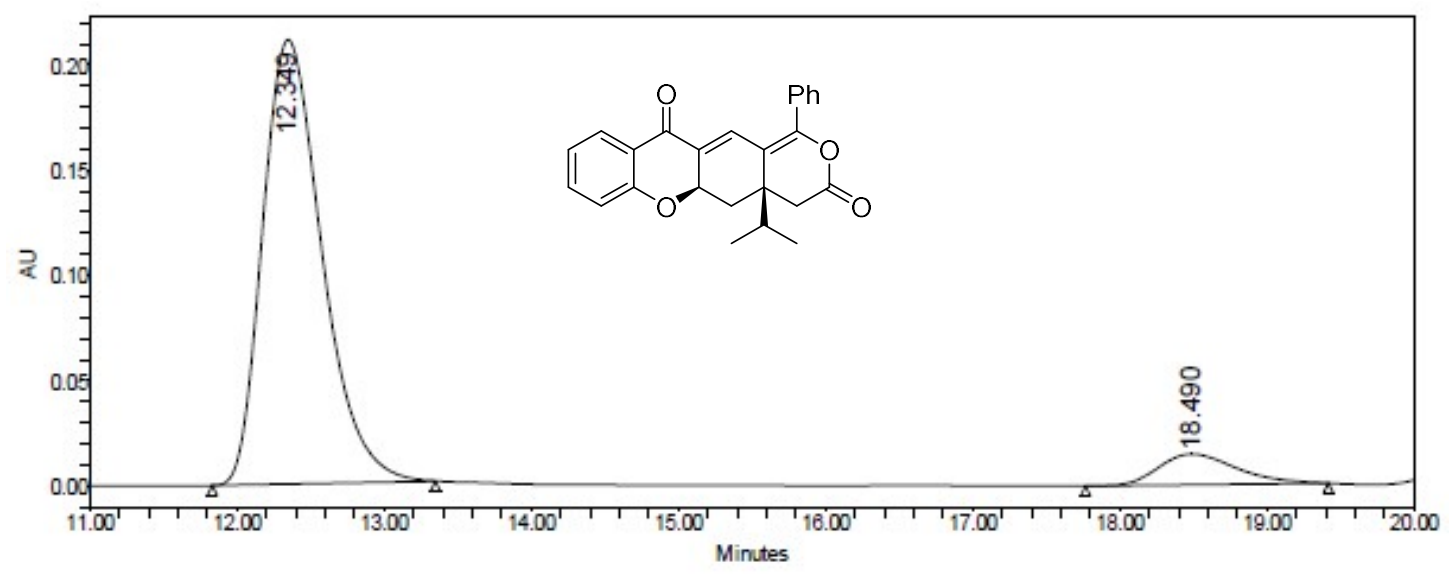

\begin{tabular}{|c|l|l|c|c|l|l|}
\hline & RT (min) & Int Type & $\begin{array}{c}\text { Width } \\
(\mathrm{sec})\end{array}$ & Area & Height & \% Area \\
\hline 1 & 12.349 & bb & 91.000 & 5927774 & 211425 & 91.74 \\
\hline 2 & 18.490 & bb & 99.000 & 533844 & 14546 & 8.26 \\
\hline Sum & & & & 6461618.0 & 225970.7 & 100.0 \\
\hline
\end{tabular}


Racemic 3m

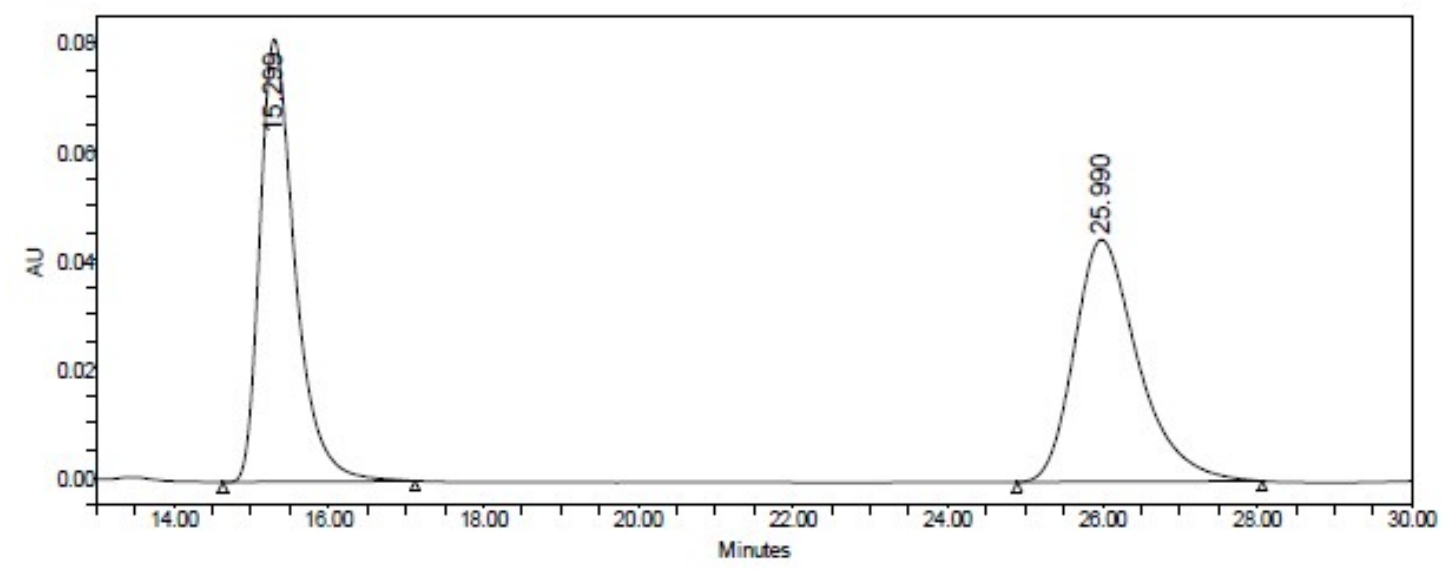

\begin{tabular}{|c|c|c|c|c|c|c|}
\hline & $R T(m i n)$ & Int Type & $\begin{array}{c}\text { Width } \\
(\mathrm{sec})\end{array}$ & Area & Height & \% Area \\
\hline 1 & 15.299 & bb & 149.000 & 2573346 & 81521 & 50.18 \\
\hline 2 & 25.990 & bb & 190.000 & 2554770 & 44587 & 49.82 \\
\hline Sum & & & & 5128115.8 & 126107.4 & 100.0 \\
\hline
\end{tabular}

\section{Enantioenriched 3m}

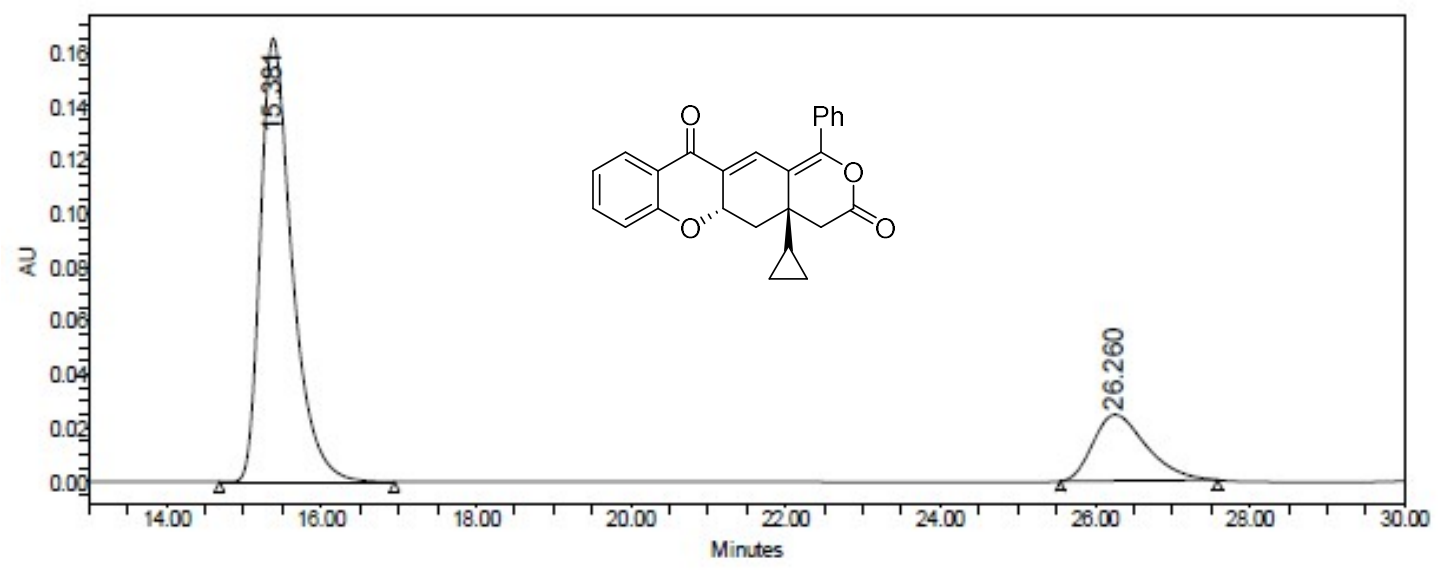

\begin{tabular}{|c|l|l|c|c|l|l|}
\hline & $R T(\min )$ & Int Type & $\begin{array}{c}\text { Width } \\
(\mathrm{sec})\end{array}$ & \multicolumn{1}{|c|}{ Area } & Height & \% Area \\
\hline 1 & 15.381 & bb & 136.000 & 4691330 & 165319 & 79.83 \\
\hline 2 & 26.260 & bb & 122.000 & 1185241 & 24693 & 20.17 \\
\hline Sum & & & & 5876570.8 & 190011.7 & 100.0 \\
\hline
\end{tabular}




\section{Racemic 3m'}

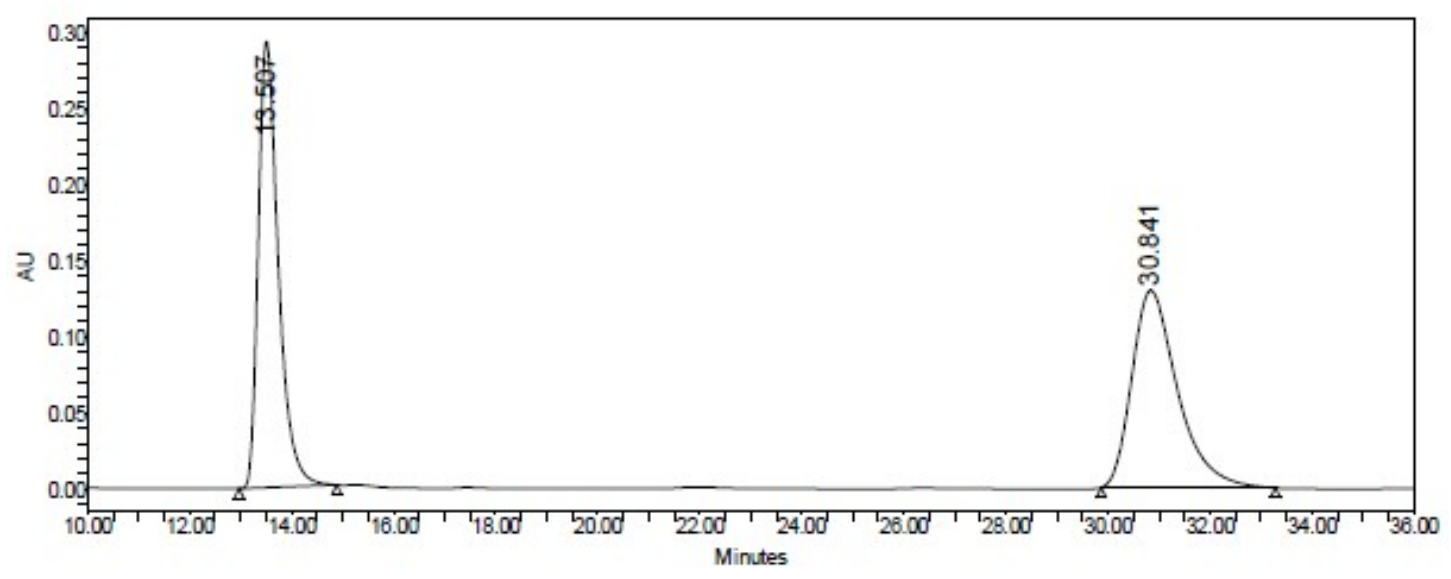

\begin{tabular}{|c|c|l|c|c|c|c|}
\hline & RT (min) & Int Type & $\begin{array}{c}\text { Width } \\
(\mathrm{sec})\end{array}$ & Area & Height & $\%$ Area \\
\hline 1 & 13.507 & $\mathrm{bb}$ & 115.000 & 8023894 & 293366 & 49.89 \\
\hline 2 & 30.841 & $\mathrm{bb}$ & 205.000 & 8059694 & 129634 & 50.11 \\
\hline Sum & & & & 16083587.9 & 422999.6 & 100.0 \\
\hline
\end{tabular}

\section{Enantioenriched 3m'}

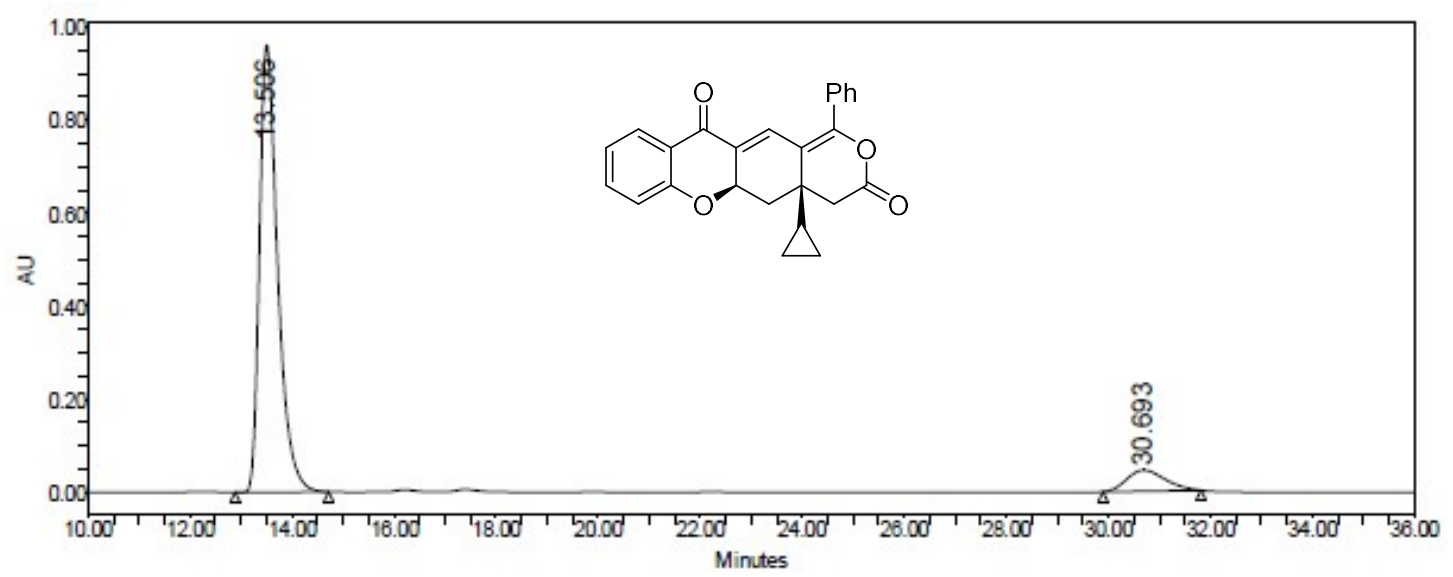

\begin{tabular}{|c|l|l|c|c|l|l|}
\hline & $R T(m i n)$ & Int Type & $\begin{array}{c}\text { Width } \\
(\text { sec })\end{array}$ & Area & Height & $\%$ Area \\
\hline 1 & 13.506 & bb & 110.000 & 23824843 & 962499 & 90.96 \\
\hline 2 & 30.693 & bb & 115.000 & 2366585 & 45293 & 9.04 \\
\hline Sum & & & & 26191428.0 & 1007792.7 & 100.0 \\
\hline
\end{tabular}




\section{Racemic 3n}

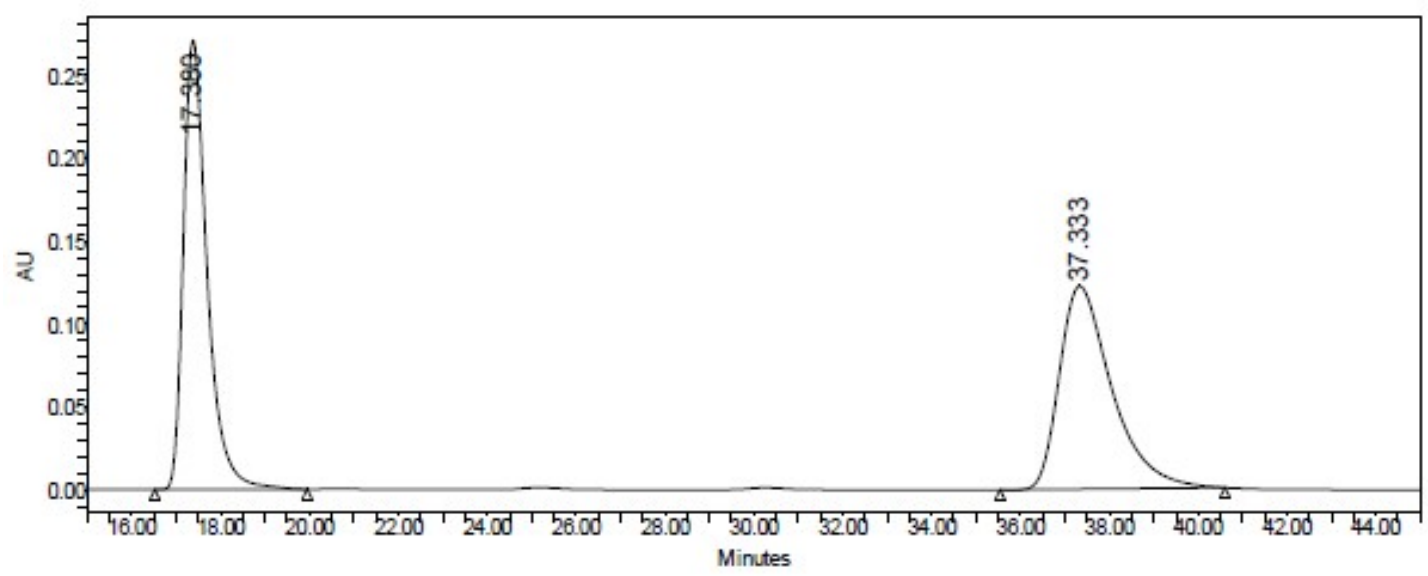

\begin{tabular}{|c|c|c|c|c|c|c|}
\hline & RT (min) & Int Type & $\begin{array}{c}\text { Width } \\
(\mathrm{sec})\end{array}$ & Area & Height & \% Area \\
\hline 1 & 17.380 & bb & 206.000 & 10030256 & 270715 & 50.03 \\
\hline 2 & 37.333 & bb & 304.000 & 10019836 & 122319 & 49.97 \\
\hline Sum & & & & 20050091.4 & 393034.1 & 100.0 \\
\hline
\end{tabular}

\section{Enantioenriched 3n}

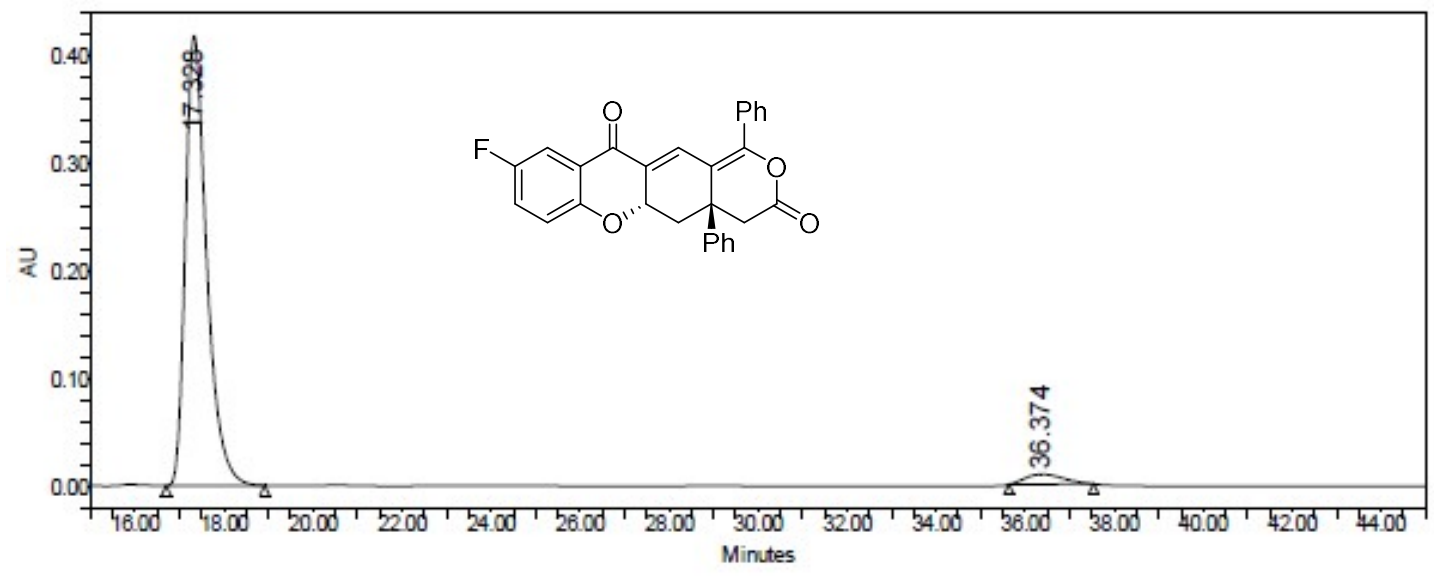

\begin{tabular}{|c|c|c|c|c|l|l|}
\hline & $R T(\min )$ & Int Type & $\begin{array}{c}\text { Width } \\
(\mathrm{sec})\end{array}$ & Area & Height & \% Area \\
\hline 1 & 17.328 & $\mathrm{bb}$ & 133.000 & 13953494 & 418793 & 96.13 \\
\hline 2 & 36.374 & $\mathrm{bb}$ & 114.000 & 561185 & 9411 & 3.87 \\
\hline Sum & & & & 14514678.9 & 428203.7 & 100.0 \\
\hline
\end{tabular}


Racemic 3n'

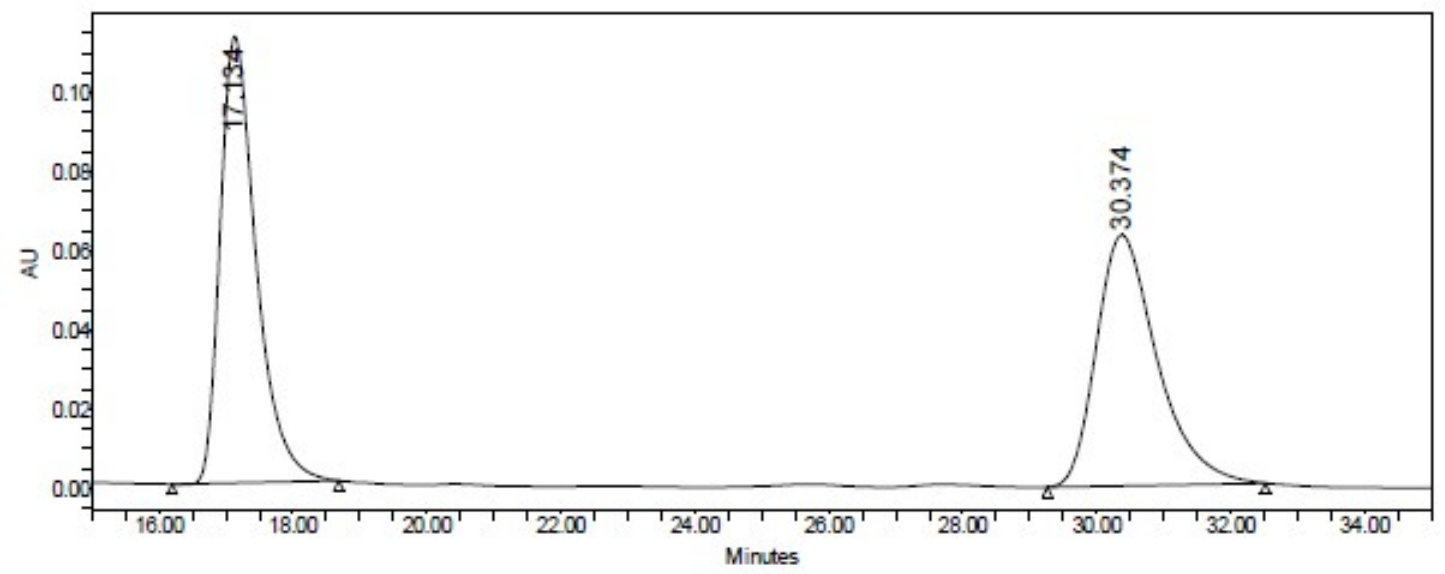

\begin{tabular}{|c|c|l|c|c|c|c|}
\hline & RT (min) & Int Type & $\begin{array}{c}\text { Width } \\
(\mathrm{sec})\end{array}$ & Area & Height & \% Area \\
\hline 1 & 17.134 & bb & 149.000 & 4219776 & 112836 & 51.38 \\
\hline 2 & 30.374 & bb & 195.000 & 3993689 & 63396 & 48.62 \\
\hline Sum & & & & 8213464.7 & 176232.2 & 100.0 \\
\hline
\end{tabular}

\section{Enantioenriched 3n'}

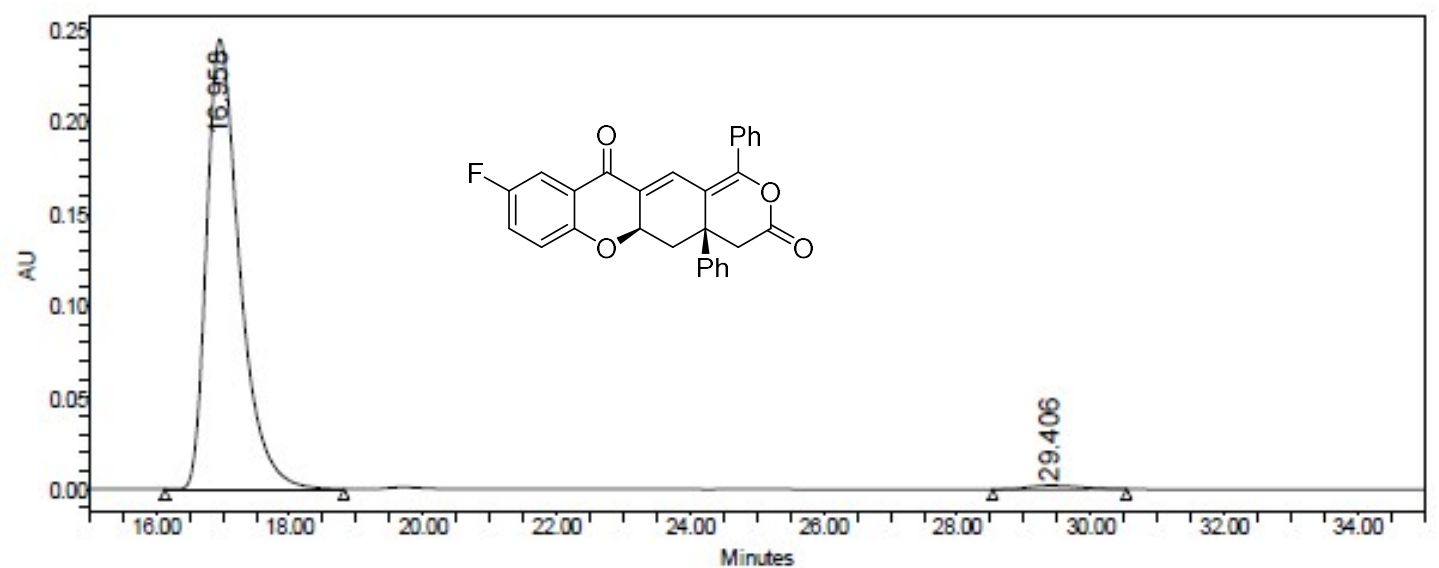

\begin{tabular}{|c|l|l|c|c|c|l|}
\hline & RT (min) & Int Type & $\begin{array}{c}\text { Width } \\
(\sec )\end{array}$ & Area & Height & $\%$ Area \\
\hline 1 & 16.958 & bb & 161.000 & 8426573 & 245241 & 98.69 \\
\hline 2 & 29.406 & bb & 120.000 & 111576 & 2108 & 1.31 \\
\hline Sum & & & & 8538148.4 & 247348.9 & 100.0 \\
\hline
\end{tabular}


Racemic 30

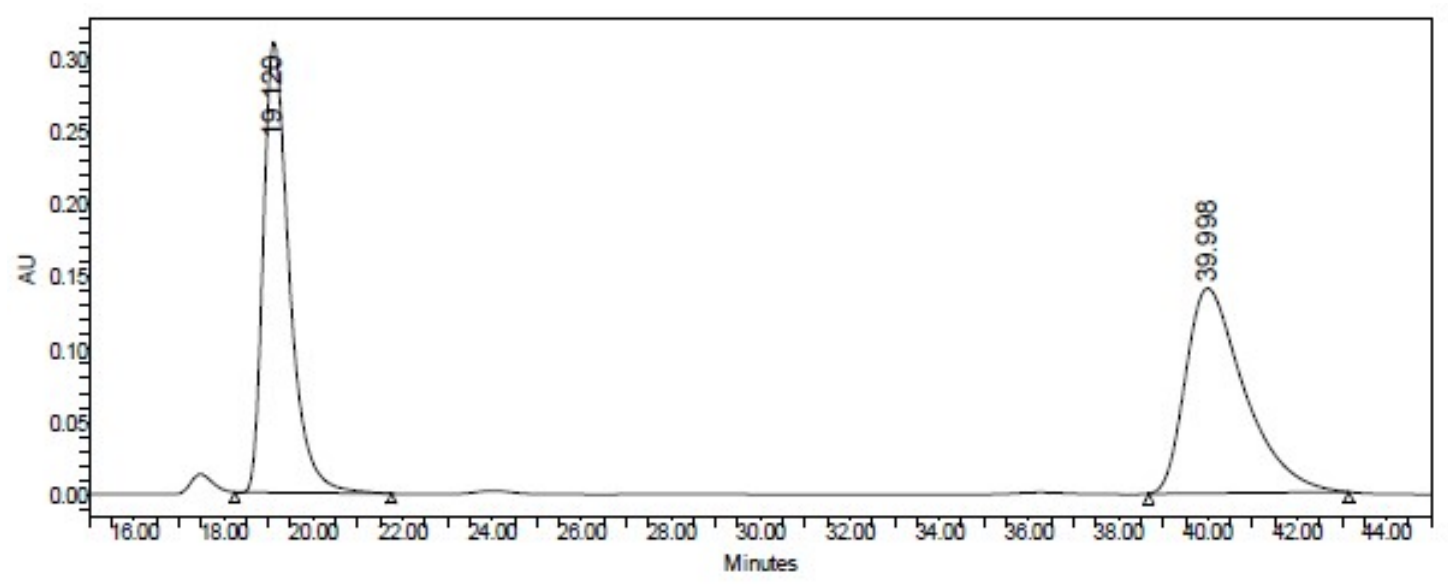

\begin{tabular}{|c|c|c|c|c|c|c|}
\hline & $R T(m i n)$ & Int Type & $\begin{array}{c}\text { Width } \\
(\mathrm{sec})\end{array}$ & Area & Height & $\%$ Area \\
\hline 1 & 19.120 & bb & 210.000 & 12483068 & 309644 & 49.26 \\
\hline 2 & 39.998 & bb & 268.000 & 12856969 & 140729 & 50.74 \\
\hline Sum & & & & 25340037.3 & 450373.0 & 100.0 \\
\hline
\end{tabular}

\section{Enantioenriched 30}

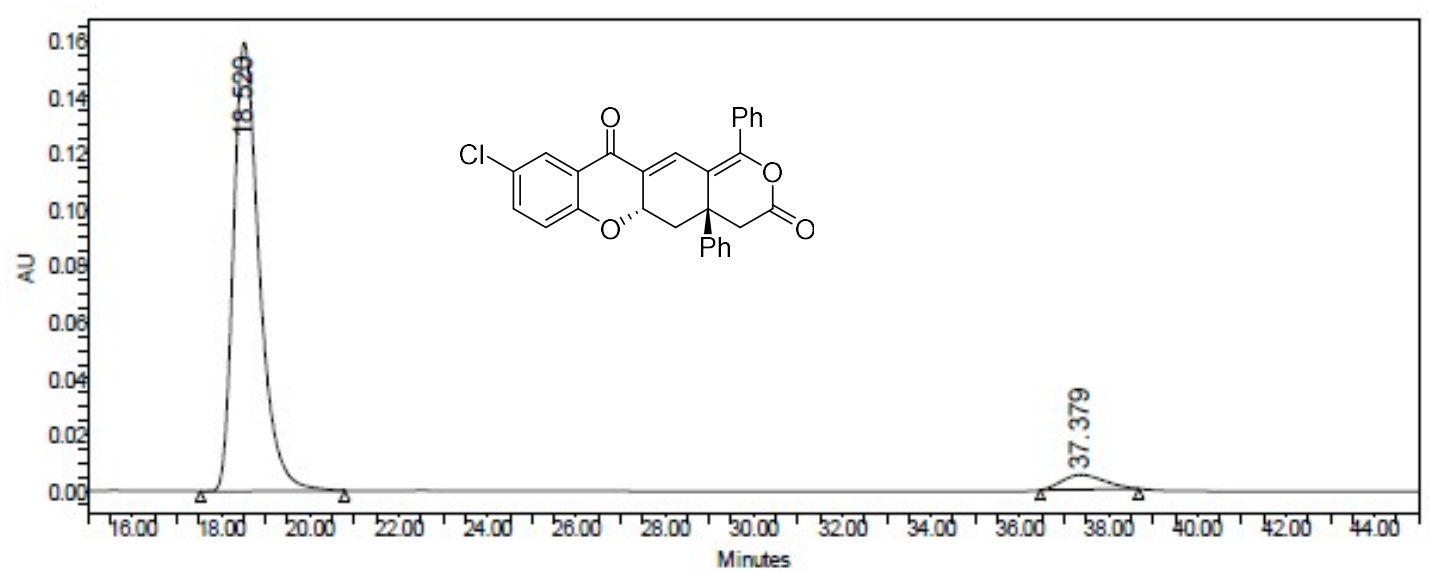

\begin{tabular}{|c|l|l|c|c|l|l|}
\hline & RT (min) & Int Type & $\begin{array}{c}\text { Width } \\
(\mathrm{sec})\end{array}$ & Area & Height & \% Area \\
\hline 1 & 18.520 & bb & 195.000 & 6439981 & 159234 & 94.88 \\
\hline 2 & 37.379 & bb & 133.000 & 347818 & 5205 & 5.12 \\
\hline Sum & & & & 6787798.8 & 164438.7 & 100.0 \\
\hline
\end{tabular}


Racemic 30'

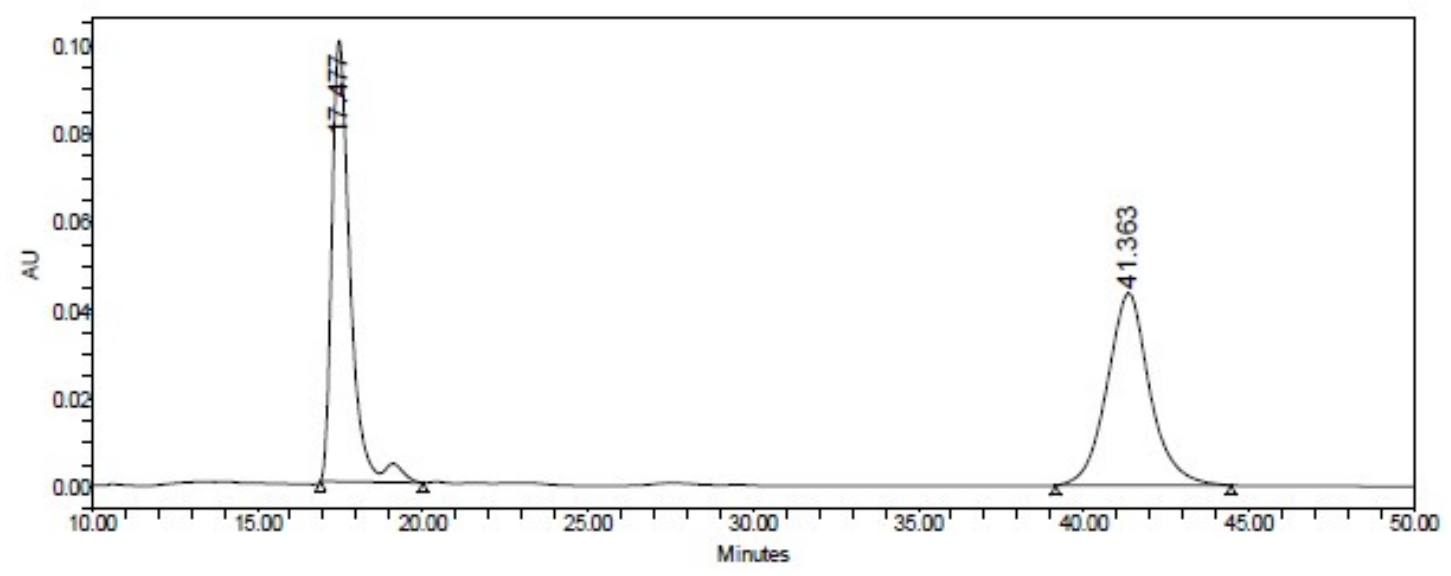

\begin{tabular}{|c|l|l|c|c|l|l|}
\hline & RT(min) & Int Type & $\begin{array}{c}\text { Width } \\
(\mathrm{sec})\end{array}$ & Area & Height & \% Area \\
\hline 1 & 17.477 & bb & 186.000 & 3891330 & 100010 & 49.88 \\
\hline 2 & 41.363 & bb & 319.000 & 3910031 & 43563 & 50.12 \\
\hline Sum & & & & 7801361.8 & 143573.5 & 100.0 \\
\hline
\end{tabular}

\section{Enantioenriched 30'}

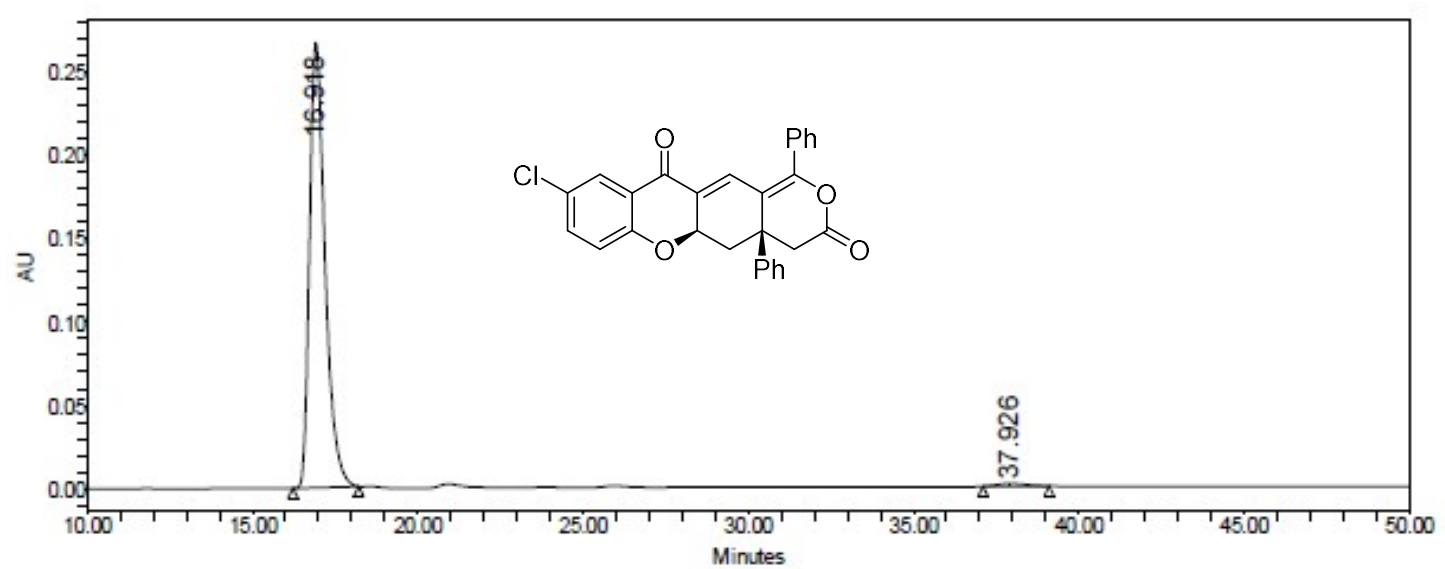

\begin{tabular}{|c|l|l|c|c|l|l|}
\hline & RT (min) & Int Type & $\begin{array}{c}\text { Width } \\
(\mathrm{sec})\end{array}$ & Area & Height & \% Area \\
\hline 1 & 16.918 & bb & 120.000 & 8666506 & 266321 & 98.84 \\
\hline 2 & 37.926 & bb & 120.000 & 101840 & 1616 & 1.16 \\
\hline Sum & & & & 8768346.0 & 267936.6 & 100.0 \\
\hline
\end{tabular}


Racemic 3p

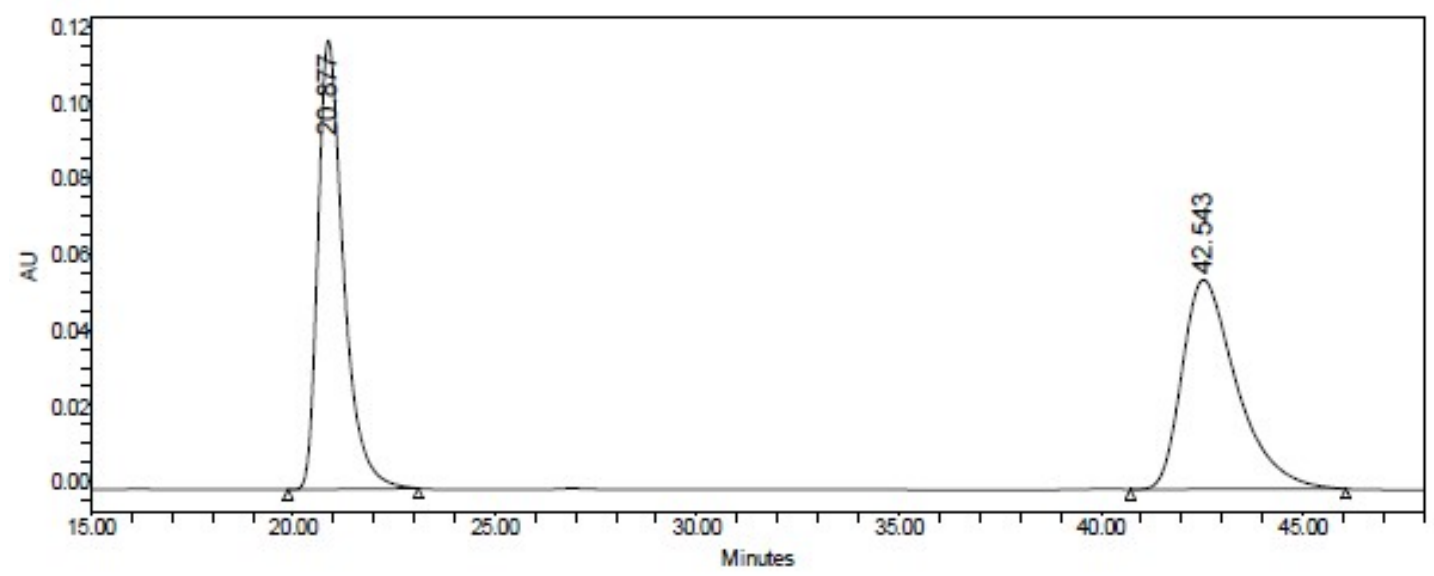

\begin{tabular}{|c|c|c|c|c|c|c|}
\hline & RT (min) & Int Type & $\begin{array}{c}\text { Width } \\
(\mathrm{sec})\end{array}$ & Area & Height & \% Area \\
\hline 1 & 20.877 & bb & 193.000 & 5218041 & 118400 & 50.16 \\
\hline 2 & 42.543 & bb & 320.000 & 5185125 & 55172 & 49.84 \\
\hline Sum & & & & 10403165.3 & 173572.1 & 100.0 \\
\hline
\end{tabular}

\section{Racemic 3p}

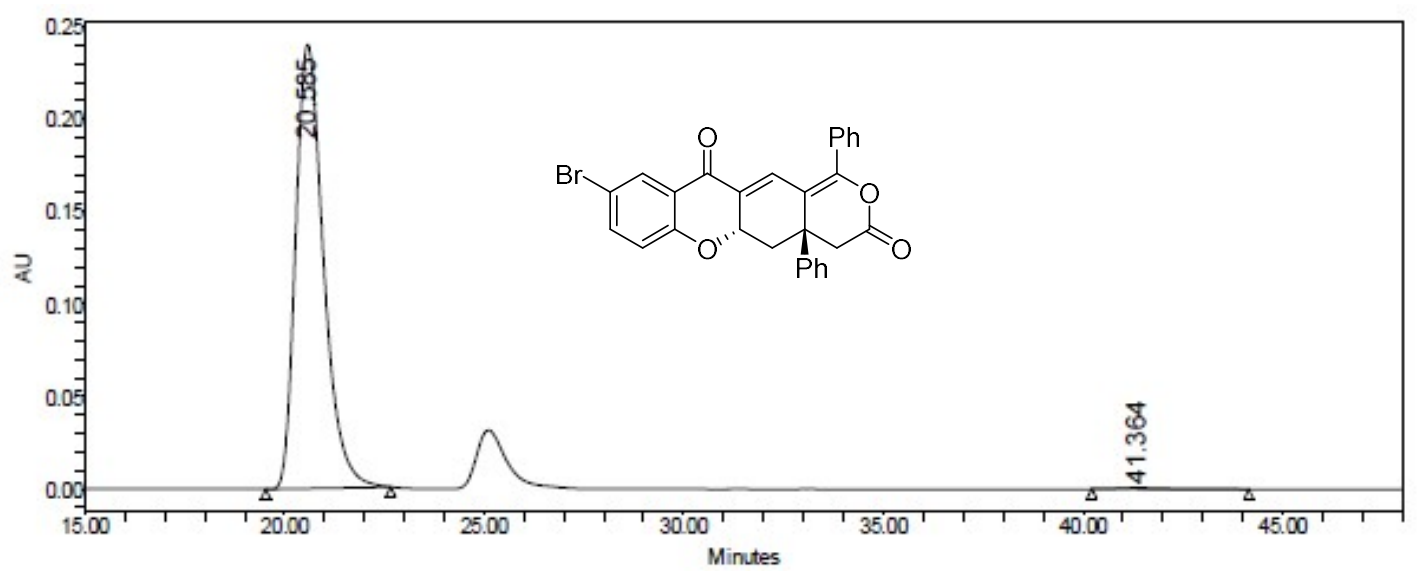

\begin{tabular}{|c|l|l|c|c|l|l|}
\hline & RT (min) & Int Type & $\begin{array}{c}\text { Width } \\
(\mathrm{sec})\end{array}$ & Area & Height & \% Area \\
\hline 1 & 20.585 & bb & 188.000 & 11649099 & 240056 & 99.32 \\
\hline 2 & 41.364 & bb & 236.000 & 80324 & 798 & 0.68 \\
\hline Sum & & & & 11729422.9 & 240853.1 & 100.0 \\
\hline
\end{tabular}


Racemic 3p'

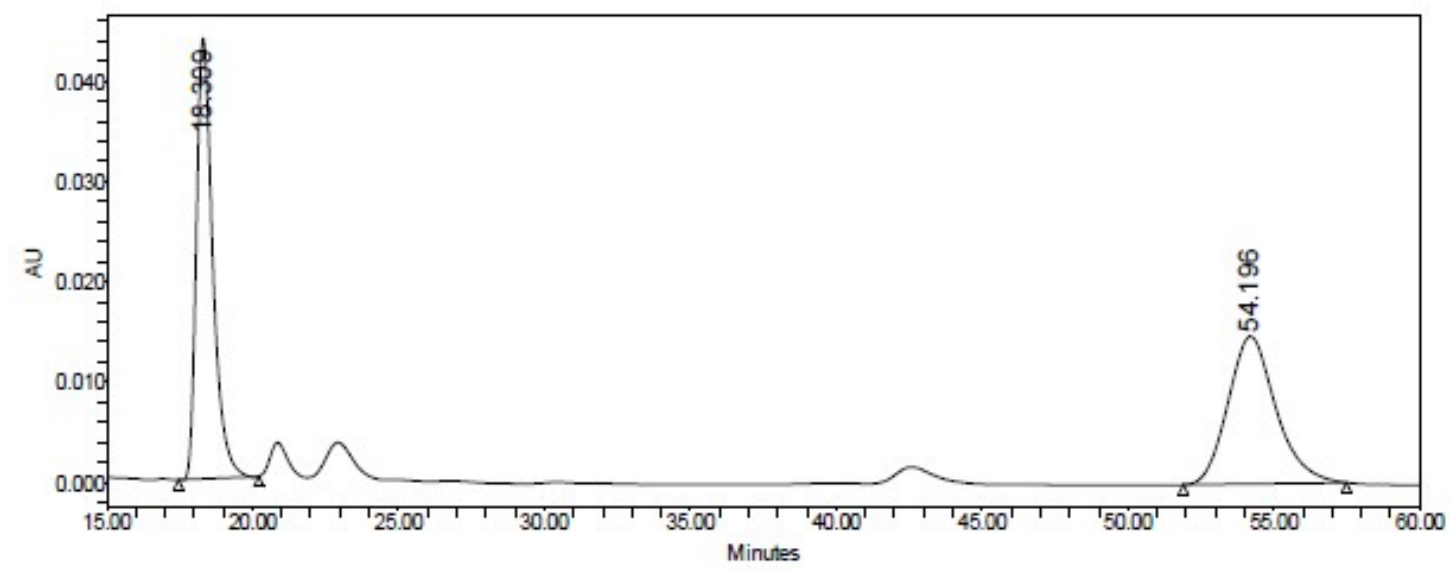

\begin{tabular}{|c|c|l|c|c|c|c|}
\hline & $R T(\min )$ & Int Type & $\begin{array}{c}\text { Width } \\
(\mathrm{sec})\end{array}$ & Area & Height & $\%$ Area \\
\hline 1 & 18.309 & $\mathrm{bb}$ & 165.000 & 1709049 & 43955 & 50.41 \\
\hline 2 & 54.196 & $\mathrm{bb}$ & 335.000 & 1680945 & 14707 & 49.59 \\
\hline Sum & & & & 3389994.6 & 58662.3 & 100.0 \\
\hline
\end{tabular}

\section{Enantioenriched 3p'}

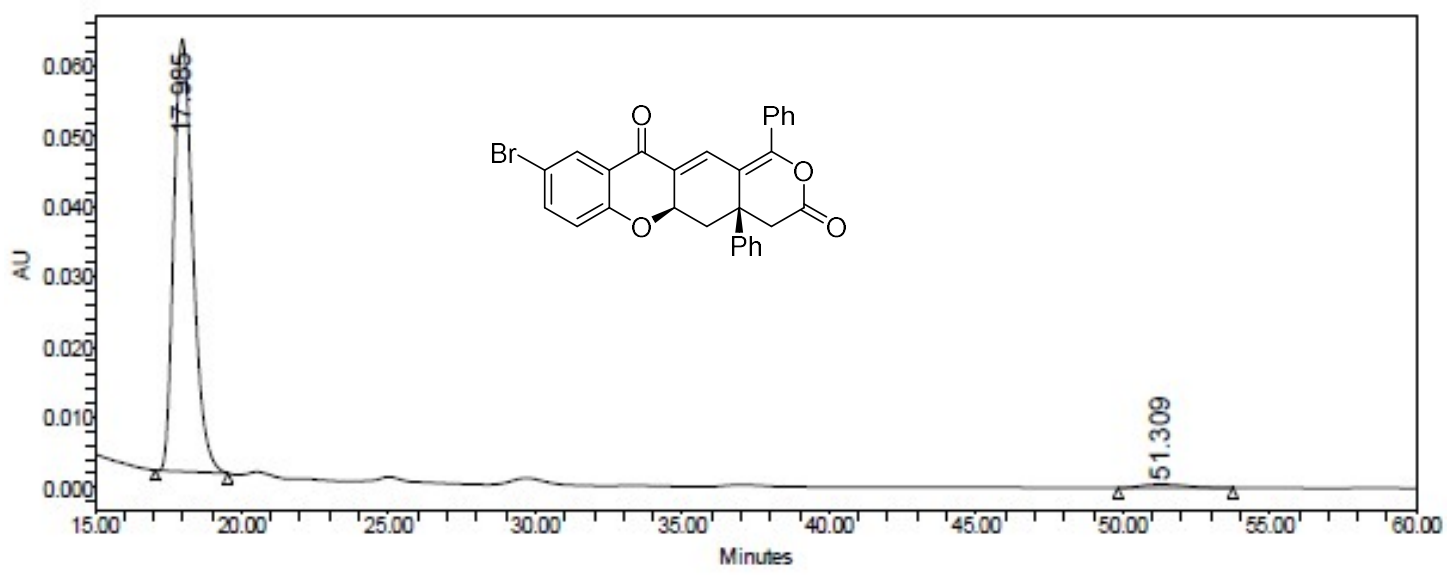

\begin{tabular}{|c|c|l|c|c|l|l|}
\hline & RT (min) & Int Type & $\begin{array}{c}\text { Width } \\
(\mathrm{sec})\end{array}$ & Area & Height & $\%$ Area \\
\hline 1 & 17.985 & $\mathrm{bb}$ & 148.000 & 2763912 & 61607 & 98.21 \\
\hline 2 & 51.309 & $\mathrm{bb}$ & 234.000 & 50471 & 507 & 1.79 \\
\hline Sum & & & & 2814383.6 & 62114.0 & 100.0 \\
\hline
\end{tabular}




\section{Racemic 3q}

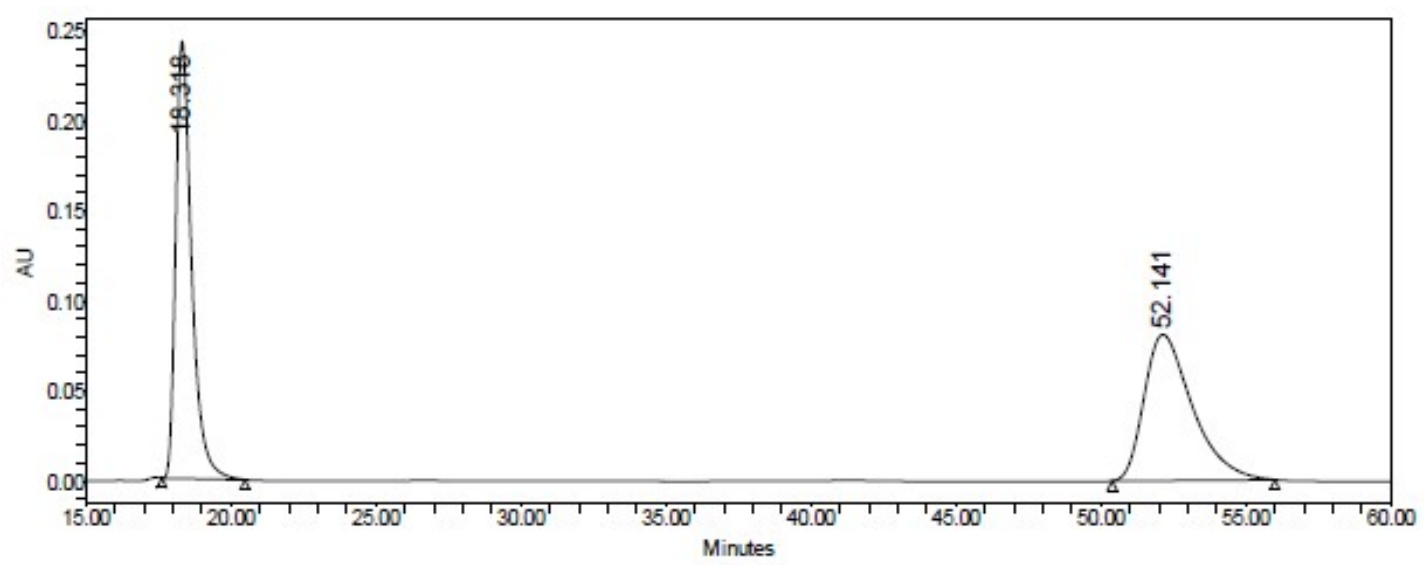

\begin{tabular}{|c|c|l|c|c|c|c|}
\hline & RT (min) & Int Type & $\begin{array}{c}\text { Width } \\
(\mathrm{sec})\end{array}$ & Area & Height & \% Area \\
\hline 1 & 18.318 & bb & 172.000 & 9287024 & 242515 & 49.81 \\
\hline 2 & 52.141 & bb & 337.000 & 9358879 & 81037 & 50.19 \\
\hline Sum & & & & 18645902.9 & 323552.1 & 100.0 \\
\hline
\end{tabular}

\section{Enantioenriched 3q}

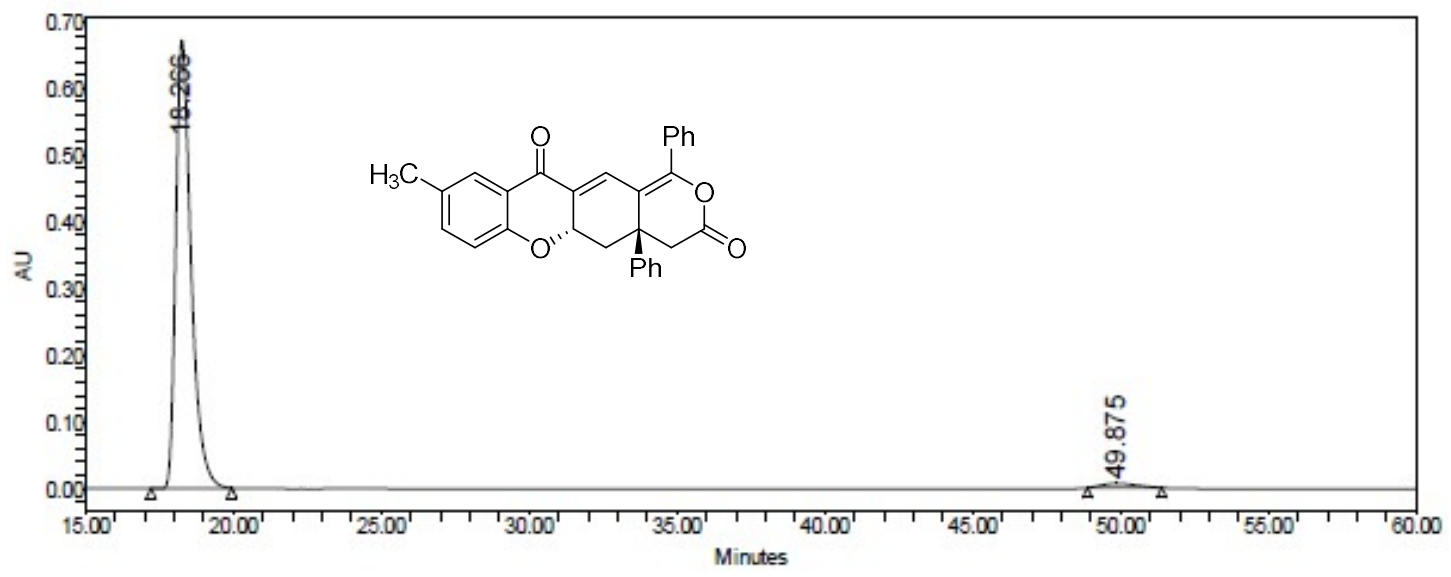

\begin{tabular}{|c|c|l|c|c|l|l|}
\hline & RT (min) & Int Type & $\begin{array}{c}\text { Width } \\
(\mathrm{sec})\end{array}$ & Area & Height & $\%$ Area \\
\hline 1 & 18.266 & bb & 164.000 & 24244784 & 671712 & 97.83 \\
\hline 2 & 49.875 & bb & 151.000 & 536882 & 6603 & 2.17 \\
\hline Sum & & & & 24781666.2 & 678315.0 & 100.0 \\
\hline
\end{tabular}




\section{Racemic 3q'}

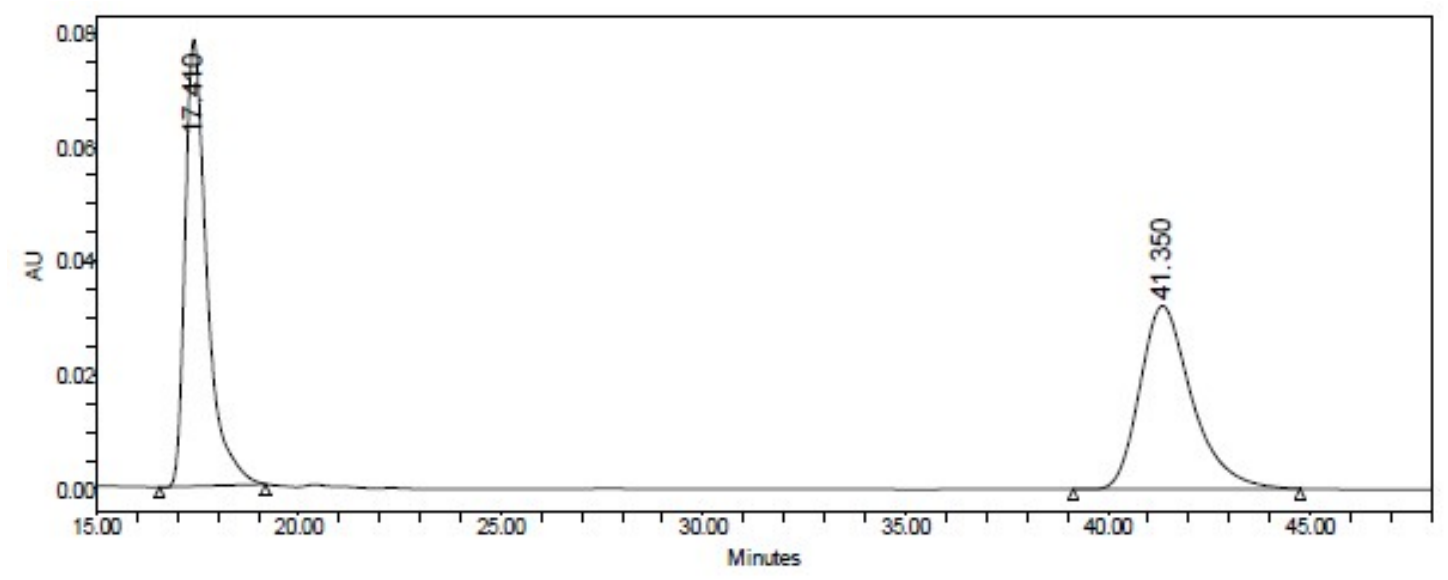

\begin{tabular}{|c|l|l|c|c|c|c|}
\hline & RT $(\min )$ & Int Type & $\begin{array}{c}\text { Width } \\
(\mathrm{sec})\end{array}$ & Area & Height & \% Area \\
\hline 1 & 17.410 & bb & 157.000 & 2932647 & 78356 & 50.54 \\
\hline 2 & 41.350 & bb & 336.000 & 2869501 & 32130 & 49.46 \\
\hline Sum & & & & 5802148.9 & 110485.2 & 100.0 \\
\hline
\end{tabular}

\section{Enantioenriched 3q'}

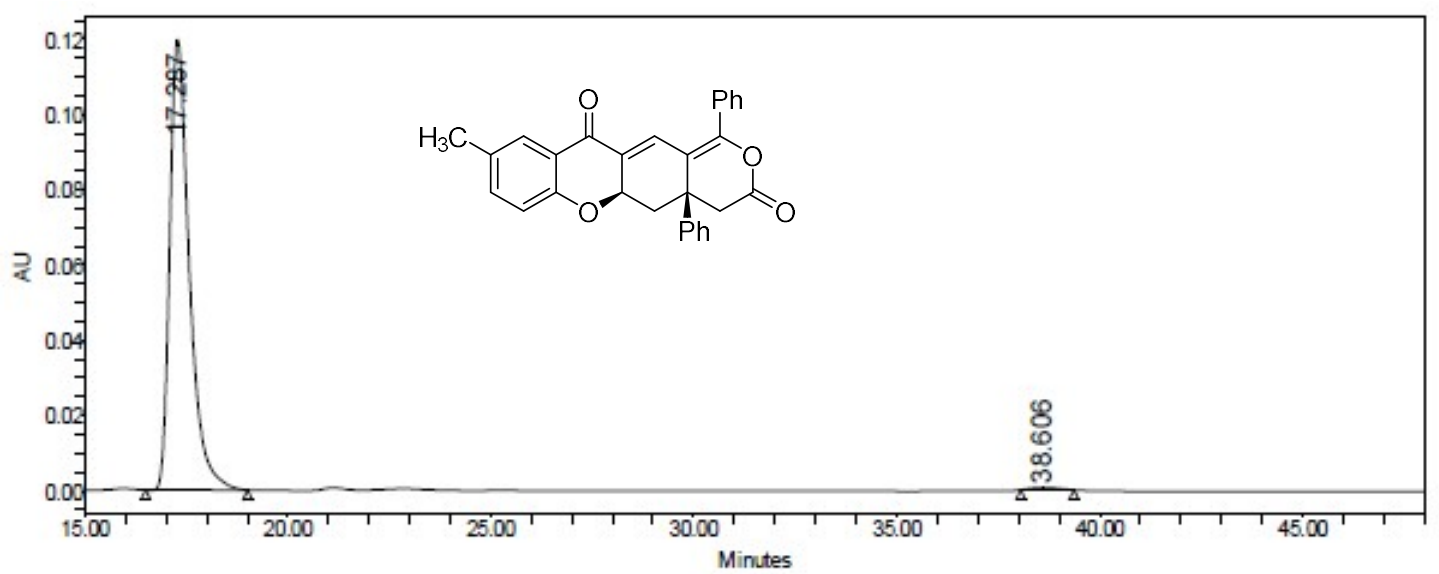

\begin{tabular}{|c|l|l|c|c|c|l|}
\hline & RT (min) & Int Type & $\begin{array}{c}\text { Width } \\
(\mathrm{sec})\end{array}$ & Area & Height & \% Area \\
\hline 1 & 17.287 & $\mathrm{bb}$ & 151.000 & 4000289 & 119518 & 99.35 \\
\hline 2 & 38.606 & $\mathrm{bb}$ & 79.000 & 26106 & 557 & 0.65 \\
\hline Sum & & & & 4026394.3 & 120075.2 & 100.0 \\
\hline
\end{tabular}


Racemic 3r

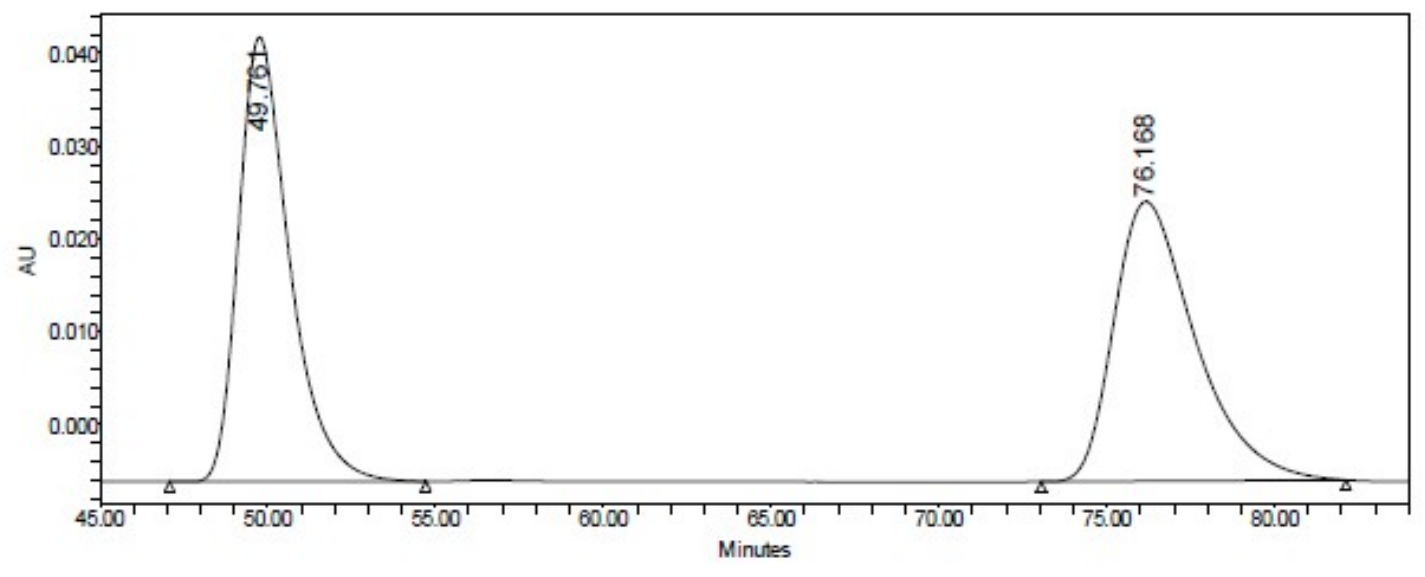

\begin{tabular}{|c|c|l|c|c|c|c|}
\hline & RT (min) & Int Type & $\begin{array}{c}\text { Width } \\
(\mathrm{sec})\end{array}$ & Area & Height & $\%$ Area \\
\hline 1 & 49.761 & bb & 458.000 & 5071237 & 47940 & 49.93 \\
\hline 2 & 76.168 & bb & 545.000 & 5085348 & 30142 & 50.07 \\
\hline Sum & & & & 10156585.2 & 78081.9 & 100.0 \\
\hline
\end{tabular}

\section{Enantioenriched 3r}

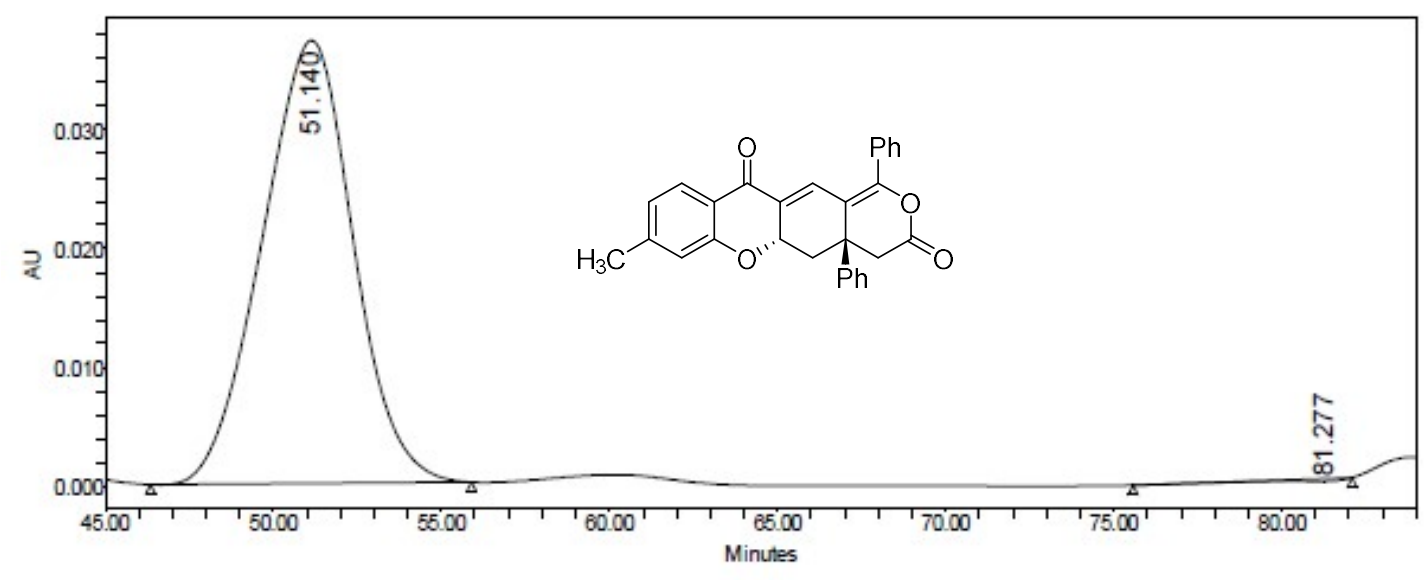

\begin{tabular}{|c|c|c|c|c|c|l|}
\hline & RT (min) & Int Type & $\begin{array}{c}\text { Width } \\
(\mathrm{sec})\end{array}$ & Area & Height & $\%$ Area \\
\hline 1 & 51.140 & bb & 573.000 & 7031642 & 37317 & 99.58 \\
\hline 2 & 81.277 & bb & 391.000 & 29621 & -234 & 0.42 \\
\hline Sum & & & & 7061263.4 & 37082.2 & 100.0 \\
\hline
\end{tabular}


Racemic 3r'

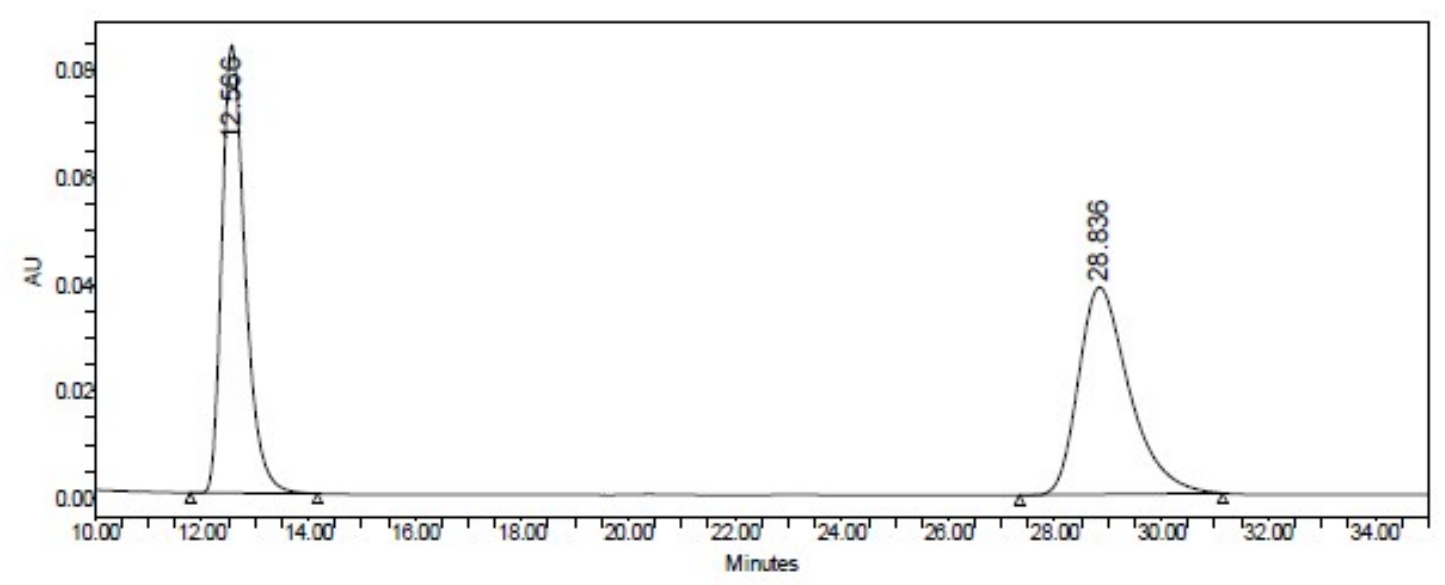

\begin{tabular}{|c|c|l|c|c|c|c|}
\hline & $R T(\min )$ & Int Type & $\begin{array}{c}\text { Width } \\
(\mathrm{sec})\end{array}$ & Area & Height & $\%$ Area \\
\hline 1 & 12.566 & bb & 143.000 & 2537596 & 83760 & 49.75 \\
\hline 2 & 28.836 & bb & 228.500 & 2563086 & 38739 & 50.25 \\
\hline Sum & & & & 5100681.9 & 122499.0 & 100.0 \\
\hline
\end{tabular}

\section{Enantioenriched 3r'}

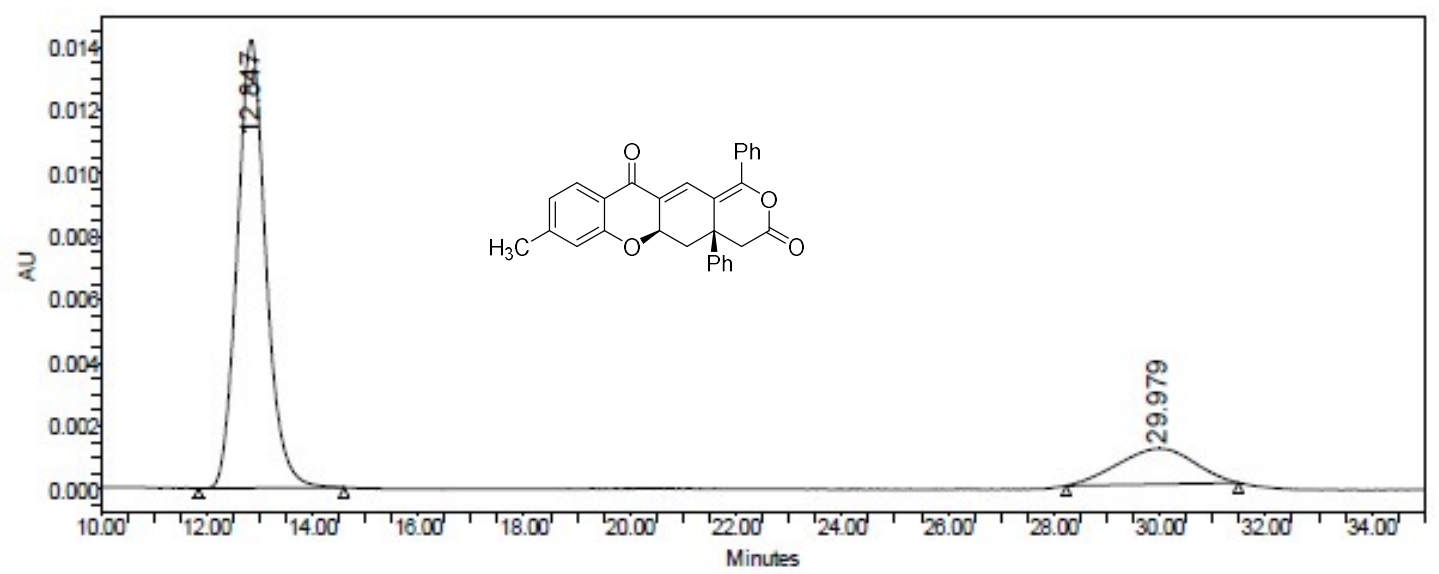

\begin{tabular}{|c|c|l|c|c|l|l|}
\hline & $R T(\min )$ & Int Type & $\begin{array}{c}\text { Width } \\
(\mathrm{sec})\end{array}$ & Area & Height & $\%$ Area \\
\hline 1 & 12.847 & bb & 164.500 & 531038 & 14170 & 82.53 \\
\hline 2 & 29.979 & bb & 195.000 & 112426 & 1122 & 17.47 \\
\hline Sum & & & & 643464.1 & 15291.6 & 100.0 \\
\hline
\end{tabular}


Racemic 3s

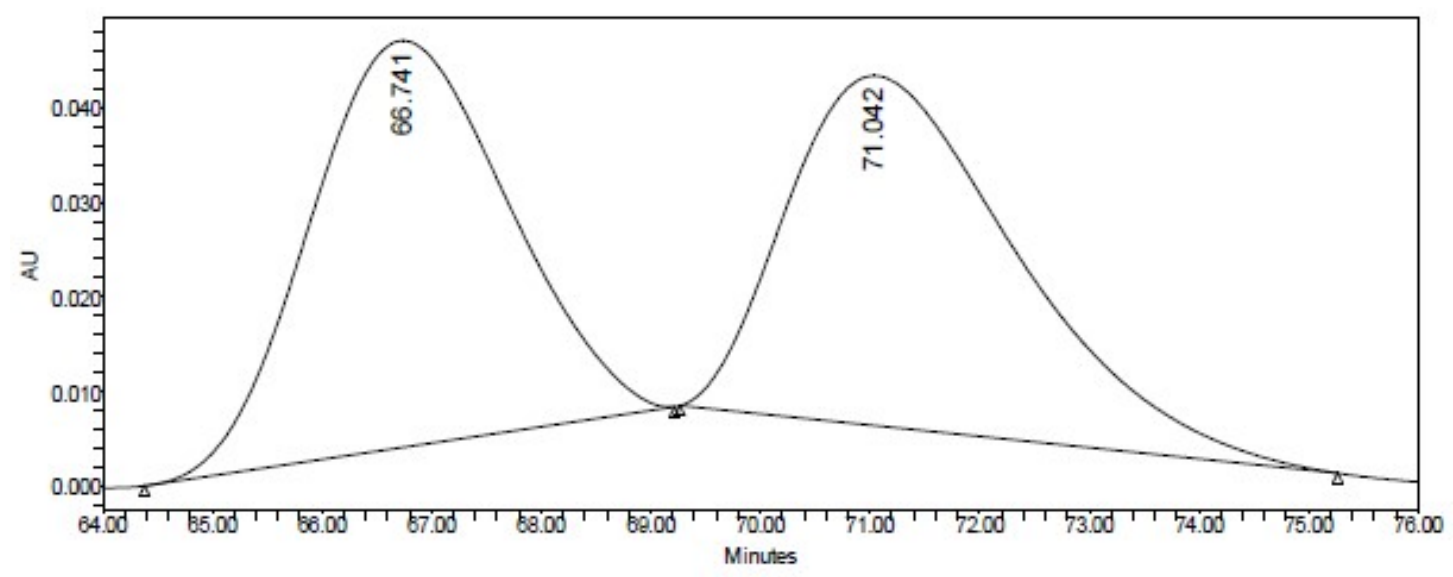

\begin{tabular}{|c|c|c|c|c|c|c|}
\hline & RT (min) & Int Type & $\begin{array}{c}\text { Width } \\
(\mathrm{sec})\end{array}$ & Area & Height & $\%$ Area \\
\hline 1 & 66.741 & bb & 290.000 & 5374152 & 42863 & 49.97 \\
\hline 2 & 71.042 & bb & 360.000 & 5380017 & 36867 & 50.03 \\
\hline Sum & & & & 10754169.7 & 79729.2 & 100.0 \\
\hline
\end{tabular}

\section{Enantioenriched 3s}

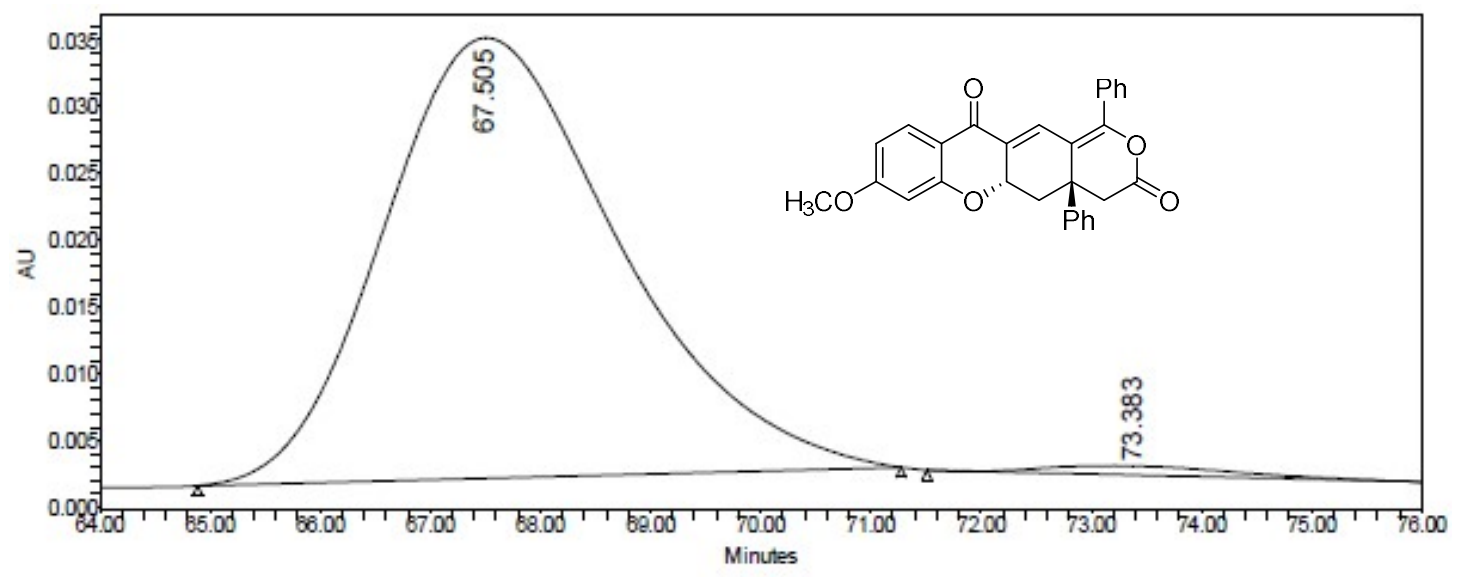

\begin{tabular}{|c|l|l|c|c|l|l|}
\hline & RT (min) & Int Type & $\begin{array}{c}\text { Width } \\
(\mathrm{sec})\end{array}$ & Area & Height & \% Area \\
\hline 1 & 67.505 & $\mathrm{bb}$ & 383.000 & 4888247 & 32910 & 98.37 \\
\hline 2 & 73.383 & $\mathrm{bb}$ & 333.500 & 80840 & 675 & 1.63 \\
\hline Sum & & & & 4969087.4 & 33584.9 & 100.0 \\
\hline
\end{tabular}


Racemic 3s'

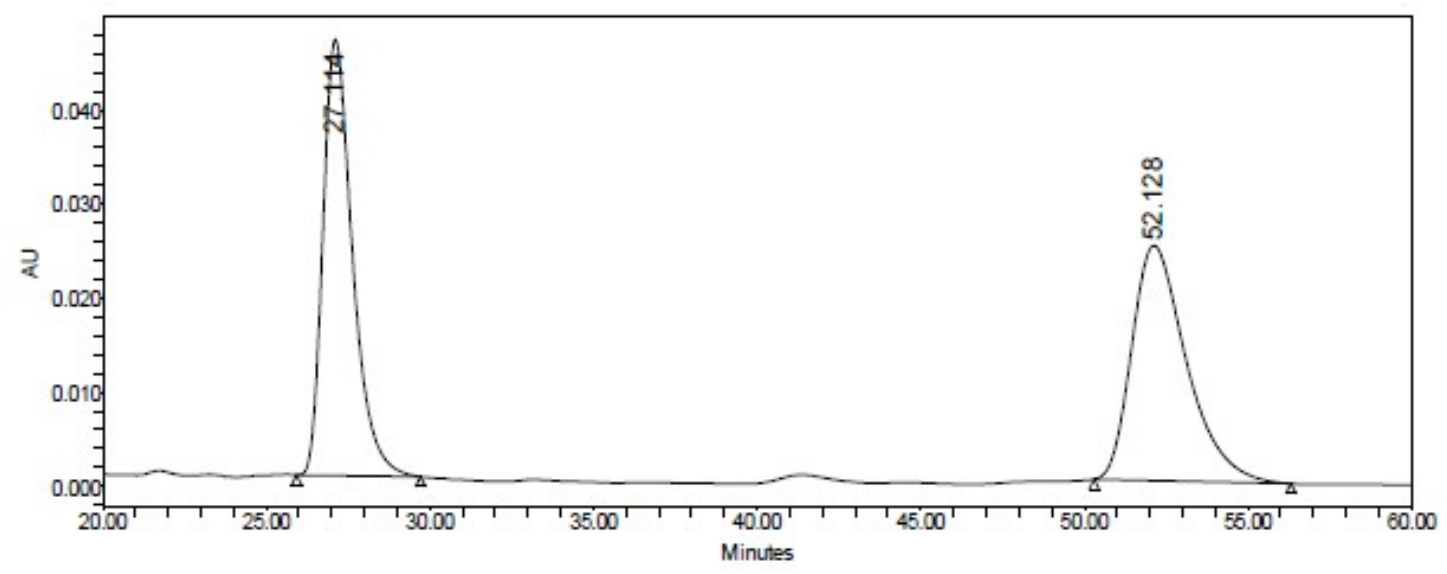

\begin{tabular}{|c|c|l|c|c|c|c|}
\hline & RT (min) & Int Type & $\begin{array}{c}\text { Width } \\
(\mathrm{sec})\end{array}$ & Area & Height & $\%$ Area \\
\hline 1 & 27.114 & bb & 227.000 & 2907333 & 46381 & 50.04 \\
\hline 2 & 52.128 & bb & 361.000 & 2902855 & 24991 & 49.96 \\
\hline Sum & & & & 5810188.4 & 71372.6 & 100.0 \\
\hline
\end{tabular}

\section{Enantioenriched 3s'}

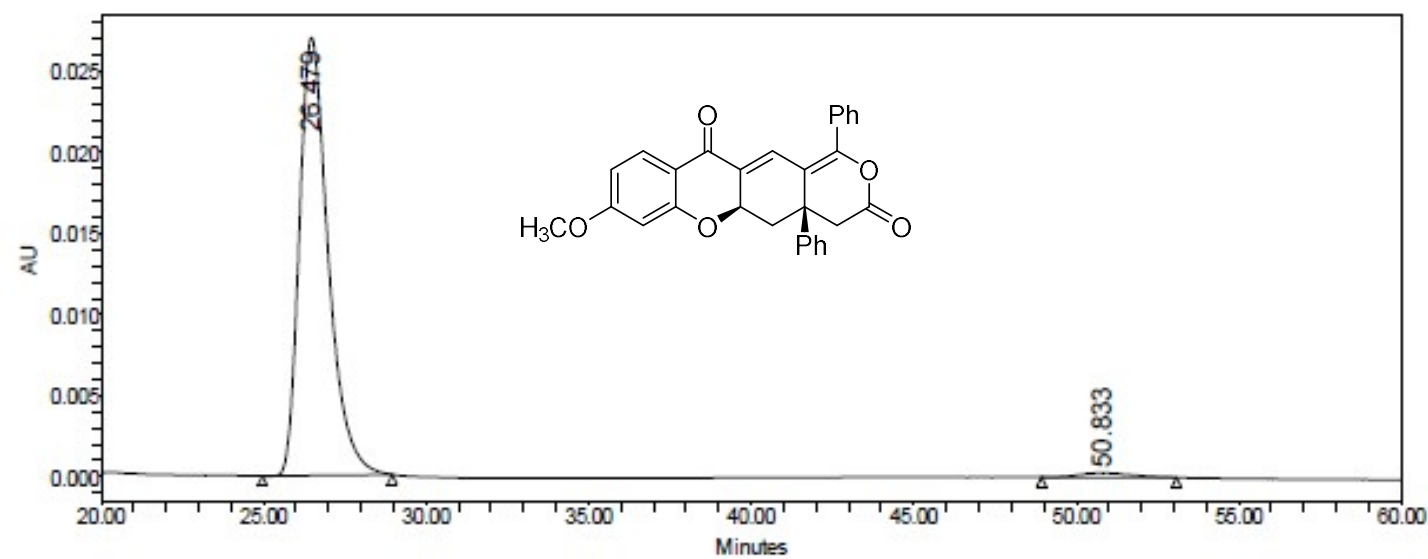

\begin{tabular}{|c|l|l|c|c|l|l|}
\hline & RT (min) & Int Type & $\begin{array}{c}\text { Width } \\
(\mathrm{sec})\end{array}$ & Area & Height & \% Area \\
\hline 1 & 26.479 & bb & 240.000 & 1661971 & 26985 & 98.19 \\
\hline 2 & 50.833 & bb & 249.000 & 30690 & 275 & 1.81 \\
\hline Sum & & & & 1692660.9 & 27260.7 & 100.0 \\
\hline
\end{tabular}




\section{Racemic 3t}

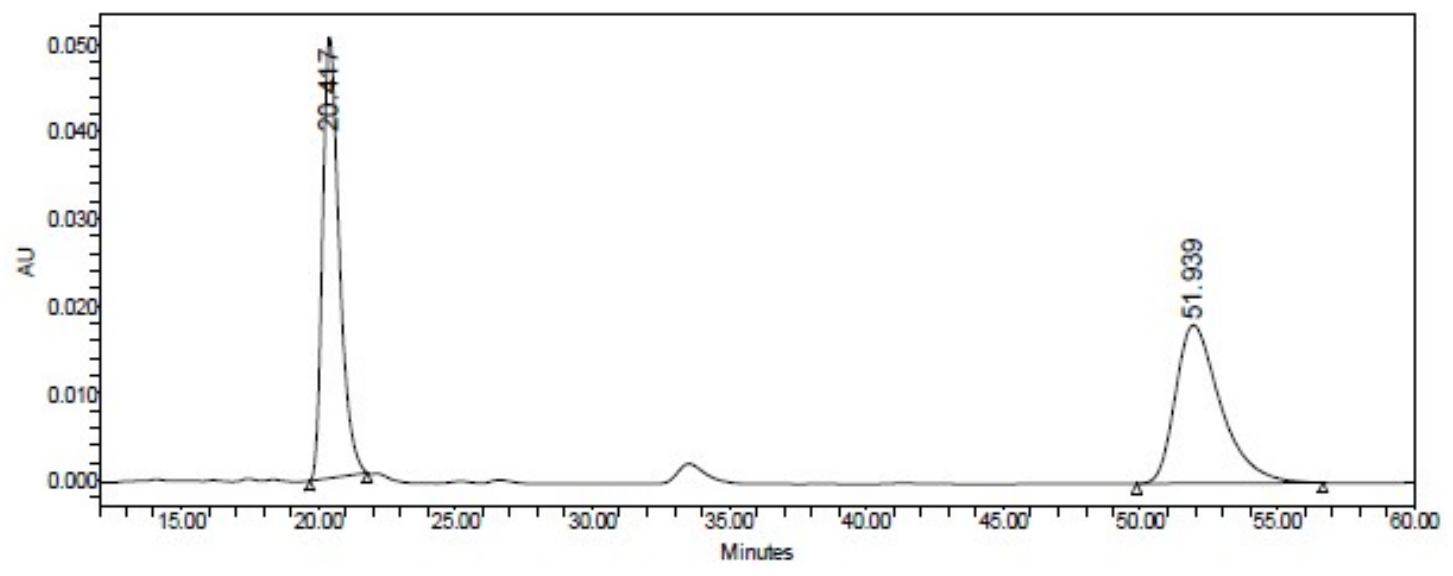

\begin{tabular}{|c|c|l|c|c|c|c|}
\hline & RT (min) & Int Type & $\begin{array}{c}\text { Width } \\
(\mathrm{sec})\end{array}$ & Area & Height & $\%$ Area \\
\hline 1 & 20.417 & bb & 125.000 & 2138583 & 50478 & 50.79 \\
\hline 2 & 51.939 & bb & 408.000 & 2071964 & 18111 & 49.21 \\
\hline Sum & & & & 4210546.9 & 68588.4 & 100.0 \\
\hline
\end{tabular}

\section{Enantioenriched 3t}

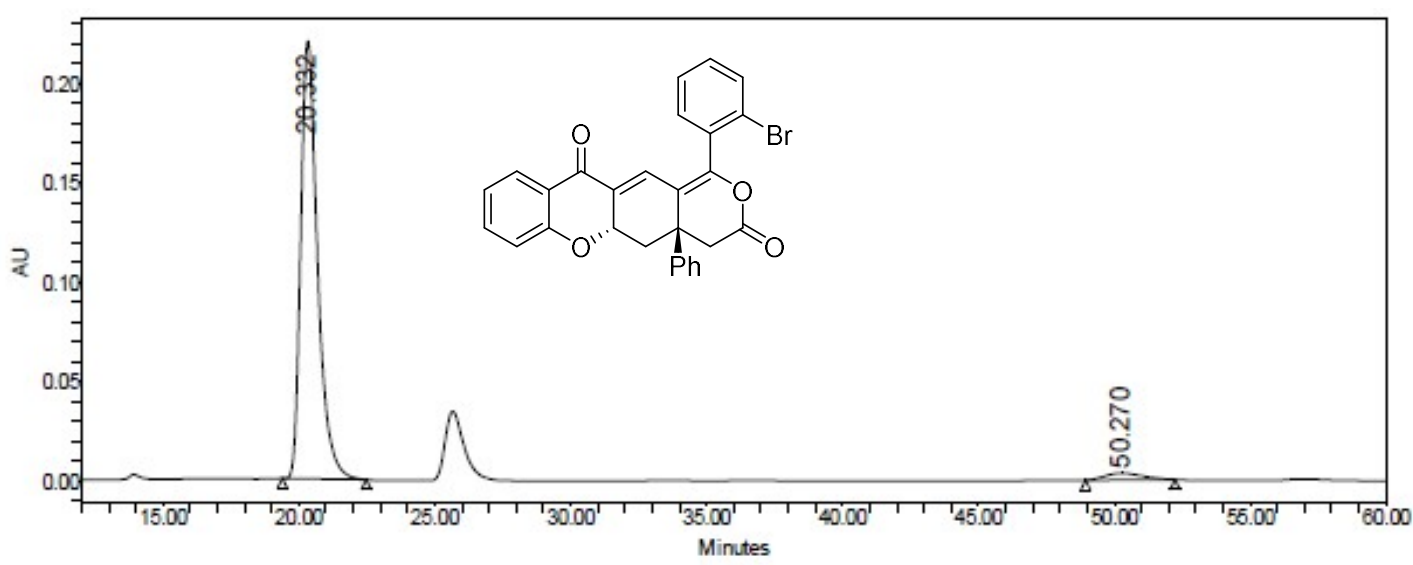

\begin{tabular}{|c|l|l|c|c|c|l|}
\hline & $R T(\min )$ & Int Type & $\begin{array}{c}\text { Width } \\
(\mathrm{sec})\end{array}$ & Area & Height & $\%$ Area \\
\hline 1 & 20.332 & bb & 185.000 & 9449504 & 220260 & 96.67 \\
\hline 2 & 50.270 & bb & 197.000 & 325481 & 3410 & 3.33 \\
\hline Sum & & & & 9774985.6 & 223669.8 & 100.0 \\
\hline
\end{tabular}


Racemic 3t'

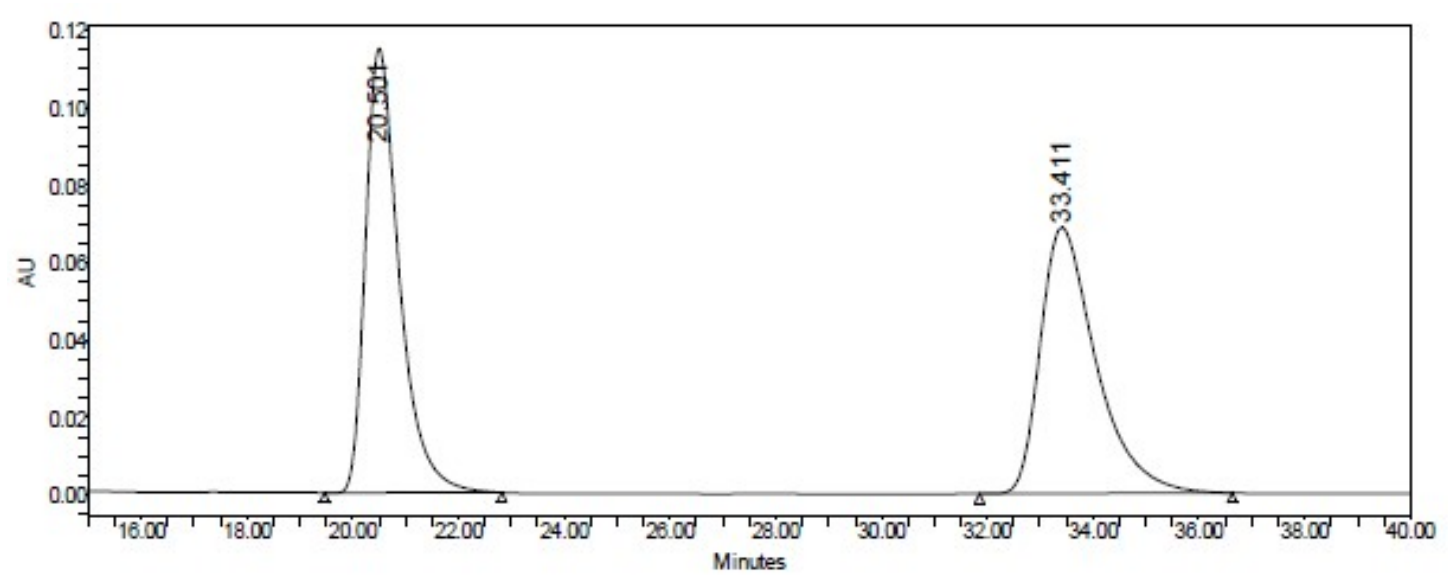

\begin{tabular}{|c|l|l|c|c|l|l|}
\hline & $R T(\min )$ & Int Type & $\begin{array}{c}\text { Width } \\
(\mathrm{sec})\end{array}$ & \multicolumn{1}{|c|}{ Area } & Height & $\%$ Area \\
\hline 1 & 20.501 & bb & 201.000 & 5104648 & 114800 & 50.39 \\
\hline 2 & 33.411 & bb & 287.000 & 5026287 & 68658 & 49.61 \\
\hline Sum & & & & 10130934.9 & 183458.5 & 100.0 \\
\hline
\end{tabular}

\section{Enantioenriched 3t'}

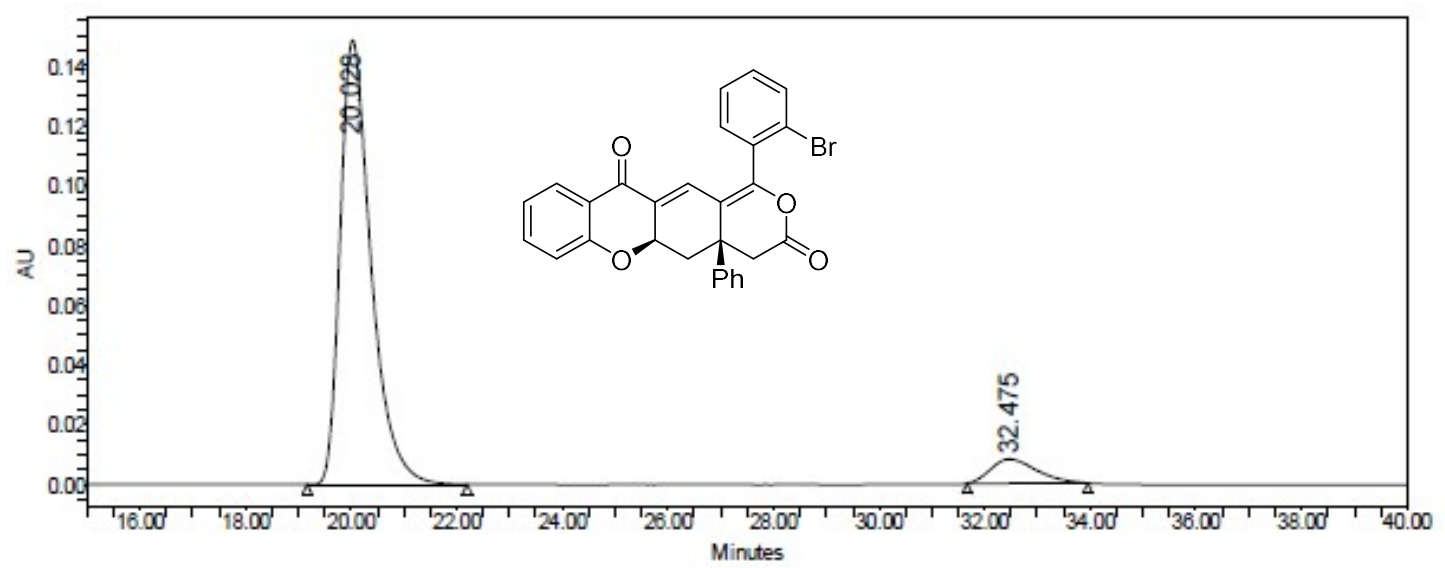

\begin{tabular}{|c|c|l|c|c|c|l|}
\hline & RT (min) & Int Type & $\begin{array}{c}\text { Width } \\
(\mathrm{sec})\end{array}$ & Area & Height & \% Area \\
\hline 1 & 20.028 & bb & 182.000 & 6033532 & 148526 & 92.51 \\
\hline 2 & 32.475 & bb & 137.000 & 488408 & 8040 & 7.49 \\
\hline Sum & & & & 6521939.9 & 156565.9 & 100.0 \\
\hline
\end{tabular}


Racemic 3u

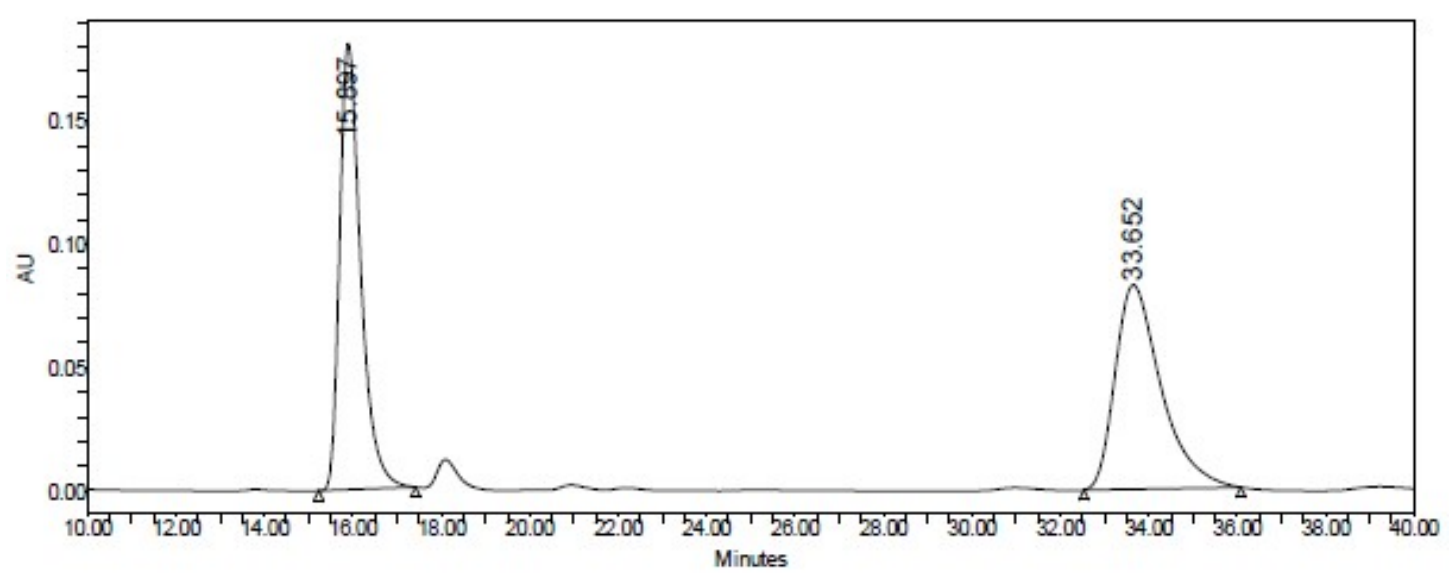

\begin{tabular}{|c|l|l|c|c|l|l|}
\hline & RT (min) & Int Type & $\begin{array}{c}\text { Width } \\
(\mathrm{sec})\end{array}$ & Area & Height & $\%$ Area \\
\hline 1 & 15.897 & bb & 131.000 & 5996214 & 180826 & 50.08 \\
\hline 2 & 33.652 & bb & 213.000 & 5976731 & 82772 & 49.92 \\
\hline Sum & & & & 11972944.7 & 263597.6 & 100.0 \\
\hline
\end{tabular}

\section{Enantioenriched 3u}

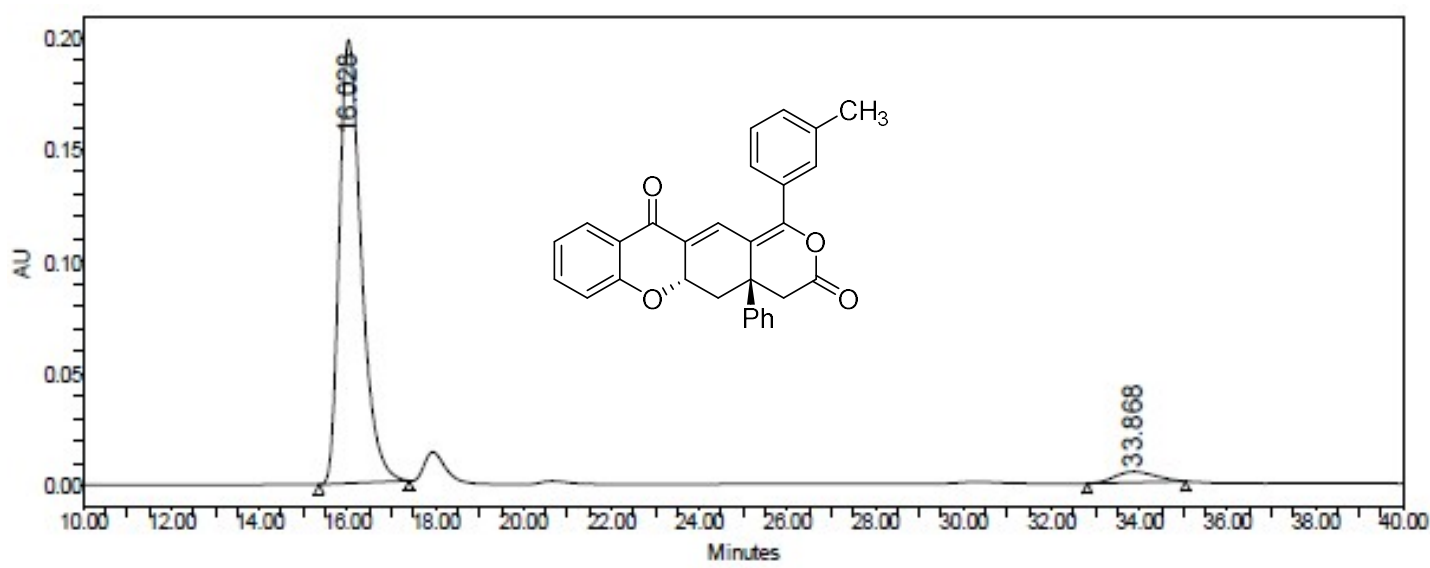

\begin{tabular}{|c|l|l|c|c|l|l|}
\hline & RT (min) & Int Type & $\begin{array}{c}\text { Width } \\
(\mathrm{sec})\end{array}$ & Area & Height & $\%$ Area \\
\hline 1 & 16.028 & bb & 123.000 & 6902642 & 198182 & 95.75 \\
\hline 2 & 33.868 & bb & 134.000 & 306167 & 4828 & 4.25 \\
\hline Sum & & & & 7208808.7 & 203009.6 & 100.0 \\
\hline
\end{tabular}


Racemic 3u'

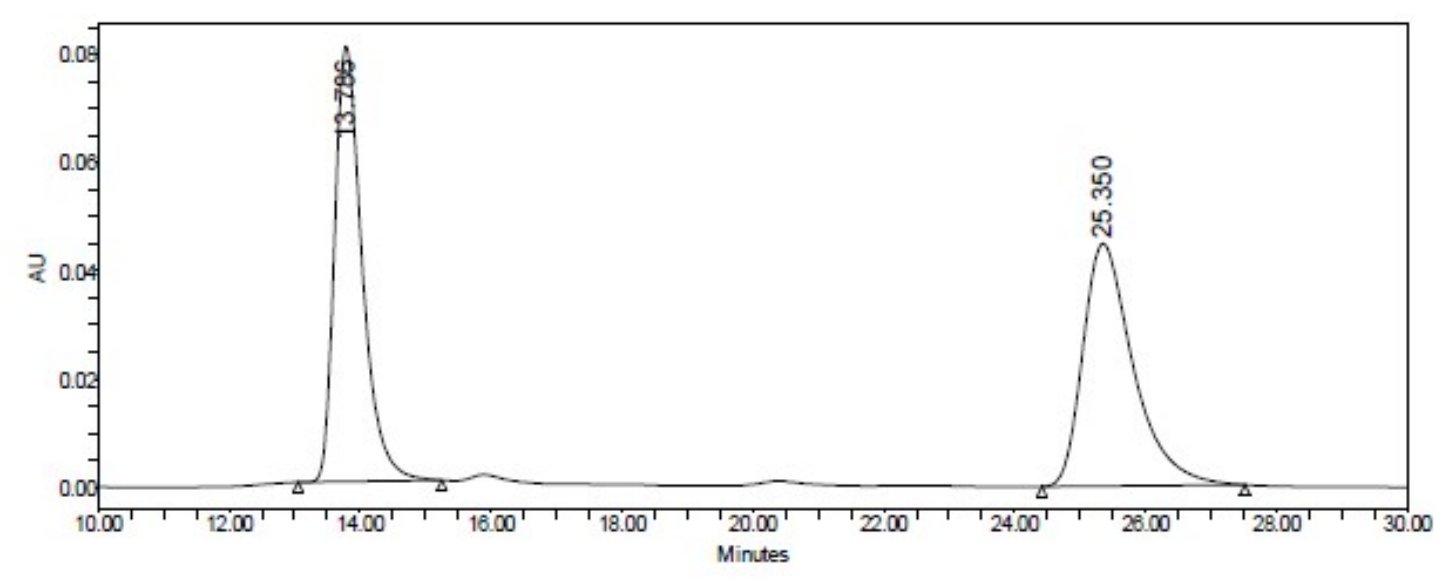

\begin{tabular}{|c|l|l|c|c|c|c|}
\hline & RT (min) & Int Type & $\begin{array}{c}\text { Width } \\
(\mathrm{sec})\end{array}$ & Area & Height & $\%$ Area \\
\hline 1 & 13.786 & bb & 132.000 & 2355906 & 80453 & 49.56 \\
\hline 2 & 25.350 & bb & 186.000 & 2398173 & 44822 & 50.44 \\
\hline Sum & & & & 4754079.2 & 125274.7 & 100.0 \\
\hline
\end{tabular}

\section{Enantioenriched 3u'}

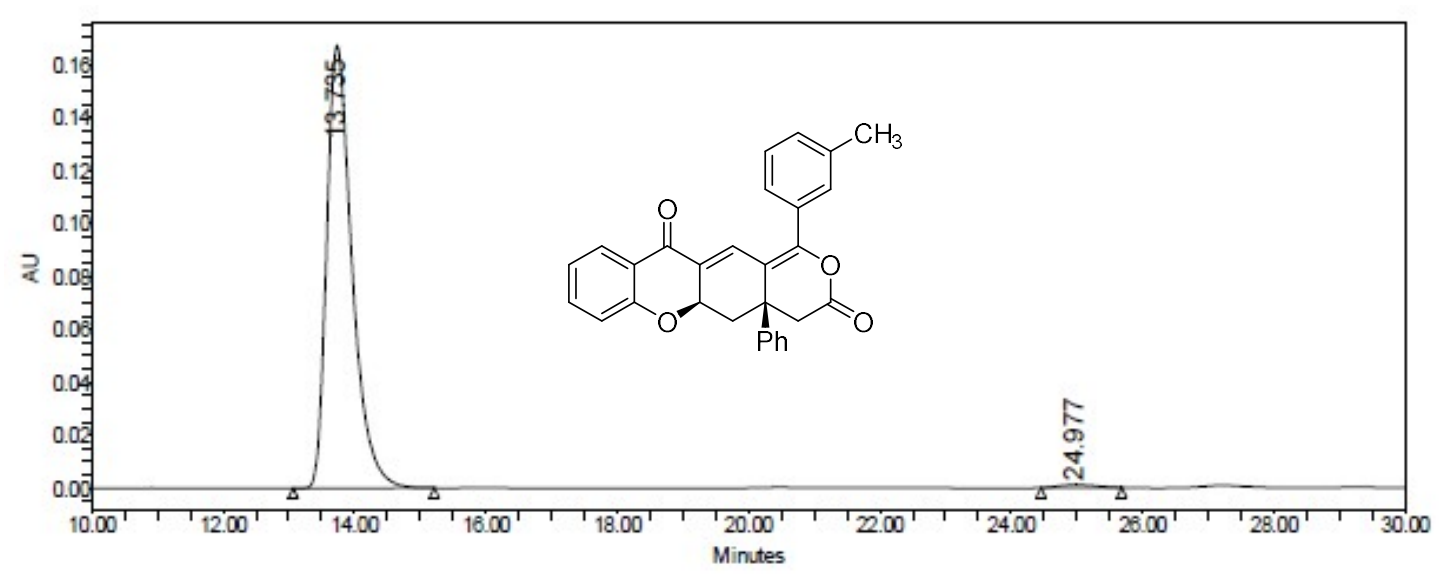

\begin{tabular}{|c|l|l|c|c|l|l|}
\hline & RT (min) & Int Type & $\begin{array}{c}\text { Width } \\
(\mathrm{sec})\end{array}$ & Area & Height & \% Area \\
\hline 1 & 13.735 & bb & 129.000 & 4481398 & 167092 & 99.05 \\
\hline 2 & 24.977 & bb & 74.000 & 42852 & 1086 & 0.95 \\
\hline Sum & & & & 4524249.8 & 168178.6 & 100.0 \\
\hline
\end{tabular}




\section{Racemic 3v}

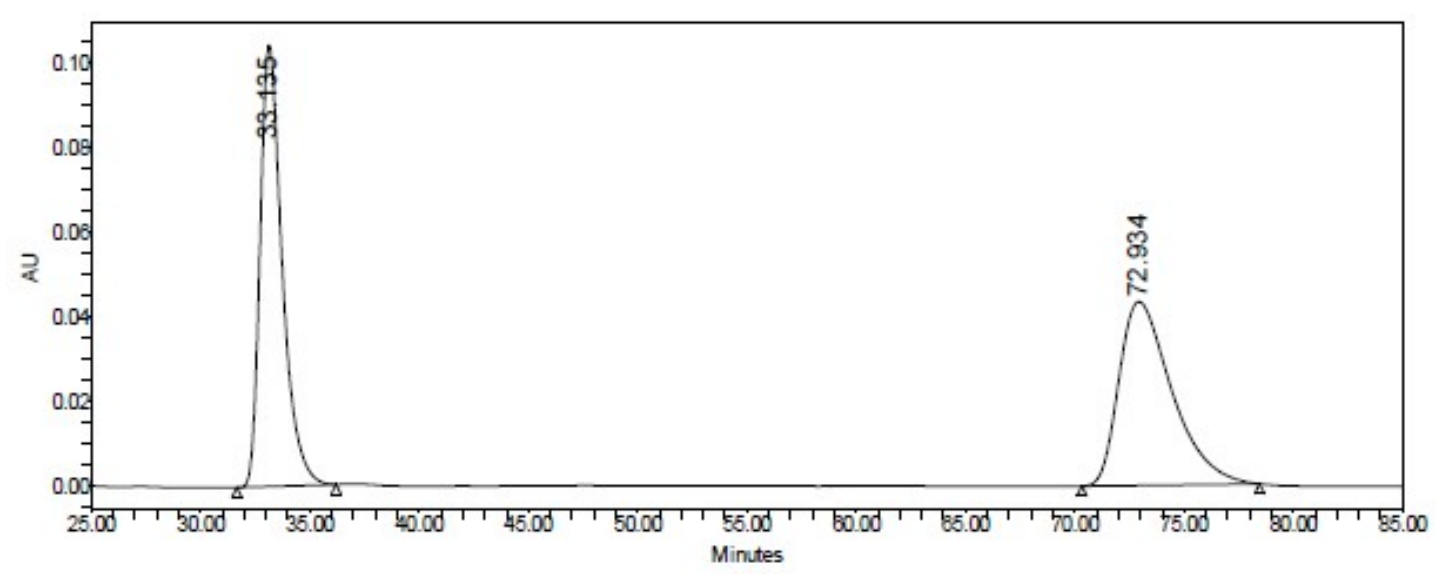

\begin{tabular}{|c|l|l|c|c|c|c|}
\hline & RT (min) & Int Type & $\begin{array}{c}\text { Width } \\
(\mathrm{sec})\end{array}$ & Area & Height & \% Area \\
\hline 1 & 33.135 & bb & 273.000 & 7297675 & 104013 & 50.05 \\
\hline 2 & 72.934 & bb & 488.000 & 7284204 & 43337 & 49.95 \\
\hline Sum & & & & 14581878.9 & 147350.0 & 100.0 \\
\hline
\end{tabular}

\section{Enantioenriched 3v}

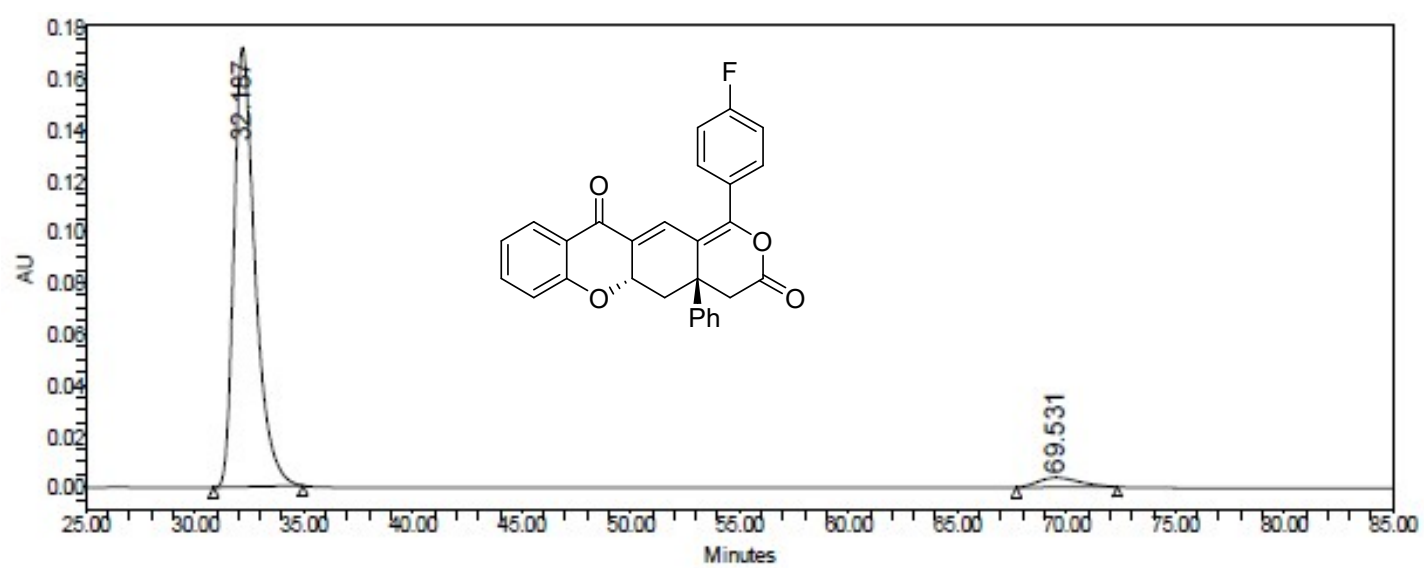

\begin{tabular}{|c|c|l|c|c|l|l|}
\hline & RT (min) & Int Type & $\begin{array}{c}\text { Width } \\
(\mathrm{sec})\end{array}$ & Area & Height & \% Area \\
\hline 1 & 32.187 & bb & 245.000 & 11643258 & 171999 & 95.91 \\
\hline 2 & 69.531 & bb & 279.000 & 496415 & 3748 & 4.09 \\
\hline Sum & & & & 12139672.7 & 175747.2 & 100.0 \\
\hline
\end{tabular}




\section{Racemic 3v'}

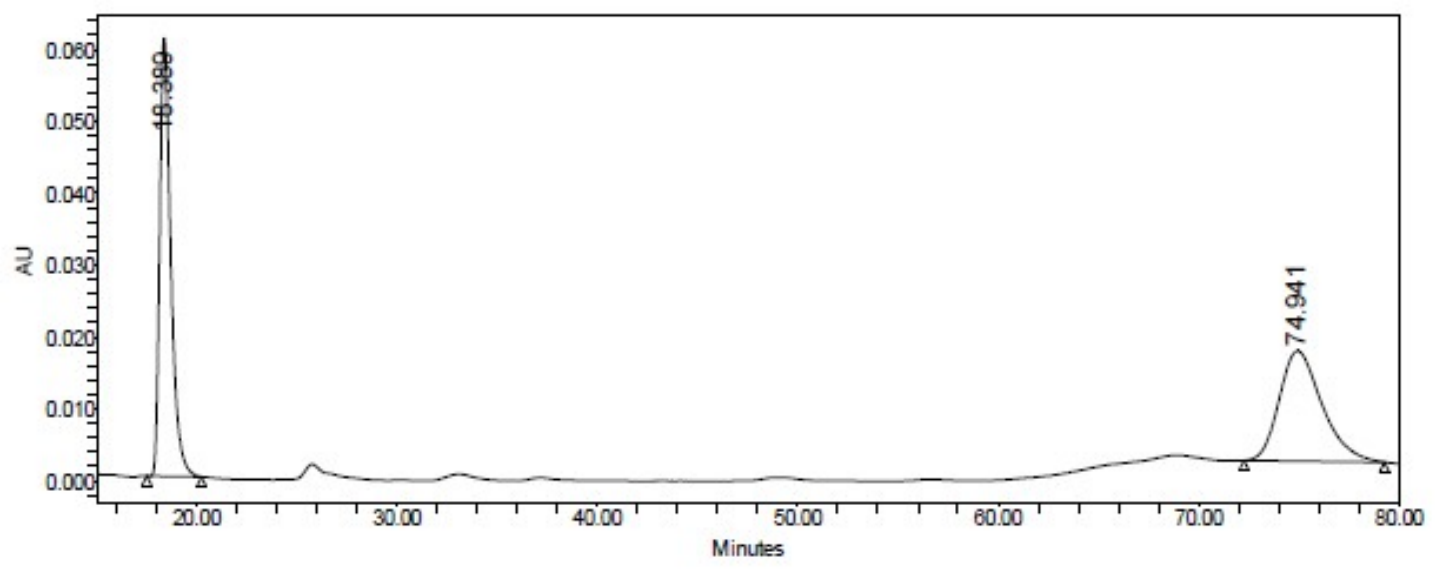

\begin{tabular}{|c|c|c|c|c|c|c|}
\hline & RT (min) & Int Type & $\begin{array}{c}\text { Width } \\
(\text { sec })\end{array}$ & Area & Height & $\%$ Area \\
\hline 1 & 18.389 & bb & 164.000 & 2348483 & 60988 & 50.86 \\
\hline 2 & 74.941 & bb & 421.000 & 2268694 & 15324 & 49.14 \\
\hline Sum & & & & 4617176.4 & 76311.3 & 100.0 \\
\hline
\end{tabular}

\section{Enantioenriched 3v'}

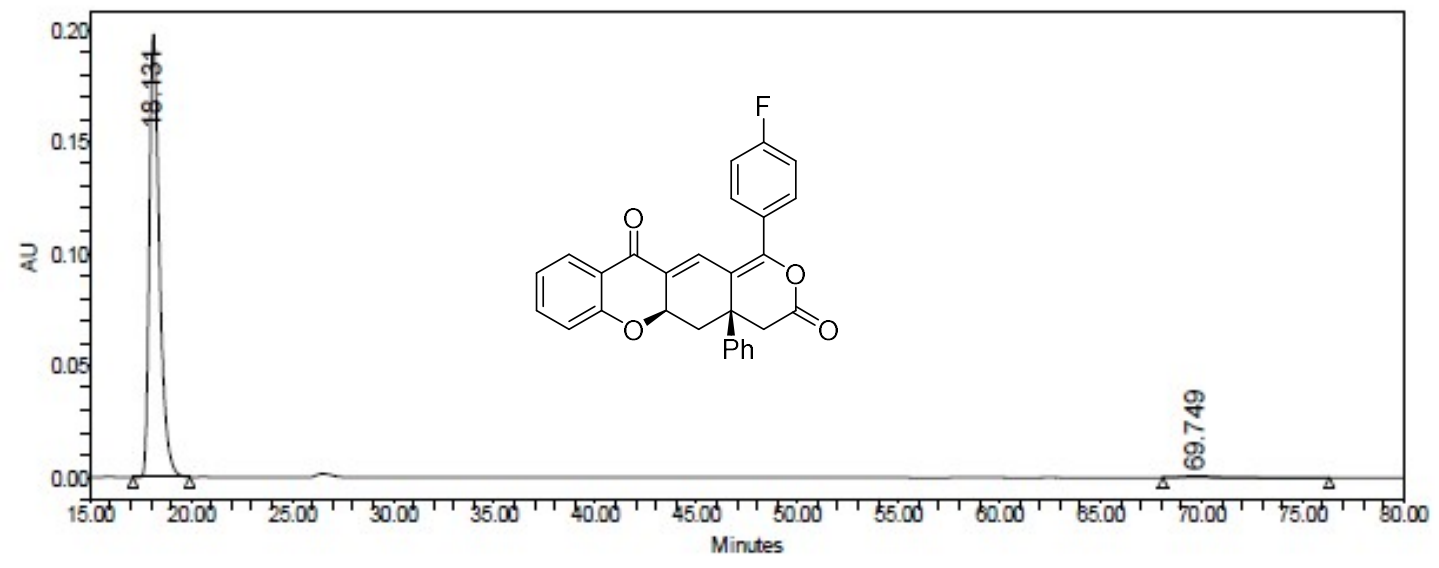

\begin{tabular}{|c|l|l|c|c|l|l|}
\hline & RT (min) & Int Type & $\begin{array}{c}\text { Width } \\
(\mathrm{sec})\end{array}$ & Area & Height & \% Area \\
\hline 1 & 18.131 & $\mathrm{bb}$ & 168.000 & 6908690 & 197641 & 98.55 \\
\hline 2 & 69.749 & $\mathrm{bb}$ & 494.000 & 101370 & 754 & 1.45 \\
\hline Sum & & & & 7010059.4 & 198394.7 & 100.0 \\
\hline
\end{tabular}




\section{Racemic 3w}

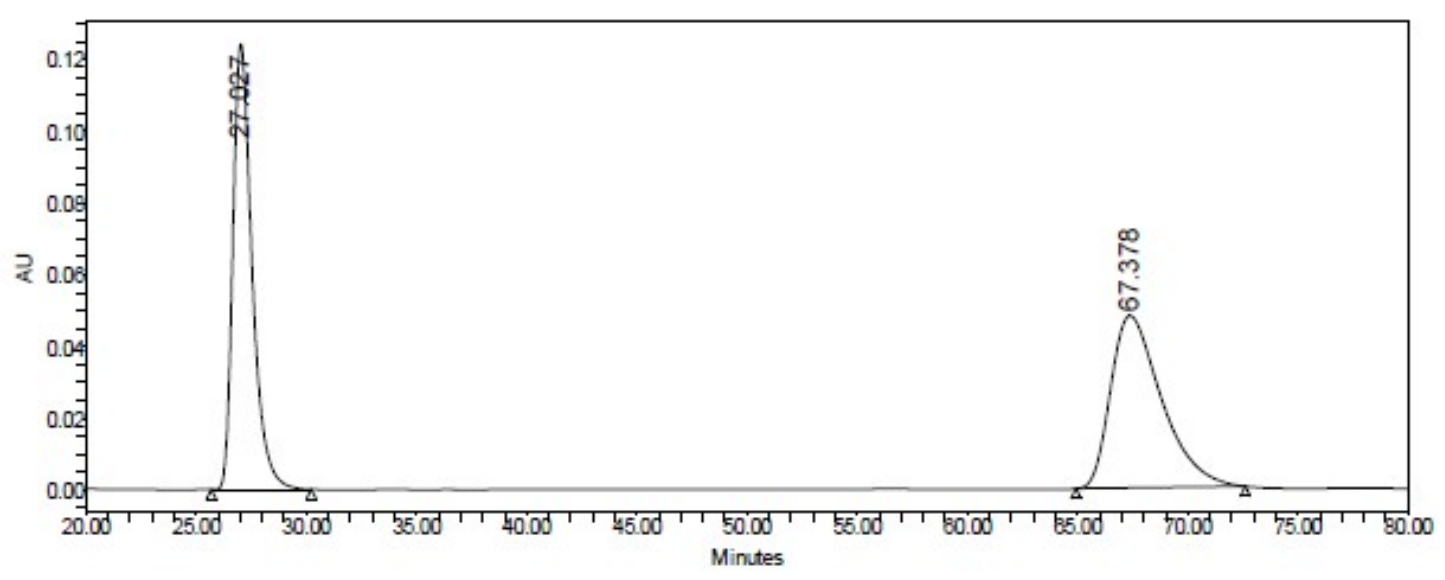

\begin{tabular}{|c|l|l|c|c|c|c|}
\hline & $R T(\min )$ & Int Type & $\begin{array}{c}\text { Width } \\
(\mathrm{sec})\end{array}$ & Area & Height & $\%$ Area \\
\hline 1 & 27.027 & bb & 272.000 & 7504814 & 124159 & 49.30 \\
\hline 2 & 67.378 & bb & 461.000 & 7717354 & 48029 & 50.70 \\
\hline Sum & & & & 15222168.1 & 172187.3 & 100.0 \\
\hline
\end{tabular}

\section{Enantioenriched 3w}

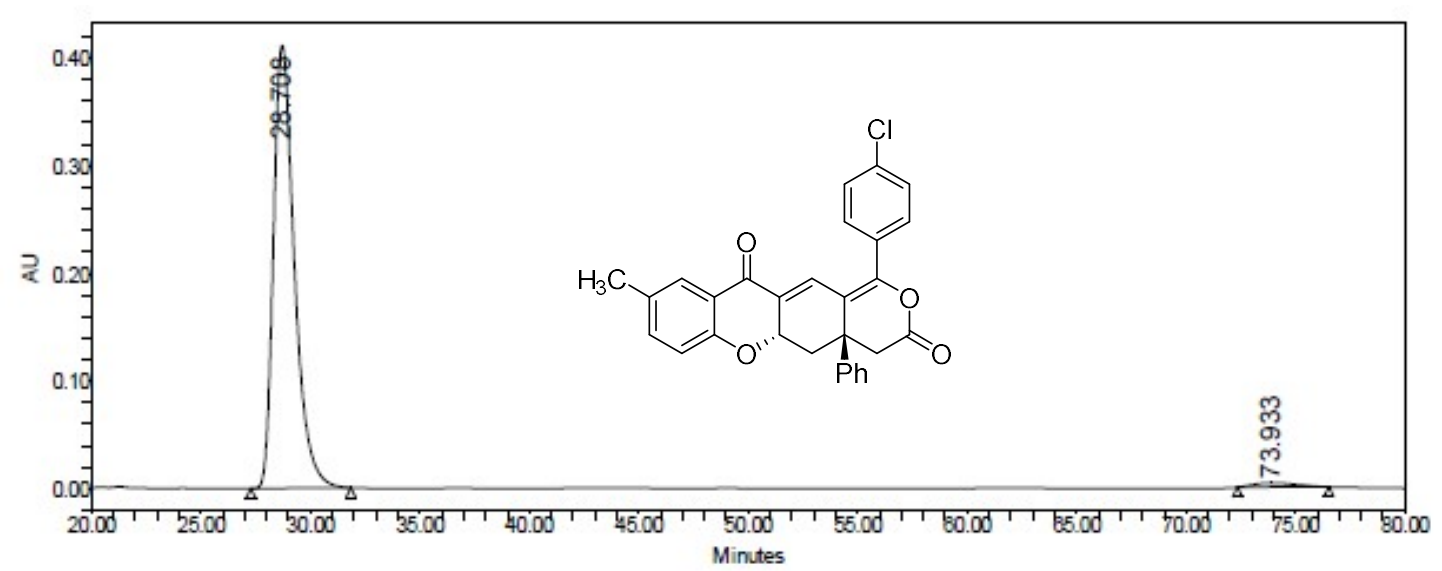

\begin{tabular}{|c|l|l|c|c|c|l|}
\hline & RT (min) & Int Type & $\begin{array}{c}\text { Width } \\
(\mathrm{sec})\end{array}$ & Area & Height & \% Area \\
\hline 1 & 28.708 & bb & 274.000 & 27340586 & 411447 & 98.10 \\
\hline 2 & 73.933 & bb & 250.000 & 529080 & 4017 & 1.90 \\
\hline Sum & & & & 27869666.1 & 415463.5 & 100.0 \\
\hline
\end{tabular}




\section{Racemic 3w'}

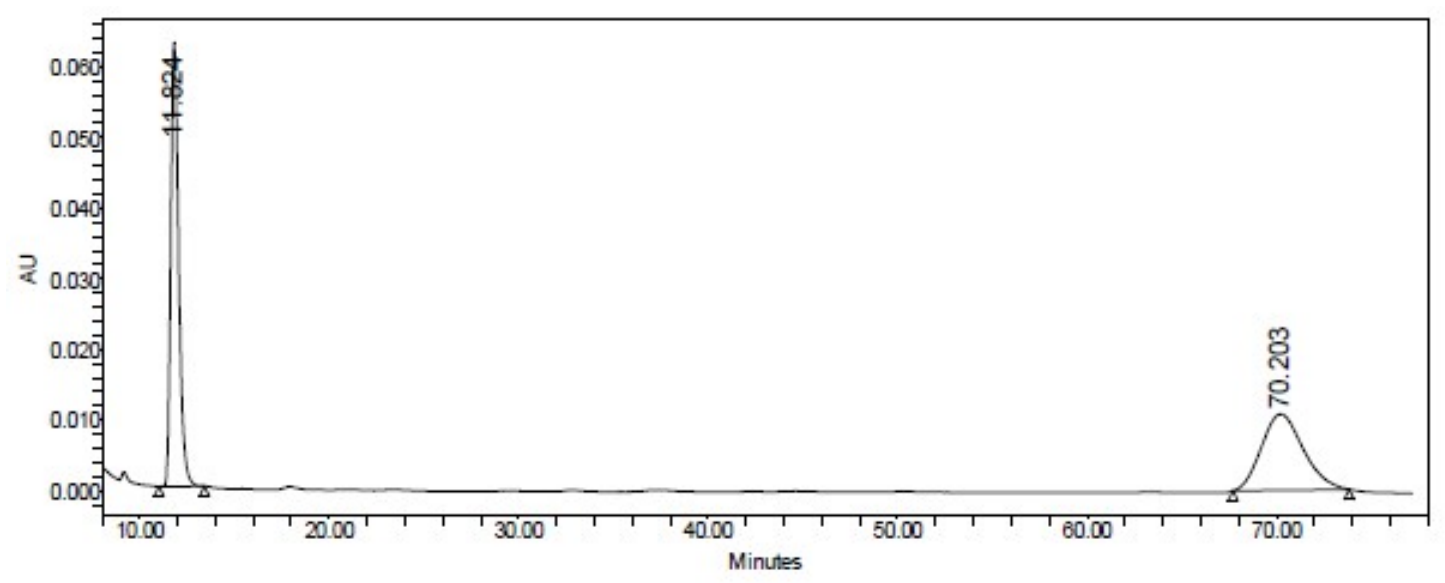

\begin{tabular}{|c|c|l|c|c|c|c|}
\hline & RT (min) & Int Type & $\begin{array}{c}\text { Width } \\
(\mathrm{sec})\end{array}$ & Area & Height & $\%$ Area \\
\hline 1 & 11.824 & bb & 143.500 & 1835646 & 62870 & 52.47 \\
\hline 2 & 70.203 & bb & 367.000 & 1662510 & 10816 & 47.53 \\
\hline Sum & & & & 3498155.4 & 73685.6 & 100.0 \\
\hline
\end{tabular}

\section{Enantioenriched 3w'}

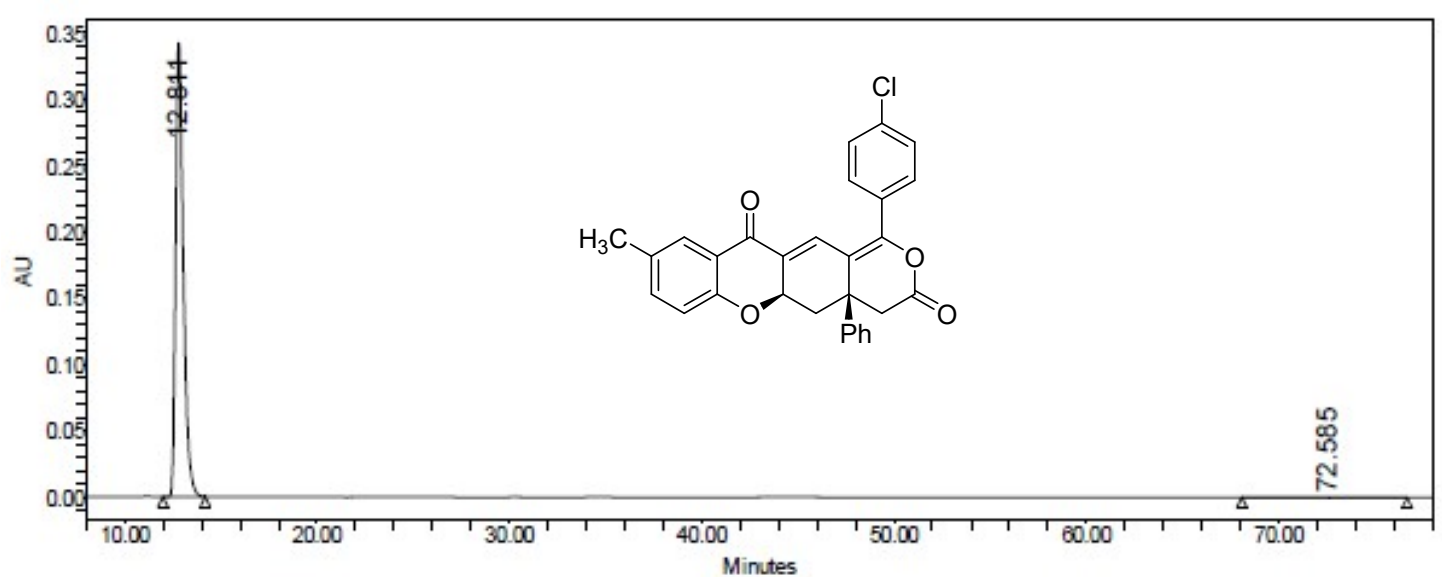

\begin{tabular}{|c|c|l|c|c|c|l|}
\hline & RT (min) & Int Type & $\begin{array}{c}\text { Width } \\
(\mathrm{sec})\end{array}$ & Area & Height & \% Area \\
\hline 1 & 12.811 & $\mathrm{bb}$ & 131.500 & 9605910 & 342205 & 99.91 \\
\hline 2 & 72.585 & $\mathrm{bb}$ & 514.500 & 8918 & 44 & 0.09 \\
\hline Sum & & & & 9614827.3 & 342249.1 & 100.0 \\
\hline
\end{tabular}




\section{Racemic 3x}

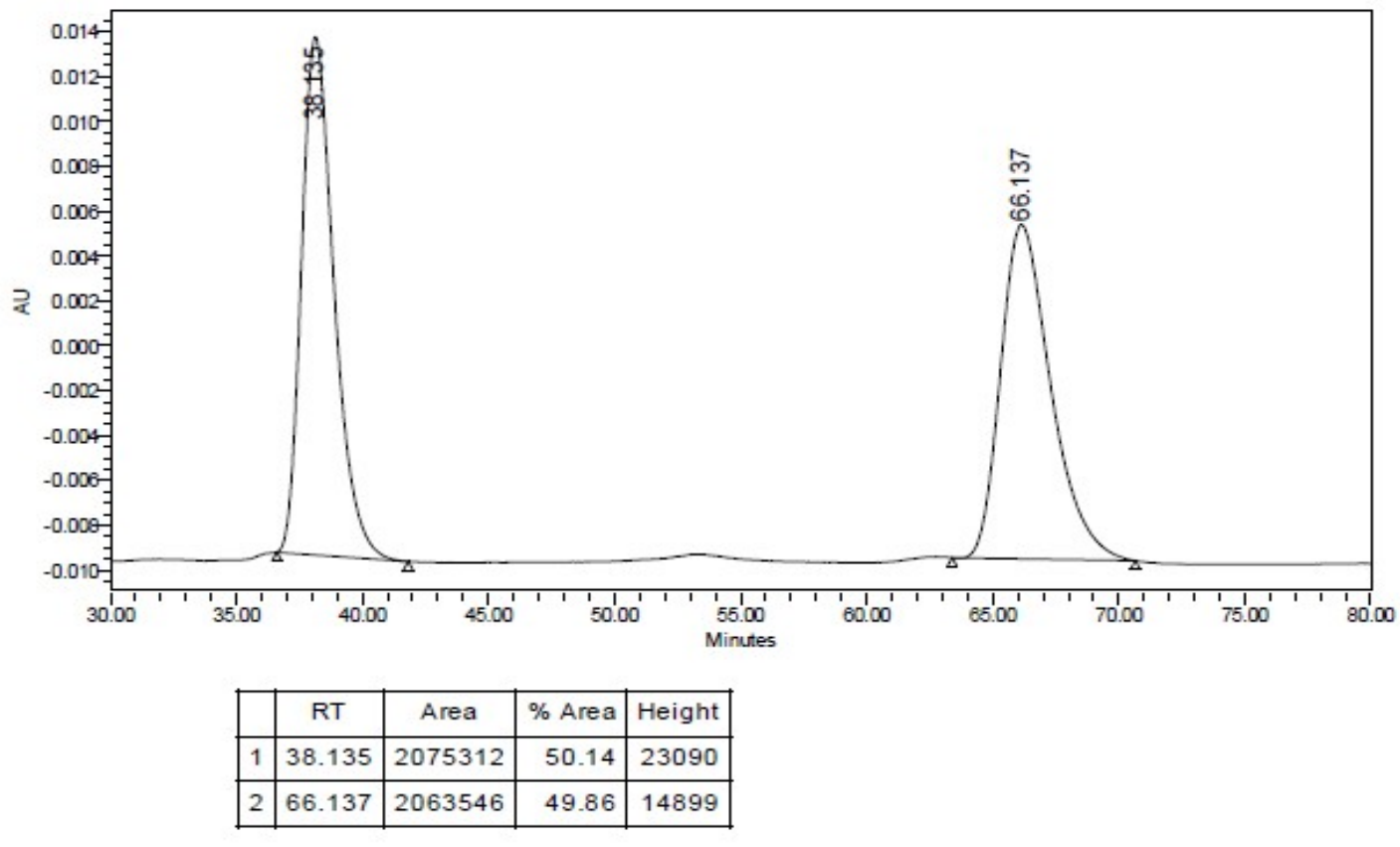

Enantioenriched 3x

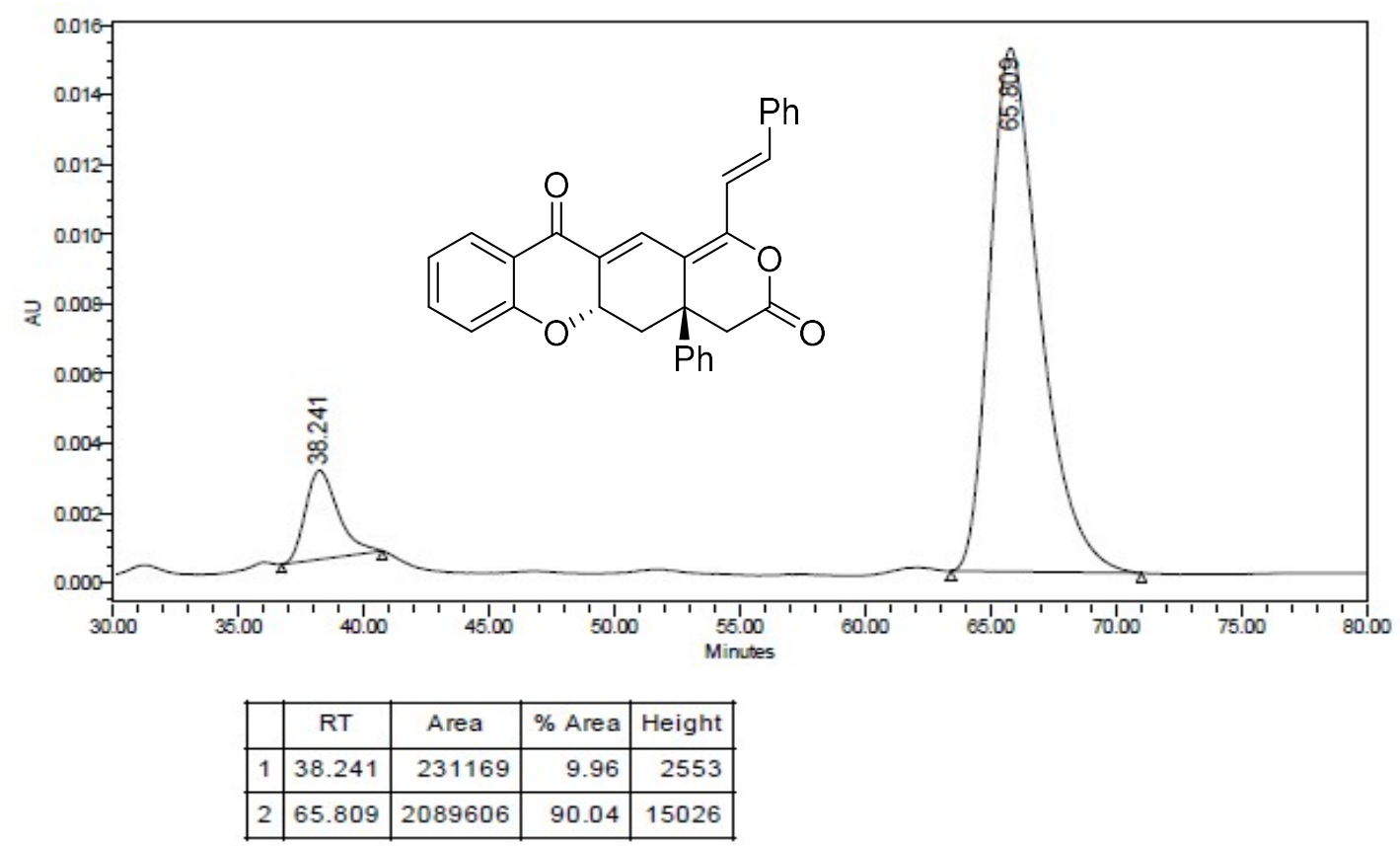




\section{Racemic 3x'}

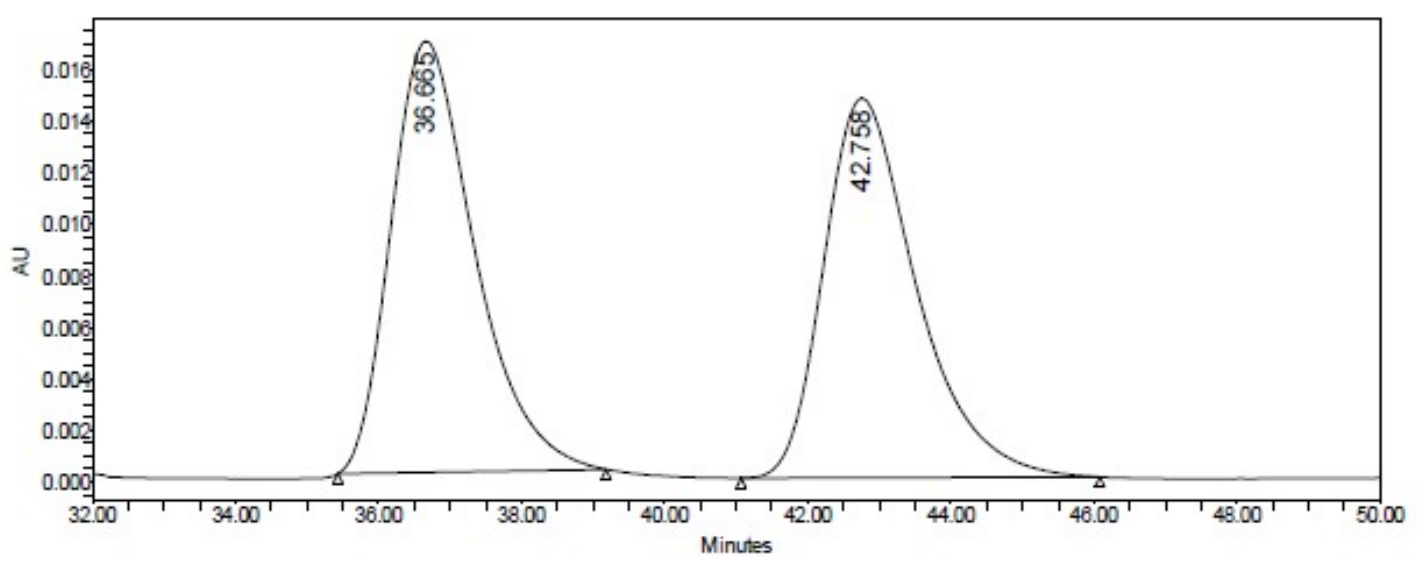

\begin{tabular}{|c|c|c|c|c|c|c|}
\hline & RT (min) & Int Type & $\begin{array}{c}\text { Width } \\
(\mathrm{sec})\end{array}$ & Area & Height & $\%$ Area \\
\hline 1 & 36.665 & bb & 225.000 & 1359250 & 16725 & 50.65 \\
\hline 2 & 42.758 & bb & 301.000 & 1324106 & 14711 & 49.35 \\
\hline Sum & & & & 2683355.8 & 31435.8 & 100.0 \\
\hline
\end{tabular}

\section{Enantioenriched 3x'}

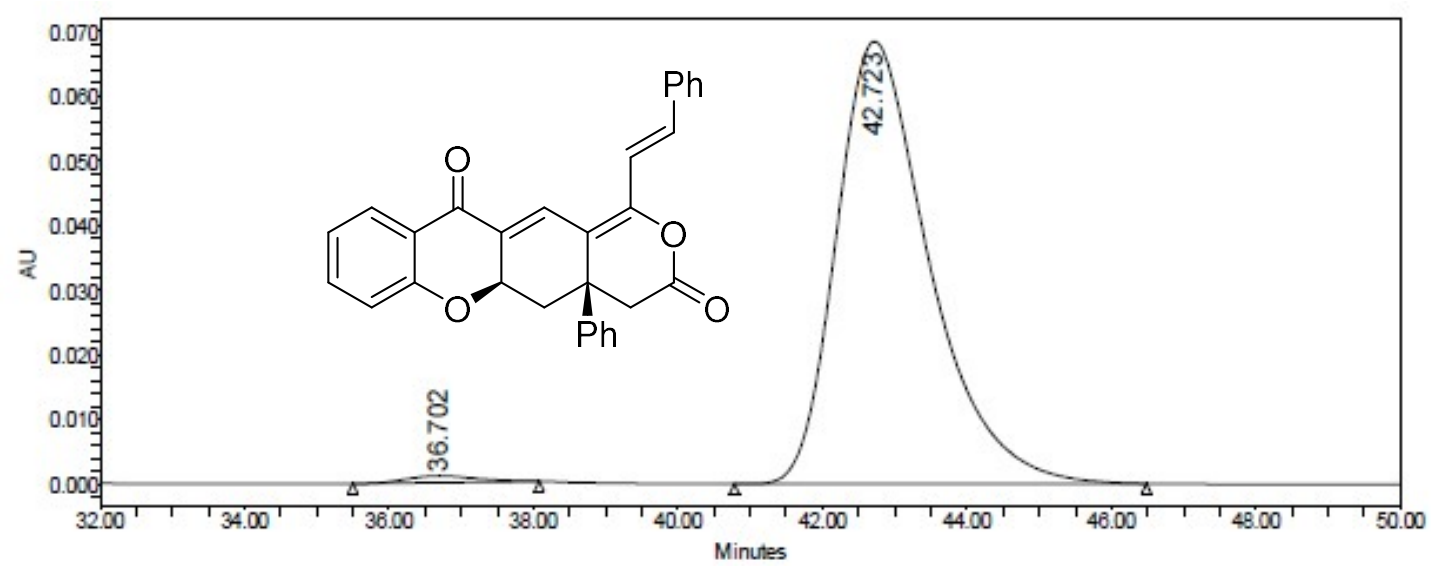

\begin{tabular}{|c|l|l|c|c|l|l|}
\hline & RT (min) & Int Type & $\begin{array}{c}\text { Width } \\
(\mathrm{sec})\end{array}$ & Area & Height & $\%$ Area \\
\hline 1 & 36.702 & bb & 155.000 & 71023 & 1049 & 1.15 \\
\hline 2 & 42.723 & bb & 342.000 & 6111318 & 68425 & 98.85 \\
\hline Sum & & & & 6182341.3 & 69474.0 & 100.0 \\
\hline
\end{tabular}




\section{Racemic 3y}

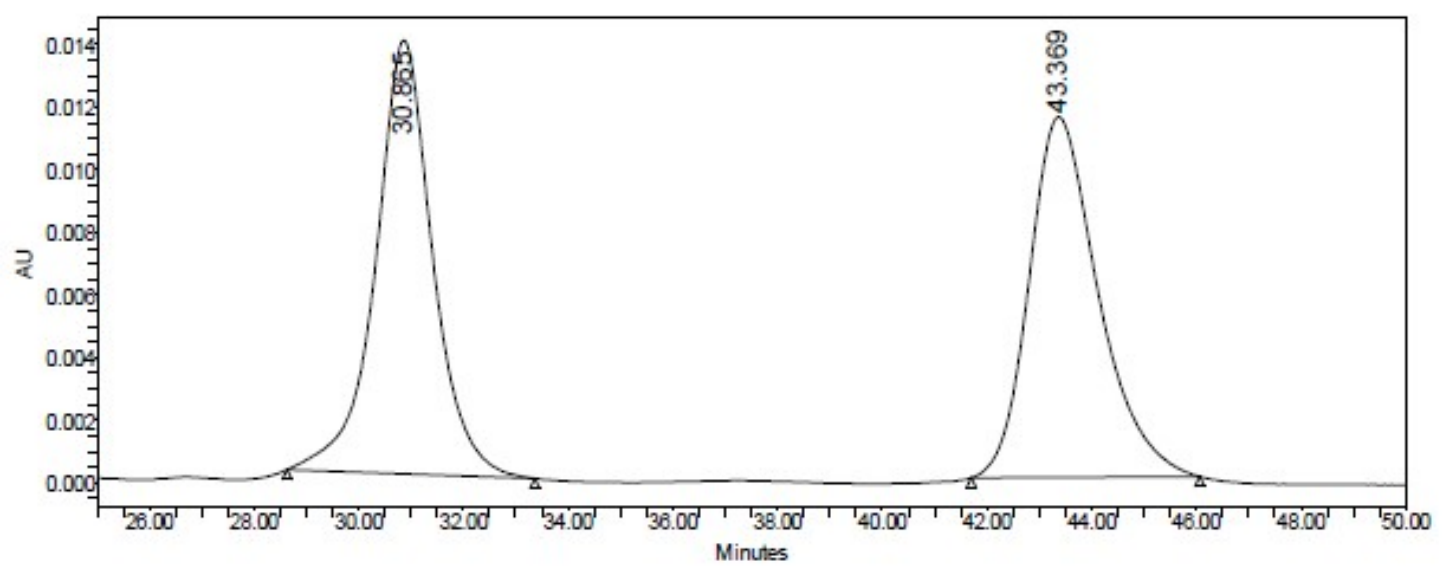

\begin{tabular}{|c|c|c|c|c|c|c|}
\hline & RT (min) & Int Type & $\begin{array}{c}\text { Width } \\
(\mathrm{sec})\end{array}$ & Area & Height & $\%$ Area \\
\hline 1 & 30.865 & $\mathrm{bb}$ & 284.000 & 1050946 & 13849 & 49.88 \\
\hline 2 & 43.369 & $\mathrm{bb}$ & 262.000 & 1056195 & 11514 & 50.12 \\
\hline Sum & & & & 2107140.8 & 25362.9 & 100.0 \\
\hline
\end{tabular}

\section{Enantioenriched 3y}

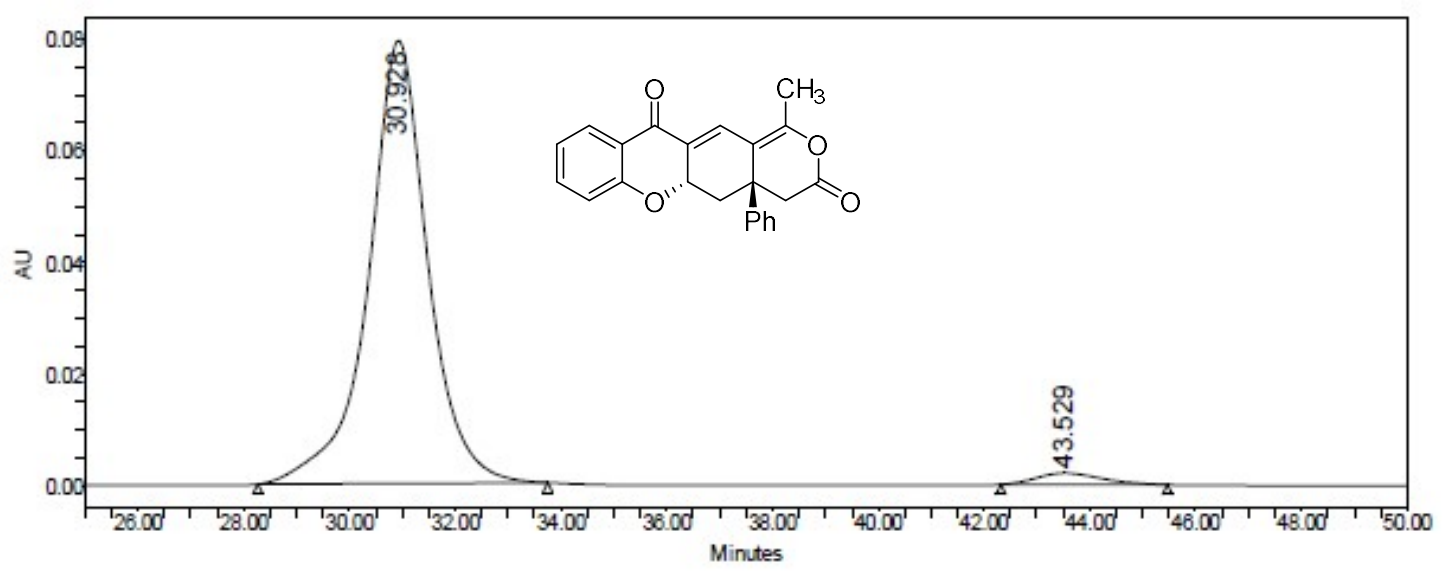

\begin{tabular}{|c|l|l|c|c|l|l|}
\hline & RT (min) & Int Type & $\begin{array}{c}\text { Width } \\
(\mathrm{sec})\end{array}$ & \multicolumn{1}{|c|}{ Area } & Height & \% Area \\
\hline 1 & 30.928 & bb & 329.000 & 6204594 & 79323 & 97.28 \\
\hline 2 & 43.529 & bb & 189.000 & 173663 & 2058 & 2.72 \\
\hline Sum & & & & 6378257.2 & 81381.7 & 100.0 \\
\hline
\end{tabular}




\section{Racemic 3y'}

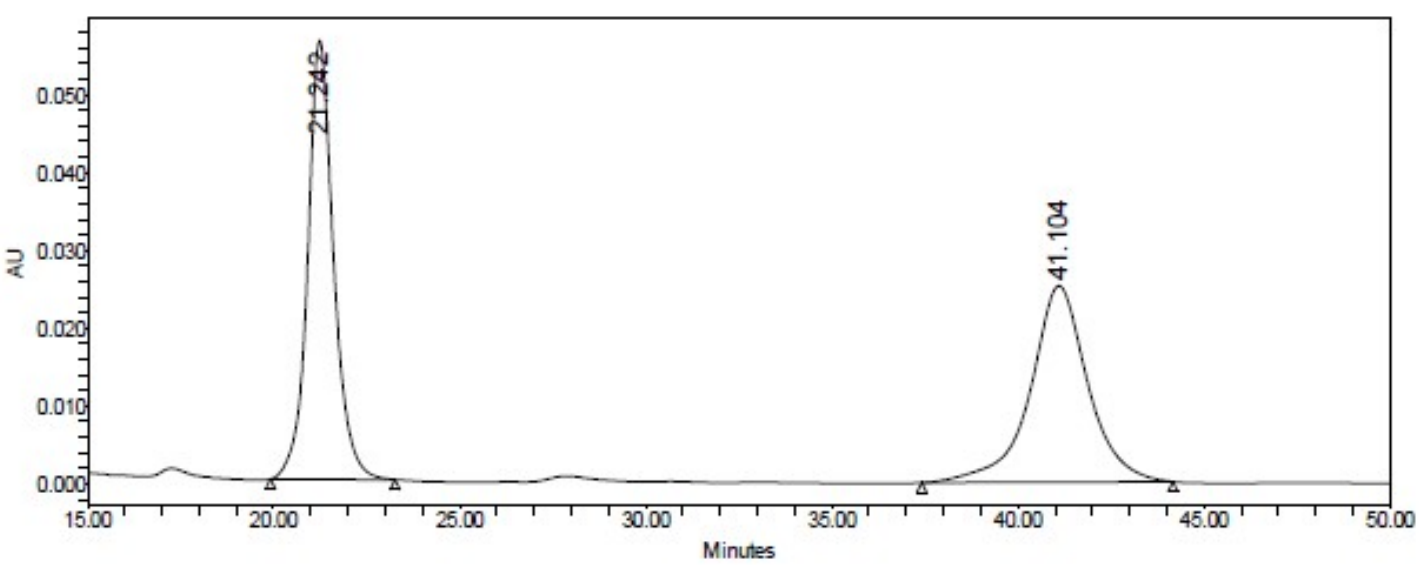

\begin{tabular}{|c|c|l|c|c|c|l|}
\hline & RT (min) & Int Type & $\begin{array}{c}\text { Width } \\
(\mathrm{sec})\end{array}$ & Area & Height & $\%$ Area \\
\hline 1 & 21.242 & bb & 201.000 & 2730970 & 56320 & 50.13 \\
\hline 2 & 41.104 & bb & 405.000 & 2716539 & 25224 & 49.87 \\
\hline Sum & & & & 5447509.5 & 81543.9 & 100.0 \\
\hline
\end{tabular}

\section{Enantioenriched 3y'}

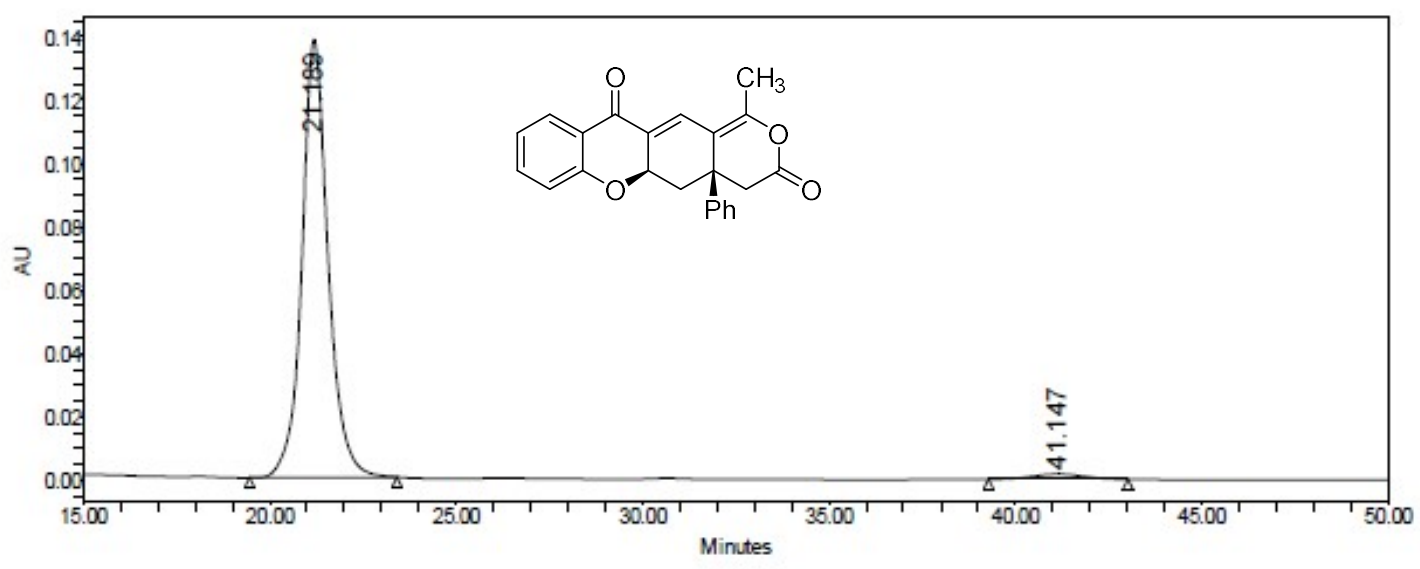

\begin{tabular}{|c|l|l|c|c|l|l|}
\hline & RT (min) & Int Type & $\begin{array}{c}\text { Width } \\
(\mathrm{sec})\end{array}$ & Area & Height & \% Area \\
\hline 1 & 21.189 & $\mathrm{bb}$ & 237.000 & 6741282 & 138243 & 97.80 \\
\hline 2 & 41.147 & bb & 224.000 & 151849 & 1670 & 2.20 \\
\hline Sum & & & & 6893131.3 & 139912.7 & 100.0 \\
\hline
\end{tabular}




\section{Racemic 5}

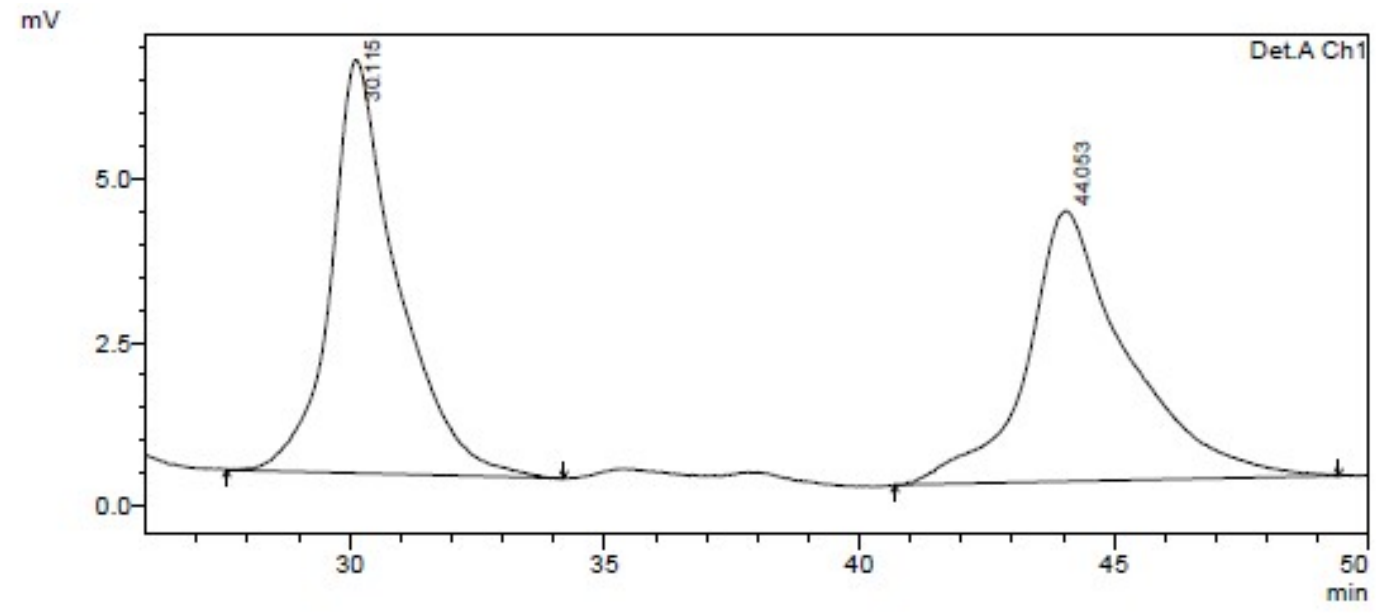

Detector A Chl 254mm
\begin{tabular}{|r|r|r|r|r|r|}
\hline Peak = & Ret. Time & Area & Height & Area \% & \multicolumn{1}{c|}{ Height \% } \\
\hline 1 & 30.115 & 575963 & 6304 & 50.229 & 60.440 \\
\hline 2 & 44.053 & 570701 & 4126 & 49.771 & 39.560 \\
\hline Total & & 1146665 & 10431 & 100.000 & 100.000 \\
\hline
\end{tabular}

\section{Enantioenriched 5}

$\mathrm{mv}$

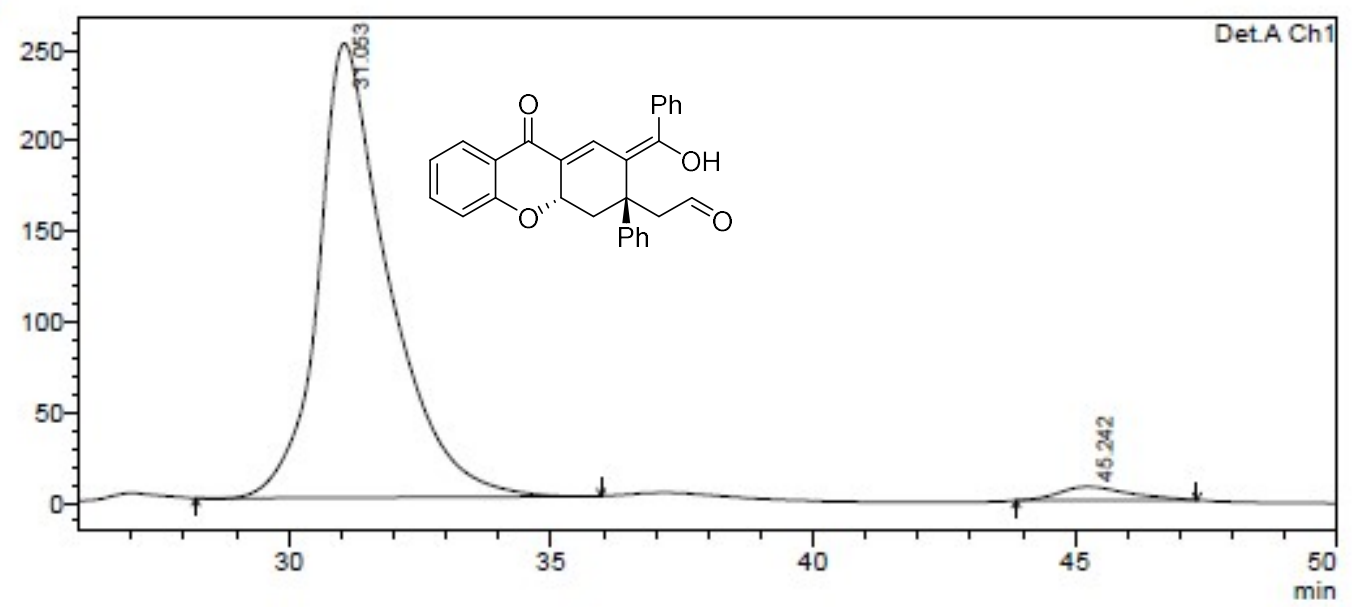

\begin{tabular}{|r|r|r|r|r|r|}
\hline \multicolumn{1}{|c|}{ Peak \# } & Ret. Time & \multicolumn{1}{c|}{ Area } & Height & Area \% & \multicolumn{1}{c|}{ Height \% } \\
\hline 1 & 31.053 & 23364518 & 250998 & 97.097 & 97.070 \\
\hline 2 & 45.242 & 698462 & 7578 & 2.903 & 2.930 \\
\hline Total & & 24062980 & 258575 & 100.000 & 100.000 \\
\hline
\end{tabular}




\section{Racemic 6}

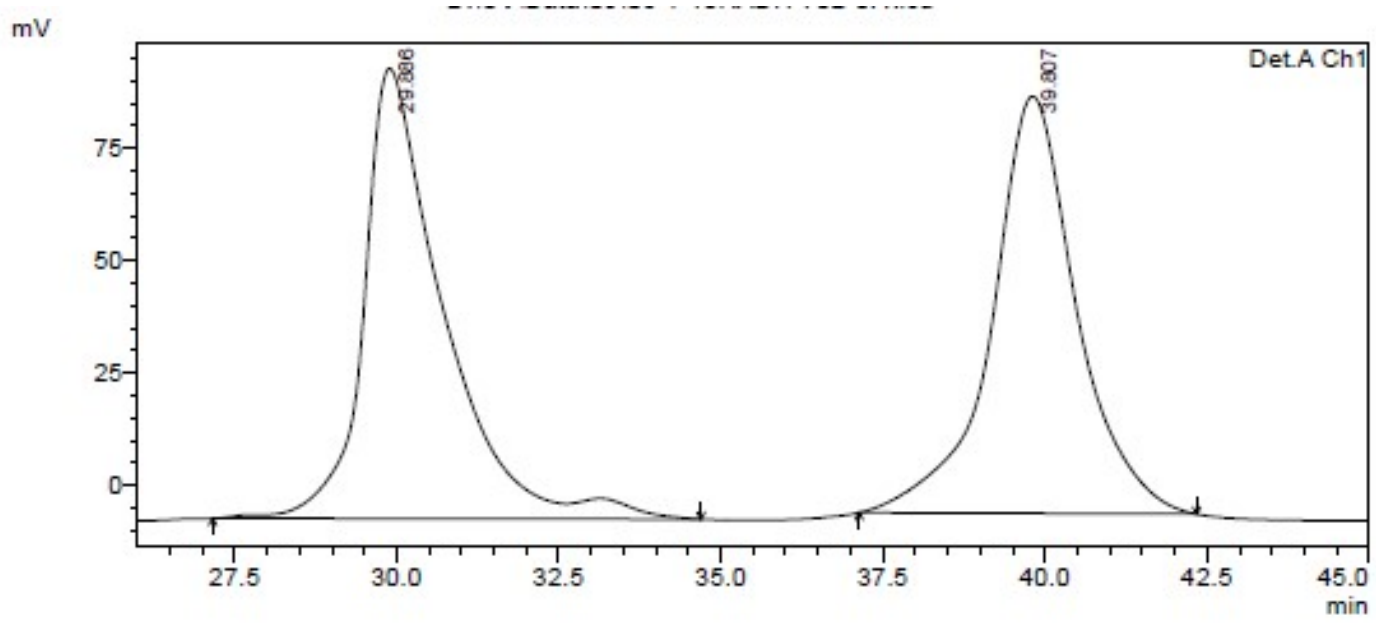

Detector A Chl 254nm
\begin{tabular}{|r|r|r|r|r|r|}
\hline Peals= & Ret. Time & \multicolumn{1}{|c|}{ Area } & Height & Area \% & Height $\%$ \\
\hline 1 & 29.886 & 8615224 & 100225 & 50.425 & 51.905 \\
\hline 2 & 39.807 & 8469971 & 92867 & 49.575 & 48.095 \\
\hline Total & & 17085196 & 193092 & 100.000 & 100.000 \\
\hline
\end{tabular}

\section{Enantioenriched 6}

$\mathrm{mV}$

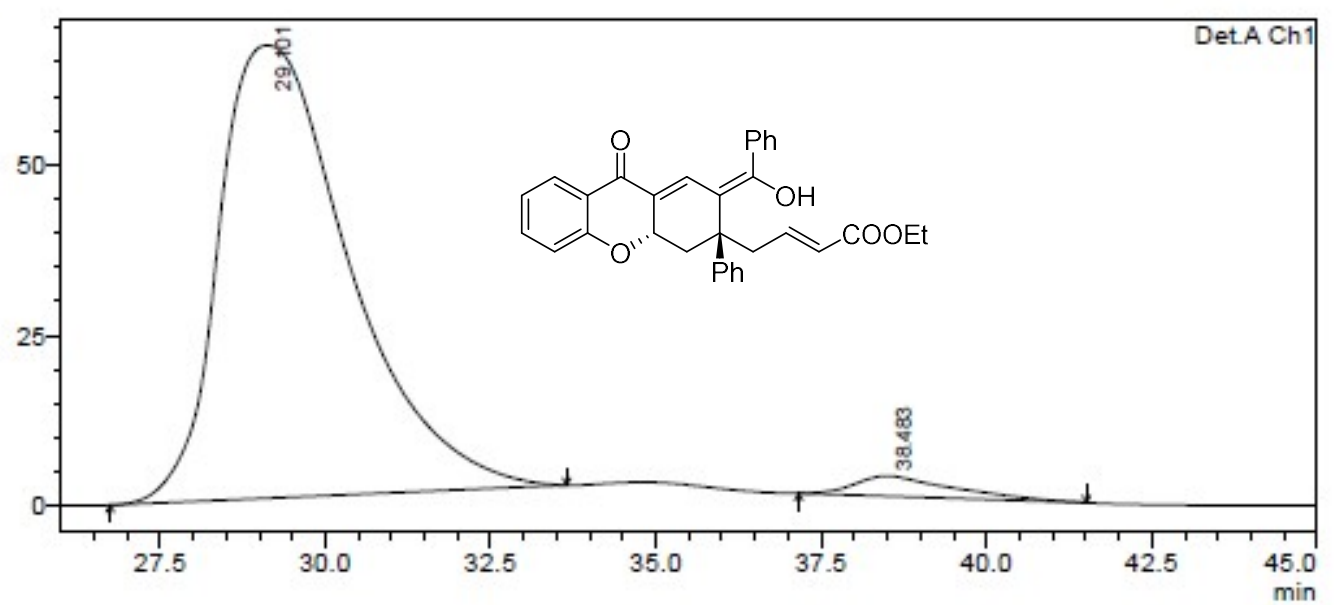

Detector A Chl 254nm

\begin{tabular}{|r|r|r|r|r|r|}
\hline \multicolumn{1}{|c|}{ Peak= } & Ret. Time & \multicolumn{1}{|c|}{ Area } & Height & Area \% & \multicolumn{1}{|c|}{ Height \% } \\
\hline 1 & 29.101 & 9130232 & 66339 & 96.780 & 95.777 \\
\hline 2 & 38.483 & 303805 & 2925 & 3.220 & 4.223 \\
\hline Total & & 9434037 & 69264 & 100.000 & 100.000 \\
\hline
\end{tabular}




\section{Racemic 8a}

$\mathrm{mV}$

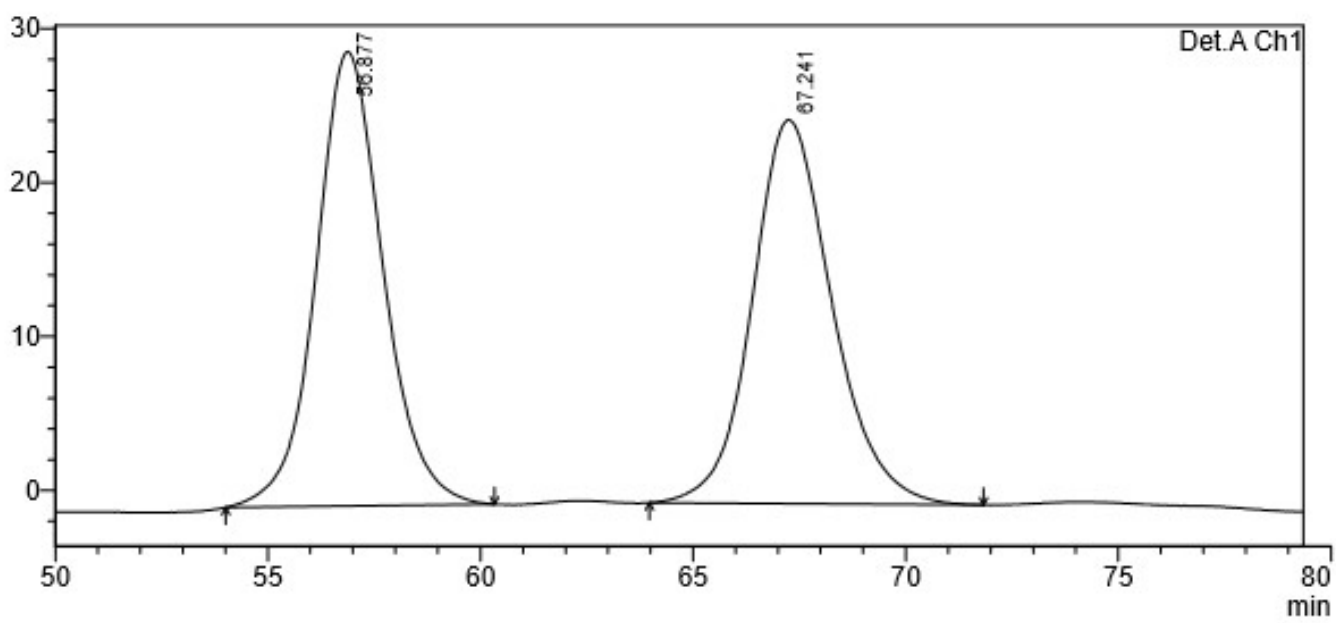

PeakTable

\begin{tabular}{|c|c|c|c|c|c|}
\hline & $4 \mathrm{~nm}$ & & & & \\
\hline Pealk\# & Ret. Time & Area & Height & Area \% & Height \% \\
\hline 1 & 56.877 & 3242029 & 29514 & 49.827 & 54.196 \\
\hline 2 & 67.241 & 3264493 & 24944 & 50.173 & 45.804 \\
\hline Total & & 6506521 & 54458 & 100.000 & 100.000 \\
\hline
\end{tabular}

\section{Enantioenriched 8a}

$\mathrm{mV}$

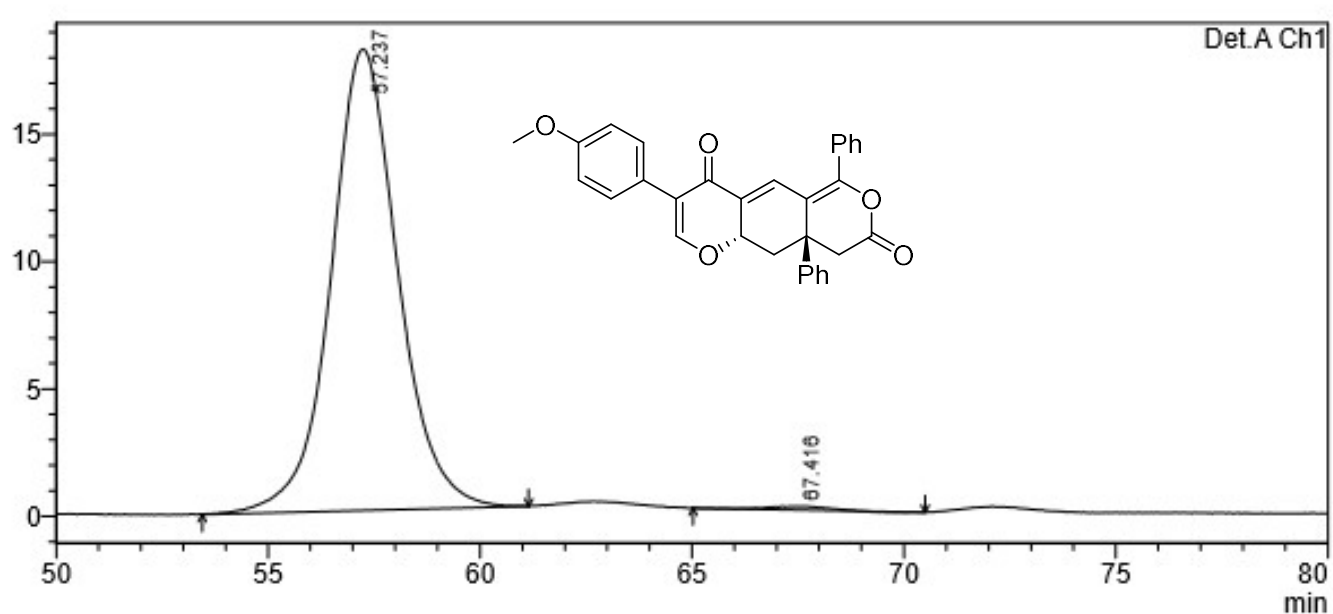

PeakTable

\begin{tabular}{|c|c|c|c|c|c|}
\hline \multicolumn{6}{|c|}{ etector $\mathrm{A} \mathrm{Cl}$} \\
\hline Peak\# & Ret. Time & Area & Height & Area \% & Height \% \\
\hline 1 & 57.237 & 1987868 & 18109 & 99.386 & 99.231 \\
\hline 2 & 67.416 & 12271 & 140 & 0.614 & 0.769 \\
\hline Total & & 2000139 & 18249 & 100.000 & 100.000 \\
\hline
\end{tabular}




\section{Racemic 8b}

$\mathrm{mV}$

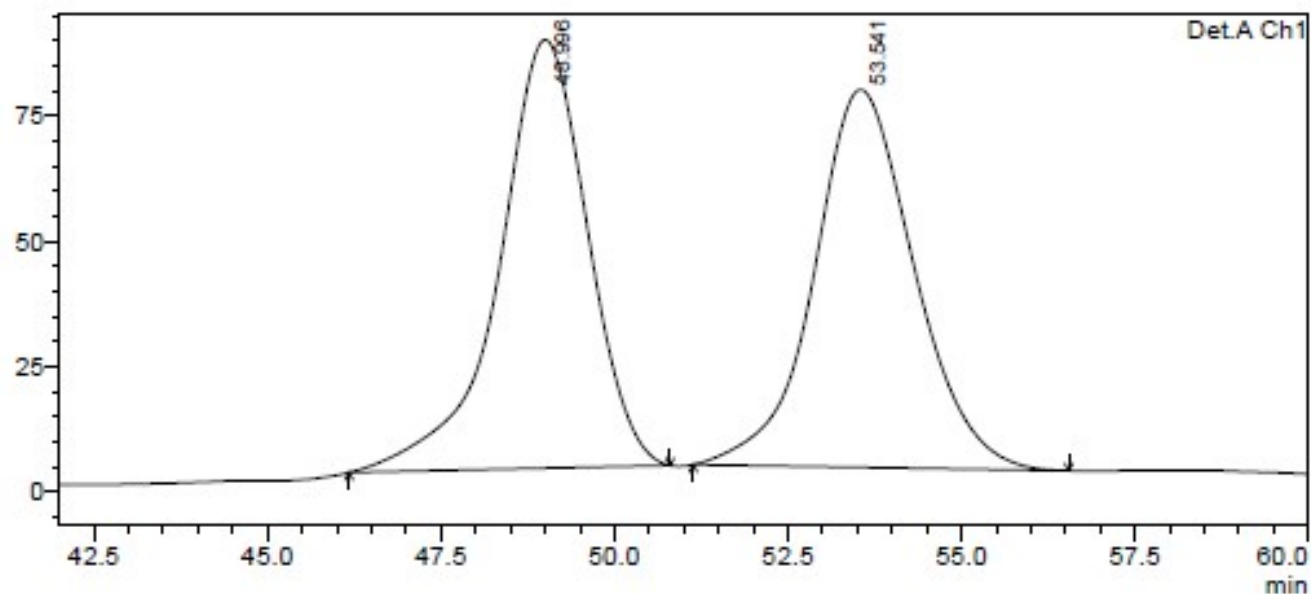

\begin{tabular}{|r|r|r|r|r|r|}
\hline Peak $\#$ & Ret. Time & \multicolumn{1}{|c|}{ Area } & Height & Area \% & \multicolumn{1}{c|}{ Height \% } \\
\hline 1 & 48.996 & 7565306 & 85366 & 50.029 & 53.145 \\
\hline 2 & 53.541 & 7556602 & 75262 & 49.971 & 46.855 \\
\hline Total. & & 15121908 & 160628 & 100.000 & 100.000 \\
\hline
\end{tabular}

\section{Enantioenriched 8b}

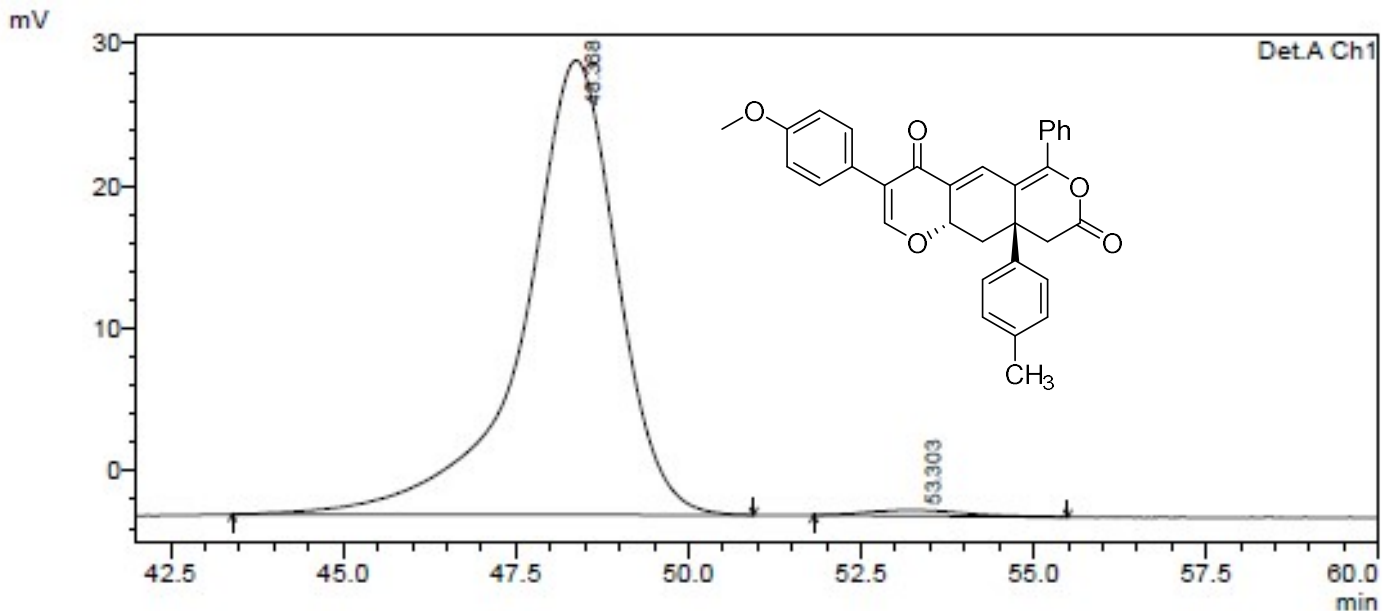

\begin{tabular}{|r|r|r|r|r|r|}
\hline Pealk\# & Ret. Time & \multicolumn{1}{|c|}{ Area } & Height & Area \% & \multicolumn{1}{c|}{ Height \% } \\
\hline 1 & 48.368 & 3013912 & 31905 & 98.755 & 98.714 \\
\hline 2 & 53.303 & 37983 & 415 & 1.245 & 1.286 \\
\hline Total & & 3051895 & 32321 & 100.000 & 100.000 \\
\hline
\end{tabular}


Racemic 8c

$\mathrm{mV}$

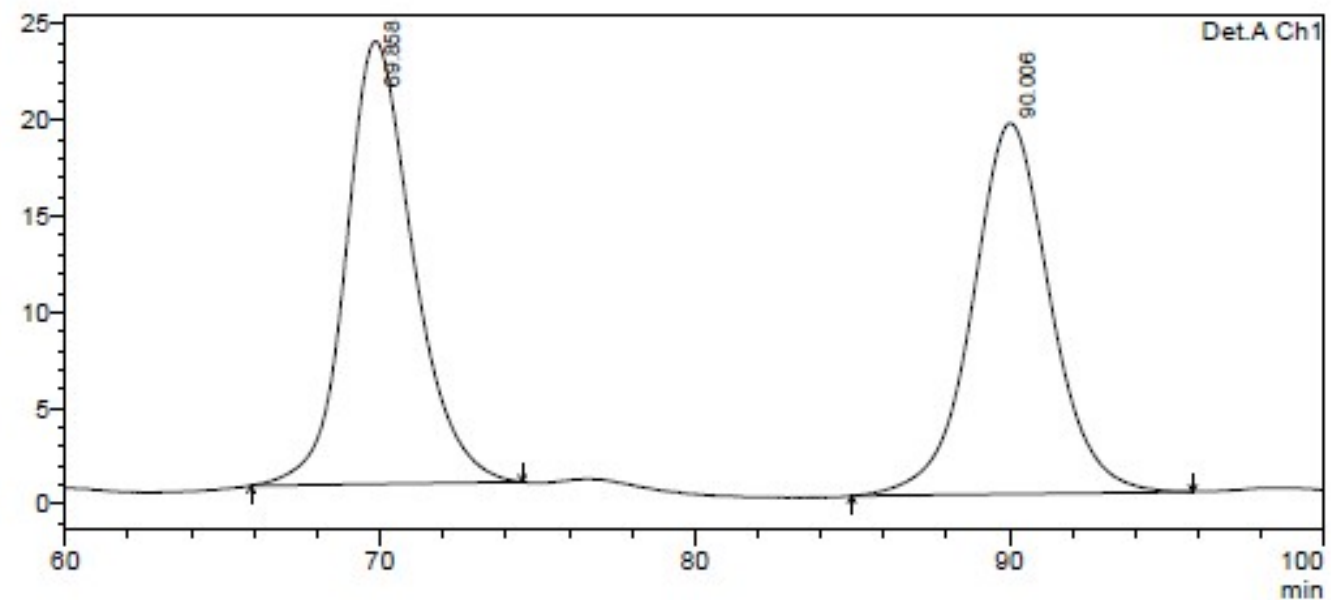

Detector A Chl 254 mm
\begin{tabular}{|r|r|r|r|r|r|}
\hline Peak\#\# & Ret. Time & Area & Height & Area \% & \multicolumn{1}{c|}{ Height \% } \\
\hline 1 & 69.858 & 3430980 & 23064 & 50.751 & 54.426 \\
\hline 2 & 90.006 & 3329486 & 19313 & 49.249 & 45.574 \\
\hline Total & & 6760466 & 42377 & 100.000 & 100.000 \\
\hline
\end{tabular}

\section{Enantioenriched 8c}

$\mathrm{mV}$

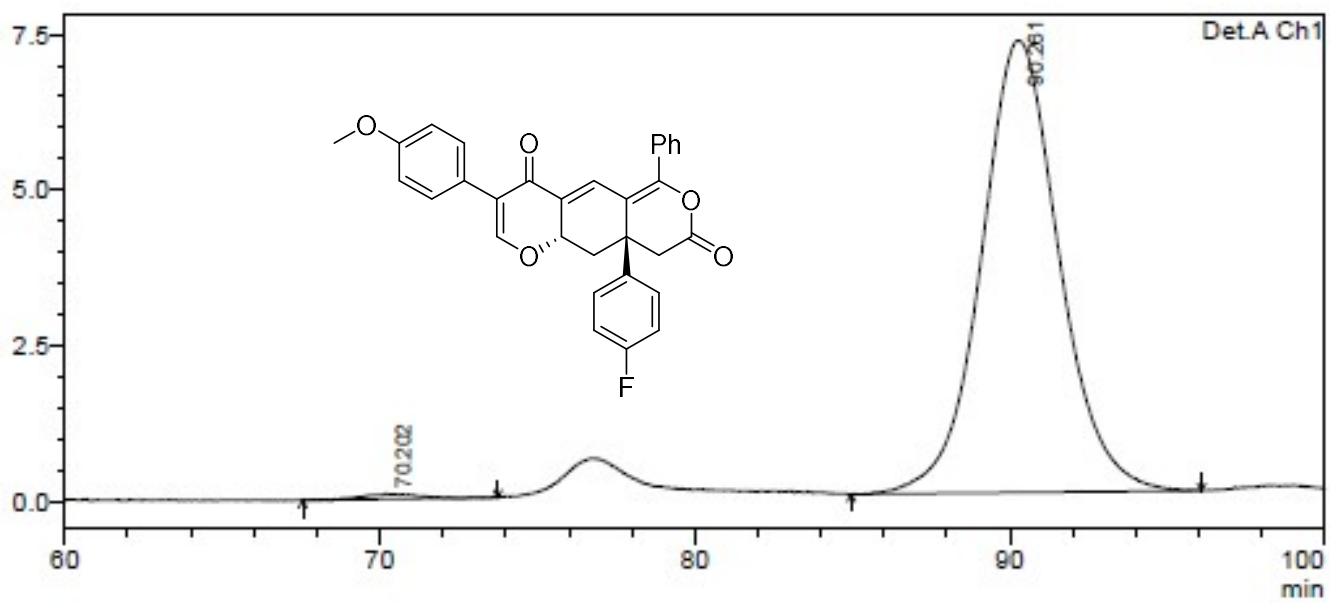

\begin{tabular}{|c|c|c|c|c|c|}
\hline \multicolumn{6}{|c|}{ Detector A Chl $254 \mathrm{~nm}$} \\
\hline Peak $=$ & Ret. Time & Area & Height & Area $\%$ & Height $\%$ \\
\hline 1 & 70.202 & 9497 & 87 & 0.756 & 1.188 \\
\hline 2 & 90.261 & 1246180 & 7253 & 99.244 & 98.812 \\
\hline Total & & 1255678 & 7341 & 100.000 & 100.000 \\
\hline
\end{tabular}




\section{Racemic 8d}

$\mathrm{mv}$

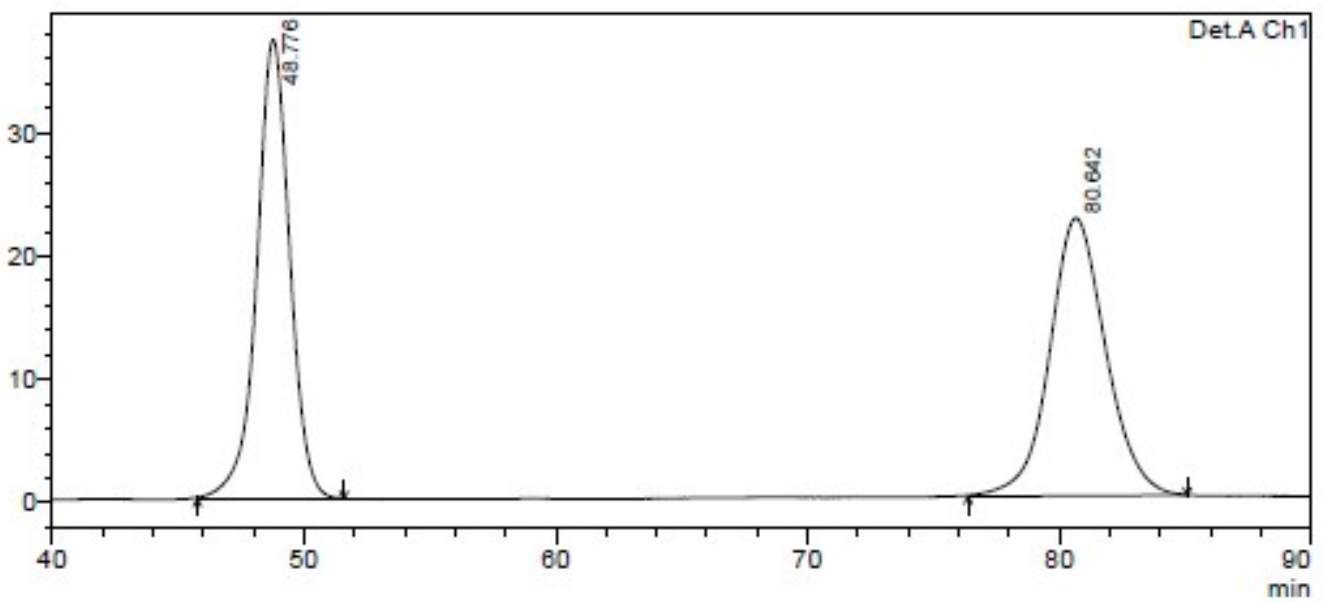

Detector A Ch1 254mm
\begin{tabular}{r|r|c|r|r|r|}
\hline \multicolumn{1}{|c|}{ Peak= $=$} & Ret. Time & Area & Height & Area \% & \multicolumn{1}{c|}{ Height \% } \\
\hline 1 & 48.776 & 3401122 & 37284 & 49.331 & 62.317 \\
\hline 2 & 80.642 & 3493409 & 22546 & 50.669 & 37.683 \\
\hline Total & & 6894531 & 59830 & 100.000 & 100.000 \\
\hline
\end{tabular}

\section{Enantioenriched 8d}

$\mathrm{mV}$

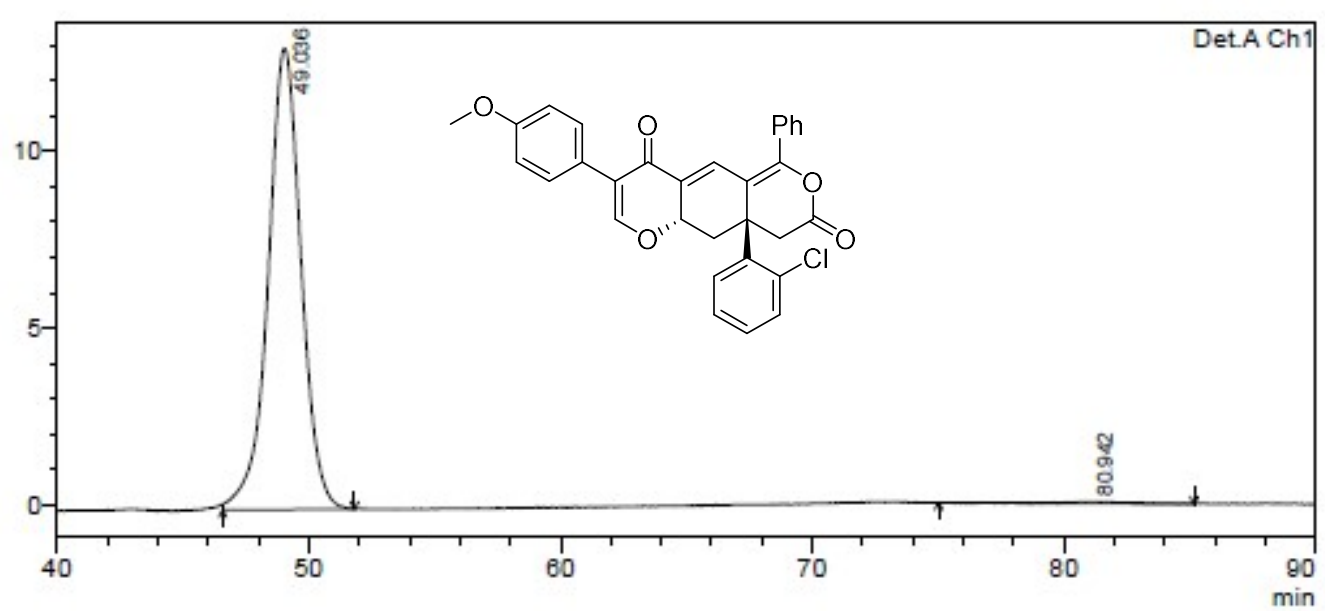

Detector A Chl 254nm

\begin{tabular}{|r|r|r|r|r|r|}
\hline Peak: & Ret. Time & \multicolumn{1}{|c|}{ Area } & Height & Area \% & \multicolumn{1}{c|}{ Height \% } \\
\hline 1 & 49.036 & 1191424 & 13051 & 99.476 & 99.674 \\
\hline 2 & 80.942 & 6271 & 43 & 0.524 & 0.326 \\
\hline Total & & 1197695 & 13094 & 100.000 & 100.000 \\
\hline
\end{tabular}




\section{Racemic 8e}

$\mathrm{mV}$

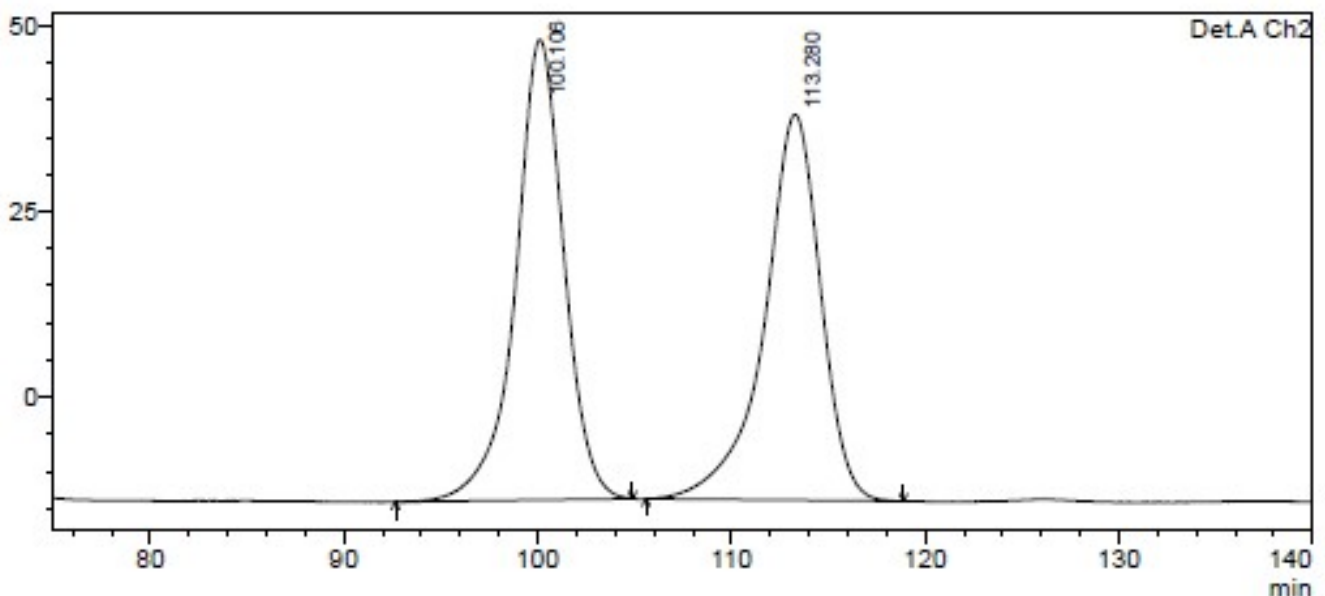

\begin{tabular}{|c|c|c|c|c|c|}
\hline \multicolumn{6}{|c|}{ Detector A Ch2 220 am } \\
\hline Peak $=$ & Ret. Time & Area & Height & Area $\%$ & Height \% \\
\hline 1 & 100.106 & 10744258 & 61961 & 50.590 & 54.396 \\
\hline 2 & 113.280 & 10493675 & 51946 & 49.410 & 45.604 \\
\hline Total & & 21237933 & 113906 & 100.000 & 100.000 \\
\hline
\end{tabular}

\section{Enantioenriched 8e}

$\mathrm{mV}$

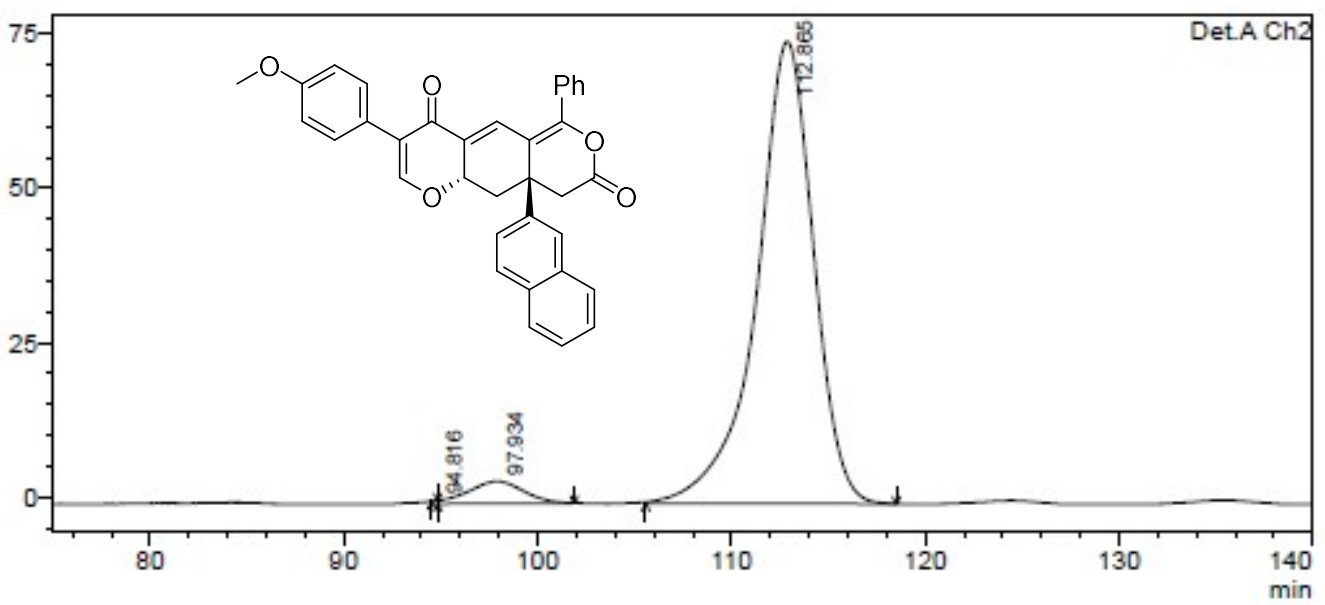

PeakTable

\begin{tabular}{|c|c|c|c|c|c|}
\hline \multicolumn{6}{|c|}{ Detector A Ch2 220mm } \\
\hline Pealk $=$ & Ret. Time & Area & Height & Area $\%$ & Height \% \\
\hline 1 & 100.106 & 10744258 & 61961 & 50.590 & 54.396 \\
\hline 2 & 113.280 & 10493675 & 51946 & 49.410 & 45.604 \\
\hline Total & & 21237933 & 113906 & 100.000 & 100.000 \\
\hline
\end{tabular}




\section{Racemic 8f}

$\mathrm{mV}$

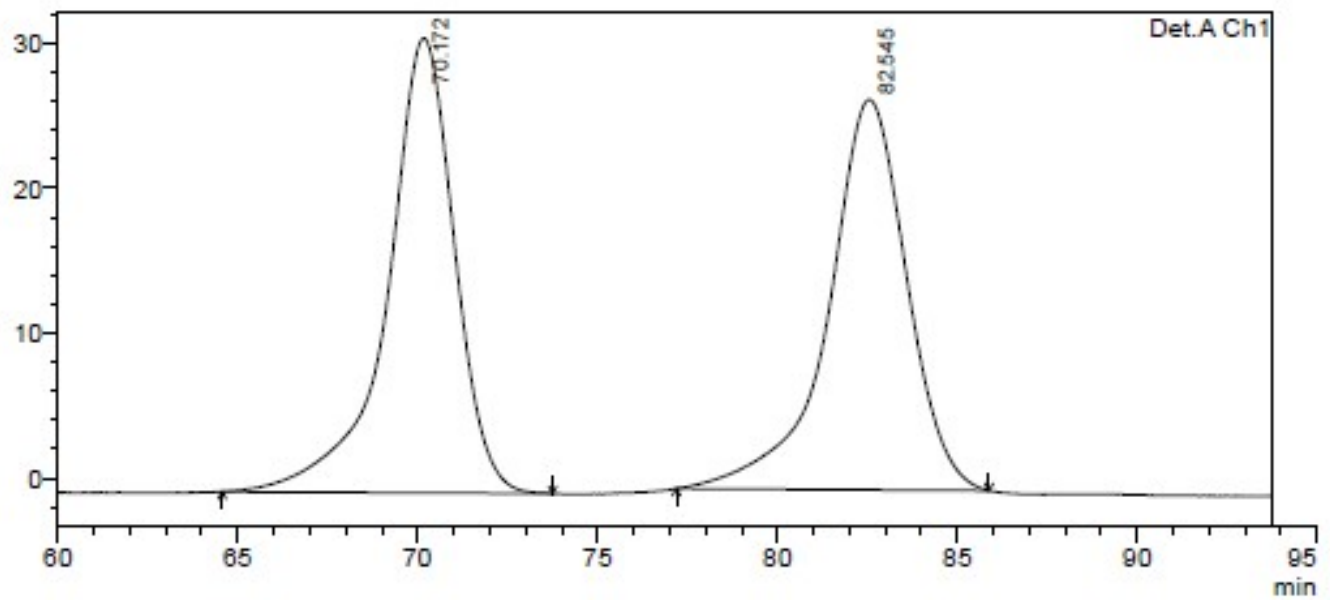

\begin{tabular}{|c|c|c|c|c|c|}
\hline \multicolumn{6}{|c|}{ Detector A Chl 254mm } \\
\hline Pealk \# & Ret. Time & Area & Height & Area \% & Height \% \\
\hline 1 & 70.172 & 4074180 & 31372 & 49.871 & 53.825 \\
\hline 2 & 82.545 & 4095178 & 26913 & 50.129 & 46.175 \\
\hline Total & & 8169358 & 58285 & 100.000 & 100.000 \\
\hline
\end{tabular}

\section{Enantioenriched $8 f$}

$\mathrm{mV}$

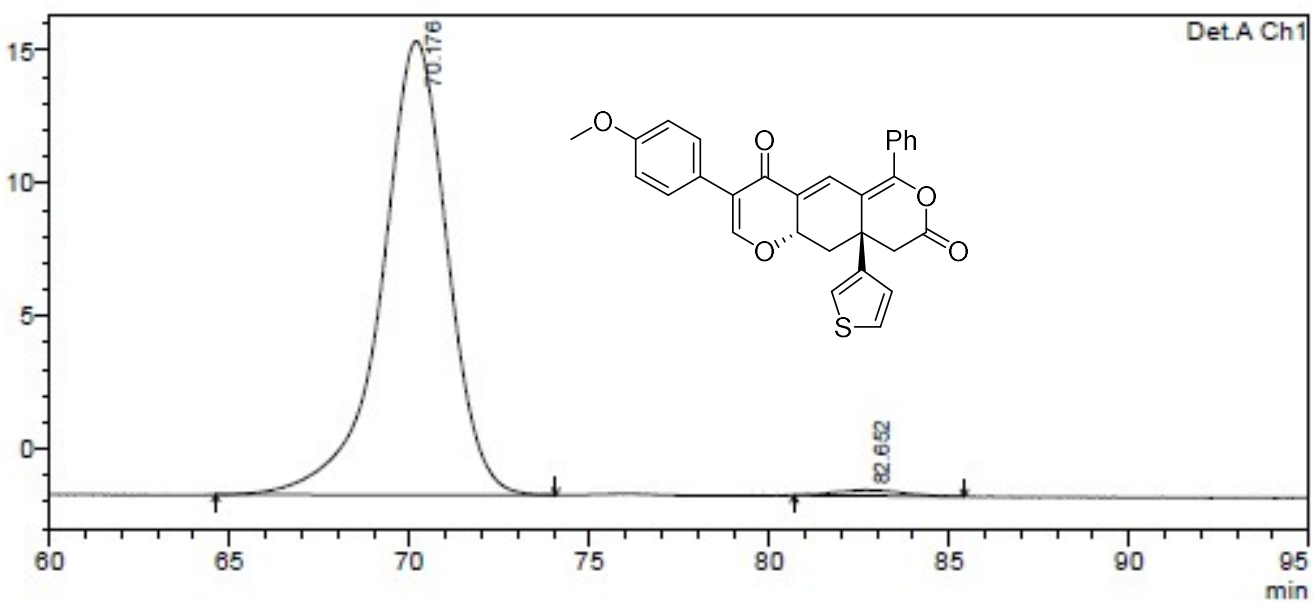

PeakTable

Detector A Chl 254nm
\begin{tabular}{|r|r|r|r|r|r|}
\hline Peak\#\# & Ret. Time & \multicolumn{1}{|c|}{ Area } & Height & Area \% & Height \% \\
\hline 1 & 70.176 & 2172901 & 17089 & 98.726 & 98.709 \\
\hline 2 & 82.652 & 28036 & 224 & 1.274 & 1.291 \\
\hline Total. & & 2200937 & 17312 & 100.000 & 100.000 \\
\hline
\end{tabular}




\section{Racemic 8g}

$\mathrm{mV}$

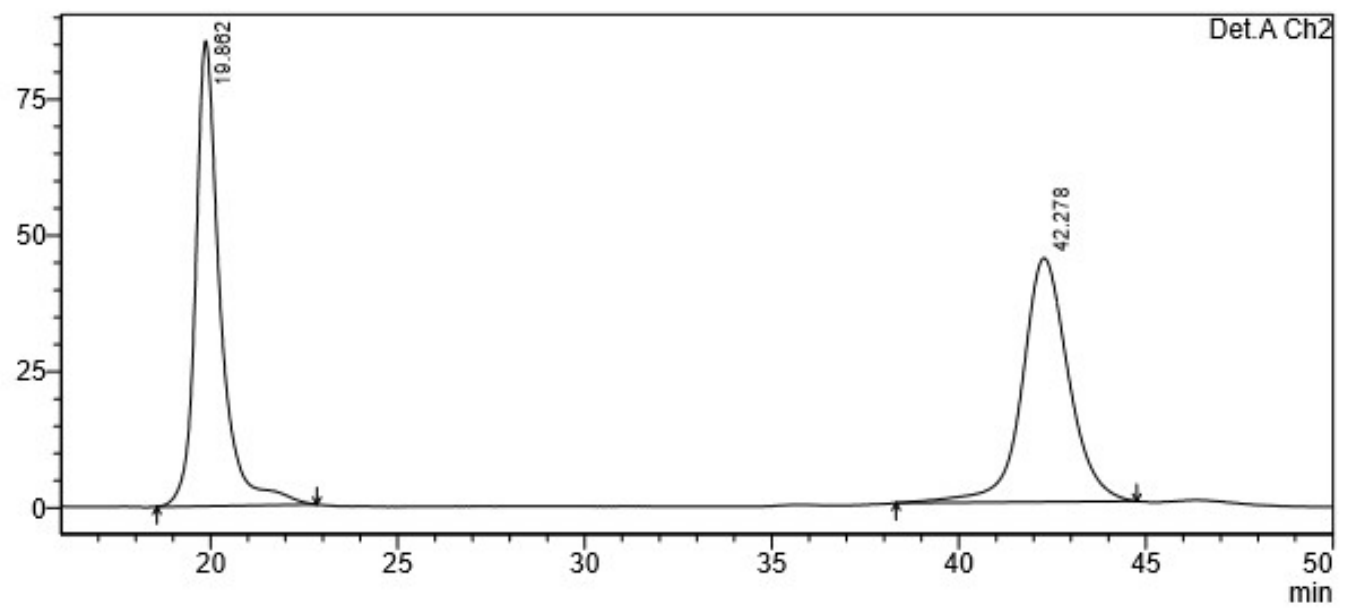

\begin{tabular}{|c|c|c|c|c|c|}
\hline \multicolumn{6}{|l|}{ Detector } \\
\hline Peak\# & Ret. Time & Area & Height & Area \% & Height \% \\
\hline 1 & 19.862 & 3914597 & 85413 & 50.328 & 65.615 \\
\hline 2 & 42.278 & 3863580 & 44761 & 49.672 & 34.385 \\
\hline Total & & 7778177 & 130173 & 100.000 & 100.000 \\
\hline
\end{tabular}

\section{Enantioenriched 8g}

$\mathrm{mV}$

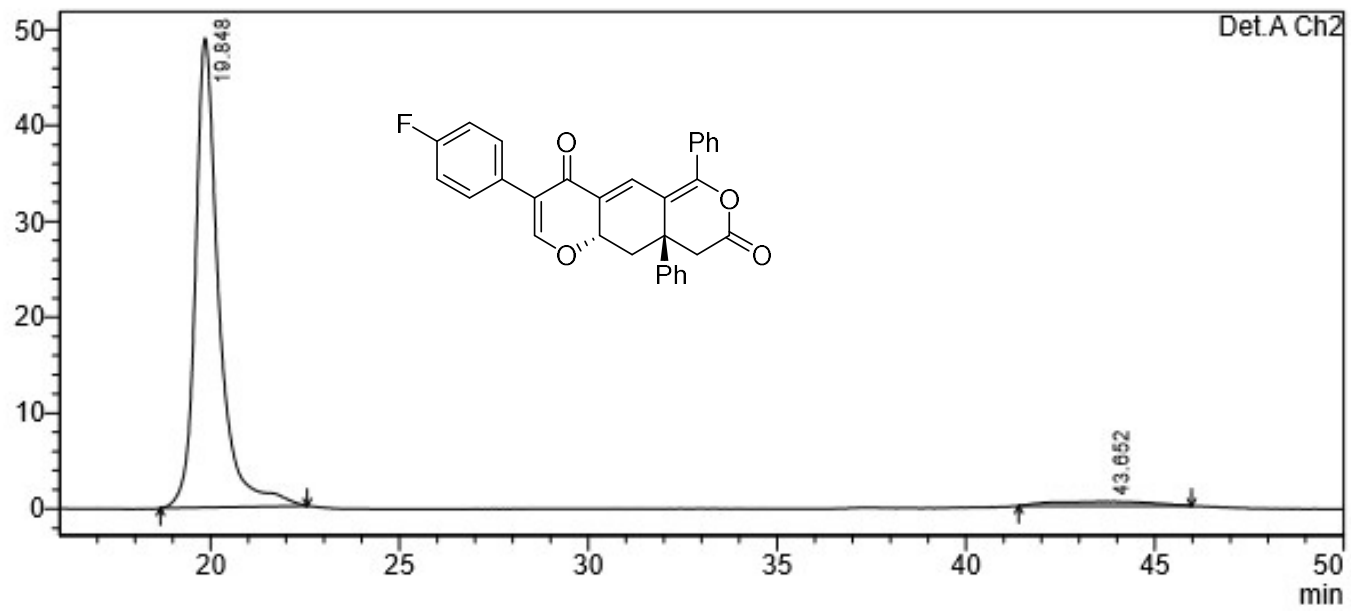

PeakTable

Detector A Ch2 220nm

\begin{tabular}{|r|r|r|r|r|r|}
\hline PeakF & Ret. Time & \multicolumn{1}{|c|}{ Area } & Height & Area \% & \multicolumn{1}{c|}{ Height \% } \\
\hline 1 & 19.848 & 2122048 & 49007 & 96.341 & 99.130 \\
\hline 2 & 43.652 & 80593 & 430 & 3.659 & 0.870 \\
\hline Total & & 2202641 & 49437 & 100.000 & 100.000 \\
\hline
\end{tabular}




\section{Racemic 8h}

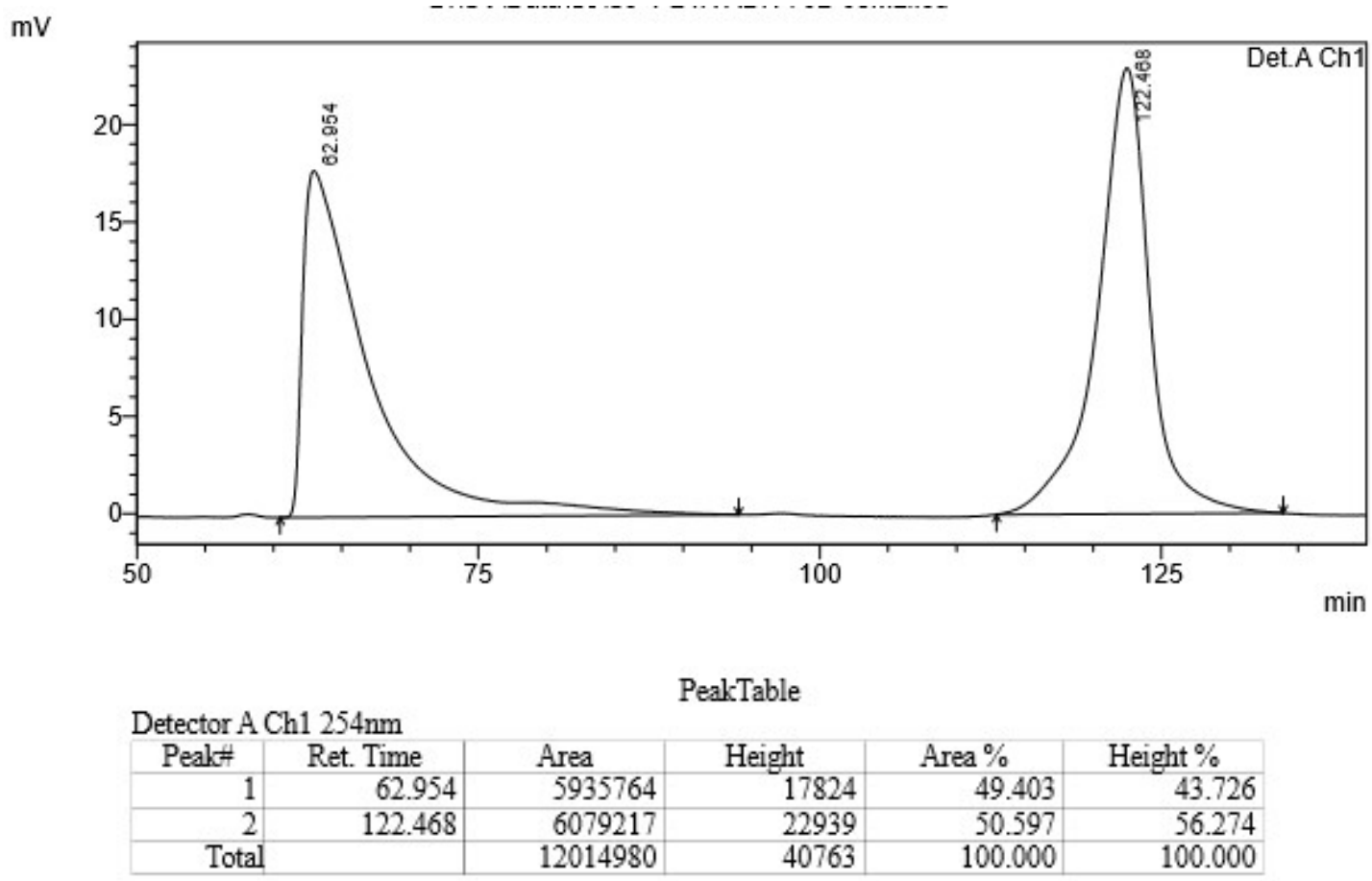

\section{Enantioenriched 8h}

$\mathrm{mV}$

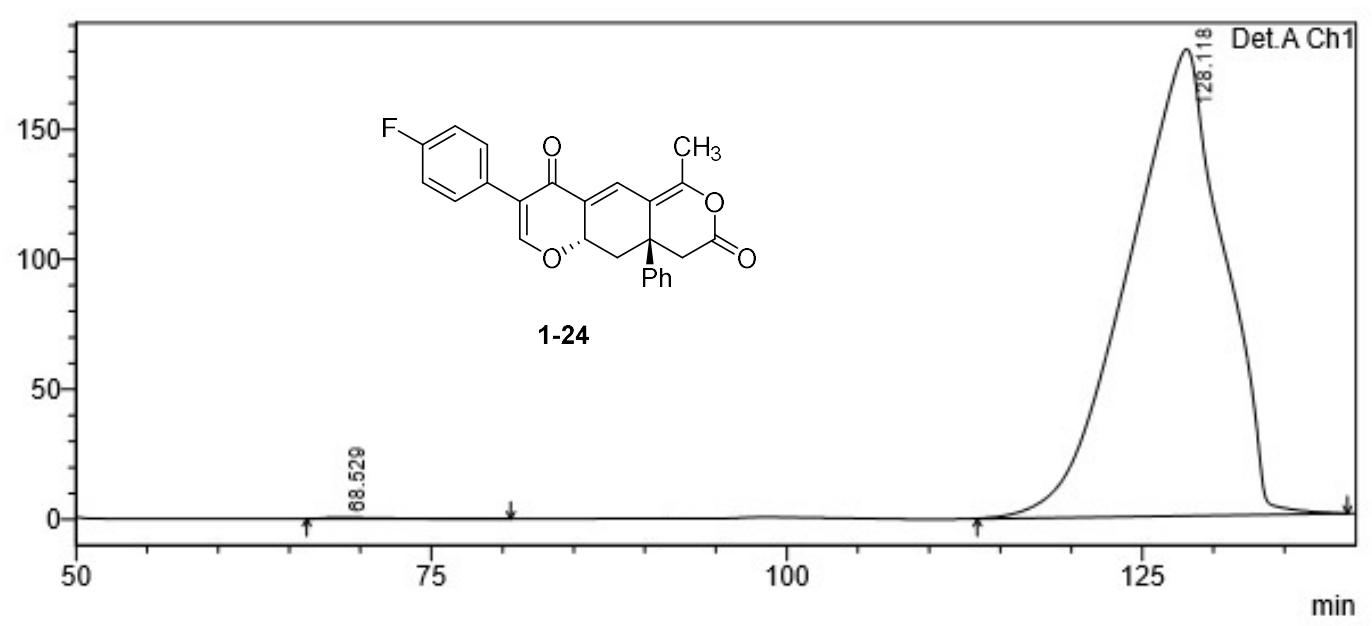

\begin{tabular}{|c|c|c|c|c|c|}
\hline \multicolumn{6}{|c|}{ cTable } \\
\hline Peak\# & Ret. Time & Area & Height & Area $\%$ & Height \% \\
\hline 1 & 68.529 & 149379 & 478 & 0.178 & 0.266 \\
\hline 2 & 128.118 & 83536774 & 179480 & 99.822 & 99.734 \\
\hline Total & & 83686153 & 179958 & 100.000 & 100.000 \\
\hline
\end{tabular}


Racemic 8i

$\mathrm{mV}$

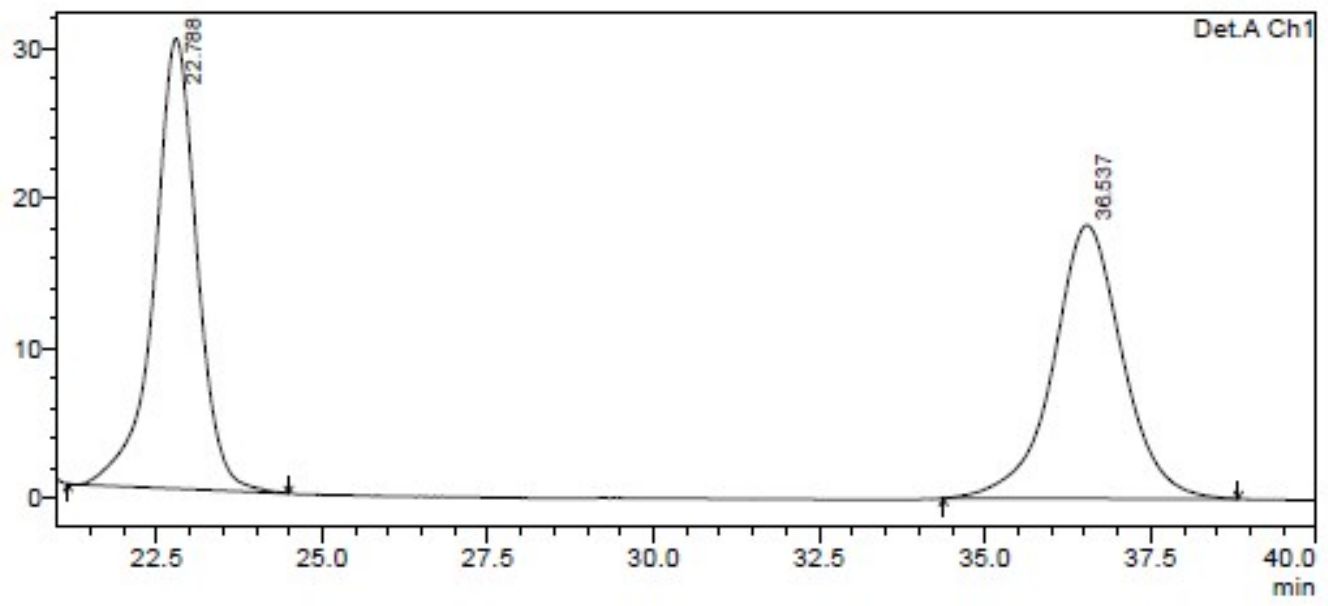

\begin{tabular}{|c|c|c|c|c|c|}
\hline Detector A & \multicolumn{5}{|c|}{ PeakTable } \\
\hline Peak\#\# & Ret. Time & Area & Height & Area \% & Height \% \\
\hline 1 & 22.788 & 1353877 & 30037 & 50.478 & 62.216 \\
\hline 2 & 36.537 & 1328253 & 18242 & 49.522 & 37.784 \\
\hline Total & & 2682130 & 48279 & 100.000 & 100.000 \\
\hline
\end{tabular}

\section{Enantioenriched 8i}

$\mathrm{mV}$

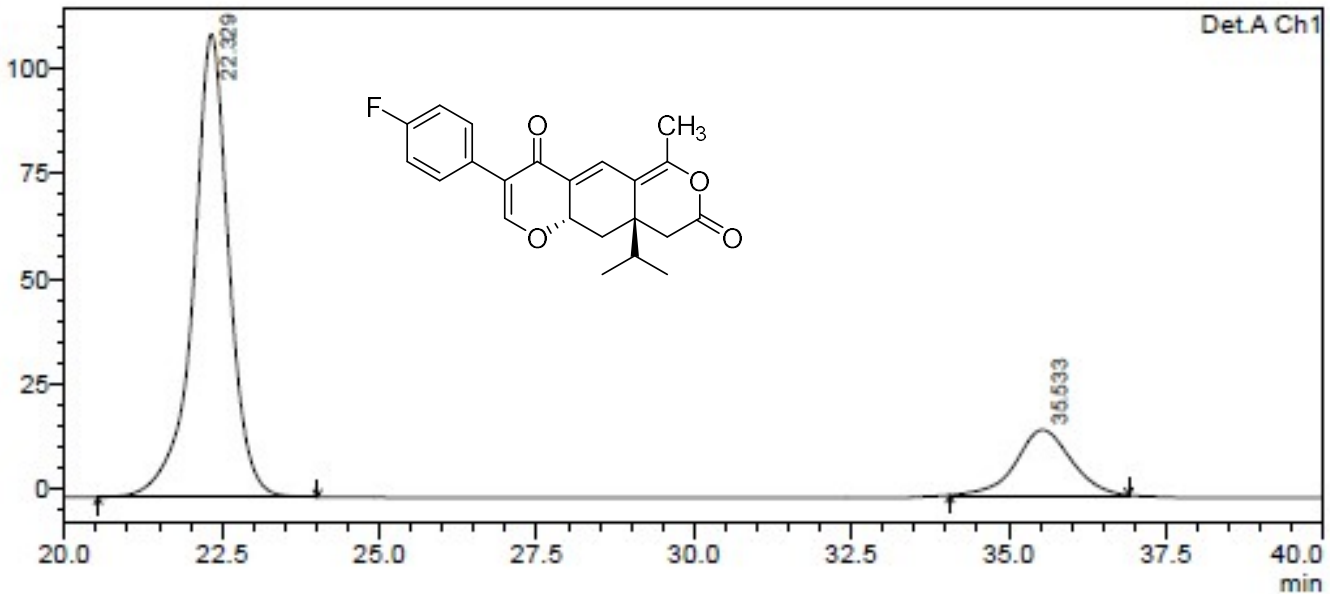

\begin{tabular}{|c|c|c|c|c|c|}
\hline \multicolumn{6}{|c|}{ Detector A Chl $254 \mathrm{~nm}$} \\
\hline Peak $\#$ & Ret. Time & Area & Height & Area $\%$ & Height \% \\
\hline 1 & 22.329 & 4331565 & 109767 & 82.073 & 87.631 \\
\hline 2 & 35.533 & 946165 & 15493 & 17.927 & 12.369 \\
\hline Total & & 5277730 & 125260 & 100.000 & 100.000 \\
\hline
\end{tabular}




\section{3s HRMS}

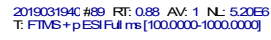

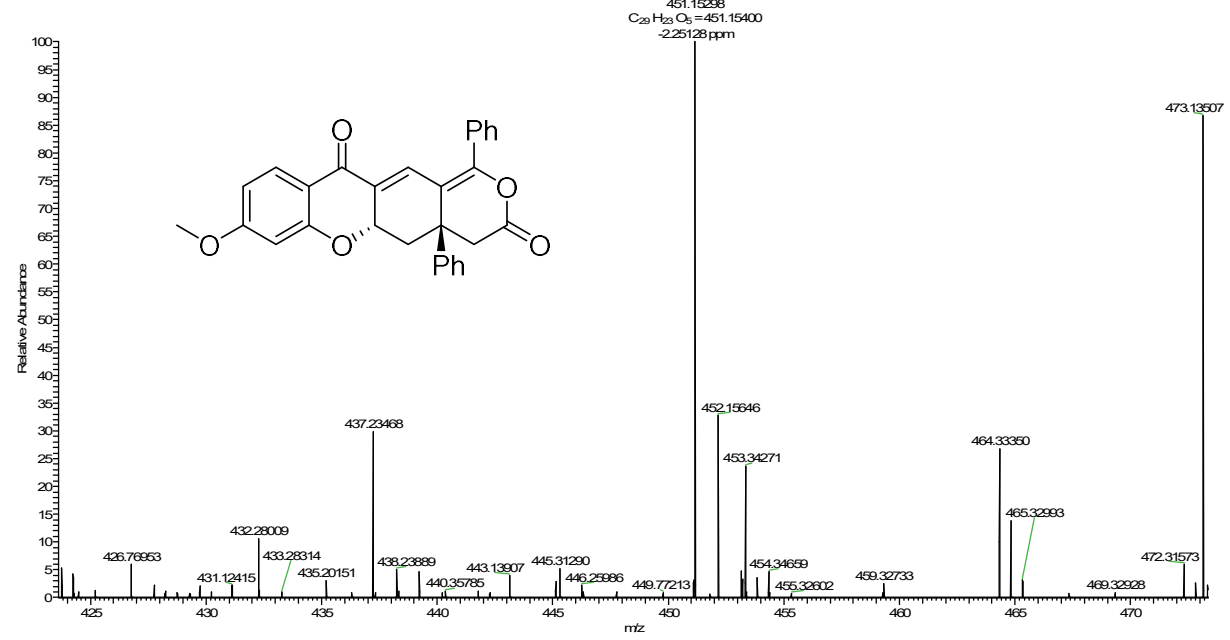

\section{3s' HRMS}

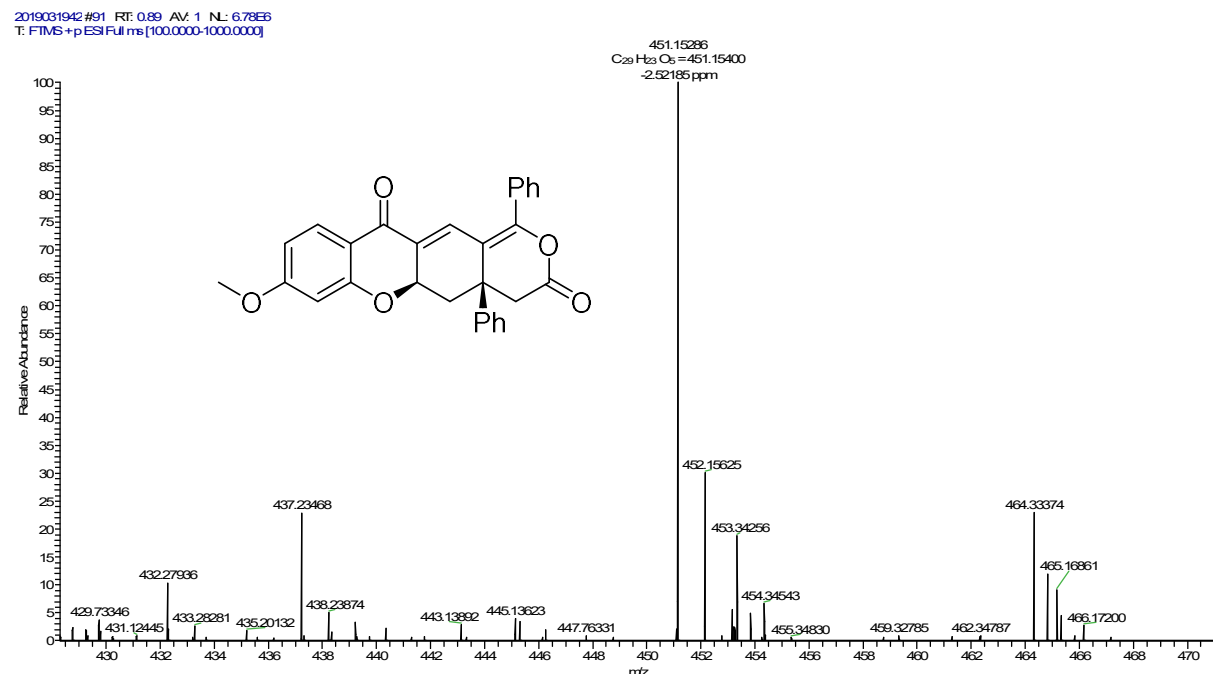

\section{3w HRMS}

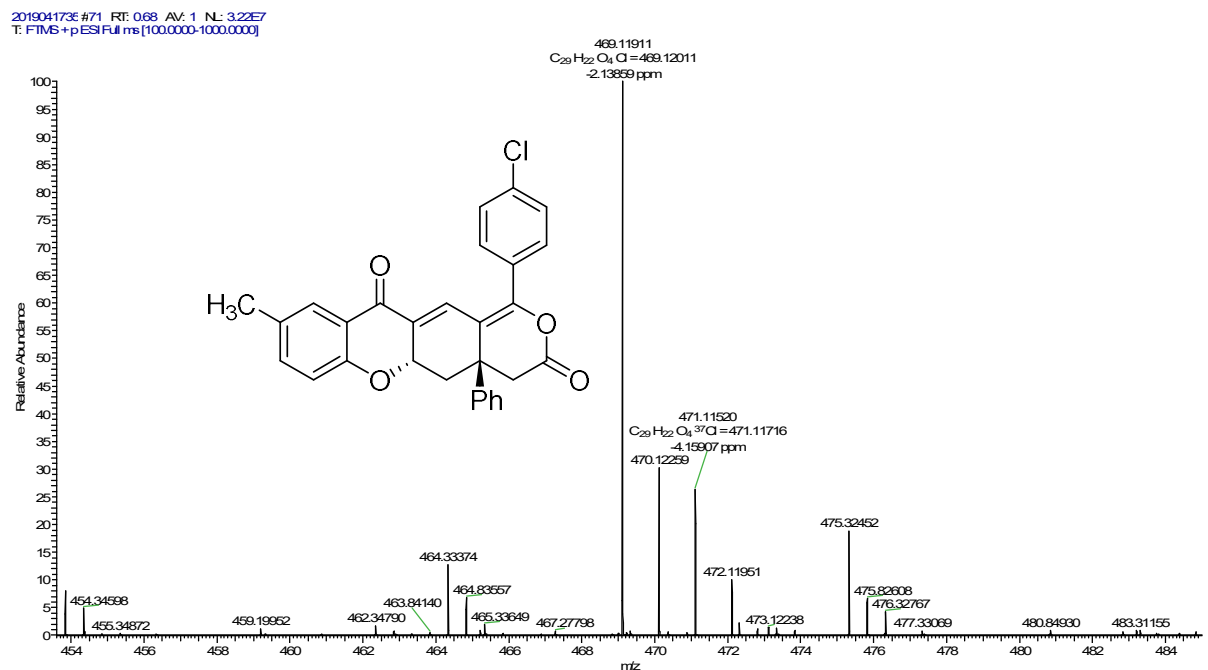

